\title{
A General Duality Theory for Clones
}

\section{DisSERTATION}

zur Erlangung des akademischen Grades

Doctor rerum naturalium

(Dr. rer. nat.)

vorgelegt

der Fakultät Mathematik und Naturwissenschaften

der Technischen Universität Dresden

von

Dipl.-Math. Sebastian Kerkhoff

geboren am 01. Juli 1982 in Gronau (Westf.)

Gutachter: $\quad$ Prof. Dr. rer. nat. habil. Reinhard Pöschel (TU Dresden)

Prof. Dr. Dragan Mašulović (University of Novi Sad)

Eingereicht am: $\quad$ 07. April 2011

Tag der Disputation: 28. Juni 2011 



\section{Abstract}

In this thesis, we generalize clones (as well as their relational counterparts and the relationship between them) to categories. Based on this framework, we introduce a general duality theory for clones and apply it to obtain new results for clones on finite sets. 



\section{Acknowledgements}

First and foremost, I have to thank Reinhard Pöschel. To put it short and simple, I could not have asked for a better supervisor. He gave me a topic that I enjoyed working on, he always found the time to discuss my ideas, and his sincere interest in my work was a huge motivation. Besides all that, working with him was also a lot of fun.

I also thank Dragan Mašulović from the University of Novi Sad. I profited very much from the e-mail correspondence with him and the talks we had on the rare occasions we have met in person were enjoyable, informative and inspiring.

Furthermore, I want to express my gratitude towards the Institute of Algebra at my university. Working at this place was and still is a pleasure every single day. I feel nothing but privileged that I can leave the house every morning to go to a work about which I like almost everything, surrounded by people I enjoy spending time with. In such an inspiring, entertaining and exceedingly friendly environment, writing a thesis becomes as easy as it gets.

Moreover, I thank Cynthia Glodeanu for proof-reading drafts of my thesis again and again, although she had more than enough work to do herself.

Finally, I thank all the people that believed in me and supported me. Among them, I am particularly indebted to my parents, Erika and Reinhold. I am grateful for everything they have done for me and for everything they are still doing for me. 


\section{Contents}

\begin{tabular}{lr}
\hline Introduction & 8
\end{tabular}

$\begin{array}{ll}\text { 1. Preliminaries } & 11\end{array}$

1.1. Structures . . . . . . . . . . . . . . . . . . . . . . . 11

1.2. Clones over Sets . . . . . . . . . . . . . . . . . . . . . 13

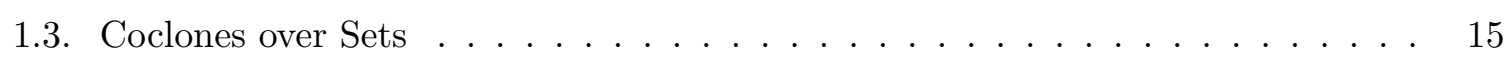

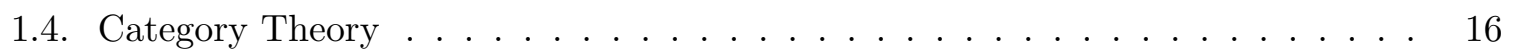

1.5. Abstract Clones and Lawvere Theories . . . . . . . . . . . . . . . . . 23

1.6. Natural Dualities . . . . . . . . . . . . . . . . . . . . . . . 24

\begin{tabular}{lr}
\hline 2. Clones of Operations & 27
\end{tabular}

2.1. Operations and their Clones . . . . . . . . . . . . . . . . 28

2.2. Clones over Objects vs. Abstract Clones and Lawvere Theories . . . . . . . . . 31

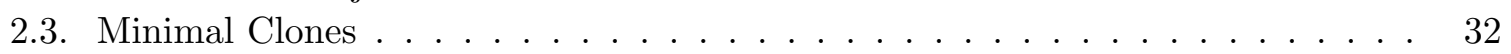

3. Clones of Dual Operations 35

3.1. Dual Operations and their Clones . . . . . . . . . . . . . . . . . . . . . 35

3.2. Minimal Clones . . . . . . . . . . . . . . . . . . . . . 38

3.3. Clones of Dual Operations and Abstract Clones . . . . . . . . . . . . . . 38

\begin{tabular}{|lr}
\hline 4. A Duality for Clones & 40
\end{tabular}

4.1. From Dual Equivalences to Dualizing Clones . . . . . . . . . . . . . . . 40

4.2. Dualizing Clones over Structures . . . . . . . . . . . . . . . . . . . . . 44

4.2.1. Clones over Distributive Lattices . . . . . . . . . . . . . . . . . . 48

4.2.2. Clones over Boolean Algebras . . . . . . . . . . . . . . . . . . 53

4.2.3. Clones over Median Algebras . . . . . . . . . . . . . . . . . 55

$4.2 .4 . \quad$ Clones over Boolean Rings . . . . . . . . . . . . . . . . . . 59

4.2.5. Clones over Boolean Lattices . . . . . . . . . . . . . . . . . . . . . . . . . . . . 62

4.2.6. Clones over Boolean Groups. . . . . . . . . . . . . . . . . 64

4.2.7. Clones over Semilattices . . . . . . . . . . . . . . . . . . . 70

4.2.8. Summary of Examples . . . . . . . . . . . . . . . . . 76

$\begin{array}{ll}\text { 5. Generalizing and Dualizing Pol-Inv } & \mathbf{7 7}\end{array}$

5.1. A General Galois Theory for Operations and Relations in Categories . . . . . . 78

5.1.1. Generalized Relations . . . . . . . . . . . . . . . . . . 78

5.1.2. The Generalized Galois Connection $\mathrm{Pol}_{\mathbf{A}}-\operatorname{Inv}_{\mathbf{A}}^{\mathbb{T}}$. . . . . . . . . . . . 81

5.1.3. A Remark on the Choice of $\mathbb{T}$. . . . . . . . . . . . . . . . . . . . 91

5.1.4. Pol $_{\mathbf{A}}-\operatorname{Inv}_{\mathbf{A}}^{\mathbb{T}}$ in Concrete Categories . . . . . . . . . . . . . . . 92 
5.2. A General Galois Theory for Dual Operations and Dual Relations . . . . . . . 94

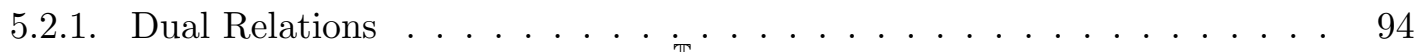

5.2.2. The Galois Connection $\overline{\mathrm{Pol}}_{\mathbf{X}}-\overline{\operatorname{Inv}}_{\mathbf{X}}^{T} \ldots \ldots \ldots$. . . . . . . . . . . . . . . . . 95

5.2.3. $\overline{\mathrm{Pol}}_{\mathrm{X}}-\overline{\mathrm{Inv}}_{\mathbf{X}}^{\mathbb{1}}$ in Concrete Categories . . . . . . . . . . . . . . . . . . . . . . . . 99

5.3. Completing the Duality for Clones . . . . . . . . . . . . . . . 100

$5.4 . \quad$ Example . . . . . . . . . . . . . . . . . . . . . 106

6. Clones of Dual Operations in Concrete Categories 112

6.1 . Respecting the Images of the Injection Morphisms . . . . . . . . . . . . . . . . 112

6.2. The Essential Arity of Clones of Dual Operations . . . . . . . . . . . . . . . . . 114

$6.3 . \quad$ Identities of Clones of Dual Operations . . . . . . . . . . . . . . . . . 118

6.4. Dual Idempotent Operations . . . . . . . . . . . . . . . . . . . . . . . . . 119

6.5. Minimal Clones . . . . . . . . . . . . . . . . . . . . . . 123

$\begin{array}{ll}\text { 7. Applying the Duality for Clones over Sets } & 125\end{array}$

7.1. Respecting the Images of the Injection Morphisms and Clones over Structures . 126

7.2. The Essential Arity of Clones over Structures . . . . . . . . . . . . . . . . 129

7.2.1. The Essential Arity of Clones over Boolean Algebras . . . . . . . . . . . 130

7.2.2. The Essential Arity of Clones over Boolean Rings . . . . . . . . . . . . . . 130

7.2.3. The Essential Arity of Clones over Median Algebras . . . . . . . . . . . 130

7.3. Identities of Clones over Structures . . . . . . . . . . . . . . . . . . . 132

7.4. Idempotent Operations . . . . . . . . . . . . . . . . . . . 133

7.4.1. The Duals of Idempotent Operations over Boolean Groups . . . . . . . 135

7.4.2. The Duals of Idempotent Operations over Semilattices . . . . . . . . . . 137

7.5. Minimal Clones . . . . . . . . . . . . . . . . . . . . . . . . . . 139

7.5.1. The Minimal Clones over Boolean Groups . . . . . . . . . . . . . . . . . 141

7.5.2. The Minimal Clones over Semilattices . . . . . . . . . . . . . . . . . 144

8. The Lattice of Clones over Distributive Lattices 146

8.1. Three Cases of Distributive Lattices . . . . . . . . . . . . . . . . . . . 149

8.2. The Essential Arity of Operations in $O_{\mathbf{A}} \ldots \ldots \ldots \ldots \ldots \ldots$

8.3. Idempotent Operations in $O_{\mathbf{A}} \ldots \ldots \ldots \ldots \ldots \ldots \ldots$

8.4. $\quad$ 0-Homomorphisms and 1-Homomorphisms over A $\ldots \ldots \ldots$

8.5. Unary Clones and Clones Containing End A . . . . . . . . . . . . 165

8.6. Minimal Clones . . . . . . . . . . . . . . . . . . . . . . . 168

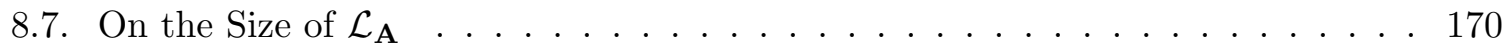

$\begin{array}{ll}\text { 9. Remarks and Conclusion } & 175\end{array}$

9.1. Clones in Quasi-Categories . . . . . . . . . . . . . . . . . . 175

9.2. Clones with Constants . . . . . . . . . . . . . . . . . . . 175

9.3. Further Applications for Clones over Sets . . . . . . . . . . . . . . 176

\begin{tabular}{ll}
\hline A. Appendix & 177
\end{tabular}

$\begin{array}{ll}\text { Nomenclature } & 182\end{array}$ 


\section{Introduction}

The aim of this thesis is to introduce a general duality theory for clones.

A clone is a set of (finitary) operations on a set $A$ that is closed under composition and contains all the projection mappings. The interest in clones is driven by the fact that clones represent the behaviour of algebras. Roughly speaking, if one understands all clones on a finite set $A$, one understands all algebras on $A$. However, as long as $A$ contains at least three elements, very little is known about the structure of all clones on $A$, despite intensive research for several decades.

Each clone also has a relational side: Built on the notion of invariance between operations and relations, a general Galois theory for operations and relations leads to the "most basic Galois connection in algebra" [MMT87, Pol-Inv, whose Galois closed classes are local closures of the clones and local closures of so-called clones of relations.

The principle of Duality is "a very pervasive and important concept in (modern) mathematics" Haz95] and "an important general theme that has manifestations in almost every area of mathematics" GBGL08. However, for clones, a general duality theory seems to be non-existent. When it comes to dualizing clones, the usual approach is to consider a clone as a term algebra and then try to prove or disprove that the algebra is dualizable. Another approach was introduced by D. MAŠUlović in [Maš06], where clones are dualized by treating them as sets of homomorphisms in a quasivariety that is then understood and dualized as a category. Although some results were obtained by using this duality, the approach has a major drawback: It only works for finite centralizer clones, and only a tiny fraction of clones are centralizer clones. Furthermore, the approach does not give us any information about what happens to the Galois connection Pol-Inv and the relational side of a clone. Nonetheless, Maš06] is the foundation of this thesis. Everything we obtain is based on the idea of treating (and dualizing) clones as sets of morphisms in a category.

After the preliminaries, the actual work starts in Chapter 2 where we will build our framework by introducing the notion of clones over objects in arbitrary categories, merging clones over sets and abstract clones into one concept. As we will see, all results that hold for abstract clones and many results that hold for clones over sets can be generalized to this framework. Among the latter is the Galois connection Pol-Inv. In Chapter 5, we will present a general Galois theory for operations over objects and something that we will introduce as the category-theoretic generalization of relations. This will lead us to the Galois connection $\operatorname{Pol}_{\mathbf{A}}-\operatorname{Inv}_{\mathbf{A}}^{\mathbb{T}}$, where the Galois closed classes are characterized by local closures of the clones of operations and local closures of what we will introduce as clones of relations. This Galois connection is applicable in any category and will coincide with Pol-Inv in the category of sets. Consequently, even if we treat clones at the most abstract level, which has proven itself to be advantageous in several situation, we can 
still use (almost) all the techniques that Pol-Inv provides for clones over sets.

Furthermore, the framework allows us to apply techniques from the field of category theory, such as the Duality Principle. As outlined in Chapter 3 , dualizing our notion of clones of operations gives rise to the notion of clones of dual operations, which generalizes the notion of so-called coclones. By the nature of the Duality Principle, this means that each result that holds for a clone of operations has a dual counterpart for a clone of dual operations and vice versa. As a consequence, it is no longer necessary to treat clones and coclones as if they were essentially different concepts. In fact, with the duality theory obtained in this thesis, several proofs that have been done for coclones become obsolete as they simply become the dual of a result that holds for clones of operations. For instance, instead of introducing a Galois theory between cofunctions and so-called corelations as done in PR00, we can simply dualize our generalized Galois connection $\mathrm{Pol}_{\mathbf{A}}-\operatorname{Inv}_{\mathbf{A}}^{\mathbb{T}}$ and obtain a general Galois theory for dual operations and what we will call dual relations. This Galois theory will then coincide with the one introduced in [PR00] if the underlying category is the category of sets.

However, the Duality Principle itself only establishes a duality theory for clones at the most abstract level. It allows us to dualize any clone of operations in a category $\mathcal{A}$ to a clone of dual operations in the category $\mathcal{A}^{o p}$ (the so-called opposite category, obtained from $\mathcal{A}$ by reversing the direction of each morphism). But $\mathcal{A}^{o p}$ is a rather abstract concept, and it is by no means clear how to interpret it. In practice, one wants to dualize a clone of operations to a clone of dual operations that is as easy as possible. In Chapter 4, we will show how to dualize a clone of operations over some object $\mathbf{A}$ in a category $\mathcal{A}$ to a clone of dual operations over some object $\mathbf{X}$ in any category $\mathcal{X}$ that is dually equivalent to $\mathcal{A}$. In particular, we will discuss how to dualize clones over sets, and we will illustrate this technique with several examples. As one of the main results of this thesis, we will obtain the following theorem:

Theorem 4.2.1. For each clone $C$ over a finite set $A$, there exists a category of finite structures $\mathcal{X}$ and some $\mathbf{X} \in \mathcal{X}$ such that the order-ideal $\langle C]$ is isomorphic to the lattice of clones of dual operations over $\mathbf{X}$.

In other words, we will be able to dualize any clone over a finite set to the full clone of dual operations over a finite structure (for clones over infinite sets we will still be able to formulate a slightly weaker result). Thus, we can study clones over sets by examining their duals - for which we also have the general Galois theory for dual operations and dual relations. This is exactly what we will do in Chapters 6 to 8 , where the application of our duality theory will yield several new results for clones over sets.

In Chapter 6, we will study clones of dual operations in concrete categories in a very general way. We will see that the structure of the copowers of the object $\mathbf{X}$ gives us a remarkable lot of information about the clones of dual operations over $\mathbf{X}$. For instance, under some assumptions on the copowers of $\mathbf{X}$, which will be satisfied in many wellknown categories, we will be able to characterize all minimal clones of dual operations over $\mathbf{X}$.

In Chapter 7, we will apply the results from the previous chapter by dualizing them back to clones of operations. In particular, we will take the examples we introduced in 
Chapter 4 and see what each result from Chapter 6 means for these particular clones.

Chapter 8 will serve as an illustration of how to use the clone duality to obtain some very specific, in-depth results about parts of the lattice of clones over sets. We will take clones over finite distributive lattices and examine the order-ideals generated by them. Equipped with the clone duality and the Galois theory for dual operations and dual relations, we will repeatedly see how our new techniques yield a surprising shortcut to the solution of a problem.

We will conclude with some remarks and suggestions for further research in the final Chapter 9 . 


\section{Preliminaries}

We assume that the reader is familiar with the basic notions of universal algebra. Therefore, we will omit the introduction of well-known algebraic terminology. For the reader who might need an introduction to universal algebra, we refer to standard texts such as [BS81, Grä79], MMT87].

For the whole thesis, we will denote by $\mathbb{N}_{+}$the set of natural numbers not including 0 .

\subsection{Structures}

The notion of a structure generalizes algebras, partial algebras and relational structures.

1.1.1 Definition. Let $X$ be a set, let $F$ be a set of finitary (total) operation symbols, let $H$ be a set of finitary partial operation symbols, and let $R$ be a set of finitary relation symbols. A structure of type $\langle F, H, R\rangle$ is a structure

$$
\mathbf{X}=\left\langle X, F^{\mathbf{x}}, H^{\mathbf{x}}, R^{\mathbf{x}}\right\rangle,
$$

where

(i) $F^{\mathbf{X}}$ consists of an $n$-ary (total) operation $f^{\mathbf{X}}: X^{n} \rightarrow X$ for each $n$-ary (total) operation symbol $f \in F$,

(ii) $H^{\mathbf{X}}$ consists of an $n$-ary partial operation $h^{\mathbf{x}}: \operatorname{dom}\left(h^{\mathbf{x}}\right) \rightarrow X$ for each $n$-ary partial operation symbol $h \in H$,

(iii) $R^{\mathbf{X}}$ consists of an $n$-ary relation $r^{\mathbf{X}} \subseteq X^{n}$ for each $n$-ary relation symbol $r \in R$.

The set $X$ is called the universe or carrier set of $\mathbf{X}$.

By abuse of notation, we will sometimes write $\mathbf{X}$ when we actually refer to the underlying set of $\mathbf{X}$. For instance, we write $x \in \mathbf{X}$ to indicate that $x$ is in the carrier set of the structure $\mathbf{X}$, and we will sometimes refer to the cardinality of the carrier set of the structure $\mathbf{X}$ by $|\mathbf{X}| \cdot 1$

Note that a structure of type $\langle F, \emptyset, \emptyset\rangle$ is an algebra and that a structure of type $\langle\emptyset, \emptyset, R\rangle$ is a so-called relational structure. Any structure of type $\langle F, \emptyset, R\rangle$ (that is, a structure without partial operations) is called a total structure.

\footnotetext{
${ }^{1}$ The reason for this is to avoid confusion when we refer to coproducts or products of a structure. If we always used the convention of using bold letters for structures and normal letters for their underlying carrier set, the carrier set of $\mathbf{X}^{n}$ would be denoted by $X^{n}$. However, the carrier set of $\mathbf{X}^{n}$ is not necessarily the $n$-th Cartesian power of the set $X$, so confusion would arise. The other (and formally correct) option would be to use the forgetful functor, but this would be at the expense of notational simplicity.
} 
1.1.2 Definition. A structure $\mathbf{Y}=\left\langle Y, F^{\mathbf{Y}}, H^{\mathbf{Y}}, R^{\mathbf{Y}}\right\rangle$ is called a substructure of a structure $\mathbf{X}=\left\langle X, F^{\mathbf{X}}, H^{\mathbf{X}}, R^{\mathbf{X}}\right\rangle$ provided that $Y \subseteq X$ and

(i) for each $n$-ary $f \in F$, the operations $f^{\mathbf{Y}}$ and $f^{\mathbf{X}}$ agree on $Y^{n}$,

(ii) for each $n$-ary $h \in H$, we have $\operatorname{dom}\left(h^{\mathbf{Y}}\right)=\operatorname{dom}\left(h^{\mathbf{X}}\right) \cap Y^{n}$ and $h^{\mathbf{Y}}$ and $h^{\mathbf{X}}$ agree on $\operatorname{dom}\left(h^{\mathbf{Y}}\right)$,

(iii) for each $n$-ary $r \in R$, we have $r^{\mathbf{Y}}=r^{\mathbf{X}} \cap Y^{n}$.

Note that, for a structure of type $(F, \emptyset, \emptyset)$, this definition coincides with the usual definition of a subalgebra.

1.1.3 Definition. Let $\mathbf{X}=\left\langle X, F^{\mathbf{X}}, H^{\mathbf{X}}, R^{\mathbf{X}}\right\rangle$ and $\mathbf{Y}=\left\langle Y, F^{\mathbf{Y}}, H^{\mathbf{Y}}, R^{\mathbf{Y}}\right\rangle$ be structures. A mapping $\varphi: X \rightarrow Y$ is said to be a homomorphism from $\mathbf{X}$ to $\mathbf{Y}$ provided that

(i) for each $n$-ary $f \in F$ and each $\left(x_{1}, \ldots, x_{n}\right) \in X^{n}$, we have

$$
\varphi\left(f^{\mathbf{X}}\left(x_{1}, \ldots, x_{n}\right)\right)=f^{\mathbf{Y}}\left(\varphi\left(x_{1}\right), \ldots, \varphi\left(x_{n}\right)\right),
$$

(ii) for each $n$-ary $h \in H$ and each $\left(x_{1}, \ldots, x_{n}\right) \in \operatorname{dom}\left(h^{\mathbf{x}}\right)$, we have

$$
\varphi\left(h^{\mathbf{X}}\left(x_{1}, \ldots, x_{n}\right)\right)=h^{\mathbf{Y}}\left(\varphi\left(x_{1}\right), \ldots, \varphi\left(x_{n}\right)\right),
$$

(iii) for each $n$-ary $r \in R$ and each $\left(x_{1}, \ldots, x_{n}\right) \in r^{\mathbf{X}}$, we have $\left(\varphi\left(x_{1}\right), \ldots, \varphi\left(x_{n}\right)\right) \in r^{\mathbf{Y}}$.

For $\operatorname{dom}(h) \subseteq X$, a mapping $h: \operatorname{dom}(h) \rightarrow Y$ is a partial homomorphism from $\mathbf{X}$ to $\mathbf{Y}$ if the above conditions are satisfied for all $\left(x_{1}, \ldots, x_{n}\right) \in \operatorname{dom}(h)$.

In other words, a mapping $\varphi: X \rightarrow Y$ is a homomorphism whenever $\varphi$ preserves each member of $r$ and the graph of each member of $F \cup H$ (we will see in Definition 1.2.6 what it means that an operation preserves a relation).

For a class $\mathcal{K}$ of structures of the same type, we denote by $\mathbb{H}(\mathcal{K}), \mathbb{I}(\mathcal{K}), \mathbb{S}(\mathcal{K})$ and $\mathbb{P}(\mathcal{K})$ the class of homomorphic images, isomorphic images, non-empty substructures and (Cartesian) products of structures from $\mathcal{K}$, respectively. Furthermore, we denote by $\mathbb{S}^{0}(\mathcal{K})$ the class of all substructures (including the empty one if $F$ contains no nullary operation symbols), and we denote by $\mathbb{P}^{+}(\mathcal{K})$ the class of all products of structures from $\mathcal{K}$ over a non-empty index set.

1.1.4 Definition. For a class $\mathcal{K}$ of structures, the class $\mathbb{I S P}(\mathcal{K})$ is called the quasivariety generated by $\mathcal{K}$.

A class of structures $\mathcal{K}$ of the same type is a quasivariety if and only if there exists a set of quasi-identities $\mathcal{E}$ such that $\mathcal{K}$ is the class of all models of $\mathcal{E}$.

Sometimes, we will equip a structure with a topology. In this case, we denote by $\mathbb{S}_{c}(\mathcal{K})$ the class of topologically closed substructures of structures from $\mathcal{K}$. 


\subsection{Clones over Sets}

In this section, we will outline the rudimentary basics of clone theory. For a further reading, we refer to [PK79] and [Sze86].

Until the end of this section, let $A$ be a non-empty finite set. For $n \in \mathbb{N}_{+}$, denote by $O_{A}^{(n)}$ the set of all $n$-ary operations over $A$. Let $O_{A}:=\bigcup_{n \in \mathbb{N}_{+}} O_{A}^{(n)}$ and, for a set of operations $F \subseteq O_{A}$, set $F^{(n)}:=F \cap O_{A}^{(n)}$. Moreover, for $f \in O_{A}$, we denote by $\operatorname{ar}(f)$ the arity of $f$. Note that $O_{A}$ does not contain nullary operations (i.e., $\operatorname{ar}(f) \geq 1$ for all $\left.f \in O_{A}\right)$.

1.2.1 Definition. A subset $C \subseteq O_{A}$ is a called a clone (or clone of operations) if it contains all the projection mappings

$$
\pi_{i}^{k}: A^{k} \rightarrow A:\left(x_{1}, \ldots, x_{k}\right) \mapsto x_{i}
$$

and is closed with respect to superposition of operations in the following sense: For $f \in O_{A}^{(n)}$ and $f_{1}, \ldots, f_{n} \in O_{A}^{(k)}$, the $k$-ary operation $f\left(f_{1}, \ldots, f_{n}\right)$, defined by setting

$$
f\left(f_{1}, \ldots, f_{n}\right)\left(x_{1}, \ldots, x_{k}\right):=f\left(f_{1}\left(x_{1}, \ldots, x_{k}\right), \ldots, f_{n}\left(x_{1}, \ldots, x_{k}\right)\right),
$$

is also in $C$.

For each $F \subseteq O_{A}$, there is a least clone containing $F$. We denote this clone by $\operatorname{Clo}(F)$, and we say that $F$ generates $\operatorname{Clo}(F)$. For operations $f_{1}, \ldots, f_{k}$, we briefly write $\mathrm{Clo}\left(f_{1}, \ldots, f_{k}\right)$ instead of $\operatorname{Clo}\left(\left\{f_{1}, \ldots, f_{k}\right\}\right)$. Note that if $C \subseteq O_{\mathbf{A}}$ is a clone generated by a set of operations $F$, then $C$ can be interpreted as the term algebra of $\langle A, F\rangle$.

We also define the so-called expanded superposition of operations, where $f_{1}, \ldots, f_{n}$ do not need to have the same arity.

1.2.2 Definition. Let $f$ be an $n$-ary operation and $f_{1}, \ldots, f_{n}$ operations over $A$ where $k_{i}$ is the arity of $f_{i}$. The expanded superposition of $f$ and $f_{1}, \ldots, f_{n}$ is the $\left(\sum_{i=1}^{n} k_{i}\right)$-ary operation

$$
f\left(f_{1}\left(x_{1}, \ldots, x_{k_{1}}\right), f_{2}\left(x_{k_{1}+1}, \ldots, x_{k_{1}+k_{2}}\right), \ldots, f_{n}\left(x_{\left(\sum_{i=1}^{n-1} k_{i}\right)+1}, \ldots, x_{\sum_{i=1}^{n} k_{i}}\right)\right) .
$$

Clones are closed under expanded superposition. Indeed, it is justified to say that clones are closed under all meaningful compositions.

1.2.3 Definition. The $i$-th argument of an $n$-ary operation $f$ is said to be non-essential if $f\left(x_{1}, \ldots, x_{n}\right) \approx f\left(x_{1}, \ldots, x_{i-1}, y, x_{i+1}, \ldots, x_{n}\right)$. An argument is called essential if it is not nonessential. Moreover, an operation is said to be essentially $k$-ary if it has exactly $k$ essential arguments.

It is easy to see that the clones over a set $A$ form a lattice.

1.2.4 Definition. Denote by $L_{A}$ the set of clones over $A$. Then, $\mathcal{L}_{A}:=\left\langle L_{A}, \subseteq\right\rangle$ is called the clone lattice over $A$ or the lattice of clones over $A$. 


\section{Preliminaries}

Clearly, $O_{A}$ is the greatest element of the clone lattice, while the least clone is the clone $J_{A}$ that contains only the projections. On a one-element set, we have $O_{A}=J_{A}$, so the clone lattice is trivial. On a two-element set, there are countably many clones, and the lattice was completely described by E. Post in [Pos41]. However, for $|A| \geq 3$, there are continuum many clones, and a full description of these lattices seems to be hopeless, even for $|A|=3$.

Some clones are of particular interest. For example, it is an interesting problem to determine all minimal clones in a given clone lattice.

1.2.5 Definition. Let $\mathcal{L}$ be a lattice of clones. A clone $C \in \mathcal{L}$ is said to be minimal in $\mathcal{L}$ if $C$ is an atom of $\mathcal{L}$. An operation $f$ is called minimal if $\operatorname{Clo}(f)$ is a minimal clone and $f$ has minimum arity among all operations that generate $\mathrm{Clo}(f)$.

Evidently, each minimal clone is uniquely described by any minimal operation that it contains. All minimal operations over $A$ and, consequently, all minimal clones in $\mathcal{L}_{A}$ are only known for $|A| \leq 3$, described in Csá83. For $|A| \geq 4$, only partial results are known (see [Csá05] or Qua95 for a survey on minimal clones).

We will now see that there is a correspondence between clones of operations and certain sets of relations.

We denote by $R_{A}^{(n)}$ the set of all $n$-ary relations on the set $A$. Moreover, we set $R_{A}:=\bigcup_{n \in \mathbb{N}_{+}} O_{A}^{(n)}$ and, for a set of relations $R \subseteq R_{A}$, we set $R^{(n)}:=R \cap R_{A}^{(n)}$.

1.2.6 Definition. An operation $f \in O_{A}^{(n)}$ is said to preserve a relation $\sigma \in R_{A}^{(k)}$, written $f \triangleright \sigma$, if

$$
\left(\begin{array}{c}
\nu_{11} \\
\nu_{12} \\
\vdots \\
\nu_{1 k}
\end{array}\right), \ldots,\left(\begin{array}{c}
\nu_{n 1} \\
\nu_{n 2} \\
\vdots \\
\nu_{n k}
\end{array}\right) \in \sigma \Longrightarrow\left(\begin{array}{c}
f\left(\nu_{11}, \nu_{21}, \ldots, \nu_{n 1}\right) \\
f\left(\nu_{12}, \nu_{22}, \ldots, \nu_{n 2}\right) \\
\vdots \\
f\left(\nu_{1 k}, \nu_{2 k}, \ldots, \nu_{n k}\right)
\end{array}\right) \in \sigma .
$$

In terms of algebras, an $n$-ary relation $\sigma$ is preserved by $C$ if and only if it forms a subalgebra of $\langle A, F\rangle^{n}$.

1.2.7 Definition. For $F \subseteq O_{A}$ and $R \subseteq R_{A}$, define

$$
\begin{aligned}
& \operatorname{Inv} F:=\left\{\sigma \in R_{A} \mid \forall f \in F: f \triangleright \sigma\right\}, \\
& \operatorname{Pol} R:=\left\{f \in O_{A} \mid \forall \sigma \in R: f \triangleright \sigma\right\} .
\end{aligned}
$$

Furthermore, let $\operatorname{Inv}^{(m)} F:=(\operatorname{Inv} F)^{(m)}$ and define $\mathrm{Pol}^{(m)} R$ analogously.

Pol-Inv is a Galois connection between operations and relations, where the Galois closed classes of operations are the clones of operations and the Galois closed classes of relations are so-called clones of relations.

1.2.8 Definition. A relation $\sigma \in R_{A}^{(n)}$ is called a diagonal relation on $A$ if there exists an equivalence relation $\theta \subseteq A \times A$ with

$$
\sigma=\left\{\left(\begin{array}{c}
a_{1} \\
\vdots \\
a_{n}
\end{array}\right) \mid \forall(i, j) \in \theta: a_{i}=a_{j}\right\} .
$$


A clone of relations on $A$ is a set of relations $R \subseteq R_{A}$ that contains all diagonal relations and is closed under direct (Cartesian) product and under permutation and identification of components.

It is a direct consequence that clones of relations on $A$ form a lattice with respect to inclusion and that this lattice is dual to the clone lattice $\mathcal{L}_{A}$. In the case $|A|=\infty$, the Galois closed classes of Pol-Inv are local closures of clones of operations and clones of relations, and the lattices formed by these locally closed sets are the ones that are dually isomorphic. For more details on Pol-Inv, we refer to [Pös79] and [Pös80].

There is a special kind of operation that has, among other properties, an important consequence for the Galois connection Pol-Inv.

1.2.9 Definition. For $n \geq 3$, an $n$-ary operation $f$ is called a near-unanimity operation provided that

$$
f(y, x, \ldots, x) \approx f(x, y, x, \ldots, x) \approx \ldots \approx f(x, \ldots, x, y) \approx x .
$$

1.2.10 Theorem (Baker-Pixley [BP75]). Let $C$ be a clone that has a $(k+1)$-ary near-unanimity operation. Then Pol Inv ${ }^{(k)} C=\operatorname{Pol} \operatorname{Inv} C$.

From the Baker-Pixley Theorem, one can easily deduce that clones which contain a near-unanimity operation are finitely generated and have only finitely many subclones. As we will see later, the Baker-Pixley Theorem also effects duality theory.

Before we end this section, we will introduce an important example of clones of operations, namely the so-called centralizer clones.

1.2.11 Definition. Let $\mathbf{A}$ be an algebra. The centralizer clone of $\mathbf{A}$ is the set of all homomorphisms from finite non-empty powers of $\mathbf{A}$ to $\mathbf{A}$. A clone $C$ is said to be a clone over the algebra $\mathbf{A}$ if it is a subclone of the centralizer clone of $\mathbf{A}$.

It was shown in BW87 that there are only finitely many centralizer clones over a finite set.

\subsection{Coclones over Sets}

Coclones as introduced in this section are often understood as the dual notion of a clone. Although we will later argue that this understanding can be somewhat misleading, it is justified in the sense that the notion of a coclone is obtained from the notion of a clone by reversing the direction of the operations and by replacing the products by coproducts.

1.3.1 Definition. Let $X$ be a non-empty set. An $n$-ary dual operation (or cooperation or cofunction) over $X$ is a mapping from $X$ to $n \cdot X:=\{\langle i, x\rangle \mid i \in\{1, \ldots, n\}, x \in X\}$. Denote the set of all $k$-ary dual operations over $X$ by $\bar{O}_{X}^{(k)}$ and set $\bar{O}_{X}:=\bigcup_{k \in \mathbb{N}_{+}} \bar{O}_{X}^{(k)}$.

For $g_{1}, \ldots, g_{n} \in \bar{O}_{X}^{(k)}$, we define $\left[g_{1}, \ldots, g_{n}\right]$ to be the mapping

$$
\left[g_{1}, \ldots, g_{n}\right]: n \cdot X \rightarrow k \cdot X:\langle i, x\rangle \mapsto g_{i}(x) .
$$

The following notion of a coclone was introduced by B. CsÁKÁNY in [Csá85]: 
1.3.2 Definition. A subset $C \subseteq \bar{O}_{X}$ is called a coclone if it contains all the injection mappings

$$
\iota_{i}^{k}: X \rightarrow k \cdot X: x \mapsto\langle i, x\rangle
$$

and is closed with respect to superposition of dual operations in the following sense: For $g \in \bar{O}_{X}^{(n)}$ and $g_{1}, \ldots, g_{n} \in \bar{O}_{X}^{(k)}$, the $k$-ary mapping $\left[g_{1}, \ldots, g_{n}\right] \circ g$ is also in $C$.

Coclones over a set $X$ form a lattice that is finite whenever $X$ is finite. This lattice was studied in [Csá85, Szé89, Drb71, Maš99].

Also, a Galois connection cPol-cInv between sets of dual operations over some, not necessarily finite, set $X$ and sets of so-called corelations on $X$ was introduced in [PR00]. Analogue to Pol-Inv, the Galois closed classes of cPol-cInv can be characterized as local closures of coclones and local closures of so-called clones of corelations, where the local closure operators can be omitted in the case of $X$ being finite.

\subsection{Category Theory}

In this section, we will turn to the field of category theory and introduce the notions, terminology and facts needed for our upcoming task. For additional information and the omitted proofs of the statements given in this section, we refer to [AHS90] and [Bor94.

In the following, we will always assume the Axiom of Choice for classes (that is, every collection of non-empty classes has a choice function).

1.4.1 Definition. Let $\mathcal{A}$ be a class consisting of:

- a class of objects,

- a set $\mathcal{A}(\mathbf{A}, \mathbf{B})$ for each pair of objects $\mathbf{A}, \mathbf{B}$, called the morphisms from $\mathbf{A}$ to $\mathbf{B}$,

- a binary operation $\circ$, called composition, on the class of all morphisms of $\mathcal{A}$ such that $u \in \mathcal{A}(\mathbf{A}, \mathbf{B})$ and $v \in \mathcal{A}(\mathbf{B}, \mathbf{C})$ implies $v \circ u \in \mathcal{A}(\mathbf{A}, \mathbf{C})$.

We write $\mathbf{A} \in \mathcal{A}$ to indicate that $\mathbf{A}$ is an object in $\mathcal{A}$, and $u: \mathbf{A} \rightarrow \mathbf{B}$ to mean that $u \in \mathcal{A}(\mathbf{A}, \mathbf{B})$. Then, $\mathcal{A}$ is a category if it satisfies the following axioms:

(i) For each object $\mathbf{A}$ of $\mathcal{A}$ there is a morphism $i d_{\mathbf{A}}: \mathbf{A} \rightarrow \mathbf{A}$, called the identity on $\mathbf{A}$, such that $i d_{\mathbf{A}} \circ u=u$ and $v \circ i d_{\mathbf{A}}=v$ for all $\mathbf{B}, \mathbf{C} \in \mathcal{A}$ and $u: \mathbf{B} \rightarrow \mathbf{A}, v: \mathbf{A} \rightarrow \mathbf{C}$,

(ii) for objects $\mathbf{A}, \mathbf{B}, \mathbf{C}, \mathbf{D}$ of $\mathcal{A}$ and morphisms $u: \mathbf{A} \rightarrow \mathbf{B}, v: \mathbf{B} \rightarrow \mathbf{C}, w: \mathbf{C} \rightarrow \mathbf{D}$, the equation $w \circ(v \circ u)=(w \circ v) \circ u$ holds (i.e., $\circ$ is associative).

Sometimes, we will write $\mathbf{B}^{\mathbf{A}}$ instead of $\mathcal{A}(\mathbf{A}, \mathbf{B})$.

The most obvious example for a category is the category Set that contains all sets as objects, all functions as morphisms and the usual composition. The following examples are also of particular interest for the content of this thesis:

\subsubsection{Examples.}

(i) Let $\mathbf{A}$ be a structure. The quasivariety $\mathcal{A}:=\mathbb{I S P}(\mathbf{A})$ can be interpreted as a category with the structures as objects, the homomorphisms between the structures as morphisms and the usual composition. 
(ii) Let $\mathbf{X}$ be a structure equipped with a topology. Then, $x:=\mathbb{I S}_{c}^{0} \mathbb{P}^{+}(\mathbf{X})$ can be interpreted as a category with the structures as objects and the continuous homomorphisms between them as morphisms. The composition is again the usual one.

1.4.3 Definition. A category $\mathcal{B}$ is called a subcategory of $\mathcal{A}$ if the objects of $\mathcal{B}$ form a subclass of the objects of $\mathcal{A}$ and $\mathcal{B}\left(\mathbf{B}_{1}, \mathbf{B}_{2}\right) \subseteq \mathcal{A}\left(\mathbf{B}_{1}, \mathbf{B}_{2}\right)$ for all $\mathbf{B}_{1}, \mathbf{B}_{2} \in \mathcal{B}$. A subcategory is called a full subcategory provided that $\mathcal{B}\left(\mathbf{B}_{1}, \mathbf{B}_{2}\right)=\mathcal{A}\left(\mathbf{B}_{1}, \mathbf{B}_{2}\right)$ for all $\mathbf{B}_{1}, \mathbf{B}_{2} \in \mathcal{B}$.

One kind of full subcategory will be of particular importance in this thesis: For a category $\mathcal{A}$ of structures, we denote by $\mathcal{A}_{\text {fin }}$ the full subcategory of finite objects among $\mathcal{A}$.

We will now introduce certain types of morphisms:

1.4.4 Definition. Let $u: \mathrm{A} \rightarrow \mathrm{B}$ be a morphism.

(i) $u$ is called an isomorphism if there exists a morphism $v: \mathbf{B} \rightarrow \mathbf{A}$ such that $v \circ u=i d_{\mathbf{A}}$ and $u \circ v=i d_{\mathbf{B}}$. Since $v$ is unique whenever it exists, we call it the inverse of $u$ and denote it by $u^{-1}$.

(ii) $u$ is said to be factorizable over an object $\mathbf{C} \in \mathcal{A}$ if there exist two morphisms $u_{1}: \mathbf{A} \rightarrow \mathbf{C}$ and $u_{2}: \mathbf{C} \rightarrow \mathbf{B}$ such that $u=u_{2} \circ u_{1}$.

(iii) $u$ is called a monomorphism if, for all morphisms $g_{1}, g_{2}: \mathbf{C} \rightarrow \mathbf{A}, u \circ g_{1}=u \circ g_{2}$ implies $g_{1}=g_{2}$.

(iv) $u$ is called an epimorphism if, for all morphisms $g_{1}, g_{2}: \mathbf{B} \rightarrow \mathbf{C}, g_{1} \circ u=g_{2} \circ u$ implies $g_{1}=g_{2}$.

Note that the notion of monomorphisms generalizes the notion of injective functions, while the notion of epimorphisms generalizes the notion of surjective functions. In Set, a morphism is a monomorphism if and only if it is injective and an epimorphism if and only if it is surjective. In other categories, however, monomorphisms and epimorphisms are not necessarily injective and surjective, respectively.

For two objects $\mathbf{A}, \mathbf{B}$, we write $\mathbf{A} \cong \mathbf{B}$ if there exists an isomorphism from $\mathbf{A}$ to $\mathbf{B}, \mathbf{A} \leqslant \mathbf{B}$ if there exists an epimorphism from $\mathbf{B}$ to $\mathbf{A}$ and $\mathbf{A}<\mathbf{B}$ if there exists a monomorphism from $\mathbf{A}$ to $\mathbf{B}$. In the category of sets (and many other categories), we have $\mathbf{A} \leqslant \mathbf{B}$ if and only if $\mathbf{A}<\mathbf{B}$. In general, however, this is not true.

1.4.5 Definition. A skeleton of a category is a full, isomorphism-dense subcategory in which no two distinct objects are isomorphic.

Since we assume the Axiom of Choice for classes, every category has a skeleton. For instance, in Set, the class of all cardinal numbers is a skeleton.

1.4.6 Definition. An object $\mathbf{A}$ is a retract of an object $\mathbf{B}$ if there exist morphisms $b: \mathbf{A} \rightarrow \mathbf{B}$ and $d: \mathbf{B} \rightarrow \mathbf{A}$ such that $d \circ b=i d_{\mathbf{A}}$.

It is easy to see that the morphisms $b$ and $d$ from this definition are a monomorphism and an epimorphism, respectively. 
1.4.7 Definition. Let $\left(\mathbf{A}_{i}\right)_{i \in I}$ be a family of objects in a category $\mathcal{A}$. An object $\mathbf{C}$ is a product of $\left(\mathbf{A}_{i}\right)_{i \in I}$ if there exists a collection of associated projection morphisms $\pi_{i}: \mathbf{C} \rightarrow \mathbf{A}_{i}$ such that, for each object $\mathbf{D} \in \mathcal{A}$ and each collection of morphisms $f_{i}: \mathbf{D} \rightarrow \mathbf{A}_{i}$, there is a unique morphism $u: \mathbf{D} \rightarrow \mathbf{C}$, called the tupling of $\left(f_{i}\right)_{i \in I}$, such that $\pi_{i} \circ u=f_{i}$ for all $i \in I$.

It is straightforward to check that products of objects, if they exist at all, are determined up to isomorphism. In practice, one usually considers one object (with its associated projection morphisms) to be the product and the other objects that fulfil the condition above to be isomorphic to the product. For instance, in the category Set, the Cartesian product (with the usual projection mappings) is considered to be the product.

For objects $\mathbf{A}_{1}, \ldots, \mathbf{A}_{n} \in \mathcal{A}$ such that the product of the family $\left(\mathbf{A}_{i}\right)_{i \in\{1, \ldots, n\}}$ exists, we write $\mathbf{A}_{1} \times \ldots \times \mathbf{A}_{n}$ for the product of $\mathbf{A}_{1}, \ldots, \mathbf{A}_{n}$, and for morphisms $f_{i}: \mathbf{D} \rightarrow \mathbf{A}_{i}$, we denote the tupling of $\left(f_{i}\right)_{i \in\{1, \ldots, n\}}$ by $\left\langle f_{1}, \ldots, f_{n}\right\rangle$. For $\mathbf{A}_{1}=\ldots=\mathbf{A}_{n}$, we write $\mathbf{A}^{n}$ instead of $\mathbf{A} \times \ldots \times \mathbf{A}$, and we denote by $\pi_{i}^{n}: \mathbf{A}^{n} \rightarrow \mathbf{A}$ the associated projection morphisms.

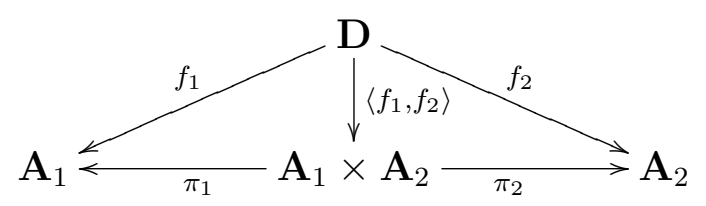

Figure 1.1.: The binary product

As explained above, when we write $\mathbf{A}_{1} \times \ldots \times \mathbf{A}_{n}$ or $\mathbf{A}^{n}$, we will refer to one specific object in the category and not to the class of all (isomorphic) objects that fulfil the condition of the definition above.

1.4.8 Definition. Call $\mathbf{A}^{n}$ the $n$-th power of $\mathbf{A}$, and call $\pi_{i}^{n}: \mathbf{A}^{n} \rightarrow \mathbf{A}$ the $i$-th projection on $\mathbf{A}$.

Note that, in categories different from Set, products can be very complicated. In fact, a category may not even have a product of any two of its objects.

The 0 -th power of $\mathbf{A}$ is defined to be a so-called terminal object.

1.4.9 Definition. An object $\mathbf{T} \in \mathcal{A}$ is said to be terminal (or final) if, for each $\mathbf{B} \in \mathcal{A}$, there is exactly one morphism from $\mathbf{B}$ to $\mathbf{T}$.

It is easy to see that terminal objects are unique up to isomorphism. In the category of sets, each one-element set is a terminal object. If a category does not contain a terminal object, then no object has a 0 -th power.

For working with tuplings, the following proposition is useful:

1.4.10 Proposition. Let $f_{i}: \mathbf{D} \rightarrow \mathbf{A}_{i}$ for $i \in\{1, \ldots, n\}$, and let $g: \mathbf{C} \rightarrow \mathbf{D}$. Then

$$
\left\langle f_{1} \circ g, \ldots, f_{n} \circ g\right\rangle=\left\langle f_{1}, \ldots, f_{n}\right\rangle \circ g .
$$


We will now extend the notion of a tupling.

1.4.11 Definition. For all $i \in\{1, \ldots, n\}$, let $k_{i} \in \mathbb{N}_{+}$and let $f_{i}: \mathbf{A}^{k_{i}} \rightarrow \mathbf{B}_{i}$. Furthermore, for $j \in\{1, \ldots, n-1\}$, set $m_{j}:=\sum_{i=1}^{j} k_{i}$ and $m:=\sum_{i=1}^{n} k_{i}$. The expanded tupling of $f_{1}, \ldots, f_{n}$ is the morphism from $\mathbf{A}^{m}$ to $\mathbf{B}_{1} \times \ldots \times \mathbf{B}_{n}$ defined as follows:

$$
\left\langle\left\langle f_{1}, \ldots, f_{n}\right\rangle\right\rangle:=\left\langle f_{1} \circ\left\langle\pi_{1}^{m}, \ldots, \pi_{m_{1}}^{m}\right\rangle, f_{2} \circ\left\langle\pi_{m_{1}+1}^{m}, \ldots, \pi_{m_{2}}^{m}\right\rangle, \ldots, f_{n} \circ\left\langle\pi_{m_{n-1}+1}^{m}, \ldots, \pi_{m}^{m}\right\rangle\right\rangle .
$$

The following proposition provides us with a useful equation:

1.4.12 Proposition. Let $f_{1}, \ldots, f_{n}$ and $m$ be as above. Then

$$
\left\langle\left\langle f_{1}, \ldots, f_{n}\right\rangle\right\rangle \circ\left\langle h_{1}, \ldots, h_{m}\right\rangle=\left\langle f_{1} \circ\left\langle h_{1}, \ldots, h_{m_{1}}\right\rangle, \ldots, f_{n} \circ\left\langle h_{m_{n-1}+1}, \ldots, h_{m}\right\rangle\right\rangle
$$

for all $h_{1}, \ldots, h_{m}: \mathbf{C} \rightarrow \mathbf{A}$.

Dual to the notion of the product is the notion obtained by reversing the directions of the morphisms in its definition.

1.4.13 Definition. Let $\left(\mathrm{A}_{i}\right)_{i \in I}$ be a family of objects in a category $\mathcal{A}$. An object $\mathbf{C}$ is a coproduct of $\left(\mathbf{A}_{i}\right)_{i \in I}$ if there exists a collection of associated injection morphisms $\iota_{i}: \mathbf{A}_{i} \rightarrow \mathbf{C}$ such that, for each object $\mathbf{D} \in \mathcal{A}$ and each collection of morphisms $f_{i}: \mathbf{A}_{i} \rightarrow \mathbf{D}$, there is a unique morphism $v: \mathbf{C} \rightarrow \mathbf{D}$, called the cotupling of $\left(f_{i}\right)_{i \in I}$, such that $v \circ \iota_{i}=f_{i}$ for all $i \in I$.

Again, coproducts of objects, if they exist at all, are determined up to isomorphism, but in practice, one usually considers one object (with its associated projection morphisms) to be the coproduct and the other objects that fulfil the condition above to be isomorphic to the coproduct. For instance, in the category Set, the disjoint union $\bullet$ (with the usual injection mappings) is considered to be the coproduct.

For objects $\mathbf{A}_{1}, \ldots, \mathbf{A}_{n} \in \mathcal{A}$ such that the coproduct of the family $\left(\mathbf{A}_{i}\right)_{i \in\{1, \ldots, n\}}$ exists, we write $\mathbf{A}_{1} * \ldots * \mathbf{A}_{n}$ for the coproduct of $\mathbf{A}_{1}, \ldots, \mathbf{A}_{n}$, and for morphisms $f_{i}: \mathbf{A}_{i} \rightarrow \mathbf{D}$, we denote the cotupling of $\left(f_{i}\right)_{i \in\{1, \ldots, n\}}$ by $\left[f_{1}, \ldots, f_{n}\right]$. For $\mathbf{A}_{1}=\ldots=\mathbf{A}_{n}$, we write $n \cdot \mathbf{A}$ instead of $\mathbf{A} * \ldots * \mathbf{A}$, and we denote by $\iota_{i}^{n}: \mathbf{A} \rightarrow n \cdot \mathbf{A}$ the associated injection morphisms.

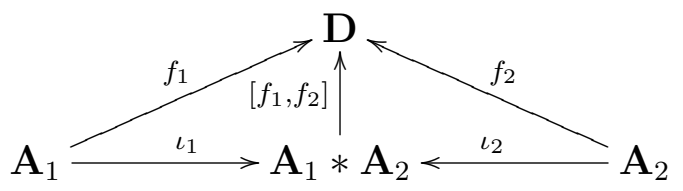

Figure 1.2.: The binary coproduct

Similar as for products, when we write $\mathbf{A}_{1} * \ldots * \mathbf{A}_{n}$ or $n \cdot \mathbf{A}$, we will refer to one specific object in the category and not to the class of all (isomorphic) objects that fulfil the condition of the definition above. 


\section{Preliminaries}

1.4.14 Definition. Call $n \cdot \mathbf{A}$ the $n$-th copower of $\mathbf{A}$, and call $\iota_{i}^{n}: \mathbf{A} \rightarrow n \cdot \mathbf{A}$ the $i$-th injection on $\mathbf{A}$.

In Set, we have $n \cdot \mathbf{A}:=\{\langle i, a\rangle \mid i \in\{1, \ldots, n\}, a \in \mathbf{A}\}$. Copowers with such an easy structure are a convenient but unfortunately uncommon occurrence. Indeed, in the upcoming chapters of this thesis, we will see many copowers that have a far more complex structure.

The 0-th copower of an object $\mathbf{A}, 0 \cdot \mathbf{A}$, leads us to the definition of initial objects.

1.4.15 Definition. An object $\mathbf{I} \in \mathcal{A}$ is said to be initial if, for each $\mathbf{B} \in \mathcal{A}$, there is exactly one morphism from $\mathbf{I}$ to $\mathbf{B}$.

Analogue to terminal objects, initial objects are unique up to isomorphism, and the 0 -th copower of an object is defined to be an initial object (if it exists in the category). In Set, there is exactly one initial object, the empty set.

1.4.16 Proposition. Let $f_{i}: \mathbf{A}_{i} \rightarrow \mathbf{D}$ for $i \in\{1, \ldots, n\}$, and let $g: \mathbf{D} \rightarrow \mathbf{C}$. Then

$$
\left[g \circ f_{1}, \ldots, g \circ f_{n}\right]=g \circ\left[f_{1}, \ldots, f_{n}\right] .
$$

We will now extend the notion of a cotupling.

1.4.17 Definition. For all $i \in\{1, \ldots, n\}$, let $k_{i} \in \mathbb{N}_{+}$and let $g_{i}: \mathbf{B}_{i} \rightarrow k_{i} \cdot \mathbf{A}$. Furthermore, for $j \in\{1, \ldots, n-1\}$, set $m_{j}:=\sum_{i=1}^{j} k_{i}$ and $m:=\sum_{i=1}^{n} k_{i}$. The expanded cotupling of $g_{1}, \ldots, g_{n}$ is the morphism from $\mathbf{B}_{1} * \ldots * \mathbf{B}_{n}$ to $m \cdot \mathbf{A}$ defined as follows:

$$
\llbracket g_{1}, \ldots, g_{n} \rrbracket:=\left[\left[\iota_{1}^{m}, \ldots, \iota_{m_{1}}^{m}\right] \circ g_{1},\left[\iota_{m_{1}+1}^{m}, \ldots, \iota_{m_{2}}^{m}\right] \circ g_{2}, \ldots,\left[\iota_{m_{n-1}+1}^{m}, \ldots, \iota_{m}^{m}\right] \circ g_{n}\right] .
$$

1.4.18 Proposition. Let $g_{1}, \ldots, g_{n}$ and $m$ be as above. Then

$$
\left[h_{1}, \ldots, h_{m}\right] \circ \llbracket g_{1}, \ldots, g_{n} \rrbracket=\left[\left[h_{1}, \ldots, h_{m_{1}}\right] \circ g_{1}, \ldots,\left[h_{m_{n-1}+1}, \ldots, h_{m}\right] \circ g_{n}\right]
$$

for all $h_{1}, \ldots, h_{m}: \mathbf{A} \rightarrow \mathbf{C}$.

1.4.19 Definition. Let $\mathcal{A}$ and $x$ be categories. A covariant functor $F: \mathcal{A} \rightarrow \mathcal{X}$ associates to each object $\mathbf{A} \in \mathcal{A}$ an object $F(\mathbf{A}) \in X$ and to each morphism $u: \mathbf{A} \rightarrow \mathbf{B}$ a morphism $F(u): F(\mathbf{A}) \rightarrow F(\mathbf{B})$ such that

(i) $F\left(i d_{\mathbf{A}}\right)=i d_{F(\mathbf{A})}$ for all objects $\mathbf{A} \in \mathcal{A}$,

(ii) $F(v \circ u)=F(v) \circ F(u)$ for all morphisms $u: \mathbf{A} \rightarrow \mathbf{B}$ and $v: \mathbf{B} \rightarrow \mathbf{C}$.

A functor that reverses the direction of the morphisms is called a contravariant functor. In other words, a contravariant functor $D: \mathcal{A} \rightarrow X$ associates to each object $\mathbf{A} \in \mathcal{A}$ an object $D(\mathbf{A}) \in \mathcal{X}$ and to each morphism $u: \mathbf{A} \rightarrow \mathbf{B}$ a morphism $D(u): D(\mathbf{B}) \rightarrow D(\mathbf{A})$ such that

(i) $D\left(i d_{\mathbf{A}}\right)=i d_{D(\mathbf{A})}$ for all objects $\mathbf{A} \in \mathcal{A}$,

(ii) $D(v \circ u)=D(u) \circ D(v)$ for all morphisms $u: \mathbf{A} \rightarrow \mathbf{B}$ and $v: \mathbf{B} \rightarrow \mathbf{C}$. 

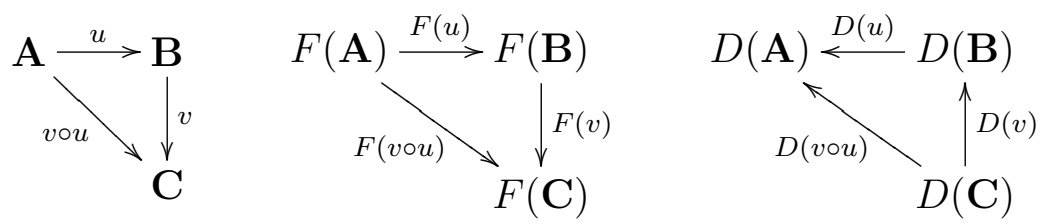

Figure 1.3.: A covariant functor $F$ and a contravariant functor $D$

It is easy to see that functors (covariant and contravariant) map isomorphisms to isomorphisms (indeed, $F(u)^{-1}=F\left(u^{-1}\right)$ ). In contrast, retractions, monomorphisms and epimorphisms are not necessarily preserved.

1.4.20 Definition. A covariant functor $F: \mathcal{A} \rightarrow X$ is said to be faithful if it is oneto-one on each class of morphisms $\mathcal{A}(\mathbf{A}, \mathbf{B})$ and full if it maps each $\mathcal{A}(\mathbf{A}, \mathbf{B})$ onto $\chi(F(\mathbf{A}), F(\mathbf{B}))$. A contravariant functor $D: \mathcal{A} \rightarrow \chi$ is said to be faithful if it is one-to-one on each class of morphisms $\mathcal{A}(\mathbf{A}, \mathbf{B})$ and full if it maps each $\mathcal{A}(\mathbf{A}, \mathbf{B})$ onto $\mathcal{A}(D(\mathbf{B}), D(\mathbf{A}))$.

We now have the terminology to introduce the notion of concrete categories:

1.4.21 Definition. A concrete category $(\mathcal{A}, U)$ is a category $\mathcal{A}$ equipped with a faithful covariant functor $U: \mathcal{A} \rightarrow$ Set, the so-called forgetful functor.

If a category $\mathcal{A}$ can be equipped with a faithful functor $U: \mathcal{A} \rightarrow \operatorname{Set}$, it is called concretizable. A possible definition of the forgetful functor is often obvious. For instance, the categories in Examples 1.4.2 (page 16) are concretizable by defining the forgetful functor to map each structure to its underlying set. In such cases, we will refer to the forgetful functor as the obvious forgetful functor. In a concrete category, objects can be thought of as sets (their images under the forgetful functor) with some additional structure, and morphisms can the thought of as structure-preserving functions.

1.4.22 Definition. Let $\mathcal{A}, x$ be categories, and let $F: \mathcal{A} \rightarrow x, G: \mathcal{A} \rightarrow x$ be two covariant functors. A natural transformation $\tau$ from $F$ to $G$, written $\tau: F \rightarrow G$, is a family of morphisms $\left(\tau_{\mathbf{A}}\right)_{\mathbf{A} \in \mathfrak{A}}, \tau_{\mathbf{A}}: F(\mathbf{A}) \rightarrow G(\mathbf{A})$ such that the following diagram commutes for any two objects $\mathbf{A}, \mathbf{B} \in \mathcal{A}$ and each morphism $u: \mathbf{A} \rightarrow \mathbf{B}$ :

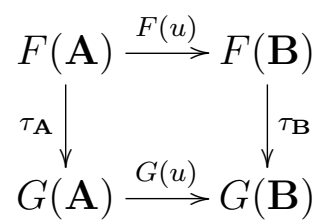

For two contravariant functors $D: \mathcal{A} \rightarrow \mathcal{X}, E: \mathcal{A} \rightarrow \mathcal{X}$, a natural transformation $\tau$ from $D$ to $E$, written $\tau: D \rightarrow E$, is a family of morphisms $\left(\tau_{\mathbf{A}}\right)_{\mathbf{A} \in \mathcal{A}}, \tau_{\mathbf{A}}: D(\mathbf{A}) \rightarrow E(\mathbf{A})$ such that the following diagram commutes for any two objects $\mathbf{A}, \mathbf{B} \in \mathcal{A}$ and each morphism 
$u: \mathbf{A} \rightarrow \mathbf{B}:$

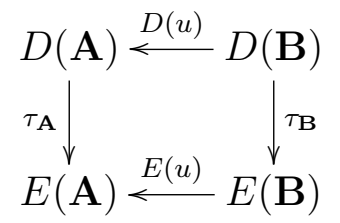

We are now interested in contravariant functors that are in a general sense invariant to each other.

1.4.23 Definition. Let $\mathcal{A}$ and $\mathcal{X}$ be categories, and let $D: \mathcal{A} \rightarrow \mathcal{X}$ and $E: \mathcal{X} \rightarrow \mathcal{A}$ be contravariant functors. Furthermore, let $e: i d_{\mathcal{A}} \rightarrow E D$ and $\epsilon: i d_{\chi} \rightarrow D E$ be natural transformations. The quadruple $\langle D, E, e, \epsilon\rangle$ is called a dual adjunction between $\mathcal{A}$ and $\mathcal{X}$ if, for all objects $\mathbf{A} \in \mathcal{A}$ and $\mathbf{X} \in \mathcal{X}$, there is a bijection between $\mathcal{A}(\mathbf{A}, E(\mathbf{X}))$ and $x(\mathbf{X}, D(\mathbf{A}))$ associating $u$ and $\varphi$ as given in the triangles of Figure 1.4. That is, $u=E\left(D(u) \circ \epsilon_{\mathbf{X}}\right) \circ e_{\mathbf{A}}$ and $\varphi=D\left(E(\varphi) \circ e_{\mathbf{A}}\right) \circ \epsilon_{\mathbf{X}}$.
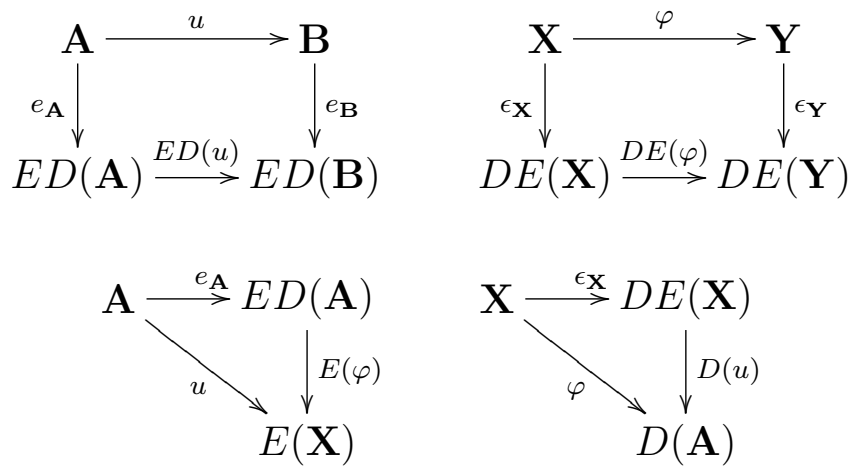

Figure 1.4.: A dual adjunction

If $\langle D, E, e, \epsilon\rangle$ is a dual adjunction between $\mathcal{A}$ and $\mathcal{X}$, then we say that $\mathcal{A}$ and $\mathcal{X}$ are dually adjoint.

If one has a category $\mathcal{A}$ and a dually adjoint category $\mathcal{X}$, one might have the wish to find information about the objects of $\mathcal{A}$ by looking into $\mathcal{X}$. To do so, each object $\mathbf{A} \in \mathcal{A}$ must be represented as $E(\mathbf{X})$ for some object $\mathbf{X} \in X$ (i.e., $\mathbf{A} \cong E(\mathbf{X})$ ). This is the idea behind the definition of a dual representation.

1.4.24 Definition. A dual adjunction $\langle D, E, e, \epsilon\rangle$ between categories $\mathcal{A}$ and $\mathcal{X}$ is called a dual representation if, for every object $\mathbf{A} \in \mathcal{A}$, the morphism $e_{\mathbf{A}}: \mathbf{A} \rightarrow E D(\mathbf{A})$ is an isomorphism.

A dual representation offers us a possibility to construct the objects of $\mathcal{A}$ from the objects of $\mathcal{X}$, but it does not provide a close connection between $X$ and $\mathcal{A}$ as categories. However, we do obtain this if we additionally require that each object $\mathbf{X} \in X$ is represented as $D(\mathbf{A})$ for some object $\mathbf{A} \in \mathcal{A}$. 
1.4.25 Definition. A dual representation $\langle D, E, e, \epsilon\rangle$ is called a dual equivalence between the categories $\mathcal{A}$ and $\mathcal{X}$ if, for every object $\mathbf{X} \in \mathcal{X}$, the morphism $\epsilon_{\mathbf{X}}: \mathbf{X} \rightarrow D E(\mathbf{X})$ is an isomorphism.

In other words, a dual adjunction $\langle D, E, e, \epsilon\rangle$ is a dual equivalence if, for all objects $\mathbf{A} \in \mathcal{A}$ and $\mathbf{X} \in \mathcal{X}$, the morphisms $e_{\mathbf{A}}$ and $\epsilon_{\mathbf{X}}$ are isomorphisms. In this case, we also say that $\mathcal{A}$ and $X$ are dually equivalent. The notion 'dual equivalence' is justified since $D$ and $E$ are full, faithful and preserve all purely category-theoretic properties. However, they reverse the order of the morphisms. For instance, monomorphisms become epimorphisms and products become coproducts. In particular, we have $\mathbf{A}^{n} \in \mathcal{A}$ if and only if $n \cdot D(\mathbf{A}) \in \mathcal{X}$. Thus, every result that is obtained for a category $\mathcal{A}$ and relies on purely category-theoretic properties has its dual counterpart in each dually equivalent category $x$. This allows us to transfer problems from a category to any dually equivalent category where it might be easier to solve. This is one of the most effective tools in category theory, and we will use it many times in our upcoming work.

For each category $\mathcal{A}$, one dually equivalent category can be obtained by simply reversing the direction of each morphism.

1.4.26 Definition. For a category $\mathcal{A}$, the opposite category of $\mathcal{A}$ is the category $\mathcal{A}^{o p}$ that has the same class of objects and $\mathcal{A}^{o p}(\mathbf{A}, \mathbf{B}):=\mathcal{A}(\mathbf{B}, \mathbf{A})$ for all $\mathbf{A}, \mathbf{B} \in \mathcal{A}^{o p}$. Denote by $(-)^{o p}$ the contravariant functor that carries $\mathcal{A}$ to $\mathcal{A}^{o p}$ in the obvious way.

Evidently, $\left\langle(-)^{o p},(-)^{o p}, i d, i d\right\rangle$ is a dual equivalence between $\mathcal{A}$ and $\mathcal{A}^{o p}$.

Analogue to the notion of a dual equivalence is the notion of an equivalence between categories. In this case, the functors $D, E$ are covariant instead of contravariant and all definitions are adjusted accordingly. As one would expect, two categories are equivalent if and only if all their dually equivalent categories are equivalent.

\subsection{Abstract Clones and Lawvere Theories}

It is a natural question whether clones can also be defined abstractly. In this section, we will present two different ways to do so. We will present so-called abstract clones which define clones axiomatically and so-called Lawvere Theories which are the categorical equivalent to an abstract clone.

1.5.1 Definition ([Tay73]). Let $T$ be a heterogeneous algebra on a series of base sets $T_{1}, T_{2}, \ldots$ equipped with composition operations

$$
C_{m}^{r}: T_{r} \times T_{m}^{r} \rightarrow T_{m}\left(m, r \in \mathbb{N}_{+}\right)
$$

and constants $e_{i}^{n} \in T_{n}\left(n \in \mathbb{N}_{+}, i \in\{1, \ldots, n\}\right)$ satisfying the following three identities:

(i) $C_{m}^{r}\left(t, C_{m}^{n}\left(u_{1}, v_{1}, \ldots, v_{n}\right), \ldots, C_{m}^{n}\left(u_{r}, v_{1}, \ldots, v_{n}\right)\right)=C_{m}^{n}\left(C_{n}^{r}\left(t, u_{1}, \ldots, u_{r}\right), v_{1}, \ldots, v_{n}\right)$,

(ii) $C_{m}^{n}\left(e_{i}^{n}, t_{1}, \ldots, t_{n}\right)=t_{i}$,

(iii) $C_{n}^{n}\left(t, e_{1}^{n}, \ldots, e_{i}^{n}\right)=t$, 


\section{Preliminaries}

where $m, n, r \in \mathbb{N}_{+}$and $i \in\{1, \ldots, n\}$. Such a many-sorted algebra is called an abstract clone.

To distinguish between abstract clones and the usual definition of a clone, clones in the usual sense are sometimes called concrete clones. Note that the constants and the identities of abstract clones imitate the behaviour of the projections and the composition in a concrete clone. In fact, every concrete clone is isomorphic to some abstract clone, and it was shown in Coh65 that the other direction is also true: Every abstract clone is isomorphic to a concrete clone (although the isomorphism is, as B. CsÁkÁNY Csá05] noted, far from natural). In other words, abstract clones and concrete clones stand in the same relation to each other as groups and permutation groups. For more information on abstract clones we refer to Tay93.

1.5.2 Definition ([Law63]). A Lawvere Theory $\mathcal{T}$ is a category with finitely many objects $t_{1}, t_{2}, \ldots$ such that $t_{i}$ is the $i$-th power of $t_{1} .^{2}$

It was shown in Tay73 that the notions of abstract clones and Lawvere Theories are equivalent.

\subsection{Natural Dualities}

In Section 1.4, we have introduced the notion of dual equivalences. Arguably the moststudied dualities are so-called natural dualities for which we will present a brief introduction in this section. For more details, we refer the reader to [CD98] and [Dav06].

1.6.1 Definition. Let $\mathbf{M}=\langle M, F, H, R\rangle$ and $\mathbf{M}^{\prime}=\left\langle M, F^{\prime}, H^{\prime}, R^{\prime}\right\rangle$ be structures with the same underlying set. $\mathbf{M}$ is said to be compatible with $\mathbf{M}^{\prime}$ if

(i) each $n$-ary operation $g \in F^{\prime}$ is a homomorphism from $\mathbf{M}^{n}$ to $\mathbf{M}$,

(ii) for each $n$-ary partial operation $h \in H^{\prime}$, the domain of $h$ forms a substructure $\operatorname{dom}(\mathbf{h})$ of $\mathbf{M}^{n}$ and $h$ is a homomorphism from $\operatorname{dom}(\mathbf{h})$ to $\mathbf{M}$,

(iii) each $n$-ary $r \in R^{\prime}$ forms a substructure of $\mathbf{M}^{n}$.

Compatibility is a symmetric relation, so $\mathbf{M}$ is compatible with $\mathbf{M}^{\prime}$ if and only if $\mathbf{M}^{\prime}$ is compatible with $\mathbf{M}$.

1.6.2 Example. Let $F$ be a set of operations over $M$, and let $R$ be a set of relations such that $\operatorname{Pol} \operatorname{Inv} F=\operatorname{Inv} \operatorname{Pol} R$. Then, $\mathbf{M}:=\langle M, F\rangle$ and $\mathbf{M}^{\prime}:=\langle M, R\rangle$ are compatible structures.

1.6.3 Definition. Let $\mathbf{M}=\langle M, F, H, R\rangle$ be a structure, and let $\mathbf{M}:=\left\langle M, F^{\prime}, H^{\prime}, R^{\prime}, \mathcal{T}\right\rangle$ be a structure equipped with the discrete topology $\mathcal{T}$. The topological structure $\mathbf{M}$ is called an alter ego of $\mathbf{M}$ provided that $\mathbf{M}$ and $\left\langle M, F^{\prime}, H^{\prime}, R^{\prime}\right\rangle$ are compatible.

${ }^{2}$ In the standard case, a Lawvere Theory is defined to also contain the 0 -th power $t_{0}$ (recall that $t_{0}$ is then a terminal object). Here, as done in many other papers, we have slightly adjusted the definition to have a more straight-forward connection between Lawvere Theories and clones, which do not contain nullary operations (this will be further discussed in Section 9.2). 
Let $\mathbf{M}$ be a finite structure and let $\mathbf{M}$ be an alter ego of $\mathbf{M}$. As explained in Example 1.4 .2 (page 16),

$$
\mathcal{A}:=\mathbb{I S P}(\mathbf{M}) \text { and } x:=\mathbb{I} \mathbb{S}_{c}^{0} \mathbb{P}^{+}(\underline{\mathbf{M}})
$$

form categories where the objects are the structures and the morphisms are the (continuous) homomorphisms. Recall that $\mathbb{I S P}(\mathbf{M})$ is a quasivariety, so it can be described as the class of all models of a set of quasi-identities. The question of how to describe $\mathbb{I S}_{c}^{0} \mathbb{P}^{+}(\mathbf{M})$ is addressed in $\mathrm{CDH}^{+} 03$, CDF 04, DT05, CDJP08.

Let $\langle D, E, e, \epsilon\rangle$ be the quadruple consisting of two functors $D: \mathcal{A} \rightarrow \mathcal{X}, E: \mathcal{X} \rightarrow \mathcal{A}$ and two natural transformations $e: i d_{\mathcal{A}} \rightarrow E D, \epsilon: i d_{\chi} \rightarrow D E$ defined as follows:

$$
\begin{aligned}
D(\mathbf{A}) & :=\mathcal{A}(\mathbf{A}, \mathbf{M}), D(u)(x):=x \circ u, \\
E(\mathbf{X}) & :=x(\mathbf{X}, \mathbf{M}), E(\varphi)(\alpha):=\alpha \circ \varphi, \\
e_{\mathbf{A}}(a)(x) & :=x(a), \\
\epsilon_{\mathbf{X}}(x)(\alpha) & :=\alpha(x) .
\end{aligned}
$$

The fact that $D, E, e, \epsilon$ are well-defined follows from the compatibility of $\mathbf{M}$ and $\mathbf{M}$. Indeed, $\langle D, E, e, \epsilon\rangle$ forms a dual adjunction between $\mathcal{A}$ and $\mathcal{X}$, and it is easy to see that $e_{\mathbf{A}}$ and $\epsilon_{\mathbf{X}}$ are injective for all $\mathbf{A} \in \mathcal{A}, \mathbf{X} \in X$. There are many theorems that give conditions under which $\langle D, E, e, \epsilon\rangle$ is a dual representation (i.e., $e_{\mathbf{A}}$ is also surjective for each $\mathbf{A} \in \boldsymbol{X}$ ). In particular, if $\mathbf{M}$ is a total structure (recall that this means that $\mathbf{M}$ does not have any partial operations), then there always exists an alter ego of $\mathbf{M}$ for which $\langle D, E, e, \epsilon\rangle$ defined as above is a dual representation between $\mathbb{I S P}(\mathbf{M})$ and $\mathbb{S}_{c}^{0} \mathbb{P}^{+}(\mathbf{M})$.

1.6.4 Definition. Let $\mathbf{M}$ be a finite structure and let $\mathbf{M}$ be an alter ego of $\mathbf{M}$. If $\langle D, E, e, \epsilon\rangle$ defined as above is a dual representation between $\mathcal{A}:=\mathbb{I S P}(\mathbf{M})$ and $x:=\mathbb{I} \mathbb{S}_{c}^{0} \mathbb{P}^{+}(\underline{\mathbf{M}})$, then we say that $\mathbf{M}$ yields a natural duality on $\mathcal{A}$. In this case, we call $\langle D, E, e, \epsilon\rangle$ the natural dual representation between $\mathcal{A}$ and $\boldsymbol{X}$.

1.6.5 Theorem (Brute Force Duality Theorem [Dav06]). Let $\mathbf{M}$ be a finite total structure. Let $\mathbf{M}:=\langle M, R, \mathcal{T}\rangle$ where $\mathcal{T}$ is the discrete topology and $R$ is the set of all universes of substructures of finite powers of $\mathbf{M}$. Then, $\mathbf{M}$ yields a natural duality on $\mathcal{A}$.

Note that the set of all universes of substructures of finite powers of a total structure $\mathbf{M}=\langle M, F, R\rangle$ is precisely Inv $F$, which can be very big. In particular, it contains all subuniverses if $\mathbf{M}$ contains no operations. An easy argument yields that it is enough to consider a set of relations $R^{\prime}$ for which we have Pol $R^{\prime}=F$. Furthermore, the brute force construction can be tamed if $\mathbf{M}$ has a $(k+1)$-ary near-unanimity term for some $k \geq 2$. In this case, the Baker-Pixley Theorem 1.2.10, page 15 establishes that it is sufficient to consider all subuniverses of the $k$-th power of $\mathbf{M}$. Although a brute force construction never fails to construct an alter ego $\mathbf{M}$, it might not give us a duality that we like to work with, as the set of relations $R$ can be very big and/or very complicated. There are more elegant ways to obtain a much more feasible duality. Unfortunately, all of the known theorems require some additional conditions and only work in certain scenarios. 
Provided that $\langle D, E, e, \epsilon\rangle$ is a dual representation, restricting $E$ and $\epsilon$ to the full subcategory $\mathbb{I} D(\mathcal{A}) \subseteq \mathcal{X}$ gives a dual equivalence between $\mathcal{A}$ and $\mathbb{I} D(\mathcal{A})$.

1.6.6 Definition. Let $\mathbf{M}$ be a finite structure and let $\mathbf{M}$ be an alter ego of $\mathbf{M}$. If $\langle D, E, e, \epsilon\rangle$ as above is a dual equivalence between $\mathcal{A}:=\mathbb{I S P}(\mathbf{M})$ and some full subcategory $\mathcal{X}$ of $\mathbb{I} \mathbb{S}_{c}^{0} \mathbb{P}^{+}(\underline{\mathbf{M}})$, then we say that $\mathbf{M}$ yields a full natural duality between $\mathcal{A}$ and $\mathcal{X}$. In this case, we call $\left\langle D, E^{\prime}, e, \epsilon^{\prime}\right\rangle$ the natural dual equivalence between $\mathcal{A}$ and $\mathcal{X}$, where $E^{\prime}$ and $\epsilon^{\prime}$ are the restrictions of $E$ and $\epsilon^{\prime}$ to $X$.

Sometimes, $\underline{\mathbf{M}}$ yields a full natural duality between $\mathcal{A}$ and $\mathbb{S}_{c}^{0} \mathbb{P}^{+}(\underline{\mathbf{M}})$ itself. Several criteria for this event (it is not always possible to choose $\mathbf{M}$ in this way) can be found in [CD98] and Dav06].

We will now state a triviality that will be of some importance for the remainder of this thesis: If $\mathbb{M}$ yields a full natural duality between $\mathcal{A}$ and $\mathcal{X}$, then we obtain a dual equivalence between the full subcategories $\mathcal{A}_{\text {fin }}$ and $x_{\text {fin }}$ by restricting $\langle D, E, e, \epsilon\rangle$ to the finite parts of the categories. In this case, we can neglect the topology as it is anyhow discrete.

It should be noted that there is an obvious asymmetry between the two classes $\mathbb{I S P}(\mathbf{M})$ and $\mathbb{I S}_{c}^{0} \mathbb{P}^{+}(\underline{\mathbf{M}})$ : $\mathbb{I S P}(\mathbf{M})$ excludes the empty substructure, whereas $\mathbb{S}_{c}^{0} \mathbb{P}(\underline{\mathbf{M}})$ excludes products over an empty index set. For readers more interested in the background of this asymmetry (and how to get around it), we refer to [DPW] where B. A. DAVEY, J. G. Pitkethly and R. Willard discuss how to shift to a completely symmetric setting. 


\section{Clones of Operations}

In this chapter, we will generalize clones over sets to clones over objects in categories. As we will see in the upcoming chapters, there are three major advantages of this approach:

- Many results that hold for clones over sets can be generalized to clones over objects in categories. This is true for all results that hold for abstract clones, but also for some results that only hold for concrete clones. For instance, we will show in Chapter 5 that the Galois connection Pol-Inv can be generalized to our scenario. Although this might be a worthwhile task in itself, it is also interesting for those who mostly care about clones over sets, as this allows us to treat clones over sets abstractly (which has proven itself to be advantageous in several situations) without losing powerful tools such as the Galois theory between operations and relations.

- We can apply tools from the field of category theory to clones. For instance, we can use functors to shift the clones from one category to another. Sometimes, this will make a problem much easier to solve. Again, this is also relevant for those who are only interested in clones over sets, as the category Set is not always the most suitable category to solve some clone-theoretic problems in. In Chapter 7 and 8 , we will see how to obtain new results for certain clones over sets by moving them into another categorical environment.

- Concerning the tools from category theory, it is of particular interest that we will be able to apply the Duality Principle. Dualizing our generalized notion of a clone will give us the notion of what we will call a clone of dual operations (which generalizes the notion of coclones over sets). Since the two notions are dually equivalent, each result for clones of operations holds in its dualized version for clones of dual operations and vice versa. With some technical adjustments, this gives rise to a general duality theory for clones, which will ultimately allow us to dualize any clone together with its relational counterpart.

Besides these advantages, the categorical abstraction will also give us new insight into some well-known results for clones over sets. It is of regular occurrence that a result for clones over sets holds under an assumption that coincides with several categorical properties that are essentially different but happen to be equivalent in the category of sets. By lifting the result in question to clones over objects in other categories (if possible), we will see which of these properties was actually providing the desired result. 


\subsection{Operations and their Clones}

Operations and clones over sets can be generalized to categories. Until the end of this section, let $\mathcal{A}$ be a category that contains an object $\mathbf{A}$ and all non-empty finite powers of $\mathbf{A}$, that is, $\left\{\mathbf{A}^{n} \mid n \in \mathbb{N}_{+}\right\} \subseteq \mathcal{A}$.

2.1.1 Definition. Let $n \in \mathbb{N}_{+}$. A morphism $f: \mathbf{A}^{n} \rightarrow \mathbf{A}$ is called an $n$-ary operation over $\mathbf{A}$. Denote by $O_{\mathbf{A}}^{(n)}$ the set of all $n$-ary operations over $\mathbf{A}$, define $O_{\mathbf{A}}:=\bigcup_{n \in \mathbb{N}_{+}} O_{\mathbf{A}}^{(n)}$, and for $F \subseteq O_{\mathbf{A}}$, set $F^{(n)}:=F \cap O_{\mathbf{A}}^{(n)}$.

In this setting, the projection mappings are generalized to the projection morphisms $\pi_{i}^{n}: \mathbf{A}^{n} \rightarrow \mathbf{A}$ that are associated to the product $\mathbf{A}^{n}$.

\subsubsection{Examples.}

(i) If $\mathcal{A}=$ Set and $\mathbf{A} \in \mathcal{A}$, then $O_{\mathbf{A}}$ is just the set of all operations over the set $\mathbf{A}$ as defined at the beginning of Section 1.2 (page 13).

(ii) If $\mathcal{A}$ is a quasivariety of algebras and $\mathbf{A} \in \mathcal{A}$, then $O_{\mathbf{A}}$ is the centralizer clone of $\mathbf{A}$.

(iii) If $\mathcal{A}$ is a quasivariety of relational structures and $\mathbf{A}=\langle A, R\rangle \in \mathcal{A}$, then $O_{\mathbf{A}}$ is $\mathrm{Pol} R$.

Note that, strictly speaking, it is incorrect to say that $O_{\mathbf{A}}$ is the centralizer clone of A or that $O_{\mathbf{A}}$ is $\mathrm{Pol} R$. While $O_{\mathbf{A}}$ is a set of morphisms, the centralizer clone of $\mathbf{A}$ and $\mathrm{Pol} R$ consist of functions. Therefore, it is more accurate to say that the image of $O_{\mathbf{A}}$ under the obvious forgetful functor is the centralizer clone of $\mathbf{A}$ or $\operatorname{Pol} R$. However, we will sometimes relax the terminology and identify morphisms between two structures directly with their underlying functions.

We can also generalize the notion of nonessential and essential arguments.

2.1.3 Definition. Let $\widetilde{\pi}_{i}^{n}: \mathbf{A}^{n} \rightarrow \mathbf{A}^{n-1}$ denote the morphism defined by setting

$$
\widetilde{\pi}_{i}^{n}:=\left\langle\pi_{1}^{n}, \ldots, \pi_{i-1}^{n}, \pi_{i+1}^{n}, \ldots, \pi_{n}^{n}\right\rangle .
$$

The $i$-th argument of an $n$-ary operation $f$ is said to be nonessential if there exists an $(n-1)$-ary operation $f^{\prime} \in O_{\mathbf{A}}$ such that $f=f^{\prime} \circ \widetilde{\pi}_{i}^{n}$. An argument is called essential if it is not nonessential.

For $\mathcal{A}=$ Set, this definition coincides with the usual definition of nonessential and essential arguments.

2.1.4 Definition. An operation $f \in O_{\mathbf{A}}$ is said to be essentially $n$-ary if it has exactly $n$ essential arguments.

Note that an operation might have no essential arguments. Hence, an operation can be essentially (but not actually) nullary.

2.1.5 Definition. Let $n \geq 2$, let $f \in O_{\mathbf{A}}^{(n)}$ and let $i, j \in\{1, \ldots, n\}$ with $i<j$. We say that $f^{\prime} \in O_{\mathbf{A}}^{(n-1)}$ arises from $f$ by identification of the $i$-th and $j$-th argument if we have $f^{\prime}=f \circ\left\langle\pi_{1}^{n-1}, \ldots, \pi_{j-1}^{n-1}, \pi_{i}^{n-1}, \pi_{j}^{n-1}, \ldots, \pi_{n-1}^{n-1}\right\rangle$. 
Note that if the $t$-th argument of an operation $f \in O_{\mathbf{A}}^{(n)}$ is nonessential, then the operation $f^{\prime} \in O_{\mathbf{A}}^{(n-1)}$ from Definition 2.1.3 can be obtained by identifying the $t$-th argument with any of the other arguments.

2.1.6 Definition. A nonessential argument is said to be eliminated from an operation if it is identified with an essential argument.

2.1.7 Definition. Let $f, f^{\prime} \in O_{\mathbf{A}}^{(n)}$. We say that $f^{\prime}$ arises from $f$ by permutation of arguments if there exists a permutation $p$ on $\{1, \ldots, n\}$ such that $f^{\prime}=f \circ\left\langle\pi_{p(1)}^{n}, \ldots, \pi_{p(n)}^{n}\right\rangle$.

With the last two notions, we can define what we mean when we say that two operations are essentially the same.

2.1.8 Definition. Two essentially at least unary operations are said to be essentially the same operation if they arise from each other by permutation of arguments and elimination of nonessential arguments. Two essentially nullary operations are said to be essentially the same operation if they coincide with each other after identifying all arguments. Two operations are said to be essentially different if they are not essentially the same.

With the generalization of operations, we can now generalize the notion of a clone.

2.1.9 Definition. A set $C$ of operations over $\mathbf{A}$ is called a clone of operations, written $C \leq O_{\mathbf{A}}$, if $C$ contains all the projection morphisms $\pi_{i}^{n}: \mathbf{A}^{n} \rightarrow \mathbf{A}$ and, for $f \in C^{(n)}$ and $f_{1}, \ldots, f_{n} \in C^{(k)}$, the superposition $f \circ\left\langle f_{1}, \ldots, f_{n}\right\rangle$ is also in $C$.

\subsubsection{Examples.}

(i) If $\mathcal{A}=\operatorname{Set}$, then each clone over $\mathbf{A} \in \mathcal{A}$ is just a clone in the usual sense.

(ii) If $\mathcal{A}$ is a quasivariety of algebras, then the set of clones over $\mathbf{A} \in \mathcal{A}$ is the set of subclones of the centralizer clone of $\mathbf{A}$.

(iii) If $\mathcal{A}$ is a quasivariety of relational structures, then the set of clones over $\langle A, R\rangle \in \mathcal{A}$ is the set of subclones of $\mathrm{Pol} R$.

(iv) If $\mathcal{A}$ is a the category of topological spaces and $\mathbf{A} \in \mathcal{A}$, then $O_{\mathbf{A}}$ is the clone of the topological space $\mathbf{A}$ as investigated by W. TAYLOR in TTay86.

These examples show that we can investigate clones over sets by treating them as clones over objects in categories different from Set.

We can also generalize the expanded superposition in which the operations $f_{1}, \ldots, f_{n}$ do not need to have the same arity. Recall that $\left\langle\left\langle f_{1}, \ldots, f_{n}\right\rangle\right\rangle$ denotes the expanded superposition (see Definition 1.2.2, page 13.

2.1.11 Definition. Let $f$ be an $n$-ary operation over $\mathbf{A}$, and let $f_{1}, \ldots, f_{n}$ be operations over $\mathbf{A}$ where $k_{i}$ is the arity of $f_{i}$. The expanded superposition of $f$ and $f_{1}, \ldots, f_{n}$ is the $\left(\sum_{i=1}^{n} k_{i}\right)$-ary operation $f \circ\left\langle\left\langle f_{1}, \ldots, f_{n}\right\rangle\right\rangle$.

2.1.12 Proposition. Clones of operations are closed under expanded superposition. 


\section{Clones of Operations}

Proof. Let $C \leq O_{\mathbf{A}}$, let $f \in O_{\mathbf{A}}^{(n)}$ and let $f_{i} \in O_{\mathbf{A}}^{\left(k_{i}\right)}$ for $i \in\{1, \ldots, n\}$. Moreover, for $j \in\{1, \ldots, n-1\}$, set $m_{j}:=\sum_{i=1}^{j} k_{i}$ and $m:=\sum_{i=1}^{n} k_{i}$. By Proposition 1.4.12 (page 19), we have

$$
\begin{aligned}
f \circ\left\langle\left\langle f_{1}, \ldots, f_{n}\right\rangle\right\rangle & =f \circ\left\langle\left\langle f_{1}, \ldots, f_{n}\right\rangle\right\rangle \circ\left\langle\pi_{1}^{m}, \pi_{2}^{m}, \ldots, \pi_{m}^{m}\right\rangle \\
& =f \circ\langle\underbrace{f_{1} \circ\left\langle\pi_{1}^{m}, \ldots, \pi_{m_{1}}^{m}\right\rangle}_{\in C}, \ldots, \underbrace{f_{n} \circ\left\langle\pi_{m_{n-1}+1}^{m}, \ldots, \pi_{m}^{m}\right\rangle}_{\in C}\rangle \in C .
\end{aligned}
$$

It is easy to verify that the intersection of clones of operations is again a clone of operations. Moreover, $O_{\mathbf{A}}$ is always a clone, so we can extend the definition of the closure operator Clo: For a set of operation $F \subseteq O_{\mathbf{A}}$, we denote by $\operatorname{Clo}(F)$ the least clone over A that contains $F$.

2.1.13 Definition. A set of operations $F \subseteq O_{\mathbf{A}}$ is said to generate an operation $f \in O_{\mathbf{A}}$ provided that $f \in \mathrm{Clo}(F)$.

It also follows that the clones over an object $\mathbf{A}$ form a complete lattice with respect to inclusion (recall that a lattice is complete if every set has a supremum and an infimum). The top element of the lattice is the aforementioned full clone $O_{\mathbf{A}}$ and the bottom element is the clone that contains only the projection morphisms. Denote the latter by $J_{\mathbf{A}}$.

2.1.14 Definition. Denote by $L_{\mathbf{A}}$ the set of clones over A. Then, $\mathcal{L}_{\mathbf{A}}:=\left\langle L_{\mathbf{A}}, \subseteq\right\rangle$ is called the lattice of clones over $\mathbf{A}$.

\subsubsection{Examples.}

(i) If $\mathcal{A}=$ Set and $\mathbf{A} \in \mathcal{A}$, then $\mathcal{L}_{\mathbf{A}}$ is simply the usual clone lattice over the set $\mathbf{A}$.

(ii) If $\mathcal{A}$ is a quasivariety of structures and $\mathbf{A} \in \mathcal{A}$, then $\mathcal{L}_{\mathbf{A}}$ is order-isomorphic to the ideal $\left\langle O_{\mathbf{A}}\right]$ in the clone lattice $\mathcal{L}_{A}$, where $A$ is the universe of $\mathbf{A}$.

We will now introduce certain types of operations that will be useful in the sequel.

\subsubsection{Definition.}

(i) A unary operation $f \in O_{\mathbf{A}}$ is called a retraction if $f \circ f=f$.

(ii) For $n \geq 3$, an $n$-ary operation $f \in O_{\mathbf{A}}$ is called a near-unanimity operation if

$$
\begin{gathered}
f \circ\left\langle\pi_{2}^{n}, \pi_{1}^{n}, \pi_{1}^{n}, \ldots, \pi_{1}^{n}\right\rangle=\pi_{1}^{n}, \\
f \circ\left\langle\pi_{1}^{n}, \pi_{2}^{n}, \pi_{1}^{n}, \ldots, \pi_{1}^{n}\right\rangle=\pi_{1}^{n}, \\
\vdots \\
f \circ\left\langle\pi_{1}^{n}, \pi_{1}^{n}, \ldots, \pi_{1}^{n}, \pi_{2}^{n}\right\rangle=\pi_{1}^{n} .
\end{gathered}
$$

Ternary near-unanimity operations are called majority operations.

(iii) A ternary operation $f \in O_{\mathbf{A}}$ is called a minority operation if

$$
f \circ\left\langle\pi_{1}^{3}, \pi_{2}^{3}, \pi_{2}^{3}\right\rangle=f \circ\left\langle\pi_{2}^{3}, \pi_{1}^{3}, \pi_{2}^{3}\right\rangle=f \circ\left\langle\pi_{2}^{3}, \pi_{2}^{3}, \pi_{1}^{3}\right\rangle=\pi_{1}^{3} .
$$


(iv) An $n$-ary operation $f \in O_{\mathbf{A}}$ is called idempotent if $f \circ\left\langle\pi_{1}^{n}, \ldots, \pi_{1}^{n}\right\rangle=\pi_{1}^{n}$.

(v) An $n$-ary operation $f$ is called a semiprojection if there exists $i \in\{1, \ldots, n\}$ such that

$$
f \circ\left\langle\pi_{j_{1}}^{n}, \ldots, \pi_{j_{n}}^{n}\right\rangle=\pi_{j_{i}}^{n}
$$

whenever $j_{1}, \ldots, j_{n}$ are not pairwise distinct. If $f$ is also nontrivial, then we say that $f$ is a proper semiprojection.

If $\mathcal{A}$ is the category of sets (or any category where the product is the usual Cartesian product), then the above definitions coincide with the usual definitions of these kind of operations. For example, the notion of a near-unanimity operation as presented here coincides with the one presented in the preliminaries (see Definition 1.2.9, page 15). With the exception of idempotent operations, all operations above are defined by socalled irregular identities.

2.1.17 Definition. Let $f \in O_{\mathbf{A}}^{(n)}$ and $h \in O_{\mathbf{A}}^{(r)}$. For $t \in\{1, \ldots, n\}$, the identity

$$
f \circ\left\langle\pi_{i_{1}}^{n}, \ldots, \pi_{i_{n}}^{n}\right\rangle=h \circ\left\langle\pi_{j_{1}}^{n}, \ldots, \pi_{j_{r}}^{n}\right\rangle
$$

is called irregular in its $t$-th argument if $i_{t} \notin\left\{j_{1}, \ldots, j_{r}\right\}$.

For instance, the identity $f \circ\left\langle\pi_{2}^{3}, \pi_{2}^{3}, \pi_{1}^{3}\right\rangle=\pi_{1}^{3}$ is irregular in its first and second argument.

\subsection{Clones over Objects vs. Abstract Clones and Lawvere Theories}

In this section, we compare the notions of abstract clones and Lawvere Theories (see Section 1.5) with the notion of clones of operations over objects as introduced in this chapter.

Since Lawvere Theories are also defined categorically, one might expect a strong connection to the clones of operations as introduced here. In fact, a subset $C \subseteq O_{\mathbf{A}}$ is a clone of operations if and only if

$$
C=\left\{M(f) \mid f \in \mathcal{T}\left(t_{n}, t_{1}\right)\right\}
$$

for some Lawvere Theory $\mathcal{T}$ and some product-preserving functor $M: \mathcal{T} \rightarrow \mathcal{A}(M$ is then called a model of $\mathcal{T}$ ). Roughly speaking, what we call a clone of operations over A becomes a Lawvere Theory if we eliminate everything from the category except the finite non-empty powers of $\mathbf{A}$ and the morphisms that belong to $O_{\mathbf{A}}$. This also explains the connection between our clones and abstract clones: The notions are equivalent up to the categorical environment of the clone.

The following proposition is a direct consequence:

2.2.1 Proposition. Every clone of operations over an object $\mathbf{A}$ is isomorphic to some clone of operations over a set and vice versa. 


\section{Clones of Operations}

Furthermore, every result that is formulated for abstract clones or Lawvere Theories can easily be adopted for clones over an object A. For Lawvere Theories, this is obvious. For abstract clones, we simply have to replace each $T_{n}$ with $O_{\mathbf{A}}^{(n)}$, each $e_{i}^{n}$ with $\pi_{i}^{n}$ and each $C_{m}^{r}\left(t, u_{1}, \ldots, u_{r}\right)$ with $t \circ\left\langle u_{1}, \ldots, u_{r}\right\rangle$.

However, working with clones over objects gives us more room to obtain new results since we can use the categorical environment. For instance, we can observe the interaction of the clones with other morphisms in the category. As it will be presented in Chapter 5, this allows us to lift the Galois connection Pol-Inv to clones over objects, whereas we do not have such a thing for abstract clones or Lawvere Theories. Furthermore, as mentioned before, we can move clones from one categorical environment into another and see where the problem is the easiest to solve. We will see in Chapter 7 and 8 that this technique will give us some new results for clones over sets.

\subsection{Minimal Clones}

In the introduction to this chapter we claimed that many results that hold for clones over sets can be generalized to clones of operations over objects. In this section, we will start living up to this claim and turn to minimal clones. This area of clone theory seems very promising for generalization since, as it was noted by P. P. PÁLFY in [Pál86], the minimality of a clone is an inner property, implying that minimality is invariant under isomorphisms and can therefore be treated abstractly. In fact, some results for minimal clones were obtained by treating the clones as abstract clones (e.g. in [LP96], Wal07]). We will generalize two of the most important results, namely the Świerczkowski Lemma [Świ61] and Rosenberg's Classification Theorem for minimal clones Ros83. This will not only serve as an illustration of how to generalize results to our framework, but the two results will also be used in the remainder of this thesis.

For the whole section, let $\mathcal{A}$ be a category that contains all non-empty finite powers of an object $\mathbf{A} \in \mathcal{A}$.

2.3.1 Lemma (Generalized Świerczkowski Lemma). Let $f \in O_{\mathbf{A}}$ be an at least quaternary operation. If every operation arising from $f$ by identification of two arguments is a projection, then $f$ is a semiprojection.

Proof. This proof is a generalized version of the proof sketched in Qua95. Let $k \geq 4$ and let $f \in O_{\mathbf{A}}^{(k)}$. We distinguish two cases:

Case 1: For some $i \in\{1, \ldots, k\}$, we have

$$
f \circ\left\langle\pi_{2}^{k}, \ldots, \pi_{2}^{k}, \pi_{\substack{\uparrow \\ i}}^{k}, \pi_{2}^{k}, \ldots, \pi_{2}^{k}\right\rangle=\pi_{1}^{k} .
$$

Without loss of generality, we can assume $i=1$ so that $f \circ\left\langle\pi_{1}^{k}, \pi_{2}^{k}, \ldots, \pi_{2}^{k}\right\rangle=\pi_{1}^{k}$. But now, we must have

$$
f \circ\left\langle\pi_{1}^{k}, \pi_{2}^{k}, \pi_{3}^{k}, \pi_{3}^{k}, \pi_{5}^{k}, \ldots, \pi_{k}^{k}\right\rangle=\pi_{1}^{k}
$$


which implies

$$
f \circ\left\langle\pi_{1}^{k}, \pi_{1}^{k}, \pi_{2}^{k}, \pi_{2}^{k}, \pi_{5}^{k}, \ldots, \pi_{k}^{k}\right\rangle=\pi_{1}^{k},
$$

and consequently $f \circ\left\langle\pi_{1}^{k}, \pi_{1}^{k}, \pi_{3}^{k}, \ldots, \pi_{k}^{k}\right\rangle=\pi_{1}^{k}$. Analogously, we can show

$$
f \circ\left\langle\pi_{1}^{k}, \pi_{2}^{k}, \pi_{1}^{k}, \pi_{4}^{k}, \ldots, \pi_{k}^{k}\right\rangle=\ldots=f \circ\left\langle\pi_{1}^{k}, \pi_{2}^{k}, \ldots, \pi_{k-1}^{k}, \pi_{1}^{k}\right\rangle=\pi_{1}^{k},
$$

and we are done with case 1.

Case 2: We have

$$
f \circ\left\langle\pi_{1}^{k}, \pi_{2}^{k}, \ldots, \pi_{2}^{k}\right\rangle=f \circ\left\langle\pi_{2}^{k}, \pi_{1}^{k}, \pi_{2}^{k}, \ldots, \pi_{2}^{k}\right\rangle=\ldots=f \circ\left\langle\pi_{2}^{k}, \ldots, \pi_{2}^{k}, \pi_{1}^{k}\right\rangle=\pi_{2}^{k} .
$$

We will show that, for all $i, j \in\{1, \ldots, k\}$ with $i<j$, we must have

$$
f \circ\left\langle\pi_{1}^{k}, \ldots, \pi_{j-1}^{k}, \pi_{i}^{k}, \pi_{j+1}^{k}, \ldots, \pi_{k}^{k}\right\rangle=\pi_{i}^{k} .
$$

For contradiction, let $i, j$ be as above and assume that there exists $l \neq i$ such that

$$
f \circ\left\langle\pi_{1}^{k}, \ldots, \pi_{j-1}^{k}, \pi_{i}^{k}, \pi_{j+1}^{k}, \ldots, \pi_{k}^{k}\right\rangle=\pi_{l}^{k} .
$$

Then, we obtain

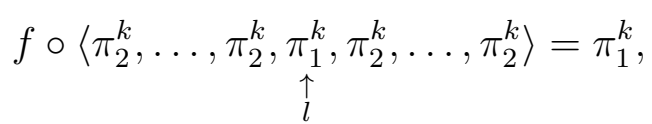

which contradicts the assumption of this case. Thus, we have

$$
f \circ\left\langle\pi_{1}^{k}, \ldots, \pi_{j-1}^{k}, \pi_{i}^{k}, \pi_{j+1}^{k}, \ldots, \pi_{k}^{k}\right\rangle=\pi_{i}^{k}
$$

for all $1 \leq i<j \leq k$. But now,

$$
\pi_{1}^{k}=f \circ\left\langle\pi_{1}^{k}, \pi_{1}^{k}, \pi_{2}^{k}, \pi_{2}^{k}, \pi_{5}^{k}, \ldots, \pi_{k}^{k}\right\rangle=\pi_{2}^{k} .
$$

Thus,

$$
f=\pi_{1}^{k} \circ\left\langle f, \pi_{1}^{k}, \ldots, \pi_{1}^{k}\right\rangle=\pi_{2}^{k} \circ\left\langle f, \pi_{1}^{k}, \ldots, \pi_{1}^{k}\right\rangle=\pi_{1}^{k},
$$

a contradiction.

Recall that an operation is called minimal if it generates a minimal clone and has minimum arity among all operations that generate this clone.

2.3.2 Theorem (Generalized Rosenberg's Classification Theorem (RCT) ${ }^{1}$ ). Every minimal operation over $\mathbf{A}$ is of one of the following five types:

1. a unary operation that is either a retraction or an automorphism of prime order,

2. a binary idempotent operation,

3. a majority operation,

4. a minority operation,

${ }^{1}$ In the original formulation, the RCT also states that, in the fourth case, the minority operation on the set $A$ is necessarily $x+y+z$ in a Boolean group. 


\section{Clones of Operations}

\section{5. a semiprojection.}

Proof. This proof is essentially a generalized version of the original proof (see [Ros83]). Assume that $f$ is unary. If $f$ is neither a retraction nor an automorphism of finite order, then we have $\left(f^{2}\right)^{n} \neq f$ for all $n \in \mathbb{N}_{+}$, whence it follows $f \notin \operatorname{Clo}\left(f^{2}\right)$. Hence, $\mathrm{Clo}\left(f^{2}\right) \subsetneq \mathrm{Clo}(f)$, a contradiction to the minimality of $f$.

Let $f$ be an automorphism of order $q$ (i.e., $f^{q}=i d_{\mathbf{A}}$ while $f^{i} \neq i d_{\mathbf{A}}$ for $i \in\{1, \ldots, q-1\}$ ). Suppose that $q$ is not prime. Let $q=r s$ where $r$ is a prime divisor of $q$. Setting $g:=f^{s} \in \mathrm{Clo}(f)$, we reach a contradiction since $f \notin \mathrm{Clo}(g)$.

Now assume that $f$ is binary. Since identification of the two arguments must yield a trivial operation, we have $f \circ\left\langle\pi_{1}^{2}, \pi_{1}^{2}\right\rangle=\pi_{1}^{2}$. That is, $f$ is idempotent.

Now assume that $f$ is ternary. For the same reasons as above, we must obtain a projection by any identification of two arguments. This leaves us with eight possible cases:

\begin{tabular}{|l|c|c|c|c|c|c|c|c|}
\hline & $(1)$ & $(2)$ & $(3)$ & $(4)$ & $(5)$ & $(6)$ & $(7)$ & $(8)$ \\
\hline$f \circ\left\langle\pi_{1}^{3}, \pi_{1}^{3}, \pi_{2}^{3}\right\rangle=$ & $\pi_{1}^{3}$ & $\pi_{1}^{3}$ & $\pi_{1}^{3}$ & $\pi_{1}^{3}$ & $\pi_{2}^{3}$ & $\pi_{2}^{3}$ & $\pi_{2}^{3}$ & $\pi_{2}^{3}$ \\
$f \circ\left\langle\pi_{1}^{3}, \pi_{2}^{3}, \pi_{1}^{3}\right\rangle=$ & $\pi_{1}^{3}$ & $\pi_{1}^{3}$ & $\pi_{2}^{3}$ & $\pi_{2}^{3}$ & $\pi_{1}^{3}$ & $\pi_{1}^{3}$ & $\pi_{2}^{3}$ & $\pi_{2}^{3}$ \\
$f \circ\left\langle\pi_{2}^{3}, \pi_{1}^{3}, \pi_{1}^{3}\right\rangle=$ & $\pi_{1}^{3}$ & $\pi_{2}^{3}$ & $\pi_{1}^{3}$ & $\pi_{2}^{3}$ & $\pi_{1}^{3}$ & $\pi_{2}^{3}$ & $\pi_{1}^{3}$ & $\pi_{2}^{3}$ \\
\hline
\end{tabular}

In the cases (1) and (8), $f$ is a majority and minority operation, respectively. In the cases (2), (3) and (5), $f$ is a ternary semiprojection. To show that the cases (4), (6) and (7) cannot occur we define

$$
\begin{aligned}
& f_{4}:=f \circ\left\langle\pi_{1}^{3}, \pi_{2}^{3}, f\right\rangle, \\
& f_{6}:=f \circ\left\langle\pi_{1}^{3}, f, \pi_{3}^{3}\right\rangle, \\
& f_{7}:=f \circ\left\langle f, \pi_{2}^{3}, \pi_{3}^{3}\right\rangle .
\end{aligned}
$$

Clearly, $f_{4}, f_{6}, f_{7} \in \mathrm{Clo}(f)$. Furthermore, it can be checked that $f_{4}, f_{6}, f_{7}$ are majority operations in the cases $(4),(6),(7)$, respectively. But now $f \notin \operatorname{Clo}\left(f_{4}\right), \operatorname{Clo}\left(f_{6}\right), \operatorname{Clo}\left(f_{7}\right)$, respectively, because it is easy to see that any ternary operation generated by a majority operation has to be a majority operation again.

Finally, let $f$ be an at least quaternary operation. By the same argument as above, $f$ turns into a projection by identification of any two arguments. But now, by the Generalized Świerczkowski Lemma, these projections have to coincide. Thus, $f$ is a semiprojection.

Using analogue arguments as in the usual version of the RCT, one can easily show that the first case ensures the minimality of the operation, whereas the other cases do not. 


\section{Clones of Dual Operations}

For the whole chapter, let $x$ be a category and let $\mathbf{X}$ be an object in $x$ such that all non-empty finite copowers of $\mathbf{X}$ are also in $X$.

\subsection{Dual Operations and their Clones}

Analogue to operations, we can define dual operations and clones of dual operations.

3.1.1 Definition. Let $n \in \mathbb{N}_{+}$. An $n$-ary dual operation over $\mathbf{X}$ (or cooperation over $\mathbf{X})$ is a morphism from $\mathbf{X}$ to $n \cdot \mathbf{X}$. Denote by $\bar{O}_{\mathbf{X}}^{(n)}$ the set of all $n$-ary dual operations over $\mathbf{X}$, define $\bar{O}_{\mathbf{X}}:=\bigcup_{n \in \mathbb{N}_{+}} \bar{O}_{\mathbf{X}}^{(n)}$ and, for a set of dual operations $G \subseteq \bar{O}_{\mathbf{X}}$, set $G^{(n)}:=G \cap \bar{O}_{\mathbf{X}}^{(n)}$.

3.1.2 Example. In the category Set, a dual operation over $\mathbf{X} \in$ Set is a cofunction as introduced in the preliminaries (see Definition 1.3.1, page 15).

We can also distinguish between essential and nonessential arguments of a dual operation.

3.1.3 Definition. Let $\widetilde{\iota}_{i}^{n}:(n-1) \cdot \mathbf{X} \rightarrow n \cdot \mathbf{X}$ denote the operation defined by setting

$$
\widetilde{\iota}_{i}^{n}:=\left[\iota_{1}^{n}, \ldots, \iota_{i-1}^{n}, \iota_{i+1}^{n}, \ldots, \iota_{n}^{n}\right] .
$$

The $i$-th argument of an $n$-ary dual operation $g$ is said to be nonessential if there exists an $(n-1)$-ary dual operation $g^{\prime}$ over $\mathbf{X}$ such that $g=\widetilde{\iota}_{i}^{n} \circ g^{\prime}$. An argument is called essential if it is not nonessential.

Analogue to operations, we say that a dual operation depends on an argument if this argument is essential, and we say that a dual operation is essentially $n$-ary if it has exactly $n$ essential arguments.

The following definitions explain what we mean when we say that we identify arguments or permute arguments in a dual operation:

3.1.4 Definition. Let $n \geq 2$, let $g \in \bar{O}_{\mathbf{X}}^{(n)}$, and let $i, j \in\{1, \ldots, n\}$ with $i<j$. We say that $g^{\prime} \in \bar{O}_{\mathbf{X}}^{(n-1)}$ arises from $g$ by identification of the $i$-th and $j$-th argument if we have $g^{\prime}=\left[\iota_{1}^{n-1}, \ldots, \iota_{j-1}^{n-1}, \iota_{i}^{n-1}, \iota_{j}^{n-1}, \ldots, \iota_{n-1}^{n-1}\right] \circ g$.

3.1.5 Definition. Let $g, g^{\prime} \in \bar{O}_{\mathbf{X}}^{(n)}$. We say that $g^{\prime}$ arises from $g$ by permutation of arguments if there exists a permutation $p$ on $\{1, \ldots, n\}$ such that $g^{\prime}=\left[\iota_{p(1)}^{n}, \ldots, \iota_{p(n)}^{n}\right] \circ g$. 


\section{Clones of Dual Operations}

Moreover, we define the notion of two dual operations to be essentially the same or essentially different exactly as we did for operations in Definition 2.1.8 (page 29).

We will now introduce clones of dual operations:

3.1.6 Definition. A set $C$ of dual operations over $\mathbf{X}$ is a clone of dual operations (or coclone) if it contains all the injection morphisms and, for $g \in C^{(n)}$ and $g_{1}, \ldots, g_{n} \in C^{(k)}$, the superposition $\left[g_{1}, \ldots, g_{n}\right] \circ g$ is also in $C$.

3.1.7 Example. If $\mathbf{X}$ is a finite set in the category of sets, then a clone of dual operations over $\mathbf{X}$ is a coclone as introduced in the preliminaries (see Definition 1.3.2, page 16).

Evidently, all clones of dual operations over $\mathbf{X}$ form a complete lattice with respect to inclusion. The greatest clone of dual operations over $\mathbf{X}$ is $\bar{O}_{\mathbf{X}}$, while the smallest clone of dual operations over $\mathbf{X}$ is the clone that contains only the injection morphisms. Denote the latter by $\bar{J}_{\mathbf{X}}$.

3.1.8 Definition. Denote by $\bar{L}_{\mathbf{X}}$ the set of clones of dual operations over $\mathbf{X}$. Then, $\overline{\mathcal{L}}_{\mathbf{X}}:=\left\langle L_{\mathbf{X}}, \subseteq\right\rangle$ is called the lattice of clones of dual operations over $\mathbf{X}$.

Analogue to the closure operator Clo on the clones of operations, we can define $\overline{\mathrm{Clo}}$ : For a set of dual operations $G \subseteq O_{\mathbf{A}}$, we denote by $\overline{\mathrm{Clo}}(G)$ the least clone of dual operations that contains $G$.

We will now explain the relationship between the notions introduced in this section and the notions introduced in the last section. Let $\mathcal{A}$ be a category and let $\mathbf{A} \in \mathcal{A}$. Recall that $\mathcal{A}$ contains all non-empty finite powers of $\mathbf{A}$ if and only if $\mathcal{A}^{o p}$ (the opposite category, see Definition 1.4.26, page 23) contains all non-empty finite copowers of $\mathbf{A}$. Furthermore, for $f \in O_{\mathbf{A}}^{(n)}$ and $f_{1}, \ldots, f_{n} \in O_{\mathbf{A}}^{(n)}$, we have

$$
\left(f \circ\left\langle f_{1}, \ldots, f_{n}\right\rangle\right)^{o p}=\left[f_{1}^{o p}, \ldots, f_{n}^{o p}\right] \circ f^{o p} .
$$

Hence, a set $C$ of operations is a clone of operations over $\mathbf{A}$ in $\mathcal{A}$ if and only if $C^{o p}$ is a clone of dual operations over $\mathbf{A}$ in $\mathcal{A}^{o p}$. In other words, we have the following proposition:

3.1.9 Proposition. Clones of dual operations over $\mathbf{X} \in X$ are clones of operations over $\mathrm{X} \in X^{o p}$ and vice versa.

Although it was already acknowledged in [Csá85] that clones and coclones can both be considered as abstract clones, they have mostly been treated separately. This proposition shows that this is not necessary. Both are clones of operations in a suitable category. Thus, every result for clones of operations has a dual counterpart for clones of dual operations and vice versa. For instance, if we define $\llbracket g_{1}, \ldots, g_{n} \rrbracket \circ g$ to be the expanded superposition of dual operations $g \in \bar{O}_{\mathbf{X}}^{(n)}$ and $g_{1}, \ldots, g_{n} \in \bar{O}_{\mathbf{X}}$, then the following proposition is the dualized version of Proposition 2.1 .12 and is therefore without any need of a proof:

3.1.10 Proposition. Clones of dual operations are closed under expanded superposition. 
Also, we can dualize the types of operations that were introduced in Definition 2.1.16 (page 30).

\subsubsection{Definition.}

(i) A unary operation $g \in \bar{O}_{\mathbf{X}}$ is called a retraction if $g \circ g=g !^{1}$

(ii) For $n \geq 3$, an $n$-ary dual operation $g \in \bar{O}_{\mathbf{X}}$ is called a dual near-unanimity operation if

$$
\begin{gathered}
{\left[\iota_{2}^{n}, \iota_{1}^{n}, \iota_{1}^{n}, \ldots, \iota_{1}^{n}\right] \circ g=\iota_{1}^{n},} \\
{\left[\iota_{1}^{n}, \iota_{2}^{n}, \iota_{1}^{n}, \ldots, \iota_{1}^{n}\right] \circ g=\iota_{1}^{n},} \\
\vdots \\
{\left[\iota_{1}^{n}, \iota_{1}^{n}, \ldots, \iota_{1}^{n}, \iota_{2}^{n}\right] \circ g=\iota_{1}^{n} .}
\end{gathered}
$$

Ternary dual near-unanimity operations are called dual majority operations.

(iii) A ternary dual operation $g \in \bar{O}_{\mathbf{X}}$ is called a dual minority operation if

$$
\left[\iota_{1}^{3}, \iota_{2}^{3}, \iota_{2}^{3}\right] \circ g=\left[\iota_{2}^{3}, \iota_{1}^{3}, \iota_{2}^{3}\right] \circ g=\left[\iota_{2}^{3}, \iota_{2}^{3}, \iota_{1}^{3}\right] \circ g=\iota_{1}^{3} .
$$

(iv) An $n$-ary dual operation $g \in \bar{O}_{\mathbf{X}}$ is called idempotent if $\left[\iota_{1}^{n}, \ldots, \iota_{1}^{n}\right] \circ g=\iota_{1}^{n}$.

(v) An $n$-ary operation $g$ is called a semiinjection if there exists $i \in\{1, \ldots, n\}$ such that

$$
\left[\iota_{j_{1}}^{n}, \ldots, \iota_{j_{n}}^{n}\right] \circ g=\iota_{j_{i}}^{n}
$$

whenever $j_{1}, \ldots, j_{n}$ are not pairwise distinct. If $g$ is also nontrivial, then we say that $g$ is a proper semiinjection.

Clearly, $f$ is an operation of one of the types given in Definition 2.1.16 (page 30) if and only if $f^{o p} \in \bar{O}_{\mathbf{A}}$ is a dual operation of the corresponding type and vice versa.

Again, except the idempotent dual operations, the dual operations from above are defined by irregular identities.

3.1.12 Definition. Let $g \in \bar{O}_{\mathbf{X}}^{(n)}$ and $h \in \bar{O}_{\mathbf{X}}^{(r)}$. For $t \in\{1, \ldots, n\}$, the identity

$$
\left[\iota_{i_{1}}^{n}, \ldots, \iota_{i_{n}}^{n}\right] \circ g=\left[\iota_{j_{1}}^{n}, \ldots, \iota_{j_{r}}^{n}\right] \circ h
$$

is called irregular in its $t$-th argument if $i_{t} \notin\left\{j_{1}, \ldots, j_{r}\right\}$.

Let us come back to something we have mentioned in the preliminaries. We claimed that it can be somewhat misleading to understand coclones in the usual sense (clones of dual operations over sets in our terminology) as the dual counterpart of a clone. Here is why: For a clone $C$ over a set $A$, the dual $C^{o p}$ is not a clone of dual operations over a set, but over some object in $\operatorname{Set}^{o p}$. Furthermore, only in very few cases, $C^{o p}$ is isomorphic to a clone of dual operations over a set (we will study these clones in Section 4.2).

\footnotetext{
${ }^{1}$ Of course, this definition is somewhat redundant since unary operations and unary dual operations are the same thing.
} 
Indeed, clones of operations over sets and clones of dual operations over sets are two essentially different structures (although some surprising similarities occur, see [Maš99]).

For instance, $\overline{\mathcal{L}}_{A}$ is finite for any finite set $A$, whereas $\mathcal{L}_{A}$ is of course infinite for $|A| \geq 2$ (and also uncountable for $|A| \geq 3$ ). Thus, even if we only want dual counterparts for the clones over sets, we still need to consider clones of dual operations in categories that are different from Set.

\subsection{Minimal Clones}

As we have mentioned in the last section, we can dualize every statement that holds for clones of operations and it will hold for clones of dual operations. Here, we present the duals of the two results from Section 2.3,

3.2.1 Lemma (Dualized Świerczkowski Lemma). Let $g \in \bar{O}_{\mathbf{X}}$ be an at least quaternary dual operation. If every dual operation arising from $g$ by identification of two arguments is an injection morphism, then $g$ is a semiinjection.

3.2.2 Theorem (Dualized Rosenberg's Classification Theorem (RCT)). Every minimal dual operation is of one of the following five types:

1. a unary (dual) operation that is either a retraction or an automorphism of prime order,

2. a binary dual idempotent operation,

3. a dual majority operation,

4. a dual minority operation,

5. a semiinjection.

Analogue to the RCT for operations, the first case ensures the minimality of the dual operation, whereas the other cases do not.

\subsection{Clones of Dual Operations and Abstract Clones}

We have mentioned in the preliminaries that every abstract clone is isomorphic to some clone over a set. This raises the question of whether there exists a specific concrete category $x$ such that every abstract clone is isomorphic to a clone of dual operations over some $\mathbf{X} \in X$. Obviously, not every abstract clone can be isomorphic to a clone of dual operations over a set. What we need is a category that is dually equivalent to the category of sets, and such a category is the category of complete atomic Boolean algebras, where the morphisms are the homomorphisms between the algebras.

3.3.1 Definition. A complete Boolean algebra is a Boolean algebra in which every subset has a supremum. It is said to be atomic if every element can be written as the supremum of a set of atoms. 
Note that the finite part of the category of complete atomic Boolean algebras is simply the category of finite Boolean algebras.

We can state the following result:

3.3.2 Proposition. Every abstract clone is isomorphic to a clone of dual operations over a complete atomic Boolean algebra. 


\section{A Duality for Clones}

Recall that the functor $(-)^{o p}$ carries a clone of operations to a clone of dual operations. Thus, (-) $)^{o p}$ enables us to dualize clones of operations to clones of dual operations and vice versa. However, for a given category $\mathcal{A}$, the category $\mathcal{A}^{o p}$ is a rather abstract concept, and one usually does not know how to interpret its morphisms. In practice, one approaches $\mathcal{A}^{o p}$ by looking at a category that is equivalent to $\mathcal{A}^{o p}$ but easier to understand, preferably a category that has functions as morphisms. For instance, instead of looking at $\operatorname{Set}_{\text {fin }}{ }^{o p}$, one usually looks at the category of finite Boolean algebras. Since a category equivalent to $\mathcal{A}^{o p}$ is necessarily dually equivalent to $\mathcal{A}$, this raises the question of how we can dualize a clone of operations in $\mathcal{A}$ to a clone of dual operations in any dually equivalent category $x$. In this chapter, we will explain how this can be done.

\subsection{From Dual Equivalences to Dualizing Clones}

Let $\langle D, E, e, \epsilon\rangle$ be a dual equivalence between the two categories $\mathcal{A}$ and $\mathcal{X}$, and let $\mathbf{A} \in \mathcal{A}$ such that all finite non-empty powers of $\mathbf{A}$ are also in $\mathcal{A}$. Set $\mathbf{X}:=D(\mathbf{A})$. Since $\mathcal{A}$ and $x$ are dually equivalent, $x$ contains all finite non-empty copowers of $\mathbf{X}$. The functor $D$ carries $\mathbf{A}$ to $\mathbf{X}$ and reverses the order of the morphisms, so wishful thinking suggests that it should map a morphism $f \in O_{\mathbf{A}}$ to a morphism in $\bar{O}_{\mathbf{X}}$. Unfortunately, $D$ only maps $f$ to a morphism from $\mathbf{X}$ to $D\left(\mathbf{A}^{n}\right)$ and the latter is only isomorphic and not necessarily equal to $n \cdot \mathbf{X}]^{1}$ However, we can get around this technical problem by finding a family of isomorphism $\left(\eta_{n}\right)_{n \in \mathbb{N}_{+}}$such that $f \mapsto \eta_{\text {ar }(f)} \circ D(f)$ becomes a clone isomorphism from $O_{\mathbf{A}}$ to $\bar{O}_{\mathbf{X}}$ (recall that $\operatorname{ar}(f)$ denotes the arity of $f$ ).

4.1.1 Lemma. For every $n \in \mathbb{N}_{+}$, there exists a unique isomorphism $\eta_{n}: D\left(\mathbf{A}^{n}\right) \rightarrow n \cdot \mathbf{X}$ such that the following diagram commutes for all $i \in\{1, \ldots, n\}$ :

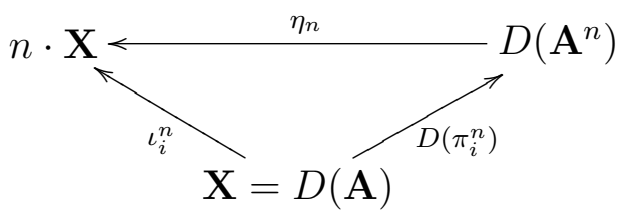

Proof. Since $\mathcal{A}$ and $x$ are dually equivalent, products in $\mathcal{A}$ are taken to coproducts in $x$. Hence, $D\left(\mathbf{A}^{n}\right)$, together with the associated morphisms $D\left(\pi_{1}^{n}\right), \ldots, D\left(\pi_{n}^{n}\right)$, fulfils the condition of being a coproduct in $X$. Therefore, we must have a unique morphism

${ }^{1}$ Of course, we could avoid the trouble by defining $n \cdot \mathbf{X}:=D\left(\mathbf{A}^{n}\right)$ for all $n \in \mathbb{N}_{+}$. But then, the copowers of $\mathbf{X}$ might not be canonical and they would depend on the choice of the dual equivalence. One usually wants to avoid both. 
$\eta_{n}: D\left(\mathbf{A}^{n}\right) \rightarrow n \cdot \mathbf{X}$ such that the above diagram commutes for all $i \in\{1, \ldots, n\}$. It remains to show that $\eta_{n}$ is an isomorphism. We will do so by showing that the morphism $\left[D\left(\pi_{1}^{n}\right), \ldots, D\left(\pi_{n}^{n}\right)\right]$ is the inverse of $\eta_{n}$ : On the one hand, we have

$$
\eta_{n} \circ\left[D\left(\pi_{1}^{n}\right), \ldots, D\left(\pi_{n}^{n}\right)\right]=\left[\eta_{n} \circ D\left(\pi_{1}^{n}\right), \ldots, \eta_{n} \circ D\left(\pi_{n}^{n}\right)\right]=\left[\iota_{1}^{n}, \ldots, \iota_{n}^{n}\right]=i d_{n} \cdot \mathbf{x} .
$$

On the other hand, we have

$$
\left[D\left(\pi_{1}^{n}\right), \ldots, D\left(\pi_{n}^{n}\right)\right] \circ \eta_{n} \circ D\left(\pi_{i}^{n}\right)=\left[D\left(\pi_{1}^{n}\right), \ldots, D\left(\pi_{n}^{n}\right)\right] \circ \iota_{i}^{n}=D\left(\pi_{i}^{n}\right)=i d_{D\left(\mathbf{A}^{n}\right)} \circ D\left(\pi_{i}^{n}\right)
$$

for all $i \in\{1, \ldots, n\}$. Since $D\left(\mathbf{A}^{n}\right)$, together with the morphisms $D\left(\pi_{1}^{n}\right), \ldots, D\left(\pi_{n}^{n}\right)$, fulfils the condition to be a coproduct, there exists a unique morphism $v$ such that $v \circ D\left(\pi_{i}^{n}\right)=D\left(\pi_{i}^{n}\right)$ for all $i \in\{1, \ldots, n\}$. But now, the uniqueness of $v$ yields the desired equation $\left[D\left(\pi_{1}^{n}\right), \ldots, D\left(\pi_{n}^{n}\right)\right] \circ \eta_{n}=i d_{D\left(\mathbf{A}^{n}\right)}$.

Note that the proof yields a way to construct $\eta_{n}$. Indeed, we can immediately state the following proposition:

4.1.2 Proposition. $\eta_{n}=\left[D\left(\pi_{1}^{n}\right), \ldots, D\left(\pi_{n}^{n}\right)\right]^{-1}$.

The following lemma holds:

4.1.3 Lemma. Let $\mathbf{B} \in \mathcal{A}, n \in \mathbb{N}_{+}$and let $f_{1}, \ldots, f_{n}: \mathbf{B} \rightarrow \mathbf{A}$. Then,

$$
D\left(\left\langle f_{1}, \ldots, f_{n}\right\rangle\right)=\left[D\left(f_{1}\right), \ldots, D\left(f_{n}\right)\right] \circ \eta_{n} .
$$

Proof. By Lemma 4.1.1, we have

$$
D\left(f_{i}\right)=D\left(\pi_{i}^{n} \circ\left\langle f_{1}, \ldots, f_{n}\right\rangle\right)=D\left(\left\langle f_{1}, \ldots, f_{n}\right\rangle\right) \circ D\left(\pi_{i}^{n}\right)=D\left(\left\langle f_{1}, \ldots, f_{n}\right\rangle\right) \circ \eta_{n}^{-1} \circ \iota_{i}^{n}
$$

for all $i \in\{1, \ldots, n\}$. Thus,

$$
\begin{aligned}
{\left[D\left(f_{1}\right), \ldots, D\left(f_{n}\right)\right] } & =\left[D\left(\left\langle f_{1}, \ldots, f_{n}\right\rangle\right) \circ \eta_{n}^{-1} \circ \iota_{1}^{n}, \ldots, D\left(\left\langle f_{1}, \ldots, f_{n}\right\rangle\right) \circ \eta_{n}^{-1} \circ \iota_{n}^{n}\right] \\
& =D\left(\left\langle f_{1}, \ldots, f_{n}\right\rangle\right) \circ \eta_{n}^{-1} \circ\left[\iota_{1}^{n}, \ldots, \iota_{n}^{n}\right] \\
& =D\left(\left\langle f_{1}, \ldots, f_{n}\right\rangle\right) \circ \eta_{n}^{-1} .
\end{aligned}
$$

We can now define the desired duality between the clones of operations over $\mathbf{A}$ and the clones of dual operations over $\mathbf{X}$.

4.1.4 Definition. The mapping $(-)^{\partial}: O_{\mathbf{A}} \rightarrow \bar{O}_{\mathbf{X}}$, defined by setting

$$
f^{\partial}:=\eta_{a r(f)} \circ D(f)
$$

is called the clone duality with respect to $D$. For $F \subseteq O_{\mathbf{A}}$, set

$$
F^{\partial}:=\left\{f^{\partial} \mid f \in F\right\} .
$$

It remains to show that $(-)^{\partial}: O_{\mathbf{A}} \rightarrow \bar{O}_{\mathbf{X}}$ has the properties that the name suggests. That is, we need to show that $(-)^{\partial}$ is a clone isomorphism and that it is uniquely determined by the functor $D$. 
4.1.5 Lemma. Let $k, n \in \mathbb{N}_{+}$. Then,

(i) $(-)^{\partial}: O_{\mathbf{A}} \rightarrow \bar{O}_{\mathbf{X}}$ is a bijection,

(ii) $\left(\pi_{i}^{n}\right)^{\partial}=\iota_{i}^{n}$ and $\left(f \circ\left\langle f_{1}, \ldots, f_{n}\right\rangle\right)^{\partial}=\left[f_{1}^{\partial}, \ldots, f_{n}^{\partial}\right] \circ f^{\partial}$ for all $f_{1}, \ldots, f_{n} \in O_{\mathbf{A}}^{(k)}$, whence it follows that $C$ is a clone of operations over $\mathbf{A}$ if and only if $C^{\partial}$ is a clone of dual operations over $\mathbf{X}$.

Proof. (i) Since $\langle D, E, e, \epsilon\rangle$ is a dual equivalence, $D$ is both full and faithful. Furthermore, we have just seen that $\eta_{n}$ is an isomorphism for all $n \in \mathbb{N}_{+}$. Thus, $f \mapsto \eta_{\operatorname{ar}(f)} \circ D(f)$ is bijective on $O_{\mathbf{A}}$.

(ii) By Lemma 4.1.1, we have $\left(\pi_{i}^{n}\right)^{\partial}=\eta_{n} \circ D\left(\pi_{i}^{n}\right)=\iota_{i}^{n}$. For the second part, according to Lemma 4.1.3, we get

$$
\begin{aligned}
\left(f \circ\left\langle f_{1}, \ldots, f_{n}\right\rangle\right)^{\partial} & =\eta_{k} \circ D\left(f \circ\left\langle f_{1}, \ldots, f_{n}\right\rangle\right) \\
& =\eta_{k} \circ D\left(\left\langle f_{1}, \ldots, f_{n}\right\rangle\right) \circ D(f) \\
& =\eta_{k} \circ\left[D\left(f_{1}\right), \ldots, D\left(f_{n}\right)\right] \circ \eta_{n} \circ D(f) \\
& =\left[\eta_{k} \circ D\left(f_{1}\right), \ldots, \eta_{k} \circ D\left(f_{n}\right)\right] \circ \eta_{n} \circ D(f) \\
& =\left[f_{1}^{\partial}, \ldots, f_{n}^{\partial}\right] \circ f^{\partial} .
\end{aligned}
$$

Now, the following theorem is an immediate consequence:

4.1.6 Theorem. $\mathcal{L}_{\mathbf{A}} \cong \overline{\mathcal{L}}_{\mathbf{X}}$, where an isomorphism between $\mathcal{L}_{\mathbf{A}}$ and $\overline{\mathcal{L}}_{\mathbf{X}}$ is given by $C \mapsto C^{\partial}$.

Moreover, $(-)^{\partial}$ is unique in the following sense: It is the only isomorphism (with respect to superposition) from $O_{\mathbf{A}}$ to $\bar{O}_{\mathbf{X}}$ such that $\left(\pi_{i}^{n}\right)^{\partial}=\iota_{i}^{n}$ for all $n \in \mathbb{N}_{+}$and $i \in\{1, \ldots, n\}$. This follows directly from the uniqueness of $\eta_{n}$. Therefore, $(-)^{\partial}$ is uniquely determined by $O_{\mathbf{A}}$ and the functor $D$. Thus, it is justified to call $(-)^{\partial}$ the clone duality with respect to $D$.

Since $(-)^{\partial}$ is simply a morphism that is obtained by applying an isomorphism after $D$, it will respect the Duality Principle. That is, a (purely category-theoretic) statement holds for a clone of operations $C \leq O_{\mathbf{A}}$ if and only if the dualized statement holds for the clone of dual operations $C^{\partial} \leq \bar{O}_{\mathbf{X}}$. For instance, an identity holds in $C$ if and only if its dualized version holds in $C^{\partial}$.

4.1.7 Lemma. Let $f \in O_{\mathbf{A}}^{(n)}$ and let $h \in O_{\mathbf{A}}^{(k)}$. For integers $i_{1}, \ldots, i_{n} \in\{1, \ldots, n\}$ and $j_{1}, \ldots, j_{k} \in\{1, \ldots n\}$, we have

$$
f \circ\left\langle\pi_{i_{1}}^{n}, \ldots, \pi_{i_{n}}^{n}\right\rangle=h \circ\left\langle\pi_{j_{1}}^{n}, \ldots, \pi_{j_{k}}^{n}\right\rangle \Longleftrightarrow\left[\iota_{i_{1}}^{n}, \ldots, \iota_{i_{n}}^{n}\right] \circ f^{\partial}=\left[\iota_{j_{1}}^{n}, \ldots, \iota_{j_{k}}^{n}\right] \circ h^{\partial} .
$$

Proof. " $\Longrightarrow$ " follows by applying $(-)^{\partial}$ to both sides of the equation and using Lemma 4.1 .5 (ii). This also implies "£" since $(-)^{\partial}$ is an isomorphism.

Thus, $f$ is an operation of one of the types given in Definition 2.1.16 (page 30) if and only if $f^{\partial}$ is the according type of dual operation given in Definition 3.1.11 (page 37). The following lemma is another direct consequence: 
4.1.8 Lemma. Let $f \in O_{\mathbf{A}}^{(n)}$. The $i$-th argument of $f$ is nonessential if and only if the $i$-th argument of $f^{\partial}$ is nonessential.

Note that the duality provides us with a new technique to examine clones: Instead of trying to solve a problem for a clone of operations $C$, we can solve the dualized problem for $C^{\partial}$, which might be easier. Indeed, being able to do this is quite possibly the main benefit of our theory.

After dualizing clones of operations over $\mathbf{A}$ to clones of dual operations over $\mathbf{X}$, one might want to dualize them back, requiring the inverse of $(-)^{\partial}$. The following proposition shows that $(-)^{\partial^{-1}}$ can be calculated with the functor $E$ and the two natural transformations $e$ and $\epsilon$ :

4.1.9 Proposition. The inverse of $(-)^{\partial}$ is given by

$$
g \mapsto e_{\mathbf{A}}^{-1} \circ E\left(\eta_{a r(g)}^{-1} \circ g\right) \circ e_{\mathbf{A}^{a r(g)}} .
$$

Proof. Let $f \in O_{\mathbf{A}}^{(n)}$ such that $g=f^{\partial}$. Then, $g=\eta_{n} \circ D(f)$. Hence, $E\left(\eta_{n}^{-1} \circ g\right)=E D(f)$. Since $\langle D, E, e, \epsilon\rangle$ is a dual equivalence, we have $E D(f)=e_{\mathbf{A}} \circ f \circ e_{\mathbf{A}^{n}}^{-1}$. Thus,

$$
E\left(\eta_{n}^{-1} \circ g\right)=e_{\mathbf{A}} \circ f \circ e_{\mathbf{A}^{n}}^{-1}
$$

That is, $f=e_{\mathbf{A}}^{-1} \circ E\left(\eta_{n}^{-1} \circ g\right) \circ e_{\mathbf{A}^{n}}$.

Due to the symmetry of the setting, we can also start with some $\mathbf{X} \in \mathcal{X}$ (provided that the copowers of $\mathbf{X}$ exist in $X$ ) and dualize the clones of dual operations over $\mathbf{X}$ to clones of operations over $\mathbf{A}:=E(\mathbf{X})$. The arguments are all analogue: For each $n \in \mathbb{N}_{+}$, there exists a unique isomorphism $\bar{\eta}_{n}: \mathbf{A}^{n} \rightarrow E(n \cdot \mathbf{X})$ such that the following diagram commutes for all $i \in\{1, \ldots, n\}$ :

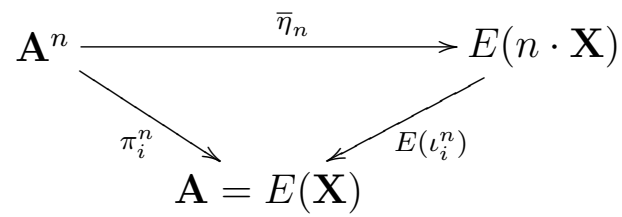

Its construction is analogue to the one of $\eta_{n}$. That is,

$$
\bar{\eta}_{n}=\left\langle E\left(\iota_{1}^{n}\right), \ldots, E\left(\iota_{n}^{n}\right)\right\rangle^{-1} .
$$

Defining $(-)^{\bar{\partial}}: \bar{O}_{\mathbf{X}} \rightarrow O_{\mathbf{A}}$ by setting

$$
g^{\bar{\partial}}:=E(g) \circ \bar{\eta}_{\operatorname{ar}(g)}
$$

gives us the desired clone isomorphism. Since it is unique in the same sense as above, it is justified to call it the clone duality with respect to $E$. Its inverse is given by

$$
f \mapsto e_{n \cdot \mathbf{X}}^{-1} \circ D\left(f \circ \bar{\eta}_{n}^{-1}\right) \circ e_{\mathbf{X}}
$$

where $n$ is the arity of $f$. 


\subsection{Dualizing Clones over Structures}

In the last section, we have presented how to dualize a clone of operations in a category $\mathcal{A}$ to a clone of dual operations in any category $\mathcal{X}$ that is dually equivalent to $\mathcal{A}$. Let us now discuss how to use our approach for clones over sets.

For this, let us assume that we have a clone $C$ over a finite set $A$. Clearly, we can dualize $C$ to a clone of dual operations $C^{\partial}$ in any category dually equivalent to Set. However, the categories that are dually equivalent to Set (such as the category of complete atomic Boolean algebras) have horrible copowers and, consequently, very complicated dual operations. Hence, dualizing $C$ in this way will probably not be of great help. It seems more promising to interpret $C$ as a clone in another category and then dualize this category instead. Preferably we want a category that is easily accessible and in which we can regard $C$ as the full clone $O_{\mathbf{A}}$ for some object $\mathbf{A}$ (so we do not have to build the clone duality in a way such that it can also dualize clones that are greater than $C$ ). A possible way to do so is to interpret $C$ as the full clone over some structure A in a quasi-variety. This is precisely the approach we will pursuit in this section.

As a first step, let us note that we can think of $C$ as the set of homomorphisms $\bigcup_{n \in \mathbb{N}_{+}} \operatorname{Hom}\left(\mathbf{A}^{n}, \mathbf{A}\right)$ for some finite structure $\mathbf{A}=\langle A, F, H, R\rangle$ (for instance, this always works if we choose $F=H=\emptyset$ and $R=\operatorname{Inv} C$ ). Now, let $\mathcal{A}$ be the finite part of any quasi-variety that contains $\mathbf{A}$. Recall that we can understand $\mathcal{A}$ as a category by defining the objects to be the structures and the morphisms to be the homomorphisms between the structures (see Example 1.4.2, page 16). In this setting, we have $O_{\mathbf{A}}=\bigcup_{n \in \mathbb{N}_{+}} \operatorname{Hom}\left(\mathbf{A}^{n}, \mathbf{A}\right)$, so $O_{\mathbf{A}}$ is essentially our clone $C$.

Moreover, we can choose $\mathcal{A}$ to be a quasi-variety that is generated by a single finite structure. In other words, there always exists a finite structure $\mathbf{M}$ such that

$$
\mathcal{A}:=\mathbb{I S P}(\mathbf{M})_{\text {fin }}=\{\mathbf{A} \in \mathbb{I S P}(\mathbf{M}) \mid \mathbf{A} \text { finite }\} .
$$

The most obvious choice would be $\mathbf{M}:=\mathbf{A}$, but we will shortly understand that other choices of $\mathbf{M}$ might be more promising.

In any way, we have succeeded in finding a category $\mathcal{A}$ of comparably easy structure such that $C$ can be written as $O_{\mathbf{A}}$ for some $\mathbf{A} \in \mathcal{A}$. All that remains to be done is to find a dual equivalence between $\mathcal{A}$ and some (preferably easy) category $\mathcal{X}$. For this, we will use the natural dualities that we have introduced in the preliminaries (see Section 1.6). We obtain the following theorem:

4.2.1 Theorem. Let $C$ be a clone over a finite set $A$. There exists a category of finite structures $X$ and some $\mathbf{X} \in X$ such that the order-ideal $\langle C]$ is isomorphic to $\overline{\mathcal{L}}_{\mathbf{X}}$.

Proof. As explained above, there always exists a finite structure $\mathbf{A}$ and a finite structure $\mathbf{M}$ such that $\mathbf{A} \in \mathbb{I S P}(\mathbf{M})_{f i n}$. Understanding $\mathcal{A}:=\mathbb{I} \mathbb{S P}(\mathbf{M})_{\text {fin }}$ as a category, $C$ is the image of $O_{\mathbf{A}}$ under the obvious forgetful functor $U: \mathcal{A} \rightarrow$ Set. Now, by the Brute Force Duality Theorem 1.6 .5 , page 25, there exists an alter ego $\mathbf{M}$ of $\mathbf{M}$ such that $\mathcal{A}$ and some full subcategory $\chi$ of $\mathbb{S}_{c}^{0} \mathbb{P}^{+}(\underline{\mathbf{M}})_{\text {fin }}$ are dually equivalent via a natural dual equivalence $\langle D, E, e, \epsilon\rangle$. Since all structures in $X$ are finite, we can safely omit the discrete topology. 
Let $\mathbf{X}:=D(\mathbf{A})$. By Theorem 4.1.6, we have an isomorphism $(-)^{\partial}: \mathcal{L}_{\mathbf{A}} \rightarrow \overline{\mathcal{L}}_{\mathbf{X}}$. Thus, $\langle C] \cong \mathcal{L}_{\mathbf{A}} \cong \overline{\mathcal{L}}_{\mathbf{X}}$

By the way the natural dualities work, it is now easy to see why choosing $\mathbf{M}:=\mathbf{A}$ might not be the best choice: A natural dual equivalence dualizes $\mathbf{A}$ to $\mathcal{A}(\mathbf{A}, \mathbf{M})$, and the latter is obviously of the easiest form if $\mathbf{M}$ is chosen to be as small as possible.

Moreover, it is easy to see that we can always choose $\mathbf{A}$ and $\mathbf{M}$ to be total structures (that is, structures without partial operations), in which case $X$ becomes a category of finite total structures. However, the theorem is not stated in this way since it is often advantageous to minimize the number of relations in $\mathbf{M}$ by including partial operations. The reason for this is the following: Partial operations, in contrast to relations, can lower the number of subalgebras of finite powers of $\mathbf{M}$, and this simplifies the alter ego $\mathbf{M}$ and consequently the category $x$.

This theorem is a generalization of a result appearing in [Maš06], where it was shown that the statement above holds for all centralizer clones $C$. In fact, it was already proposed in [Maš06] to use natural dualities to dualize centralizer clones and a clone isomorphism $(-)^{\partial}: O_{\mathbf{A}} \rightarrow \bar{O}_{\mathbf{X}}$ similar to the one we constructed in Section 4.1 was built for the specific scenario of $\mathbf{A}$ being a finite algebra. As mentioned in the introduction, Maš06] was the starting point for our general theory.

If $A$ is an infinite set, we can still state a somewhat weaker result.

4.2.2 Proposition. Let $C$ be a clone over an infinite set $A$ such that $C$ can be written as $\mathrm{Pol} R$ for some set of (finitary) relations on $A$. If the structure $\mathbf{A}:=\langle A, R\rangle$ is in a quasivariety generated by a single finite structure $\mathbf{M}$, then there exists a category of topological structures $\boldsymbol{X}$ and some $\mathbf{X} \in X$ such that the order-ideal $\langle C]$ is isomorphic to $\overline{\mathcal{L}}_{\mathbf{X}}$.

Proof. By assumption, there exists a finite structure $\mathbf{M}$ such that $\mathbf{A} \in \operatorname{ISP}(\mathbf{M})$ and $C$ is the image of $O_{\mathbf{A}}$ under the obvious forgetful functor. By the Brute Force Duality Theorem, there exists an alter ego $\mathbf{M}$ of $\mathbf{M}$ such that $\mathcal{A}:=\mathbb{I S P}(\mathbf{M})$ and some full subcategory $x$ of $\mathbb{I} \mathbb{S}_{c}^{0} \mathbb{P}^{+}(\underline{\mathbf{M}})$ are dually equivalent via a natural dual equivalence $\langle D, E, e, \epsilon\rangle$. Let $\mathbf{X}:=D(\mathbf{A})$. Again, by Theorem 4.1.6, we obtain an isomorphism $(-)^{\partial}: \mathcal{L}_{\mathbf{A}} \rightarrow \overline{\mathcal{L}}_{\mathbf{X}}$, whence it follows $\langle C] \cong \overline{\mathcal{L}}_{\mathbf{X}}$.

Note that, here, $\mathbf{X}$ is a topological structure and the morphisms in $X$ are the continuous homomorphisms. Although the assumption of the proposition is not satisfied for all clones over an infinite set $A$, it is applicable to many interesting types of clones. In particular, it is applicable if $C$ is the centralizer clone of an algebra that is in the quasivariety generated by a single finite algebra. This includes many well-known classes of algebras such as distributive lattices, semilattices or Boolean groups.

We will now explore some examples for the finite case. For this, we will take wellknown classes of finite structures, use natural dual equivalences taken from [CD98] and build the clone duality $(-)^{\partial}$.

Since the functors of a natural duality are often hard to interpret, we do not want to build our clone dualities directly on a natural dual equivalence. Hence, before we 


\section{A Duality for Clones}

construct $(-)^{\partial}$, we will use the following lemma to shift the natural dual equivalence to a more feasible dual equivalence:

4.2.3 Lemma. Let $\left\langle D^{\prime}, E^{\prime}, e^{\prime}, \epsilon^{\prime}\right\rangle$ be a dual equivalence between two categories $\mathcal{A}$ and $\boldsymbol{X}$. Let $D: \mathcal{A} \rightarrow \mathcal{X}$ and $E: X \rightarrow \mathcal{A}$ be functors. If there exist two natural transformations $\tau: D^{\prime} \rightarrow D$ and $\psi: E^{\prime} \rightarrow E$ such that $\tau_{\mathbf{A}}$ and $\psi_{\mathbf{X}}$ are isomorphisms for all $\mathbf{A} \in \mathcal{A}$ and $\mathbf{X} \in \mathcal{X}$, then $\langle D, E, e, \epsilon\rangle$ is a dual equivalence where $e$ and $\epsilon$ are defined as follows:

$$
\begin{aligned}
& \left(e_{\mathbf{A}}\right)_{\mathbf{A} \in \mathcal{A}}, e_{\mathbf{A}}: \mathbf{A} \rightarrow E D(\mathbf{A}), e_{\mathbf{A}}:=E\left(\tau_{\mathbf{A}}^{-1}\right) \circ \psi_{D(\mathbf{A})} \circ e_{\mathbf{A}}^{\prime} \\
& \left(\epsilon_{\mathbf{X}}\right)_{\mathbf{X} \in X}, \epsilon_{\mathbf{X}}: \mathbf{X} \rightarrow D E(\mathbf{X}), \epsilon_{\mathbf{X}}:=D\left(\psi_{\mathbf{X}}^{-1}\right) \circ \tau_{E(\mathbf{X})} \circ \epsilon_{\mathbf{X}}^{\prime}
\end{aligned}
$$

Proof. We start by showing that $\langle D, E, e, \epsilon\rangle$ is a dual adjunction (see Definition 1.4.23. page 22). First, we will show that $e$ is a well-defined natural transformation. Let $u \in \mathcal{A}(\mathbf{A}, \mathbf{B})$. Since $e^{\prime}$ and $\psi$ are natural transformations, we have the following commuting diagram:

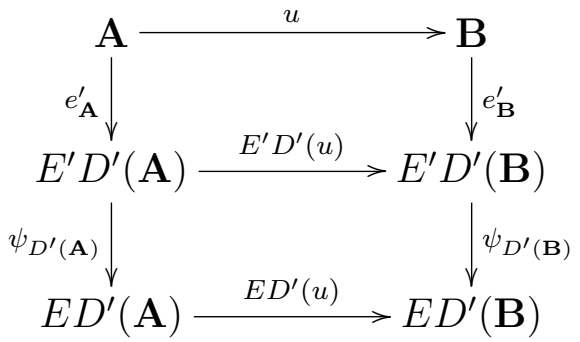

Since $\tau$ is also a natural transformation, we also have the following commuting diagram:

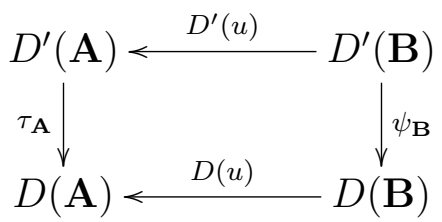

After applying the functor $E$, we obtain

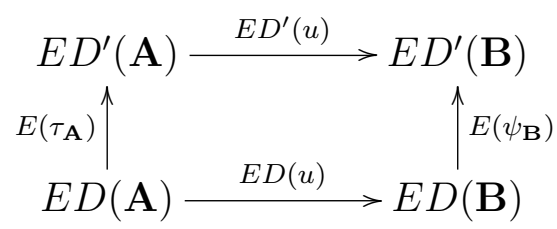

Since $\tau_{\mathbf{A}}$ and $\tau_{\mathbf{B}}$ are isomorphisms, this, in turn, implies the commutativity of the following diagram:

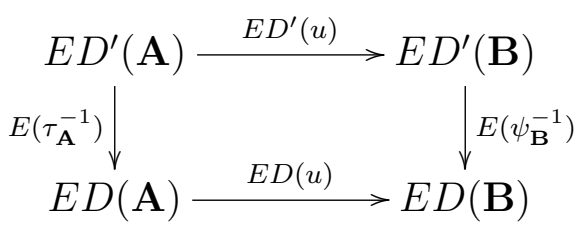


Merging this diagram with the first diagram of this proof gives us the following commuting diagram:

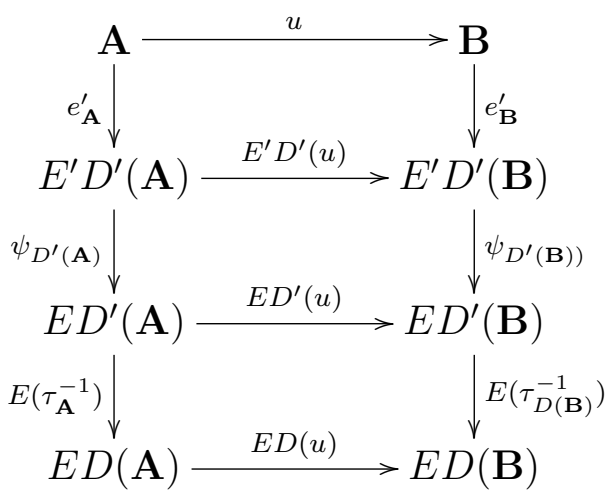

That is, $e$ is a natural transformation. An analogue proof yields that $\epsilon$ is a natural transformation. It remains to show that there exists a bijection between $\mathcal{A}(\mathbf{A}, E(\mathbf{X}))$ and $\mathcal{X}(\mathbf{X}, D(\mathbf{A}))$ associating $u$ and $\varphi$ such that $u=E(\varphi) \circ e_{\mathbf{A}}$ and $\varphi=D(u) \circ \epsilon_{\mathbf{X}}$ (see Figure 1.4, page 22). For $u \in \mathcal{A}(\mathbf{A}, E(\mathbf{X}))$, there exists a unique $u^{\prime} \in \mathcal{A}\left(\mathbf{A}, E^{\prime}(\mathbf{X})\right)$ such that $u=\psi_{\mathbf{X}} \circ u^{\prime}$. We will show $u=E(\varphi) \circ e_{\mathbf{A}}$ for $\varphi:=\tau_{\mathbf{A}} \circ \varphi^{\prime}$ where $\varphi^{\prime} \in \mathcal{X}(\mathbf{X}, D(\mathbf{A}))$ is the unique morphism that is associated with $u^{\prime}$ via the dual equivalence $\left\langle D^{\prime}, E^{\prime}, e^{\prime}, \epsilon^{\prime}\right\rangle$. We have

$$
\begin{aligned}
u & =\psi_{\mathbf{X}} \circ u^{\prime} \\
& =\psi_{\mathbf{X}} \circ E^{\prime}\left(\varphi^{\prime}\right) \circ e_{\mathbf{A}}^{\prime} \\
& =\psi_{\mathbf{X}} \circ \psi_{\mathbf{X}}^{-1} \circ E\left(\varphi^{\prime}\right) \circ \psi_{D(\mathbf{A})} \circ e_{\mathbf{A}}^{\prime} \\
& =E\left(\varphi^{\prime}\right) \circ \psi_{D(\mathbf{A})} \circ e_{\mathbf{A}}^{\prime} \\
& =E\left(\varphi^{\prime}\right) \circ \psi_{D(\mathbf{A})} \circ \psi_{D(\mathbf{A})}^{-1} \circ E\left(\tau_{\mathbf{A}}\right) \circ e_{\mathbf{A}} \\
& =E\left(\varphi^{\prime}\right) \circ E\left(\tau_{\mathbf{A}}\right) \circ e_{\mathbf{A}} \\
& =E\left(\tau_{\mathbf{A}} \circ \varphi^{\prime}\right) \circ e_{\mathbf{A}} \\
& =E(\varphi) \circ e_{\mathbf{A}},
\end{aligned}
$$

as desired. The proof of $\varphi=D(u) \circ \epsilon_{\mathbf{X}}$ is analogue. Thus, $\langle D, E, e, \epsilon\rangle$ is a dual adjunction. It remains to show that $e_{\mathbf{A}}$ and $\epsilon_{\mathbf{X}}$ are isomorphisms for each $\mathbf{A} \in \mathcal{A}$ and $\mathbf{X} \in X$. However, this follows directly from the fact that $e_{\mathbf{A}}^{\prime}, \epsilon_{\mathbf{X}}^{\prime}, \psi_{D(\mathbf{A})}, \tau_{E(\mathbf{X})}, E\left(\tau_{\mathbf{A}}^{-1}\right)$ and $D\left(\psi_{\mathbf{X}}^{-1}\right)$ are all isomorphisms.

With the dual equivalences that we obtain after applying this lemma, we will construct the clone dualities.

Since many of the structures we will choose as examples are lattices, we will apply some techniques from the field of lattice theory. Although the techniques are basic and we give definitions for most of the terminology, the reader unfamiliar with lattice theory might want to equip himself/herself with some background knowledge. For this, we refer to standard texts such as [DP02], [Bir67] and [CD73].

In the remainder of this thesis, we will always look back at the upcoming examples and use them to illustrate and apply our results. 


\subsubsection{Clones over Distributive Lattices}

First, we will dualize clones over finite distributive lattices. It is a well-known fact that the quasivariety of distributive lattices is generated by the structure $\mathbf{M}:=\langle\{0,1\}, \vee, \wedge\rangle$ with the obvious definition for $\vee$ and $\wedge$. In other words, we can understand $\mathbb{I S P}(\mathbf{M})$ as the category of distributive lattices. It was observed in Pri72] and generalized in [CD98] that

$$
\underline{\mathbf{M}}:=\langle\{0,1\}, 0,1, \leq, \mathcal{T}\rangle
$$

yields a full natural duality between $\mathbb{I S P}(\mathbf{M})$ and $\mathbb{S}_{c}^{0} \mathbb{P}^{+}(\underline{\mathbf{M}})=\mathbb{I} \mathbb{S}_{c} \mathbb{P}^{+}(\underline{\mathbf{M}})$. Since we are only interested in the duals of the finite structures in $\mathcal{A}$, we can omit the topology in $\underline{\mathbf{M}}$, and we obtain that

$$
\mathcal{A}:=\mathbb{I S P}(\mathbf{M})_{\text {fin }}=\{\mathbf{A} \in \mathbb{I S P}(\mathbf{M}) \mid \mathbf{A} \text { finite }\}
$$

and

$$
x:=\mathbb{I S P} \mathbb{P}^{+}(\underline{\mathbf{M}})_{f i n}=\left\{\mathbf{X} \in \mathbb{I S P} \mathbb{P}^{+}(\underline{\mathbf{M}}) \mid \mathbf{X} \text { finite }\right\}
$$

are dually equivalent via the natural dual equivalence $\left\langle D^{\prime}, E^{\prime}, e^{\prime}, \epsilon^{\prime}\right\rangle$.

Note that $X$ is the category of finite bounded partially ordered sets.

4.2.4 Definition. A bounded partially ordered set (or bounded poset) is a set $X$ equipped with a partial order $\leq$ and two distinguished elements 0,1 such that $0 \leq x \leq 1$ for all $x \in X$.

We will now apply Lemma 4.2 .3 to obtain a dual equivalence $\langle D, E, e, \epsilon\rangle$ in which we do not have to think of $D(\mathbf{A})$ as a set of morphisms and of $D(f)$ as a mapping of a morphism to another morphism. For this, we need some definitions and facts from the field of lattice theory. Recall that a lattice $\langle A, \vee, \wedge\rangle$ induces an order relation $\leq$ by setting $a \leq b: \Longleftrightarrow a=a \wedge b$.

4.2.5 Definition. Let $\mathbf{A}=\langle A, \vee, \wedge\rangle$ be a distributive lattice. An increasing subset of $A$ is a subset $X \subseteq A$ such that $b \in X$ whenever there exists $a \in X$ with $b \geq a$. A prime filter of $\mathbf{A}$ is a non-empty, proper, increasing subset $X \subseteq A$ that is closed with respect to $\wedge$ and where $a_{1} \vee a_{2} \in X$ implies $a_{1} \in X$ or $a_{2} \in X$. Denote by $\operatorname{Spec}(\mathbf{A})$ the set of prime filters of $\mathbf{A}$.

Note that, setting $\uparrow a:=\{b \geq a \mid b \in A\}$, every prime filter of a distributive lattice can be written as $\uparrow a$ for some join-irreducible $a \in A \backslash\{0\}$. Conversely, every join-irreducible $a \in A \backslash\{0\}$ determines a prime filter in this way. As we will see in the following wellknown lemma, the prime filters of $\mathbf{A}$ also have a very strong connection to the morphisms in $\mathcal{A}(\mathbf{A}, \mathbf{M})$.

4.2.6 Lemma ([Bir37]). Let $\mathbf{A}, \mathbf{B} \in \mathcal{A}$. A mapping $u: A \rightarrow B$ is a homomorphism between $\mathbf{A}$ and $\mathbf{B}$ if and only if $u^{-1}(x) \in\{A, \emptyset\} \cup \operatorname{Spec}(\mathbf{A})$ for all $x \in \operatorname{Spec}(\mathbf{B})$.

For $\mathbf{X} \in X$, we denote by $\operatorname{Inc}(\mathbf{X})$ the set of non-empty, proper, increasing subsets of $X$. 
4.2.7 Lemma ([Bir37]). Let $\mathbf{X}, \mathbf{Y} \in X . A$ mapping $\varphi: X \rightarrow Y$ is a homomorphism between $\mathbf{X}$ and $\mathbf{Y}$ if and only if $\varphi^{-1}(z) \in \operatorname{Inc}(\mathbf{X})$ for all $z \in \operatorname{Inc}(\mathbf{Y})$.

Now we are ready to define $\langle D, E, e, \epsilon\rangle$. The central idea is to think of every morphism to $\mathbf{M}$ and $\mathbf{M}$ as the set that is the inverse image of 1 . The duality we will obtain was discovered by G. D. BIRKHOFF in [Bir37 and was later generalized to a duality for all distributive lattices by H. A. PRIEstley [Pri72]. Although this duality is wellknown, we will give the proof to show how it arises via Lemma 4.2 .3 from the natural duality. Since we will take a very similar route for the other structures in the upcoming subsections, the proof will also serve as a prototype.

4.2.8 Lemma. Let $D: \mathcal{A} \rightarrow x, E: X \rightarrow \mathcal{A}, e: i d_{\mathcal{A}} \rightarrow E D$ and $\epsilon: i d_{x} \rightarrow D E$ be defined as follows:

$$
\begin{aligned}
& D(\mathbf{A}):=\langle\operatorname{Spec}(\mathbf{A}) \cup\{\emptyset, A\}, \emptyset, A, \subseteq\rangle, D(u)(x):=u^{-1}(x), \\
& E(\mathbf{X}):=\langle\operatorname{Inc}(\mathbf{X}), \cup, \cap\rangle, E(\varphi)(x):=\varphi^{-1}(x), \\
& e_{\mathbf{A}}(a):=\{A\} \cup\{x \in \operatorname{Spec}(\mathbf{A}) \mid a \in x\}, \\
& \epsilon_{\mathbf{X}}(x):=\{a \in \operatorname{Inc}(\mathbf{X}) \mid x \in a\} .
\end{aligned}
$$

Then, $\langle D, E, e, \epsilon\rangle$ is a dual equivalence between $\mathcal{A}$ and $\boldsymbol{X}$.

Proof. Let $\mathbf{A}, \mathbf{B} \in \mathcal{A}$ and let $u \in \mathcal{A}(\mathbf{A}, \mathbf{B})$. If follows from Lemma 4.2 .6 that the morphism $D(u): D(\mathbf{B}) \rightarrow D(\mathbf{A})$ is well-defined (note that $\{1\}$ is a prime filter of $\mathbf{M}$ and that the set-mapping $u^{-1}$ clearly commutes with the two constants and $\subseteq$ ). Now let $\mathbf{C} \in \mathcal{A}$ and $v \in \mathcal{A}(\mathbf{B}, \mathbf{C})$. We have

$$
D(v \circ u)(x)=(v \circ u)^{-1}(x)=u^{-1}\left(\left(v^{-1}\right)(x)\right)=(D(u) \circ D(v))(x) .
$$

Thus, $D$ is a well-defined contravariant functor. By Lemma 4.2.7, it follows in the same way that $E$ is well-defined. We define the following two families of morphisms:

$$
\begin{aligned}
&\left(\tau_{\mathbf{A}}\right)_{\mathbf{A} \in \mathcal{A}}, \tau_{\mathbf{A}}: D^{\prime}(\mathbf{A}) \rightarrow D(\mathbf{A}), \tau_{\mathbf{A}}(f):=f^{-1}(1), \\
&\left(\psi_{\mathbf{X}}\right)_{\mathbf{X} \in \mathcal{X}}, \psi_{\mathbf{X}}: E^{\prime}(\mathbf{X}) \rightarrow E(\mathbf{X}), \psi_{\mathbf{X}}(g):=g^{-1}(1)
\end{aligned}
$$

Let $\mathbf{A} \in \mathcal{A}$ and $\mathbf{X} \in \mathcal{X}$. By Lemma 4.2.6 and Lemma 4.2.7, $\tau_{\mathbf{A}}$ and $\psi_{\mathbf{X}}$ are well-defined mappings. It is easy to see that they are also homomorphisms and that they are injective. Furthermore, it follows by Lemma 4.2.6 and Lemma 4.2.7 that $\tau_{\mathbf{A}}$ and $\psi_{\mathbf{X}}$ are surjective, so they are isomorphisms. For $\mathbf{B} \in \mathcal{A}$ and $u \in \mathcal{A}(\mathbf{A}, \mathbf{B})$, we have

$$
\begin{aligned}
\left(\tau_{\mathbf{A}}^{-1} \circ D(u) \circ \tau_{\mathbf{B}}\right)(f) & =\left(\tau_{\mathbf{A}}^{-1} \circ D(u)\right)\left(f^{-1}(1)\right) \\
& =\tau_{\mathbf{A}}^{-1}\left((f \circ u)^{-1}(1)\right) \\
& =f \circ u \\
& =D^{\prime}(u)(f)
\end{aligned}
$$




\section{A Duality for Clones}

for all $f \in D^{\prime}(\mathbf{B})$. Thus, $\tau$ is a natural transformation. An analogue argument establishes that $\psi$ is a natural transformation. By Lemma 4.2.3, it remains to show that we have

$$
\begin{aligned}
& e_{\mathbf{A}}=E\left(\tau_{\mathbf{A}}^{-1}\right) \circ \psi_{D(\mathbf{A})} \circ e_{\mathbf{A}}^{\prime}, \\
& \epsilon_{\mathbf{X}}=D\left(\psi_{\mathbf{X}}^{-1}\right) \circ \tau_{E(\mathbf{X})} \circ \epsilon_{\mathbf{A}}^{\prime} .
\end{aligned}
$$

Let $a \in \mathbf{A}$. The mapping $e_{\mathbf{A}}^{\prime}(a): D^{\prime}(\mathbf{A}) \rightarrow \mathbf{M}$ is given by $z \mapsto z(a)$. Thus, $\psi_{D(\mathbf{A})}\left(e_{\mathbf{A}}^{\prime}(a)\right)$ is the set of mappings $u \in \mathbf{M}^{\mathbf{A}}$ with $u(a)=1$. In other words,

$$
\left(\psi_{D(\mathbf{A})} \circ e_{\mathbf{A}}^{\prime}\right)(a)=e_{\mathbf{A}}^{\prime}(a)^{-1}(1)=\{u \in \mathcal{A}(\mathbf{A}, \mathbf{M}) \mid u(a)=1\} .
$$

Note that $E\left(\tau_{\mathbf{A}}^{-1}\right)$ is a mapping from $\mathfrak{P}\left(\mathbf{M}^{\mathbf{A}}\right)$ to $\mathfrak{P}(\operatorname{Spec}(\mathbf{A}) \cup\{A, \emptyset\})$. More particular, it maps a set $F \subseteq \mathbf{M}^{\mathbf{A}}$ to the set of elements of $D(\mathbf{A})$ that are the inverse image of 1 under some $f \in F$. That is,

$$
E\left(\tau_{\mathbf{A}}^{-1}\right)(F)=\left\{x \in \operatorname{Spec}(\mathbf{A}) \cup\{A, \emptyset\} \mid \exists f \in F: f^{-1}(1)=x\right\} .
$$

Thus, $\left(E\left(\tau_{\mathbf{A}}^{-1}\right) \circ \psi_{D(\mathbf{A})} \circ e_{\mathbf{A}}^{\prime}\right)(a)$ is the set of all elements among $x \in \operatorname{Spec}(\mathbf{A}) \cup\{A\}$ that contain $a$. This is precisely $e_{\mathbf{A}}(a)$. A similar argument yields the desired equation for the other natural transformation $\epsilon$.

We have now obtained a dual equivalence $\langle D, E, e, \epsilon\rangle$ between the category of finite distributive lattices and the category of finite bounded partially ordered sets. The original duality was formulated in exactly this way, while it was then observed in CD98] that Priestley's duality can be generalized to the concept of natural dualities.

The following example illustrates $\langle D, E, e, \epsilon\rangle$ :

4.2.9 Example. Take the following two distributive lattices:

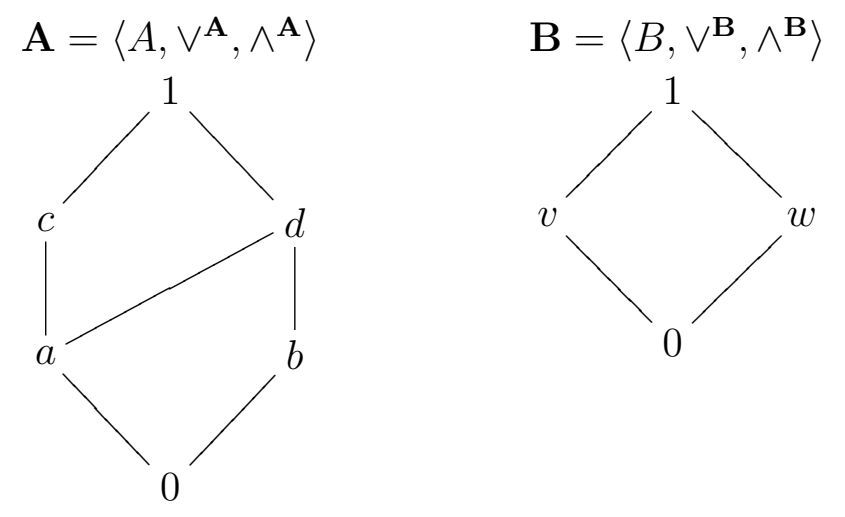

Let the morphisms $f_{1}, f_{2}: \mathbf{A} \rightarrow \mathbf{B}$ be defined as follows:

\begin{tabular}{|c||c|c|c|c|c|c|}
\hline$x$ & 0 & $a$ & $b$ & $c$ & $d$ & 1 \\
\hline \hline$f_{1}(x)$ & 0 & 0 & 0 & $v$ & 0 & $v$ \\
\hline$f_{2}(x)$ & 0 & 0 & $v$ & $w$ & $v$ & 1 \\
\hline
\end{tabular}


Under $D, \mathbf{A}$ and $\mathbf{B}$ dualize to the bounded poset of their prime filters and trivial subsets, while $f_{1}$ and $f_{2}$ dualize to the set-mappings $f_{1}^{-1}$ and $f_{2}^{-1}$ from the prime filters and trivial subsets of $\mathbf{B}$ to those of $\mathbf{A}$.
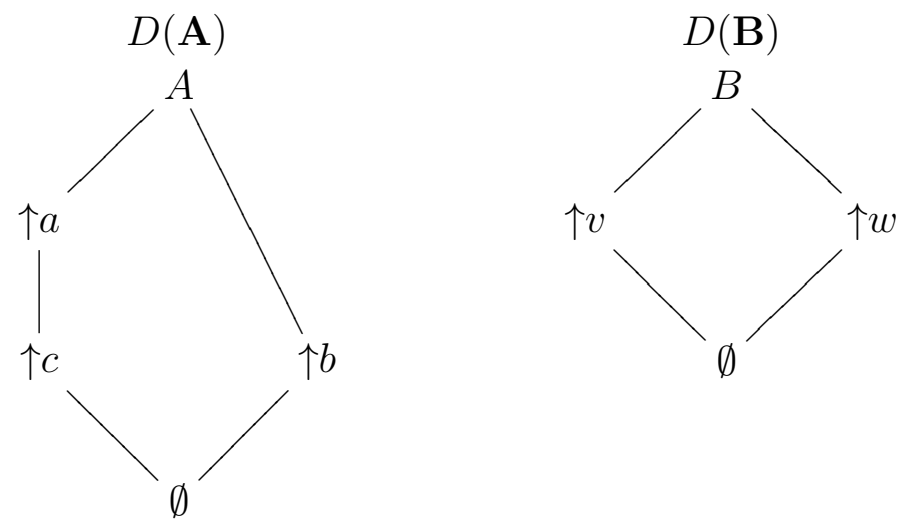

\begin{tabular}{|c||c|c|c|c|}
\hline$x$ & $\emptyset$ & $\uparrow v$ & $\uparrow w$ & $B$ \\
\hline \hline$D\left(f_{1}\right)(x)$ & $\emptyset$ & $\uparrow c$ & $\emptyset$ & $A$ \\
\hline$D\left(f_{2}\right)(x)$ & $\emptyset$ & $\uparrow b$ & $\uparrow c$ & $A$ \\
\hline
\end{tabular}

Note that $f_{1}$ is not a 1 -homomorphism which causes $D\left(f_{1}\right)^{-1}(\{\emptyset\}) \neq\{\emptyset\}$. In fact, it is easy to see that a morphism $u: \mathbf{A} \rightarrow \mathbf{B}$ is a 1-homomorphism if and only if $D(u)^{-1}(\{\emptyset\})=\{\emptyset\}$ and a 0-homomorphism if and only if $D(u)^{-1}(\{B\})=\{A\}$. Consequently, $u$ is a 01-homomorphism (that is, a homomorphism that preserves the maximum and minimum element) if and only if $D(u)^{-1}(\{\emptyset\})=\{\emptyset\}$ and $D(u)^{-1}(\{B\})=\{A\}$.

If we apply $E$ to $D(\mathbf{A})$, we obtain the lattice formed by the nontrivial increasing subsets of $D(\mathbf{A})$.

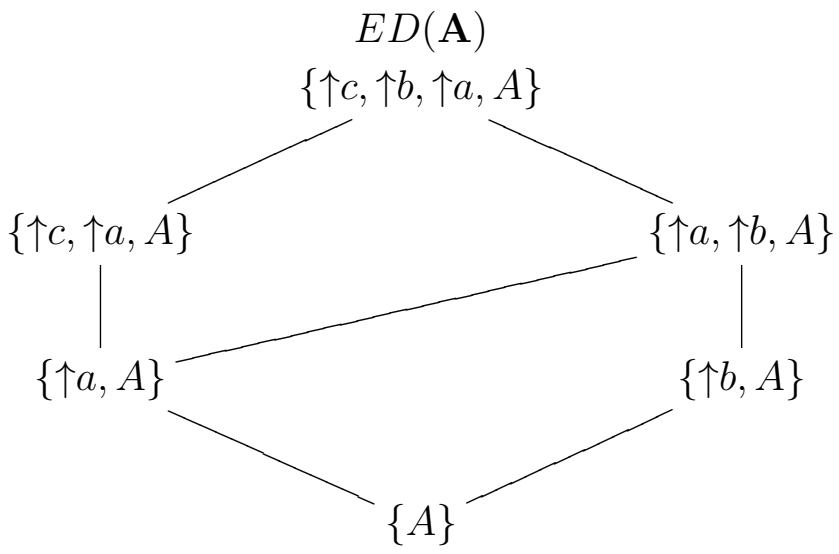

It is now easy to see that this lattice is isomorphic to $\mathbf{A}$ via $e_{\mathbf{A}}$ : For example, the element $\{\uparrow c, \uparrow a, A\}$ is exactly the set of all prime filters and trivial subsets of $\mathbf{A}$ that contain $c$. That is, $e_{\mathbf{A}}(c)=\{\uparrow c, \uparrow a, A\}$.

Ultimately, we want to use the dual equivalence $\langle D, E, e, \epsilon\rangle$ to dualize clones over finite distributive lattices. Therefore, we need to understand how an $n$-ary operation over some 
$\mathbf{A} \in \mathcal{A}$ dualizes under $D$. Since $f: \mathbf{A}^{n} \rightarrow \mathbf{A}$ dualizes to $D(f): D(\mathbf{A}) \rightarrow D\left(\mathbf{A}^{n}\right)$, this means that we have to understand how the powers of $\mathbf{A}$ are dualized. Since we have

$$
D\left(\mathbf{A}^{n}\right)=\left\langle\operatorname{Spec}\left(\mathbf{A}^{n}\right) \cup\left\{\emptyset, A^{n}\right\}, \emptyset, A^{n}, \subseteq\right\rangle,
$$

this is a matter of understanding the prime filters of $\mathbf{A}^{n}$.

4.2.10 Lemma. Let $n \in \mathbb{N}_{+}$. The prime filters of $\mathbf{A}^{n}$ are exactly the Cartesian product of $n$ sets, where $n-1$ of these sets are $A$ and the remaining set is a prime filter of $\mathbf{A}$. In other words,

$$
\operatorname{Spec}\left(\mathbf{A}^{n}\right)=\left\{A^{i-1} \times x \times A^{n-i} \mid i \in\{1, \ldots, n\}, x \in \operatorname{Spec}(\mathbf{A})\right\} .
$$

Proof. Since it is straightforward to check that each element of the set on the right hand side of the equation is a prime filter, we only show " $\subseteq$ ". We will do so in three steps. First we show that each $F \in \operatorname{Spec}\left(\mathbf{A}^{n}\right)$ must be the Cartesian product of $n$ sets $F_{1}, \ldots, F_{n}$, then we show that $F_{i} \neq A$ implies that $F_{i}$ is a prime filter, and finally we show that exactly $n-1$ of the sets $F_{1}, \ldots, F_{n}$ must be $A$. For the first part, suppose $\left(a_{1}, \ldots, a_{n}\right),\left(b_{1}, \ldots, b_{n}\right) \in F$. For $c_{i} \in\left\{a_{i}, b_{i}\right\}$, we have

$$
\left(c_{1}, \ldots, c_{n}\right) \geq\left(a_{1} \wedge b_{1}, \ldots, a_{n} \wedge b_{n}\right)=\left(a_{1}, \ldots, a_{n}\right) \wedge\left(b_{1}, \ldots, b_{n}\right) \in F
$$

and consequently $\left(c_{1}, \ldots, c_{n}\right) \in F$. This proves that $F$ is the Cartesian product of $n$ sets. Let $F=F_{1} \times \ldots \times F_{n}$. Suppose that there exists $i \in\{1, \ldots, n\}$ such that $F_{i} \neq A$ but $F_{i} \notin \operatorname{Spec}(\mathbf{A})$. Without loss of generality, we can assume $i=1$. Hence, there must exist two elements $a, b \in F_{1}$ that violate one of the conditions from the definition of a prime filter. Let $\left(x_{2}, \ldots, x_{n}\right) \in F_{2} \times \ldots \times F_{n}$. We have $\left(a, x_{2}, \ldots, x_{n}\right),\left(b, x_{2}, \ldots, x_{n}\right) \in F$, and it is evident that these two elements violate the same condition for $F$ to be a prime filter of $\mathbf{A}^{n}$. Moreover, it is obvious that at least one of the sets $F_{1}, \ldots, F_{n}$ must be a proper subset of $A$ since otherwise it would follow $F=A^{n}$, which is impossible. It remains to show that we have $F_{i} \neq A$ for no more than one $i \in\{1, \ldots, n\}$. Assume that there exist $i, j \in\{1, \ldots, n\}, i \neq j$, such that $F_{i} \neq A$ and $F_{j} \neq A$. This implies that $F_{i}$ and $F_{j}$ are prime filters and, hence, $0^{\mathbf{A}} \notin F_{i}, F_{j}$. Now let $\left(a_{1}, \ldots, a_{n}\right) \in F$. We have

$$
\left(a_{1}, \ldots, a_{i-1}, 0^{\mathbf{A}}, a_{i+1}, \ldots, a_{n}\right),\left(a_{1}, \ldots, a_{j-1}, 0^{\mathbf{A}}, a_{j+1}, \ldots, a_{n}\right) \notin F .
$$

But now, the join of these two elements gives $\left(a_{1}, \ldots, a_{n}\right)$ which is a contradiction to $F \in \operatorname{Spec}\left(\mathbf{A}^{n}\right)$. This finishes the proof.

It remains to understand how the projection morphisms dualize. For $n \in \mathbb{N}_{+}$and $i \in\{1, \ldots, n\}$, it is straightforward to check that $D\left(\pi_{i}^{n}\right)$ is given as follows:

$$
D\left(\pi_{i}^{n}\right): D(\mathbf{A}) \rightarrow D\left(\mathbf{A}^{n}\right): x \mapsto A^{i-1} \times x \times A^{n-i} .
$$

We will now look at what is usually defined as the $n$-th copower of a bounded partially ordered set. For $\mathbf{X} \in X$ denote by $X^{\prime}$ the underlying set not including $0^{\mathbf{X}}$ and $1^{\mathbf{X}}$, 
and recall that $n \cdot X^{\prime}$ denotes the $n$-th copower of $X^{\prime}$ in the category of sets (that is, $\left.n \cdot X^{\prime}=\left\{\langle i, x\rangle \mid i \in\{1, \ldots, n\}, x \in X^{\prime}\right\}\right)$. Then,

$$
n \cdot \mathbf{X}:=\left\langle\left\{0^{n \cdot \mathbf{X}}, 1^{n \cdot \mathbf{X}}\right\} \cup n \cdot X^{\prime}, 0^{n \cdot \mathbf{X}}, 1^{n \cdot \mathbf{X}}, \leq\right\rangle,
$$

where $z_{1} \leq z_{2}$ whenever $z_{1}=0^{n \cdot \mathbf{X}}$ or $z_{2}=1^{n \cdot \mathbf{X}}$ or there exists $i \in\{1, \ldots, n\}$ such that $z_{1}=\left\langle i, x_{1}\right\rangle, z_{2}=\left\langle i, x_{2}\right\rangle$ and $x_{1} \leq^{\mathbf{X}} x_{2}$. The associated injection morphisms are given by

$$
\iota_{i}^{n}(x):= \begin{cases}0^{n \cdot \mathbf{X}} & \text { if } x=0^{\mathbf{X}} \\ 1^{n \cdot \mathbf{X}} & \text { if } x=1^{\mathbf{x}} \\ \langle i, x\rangle & \text { otherwise. }\end{cases}
$$

Set $\mathbf{X}:=D(\mathbf{A})$. In Lemma 4.1.1 (page 40), we have seen that, for each $n \in \mathbb{N}_{+}$, there exists an isomorphism $\eta_{n}: D\left(\mathbf{A}^{n}\right) \rightarrow n \cdot \mathbf{X}$ such that $\iota_{i}^{n}=\eta_{n} \circ D\left(\pi_{i}^{n}\right)$ for all $i \in\{1, \ldots, n\}$. In this case (and all other cases in this chapter), it is fairly obvious how we have to define $\eta_{n}$. For not so obvious cases, recall that Proposition 4.1 .2 yields a construction of $\eta_{n}$ : It is the inverse of $\left[D\left(\pi_{1}^{n}\right), \ldots, D\left(\pi_{n}^{n}\right)\right]$. In any case, we have to set

$$
\eta_{n}(x):= \begin{cases}0^{n \cdot \mathbf{X}} & \text { if } x=\emptyset \\ 1^{n \cdot \mathbf{X}} & \text { if } x=A^{n} \\ \langle i, y\rangle & \text { if } x=A^{i-1} \times y \times A^{n-i} \text { for some } y \in \operatorname{Spec}(\mathbf{A}) .\end{cases}
$$

As outlined in Section 4.2, we now obtain the required isomorphism $(-)^{\partial}: O_{\mathbf{A}} \rightarrow \bar{O}_{\mathbf{X}}$ by setting $f^{\partial}:=\eta_{n} \circ D(f)$ for $f \in O_{\mathbf{A}}^{(n)}$. Stating this explicitly, $(-)^{\partial}$ maps each $f \in O_{\mathbf{A}}^{(n)}$ to

$$
f^{\partial}(x)= \begin{cases}0^{n \cdot \mathbf{X}} & \text { if } f^{-1}(x)=\emptyset \\ 1^{n \cdot \mathbf{X}} & \text { if } f^{-1}(x)=A^{n} \\ \langle i, y\rangle & \text { if } f^{-1}(x)=A^{i-1} \times y \times A^{n-i} \text { for some } y \in \operatorname{Spec}(\mathbf{A})\end{cases}
$$

In Chapter 8, we will get very familiar with this duality when we use it to examine clones over distributive lattices.

\subsubsection{Clones over Boolean Algebras}

After dualizing distributive lattices, we will now take a look at a somewhat simpler example. Clones over finite Boolean algebras are the only example among the cases in this chapter that were already investigated by dualizing them. This was done in [Maš06] and Maš08], where many results are presented (e.g. a full description of the maximal and minimal clones in the lattice of clones over a finite Boolean algebra).

The structure of a Boolean algebra is basically the structure of a complemented distributive lattice. However, it does not fall under the case of clones over distributive lattices since the morphisms also have to commute with $\neg, 0$ and 1 .

For a Boolean algebra $\mathbf{A}$, we denote by $\operatorname{At}(\mathbf{A})$ the set of atoms of $\mathbf{A}$. For $a \in \mathbf{A}$, we define $\operatorname{At}(a)$ to be the set of atoms below $a$, that is,

$$
\operatorname{At}(a)=\left\{a^{\prime} \in \operatorname{At}(\mathbf{A}) \mid a^{\prime} \leq a\right\} .
$$

The following observations can be made: 
4.2.11 Lemma. Let $\mathbf{M}=\langle\{0,1\}, 0,1, \neg, \vee, \wedge\rangle$ with the obvious definitions for the three operations $\vee, \wedge$ and $\neg$. An algebra $\mathbf{A}$ is a Boolean algebra if an only if it is isomorphic to a subalgebra of a direct power of $\mathbf{M}$.

Thus, the category of finite Boolean algebras is given by

$$
\mathcal{A}:=\mathbb{I S P P}(\mathbf{M})_{f i n}=\{\mathbf{A} \in \mathbb{I S P P}(\mathbf{M}) \mid \mathbf{A} \text { finite }\} .
$$

By M. H. STONE's well-known representation theorem for Boolean algebras [Sto36], the category $\mathcal{A}$ and the category $x:=\operatorname{Set}_{\text {fin }}$ of finite sets are dually equivalent, where the dual equivalence is given as follows:

$$
\begin{aligned}
D(\mathbf{A}) & :=\operatorname{At}(\mathbf{A}), D(u)(x):=\bigwedge u^{-1}(\uparrow x), \\
E(\mathbf{X}) & :=\langle\mathfrak{P}(X), \emptyset, X, \neg, \cup, \cap\rangle, E(\varphi)(x):=\varphi^{-1}(x), \\
e_{\mathbf{A}}(a) & :=\operatorname{At}(a), \\
\epsilon_{\mathbf{X}} & :=i d_{\mathbf{X}} .
\end{aligned}
$$

This dual equivalence is called the Stone Duality. It also fits with the concept of natural dualities: It was shown in [CD98] that $\mathbf{M}:=\langle\{0,1\}, \mathcal{T}\rangle$ yields a full natural duality between $\mathbb{I S P}(\mathbf{M})$ and $\mathbb{S}_{c}^{0} \mathbb{P}^{+}(\underline{\mathbf{M}})$, and we leave it to the reader to verify that the Stone Duality can be obtained from the natural duality $\left\langle D^{\prime}, E^{\prime}, e^{\prime}, \epsilon^{\prime}\right\rangle$ by using Lemma 4.2.3 with the natural transformations

$$
\begin{aligned}
&\left(\tau_{\mathbf{A}}\right)_{\mathbf{A} \in \mathcal{A}}, \tau_{\mathbf{A}}: D^{\prime}(\mathbf{A}) \rightarrow D(\mathbf{A}), \tau_{\mathbf{A}}(f):=\bigwedge f^{-1}(1) \\
&\left(\psi_{\mathbf{X}}\right)_{\mathbf{X} \in \mathcal{X}}, \psi_{\mathbf{X}}: E^{\prime}(\mathbf{X}) \rightarrow E(\mathbf{X}), \psi_{\mathbf{X}}(g):=g^{-1}(1) .
\end{aligned}
$$

Since we are ultimately interested in dualizing operations over $\mathbf{A} \in \mathcal{A}$, we have to examine the structure of $D\left(\mathbf{A}^{n}\right)=\operatorname{At}\left(\mathbf{A}^{n}\right)$. For $a \in \mathbf{A}$ and $j \in\{1, \ldots, n\}$, set

$$
a^{j}:=(0, \ldots, \underset{\substack{\uparrow \\ j}}{a}, 0, \ldots, 0) .
$$

It is easy to see that we have

$$
D\left(\mathbf{A}^{n}\right)=\operatorname{At}\left(\mathbf{A}^{n}\right)=\left\{a^{j} \mid a \in \operatorname{At}(\mathbf{A}), j \in\{1, \ldots, n\}\right\} .
$$

Furthermore, for $n \in \mathbb{N}_{+}$and $i \in\{1, \ldots, n\}$, we obtain

$$
D\left(\pi_{i}^{n}\right): D(\mathbf{A}) \rightarrow D\left(\mathbf{A}^{n}\right): x \mapsto x^{i}
$$

Set $\mathbf{X}:=D(\mathbf{A})$. The $n$-th copower of $\mathbf{X}$ in $X$ is simply its $n$-th disjoint union. It is now easy to see that the isomorphism $\eta_{n}: D\left(\mathbf{A}^{n}\right) \rightarrow n \cdot \mathbf{X}$ is given as follows:

$$
\eta_{n}(x)=\langle j, y\rangle: \Longleftrightarrow x=y^{j} .
$$

We get the clone duality $(-)^{\partial}: O_{\mathbf{A}} \rightarrow \bar{O}_{\mathbf{X}}$ by setting $f^{\partial}:=\eta_{\operatorname{ar}(f)} \circ D(f)$. That is,

$$
f^{\partial}(x)=\langle j, y\rangle \Longleftrightarrow x \leq f\left(y^{j}\right) .
$$


This means that each $f \in O_{\mathbf{A}}$ dualizes to a cofunction as introduced in Section 1.3 and vice versa. Consequently, $\overline{\mathcal{L}}_{\mathbf{X}}$ is the well-known lattice of coclones over the set $\mathbf{X}=\operatorname{At}(\mathbf{A})$, and we have established something we have already mentioned in Chapter 3. Coclones (in the usual sense) are not the dual counterpart of clones over sets - they are the dual counterpart of clones over Boolean algebras. Thus, every result that appears in the literature for coclones over sets is also a result about clones of operations over Boolean algebras.

\subsubsection{Clones over Median Algebras}

4.2.12 Definition. An median algebra is an algebra $\mathbf{A}=\langle A, m\rangle$ with a single ternary operation $m$ for which the following identities hold:

(1) $m(x, y, z) \approx m(y, x, z) \approx m(y, z, x)$,

(2) $m(x, x, y) \approx x$

(3) $m(m(x, y, z), u, v) \approx m(x, m(y, u, v), m(z, u, v))$.

An operation $m$ that satisfies (i)-(iii) is called a median operation.

For a distributive lattice $\langle D, \vee, \wedge\rangle$, the ternary function

$$
m(x, y, z):=(x \vee y) \wedge(y \vee z) \wedge(z \vee x)
$$

is a median operation. Hence, median algebras can be thought of as generalized distributive lattices.

As the following lemma shows, the category of median algebras is the quasivariety generated by the (up to isomorphism) unique two-element median algebra.

4.2.13 Lemma ([Isb80]). Let $\mathbf{M}=\langle\{0,1\}, m\rangle$ be the unique median algebra on the carrier set $\{0,1\}$. Then, $\mathcal{A}:=\mathbb{I} \mathbb{S P}(\mathbf{M})$ is the class of all median algebras.

Since we will only consider finite median algebras, we define $\mathcal{A}$ by setting

$$
\mathcal{A}:=\mathbb{I} \mathbb{S P}(\mathbf{M})_{\text {fin }}=\{\mathbf{A} \in \mathbb{I} \mathbb{S P}(\mathbf{M}) \mid \mathbf{A} \text { finite }\} .
$$

In [CD98], it was shown that

$$
\underline{\mathbf{M}}:=\langle\{0,1\}, 0,1, \neg, \leq, \mathcal{T}\rangle
$$

(with the obvious definitions for $\leq$ and $\neg$ ) yields a full natural duality between $\mathbb{I S P}(\mathbf{M})$ and $\mathbb{I S}_{c}^{0} \mathbb{P}^{+}(\underline{\mathbf{M}})=\mathbb{I S}_{c} \mathbb{P}^{+}(\underline{\mathbf{M}})$. Thus, omitting the discrete topology in $\underline{\mathbf{M}}$, the categories $\mathcal{A}$ and

$$
x:=\mathbb{I S P} \mathbb{P}^{+}(\underline{\mathbf{M}})_{f i n}=\left\{\mathbf{X} \in \mathbb{I S P} \mathbb{P}^{+}(\underline{\mathbf{M}}) \mid \mathbf{X} \text { finite }\right\}
$$

are dually equivalent via the natural dual equivalence $\left\langle D^{\prime}, E^{\prime}, e^{\prime}, \epsilon^{\prime}\right\rangle$.

Note that $X$ is the category of finite bounded partially ordered sets that are in a special way complemented. Roughly speaking, the objects in $X$ are bounded partially ordered sets equipped with a complement operation that acts like the set-complement. 
4.2.14 Definition. A structure $\langle X, 0,1, \neg, \leq\rangle$ is called a strongly complemented bounded partially ordered set if $\langle X, 0,1, \leq\rangle$ is a bounded partially ordered set and $\neg$ is an order-reversing involution (i.e., $\neg \circ \neg=i d$ ) for which $x \leq \neg x$ implies $x=0$.

4.2.15 Proposition ([CD98]). $x$ is the category of finite strongly complemented bounded partially ordered sets.

Again, we want to apply Lemma 4.2 .3 to obtain a more feasible dual equivalence.

4.2.16 Definition. Let $\mathbf{A}=\langle A, m\rangle$ be a finite median algebra. An ideal of $\mathbf{A}$ is a subset $X \subseteq A$ such that, for all $a_{1}, a_{2}, a_{3} \in A$, we have $m\left(a_{1}, a_{2}, a_{3}\right) \in X$ if and only if $a_{i} \in X$ for at least two $i \in\{1,2,3\}$. Denote the set of all ideals of $\mathbf{A}$ by $\operatorname{Spec}(\mathbf{A})$.

Note that we have $\emptyset, A \in \operatorname{Spec}(\mathbf{A})$ and that $x \in \operatorname{Spec}(\mathbf{A})$ implies $A \backslash x \in \operatorname{Spec}(\mathbf{A})$.

4.2.17 Lemma ([Isb80]). Let $\mathbf{A}, \mathbf{B} \in \mathcal{A} . A$ mapping $u: A \rightarrow B$ is a homomorphism between $\mathbf{A}$ and $\mathbf{B}$ if and only if $u^{-1}(x) \in \operatorname{Spec}(\mathbf{A})$ for all $x \in \operatorname{Spec}(\mathbf{B})$.

For $\mathbf{X}=\langle X, 0,1, \neg, \leq\rangle \in X$, we denote by $\operatorname{dInc}(\mathbf{X})$ the set of increasing subsets $a \subseteq X$ where, for all $x \in X$, exactly one of the two elements $x, \neg x$ belongs to $a$.

4.2.18 Lemma ([BH83]). Let $\mathbf{X}, \mathbf{Y} \in X$. A mapping $\varphi: X \rightarrow Y$ is a homomorphism between $\mathbf{X}$ and $\mathbf{Y}$ if and only if $\varphi^{-1}(a) \in \mathrm{d} \operatorname{Inc}(\mathbf{X})$ for all $a \in \operatorname{dInc}(\mathbf{Y})$. Moreover, $\varphi^{-1}: \operatorname{dInc}(\mathbf{Y}) \rightarrow \operatorname{dInc}(\mathbf{X})$ commutes with the mapping

$$
m\left(X_{1}, X_{2}, X_{3}\right):=\left(X_{1} \cap X_{2}\right) \cup\left(X_{1} \cap X_{3}\right) \cup\left(X_{2} \cap X_{3}\right) .
$$

We can now apply Lemma 4.2 .3 .

4.2.19 Lemma. Let $D: \mathcal{A} \rightarrow \mathcal{X}, E: X \rightarrow \mathcal{A}, e: i d_{\mathcal{A}} \rightarrow E D$ and $\epsilon: i d_{X} \rightarrow D E$ be defined as follows:

$$
\begin{aligned}
& D(\mathbf{A}):=\left\langle\operatorname{Spec}(\mathbf{A}), \emptyset, A,(-)^{c}, \subseteq\right\rangle, D(f)(x):=f^{-1}(x) \\
& E(\mathbf{X}):=\left\langle\operatorname{dInc}(\mathbf{X}), m_{E(\mathbf{X})}\right\rangle, E(\varphi)(x):=\varphi^{-1}(x) \\
& e_{\mathbf{A}}(a):=\{x \in \operatorname{Spec}(\mathbf{A}) \mid a \in x\} \\
& \epsilon_{\mathbf{X}}(x):=\{a \in \operatorname{dInc}(\mathbf{X}) \mid x \in a\}
\end{aligned}
$$

where

$$
(-)^{c}: \operatorname{Spec}(\mathbf{A}) \rightarrow \operatorname{Spec}(\mathbf{A}): x \mapsto A \backslash x
$$

and

$$
m_{E(\mathbf{X})}\left(X_{1}, X_{2}, X_{3}\right):=\left(X_{1} \cap X_{2}\right) \cup\left(X_{1} \cap X_{3}\right) \cup\left(X_{2} \cap X_{3}\right) .
$$

Then, $\langle D, E, e, \epsilon\rangle$ is a dual equivalence between $\mathcal{A}$ and $X$.

Proof. The proof is essentially the same as the one of Lemma 4.2.8 (page 49). In fact, it follows from Lemma 4.2 .3 with the same natural transformations by analogue arguments. Note that, this time, we need Lemma 4.2.17 and 4.2.18 instead of Lemma 4.2.6 and 4.2 .7 . 
This dual equivalence was first discovered by J. IsBELL in [Isb80].

4.2.20 Example. Let $\mathbf{A}=\left\langle\{0,1,2,3\}, m_{\mathbf{A}}\right\rangle$ and $\mathbf{B}=\left\langle\{0,1,2\}, m_{\mathbf{B}}\right\rangle$ be two median algebras where $m_{\mathbf{B}}$ maps a triple $\left(b_{1}, b_{2}, b_{3}\right)$ to 0 whenever $\left(b_{1}, b_{2}, b_{3}\right)$ is not a near-unanimity sequence and $m_{\mathbf{A}}$ maps a triple $\left(a_{1}, a_{2}, a_{3}\right)$ that is not a near-unanimity sequence to the second greatest integer in the sequence (e.g. $\left.m_{\mathbf{A}}(0,2,3)=2\right)$. Furthermore, define the morphism $f: \mathbf{A} \rightarrow \mathbf{B}$ as follows

\begin{tabular}{|c||c|c|c|c|}
\hline$x$ & 0 & 1 & 2 & 3 \\
\hline$f(x)$ & 2 & 0 & 0 & 1 \\
\hline
\end{tabular}

Under $D, \mathbf{A}$ and $\mathbf{B}$ dualize to the following strongly complemented bounded posets:
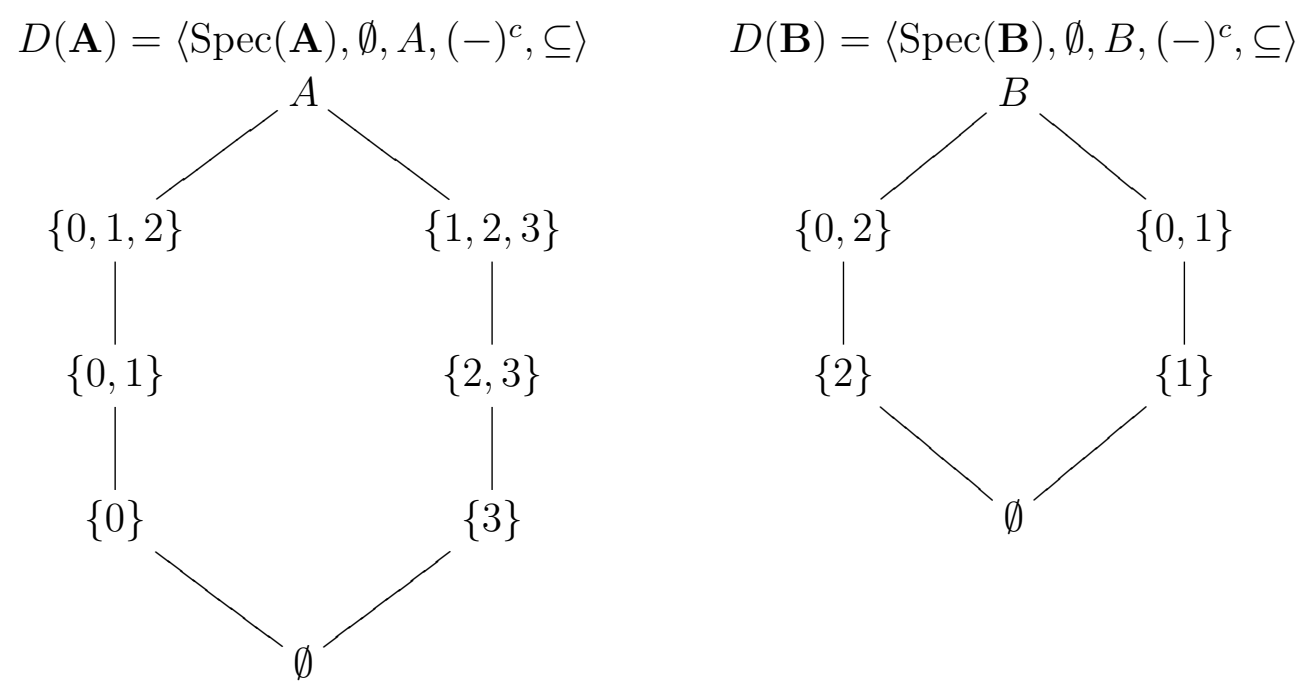

$\{2\}$

Furthermore, $f$ dualizes to the set-mapping $f^{-1}$ that maps the ideals of $\mathbf{B}$ on the ideals of $\mathbf{A}$.

\begin{tabular}{|c||c|c|c|c|c|c|}
\hline$x$ & $\emptyset$ & $\{1\}$ & $\{2\}$ & $\{0,2\}$ & $\{0,1\}$ & $B$ \\
\hline$D(f)$ & $\emptyset$ & $\{3\}$ & $\{0\}$ & $\{0,1,2\}$ & $\{1,2,3\}$ & $A$ \\
\hline
\end{tabular}

Note that $D(f)$ commutes with $(-)^{c}$ and must preserve the maximum and minimum element. Hence, it is already uniquely determined by its values on $\{1\}$ and $\{2\}$.

If we apply $E$ to $D(\mathbf{A})$, we obtain the algebra

$$
E D(\mathbf{A})=\left\langle\operatorname{dInc}(D(\mathbf{A})), m_{E D(\mathbf{A})}\right\rangle,
$$

where $\operatorname{dInc}(D(\mathbf{A}))=\left\{a_{0}, \ldots, a_{3}\right\}$ with

$$
\begin{aligned}
& a_{0}:=\{A,\{0,1,2\},\{0,1\},\{0\}\}, \\
& a_{1}:=\{A,\{0,1,2\},\{1,2,3\},\{0,1\}\}, \\
& a_{2}:=\{A,\{0,1,2\},\{1,2,3\},\{2,3\}\}, \\
& a_{3}:=\{A,\{1,2,3\},\{2,3\},\{3\}\},
\end{aligned}
$$




\section{A Duality for Clones}

and the majority operation $m_{E D(\mathbf{A})}$ is defined by

$$
m_{E D(\mathbf{A})}\left(A_{1}, A_{2}, A_{3}\right):=\left(A_{1} \cap A_{2}\right) \cup\left(A_{1} \cap A_{3}\right) \cup\left(A_{2} \cap A_{3}\right) .
$$

This median algebra is isomorphic to $\mathbf{A}$ via $e_{\mathbf{A}}$ : For $i \in\{0,1,2,3\}$, the element $a_{i}$ is exactly the set of all $x \in \operatorname{Spec}(\mathbf{A})$ that contain $i$. Thus, $e_{\mathbf{A}}(i)=a_{i}$.

Now we want to use this dual equivalence to dualize clones over a finite median algebra A. As in the cases we examined in the previous subsections, we have to understand how the powers of an object $\mathbf{A} \in \mathcal{A}$ dualize under $D$. Since we have

$$
D\left(\mathbf{A}^{n}\right)=\left\langle\operatorname{Spec}\left(\mathbf{A}^{n}\right), \emptyset, A^{n},(-)^{c}, \subseteq\right\rangle,
$$

this is a matter of understanding which subsets of $A^{n}$ are ideals of $\mathbf{A}^{n}$.

4.2.21 Lemma. For $n \in \mathbb{N}_{+}$, we have

$$
\operatorname{Spec}\left(\mathbf{A}^{n}\right)=\left\{A^{i-1} \times x \times A^{n-i} \mid i \in\{1, \ldots, n\}, x \in \operatorname{Spec}(\mathbf{A})\right\} .
$$

Proof. " $\supseteq$ " is easy to see. To show "C", assume $F \in \operatorname{Spec}\left(\mathbf{A}^{n}\right)$. First we show that $F$ is the Cartesian product of $n$ sets $F_{1}, \ldots, F_{n}$, then we show that we have $F_{i} \in \operatorname{Spec}(\mathbf{A})$ for all $i \in\{1, \ldots, n\}$, and finally we show that we have $F=\emptyset$ or $F=A^{n}$ or $F_{i}=A$ for all except one $i \in\{1, \ldots, n\}$. For the first part, suppose $\left(a_{1}, \ldots, a_{n}\right),\left(b_{1}, \ldots, b_{n}\right) \in F$. Then, for $c_{i} \in\left\{a_{i}, b_{i}\right\}(i \in\{1, \ldots, n\})$, we obtain

$$
\left(m_{\mathbf{A}}, \ldots, m_{\mathbf{A}}\right)(\underbrace{\left(a_{1}, \ldots, a_{n}\right)}_{\in F}, \underbrace{\left(b_{1}, \ldots, b_{n}\right)}_{\in F},\left(c_{1}, \ldots, c_{n}\right))=\left(c_{1}, \ldots, c_{n}\right) \in F .
$$

Thus, there exist $F_{1}, \ldots, F_{n}$ such that $F=F_{1} \times \ldots \times F_{n}$. Suppose that $F_{i} \notin \operatorname{Spec}(\mathbf{A})$ for some $i \in\{1, \ldots, n\}$. Without loss of generality, let $i=1$. Then, there exist three elements $a, b, c \in A$ such that $a, b \in F_{1}$ and $m_{\mathbf{A}}(a, b, c) \notin F_{1}$. Since $F$ is a Cartesian product, there exist $x_{2}, \ldots, x_{n} \in A$ such that $\left(a, x_{2}, \ldots, x_{n}\right),\left(b, x_{2}, \ldots, x_{n}\right) \in F$. But now, we reach a contradiction by

$$
\begin{aligned}
& \left(m_{\mathbf{A}}, \ldots, m_{\mathbf{A}}\right)\left(\left(a, x_{2}, \ldots, x_{n}\right),\left(b, x_{2}, \ldots, x_{n}\right),\left(c, x_{2}, \ldots, x_{n}\right)\right) \\
= & \left(m_{\mathbf{A}}(a, b, c), x_{2}, \ldots, x_{n}\right) \notin F .
\end{aligned}
$$

Finally, assume that we have $F \notin\left\{\emptyset, A^{n}\right\}$ and $F_{i}, F_{j} \neq A$ for some $i \neq j$. Then, $A^{n} \backslash F$ is not a Cartesian product of $n$ sets and consequently not an ideal of $A^{n}$, a contradiction.

For $n \in \mathbb{N}_{+}$and $i \in\{1, \ldots, n\}$, the projection morphism $\pi_{i}^{n}$ dualizes to

$$
D\left(\pi_{i}^{n}\right): D(\mathbf{A}) \rightarrow D\left(\mathbf{A}^{n}\right): x \mapsto A^{i-1} \times x \times A^{n-i} .
$$

The usual definition of a copower in $X$ is given as follows: Let $\mathbf{X} \in X$ and let $X^{\prime}$ be the underlying set not including $0^{\mathbf{X}}$ and $1^{\mathbf{X}}$. Then

$$
n \cdot \mathbf{X}:=\left\langle\left\{0^{n \cdot \mathbf{X}}, 1^{n \cdot \mathbf{X}}\right\} \cup n \cdot X^{\prime}, 0^{n \cdot \mathbf{X}}, 1^{n \cdot \mathbf{X}}, \neg, \leq\right\rangle,
$$


where $\leq$ is defined as in the copowers of the bounded partially ordered sets and

$$
\neg x:= \begin{cases}0^{n \cdot \mathbf{X}} & \text { if } x=1^{n \cdot \mathbf{X}}, \\ 1^{n \cdot \mathbf{X}} & \text { if } x=0^{n \cdot \mathbf{X}}, \\ \left\langle i, \neg^{\mathbf{x}} x^{\prime}\right\rangle & \text { if } x=\left\langle i, x^{\prime}\right\rangle .\end{cases}
$$

The associated injection morphisms are given by

$$
\iota_{i}^{n}(x):= \begin{cases}0^{n \cdot \mathbf{X}} & \text { if } x=0^{\mathbf{X}} \\ 1^{n \cdot \mathbf{X}} & \text { if } x=1^{\mathbf{X}} \\ \langle i, x\rangle & \text { otherwise }\end{cases}
$$

We now have the desired dual equivalence, we know how the powers of $\mathbf{A}$ dualize under $D$, and we know how the copowers in $\mathcal{X}$ look like. Thus, finally, we can dualize clones over $\mathbf{A}$. Set $\mathbf{X}:=D(\mathbf{A})$. We obtain the isomorphism $\eta_{n}: D\left(\mathbf{A}^{n}\right) \rightarrow n \cdot \mathbf{X}$ by setting

$$
\eta_{n}(x):= \begin{cases}0^{n \cdot \mathbf{X}} & \text { if } x=\emptyset \\ 1^{n \cdot \mathbf{X}} & \text { if } x=A^{n} \\ \langle i, y\rangle & \text { if } x=A^{i-1} \times y \times A^{n-i} \text { for some } y \in \operatorname{Spec}(\mathbf{A})\end{cases}
$$

Again, we obtain the clone duality $(-)^{\partial}: O_{\mathbf{A}} \rightarrow \bar{O}_{\mathbf{X}}$ by setting $f^{\partial}:=\eta_{n} \circ D(f)$ for $f \in O_{\mathbf{A}}^{(n)}$. Stating this explicitly, we have

$$
f^{\partial}(x)= \begin{cases}0^{n \cdot \mathbf{X}} & \text { if } f^{-1}(x)=\emptyset \\ 1^{n \cdot \mathbf{X}} & \text { if } f^{-1}(x)=A^{n} \\ \langle i, y\rangle & \text { if } f^{-1}(x)=A^{i-1} \times y \times A^{n-i} \text { for some } y \in \operatorname{Spec}(\mathbf{A})\end{cases}
$$

\subsubsection{Clones over Boolean Rings}

4.2.22 Definition. A Boolean ring is a ring $\mathbf{A}=\langle A, \wedge,+, 0\rangle$ with $x \wedge x \approx x$.

Note that in our definition of a Boolean ring, the identity of the monoid $\langle A, \wedge\rangle$ is not a constant in the structure. If it were a constant in the structure, then the category of Boolean rings and the category of Boolean algebras would be isomorphic.

It is an easy exercise to show that, in a Boolean ring, the operation $\wedge$ is commutative and we have $x+x \approx 0$ (that is, every Boolean ring has characteristic two). Moreover, a Boolean ring can be thought of as the lattice $\langle A, \wedge, \vee, 0\rangle$, where $x \vee y:=x+y+(x \wedge y)$. Note that the number of atoms and coatoms of this lattice coincide.

4.2.23 Lemma. Let $\mathbf{M}=\langle\{0,1\}, \wedge,+, 0\rangle$ be the Boolean ring with the obvious definitions for $\wedge$ and + . An algebra $\mathbf{A}$ is a Boolean ring if an only if it is in the quasivariety generated by $\mathbf{M}$. Furthermore, $\mathbb{I S P}(\mathbf{M})_{\text {fin }}=\mathbb{I P}(\mathbf{M})_{\text {fin }}$.

Note that this implies that every finite Boolean ring has a cardinality that is a power of 2 . 
By [CD98],

$$
\underline{\mathbf{M}}:=\langle\{0,1\}, 1, \mathcal{T}\rangle
$$

yields a full natural duality between $\mathbb{I S P}(\mathbf{M})$ and $\mathbb{I S}_{c}^{0} \mathbb{P}^{+}(\underline{\mathbf{M}})=\mathbb{I} \mathbb{S}_{c} \mathbb{P}^{+}(\underline{\mathbf{M}})$. Thus, omitting the discrete topology in $\mathbf{M}$, the two categories

$$
\mathcal{A}:=\mathbb{I S P}(\mathbf{M})_{\text {fin }}=\mathbb{I P}(\mathbf{M})_{\text {fin }}
$$

and

$$
x:=\mathbb{I S P} \mathbb{P}^{+}(\underline{\mathbf{M}})_{f i n}=\left\{\mathbf{X} \in \mathbb{I S P} P^{+}(\underline{\mathbf{M}}) \mid \mathbf{X} \text { finite }\right\}
$$

are dually equivalent via the natural dual equivalence $\left\langle D^{\prime}, E^{\prime}, e^{\prime}, \epsilon^{\prime}\right\rangle$.

Note that $X$ is the category of finite sets with one distinguished element, also called pointed sets.

We will now use the same ideas as in the previous subsections to obtain a more feasible dual equivalence.

4.2.24 Definition. A prime ideal of a commutative ring $\langle R, \wedge,+, 0\rangle$ is a proper, nonempty subset $I \subseteq R$ for which the following three conditions hold:

(i) $(I,+, 0)$ is a subgroup of $(R,+, 0)$,

(ii) $x \wedge r \in I$ for all $x \in I, r \in R$,

(iii) $r \wedge s \in I$ implies $r \in I$ or $s \in I$.

Denote the set of prime ideals by $\operatorname{Spec}(\mathbf{A})$.

The set of prime ideals can alternatively be characterized as the set of maximal, nontrivial ideals. That is, a proper, non-empty subset $I \subseteq A$ is a prime ideal if and only if (i) and (ii) from above hold and $I$ is maximal among all such proper, non-empty subsets.

Note that a subset of $A$ is a prime ideal of $\mathbf{A}$ if and only if it can be written as $\{x \leq a \mid x \in A\}$ for some coatom $a$ of the lattice $\langle A, \wedge, \vee, 0\rangle$. Thus, the cardinality of $\operatorname{Spec}(\mathbf{A})$ is precisely the number of atoms (or coatoms) in the lattice $\langle A, \wedge, \vee, 0\rangle$.

4.2.25 Lemma. Let $\mathbf{A}, \mathbf{B} \in \mathcal{A}$. A mapping $\varphi: A \rightarrow B$ is a homomorphism between $\mathbf{A}$ and $\mathbf{B}$ if and only if $u^{-1}(x) \in \operatorname{Spec}(\mathbf{A}) \cup\{A\}$ for all $x \in \operatorname{Spec}(\mathbf{B}) \cup\{B\}$.

This time, a characterization of the morphisms in $X$ is trivial.

4.2.26 Lemma. Let $\mathbf{X}, \mathbf{Y} \in X$. A mapping $\varphi: X \rightarrow Y$ is a homomorphism between $\mathbf{X}$ and $\mathbf{Y}$ if and only if $1^{\mathbf{X}} \in \varphi^{-1}\left(1^{\mathbf{Y}}\right)$.

4.2.27 Lemma. Let $D: \mathcal{A} \rightarrow \mathcal{X}, E: X \rightarrow \mathcal{A}, e: i d_{\mathcal{A}} \rightarrow E D$ and $\epsilon: i d_{X} \rightarrow D E$ be defined as follows:

$$
\begin{aligned}
D(\mathbf{A}) & :=\langle\operatorname{Spec}(\mathbf{A}) \cup\{A\}, A\rangle, D(u)(x):=u^{-1}(x), \\
E(\mathbf{X}) & :=\left\langle\mathfrak{P}\left(X \backslash\left\{1^{\mathbf{X}}\right\}\right), \cap, \uplus, \emptyset\right\rangle, E(\varphi)(x):=\varphi^{-1}(x), \\
e_{\mathbf{A}}(a) & :=\{x \in \operatorname{Spec}(\mathbf{A}) \mid a \in x\}, \\
\epsilon_{\mathbf{X}}(x) & :=\left\{a \in \mathfrak{P}\left(\mathbf{X} \backslash\left\{1^{\mathbf{X}}\right\}\right) \mid x \in a\right\},
\end{aligned}
$$


where $\uplus$ is the binary operation defined by

$$
X_{1} \uplus X_{2}:=\left(X_{1} \cup X_{2}\right) \backslash\left(X_{1} \cap X_{2}\right) .
$$

Then, $\langle D, E, e, \epsilon\rangle$ is a dual equivalence between $\mathcal{A}$ and $\mathcal{X}$.

Proof. Again, the proof is essentially the same as the one of Lemma 4.2.8 (page 49). However, there is one small technical difference. This time, we identify the morphisms in $\mathbf{M}^{\mathbf{A}}$ with the sets that are the inverse images of 0 . In other words, we apply Lemma 4.2 .3 with the two natural transformations $\tau$ and $\psi$ defined as follows:

$$
\begin{aligned}
&\left(\tau_{\mathbf{A}}\right)_{\mathbf{A} \in \mathcal{A}}, \tau_{\mathbf{A}}: D^{\prime}(\mathbf{A}) \rightarrow D(\mathbf{A}), \tau_{\mathbf{A}}(f):=f^{-1}(0), \\
&\left(\psi_{\mathbf{X}}\right)_{\mathbf{X} \in \mathcal{X}}, \psi_{\mathbf{X}}: E^{\prime}(\mathbf{X}) \rightarrow E(\mathbf{X}), \psi_{\mathbf{X}}(g):=g^{-1}(1)
\end{aligned}
$$

The small difference aside, all arguments are analogue, so we will not present a detailed proof.

We have obtained the desired dual equivalence. As we always did before, we will now examine how the powers in $\mathcal{A}$ dualize under $D$.

4.2.28 Lemma. For $n \in \mathbb{N}_{+}$, we have

$$
\operatorname{Spec}\left(\mathbf{A}^{n}\right)=\left\{A^{i-1} \times x \times A^{n-i} \mid i \in\{1, \ldots, n\}, x \in \operatorname{Spec}(\mathbf{A})\right\} .
$$

Proof. Since it is straightforward to check that each element of the set on the right hand side of the equation is a prime ideal, we only show " $\subseteq$ ". First, we can use the same arguments as we used in the proof of Lemma 4.2.10 (page 52) to conclude that every prime ideal of $\mathbf{A}^{n}$ must be the Cartesian product of $n$ sets $F_{1}, \ldots, F_{n}$ and that $F_{i} \neq A$ implies $F_{i} \in \operatorname{Spec}(\mathbf{A})$. Assuming that we have $F_{i}=A$ for all $i \in\{1, \ldots, n\}$ implies $F=A^{n}$ (which is impossible), and assuming that we have $F_{i}, F_{j} \neq A$ for some $i \neq j$ contradicts the maximality of the ideal $F_{1} \times \ldots \times F_{n}$ since it would then be properly contained in the ideal $A^{i-1} \times F_{i} \times A^{n-i}$. The claim follows.

Once again, for each $n \in \mathbb{N}_{+}$and $i \in\{1, \ldots, n\}$, the projection morphism $\pi_{i}^{n}$ dualizes to

$$
D\left(\pi_{i}^{n}\right): D(\mathbf{A}) \rightarrow D\left(\mathbf{A}^{n}\right): x \mapsto A^{i-i} \times x \times A^{n-i} .
$$

The copowers of pointed sets are usually defined as follows: For $\mathbf{X} \in \mathcal{X}$, denote by $X^{\prime}$ the underlying set not including $1^{\mathbf{X}}$. Then,

$$
n \cdot \mathbf{X}:=\left\langle\left\{1^{n \cdot \mathbf{X}}\right\} \cup n \cdot X^{\prime}, 1^{n \cdot \mathbf{X}}\right\rangle,
$$

with its associated injection morphisms

$$
\iota_{i}^{n}(x):= \begin{cases}1^{n \cdot \mathbf{X}} & \text { if } x=1^{\mathbf{X}} \\ \langle i, x\rangle & \text { otherwise }\end{cases}
$$


We are ready to dualize the clones over a finite Boolean ring $\mathbf{A}$. Set $\mathbf{X}:=D(\mathbf{A})$. We can make the isomorphism $\eta_{n}: D\left(\mathbf{A}^{n}\right) \rightarrow n \cdot \mathbf{X}$ precise by setting

$$
\eta_{n}(x):= \begin{cases}\langle i, y\rangle & \text { if } x=A^{i-1} \times y \times A^{n-i} \text { for some } y \in \operatorname{Spec}(\mathbf{A}), \\ 1^{n \cdot \mathbf{X}} & \text { if } x=A^{n} .\end{cases}
$$

Again, we obtain the clone duality $(-)^{\partial}: O_{\mathbf{A}} \rightarrow \bar{O}_{\mathbf{X}}$ by setting $f^{\partial}:=\eta_{n} \circ D(f)$ for $f \in O_{\mathbf{A}}^{(n)}$. Stating this explicitly, we have

$$
f^{\partial}(x)= \begin{cases}\langle i, y\rangle & \text { if } f^{-1}(x)=A^{i-1} \times y \times A^{n-i} \text { for some } y \in \operatorname{Spec}(\mathbf{A}) \\ 1^{n \cdot \mathbf{X}} & \text { if } f^{-1}(x)=A^{n}\end{cases}
$$

\subsubsection{Clones over Boolean Lattices}

4.2.29 Definition. A Boolean lattice $\mathbf{A}=\langle A, \vee, \wedge\rangle$ is a complemented distributive lattice. That is, $\mathbf{A}$ has a bottom element 0 and a top element 1 such that, for each $a \in A$, there exists a unique element $\bar{a} \in A$ such that $a \vee \bar{a}=1$ and $a \wedge \bar{a}=0$.

To dualize Boolean lattices, we can use the duality we obtained for distributive lattices and see how it simplifies. Let $\mathcal{A}^{*}$ be the category of finite distributive lattices, let $X^{*}$ be the category of finite bounded partially ordered sets, and let $\left\langle D^{*}, E^{*}, e^{*}, \epsilon^{*}\right\rangle$ be the dual equivalence from Lemma 4.2.8 (page 49.

4.2.30 Lemma. A distributive lattice $\mathbf{A} \in \mathcal{A}^{*}$ is a Boolean lattice if and only if the order relation $\leq^{D^{*}(\mathbf{A})}$ is given as follows:

$$
\leq^{D^{*}(\mathbf{A})}=\left\{\left(x_{1}, x_{2}\right) \mid x_{1}=0^{D^{*}(\mathbf{A})} \text { or } x_{2}=1^{D^{*}(\mathbf{A})}\right\}
$$

Proof. Under $D^{*}$, A dualizes to the bounded poset of its prime filters and trivial subsets. But now, the prime filters of a Boolean lattice are precisely the sets of the form $\uparrow a$, where $a$ is an atom of $\mathbf{A}$. Hence, two distinct prime filters of a Boolean lattice are incomparable with respect to $\subseteq$. The claim follows.

Thus, if we restrict $\mathcal{A}^{*}, D^{*}$ and $e^{*}$ to the full subcategory of finite Boolean lattices and $x^{*}, E^{*}$ and $\epsilon^{*}$ to the full subcategory of finite bounded partially ordered sets with the trivial order relation $(x \leq y$ if and only if $x=0$ or $y=1$ ), then we obtain a dual equivalence $\langle D, E, e, \epsilon\rangle$ between the two subcategories that we may denote by $\mathcal{A}$ and $\boldsymbol{X}$, respectively.

Let $\mathbf{X} \in X$. A subset of $\mathbf{X}$ is a proper, non-empty increasing subset of $X$ if and only if it contains $1^{\mathbf{X}}$, but not $0^{\mathbf{X}}$. Hence,

$$
\operatorname{Inc}(\mathbf{X})=\left\{1^{\mathbf{X}}\right\} \cup \mathfrak{P}\left(X \backslash\left\{0^{\mathbf{X}}, 1^{\mathbf{X}}\right\}\right) .
$$

Consequently, if $\mathbf{X}=D(\mathbf{A})$, then $\operatorname{Inc}(\mathbf{X})=\{A\} \cup \mathfrak{P}(\operatorname{Spec}(\mathbf{A}))$. Furthermore, for $\mathbf{Y} \in \mathcal{X}$, a mapping $u: X \rightarrow Y$ that maps $0^{\mathbf{X}}$ to $0^{\mathbf{Y}}$ and $1^{\mathbf{X}}$ to $1^{\mathbf{Y}}$ automatically commutes with $\leq$. Thus, we can safely dismiss the order relation from the objects in $X$, making $X$ the 
category of finite sets with two distinguished elements, also called doubly pointed sets. We can describe $\langle D, E, e, \epsilon\rangle$ as follows:

$$
\begin{aligned}
& D(\mathbf{A}):=\langle\operatorname{Spec}(\mathbf{A}) \cup\{\emptyset, A\}, \emptyset, A\rangle, D(u)(x):=u^{-1}(x), \\
& E(\mathbf{X}):=\left\langle\left\{1^{\mathbf{X}}\right\} \cup \mathfrak{P}\left(X \backslash\left\{0^{\mathbf{X}}, 1^{\mathbf{X}}\right\}\right), \cup, \cap\right\rangle, E(\varphi)(a):=\varphi^{-1}(a), \\
& e_{\mathbf{A}}(a):=\{A\} \cup\{x \in \operatorname{Spec}(\mathbf{A}) \mid a \in x\}, \\
& \epsilon_{\mathbf{X}}(x):=\{a \in \operatorname{Inc}(\mathbf{X}) \mid x \in a\} .
\end{aligned}
$$

As noted above, the prime filters of a Boolean lattice $\mathbf{A}$ are precisely the sets of the form $\uparrow a$, where $a \in \operatorname{At}(\mathbf{A})$. Thus, we can think of $\operatorname{Spec}(\mathbf{A})$ as the set of atoms of $\mathbf{A}$.

For $n \in \mathbb{N}_{+}$, we have $D\left(\mathbf{A}^{n}\right)=\left\langle\operatorname{Spec}\left(\mathbf{A}^{n}\right) \cup\left\{\emptyset, A^{n}\right\}, \emptyset, A^{n}\right\rangle$, and with the help of Lemma 4.2 .10 (page 52 ) we can immediately conclude

$$
\operatorname{Spec}\left(\mathbf{A}^{n}\right)=\left\{A^{i-1} \times x \times A^{n-i} \mid x \in \operatorname{Spec}(\mathbf{A}), i \in\{1, \ldots, n\}\right\} .
$$

Moreover, for $i \in\{1, \ldots, n\}$, we have

$$
D\left(\pi_{i}^{n}\right)(x)=A^{i-1} \times x \times A^{n-i} .
$$

Evidently, we can think of $\operatorname{Spec}\left(\mathbf{A}^{n}\right)$ as the set of atoms of $\mathbf{A}^{n}$.

For $\mathbf{X} \in \mathcal{X}$, denote by $X^{\prime}$ the underlying set of $\mathbf{X}$ not including $0^{\mathbf{X}}$ and $1^{\mathbf{X}}$. The $n$-th copower of $\mathbf{X}$ is usually defined by setting

$$
n \cdot \mathbf{X}:=\left\langle\left\{0^{n \cdot \mathbf{X}}, 1^{n \cdot \mathbf{X}}\right\} \cup n \cdot X^{\prime}, 0^{n \cdot \mathbf{X}}, 1^{n \cdot \mathbf{X}}\right\rangle
$$

and

$$
\iota_{i}^{n}(x):= \begin{cases}0^{n \cdot \mathbf{X}} & \text { if } x=0^{\mathbf{X}} \\ 1^{n \cdot \mathbf{X}} & \text { if } x=1^{\mathbf{x}} \\ \langle i, x\rangle & \text { otherwise }\end{cases}
$$

We have collected all the facts to dualize the clones over $\mathbf{A}$. Setting $\mathbf{X}:=D(\mathbf{A})$, we can make the isomorphisms $\eta_{n}: D\left(\mathbf{A}^{n}\right) \rightarrow n \cdot \mathbf{X}$ precise by defining it as follows:

$$
\eta_{n}(x):= \begin{cases}0^{n \cdot \mathbf{X}} & \text { if } x=\emptyset \\ 1^{n \cdot \mathbf{X}} & \text { if } x=A^{n} \\ \langle i, y\rangle & \text { if } x=A^{i-1} \times y \times A^{n-i} \text { for some } y \in \operatorname{Spec}(\mathbf{A}) .\end{cases}
$$

Again, we obtain $f \mapsto f^{\partial}$ by setting $f^{\partial}:=\eta_{n} \circ D(f)$ for all $f \in O_{\mathbf{A}}^{(n)}$. That is,

$$
f^{\partial}(x)= \begin{cases}0^{n \cdot \mathbf{X}} & \text { if } f^{-1}(x)=\emptyset \\ 1^{n \cdot \mathbf{X}} & \text { if } f^{-1}(x)=A^{n} \\ \langle i, y\rangle & \text { if } f^{-1}(x)=A^{i-1} \times y \times A^{n-i} \text { for some } y \in \operatorname{Spec}(\mathbf{A})\end{cases}
$$




\subsubsection{Clones over Boolean Groups}

So far, we have only looked at cases in which $\mathbf{A}$ was in a quasivariety generated by a structure $\mathbf{M}$ that had a near-unanimity term. As it was presented in Section 1.6, it is a consequence of the Baker-Pixley Theorem that $\mathbf{M}$ having a near-unanimity term simplifies the brute force construction from Theorem 1.6.5 (page 25). In fact, a near-unanimity term in the term algebra of $\mathbf{M}$ guarantees that the alter ego $\mathbf{M}$ has a comparatively small type. That is why many of the well-known natural dualities rely on an algebra with near-unanimity term. We will now look at a scenario, in which this is not the case.

4.2.31 Definition. A Boolean group $\mathbf{A}=\langle A,+, 0\rangle$ is a group in which every element has order 2 , that is, $x+x \approx 0$.

It is a well-known fact that an algebra $\mathbf{A}$ is a Boolean group if and only if it is in the quasivariety generated by

$$
\mathbf{M}:=\langle\{0,1\},+, 0\rangle,
$$

where + is the addition modulo 2. Furthermore, we have $\mathbb{I S P}(\mathbf{M})=\mathbb{I P}(\mathbf{M})$. Note that this implies that every finite Boolean group must have a cardinality that is a power of 2 .

By CD98,

$$
\underline{\mathbf{M}}:=\langle\{0,1\},+, 0, \mathcal{T}\rangle
$$

yields a full duality between $\operatorname{ISP}(\mathbf{M})$ and $\mathbb{S S}_{c}^{0} \mathbb{P}^{+}(\underline{\mathbf{M}})=\mathbb{I S}_{c} \mathbb{P}^{+}(\underline{\mathbf{M}})$. Thus, omitting the topology $\mathcal{T}$, the categories

$$
\mathcal{A}:=\mathbb{I S P}(\mathbf{M})_{\text {fin }}=\mathbb{I P}(\mathbf{M})_{\text {fin }}=\{\mathbf{A} \in \mathbb{I P}(\mathbf{M}) \mid \mathbf{A} \text { finite }\},
$$

and

$$
x:=\mathbb{S S P}^{+}(\underline{\mathbf{M}})_{f i n}=\mathbb{I S P}^{+}(\mathbf{M})_{f i n}=\mathbb{I P}(\mathbf{M})_{\text {fin }}=\mathcal{A}
$$

are dually equivalent via the natural dual equivalence $\left\langle D^{\prime}, E^{\prime}, e^{\prime}, \epsilon^{\prime}\right\rangle$. Note that $\mathcal{A}$ and $X$ are the same category, so the category of finite Boolean groups is dually equivalent to itself. In such cases, we say that the category is self-dual. This has some interesting consequences. For instance, in a self-dual category, products and coproducts coincide (note that they still have different associated morphisms).

Again, we want to use Lemma 4.2.3 to obtain a more feasible dual equivalence. Before we do so, we need to introduce some notation.

For each finite Boolean group $\mathbf{A}$, denote by $B_{\mathbf{A}}$ an arbitrary but fixed basis of $\mathbf{A}$. If $\mathbf{A}$ has $2^{n}$ elements, then $B_{\mathbf{A}}$ will have $n$ elements. Choose the basis of the powers of $\mathbf{A}$ such that

$$
B_{\mathbf{A}^{n}}=\left\{\left(0^{\mathbf{A}}, \ldots, 0^{\mathbf{A}}, \underset{\substack{\uparrow \\ i}}{a}, 0^{\mathbf{A}}, \ldots, 0^{\mathbf{A}}\right) \mid i \in\{1, \ldots, n\}, a \in B_{\mathbf{A}}\right\} .
$$

We define the mapping

$$
\chi_{\mathbf{A}}: \mathbf{A} \rightarrow \mathfrak{P}\left(B_{\mathbf{A}}\right)
$$


by setting $\chi_{\mathbf{A}}(a)$ to be the (unique) subset $B^{\prime} \subseteq B_{\mathbf{A}}$ such that $a=\sum B^{\prime}$ (where $\sum \emptyset$ may be defined as 0$)$. Denote by $1_{\mathbf{A}}$ the element $\chi_{\mathbf{A}}^{-1}\left(B_{\mathbf{A}}\right)$, that is, $1_{\mathbf{A}}$ is the sum of all the elements in the basis.

Furthermore, for a subset $B \subseteq B_{\mathbf{A}}$, we denote by $[B]_{\text {odd }}$ the set of all elements $a \in A$ that can be written as a sum of base elements among which an odd number of elements is from $B$, and we define $[B]_{\text {even }}$ accordingly. In other words, $x \in[B]_{\text {odd }}$ whenever $\left|\chi_{\mathbf{A}}(x) \cap B\right|$ is odd, and $x \in[B]_{\text {even }}$ whenever $\left|\chi_{\mathbf{A}}(x) \cap B\right|$ is even. Note that $x \in[B]_{\text {odd }}$ is equivalent to $x \notin[B]_{\text {even }}$. Moreover, we have $[\emptyset]_{\text {odd }}=\emptyset$ and $[\emptyset]_{\text {even }}=A$.

4.2.32 Example. Let $\mathbf{A}=\left\langle\{0,1\}^{3},+,(0,0,0)\right\rangle$, where + denotes the addition modulo 2 (applied component-wise). Set $B_{\mathbf{A}}:=\{(1,0,0),(0,1,0),(0,0,1)\}$. Then

$$
[\{(1,0,0),(0,1,0)\}]_{\text {odd }}=\{(1,0,0),(0,1,0),(1,0,1),(0,1,1)\} .
$$

The following lemma is a technicality, but it will be needed to build our desired dual equivalence:

4.2.33 Lemma. Let $\mathbf{B}, \mathbf{C}$ be finite Boolean groups, let $v: \mathbf{B} \rightarrow \mathbf{C}$ and let $x \in \mathbf{C}$. Then,

$$
\left[v^{-1}\left(\left[\chi_{\mathbf{C}}(x)\right]_{o d d}\right) \cap B_{\mathbf{B}}\right]_{o d d}=v^{-1}\left(\left[\chi_{\mathbf{C}}(x)\right]_{o d d}\right) .
$$

Proof. " $\subseteq$ ". Let $y \in\left[v^{-1}\left(\left[\chi_{\mathbf{C}}(x)\right]_{\text {odd }}\right) \cap B_{\mathbf{B}}\right]_{\text {odd }}$. There exist $b_{1}, \ldots, b_{n}, c_{1}, \ldots, c_{k} \in B_{\mathbf{B}}$ such that

$$
y=b_{1}+\ldots+b_{n}+c_{1}+\ldots+c_{k}
$$

where $b_{1}, \ldots, b_{n} \in v^{-1}\left(\left[\chi_{\mathbf{C}}(x)\right]_{o d d}\right)$ and $c_{1}, \ldots, c_{k} \notin v^{-1}\left(\left[\chi_{\mathbf{C}}(x)\right]_{o d d}\right)$. By assumption, $n$ is odd. We have

$$
v(y)=v\left(b_{1}\right)+\ldots+v\left(b_{n}\right)+v\left(c_{1}\right)+\ldots v\left(c_{k}\right) .
$$

Each of the sets $\chi_{\mathbf{C}}\left(v\left(b_{1}\right)\right), \ldots, \chi_{\mathbf{C}}\left(v\left(b_{n}\right)\right)$ contains an odd number of elements among $\chi_{\mathbf{C}}(x)$, whereas $\chi_{\mathbf{C}}\left(v\left(c_{1}\right)\right), \ldots, \chi_{\mathbf{C}}\left(v\left(c_{k}\right)\right)$ all contain an even number of elements among $\chi_{\mathbf{C}}(x)$. Thus, $\chi_{\mathbf{C}}(v(y))$ contains an odd number of elements among $\chi_{\mathbf{C}}(x)$, and we obtain $v(y) \in\left[\chi_{\mathbf{C}}(x)\right]_{\text {odd }}$. That is, $y \in v^{-1}\left(\left[\chi_{\mathbf{C}}(x)\right]_{\text {odd }}\right)$.

"卫". Let $y \in v^{-1}\left(\left[\chi_{\mathbf{C}}(x)\right]_{\text {odd }}\right)$. There exist $b_{1}, \ldots, b_{n} \in B_{\mathbf{B}}$ such that

$$
y=b_{1}+\ldots+b_{n} .
$$

We need to show that an odd number of elements among the set $\left\{b_{1}, \ldots, b_{n}\right\}$ is in $v^{-1}\left(\left[\chi_{\mathbf{C}}(x)\right]_{\text {odd }}\right)$. For contradiction, we assume the contrary. Without loss of generality, let $k \leq n$ be an even integer such that

$$
v\left(b_{1}\right), \ldots, v\left(b_{k}\right) \in\left[\chi_{\mathbf{C}}(x)\right]_{\text {odd }} \text { and } v\left(b_{k+1}\right), \ldots, v\left(b_{n}\right) \in\left[\chi_{\mathbf{C}}(x)\right]_{\text {even }} .
$$

But now,

$$
v(y)=\underbrace{\underbrace{v\left(b_{1}\right)+\ldots+v\left(b_{k}\right)}_{\in\left[\chi_{\mathbf{C}}(x)\right]_{\text {even }}}+\underbrace{v\left(b_{k+1}\right)+\ldots+v\left(b_{n}\right)}_{\in\left[\chi_{\mathbf{C}}(x)\right]_{\text {even }}}}_{\in\left[\chi_{\mathbf{C}}(x)\right]_{\text {even }}}
$$

contradicts $v(y) \in\left[\chi_{\mathbf{C}}(x)\right]_{\text {odd }}$. 


\section{A Duality for Clones}

We are ready to build our dual equivalence. This time, the proof is comparably long and rather technical.

4.2.34 Lemma. Let $D: \mathcal{A} \rightarrow \mathcal{X}, E: \mathcal{X} \rightarrow \mathcal{A}, e: i d_{\mathcal{A}} \rightarrow E D$ and $\epsilon: i d_{\mathcal{X}} \rightarrow D E$ be defined as follows (where $u \in \mathcal{A}(\mathbf{A}, \mathbf{B})$ and $\varphi \in \mathcal{X}(\mathbf{X}, \mathbf{Y})$ ):

$$
\begin{aligned}
D(\mathbf{A}) & :=\mathbf{A}, D(u)(x):=\sum\left(u^{-1}\left(\left[\chi_{\mathbf{B}}(x)\right]_{\text {odd }}\right) \cap B_{\mathbf{A}}\right), \\
E(\mathbf{X}) & :=\mathbf{X}, E(\varphi)(x):=\sum\left(\varphi^{-1}\left(\left[\chi_{\mathbf{Y}}(x)\right]_{o d d}\right) \cap B_{\mathbf{X}}\right), \\
e_{\mathbf{A}} & :=i d_{\mathbf{A}}, \\
\epsilon_{\mathbf{X}} & :=i d_{\mathbf{X}} .
\end{aligned}
$$

Then, $\langle D, E, e, \epsilon\rangle$ is a dual equivalence between $\mathcal{A}$ and $\mathcal{X}$.

Proof. For the whole proof, let $\mathbf{A}, \mathbf{B} \in \mathcal{A}$ and let $u \in \mathcal{A}(\mathbf{A}, \mathbf{B})$. Since the definitions of $D$ and $E$ are analogue, we will only show that $D$ is a functor. Evidently, the object $D(\mathbf{A})$ is well-defined and $D(u)$ is a well-defined mapping. Let us show that $D(u)$ commutes with + . For this, recall that $\uplus$ denotes the exclusive union, that is,

$$
X_{1} \uplus X_{2}=\left(X_{1} \cup X_{2}\right) \backslash\left(X_{1} \cap X_{2}\right) .
$$

We have

$$
\begin{aligned}
D(u)(x+y) & =\sum\left(u^{-1}\left(\left[\chi_{\mathbf{B}}(x+y)\right]_{\text {odd }}\right) \cap B_{\mathbf{A}}\right) \\
& =\sum\left(u^{-1}\left(\left[\chi_{\mathbf{B}}(x) \uplus \chi_{\mathbf{B}}(y)\right]_{\text {odd }}\right) \cap B_{\mathbf{A}}\right) \\
& =\sum\left(u^{-1}\left(\left[\chi_{\mathbf{B}}(x)\right]_{\text {odd }} \uplus\left[\chi_{\mathbf{B}}(y)\right]_{\text {odd }}\right) \cap B_{\mathbf{A}}\right) \\
& =\sum\left(\left(u^{-1}\left(\left[\chi_{\mathbf{B}}(x)\right]_{\text {odd }}\right) \uplus u^{-1}\left(\left[\chi_{\mathbf{B}}(y)\right]_{\text {odd }}\right)\right) \cap B_{\mathbf{A}}\right) \\
& =\sum\left(u^{-1}\left(\left[\chi_{\mathbf{B}}(x)\right]_{\text {odd }}\right) \cap B_{\mathbf{A}}\right)+\sum\left(u^{-1}\left(\left[\chi_{\mathbf{B}}(y)\right]_{\text {odd }}\right) \cap B_{\mathbf{A}}\right) \\
& =D(u)(x)+D(u)(y) .
\end{aligned}
$$

Thus, $D(u)$ is a well-defined morphism. To complete the proof that $D$ is a functor, let $\mathbf{C} \in \mathcal{A}$ and let $v \in \mathcal{A}(\mathbf{B}, \mathbf{C})$. We need to show $D(u) \circ D(v)=D(v \circ u)$. For $x \in \mathbf{C}$, Lemma 4.2.33 yields the following equation:

$$
\left[v^{-1}\left(\left[\chi_{\mathbf{C}}(x)\right]_{\text {odd }}\right) \cap B_{\mathbf{B}}\right]_{\text {odd }}=v^{-1}\left(\left[\chi_{\mathbf{C}}(x)\right]_{\text {odd }}\right) .
$$

We can conclude

$$
\begin{aligned}
(D(u) \circ D(v))(x) & =\sum\left(u^{-1}\left(\left[\chi_{\mathbf{B}}\left(\sum\left(v^{-1}\left(\left[\chi_{\mathbf{C}}(x)\right]_{\text {odd }}\right) \cap B_{\mathbf{B}}\right)\right)\right]_{\text {odd }}\right) \cap B_{\mathbf{A}}\right) \\
& =\sum\left(u^{-1}\left(\left[v^{-1}\left(\left[\chi_{\mathbf{C}}(x)\right]_{\text {odd }}\right) \cap B_{\mathbf{B}}\right]_{\text {odd }}\right) \cap B_{\mathbf{A}}\right) \\
& \stackrel{\text { 4.1. }}{=} \sum\left(u^{-1}\left(v^{-1}\left(\left[\chi_{\mathbf{C}}(x)\right]_{\text {odd }}\right)\right) \cap B_{\mathbf{A}}\right) \\
& =\sum\left((v \circ u)^{-1}\left(\left[\chi_{\mathbf{C}}(x)\right]_{\text {odd }}\right) \cap B_{\mathbf{A}}\right) \\
& =D(v \circ u)(x) .
\end{aligned}
$$


Thus, $D$ is a functor. Now, we will use Lemma 4.2 .3 with the following two natural transformations:

$$
\begin{aligned}
& \tau_{\mathbf{A}}: D^{\prime}(\mathbf{A}) \rightarrow D(\mathbf{A}), \tau_{\mathbf{A}}(f):=\sum\left(f^{-1}(1) \cap B_{\mathbf{A}}\right) \\
& \psi_{\mathbf{X}}: E^{\prime}(\mathbf{X}) \rightarrow E(\mathbf{X}), \psi_{\mathbf{X}}(g):=\sum\left(g^{-1}(1) \cap B_{\mathbf{X}}\right)
\end{aligned}
$$

Since the definitions of $\tau$ and $\psi$ are clearly analogue, we will only show that $\tau$ has the desired properties. For $\mathbf{A} \in \mathcal{A}$, it is straightforward to check that $\tau_{\mathbf{A}}$ is a welldefined morphism. Since every morphism $f: \mathbf{A} \rightarrow \mathbf{M}$ is uniquely determined by the base elements of $\mathbf{A}$ that are mapped to $1, \tau_{\mathbf{A}}$ is also injective. We will now show that $\tau_{\mathbf{A}}$ is surjective. For $a \in A$, set $f(x):=1$ for all $x \in\left[\chi_{\mathbf{A}}(a)\right]_{o d d}$ and $f(x):=0$ otherwise. We obtain

$$
\tau_{\mathbf{A}}(f)=\sum\left(f^{-1}(1) \cap B_{\mathbf{A}}\right)=\sum\left(\left[\chi_{\mathbf{A}}(a)\right]_{o d d} \cap B_{\mathbf{A}}\right)=\sum \chi_{\mathbf{A}}(a)=a .
$$

Thus, $\tau_{\mathbf{A}}$ is an isomorphism. Next, we will show that $\tau$ is a natural transformation. For $x \in \mathcal{A}(\mathbf{B}, \mathbf{M})$, the following equation follows directly from Equation (4.1) (note that $\left.\left[\chi_{\mathbf{M}}(1)\right]_{\text {odd }}=[\{1\}]_{\text {odd }}=\{1\}\right)$ :

$$
\left[x^{-1}(1) \cap B_{\mathbf{B}}\right]_{o d d}=x^{-1}(1) .
$$

We can infer

$$
\begin{aligned}
\left(D(u) \circ \tau_{\mathbf{B}}\right)(x) & =D(u)\left(\sum\left(x^{-1}(1) \cap B_{\mathbf{B}}\right)\right) \\
& =\sum\left(u^{-1}\left(\left[\chi_{\mathbf{B}}\left(\sum\left(x^{-1}(1) \cap B_{\mathbf{B}}\right)\right)\right]_{o d d}\right) \cap B_{\mathbf{A}}\right) \\
& =\sum\left(u^{-1}\left(\left[x^{-1}(1) \cap B_{\mathbf{B}}\right]_{o d d}\right) \cap B_{\mathbf{A}}\right) \\
& \stackrel{4.2}{=} \sum\left(u^{-1}\left(x^{-1}(1)\right) \cap B_{\mathbf{A}}\right) \\
& =\sum\left((x \circ u)^{-1}(1) \cap B_{\mathbf{A}}\right) \\
& =\sum\left(\left(D^{\prime}(u)(x)\right)^{-1}(1) \cap B_{\mathbf{A}}\right) \\
& =\left(\tau_{\mathbf{A}} \circ D^{\prime}(u)\right)(x) .
\end{aligned}
$$

Thus, $\tau_{\mathbf{A}}$ is a natural transformation. It remains to show that we have

$$
e_{\mathbf{A}}=E\left(\tau_{\mathbf{A}}^{-1}\right) \circ \psi_{D(\mathbf{A})} \circ e_{\mathbf{A}}^{\prime} .
$$

Before we show the equation, we need to collect a few identities. The morphism $e_{\mathbf{A}}^{\prime}(a): D^{\prime}(\mathbf{A}) \rightarrow \mathbf{M}$ is given by $z \mapsto z(a)$. Hence, $\psi_{D(\mathbf{A})}\left(e_{\mathbf{A}}^{\prime}(a)\right)$ is the sum of all $f \in B_{\mathbf{M}^{\mathbf{A}}}$ with $f(a)=1$. In other words,

$$
\psi_{D(\mathbf{A})}\left(e_{\mathbf{A}}^{\prime}(a)\right)=\sum\left\{f \in B_{\mathbf{M}^{\mathbf{A}}} \mid f(a)=1\right\} .
$$




\section{A Duality for Clones}

Moreover, it is easy to see that we have

$$
\left[\left\{f \in B_{\mathbf{M}^{\mathbf{A}}} \mid f(a)=1\right\}\right]_{\text {odd }}=\left\{f \in \mathbf{M}^{\mathbf{A}} \mid f(a)=1\right\} .
$$

For $a \in \mathbf{A}$ and $f \in \mathbf{M}^{\mathbf{A}}$, we have $f(a)=1$ if and only if an odd number of elements among $\chi_{\mathbf{A}}(a)$ get mapped to 1 . Hence,

$$
\tau_{\mathbf{A}}\left(\left\{f \in \mathbf{M}^{\mathbf{A}} \mid f(a)=1\right\}\right)=\left[\chi_{\mathbf{A}}(a)\right]_{\text {odd }} .
$$

Combining the last two displayed equations gives us

$$
\tau_{\mathbf{A}}\left(\left[\left\{f \in B_{\mathbf{M}^{\mathbf{A}}} \mid f(a)=1\right\}\right]_{o d d}\right)=\left[\chi_{\mathbf{A}}(a)\right]_{\text {odd }} .
$$

For brevity, let $h:=\sum\left\{f \in B_{\mathbf{M}^{\mathbf{A}}} \mid f(a)=1\right\}$. We have

$$
\begin{aligned}
\left(E\left(\tau_{\mathbf{A}}^{-1}\right) \circ \psi_{D(\mathbf{A})} \circ e_{\mathbf{A}}^{\prime}\right)(a) & \stackrel{4.3]}{=} E\left(\tau_{\mathbf{A}}^{-1}\right)(h) \\
& =\sum\left(\tau_{\mathbf{A}}\left(\left[\chi_{\mathbf{M}^{\mathbf{A}}}(h)\right]_{\text {odd }}\right) \cap B_{\mathbf{A}}\right) \\
& =\sum\left(\tau_{\mathbf{A}}\left(\left[\left\{f \in B_{\mathbf{M}^{\mathbf{A}}} \mid f(a)=1\right\}\right]_{\text {odd }}\right) \cap B_{\mathbf{A}}\right) \\
& \stackrel{4.4}{=} \sum\left(\left[\chi_{\mathbf{A}}(a)\right]_{\text {odd }} \cap B_{\mathbf{A}}\right) \\
& =\sum \chi_{\mathbf{A}}(a) \\
& =a \\
& =e_{\mathbf{A}}(a) .
\end{aligned}
$$

Since $D(\mathbf{A})=\mathbf{A}$, each finite power of $\mathbf{A}$ dualizes to itself, that is, $D\left(\mathbf{A}^{n}\right)=\mathbf{A}^{n}$ for all $n \in \mathbb{N}_{+}$. It remains to examine how the projection morphisms dualize. For $n \in \mathbb{N}_{+}$ and $i \in\{1, \ldots, n\}$, we have

$$
\left(\pi_{i}^{n}\right)^{-1}\left(\left[\chi_{\mathbf{A}}(a)\right]_{\text {odd }}\right)=A^{i-1} \times\left[\chi_{\mathbf{A}}(a)\right]_{\text {odd }} \times A^{n-i},
$$

whence it follows

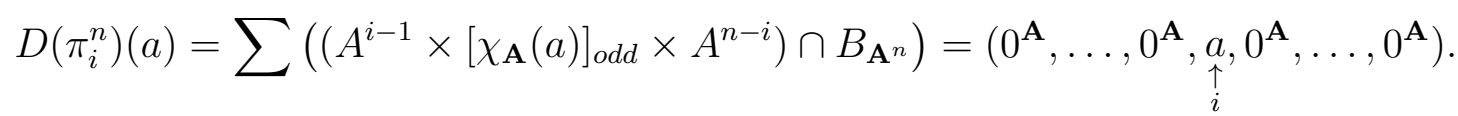

Since $\mathcal{A}$ is self-dual, we have $n \cdot \mathbf{A}=\mathbf{A}^{n}$. The associated injection morphisms are given as follows:

$$
\iota_{i}^{n}(x):=\left(0^{\mathbf{X}}, \ldots, 0^{\mathbf{X}}, \underset{\substack{\uparrow \\ i}}{x}, 0^{\mathbf{X}}, \ldots, 0^{\mathbf{X}}\right) .
$$

It follows that the cotupling of dual operations $g_{1}, \ldots, g_{n}: \mathbf{X} \rightarrow k \cdot \mathbf{X}$ is given by

$$
\begin{aligned}
{\left[g_{1}, \ldots, g_{n}\right]\left(x_{1}, \ldots, x_{n}\right) } & =\left[g_{1}, \ldots, g_{n}\right]\left(x_{1}, 0^{\mathbf{x}}, \ldots, 0^{\mathbf{X}}\right)+\ldots+\left[g_{1}, \ldots, g_{n}\right]\left(0^{\mathbf{x}}, \ldots, 0^{\mathbf{X}}, x_{n}\right) \\
& =\left[g_{1}, \ldots, g_{n}\right]\left(\iota_{1}^{n}\left(x_{1}\right)\right)+\ldots+\left[g_{1}, \ldots, g_{n}\right]\left(\iota_{n}^{n}\left(x_{n}\right)\right) \\
& =\left(\left[g_{1}, \ldots, g_{n}\right] \circ \iota_{1}^{n}\right)\left(x_{1}\right)+\ldots+\left(\left[g_{1}, \ldots, g_{n}\right] \circ \iota_{n}^{n}\right)\left(x_{n}\right) \\
& =g_{1}\left(x_{1}\right)+\ldots+g_{n}\left(x_{n}\right) .
\end{aligned}
$$


Since we have $D\left(\mathbf{A}^{n}\right)=\mathbf{A}^{n}=n \cdot \mathbf{A}=n \cdot D(\mathbf{A})$ and $\iota_{i}^{n}=D\left(\pi_{i}^{n}\right)$, the isomorphism $\eta_{n}$ is the identity morphism for all $n \in \mathbb{N}_{+}$and $(-)^{\partial}$ is given by $f \mapsto D(f)$. That is,

$$
f^{\partial}(x):=\sum\left(f^{-1}\left(\left[\chi_{\mathbf{A}}(x)\right]_{o d d}\right) \cap B_{\mathbf{A}^{n}}\right) .
$$

4.2.35 Example. Let $\mathbf{A}:=\left\langle\{0,1\}^{3},+,(0,0,0)\right\rangle$ be the Boolean group from Example 4.2.32. Recall that $B_{\mathbf{A}}:=\{(1,0,0),(0,1,0),(0,0,1)\}$. Then,

$$
B_{\mathbf{A}^{2}}=\left(B_{\mathbf{A}} \times\{(0,0,0)\}\right) \cup\left(\{(0,0,0)\} \times B_{\mathbf{A}}\right) .
$$

Let $f: \mathbf{A}^{2} \rightarrow \mathbf{A}$ be the operation that is defined as follows (note that $f$ is uniquely defined by its values on the base elements of $\mathbf{A}^{2}$ ):

\begin{tabular}{|c|c|}
\hline$\left(x_{1}, x_{2}\right)$ & $f\left(x_{1}, x_{2}\right)$ \\
\hline \hline$((1,0,0),(0,0,0))$ & $(0,0,1)$ \\
$((0,1,0),(0,0,0))$ & $(1,1,0)$ \\
$((0,0,1),(0,0,0))$ & $(1,0,1)$ \\
$((0,0,0),(1,0,0))$ & $(1,0,0)$ \\
$((0,0,0),(0,1,0))$ & $(0,1,1)$ \\
$((0,0,0),(0,0,1))$ & $(0,1,0)$ \\
\hline
\end{tabular}

Similarly, $f^{\partial}: \mathbf{X} \rightarrow 2 \cdot \mathbf{X}$ is uniquely determined by its values on the base elements of A. We have

$$
\begin{aligned}
f^{\partial}((1,0,0)) & =\sum\left(f^{-1}\left(\left[\chi_{\mathbf{A}}((1,0,0))\right]_{\text {odd }}\right) \cap B_{\mathbf{A}^{2}}\right) \\
& =\sum\left(f^{-1}\left([\{(1,0,0)\}]_{\text {odd }}\right) \cap B_{\mathbf{A}^{2}}\right)
\end{aligned}
$$

and we can see in the table above that exactly three of the elements from $B_{\mathbf{A}^{2}}$ are in

$$
f^{-1}\left([\{(1,0,0)\}]_{\text {odd }}\right)=f^{-1}(\{(1,0,0),(1,1,0),(1,0,1)\}),
$$

namely $((0,0,0),(1,0,0)),((0,1,0),(0,0,0))$ and $((0,0,1),(0,0,0))$. Thus,

$$
\begin{aligned}
f^{\partial}((1,0,0)) & =((0,0,0),(1,0,0))+((0,1,0),(0,0,0))+((0,0,1),(0,0,0)) \\
& =((0,1,1),(1,0,0)) .
\end{aligned}
$$

Repeating this procedure for the other two elements from $B_{\mathbf{A}}$, we obtain the following table that uniquely determines $f^{\partial}$ :

\begin{tabular}{|c|c|}
\hline$x$ & $f^{\partial}(x)$ \\
\hline \hline$(1,0,0)$ & $((0,1,1),(1,0,0))$ \\
$(0,1,0)$ & $((0,1,0),(0,1,1))$ \\
$(0,0,1)$ & $((1,0,1),(0,1,0))$ \\
\hline
\end{tabular}

Note that we did not need to calculate the values $f\left(x_{1}, x_{2}\right)$ for $\left(x_{1}, x_{2}\right) \notin B_{\mathbf{A}^{2}}$ to determine $f^{\partial}$. 


\subsubsection{Clones over Semilattices}

In this subsection, we will assume that $\mathbf{A}$ is a finite semilattice.

4.2.36 Definition. A semilattice is an algebra $\langle A, \vee\rangle$ consisting of a set $A$ equipped with a binary operation $\vee$, called join, that is associative, commutative and idempotent.

An algebra $\mathbf{A}$ is a finite semilattice if and only if it is isomorphic to a subalgebra of a direct power of $\langle\{0,1\}, \vee\rangle$ with the obvious definition for $\vee$. Thus, for $\mathbf{M}:=\langle\{0,1\}, \vee\rangle$, the quasivariety

$$
\mathcal{A}:=\mathbb{I S P}(\mathbf{M})_{\text {fin }}=\{\mathbf{A} \in \mathbb{I S P}(\mathbf{M}) \mid \mathbf{A} \text { finite }\}
$$

is the category of finite semilattices.

By CD98,

$$
\underline{\mathbf{M}}:=\langle\{0,1\}, \vee, 0,1, \mathcal{T}\rangle
$$

yields a full natural duality between $\mathbb{I S P}(\mathbf{M})$ and $\mathbb{S S}_{c}^{0} \mathbb{P}^{+}(\underline{\mathbf{M}})=\mathbb{S}_{c} \mathbb{P}^{+}(\underline{\mathbf{M}})$. Once again, omitting the topology, this implies that $\mathbf{M}$ yields a full natural duality between $\mathcal{A}$ and

$$
x:=\mathbb{I S P} P^{+}(\underline{\mathbf{M}})_{\text {fin }}=\left\{\mathbf{X} \in \mathbb{I S P} P^{+}(\underline{\mathbf{M}}) \mid \mathbf{X} \text { finite }\right\}
$$

Let $\left\langle D^{\prime}, E^{\prime}, e^{\prime}, \epsilon^{\prime}\right\rangle$ be the corresponding natural duality.

Note that $X$ is the category of finite bounded semilattices.

4.2.37 Definition. A bounded semilattice is an algebra $\langle A, 0,1, \vee\rangle$ such that $\langle A, \vee\rangle$ is a semilattice and $1 \vee a=1$ as well as $0 \vee a=a$ for all $a \in A$.

As usual, we use Lemma 4.2 .3 to obtain a more feasible dual equivalence $\langle D, E, e, \epsilon\rangle$.

4.2.38 Definition. A prime filter of $\mathbf{A}$ is a non-empty, proper, increasing subset $X \subseteq A$ such that $x_{1} \vee x_{2} \in X$ implies $x_{1} \in X$ or $x_{2} \in X$. Denote by $\operatorname{Spec}(\mathbf{A})$ the set of prime filters of $\mathbf{A}$.

Note that, in contrast to prime filters of distributive lattices, a prime filter of a semilattice is not necessarily generated by a single element. However, a similar version of Lemma 4.2.6 (page 48) can be stated.

4.2.39 Lemma. Let $\mathbf{A}, \mathbf{B} \in \mathcal{A}$. A mapping $u: A \rightarrow B$ is a homomorphism between $\mathbf{A}$ and $\mathbf{B}$ if and only if $u^{-1}(x) \in\{A, \emptyset\} \cup \operatorname{Spec}(\mathbf{A})$ for all $x \in \operatorname{Spec}(\mathbf{B})$.

For bounded semilattices $\mathbf{X}, \mathbf{Y} \in X$, a similar statement can be made.

4.2.40 Lemma. Let $\mathbf{X}, \mathbf{Y} \in X$. A mapping $\varphi: X \rightarrow Y$ is a homomorphism between $\mathbf{X}$ and $\mathbf{Y}$ if and only if $\varphi^{-1}(z) \in \operatorname{Spec}(\mathbf{X})$ for all $z \in \operatorname{Spec}(\mathbf{Y})$.

We are ready to apply Lemma 4.2 .3 . 
4.2.41 Lemma. Let $D: \mathcal{A} \rightarrow \mathcal{X}, E: \mathcal{X} \rightarrow \mathcal{A}, e: i d_{\mathcal{A}} \rightarrow E D$ and $\epsilon: i d_{x} \rightarrow D E$ be defined as follows:

$$
\begin{aligned}
D(\mathbf{A}) & :=\langle\operatorname{Spec}(\mathbf{A}) \cup\{\emptyset, A\}, \emptyset, A, \cup\rangle, D(u)(x):=u^{-1}(x), \\
E(\mathbf{X}) & :=\langle\operatorname{Spec}(\mathbf{X}), \cup\rangle, E(\varphi)(x):=\varphi^{-1}(x), \\
e_{\mathbf{A}}(a) & :=\{x \in \operatorname{Spec}(\mathbf{A}) \mid a \in x\}, \\
\epsilon_{\mathbf{X}}(x) & :=\{a \in \operatorname{Spec}(\mathbf{X}) \mid x \in a\} .
\end{aligned}
$$

Then, $\langle D, E, e, \epsilon\rangle$ is a dual equivalence between $\mathcal{A}$ and $X$.

Proof. Again, the proof is essentially the same as the one of Lemma 4.2.8 (page 49). We use the same natural transformations and the same arguments.

4.2.42 Example. Take the following two semilattices:
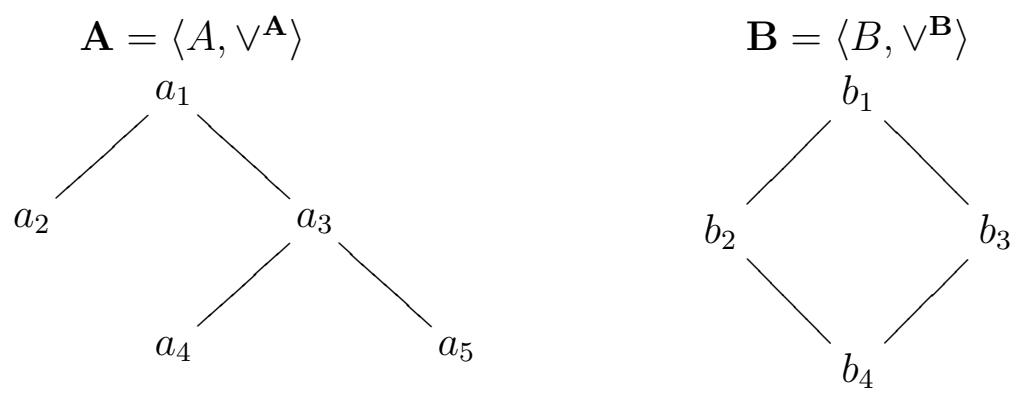

Moreover, define the morphisms $f_{1}, f_{2}: \mathbf{A} \rightarrow \mathbf{B}$ as follows:

\begin{tabular}{|c||c|c|c|c|c|}
\hline$x$ & $a_{1}$ & $a_{2}$ & $a_{3}$ & $a_{4}$ & $a_{5}$ \\
\hline \hline$f_{1}(x)$ & $b_{1}$ & $b_{2}$ & $b_{3}$ & $b_{3}$ & $b_{3}$ \\
\hline$f_{2}(x)$ & $b_{1}$ & $b_{1}$ & $b_{2}$ & $b_{2}$ & $b_{4}$ \\
\hline
\end{tabular}

Under $D, \mathbf{A}$ and $\mathbf{B}$ dualize to the set of their prime filters equipped with $\cup$ and the constants $A, B$, respectively. Thus, $D(\mathbf{A})$ and $D(\mathbf{B})$ are the following bounded semilattices:
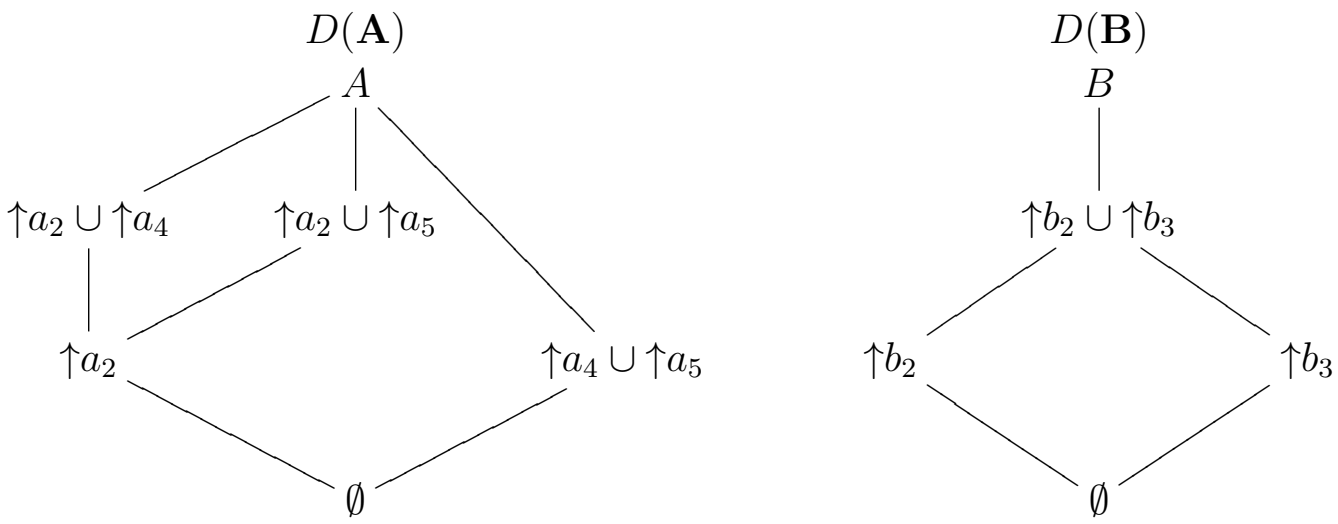
The homomorphisms $f_{1}$ and $f_{2}$ dualize to the set-mappings $f_{1}^{-1}$ and $f_{2}^{-1}$ :

\begin{tabular}{|c||c|c|c|c|c|}
\hline$x$ & $\emptyset$ & $\uparrow b_{2}$ & $\uparrow b_{3}$ & $\uparrow b_{2} \cup \uparrow b_{3}$ & $B$ \\
\hline \hline$D\left(f_{1}\right)(x)$ & $\emptyset$ & $\uparrow a_{2}$ & $\uparrow a_{4} \cup \uparrow a_{5}$ & $A$ & $A$ \\
\hline$D\left(f_{2}\right)(x)$ & $\emptyset$ & $\uparrow a_{2} \cup \uparrow a_{4}$ & $\uparrow a_{2}$ & $\uparrow a_{2} \cup \uparrow a_{4}$ & $A$ \\
\hline
\end{tabular}

Note that $D\left(f_{1}\right)$ and $D\left(f_{2}\right)$ are already determined by their values on the $\cup$-irreducible prime filters $\uparrow b_{2}$ and $\uparrow b_{3}$ (more on that later). Furthermore, it might be tempting to interpret $D(\mathbf{A})$ and $D(\mathbf{B})$ as lattices (since they have a bottom element). However, $D\left(f_{1}\right)$ and $D\left(f_{2}\right)$ do not commute with the meet that one might assign to $D(\mathbf{A})$ and $D(\mathbf{B})$ from the diagrams above. It is therefore wrong to think of $D(\mathbf{A})$ and $D(\mathbf{B})$ as lattices.

If we apply $E$ to $D(\mathbf{B})$, we obtain the semilattice formed by the prime filters of $D(\mathbf{B})$.

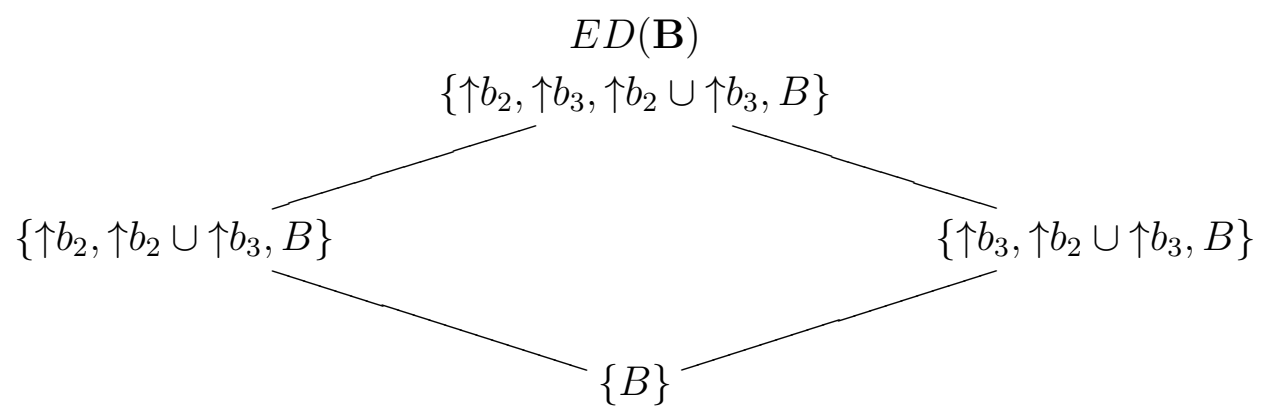

It is now easy to see that this semilattice is isomorphic to $\mathbf{B}$ via $e_{\mathbf{B}}$ : For example, the element $\left\{\uparrow b_{3}, \uparrow b_{2} \cup \uparrow b_{3}, B\right\}$ is exactly the set of all $x \in \operatorname{Spec}(\mathbf{B})$ that contain $b_{3}$. Thus, $e_{\mathbf{B}}\left(b_{3}\right)=\left\{\uparrow b_{3}, \uparrow b_{2} \cup \uparrow b_{3}, B\right\}$.

We will now check how the powers of a semilattice $\mathbf{A} \in \mathcal{A}$ dualize under $D$. Again, this is a matter of understanding $\operatorname{Spec}\left(\mathbf{A}^{n}\right)$ as we have $D\left(\mathbf{A}^{n}\right)=\left\langle\operatorname{Spec}\left(\mathbf{A}^{n}\right) \cup\{\emptyset, A\}, \emptyset, A, \cup\right\rangle$.

4.2.43 Definition. For a finite semilattice $\mathbf{A}$, denote by $\operatorname{Spec}^{*}(\mathbf{A})$ the set of prime filters of $\mathbf{A}$ that are $\cup$-irreducible among the set of prime filters of $\mathbf{A}$. That is, $x \in \operatorname{Spec}^{*}(\mathbf{A})$ if and only if $x \in \operatorname{Spec}(\mathbf{A})$ and $x_{1} \cup x_{2}=x$ implies $x \in\left\{x_{1}, x_{2}\right\}$ for all $x_{1}, x_{2} \in \operatorname{Spec}(\mathbf{A})$.

4.2.44 Lemma. The prime filters of $\mathbf{A}$ are precisely the sets that can be written as non-empty unions of sets in $\operatorname{Spec}^{*}(\mathbf{A})$, that is,

$$
\operatorname{Spec}(\mathbf{A})=\left\langle\operatorname{Spec}^{*}(\mathbf{A})\right\rangle_{\cup}:=\left\{x_{1} \cup \ldots \cup x_{n} \mid n \geq 1, x_{1}, \ldots, x_{n} \in \operatorname{Spec}^{*}(\mathbf{A})\right\} .
$$

Proof. " $\subseteq$ " is trivial. For the other direction, we only need to show that the union of two prime filters is again a prime filter of $A$. Let $x, y \in \operatorname{Spec}(\mathbf{A})$. It is obvious that $x \cup y$ is an increasing, non-empty, proper subset of $A$. It remains to show that $a \vee b \in x \cup y$ implies $a \in x \cup y$ or $b \in x \cup y$. Assume $a \notin x \cup y$ and $b \notin x \cup y$. But then, we have $a, b \notin x$ and $a, b \notin y$, which implies $a \vee b \notin x$ and $a \vee b \notin y$ since $x, y \in \operatorname{Spec}(\mathbf{A})$. Thus, $a \vee b \notin x \cup y$, a contradiction. 
4.2.45 Lemma. For $n \in \mathbb{N}_{+}$, we have

$$
\operatorname{Spec}^{*}\left(\mathbf{A}^{n}\right)=\left\{A^{i-1} \times x \times A^{n-i} \mid i \in\{1, \ldots, n\}, x \in \operatorname{Spec}^{*}(\mathbf{A})\right\} .
$$

Proof. It is straightforward to check that " $\supseteq$ " holds. Concerning the other direction, we will show " $\subseteq$ " in three parts. First we show that any $F \in \operatorname{Spec}^{*}\left(\mathbf{A}^{n}\right)$ must be the Cartesian product of $n$ sets $F_{1}, \ldots, F_{n}$, then we show that $F_{i}$ is a $\cup$-irreducible prime filter whenever $F_{i} \neq A$, and finally we show that exactly one of the sets $F_{1}, \ldots, F_{n}$ is a proper subset of $A$. The first part can be shown analogue to the corresponding part in the proof of Lemma 4.2.10 (page 52). The same applies for showing that $F_{i}$ is a prime filter for all $i \in\{1, \ldots, n\}$ with $F_{i} \neq A$. It remains to show that $F_{i}$ is $\cup$-irreducible among the prime filters of $\mathbf{A}$. For contradiction, assume that $F_{i}$ can be written as $P_{1} \cup P_{2}$ for some prime filters $P_{1}, P_{2} \in \operatorname{Spec}(\mathbf{A})$. Then, we have

$$
\bigcup_{j=1}^{2}\left(F_{1} \times \ldots \times F_{i-1} \times P_{j} \times F_{i+1} \times \ldots \times F_{n}\right)=F,
$$

a contradiction to $F \in \operatorname{Spec}^{*}\left(\mathbf{A}^{n}\right)$. Thus, $F_{i} \in \operatorname{Spec}^{*}\left(\mathbf{A}^{n}\right)$ whenever $F_{i} \neq A$. It is obvious that there exists $i \in\{1, \ldots, n\}$ such that $F_{i} \neq A$ (otherwise we obtain $F=A^{n}$, which is impossible). Assume that we have $F_{i} \neq A$ and $F_{j} \neq A$ for $i \neq j$. Without loss of generality, we may assume $i=1$ and $j=2$. Then, there exists $a \in A \backslash F_{1}$ and $b \in A \backslash F_{2}$. Hence, $\left(a, 1^{\mathbf{A}}, \ldots, 1^{\mathbf{A}}\right),\left(1^{\mathbf{A}}, b, 1^{\mathbf{A}}, \ldots, 1^{\mathbf{A}}\right) \notin F$. But now, it follows

$$
\left(1^{\mathbf{A}}, \ldots, 1^{\mathbf{A}}\right)=\left(a, 1^{\mathbf{A}}, \ldots, 1^{\mathbf{A}}\right) \vee\left(1^{\mathbf{A}}, b, 1^{\mathbf{A}}, \ldots, 1^{\mathbf{A}}\right) \notin F
$$

which contradicts that $F$ is increasing and non-empty.

With this result, we can describe $D\left(\mathbf{A}^{n}\right)$. For $i \in\{1, \ldots, n\}$, let

$$
\langle i, \operatorname{Spec}(\mathbf{A})\rangle:=\left\{A^{i-1} \times x \times A^{n-i} \mid x \in \operatorname{Spec}(\mathbf{A})\right\} .
$$

Now, the carrier set of $D\left(\mathbf{A}^{n}\right)$ is

$$
\left\{\emptyset, A^{n}\right\} \cup\left\{x_{1} \cup \ldots \cup x_{k} \mid i_{1}, \ldots, i_{k} \in\{1, \ldots, n\} \text { pairwise distinct, } x_{j} \in\left\langle i_{j}, \operatorname{Spec}(\mathbf{A})\right\rangle\right\},
$$

and the duals of the projections are given by

$$
D\left(\pi_{i}^{n}\right): D(\mathbf{A}) \rightarrow D\left(\mathbf{A}^{n}\right): x \mapsto A^{i-1} \times x \times A^{n-i} .
$$

For $n \in \mathbb{N}_{+}$, the $n$-th copower of a finite bounded semilattices is usually defined by setting

$$
n \cdot \mathbf{X}:=\left\langle\left\langle n \cdot X^{\prime}\right\rangle_{\vee^{n \cdot \mathbf{X}}} \cup\left\{0^{n \cdot \mathbf{X}}, 1^{n \cdot \mathbf{X}}\right\}, 0^{n \cdot \mathbf{X}}, 1^{n \cdot \mathbf{X}}, \vee^{n \cdot \mathbf{X}}\right\rangle,
$$

where $X^{\prime}$ denotes the carrier set of $\mathbf{X}$ without $0^{\mathbf{X}}, 1^{\mathbf{X}}$ and $\vee^{n \cdot \mathbf{X}}$ may be the commutative, associative, binary operation that is defined as follows: For $\langle i, x\rangle \in n \cdot X^{\prime}$ and $\langle j, y\rangle \in n \cdot X^{\prime}$ we define

$$
\langle i, x\rangle \vee^{n \cdot \mathbf{X}}\langle j, y\rangle:= \begin{cases}\left\langle i, x \vee^{\mathbf{X}} y\right\rangle & \text { if } i=j \\ \{\langle i, x\rangle,\langle j, y\rangle\} & \text { otherwise. }\end{cases}
$$




\section{A Duality for Clones}

For $\langle i, x\rangle \in n \cdot X^{\prime}$ and $y=\left\{\left\langle j_{1}, y_{1}\right\rangle, \ldots,\left\langle j_{k}, y_{k}\right\rangle\right\} \subseteq n \cdot X^{\prime}$ where $j_{1}, \ldots, j_{k}$ are pairwise distinct, we set

$$
\langle i, x\rangle \vee^{n \cdot \mathbf{X}} y:=\left\{\begin{array}{cc}
\left\{\left\langle j_{1}, x \vee^{\mathbf{X}} y_{1}\right\rangle,\left\langle j_{2}, y_{2}\right\rangle, \ldots,\left\langle j_{k}, y_{k}\right\rangle\right\} & \text { if } i=j_{1}, \\
\vdots & \vdots \\
\left\{\left\langle j_{1}, y_{1}\right\rangle, \ldots,\left\langle j_{k-1}, y_{k-1}\right\rangle,\left\langle j_{k}, x \vee^{\mathbf{X}} y_{k}\right\rangle\right\} & \text { if } i=j_{k}, \\
\left\{\left\langle j_{1}, y_{1}\right\rangle, \ldots,\left\langle j_{k}, y_{k}\right\rangle,\langle i, x\rangle\right\} & \text { otherwise. }
\end{array}\right.
$$

Finally, if $x$ or $y$ is one of the two distinguished elements, it is obvious how to define $x \vee y$. The remaining cases follow from the commutativity of $\mathrm{\vee}^{n \cdot \mathbf{X}}$.

The associated injection morphisms are defined as follows:

$$
\iota_{i}^{n}(x)= \begin{cases}0^{n \cdot \mathbf{X}} & \text { if } x=0^{\mathbf{X}}, \\ 1^{n \cdot \mathbf{X}} & \text { if } x=1^{\mathbf{X}}, \\ \langle i, x\rangle & \text { otherwise. }\end{cases}
$$

Concerning the cotuplings, it is straightforward to check that, for $g_{i}: \mathbf{X} \rightarrow k \cdot \mathbf{X}$ $(i \in\{1, \ldots, n\})$, we have

$$
\left[g_{1}, \ldots, g_{n}\right](x)= \begin{cases}0^{n \cdot \mathbf{X}} & \text { if } x=0^{k \cdot \mathbf{X}} \\ 1^{n \cdot \mathbf{X}} & \text { if } x=1^{k \cdot \mathbf{X}} \\ g_{i}(y) & \text { if } x=\langle i, y\rangle\end{cases}
$$

where the remaining cases for $x$ follow from the fact that $\left[g_{1}, \ldots, g_{n}\right]$ commutes with $\vee$, e.g., for $x=\{\langle 1, y\rangle,\langle n-1, z\rangle\}$, we have

$$
\left[g_{1}, \ldots, g_{n}\right](x)=\left[g_{1}, \ldots, g_{n}\right](\langle 1, y\rangle) \vee^{n \cdot \mathbf{X}}\left[g_{1}, \ldots, g_{n}\right](\langle n-1, z\rangle)=g_{1}(y) \vee^{n \cdot \mathbf{X}} g_{n-1}(z) .
$$

4.2.46 Example. Let $\mathbf{A}=\langle\{0,1,2\}, \vee\rangle$ be a semilattice where $x \vee y:=\max (x, y)$. We obtain $\mathbf{X}=D(\mathbf{A})=\langle\{\emptyset,\{2\},\{1,2\}, A\}, \emptyset, A, \cup\rangle$, and the second copower of $\mathbf{X}$ is given by the following bounded semilattice:

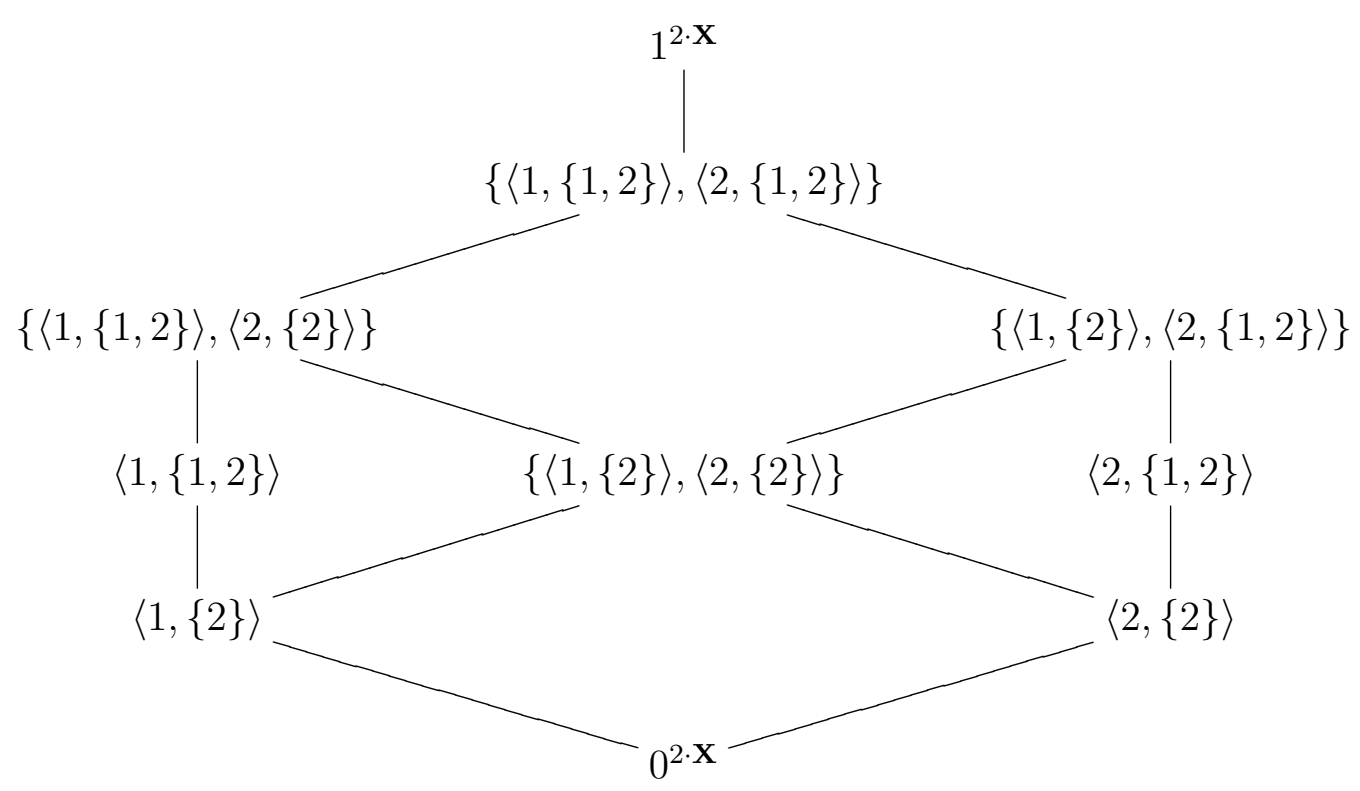


It remains to define the isomorphism $\eta_{n}: D\left(\mathbf{A}^{n}\right) \rightarrow n \cdot \mathbf{X}$. This time, this will be slightly more complicated.

4.2.47 Definition. Let $x \in \operatorname{Spec}\left(\mathbf{A}^{n}\right)$. For $k \geq 2$, a subset $\left\{x_{1}, \ldots, x_{k}\right\} \subseteq \operatorname{Spec}\left(\mathbf{A}^{n}\right)$ is said to be the prime filter partition (p.f.p.) of $x$ provided that we have $x=x_{1} \cup \ldots \cup x_{k}$ and there exist pairwise distinct $j_{1}, \ldots, j_{k} \in\{1, \ldots, n\}$ such that $x_{i} \in\left\langle j_{i}, \operatorname{Spec}(\mathbf{A})\right\rangle$ for all $i \in\{1, \ldots, k\}$.

4.2.48 Example. Let $\mathrm{A}$ be the three-element semilattice from the previous example. Then, $x:=\uparrow(0,2) \cup \uparrow(1,0)$ is a prime filter of $\mathbf{A}^{2}$ and the set $\{\uparrow(0,2), \uparrow(1,0)\}$ is the p.f.p. of $x$.

We can use the following definition of $\eta_{n}$ :

$$
\eta_{n}(x):= \begin{cases}0^{n \cdot \mathbf{X}} & \text { if } x=\emptyset \\ 1^{n \cdot \mathbf{X}} & \text { if } x=A^{n} \\ \langle i, y\rangle & \text { if } x=A^{i-1} \times y \times A^{i-n} \text { for } \operatorname{some} y \in \operatorname{Spec}(\mathbf{A}) \\ \left\{\eta_{n}\left(x_{1}\right), \ldots, \eta_{n}\left(x_{k}\right)\right\} & \text { if }\left\{x_{1}, \ldots, x_{k}\right\} \text { p.f.p. of } x\end{cases}
$$

We obtain our desired clone isomorphism $(-)^{\partial}: O_{\mathbf{A}} \rightarrow \bar{O}_{\mathbf{X}}$ by setting $f^{\partial}:=\eta_{\operatorname{ar}(f)} \circ D(f)$. Stating this explicitly, we have

$$
f^{\partial}(x)= \begin{cases}0^{n \cdot \mathbf{X}} & \text { if } x=\emptyset \\ 1^{n \cdot \mathbf{X}} & \text { if } x=A^{n} \\ \langle i, y\rangle & \text { if } f^{-1}(x)=A^{i-1} \times y \times A^{i-n} \text { for some } y \in \operatorname{Spec}(\mathbf{A}), \\ \left\{\eta_{n}\left(x_{1}\right), \ldots, \eta_{n}\left(x_{k}\right)\right\} & \text { if }\left\{x_{1}, \ldots, x_{k}\right\} \text { p.f.p. of } f^{-1}(x) .\end{cases}
$$

4.2.49 Example. Let $\mathbf{A}=\langle\{0,1,2\}, \vee\rangle$ be a semilattice where $x \vee y:=\max (x, y)$. Let $f \in O_{\mathbf{A}}^{(2)}$ be defined as follows:

$$
f(x, y):= \begin{cases}0 & \text { if } x, y \in\{0,1\} \\ 2 & \text { if } y=2 \\ 1 & \text { otherwise }\end{cases}
$$

Recall that the dual of $\mathbf{A}$ is given by

$$
\mathbf{X}=D(\mathbf{A})=\langle\{\emptyset,\{2\},\{1,2\}, A\}, \emptyset, A, \cup\rangle
$$

and that $2 \cdot \mathbf{X}$ is the bounded semilattice from Example 4.2.46. The dual operation $f^{\partial}: \mathbf{X} \rightarrow 2 \cdot \mathbf{X}$ is given as follows:

\begin{tabular}{|c|c|}
\hline$x$ & $f^{\partial}(x)$ \\
\hline \hline$\emptyset$ & $0^{2 \cdot \mathbf{X}}$ \\
\hline$\{2\}$ & $\langle 2,\{2\}\rangle$ \\
\hline$\{1,2\}$ & $\{\langle 1,\{2\}\rangle,\langle 2,\{2\}\rangle\}$ \\
\hline$A$ & $1^{2 \cdot \mathbf{X}}$ \\
\hline
\end{tabular}




\begin{tabular}{|c|c|}
\hline $\mathcal{A}$ & $\mathcal{X}$ \\
\hline \hline Distributive lattices & Bounded posets \\
\hline Boolean algebras & Sets \\
\hline Median algebras & Strongly complemented bounded posets \\
\hline Boolean rings & Pointed sets \\
\hline Boolean lattices & Doubly pointed sets \\
\hline Boolean groups & Boolean groups \\
\hline Semilattices & Bounded semilattices \\
\hline
\end{tabular}

Table 4.1.: Categories $\mathcal{A}$ and $\boldsymbol{X}$ of finite objects such that the clones of operations in $\mathcal{A}$ are dual to the clones of dual operations in $x$

\subsubsection{Summary of Examples}

Table 4.1 summarizes the results of the last seven subsections.

Of course, the dualities also work the other way around: Each clone of operations over some finite bounded poset is dual to a clone of dual operations over some finite distributive lattice, each clone over a finite set is dual to a clone of dual operations over some finite Boolean algebra, and so on.

The examples discussed in this chapter illustrate that it can be very promising to investigate clones by dualizing them. For instance, intuition suggests that it should be easier to study clones of dual operations over bounded posets than to study clones of operations over distributive lattices. However, the question arises whether we lose the opportunity to apply certain techniques from the field of clone theory when we dualize clones. Evidently, we can dualize all techniques that rely on purely category-theoretic properties. But what about other powerful tools such as the Galois connection Pol-Inv?

In the next chapter, we will generalize Pol-Inv such that it works for clones over objects (even if the underlying category is abstract), and we will show that it can be dualized as well. 


\section{Generalizing and Dualizing Pol-Inv}

As discussed in Section 1.2, we have a Galois connection Pol-Inv that we can apply to operations over $\mathbf{A}$ and relations on $\mathbf{A}$ as long as we are in the category of sets. However, we do not have such a Galois theory if $\mathcal{A}$ is another category. It is therefore a natural wish to generalize relations, the notion of preserving and the Galois connection Pol-Inv to categories. Of course, we want the generalized Galois connection to behave like Pol-Inv does in the category of sets. That is, we want that the Galois closed classes are exactly the local closures of clones of operations and the local closures of some notion that generalizes clones of relations.

For two reasons, this generalization is useful even for those only interested in clones over sets. On the one hand, it allows us to treat clones over sets abstractly while still having a tool analogue to Pol-Inv. On the other hand, although we can still use the usual Pol-Inv as long as the operations are functions and the products Cartesian, we lose the opportunity of applying Pol-Inv as soon as we dualize the clones. A general Galois theory based on purely category-theoretic properties could be dualized with the clones and would therefore still be applicable.

In this chapter, we will introduce such a Galois connection, namely $\operatorname{Pol}_{\mathbf{A}}-\operatorname{Inv}_{\mathbf{A}}^{\mathbb{T}}$, between sets of operations over some object $\mathbf{A}$ and classes of what we will define as relations on A. Furthermore, we will introduce a Galois connection $\overline{\operatorname{Pol}}_{\mathbf{X}}-\overline{\operatorname{Inv}}_{\mathbf{X}}^{\mathbb{T}}$ between sets of dual operations and classes of what we will define as dual relations. Both Galois connections will have the properties that the names suggest. Indeed, their Galois closed classes will be local closures of clones of (dual) operations and local closures of clones of (dual) relations, where the local closure operators can be omitted in certain cases that generalize the corresponding cases in the usual scenario. Furthermore, we will show how the two Galois connections correspond to the duality for clones. Indeed, we will see that there is a similar duality for the clones of relations, called $(-)^{\partial^{*}}$, such that $\operatorname{Pol}_{\mathbf{A}}, \operatorname{Inv}_{\mathbf{A}}^{\mathbb{T}}$, $\overline{\mathrm{Pol}}_{\mathbf{X}}, \overline{\operatorname{Inv}}_{\mathbf{X}}^{\mathbb{T}},(-)^{\partial}$, and $(-)^{\partial^{*}}$ commute on the Galois closed classes.

In the subsequent chapters, we will take advantage of this framework when we apply the duality theory to clones over sets. It will turn out that having relational counterparts on the dual side (that is, after we apply $(-)^{\partial}$ to a clone) is of major help. In particular, the proofs of Proposition 7.5.5 (page 142), Theorem 8.3.20 (page 159) and Lemma 8.7.3 (page 172 are illustrations of how to apply $\overline{\mathrm{Pol}}_{\mathbf{X}}-\overline{\mathrm{Inv}}_{\mathbf{X}}$ in the everyday work of a clone theorist. 


\subsection{A General Galois Theory for Operations and Relations in Categories}

As usual, let $\mathcal{A}$ be a category with an object $\mathbf{A}$ such that all finite non-empty powers of A are also in $\mathcal{A}$.

\subsubsection{Generalized Relations}

To understand the idea of our approach, let us note that one can interpret relations in the usual sense as sets of mappings. If we do so, we can say that $\sigma$ is a $k$-ary relation on the set $A$ if $\sigma$ is a subset of $A^{\{1, \ldots, k\}}$. Thus, a relation on $A$ is nothing else but a set of morphisms from the object $\{1, \ldots, k\}$ to the object $A$ in the category of sets. This is precisely the view on relations that we will now use to generalize relations on sets to relations on the object $\mathbf{A}$ : Analogue to defining $k$-ary relations to be sets of mappings from the set $\{1, \ldots, k\}$ to the set $\mathbf{A}$, we will define a relation of type $\mathbf{B} \in \mathcal{A}$ to be a set of morphisms from the object $\mathbf{B}$ to the object $\mathbf{A}$.

5.1.1 Definition. Let $\mathbf{B} \in \mathcal{A}$. A relation of type $\mathbf{B}$ on $\mathbf{A}$ is a subset of $\mathcal{A}(\mathbf{B}, \mathbf{A})=\mathbf{A}^{\mathbf{B}}$. Denote the class of all relations of type $\mathbf{B}$ on $\mathbf{A}$ by $\mathrm{R}_{\mathbf{A}}^{(\mathbf{B})}$.

We will now define the notion of invariant relations on $\mathbf{A}$ by generalizing the usual notion of invariant relations. Recall that an $n$-ary function $f$ on a set $A$ is said to preserve a $k$-ary relation $\sigma$ if

$$
\left(\begin{array}{c}
\nu_{11} \\
\nu_{12} \\
\vdots \\
\nu_{1 k}
\end{array}\right), \ldots,\left(\begin{array}{c}
\nu_{n 1} \\
\nu_{n 2} \\
\vdots \\
\nu_{n k}
\end{array}\right) \in \sigma \Longrightarrow\left(\begin{array}{c}
f\left(\nu_{11}, \nu_{21}, \ldots, \nu_{n 1}\right) \\
f\left(\nu_{12}, \nu_{22}, \ldots, \nu_{n 2}\right) \\
\vdots \\
f\left(\nu_{1 k}, \nu_{2 k}, \ldots, \nu_{n k}\right)
\end{array}\right) \in \sigma .
$$

If we interpret the relation $\sigma$ as a set of morphisms from $\{1, \ldots, k\}$ to $A$ in the category of sets, then we can express the condition of preserving by using the tupling:

$$
f \triangleright \sigma \Longleftrightarrow f \circ\left\langle r_{1}, \ldots, r_{n}\right\rangle \in \sigma \text { for all } r_{1}, \ldots, r_{n} \in \sigma .
$$

Since this notion of preserving relies on purely category-theoretic properties, we can lift it to other categories.

5.1.2 Definition. Let $\sigma$ be a relation of type $\mathbf{B}$ on $\mathbf{A}$ and let $f \in O_{\mathbf{A}}^{(n)}$. Say that $\sigma$ is invariant for $f$ or that $f$ preserves $\sigma$, written $f \triangleright \sigma$, if

$$
f \circ\left\langle r_{1}, \ldots, r_{n}\right\rangle \in \sigma
$$

whenever $r_{1}, \ldots, r_{n} \in \sigma$. Furthermore, we say that a set of operations $F \subseteq O_{\text {A }}$ preserves $\sigma$, written $F \triangleright \sigma$, if every $f \in F$ preserves $\sigma$.

Clearly, for $\mathcal{A}$ being the category of sets and $\mathbf{B}=\{1, \ldots, k\}$, this notion coincides with the usual notion of $f$ preserving a $k$-ary relation.

Note that the projection morphisms preserve any relation on $\mathbf{A}$. 
5.1.3 Definition. For $F \subseteq O_{\mathbf{A}}$ and $\sigma \in \mathrm{R}_{\mathbf{A}}^{(\mathbf{B})}$, define

$$
\Gamma_{F}(\sigma):=\bigcap\left\{\sigma^{\prime} \in \mathrm{R}_{\mathbf{A}}^{(\mathbf{B})} \mid \sigma \subseteq \sigma^{\prime}, F \triangleright \sigma^{\prime}\right\} .
$$

It is easy to see that the intersection of relations preserved by some $F \subseteq O_{\mathbf{A}}$ is again preserved by $F$. Furthermore, the full relation $\mathbf{A}^{\mathbf{B}}$ is invariant for each set of operations over A. Thus, for each $F \subseteq O_{\mathbf{A}}$ and each relation $\sigma \in \mathrm{R}_{\mathbf{A}}^{(\mathbf{B})}, \Gamma_{F}(\sigma)$ is the least relation on $\mathbf{A}$ of type $\mathbf{B}$ that is preserved by $F$ and contains $\sigma$.

We will now show that the superposition of operations preserves $\sigma$ if each operation in the superposition preserves $\sigma$.

5.1.4 Proposition. Let $f_{1}, \ldots, f_{n} \in O_{\mathbf{A}}^{(k)}, f \in O_{\mathbf{A}}^{(n)}$ and let $\sigma \in \mathrm{R}_{\mathbf{A}}^{(\mathbf{B})}$. Then

$$
f, f_{1}, \ldots, f_{n} \triangleright \sigma \Longrightarrow f \circ\left\langle f_{1}, \ldots, f_{n}\right\rangle \triangleright \sigma .
$$

Proof. Let $f_{1}, \ldots, f_{n} \in O_{\mathbf{A}}^{(k)}$ and $f \in O_{\mathbf{A}}^{(n)}$ preserve $\sigma$. For $r_{1}, \ldots, r_{n} \in \sigma$, we have

$$
f \circ\left\langle f_{1}, \ldots, f_{n}\right\rangle \circ\left\langle r_{1}, \ldots, r_{k}\right\rangle=f \circ\langle\underbrace{f_{1} \circ\left\langle r_{1}, \ldots, r_{k}\right\rangle}_{\in \sigma}, \ldots, \underbrace{f_{n} \circ\left\langle r_{1}, \ldots, r_{k}\right\rangle}_{\in \sigma}\rangle \in \sigma .
$$

Hence, $f \circ\left\langle f_{1}, \ldots, f_{n}\right\rangle$ preserves $\sigma$.

The following corollary is an almost trivial consequence, but it is important as it provides us with a very efficient technique to show that a given operation cannot generate another given operation:

5.1.5 Corollary. Let $f, f^{\prime} \in O_{\mathbf{A}}$ and let $\sigma \in \mathrm{R}_{\mathbf{A}}^{(\mathbf{B})}$. If $f \triangleright \sigma$ but $f^{\prime} \not \sigma$, then $f^{\prime} \notin \operatorname{Clo}(f)$.

Proof. Assume $f^{\prime} \in \operatorname{Clo}(f)$, that is, $f^{\prime}$ is a superposition of $f$ and the projection morphisms. Since $f$ and the projection morphisms preserve $\sigma$, it follows by Proposition 5.1.4 that we also have $f^{\prime} \triangleright \sigma$, a contradiction.

Now, we want to define clones of relations on $\mathbf{A}$ analogue to the situation in the category of sets. In [Pös79], it was observed that a clone of relations in the usual sense can be expressed as follows if we take the point of view we described above, namely to think of $k$-ary relations as sets of mappings from $\{1, \ldots, k\}$ to $A$ :

5.1.6 Proposition ([Pös79]). Let $R$ be a set of (finitary) relations on a set $A$ where each $\sigma \in R^{(k)}$ is interpreted as a set of mappings from $\{1, \ldots, k\}$ to $A$. Then, $R$ is a clone of relations on $A$ if and only if

(i) $\emptyset \in R$,

(ii) $R$ is closed under general superposition, that is, the following holds: Let $I$ be an index set, let $\sigma_{i} \in R^{\left(k_{i}\right)}(i \in I)$ and let $\varphi:\{1, \ldots, k\} \rightarrow \alpha$ and $\varphi_{i}:\left\{1, \ldots, k_{i}\right\} \rightarrow \alpha$ be mappings where $\alpha$ is some cardinal number. Then, the relation $\bigwedge_{\left(\varphi_{i}\right)_{i \in I}}^{\varphi}\left(\sigma_{i}\right)_{i \in I}$ defined by

$$
\bigwedge_{\left(\varphi_{i}\right)_{i \in I}}^{\varphi}\left(\sigma_{i}\right)_{i \in I}:=\left\{r \circ \varphi \mid \forall i \in I: r \circ \varphi_{i} \in \sigma_{i}, r \in A^{\alpha}\right\}
$$

belongs to $R$. 
To transfer this definition to our general environment, we introduce the notion of a typeclass.

5.1.7 Definition. A typeclass is a non-empty subclass $\mathbb{T} \subseteq \mathcal{A}$ in which any two different objects are non-isomorphic.

In other words, a typeclass is a non-empty subclass of a skeleton.

\subsubsection{Examples.}

(i) Each skeleton is a typeclass.

(ii) If $\mathcal{A}=\operatorname{Set}$, the set $\mathbb{T}:=\left\{\{1, \ldots, k\} \mid k \in \mathbb{N}_{+}\right\}$is a typeclass.

(iii) If $\mathcal{A}$ is the category of finite distributive lattices, then

$$
\begin{aligned}
& \mathbb{T}_{1}:=\left\{\langle\{1, \ldots, k\}, \max , \min \rangle \mid k \in \mathbb{N}_{+}\right\}, \\
& \mathbb{T}_{2}:=\left\{\langle\mathfrak{P}(\{1, \ldots, k\}), \cup, \cap\rangle \mid k \in \mathbb{N}_{+}\right\}
\end{aligned}
$$

are two typeclasses.

5.1.9 Definition. For a typeclass $\mathbb{T}$,

$$
\mathrm{R}_{\mathbf{A}}^{\mathbb{T}}:=\bigcup_{\mathbf{B} \in \mathbb{T}} \mathrm{R}_{\mathbf{A}}^{(\mathbf{B})}
$$

is called the class of relations of the typeclass $\mathbb{T}$ on $\mathbf{A}$.

For a class of relations $R \subseteq \mathrm{R}_{\mathbf{A}}^{\mathbb{T}}$ and $\mathbf{B} \in \mathbb{T}$, we write $R^{(\mathbf{B})}$ to indicate $R \cap \mathrm{R}_{\mathbf{A}}^{(\mathbf{B})}$.

Note that we have $\emptyset \in R_{\mathbf{A}}^{\mathbb{T}}$ since $\emptyset$ is a relation of type $\mathbf{B}$ for all $\mathbf{B} \in \mathbb{T}$ and $\mathbb{T}$ is non-empty by definition.

5.1.10 Examples. Let $\mathcal{A}$ be the category of sets.

(i) If we choose $\mathbb{T}:=\left\{\{1, \ldots, k\} \mid k \in \mathbb{N}_{+}\right\}$, then $\mathrm{R}_{\mathbf{A}}^{\mathbb{T}}$ coincides with the set of finitary relations in the usual sense.

(ii) If we choose $\mathbb{T}$ to be the class of all cardinal numbers (written as sets), then $\mathrm{R}_{\mathbf{A}}^{\mathbb{T}}$ coincides with the set of (possibly infinitary) relations in the usual sense.

We are now ready to define the notion of a clone of relations on $\mathbf{A}$ by generalizing Proposition 5.1.6 in a straight-forward way.

5.1.11 Definition. A class $R \subseteq \mathrm{R}_{\mathbf{A}}^{\mathbb{T}}$ is called a clone of relations of the typeclass $\mathbb{T}$ on A, written $R \leq \mathrm{R}_{\mathbf{A}}^{\mathbb{T}}$, if

(i) $\emptyset \in R$,

(ii) $R$ is closed under general superposition, that is, the following holds: Let $I$ be an index class, let $\sigma_{i} \in R^{\left(\mathbf{B}_{i}\right)}(i \in I)$ and let $\varphi: \mathbf{B} \rightarrow \mathbf{C}$ and $\varphi_{i}: \mathbf{B}_{i} \rightarrow \mathbf{C}$ be morphisms where $\mathbf{C} \in \mathcal{A}$ and $\mathbf{B} \in \mathbb{T}$. Then, the relation $\bigwedge_{\left(\varphi_{i}\right)_{i \in I}}^{\varphi}\left(\sigma_{i}\right)_{i \in I} \in \mathrm{R}_{\mathbf{A}}^{(\mathbf{B})}$ defined by

$$
\bigwedge_{\left(\varphi_{i}\right)_{i \in I}}^{\varphi}\left(\sigma_{i}\right)_{i \in I}:=\bigwedge_{\left(\varphi_{i}\right)}^{\varphi}\left(\sigma_{i}\right):=\left\{r \circ \varphi \mid \forall i \in I: r \circ \varphi_{i} \in \sigma_{i}, r \in \mathbf{A}^{\mathbf{C}}\right\}
$$

belongs to $R$. 
Note that it suffices to check the second condition for all $\mathbf{C}$ in some skeleton of $\mathcal{A}$. In fact, if such objects exist, it is enough to consider those $\mathbf{C}$ in a skeleton that are maximal with respect to $\leqslant$ (recall that we write $\mathbf{C}_{1} \leqslant \mathbf{C}_{2}$ if there exists an epimorphism from $\mathbf{C}_{2}$ to $\mathbf{C}_{1}$ ).

5.1.12 Example. Let $\mathcal{A}$ be the category of sets and let $\mathbf{A} \in \mathcal{A}$. If we choose $\mathbb{T}$ as in case (i) of Example 5.1.10, our notion of a clone of relations coincides with the usual notion of a clone of finitary relations (see Proposition 5.1.6. If we choose $\mathbb{T}$ as in case (ii), our notion coincides with the usual notion of a clone of (possibly infinitary) relations Ros72.

For a given typeclass $\mathbb{T}$, it is obvious that $\mathrm{R}_{\mathbf{A}}^{\mathbb{T}}$ is a clone of relations. Furthermore, it is easy to see that the intersection of clones of relations is again a clone of relations. Thus, for $R \subseteq \mathrm{R}_{\mathbf{A}}^{\mathbb{T}}$, there exists a clone of relations that is the least clone which contains $R$.

5.1.13 Definition. Denote by $\mathrm{Clo}^{\mathbb{T}}: \mathfrak{P}\left(\mathrm{R}_{\mathbf{A}}^{\mathbb{T}}\right) \rightarrow \mathfrak{P}\left(\mathrm{R}_{\mathbf{A}}^{\mathbb{T}}\right)$ the operator that maps each $R \subseteq \mathrm{R}_{\mathbf{A}}^{\mathbb{T}}$ to the least clone of relations that contains $R$. Say that $\mathrm{Clo}^{\mathbb{T}}(R)$ is the clone of relations generated by $R$.

Clearly, $\mathrm{R}_{\mathbf{A}}^{\mathbb{T}}$ is the greatest clone of relations on $\mathbf{A}$, whereas the least clone of relations on $\mathbf{A}$ is $\mathrm{Clo}^{\mathbb{T}}(\emptyset)$. Hence, for a given typeclass $\mathbb{T}$, the clones of relations on $\mathbf{A}$ form a complete lattice with respect to inclusion.

5.1.14 Definition. Denote by $L_{\mathbf{A}}^{* \mathbb{T}}$ the class of clones of relations of the typeclass $\mathbb{T}$ on A. Then, $\mathcal{L}_{\mathbf{A}}^{* \mathbb{T}}:=\left\langle L_{\mathbf{A}}^{* \mathbb{T}}, \subseteq\right\rangle$ is called the lattice of clones of relations of the typeclass $\mathbb{T}$ on $\mathbf{A}$.

\subsubsection{The Generalized Galois Connection $\operatorname{Pol}_{\mathbf{A}}-\operatorname{Inv}_{\mathbf{A}}^{\mathbb{T}}$}

Until the end of this section, let $\mathbb{T}$ be a typeclass of $\mathcal{A}$.

5.1.15 Definition. We define the two operators $\operatorname{Inv}_{\mathbf{A}}^{\mathbb{T}}: \mathfrak{P}\left(O_{\mathbf{A}}\right) \rightarrow \mathfrak{P}\left(\mathrm{R}_{\mathbf{A}}^{\mathbb{T}}\right)$ and $\mathrm{Pol}_{\mathbf{A}}: \mathfrak{P}\left(\mathrm{R}_{\mathbf{A}}^{\mathbb{T}}\right) \rightarrow \mathfrak{P}\left(O_{\mathbf{A}}\right)$ as follows: For $F \subseteq O_{\mathbf{A}}$ and $R \subseteq \mathrm{R}_{\mathbf{A}}^{\mathbb{T}}$, set

$$
\begin{aligned}
& \operatorname{Inv}_{\mathbf{A}}^{\mathbb{T}} F:=\left\{\sigma \in \mathrm{R}_{\mathbf{A}}^{\mathbb{T}} \mid \forall f \in F: f \triangleright \sigma\right\}, \\
& \operatorname{Pol}_{\mathbf{A}} R:=\left\{f \in O_{\mathbf{A}} \mid \forall \sigma \in R: f \triangleright \sigma\right\} .
\end{aligned}
$$

5.1.16 Proposition. The operators $\mathrm{Pol}_{\mathbf{A}}$ and $\operatorname{Inv}_{\mathbf{A}}^{\mathbb{T}}$ constitute a Galois connection between the subsets of $O_{\mathbf{A}}$ and the subclasses of $\mathrm{R}_{\mathbf{A}}^{\mathbb{T}}$.

5.1.17 Definition. For $F \subseteq O_{\mathbf{A}}, R \subseteq \mathrm{R}_{\mathbf{A}}^{\mathbb{T}}, \mathbf{B} \in \mathcal{A}$ and $n \in \mathbb{N}_{+}$, we use the following notations:

$$
\begin{aligned}
& \operatorname{Inv}_{\mathbf{A}}^{(\mathbf{B})} F:=\left\{\sigma \in \mathrm{R}_{\mathbf{A}}^{(\mathbf{B})} \mid \forall f \in F: f \triangleright \sigma\right\}, \\
& \operatorname{Pol}_{\mathbf{A}}^{(n)} R:=\operatorname{Pol}_{\mathbf{A}} R \cap O_{\mathbf{A}}^{(n)} .
\end{aligned}
$$


Note that $\operatorname{Pol}_{\mathbf{A}} R$ and $\operatorname{Inv}_{\mathbf{A}}^{(\mathbf{B})} F$ are always sets, while $\operatorname{Inv}_{\mathbf{A}}^{\mathbb{T}} F$ can be a proper class.

\subsubsection{Examples.}

(i) Let $\sigma:=\left\{i d_{\mathbf{A}}\right\}$. Then, $\operatorname{Pol}_{\mathbf{A}}\{\sigma\}$ is the set of all idempotent operations over $\mathbf{A}$.

(ii) Let $C \leq O_{\mathbf{A}}$. Note that $C^{(n)}$ is a relation of type $\mathbf{A}^{n}$. Now, $\mathrm{Pol}_{\mathbf{A}} C^{(n)}$ is the largest clone $C^{\prime}$ that agrees with $C$ on its $n$-ary part (i.e., $\left.\mathrm{Pol}_{\mathbf{A}}^{(n)} C^{(n)}=C^{(n)}\right)$.

(iii) If two operations $f, f^{\prime}$ are essentially the same, then $\operatorname{Inv}_{\mathbf{A}}^{\mathbb{T}}\{f\}=\operatorname{Inv}_{\mathbf{A}}^{\mathbb{T}}\left\{f^{\prime}\right\}$.

(iv) Let $\mathcal{A}$ be the category of finite distributive lattices and let $\mathbf{B} \in \mathcal{A}$. Let $\sigma \in \mathrm{R}_{\mathbf{A}}^{(\mathbf{B})}$ contain all morphisms $r: \mathbf{B} \rightarrow \mathbf{A}$ with $r\left(0^{\mathbf{B}}\right)=0^{\mathbf{A}}$ and $r\left(1^{\mathbf{B}}\right)=1^{\mathbf{A}}$. Then, $\operatorname{Pol}_{\mathbf{A}}\{\sigma\}$ is the set of all 01-homomorphisms over $\mathbf{A}$, that is, all operations over $\mathbf{A}$ that preserve the bottom and the top of the lattice.

(v) Again, let $\mathcal{A}$ be the category of finite distributive lattices. Let $\mathbf{B}$ be the (up to isomorphism) unique two-element distributive lattice $\langle\{0,1\}, \vee, \wedge\rangle$. Let $\sigma$ be the relation that contains each morphism $r: \mathbf{B} \rightarrow \mathbf{A}$ with $r(0) \neq r(1)$. Now, we have $f \in \operatorname{Pol}_{\mathbf{A}}\{\sigma\}$ if and only if $f\left(a_{1}, \ldots, a_{n}\right)>f\left(b_{1}, \ldots, b_{n}\right)$ whenever $a_{i}>b_{i}$ for all $i \in\{1, \ldots, n\}$ (where the order relation $>$ is obtained from the lattice in the obvious way).

Note that, in these examples, the set of polymorphisms always turned out to be a clone. This is something we know for the usual Pol-Inv, and we will shortly see that it is also true for $\mathrm{Pol}_{\mathbf{A}}-\mathrm{Inv}_{\mathbf{A}}^{\mathbb{T}}$. Indeed, we will see that we can generalize almost every definition, lemma, proposition and theorem that holds for Pol-Inv.

5.1.19 Proposition. Let $R \subseteq \mathrm{R}_{\mathbf{A}}^{\mathbb{T}}, F \subseteq O_{\mathbf{A}}, \mathbf{B}, \mathbf{C} \in \mathbb{T}$ and $s_{1}, s_{2} \in \mathbb{N}_{+}$. For $s_{1} \leq s_{2}$ and $\mathbf{B} \leqslant \mathbf{C}$, we have

(i) $\operatorname{Pol}_{\mathbf{A}} \operatorname{Inv}_{\mathbf{A}}^{(\mathbf{C})} F \subseteq \operatorname{Pol}_{\mathbf{A}} \operatorname{Inv}_{\mathbf{A}}^{(\mathbf{B})} F$,

(ii) $\operatorname{Inv}_{\mathbf{A}}^{\mathbb{T}} \operatorname{Pol}_{\mathbf{A}}^{\left(s_{2}\right)} R \subseteq \operatorname{Inv}_{\mathbf{A}}^{\mathbb{T}} \operatorname{Pol}_{\mathbf{A}}^{\left(s_{1}\right)} R$.

Proof. (i) Let $h \in \operatorname{Pol}_{\mathbf{A}} \operatorname{Inv}_{\mathbf{A}}^{(\mathbf{C})} F$ be $n$-ary and let $\sigma \in \operatorname{Inv}_{\mathbf{A}}^{(\mathbf{B})} R$. We need to show that $h$ preserves $\sigma$. Since $\mathbf{B} \leqslant \mathbf{C}$, there exists an epimorphism $e$ : $\mathbf{C} \rightarrow \mathbf{B}$. Let

$$
\sigma^{\prime}:=\{r \circ e \mid r \in \sigma\} .
$$

Note that $\sigma^{\prime}$ is a relation of type $\mathbf{C}$. First, we will show that $\sigma^{\prime}$ is preserved by $F$. Let $f \in F^{(m)}$ and let $r_{1}^{\prime}, \ldots, r_{m}^{\prime} \in \sigma^{\prime}$. Then, there exist $r_{1}, \ldots, r_{m} \in \sigma$ such that $r_{j}^{\prime}=r_{j} \circ e$ for all $j \in\{1, \ldots, m\}$. But now,

$$
f \circ\left\langle r_{1}^{\prime}, \ldots, r_{m}^{\prime}\right\rangle=f \circ\left\langle r_{1} \circ e, \ldots, r_{m} \circ e\right\rangle=\underbrace{f \circ\left\langle r_{1}, \ldots, r_{m}\right\rangle}_{\in \sigma} \circ e \in \sigma^{\prime} .
$$

Hence, $\sigma^{\prime} \in \operatorname{Inv}_{\mathbf{A}}^{(\mathbf{C})} F$, and since $h \in \operatorname{Pol}_{\mathbf{A}} \operatorname{Inv}_{\mathbf{A}}^{(\mathbf{C})} F$, it follows $h \triangleright \sigma^{\prime}$. Finally, let $r_{1}, \ldots, r_{n} \in \sigma$. We have

$$
h \circ\left\langle r_{1}, \ldots, r_{n}\right\rangle \circ e=h \circ\langle\underbrace{r_{1} \circ e}_{\in \sigma^{\prime}}, \ldots, \underbrace{r_{n} \circ e}_{\in \sigma^{\prime}}\rangle \in \sigma^{\prime} .
$$


But now $h \circ\left\langle r_{1}, \ldots, r_{n}\right\rangle \circ e \in \sigma^{\prime}$ implies that there exists $r \in \sigma$ such that

$$
h \circ\left\langle r_{1}, \ldots, r_{n}\right\rangle \circ e=r \circ e .
$$

Since $e$ is an epimorphism, this implies $h \circ\left\langle r_{1}, \ldots, r_{n}\right\rangle=r \in \sigma$, and we are done.

(ii) For $f \in \operatorname{Pol}_{\mathbf{A}}^{\left(s_{1}\right)} R$, we have $f^{\prime}:=f \circ\left\langle\pi_{1}^{s_{2}}, \ldots, \pi_{s_{1}}^{s_{2}}\right\rangle \in \mathrm{Pol}_{\mathbf{A}}^{\left(s_{2}\right)} R$. The claim now follows from the observation that a relation is preserved by $f$ if and only if it is preserved by $f^{\prime}$.

5.1.20 Definition. Let $F \subseteq O_{\mathbf{A}}, R \subseteq \mathrm{R}_{\mathbf{A}}^{\mathbb{T}}, s \geq 1$ and let $\mathbf{C} \in \mathcal{A}$. We define the following local closure operators:

$$
\begin{gathered}
\text { C-Loc } F:=\left\{f \in O_{\mathbf{A}}^{(n)} \mid n \geq 1, \forall r_{1}, \ldots, r_{n} \in \mathbf{A}^{\mathbf{C}}:\right. \\
\left.\qquad f^{\prime} \in F: f \circ\left\langle r_{1}, \ldots, r_{n}\right\rangle=f^{\prime} \circ\left\langle r_{1}, \ldots, r_{n}\right\rangle\right\}, \\
\text { s-LOC }^{\mathbb{T}} R:=\left\{\sigma \in \mathrm{R}_{\mathbf{A}}^{\mathbb{T}}|\forall B \subseteq \sigma,| B \mid \leq s: \exists \sigma^{\prime} \in R: B \subseteq \sigma^{\prime} \subseteq \sigma\right\} .
\end{gathered}
$$

Furthermore, let

$$
\operatorname{Loc}^{\mathbb{T}} F:=\bigcap_{\mathbf{C} \in \mathbb{T}} \mathbf{C}-\operatorname{Loc} F
$$

and

$$
\mathrm{LOC}^{\mathbb{T}} R:=\bigcap_{s \in \mathbb{N}_{+}} \mathrm{s}^{-\mathrm{LOC}^{\mathbb{T}}} R
$$

In other words, C-Loc $F$ is the set of all operations $f \in O_{\mathbf{A}}$ such that, for all tuplings $\left\langle r_{1}, \ldots, r_{n}\right\rangle$ of morphism from $\mathbf{C}$ to $\mathbf{A}$, there exists an operation $f^{\prime} \in F$ such that $f$ and $f^{\prime}$ cannot be distinguished if they are applied after $\left\langle r_{1}, \ldots, r_{n}\right\rangle$. Moreover, s-LOC $R$ is the class of all relations $\sigma \in \mathrm{R}_{\mathbf{A}}^{\mathbb{T}}$ such that, for every $B \subseteq \sigma$ with at most $s$ elements, there exists a member $\sigma^{\prime}$ of $R$ that agrees with $\sigma$ on $B$ and is contained in $\sigma$.

We will see later that $\operatorname{Loc}^{\mathbb{T}} C$ is a clone of operations whenever $C$ is a clone of operations (Theorem 5.1.33, page 88). Similarly, we will see that $\mathrm{LOC}^{\mathbb{T}} R$ is a clone of relations whenever $R$ is a clone of relations (Theorem 5.1.35. page 89.

5.1.21 Proposition. If $\mathbf{C}_{1} \leqslant \mathbf{C}_{2}$, then $\mathbf{C}_{2}$-Loc $F \subseteq \mathbf{C}_{1}$-Loc $F$ for all $F \subseteq O_{\mathbf{A}}$.

Proof. Let $f \in \mathbf{C}_{2}$-Loc $F$ be $n$-ary and let $r_{1}, \ldots, r_{n} \in \mathbf{A}^{\mathbf{C}_{1}}$. We need to show that there exists $f^{\prime} \in F$ such that $f \circ\left\langle r_{1}, \ldots, r_{n}\right\rangle=f^{\prime} \circ\left\langle r_{1}, \ldots, r_{n}\right\rangle$. Since $\mathbf{C}_{1} \leqslant \mathbf{C}_{2}$, there exists an epimorphism $e: \mathbf{C}_{2} \rightarrow \mathbf{C}_{1}$. For $i \in\{1, \ldots, n\}$, let $r_{i}^{\prime}:=r_{i} \circ e$. Since $r_{i}^{\prime} \in \mathbf{A}^{\mathbf{C}_{2}}$ and $f \in \mathbf{C}_{2}$-Loc $F$, there exists $f^{\prime} \in F$ such that

$$
\begin{aligned}
f \circ\left\langle r_{1}, \ldots, r_{n}\right\rangle \circ e & =f \circ\left\langle r_{1} \circ e, \ldots, r_{n} \circ e\right\rangle \\
& =f \circ\left\langle r_{1}^{\prime}, \ldots, r_{n}^{\prime}\right\rangle \\
& =f^{\prime} \circ\left\langle r_{1}^{\prime}, \ldots, r_{n}^{\prime}\right\rangle \\
& =f^{\prime} \circ\left\langle r_{1}, \ldots, r_{n}\right\rangle \circ e .
\end{aligned}
$$

But now, since $e$ is an epimorphism, this implies $f \circ\left\langle r_{1}, \ldots, r_{n}\right\rangle=f^{\prime} \circ\left\langle r_{1}, \ldots, r_{n}\right\rangle$. Thus, $f \in \mathbf{C}_{1}$-Loc $F$. 
5.1.22 Lemma. Let $n \in \mathbb{N}_{+}$and let $F \subseteq O_{\mathbf{A}}$. If we have $\mathbf{A}^{n} \leqslant \mathbf{B}$ for some $\mathbf{B} \in \mathbb{T}$, then $\left(\operatorname{Loc}^{\mathbb{T}} F\right)^{(n)}=F^{(n)}$ (that is, Loc ${ }^{\mathbb{T}} F$ and $F$ agree on their n-ary part).

Proof. $F^{(n)} \subseteq\left(\operatorname{Loc}^{\mathbb{T}} F\right)^{(n)}$ is obvious. To show $\left(\operatorname{Loc}^{\mathbb{T}} F\right)^{(n)} \subseteq F^{(n)}$, let $f \in O_{\mathbf{A}}^{(n)}$ belong to $\operatorname{Loc}^{\mathbb{T}} F$. By assumption, there exists $\mathbf{B} \in \mathbb{T}$ with $\mathbf{A}^{n} \leqslant \mathbf{B}$. Let $e: \mathbf{B} \rightarrow \mathbf{A}^{n}$ be an epimorphism. Since $f \in \operatorname{Loc}^{\mathbb{T}} F$, there exists $f^{\prime} \in F^{(n)}$ such that

$$
\begin{aligned}
f \circ e & =f \circ\left\langle\pi_{1}^{n}, \ldots, \pi_{n}^{n}\right\rangle \circ e \\
& =f \circ\left\langle\pi_{1}^{n} \circ e, \ldots, \pi_{n}^{n} \circ e\right\rangle \\
& =f^{\prime} \circ\left\langle\pi_{1}^{n} \circ e, \ldots, \pi_{n}^{n} \circ e\right\rangle \\
& =f^{\prime} \circ\left\langle\pi_{1}^{n}, \ldots, \pi_{n}^{n}\right\rangle \circ e \\
& =f^{\prime} \circ e .
\end{aligned}
$$

Since $e$ is an epimorphism, it follows $f=f^{\prime}$, which establishes $\left(\operatorname{Loc}^{\mathbb{T}} F\right)^{(n)}=F^{(n)}$.

Evidently, this implies $\operatorname{Loc}^{\mathbb{T}} F=F$ for all $F \subseteq O_{\mathbf{A}}$ if, for each $n \in \mathbb{N}_{+}$, there exists an epimorphism from some $\mathbf{B} \in \mathbb{T}$ to $\mathbf{A}^{n}$. Furthermore, if we are only interested in the local closures of the clones, a weaker condition is sufficient.

5.1.23 Corollary. If one of the following two conditions hold, then we have $\operatorname{Loc}^{\mathbb{T}} C=C$ for all $C \leq O_{\mathbf{A}}$ :

(i) For each $k \in \mathbb{N}_{+}$, there exists $n \geq k$ such that $\mathbf{A}^{n} \leqslant \mathbf{B}$ for some $\mathbf{B} \in \mathbb{T}$.

(ii) Each $f \in O_{\mathbf{A}}$ is essentially at most $n$-ary and $\mathbf{A}^{n} \leqslant \mathbf{B}$ for some $\mathbf{B} \in \mathbb{T}$.

Proof. (i) We only need to show $\operatorname{Loc}^{\mathbb{T}} C \subseteq C$. Let $k \in \mathbb{N}_{+}$and let $f \in\left(\operatorname{Loc}^{\mathbb{T}} C\right)^{(k)}$. By assumption, there exists $n \geq k$ such that $\mathbf{A}^{n} \leqslant \mathbf{B}$ for some $\mathbf{B} \in \mathbb{T}$. Let $f^{\prime}$ arise from $f$ by adding $n-k$ nonessential arguments. Clearly, $f^{\prime} \in \operatorname{Loc}^{\mathbb{T}} C$ and we can apply the last lemma to obtain $f^{\prime} \in C$, which implies $f \in C$.

(ii) As we have remarked above, $C$ and $\operatorname{Loc}^{\mathbb{T}} C$ are both clones over A. By Lemma 5.1.22, they coincide on their $n$-ary parts. Since each operation among $O_{\mathbf{A}}$ is essentially at most $n$-ary, this means $C=\operatorname{Loc}^{\mathbb{T}} C$.

For relations, the following statement is obvious:

5.1.24 Proposition. Let $R \subseteq \mathrm{R}_{\mathbf{A}}^{\mathbb{T}}$. If all relations in $R$ are finite, then $\mathrm{LOC}^{\mathbb{T}} R=R$.

Thus, we have $\mathrm{LOC}^{\mathbb{T}} R=R$ for all $R \subseteq \mathrm{R}_{\mathrm{A}}^{\mathbb{T}}$ if there are only finitely many morphisms from $\mathbf{B}$ to $\mathbf{A}$ for each $\mathbf{B} \in \mathbb{T}$. The following lemma shows that this is also a necessary condition:

5.1.25 Lemma. We have $\mathrm{LOC}^{\mathbb{T}} R=R$ for all $R \subseteq \mathrm{R}_{\mathbf{A}}^{\mathbb{T}}$ if and only if $\mathcal{A}(\mathbf{B}, \mathbf{A})$ is finite for all $\mathbf{B} \in \mathbb{T}$. 


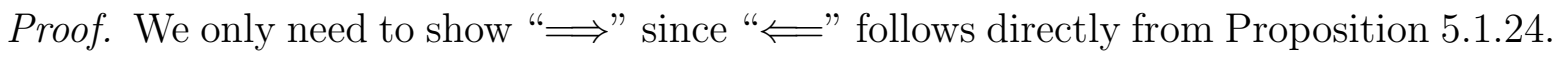
Let $\mathbf{B} \in \mathbb{T}$ such that $|\mathcal{A}(\mathbf{B}, \mathbf{A})|=\infty$. Define

$$
R:=\bigcup_{s \in \mathbb{N}_{+}}\left\{\sigma \in \mathrm{R}_{\mathbf{A}}^{(\mathbf{B})}|| \sigma \mid \leq s\right\} .
$$

Now, let $\sigma$ be the full relation $\mathcal{A}(\mathbf{B}, \mathbf{A})$ (or any other infinite relation of type $\mathbf{B}$ ). Clearly, we have $\sigma \notin R$. However, for each $s \in \mathbb{N}_{+}$and $B \subseteq \sigma$ with $|B| \leq s$, we have $B \in R$. Hence, for $\sigma^{\prime}:=B$, we obtain $B \subseteq \sigma^{\prime} \subseteq \sigma$. Thus, $\sigma \in \mathrm{LOC}^{\mathbb{T}} R$.

The following examples show that Lemma 5.1 .22 and Lemma 5.1 .25 generalize an observation for the local closures of sets of operations over sets and sets of relations in the usual sense:

\subsubsection{Examples.}

(i) If $\mathcal{A}=$ Set and $\mathbb{T}:=\left\{\{1, \ldots, k\} \mid k \in \mathbb{N}_{+}\right\}$, then Lemma 5.1.25 establishes that we have $\mathrm{LOC}^{\mathbb{T}} R=R$ for all $R \subseteq \mathrm{R}_{\mathbf{A}}^{\mathbb{T}}$ if and only if $\mathbf{A}$ is a finite set. Furthermore, Lemma 5.1 .22 yields that $\mathbf{A}$ being a finite set implies $\operatorname{Loc}^{\mathbb{T}} F=F$ for all $F \subseteq O_{\mathbf{A}}$. An easy proof shows that the other direction is also true. Thus, both local closure operators can be dismissed if and only if $\mathbf{A}$ is a finite set.

(ii) If $\mathbb{T}$ is a skeleton of $\mathcal{A}$, then Lemma 5.1 .22 establishes $\operatorname{Loc}^{\mathbb{T}} F=F$ for all $\mathbf{A} \in \mathcal{A}$ and $F \subseteq O_{\mathbf{A}}$.

(iii) If $\mathcal{A}$ is the category of finite distributive lattices and we define the typeclass by setting $\mathbb{T}:=\left\{\langle\mathfrak{P}(\{1, \ldots, k\}), \cup, \cap\rangle \mid k \in \mathbb{N}_{+}\right\}$, then Lemma 5.1 .25 yields LOC $^{\mathbb{T}} R=R$ for all $\mathbf{A} \in \mathcal{A}$ and $R \subseteq \mathrm{R}_{\mathbf{A}}^{\mathbb{T}}$. Moreover, Lemma 5.1 .22 establishes $\operatorname{Loc}^{\mathbb{T}} F=F$ for all $F \subseteq O_{\mathbf{A}}$ whenever $\mathbf{A}$ is a Boolean lattice. It is possible (but not very easy) to give a direct proof that $\mathbf{A}$ being a Boolean lattice is, in fact, equivalent to having $\operatorname{Loc}^{\mathbb{T}} F=F$ for all $F \subseteq O_{\mathbf{A}}$. However, we will see in Example 5.2.17 (page 97) that this is one of the statements that are much easier to solve after dualizing them.

As we will see in Section 5.1.4 case (i) can be generalized to a result for $\operatorname{Loc}^{\mathbb{T}}$ and LOC $^{\mathbb{T}}$ in concrete categories. Case (ii) implies that we can always choose $\mathbb{T}$ such that we have $\operatorname{Loc}^{\mathbb{T}} F=F$ for all $F \subseteq O_{\mathbf{A}}$ (i.e., $\operatorname{Loc}^{\mathbb{T}}$ becomes obsolete). However, we can not necessarily choose $\mathbb{T}$ such that we have $\operatorname{LOC}^{\mathbb{T}} R=R$ for all $R \subseteq \mathrm{R}_{\mathbf{A}}^{\mathbb{T}}$. This somewhat unsymmetrical behaviour could be avoided by allowing operations of infinite arity, that is, we had to define $O_{\mathbf{A}}$ to be the class of morphisms from any power of $\mathbf{A}$ to $\mathbf{A}$. However, we are only interested in clones (where the arity of operations is finite), so we will not elaborate this point.

Now, we will show that many lemmas that hold for Pol-Inv hold almost verbatim for $\mathrm{Pol}_{\mathbf{A}}-\operatorname{Inv}_{\mathbf{A}}^{\mathbb{T}}$.

5.1.27 Lemma. Let $R \subseteq \mathrm{R}_{\mathbf{A}}^{\mathbb{T}}$ and $F \subseteq O_{\mathbf{A}}$. Then, $\operatorname{Pol}_{\mathbf{A}} R$ and $\operatorname{Inv}_{\mathbf{A}}^{\mathbb{T}} F$ are a clone of operations and a clone of relations, respectively. That is, we have

(i) $\mathrm{Clo}\left(\mathrm{Pol}_{\mathbf{A}} R\right)=\mathrm{Pol}_{\mathbf{A}} R$, 
(ii) $\operatorname{Clo}^{\mathbb{T}}\left(\operatorname{Inv}_{\mathbf{A}}^{\mathbb{T}} F\right)=\operatorname{Inv}_{\mathbf{A}}^{\mathbb{T}} F$.

Proof. (i) Let $\sigma \in R$. Since the projection morphisms preserve $\sigma$ and the superposition of operations preserving $\sigma$ also preserves $\sigma$ (see Proposition 5.1.4, page 79), $\mathrm{Pol}_{\mathbf{A}} R$ is a clone.

(ii) It is obvious that the empty relation $\emptyset$ is preserved by each $f \in F$. It remains to show that, for $f \in F$, the general superposition of relations preserved by $f$ is again preserved by $f$. Let $I$ be an index class, let $\sigma_{i} \in R^{\left(\mathbf{B}_{i}\right)}(i \in I)$ and let $\varphi: \mathbf{B} \rightarrow \mathbf{C}$ and $\varphi_{i}: \mathbf{B}_{i} \rightarrow \mathbf{C}$ be morphisms where $\mathbf{C} \in \mathcal{A}, \mathbf{B} \in \mathbb{T}$. Assume $s_{1}, \ldots, s_{n} \in \bigwedge_{\left(\varphi_{i}\right)}^{\varphi}\left(\sigma_{i}\right)$. Then, for each $j \in\{1, \ldots, n\}$, there exists $r_{j} \in \mathbf{A}^{\mathbf{B}}$ such that $s_{j}=r_{j} \circ \varphi$ and $r_{j} \circ \varphi_{i} \in \sigma_{i}$ for all $i \in I$. Since $f$ preserves each $\sigma_{i}$, we also have

$$
f \circ\left\langle r_{1}, \ldots, r_{n}\right\rangle \circ \varphi_{i}=f \circ\langle\underbrace{r_{1} \circ \varphi_{i}}_{\in \sigma_{i}}, \ldots, \underbrace{r_{n} \circ \varphi_{i}}_{\in \sigma_{i}}\rangle \in \sigma_{i}
$$

for all $i \in I$. Thus, we have $f \circ\left\langle r_{1}, \ldots, r_{n}\right\rangle \circ \varphi \in \bigwedge_{\left(\varphi_{i}\right)}^{\varphi}\left(\sigma_{i}\right)$, whence it follows

$$
f \circ\left\langle s_{1}, \ldots, s_{n}\right\rangle=f \circ\left\langle r_{1} \circ \varphi, \ldots, r_{n} \circ \varphi\right\rangle=f \circ\left\langle r_{1}, \ldots, r_{n}\right\rangle \circ \varphi \in \bigwedge_{\left(\varphi_{i}\right)}^{\varphi}\left(\sigma_{i}\right) .
$$

Note that this proof is also valid for the case $I=\emptyset$.

5.1.28 Lemma. Let $R \subseteq \mathrm{R}_{\mathbf{A}}^{\mathbb{T}}, F \subseteq O_{\mathbf{A}}, n, s \in \mathbb{N}_{+}$and let $\mathbf{C} \in \mathbb{T}$. Then, the following statements hold for all $1 \leq n \leq s$ and $\mathbf{B} \in \mathbb{T}$ where $\mathbf{B} \leqslant \mathbf{C}$ :

(i) $\operatorname{Pol}_{\mathbf{A}}^{(n)} R=\operatorname{Pol}_{\mathbf{A}}^{(n)} \mathrm{Clo}^{\mathbb{T}}(R)=\operatorname{Pol}_{\mathbf{A}}^{(n)} \operatorname{LOC}^{\mathbb{T}} \operatorname{Clo}^{\mathbb{T}}(R)=\operatorname{Pol}_{\mathbf{A}}^{(n)}{ }_{s}-\mathrm{LOC}^{\mathbb{T}} \mathrm{Clo}^{\mathbb{T}}(R)$,

(ii) $\mathrm{Pol}_{\mathbf{A}} R=\mathrm{Pol}_{\mathbf{A}} \mathrm{Clo}^{\mathbb{T}}(R)=\mathrm{Pol}_{\mathbf{A}} \operatorname{LOC}^{\mathbb{T}} \mathrm{Clo}^{\mathbb{T}}(R)$,

(iii) $\operatorname{Inv}_{\mathbf{A}}^{(\mathbf{B})} F=\operatorname{Inv}_{\mathbf{A}}^{(\mathbf{B})} \operatorname{Clo}(F)=\operatorname{Inv}_{\mathbf{A}}^{(\mathbf{B})} \operatorname{Loc}^{\mathbb{T}} \operatorname{Clo}(F)=\operatorname{Inv}_{\mathbf{A}}^{(\mathbf{B})} \mathbf{C}-\operatorname{Loc} \operatorname{Clo}(F)$,

(iv) $\operatorname{Inv}_{\mathbf{A}}^{\mathbb{T}} F=\operatorname{Inv}_{\mathbf{A}}^{\mathbb{T}} \operatorname{Clo}(F)=\operatorname{Inv}_{\mathbf{A}}^{\mathbb{T}} \operatorname{Loc}^{\mathbb{T}} \operatorname{Clo}(F)$.

Proof. (i) It is easy to see that the sets in (i) form a decreasing chain from the left to the right. For the other direction, let $f \in \mathrm{Pol}_{\mathbf{A}}^{(n)} R$. We have to show that $f$ belongs to $\mathrm{Pol}_{\mathbf{A}}^{(n)}{ }_{\mathrm{s}}-\mathrm{LOC}^{\mathbb{T}} \mathrm{Clo}^{\mathbb{T}}(R)$, i.e., $f$ preserves each $\sigma \in \mathrm{s}_{-} \mathrm{LOC}^{\mathbb{T}} \mathrm{Clo}^{\mathbb{T}}(R)$. Since $\operatorname{Inv}_{\mathbf{A}}^{\mathbb{T}} \operatorname{Pol}_{\mathbf{A}} R$ is a clone of relations by Lemma 5.1 .27 (ii), we have

$$
\mathrm{Clo}^{\mathbb{T}}(R) \subseteq \operatorname{Inv}_{\mathbf{A}}^{\mathbb{T}} \operatorname{Pol}_{\mathbf{A}} R
$$

and hence

$$
\mathrm{Pol}_{\mathbf{A}} \mathrm{Clo}^{\mathbb{T}}(R) \supseteq \mathrm{Pol}_{\mathbf{A}} \operatorname{Inv}_{\mathbf{A}}^{\mathbb{T}} \mathrm{Pol}_{\mathbf{A}} R=\mathrm{Pol}_{\mathbf{A}} R .
$$

Thus, $f \in \operatorname{Pol}_{\mathbf{A}}^{(n)} \mathrm{Clo}^{\mathbb{T}}(R)$. Now let $\sigma \in \mathrm{s} \mathrm{LOC}^{\mathbb{T}} \mathrm{Clo}^{\mathbb{T}}(R)$ be a relation of type $\mathbf{B}$ and let $r_{1}, \ldots, r_{n} \in \sigma$. Since $n \leq s$, there exists some $\sigma^{\prime} \in \mathrm{Clo}^{\mathbb{T}}(R)$ such that $\left\{r_{1}, \ldots, r_{n}\right\} \subseteq \sigma^{\prime} \subseteq \sigma$. Hence, $f \circ\left\langle r_{1}, \ldots, r_{n}\right\rangle \in \sigma^{\prime} \subseteq \sigma$, and we are done.

(ii) By (i), we have

$$
\bigcup_{n \in \mathbb{N}_{+}} \operatorname{Pol}_{\mathbf{A}}^{(n)} R=\bigcup_{n \in \mathbb{N}_{+}} \operatorname{Pol}_{\mathbf{A}}^{(n)} \operatorname{Clo}^{\mathbb{T}}(R)=\bigcup_{n \in \mathbb{N}_{+}} \operatorname{Pol}_{\mathbf{A}}^{(n)} \operatorname{LOC}^{\mathbb{T}} \operatorname{Clo}^{\mathbb{T}}(R)
$$


and, thus, $\operatorname{Pol}_{\mathbf{A}} R=\operatorname{Pol}_{\mathbf{A}} \mathrm{Clo}^{\mathbb{T}}(R)=\operatorname{Pol}_{\mathbf{A}} \operatorname{LOC}^{\mathbb{T}} \mathrm{Clo}^{\mathbb{T}}(R)$.

(iii) Again, it is easy to see that the sets in (iii) form a decreasing chain from the left to the right. It remains to show that we have $\sigma \in \operatorname{Inv}_{\mathbf{A}}^{(\mathbf{B})} \mathbf{C}-\operatorname{Loc} \operatorname{Clo}(F)$ whenever $\sigma \in \operatorname{Inv}_{\mathbf{A}}^{(\mathbf{B})} F$. We get $\sigma \in \operatorname{Inv}_{\mathbf{A}}^{(\mathbf{B})} \mathrm{Clo}(F)$ in the same way we got $f \in \operatorname{Pol}_{\mathbf{A}}^{(n)} \operatorname{Clo}^{\mathbb{T}}(R)$ in part (i). Now let $f \in \mathrm{C}$-Loc $\mathrm{Clo}(F)$ be $n$-ary. By Proposition 5.1.21, we also have $f \in \mathrm{B}$-Loc $\operatorname{Clo}(F)$. Let $r_{1}, \ldots, r_{n} \in \sigma$. We find some $f^{\prime} \in \operatorname{Clo}(F)$ such that $f \circ\left\langle r_{1}, \ldots, r_{n}\right\rangle=f^{\prime} \circ\left\langle r_{1}, \ldots, r_{n}\right\rangle$. Since $f^{\prime} \circ\left\langle r_{1}, \ldots, r_{n}\right\rangle \in \sigma$, it follows $f \triangleright \sigma$, and thus, $\sigma \in \operatorname{Inv}_{\mathbf{A}}^{(\mathbf{B})} \mathbf{C}-\operatorname{Loc} \mathrm{Clo}(F)$.

(iv) follows from (iii) in the same way that (ii) follows from (i).

Among other results that we will see later, this lemma allows us to give a direct calculation of $\Gamma_{F}(\sigma)$.

5.1.29 Proposition. Let $F \subseteq O_{\mathbf{A}}$ and $\sigma \in \mathrm{R}_{\mathbf{A}}^{\mathbb{T}}$. Then,

$$
\Gamma_{F}(\sigma)=\left\{f \circ\left\langle r_{1}, \ldots, r_{n}\right\rangle \mid f \in \operatorname{Clo}(F)^{(n)}, n \in \mathbb{N}_{+}, r_{1}, \ldots, r_{n} \in \sigma\right\} .
$$

Proof. Let us denote the right-hand side by $\gamma$. Let $f \in F^{(n)}$ and $r_{1}, \ldots, r_{n} \in \gamma$. Then, for all $i \in\{1, \ldots, n\}$, there exist $f_{i} \in \operatorname{Clo}(F)^{\left(k_{i}\right)}$ and $r_{i, 1}, \ldots, r_{i, k_{i}} \in \sigma$ such that $r_{i}=f_{i} \circ\left\langle r_{i, 1}, \ldots, r_{i, k_{i}}\right\rangle$. By Proposition 1.4.12 (page 19), we have

$$
\begin{aligned}
f \circ\left\langle r_{1}, \ldots, r_{n}\right\rangle & =f \circ\left\langle f_{1} \circ\left\langle r_{1,1}, \ldots, r_{1, k_{1}}\right\rangle, \ldots, f_{n} \circ\left\langle r_{n, 1}, \ldots, r_{n, k_{n}}\right\rangle\right\rangle \\
& =f \circ\left\langle\left\langle f_{1}, \ldots, f_{n}\right\rangle\right\rangle \circ\left\langle r_{1,1}, \ldots, r_{n, k_{n}}\right\rangle .
\end{aligned}
$$

Since clones are closed under expanded superposition (see Proposition 2.1.12, page 29), it follows that we have $f \circ\left\langle\left\langle f_{1}, \ldots, f_{n}\right\rangle\right\rangle \in \operatorname{Clo}(F)$, and hence $f \circ\left\langle r_{1}, \ldots, r_{n}\right\rangle \in \gamma$. Thus, $\gamma \in \operatorname{Inv}_{\mathbf{A}}^{\mathbb{T}} F$. Moreover, we have $\sigma \subseteq \gamma$ since $\operatorname{Clo}(F)$ contains the projection morphisms. Thus, $\Gamma_{F}(\sigma) \subseteq \gamma$.

Conversely, we have $\Gamma_{F}(\sigma) \in \operatorname{Inv}_{\mathbf{A}}^{\mathbb{T}} F$ by definition, and by Lemma 5.1 .28 (iv), we have $\operatorname{Inv}_{\mathbf{A}}^{\mathbb{T}} F=\operatorname{Inv}_{\mathbf{A}}^{\mathbb{T}} \operatorname{Clo}(F)$. Thus, $\Gamma_{F}(\sigma) \in \operatorname{Inv}_{\mathbf{A}}^{\mathbb{T}} \operatorname{Clo}(F)$ which implies $\gamma \subseteq \Gamma_{F}(\sigma)$.

As an obvious consequence, we have the following proposition:

5.1.30 Proposition. For $\sigma=\left\{r_{1}, \ldots, r_{n}\right\}$ and a clone $C \leq O_{\mathbf{A}}$, we have

$$
\Gamma_{C}(\sigma)=\left\{f \circ\left\langle r_{1}, \ldots, r_{n}\right\rangle \mid f \in C^{(n)}\right\} .
$$

Before we start to prove our main result, we need to introduce the notion of directedness.

5.1.31 Definition. For $s \geq 1$, a family $\mathcal{F}$ of sets is called $s$-directed if, for any $X_{1}, \ldots, X_{s} \in \mathcal{F}$ and $r_{1} \in X_{1}, \ldots, r_{s} \in X_{s}$, there exists some $Z \in \mathcal{F}$ such that $\left\{r_{1}, \ldots, r_{s}\right\} \subseteq Z$.

5.1.32 Lemma. Let $R \subseteq \mathrm{R}_{\mathbf{A}}^{\mathbb{T}}$, let $\emptyset \neq \mathcal{F} \subseteq R^{(\mathbf{B})}$, and let $\mathcal{F}$ be $s$-directed for some $s \geq 1$. Then, $\bigcup \mathcal{F} \in \mathrm{s}-\mathrm{LOC}^{\mathbb{T}} R$. 
Proof. We have to show that, for all $B \subseteq \cup \mathcal{F}$ with $|B| \leq s$, there exists $\sigma^{\prime} \in R$ such that $B \subseteq \sigma^{\prime} \subseteq \bigcup \mathcal{F}$. For $B=\left\{b_{1}, \ldots, b_{s}\right\} \subseteq \cup \mathcal{F}$, there exist $X_{1}, \ldots, X_{s} \in \mathcal{F}$ such that $b_{1} \in X_{1}, \ldots, b_{s} \in X_{s}$. Since $\mathcal{F}$ is $s$-directed, this implies that there exists $Z \in \mathcal{F} \subseteq R^{(\mathbf{B})}$ such that $\left\{b_{1}, \ldots, b_{s}\right\} \subseteq Z \subseteq \bigcup \mathcal{F}$. Thus, the claim follows for $\sigma^{\prime}:=Z$.

We have prepared everything to state the main results of this section - the characterization of the Galois closed subclasses of $O_{\mathbf{A}}$ and $\mathrm{R}_{\mathbf{A}}^{\mathbb{T}}$.

5.1.33 Theorem (Galois closed sets of operations over $\mathbf{A}$ ). Let $F \subseteq O_{\mathbf{A}}$. Then,

(i) $\operatorname{Loc}^{\mathbb{T}} \mathrm{Clo}(F)=\operatorname{Pol}_{\mathbf{A}} \operatorname{Inv}_{\mathbf{A}}^{\mathbb{T}} F$,

(ii) $\mathbf{C}-\operatorname{Loc} \operatorname{Clo}(F)=\operatorname{Pol}_{\mathbf{A}} \operatorname{Inv}_{\mathbf{A}}^{(\mathbf{C})} F$ for every $\mathbf{C} \in \mathbb{T}$.

Proof. (ii) Since $\mathrm{Pol}_{\mathbf{A}}-\operatorname{Inv}_{\mathbf{A}}^{\mathbb{T}}$ is a Galois connection, we have

$$
\mathbf{C}-\operatorname{Loc} \operatorname{Clo}(F) \subseteq \operatorname{Pol}_{\mathbf{A}} \operatorname{Inv}_{\mathbf{A}}^{\mathbb{T}} \mathbf{C}-\operatorname{Loc} \operatorname{Clo}(F) \subseteq \operatorname{Pol}_{\mathbf{A}} \operatorname{Inv}_{\mathbf{A}}^{(\mathbf{C})} \mathbf{C}-\operatorname{Loc} \operatorname{Clo}(F)
$$

and, by Lemma 5.1 .28 (iii), we also have

$$
\mathrm{Pol}_{\mathbf{A}} \operatorname{Inv}_{\mathbf{A}}^{(\mathbf{C})} \mathbf{C}-\operatorname{Loc} \operatorname{Clo}(F)=\operatorname{Pol}_{\mathbf{A}} \operatorname{Inv}_{\mathbf{A}}^{(\mathbf{C})} F \text {. }
$$

For the other direction, let $f \in \operatorname{Pol}_{\mathbf{A}} \operatorname{Inv}_{\mathbf{A}}^{(\mathbf{C})} F$ be an $n$-ary operation. In order to show $f \in \mathbf{C}$-Loc $\operatorname{Clo}(F)$, let $r_{1}, \ldots, r_{n} \in \mathbf{A}^{\mathbf{C}}$ and set $\sigma:=\left\{r_{1}, \ldots, r_{n}\right\}$. We have $f \circ\left\langle r_{1}, \ldots, r_{n}\right\rangle \in \Gamma_{F}(\sigma)$. But now, by Proposition 5.1.30 we find some $f^{\prime} \in \operatorname{Clo}(F)$ such that $f \circ\left\langle r_{1}, \ldots, r_{n}\right\rangle=f^{\prime} \circ\left\langle r_{1}, \ldots, r_{n}\right\rangle$ which proves $f \in \mathbf{C}$-Loc $\operatorname{Clo}(F)$.

(i) By (ii), we have

$$
\operatorname{Loc}^{\mathbb{T}} \operatorname{Clo}(F)=\bigcap_{\mathbf{C} \in \mathbb{T}} \mathbf{C}-\operatorname{Loc} \operatorname{Clo}(F)=\bigcap_{\mathbf{C} \in \mathbb{T}} \operatorname{Pol}_{\mathbf{A}} \operatorname{Inv}_{\mathbf{A}}^{(\mathbf{C})} F=\operatorname{Pol}_{\mathbf{A}} \operatorname{Inv}_{\mathbf{A}}^{\mathbb{T}} F
$$

The following lemma will help us to prove a similar characterization for the Galois closed classes of relations:

5.1.34 Lemma. Let $\mathbf{B} \in \mathbb{T}$, let $s \geq 1$ and let $R \subseteq \mathrm{R}_{\mathbf{A}}^{\mathbb{T}}$. For $F:=\operatorname{Pol}_{\mathbf{A}} R$ and $S \subseteq \mathbf{A}^{\mathbf{B}}$, $|S| \leq s$, we have $\Gamma_{F^{(s)}}(S) \in \mathrm{Clo}^{\mathbb{T}}(R)$.

Proof. For $S=\emptyset$, we have $\Gamma_{F}(S)=\emptyset \in \mathrm{Clo}^{\mathbb{T}}(R)$, and we are done. Let $S=\left\{r_{1}, \ldots, r_{s}\right\}$ (note that $r_{1}, \ldots, r_{s}$ do not have to be pairwise distinct). We define

$$
I:=\left\{\left(r_{1}^{\prime}, \ldots, r_{s}^{\prime}, \sigma\right) \mid \sigma \in R, r_{1}^{\prime}, \ldots, r_{s}^{\prime} \in \sigma\right\} .
$$

For each $i=\left(r_{1}^{\prime}, \ldots, r_{s}^{\prime}, \sigma\right) \in I$, set $\sigma_{i}:=\sigma$, let $\mathbf{B}_{i}$ be the type of $\sigma_{i}$ and define $\varphi_{i}: \mathbf{B}_{i} \rightarrow \mathbf{A}^{s}$ by setting $\varphi_{i}:=\left\langle r_{1}^{\prime}, \ldots, r_{s}^{\prime}\right\rangle$. Moreover, define $\varphi: \mathbf{B} \rightarrow \mathbf{A}^{s}$ by setting $\varphi:=\left\langle r_{1}, \ldots, r_{s}\right\rangle$.

We shall prove that

$$
\varrho_{S}:=\bigwedge_{\left(\varphi_{i}\right)_{i \in I}}^{\varphi}\left(\sigma_{i}\right)=\left\{r \circ \varphi \mid \forall i \in I: r \circ \varphi_{i} \in \sigma_{i}, r \in \mathbf{A}^{\mathbf{A}^{s}}\right\}=\Gamma_{F^{(s)}}(S),
$$


which would finish the proof since $\varrho_{S} \in \mathrm{Clo}^{\mathbb{T}}(R)$.

Let $\varkappa \in \varrho_{S}$. We will start by showing that there exists $f \in F^{(s)}=\operatorname{Pol}_{\mathbf{A}}^{(s)} R$ such that $\varkappa=f \circ \varphi$ : Since $\varkappa \in \varrho_{S}$, there exists $r \in \mathbf{A}^{\mathbf{A}^{s}}$ such that $\varkappa=r \circ \varphi$ and $r \circ \varphi_{i} \in \sigma_{i}$ for all $i \in I$. Thus, for $f:=r$, we obtain

$$
\varkappa=r \circ \varphi=f \circ \varphi \text {. }
$$

It remains to show that we have $f \in F=\operatorname{Pol}_{\mathbf{A}} R$, i.e., $f$ preserves each $\sigma \in R$. Let $\sigma \in R$ and let $r_{1}^{\prime}, \ldots, r_{s}^{\prime} \in \sigma$. For $i:=\left(r_{1}^{\prime}, \ldots, r_{s}^{\prime}, \sigma\right)$ we have $i \in I$, and hence

$$
f \circ\left\langle r_{1}^{\prime}, \ldots, r_{s}^{\prime}\right\rangle=r \circ\left\langle r_{1}^{\prime}, \ldots, r_{s}^{\prime}\right\rangle=r \circ \varphi_{i} \in \sigma .
$$

Thus, $f \in \operatorname{Pol}_{\mathbf{A}} R=F$, as required. By Proposition 5.1.29, it follows

$$
\varkappa=f \circ \varphi=f \circ\left\langle r_{1}, \ldots, r_{s}\right\rangle \in \Gamma_{F^{(s)}}(S) .
$$

Conversely, let $\widehat{r} \in \Gamma_{F^{(s)}}(S)$. Recall that, by Lemma 5.1.27, $F=\mathrm{Pol}_{\mathbf{A}} R$ is a clone of operations. Hence, $\operatorname{Clo}\left(F^{(s)}\right) \subseteq F$. It follows by Proposition 5.1 .29 that $\widehat{r}$ is of the form $f \circ\left\langle r_{i_{1}}, \ldots, r_{i_{l}}\right\rangle$ for some $f \in F^{(l)}$ and $i_{1}, \ldots, i_{l} \in\{1, \ldots, s\}$. Set $f^{\prime}:=f \circ\left\langle\pi_{i_{1}}^{s}, \ldots, \pi_{i_{l}}^{s}\right\rangle$. Then,

$$
\widehat{r}=f \circ\left\langle r_{i_{1}}, \ldots, r_{i_{l}}\right\rangle=f \circ\left\langle\pi_{i_{1}}^{s}, \ldots, \pi_{i_{l}}^{s}\right\rangle \circ\left\langle r_{1}, \ldots, r_{s}\right\rangle=f^{\prime} \circ\left\langle r_{1}, \ldots, r_{s}\right\rangle .
$$

Now, let $r:=f^{\prime}$. Then,

$$
\widehat{r}=f^{\prime} \circ\left\langle r_{1}, \ldots, r_{s}\right\rangle=r \circ\left\langle r_{1}, \ldots, r_{s}\right\rangle=r \circ \varphi,
$$

and we can finish the proof by showing that we have $r \circ \varphi \in \varrho_{S}$. Indeed, for each $i=\left(r_{1}^{\prime}, \ldots, r_{s}^{\prime}, \sigma_{i}\right) \in I$, we get

$$
r \circ \varphi_{i}=f^{\prime} \circ \varphi_{i}=f^{\prime} \circ\left\langle r_{1}^{\prime}, \ldots, r_{s}^{\prime}\right\rangle \in \sigma_{i}
$$

since $f^{\prime} \in F=\operatorname{Pol}_{\mathbf{A}} R$ and $r_{1}^{\prime}, \ldots, r_{s}^{\prime} \in \sigma_{i} \in R$. This implies $r \circ \varphi \in \varrho_{S}$, and we have established the desired result.

5.1.35 Theorem (Galois closed classes of relations). Let $R \subseteq \mathrm{R}_{\mathbf{A}}^{\mathbb{T}}$. Then,

(i) $\operatorname{LOC}^{\mathbb{T}} \mathrm{Clo}^{\mathbb{T}}(R)=\operatorname{Inv}_{\mathbf{A}}^{\mathbb{T}} \operatorname{Pol}_{\mathbf{A}} R$,

(ii) $\mathrm{s}_{-} \mathrm{LOC}^{\mathbb{T}} \mathrm{Clo}^{\mathbb{T}}(R)=\operatorname{Inv}_{\mathbf{A}}^{\mathbb{T}} \mathrm{Pol}_{\mathbf{A}}^{(s)} R$ for every $s \geq 1$.

Proof. (ii) Since $\mathrm{Pol}_{\mathbf{A}}-\mathrm{Inv}_{\mathbf{A}}^{\mathbb{T}}$ is a Galois connection, we have

$$
\mathrm{s} \mathrm{LOC}^{\mathbb{T}} \mathrm{Clo}^{\mathbb{T}}(R) \subseteq \operatorname{Inv}_{\mathbf{A}}^{\mathbb{T}} \operatorname{Pol}_{\mathbf{A}} \mathrm{s}-\mathrm{LOC}^{\mathbb{T}} \mathrm{Clo}^{\mathbb{T}}(R) \subseteq \operatorname{Inv}_{\mathbf{A}}^{\mathbb{T}} \operatorname{Pol}_{\mathbf{A}}^{(s)} \mathrm{s} \operatorname{LOC}^{\mathbb{T}} \mathrm{Clo}^{\mathbb{T}}(R)
$$

and, by Lemma 5.1 .28 (i), we also have

$$
\operatorname{Inv}_{\mathbf{A}}^{\mathbb{T}} \mathrm{Pol}_{\mathbf{A}}^{(s)} \mathrm{s}-\mathrm{LOC}^{\mathbb{T}} \mathrm{Clo}^{\mathbb{T}}(R)=\operatorname{Inv}_{\mathbf{A}}^{\mathbb{T}} \mathrm{Pol}_{\mathbf{A}}^{(s)} R .
$$


For the other direction, let $\sigma \in \operatorname{Inv}_{\mathbf{A}}^{\mathbb{T}} \operatorname{Pol}_{\mathbf{A}}^{(s)} R$ be a relation of type $\mathbf{B}$. We have to show

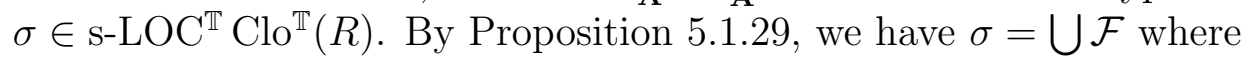

$$
\mathcal{F}:=\left\{\Gamma_{F^{(s)}}(B)|B \subseteq \sigma,| B \mid \leq s\right\}
$$

Clearly, $\mathcal{F}$ is non-empty and $s$-directed. Let $F:=\mathrm{Pol}_{\mathbf{A}} R$. By Lemma 5.1.34 we get $\Gamma_{F^{(s)}}(B) \in \mathrm{Clo}^{\mathbb{T}}(R)$ for each $B \subseteq \sigma$ with $|B| \leq s$. In other words, $\mathcal{F} \subseteq \operatorname{Clo}^{\mathbb{T}}(R)^{(\mathbf{B})}$.

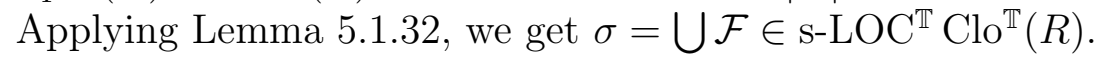

(i) By (ii), we have

$$
\mathrm{LOC}^{\mathbb{T}} \mathrm{Clo}^{\mathbb{T}}(R)=\bigcap_{s \in \mathbb{N}_{+}} \mathrm{s}_{-} \mathrm{LOC}^{\mathbb{T}} \mathrm{Clo}^{\mathbb{T}}(R)=\bigcap_{s \in \mathbb{N}_{+}} \operatorname{Inv}_{\mathbf{A}}^{\mathbb{T}} \operatorname{Pol}_{\mathbf{A}}^{(s)} R=\operatorname{Inv}_{\mathbf{A}}^{\mathbb{T}} \operatorname{Pol}_{\mathbf{A}} R
$$

The last two theorems enable us to characterize those subsets $F \subseteq O_{\mathbf{A}}$ and those subclasses $R \subseteq \mathrm{R}_{\mathbf{A}}^{\mathbb{T}}$ which can be represented as $\operatorname{Pol}_{\mathbf{A}} R^{\prime}$ and $\operatorname{Inv}_{\mathbf{A}}^{\mathbb{T}} F^{\prime}$ for some $R^{\prime} \subseteq \mathrm{R}_{\mathbf{A}}^{\mathbb{T}}$ and $F^{\prime} \subseteq O_{\mathbf{A}}$, respectively.

5.1.36 Corollary. For $F \subseteq O_{\mathbf{A}}$, the following are equivalent:

(1) $F \leq O_{\mathbf{A}}$ (i.e., $F=\mathrm{Clo}(F)$ ) and $\operatorname{Loc}^{\mathbb{T}} F=F$.

(2) $F=\operatorname{Pol}_{\mathbf{A}} \operatorname{Inv}_{\mathbf{A}}^{\mathbb{T}} F$.

(3) $\exists R \subseteq \mathrm{R}_{\mathbf{A}}^{\mathbb{T}}: F=\operatorname{Pol}_{\mathbf{A}} R$.

Proof. (1) $\Longrightarrow(2)$ by Theorem 5.1.33.

$(2) \Longrightarrow(3)$ is trivial.

$(3) \Longrightarrow(1)$. We have $F=\operatorname{Pol}_{\mathbf{A}} R \leq O_{\mathbf{A}}$ by Lemma 5.1.27. By Theorem 5.1.33, we also have $\operatorname{Loc}^{\mathbb{T}} \mathrm{Pol}_{\mathbf{A}} R=\mathrm{Pol}_{\mathbf{A}} \operatorname{Inv}_{\mathbf{A}}^{\mathbb{T}} \operatorname{Pol}_{\mathbf{A}} R=\operatorname{Pol}_{\mathbf{A}} R$.

5.1.37 Corollary. For $R \subseteq \mathrm{R}_{\mathbf{A}}^{\mathbb{T}}$, the following are equivalent:

(1) $R \leq \mathrm{R}_{\mathbf{A}}^{\mathbb{T}}$ (i.e., $R=\mathrm{Clo}^{\mathbb{T}}(R)$ ) and $\mathrm{LOC}^{\mathbb{T}} R=R$.

(2) $R=\operatorname{Inv} \operatorname{Pol}_{\mathbf{A}} R$.

(3) $\exists F \subseteq O_{\mathbf{A}}: R=\operatorname{Inv}_{\mathbf{A}}^{\mathbb{T}} F$.

Proof. (1) $\Longrightarrow(2)$ by Theorem 5.1.35.

$(2) \Longrightarrow(3)$ is trivial.

$(3) \Longrightarrow(1)$. We have $R=\operatorname{Inv}_{\mathbf{A}}^{\mathbb{T}} F \leq \mathrm{R}_{\mathbf{A}}^{\mathbb{T}}$ by Lemma 5.1.27, By Theorem 5.1.35, we also have $\operatorname{LOC}^{\mathbb{T}} \operatorname{Inv}_{\mathbf{A}}^{\mathbb{T}} F=\operatorname{Inv}_{\mathbf{A}}^{\mathbb{T}} \operatorname{Pol}_{\mathbf{A}} \operatorname{Inv}_{\mathbf{A}}^{\mathbb{T}} R=\operatorname{Inv}_{\mathbf{A}}^{\mathbb{T}} R$.

By Corollary 5.1.23 and Lemma 5.1.25 (page 84), we can also state the following corollary:

5.1.38 Corollary. Assume that the set of morphisms from any $\mathbf{B} \in \mathbb{T}$ to $\mathbf{A}$ is finite and that, for each $k \in \mathbb{N}_{+}$, there exists $n \geq k$ such that $\mathbf{A}^{n} \leqslant \mathbf{B}$ for some $\mathbf{B} \in \mathbb{T}$. Then, the Galois closed subclasses of $\mathrm{Pol}_{\mathbf{A}}-\operatorname{Inv}_{\mathbf{A}}^{\mathbb{T}}$ are precisely the clones of operations and the clones of relations, respectively. Consequently, $\mathcal{L}_{\mathbf{A}}$ and $\mathcal{L}_{\mathbf{A}}^{* \mathbb{T}}$ are dually isomorphic via $\operatorname{Inv}_{\mathbf{A}}^{\mathbb{T}}$. 
Note that this corollary generalizes the fact that the local closure operators can be omitted for the usual Galois connection Pol-Inv on a set $A$ if the set $A$ is finite (cf. Example 5.1.10, page 80.

If the condition of the above corollary is not satisfied, we have to adjust the result.

5.1.39 Definition. Denote by $\operatorname{Loc}^{\mathbb{T}} \mathcal{L}_{\mathbf{A}}$ the lattice of locally closed clones of operations over $\mathbf{A}$ and by $\operatorname{LOC}^{\mathbb{T}} \mathcal{L}_{\mathbf{A}}^{* \mathbb{T}}$ the lattice of locally closed clones of relations on $\mathbf{A}$.

5.1.40 Corollary. $\operatorname{Loc}^{\mathbb{T}} \mathcal{L}_{\mathbf{A}}$ and $\operatorname{LOC}^{\mathbb{T}} \mathcal{L}_{\mathbf{A}}^{* \mathbb{T}}$ are dually isomorphic via $\operatorname{Inv}_{\mathbf{A}}^{\mathbb{T}}$.

\subsubsection{A Remark on the Choice of $\mathbb{T}$}

As we have seen, the choice of $\mathbb{T}$ influences the local closure operators, the clones of relations, and consequently, the Galois closed classes of operations as well as those of the relations.

In this section, we will discuss how to choose $\mathbb{T}$ such that the local closure operators share a certain behaviour with the local closure operators of the usual Pol-Inv. Recall that our framework coincides with the classical case if $\mathcal{A}$ is the category of sets and we choose $\mathbb{T}=\left\{\{1, \ldots, k\} \mid k \in \mathbb{N}_{+}\right\}$. For brevity, we write $\underline{k}$ instead of $\{1, \ldots, k\}$. Evidently, we have $k_{1} \leqslant k_{2}$ (i.e., there exists an epimorphism from $k_{2}$ to $k_{1}$ ) whenever $k_{1} \leq k_{2}$. Thus, for $\overline{F \subseteq} \overline{O_{\mathbf{A}}}$, Proposition 5.1.21 (page 83) yields

$$
\underline{1-\operatorname{Loc}^{\mathbb{T}}} F \supseteq \underline{2}-\operatorname{Loc}^{\mathbb{T}} F \supseteq \underline{3}-\operatorname{Loc}^{\mathbb{T}} F \supseteq \ldots
$$

and

$$
\operatorname{Loc}^{\mathbb{T}} F=\bigcap_{k \in \mathbb{N}_{+}} \underline{\mathrm{k}-\operatorname{Loc}^{\mathbb{T}}} F
$$

Roughly speaking, $\bigcap_{k=1}^{n} \underline{\mathrm{k}-\mathrm{Loc}^{\mathbb{T}}} F$ converges to $\operatorname{Loc}^{\mathbb{T}} F$ for $n \rightarrow \infty$. A similar statement can be formulated for $\mathrm{LOC}^{\mathbb{T}}$. We have

$$
1-\mathrm{LOC}^{\mathbb{T}} R \supseteq 2-\mathrm{LOC}^{\mathbb{T}} R \supseteq 3-\mathrm{LOC}^{\mathbb{T}} R \supseteq \ldots
$$

and

$$
\mathrm{LOC}^{\mathbb{T}} R=\bigcap_{s \in \mathbb{N}_{+}} \mathrm{s}-\mathrm{LOC}^{\mathbb{T}} R
$$

The statement about $\mathrm{LOC}^{\mathbb{T}}$ holds in any category, but we can not necessarily order the objects in $\mathbb{T}$ such that we obtain a property analogue to the statement about Loc $^{\mathbb{T}}$ from above. However, we can do so if $\mathbb{T}$ is a countable set of objects that is totally ordered by $\leqslant$ and has a minimum element.

5.1.41 Proposition. Let $F \subseteq O_{\mathbf{A}}$ and let $\mathbb{T}=\left(\mathbf{C}_{i}\right)_{i \in \mathbb{N}_{+}} \subseteq \mathcal{A}$ with $\mathbf{C}_{i} \leqslant \mathbf{C}_{j}$ if and only if $i \leq j$. Then

$$
\mathbf{C}_{1} \text {-Loc } F \supseteq \mathbf{C}_{2} \text {-Loc } F \supseteq \mathbf{C}_{3} \text {-Loc } F \supseteq \ldots
$$

and

$$
\operatorname{Loc}^{\mathbb{T}} F=\bigcap_{i \in \mathbb{N}_{+}} \mathbf{C}_{\mathrm{i}^{-}} \operatorname{Loc} F
$$


Proof. Note that we can have $\mathbf{C}_{i} \leqslant \mathbf{C}_{j}$ and $\mathbf{C}_{j} \leqslant \mathbf{C}_{i}$ if and only if $i=j$. Thus, $\mathbf{C}_{j} \cong \mathbf{C}_{i}$ can only occur for $i=j$, and $\mathbb{T}$ is a typeclass. The rest follows directly from Proposition 5.1 .21 and the definition of $\operatorname{Loc}^{\mathbb{T}} F$.

\subsection{4. $\mathrm{Pol}_{\mathbf{A}}-\operatorname{Inv}_{\mathbf{A}}^{\mathbb{T}}$ in Concrete Categories}

We will now discuss the case in which $(\mathcal{A}, U)$ is concrete. In this scenario, we can assign a cardinality to each object in $\mathcal{A}$ by looking at its image under the forgetful functor. That is, for $\mathbf{B} \in \mathcal{A}$, we can define $|\mathbf{B}|:=|U(\mathbf{B})|$. In particular, we can distinguish between finite and infinite objects in $\mathcal{A}$ by defining an object $\mathbf{B}$ to be finite if $|\mathbf{B}|<\infty$ (i.e., its image under $U$ is a finite set).

We have the following result:

5.1.42 Corollary. Assume that $\mathbf{A}$ is finite. If $\mathbb{T}$ consists only of finite objects, then $\mathrm{LOC}^{\mathbb{T}} R=R$ for all $R \subseteq O_{\mathbf{A}}$.

Proof. Since $\mathbf{A}$ and each object in $\mathbb{T}$ being finite implies that every $\sigma \in \mathrm{R}_{\mathbf{A}}^{\mathbb{T}}$ is finite, we have $\mathrm{LOC}^{\mathbb{T}} R=R$ by Lemma 5.1.25 (page 84).

Thus, by Corollary 5.1.23 (page 84), we can omit the local closure operators completely if $\mathbf{A}$ is finite and $\mathbb{T}$ is a class of finite objects such that each finite power of $\mathbf{A}$ is the codomain of some epimorphism $e: \mathbf{B} \rightarrow \mathbf{A}$ for some $\mathbf{B} \in \mathbb{T}$. If we are only interested in the result that all clones are locally closed, then it is enough to require that infinitely many finite powers of $\mathbf{A}$ are the codomain of some epimorphism $e: \mathbf{B} \rightarrow \mathbf{A}$ for some $\mathbf{B} \in \mathbb{T}$.

\subsubsection{Examples.}

(i) If $\mathbf{A} \in \mathcal{A}$ is finite, we can set $\mathbb{T}:=\left\{\mathbf{A}^{n} \mid n \in \mathbb{N}_{+}\right\}$or $\mathbb{T}:=\left\{\mathbf{A}^{2 n} \mid n \in \mathbb{N}_{+}\right\}$to obtain that $\mathcal{L}_{\mathbf{A}}$ and $\mathcal{L}_{\mathbf{A}}^{* \mathbb{T}}$ are the lattices of the Galois closed classes with respect to $\mathrm{Pol}_{\mathbf{A}}-\operatorname{Inv}_{\mathbf{A}}^{\mathbb{T}}$.

(ii) If each object in $\mathcal{A}$ is finite, then choosing $\mathbb{T}$ to be a skeleton of $\mathcal{A}$ also gives us the result from (i).

(iii) For each category $\mathcal{A}$ from Section 4.2 (equipped with the obvious forgetful functor), we obtain the same results for $\mathbb{T}:=\mathbb{P}(\mathbf{M})$ or for $\mathbb{T}$ being a skeleton of the full subcategory $\mathbb{S P}(\mathbf{M})$.

Since $(\mathcal{A}, U)$ is a concrete category, we can assign arities to relations on $\mathbf{A}$ by defining the arity of a relation to be the cardinality of its type.

5.1.44 Definition. Let $\sigma \in \mathrm{R}_{\mathbf{A}}^{(\mathbf{B})}$. The arity of $\sigma$ is the cardinal number $|\mathbf{B}|$. For a cardinal number $k \geq 1$, the class

$$
\mathrm{R}_{\mathbf{A}}^{(k)}:=\bigcup_{\mathbf{B} \in \mathcal{A},|\mathbf{B}| \leq k} \mathrm{R}_{\mathbf{A}}^{(\mathbf{B})}
$$

is called the class of $k$-ary relations. The relations $\sigma$ for which we have $\sigma \notin \mathrm{R}_{\mathrm{A}}^{(k)}$ for all $k \in \mathbb{N}_{+}$are called relations of infinite arity or infinitary relations. Moreover, for $F \subseteq O_{\mathbf{A}}$, set $\operatorname{Inv}_{\mathbf{A}}^{\mathbb{T}}{ }^{(k)} F=\operatorname{Inv}_{\mathbf{A}}^{\mathbb{T}} F \cap \mathrm{R}_{\mathbf{A}}^{(k)}$. 
We can also use the notion of arities to introduce another local closure operator that uses arities instead of types.

5.1.45 Definition. Let $F \subseteq O_{\mathbf{A}}$ and let $s \geq 1$ be a cardinal number. We define the following local closure operator:

$$
\begin{aligned}
\mathrm{s}_{-} \operatorname{Loc}^{\mathbb{T}} F:=\left\{f \in O_{\mathbf{A}}^{(n)} \mid\right. & n \geq 1, \forall r_{1}, \ldots, r_{n} \in \mathbf{A}^{\mathbf{B}}, \mathbf{B} \in \mathbb{T},|\mathbf{B}| \leq s: \\
& \left.\exists f^{\prime} \in F: f \circ\left\langle r_{1}, \ldots, r_{n}\right\rangle=f^{\prime} \circ\left\langle r_{1}, \ldots, r_{n}\right\rangle\right\} .
\end{aligned}
$$

Note that we have

$$
\operatorname{Loc}^{\mathbb{T}} F=\bigcap_{s \geq 1} \operatorname{secoc}^{\mathbb{T}} F
$$

and $\mathrm{r}-\mathrm{Loc}^{\mathbb{T}} F \supseteq \mathrm{s}^{\mathrm{L}} \mathrm{Loc}^{\mathbb{T}} F$ for all $r \leq s$.

The following proposition is obvious:

5.1.46 Proposition. If $\mathbb{T}$ consists of only finite objects, then

$$
\operatorname{Loc}^{\mathbb{T}} F=\bigcap_{s \in \mathbb{N}_{+}} \operatorname{s}^{-\operatorname{Loc}^{\mathbb{T}}} F .
$$

We can show the following lemma, which formulates an equation analogue to statement (iii) from Lemma 5.1.28 (page 86):

5.1.47 Lemma. Let $n, s$ be cardinal numbers with $n \leq s$. Then,

$$
\operatorname{Inv}_{\mathbf{A}}^{\mathbb{T}}{ }^{(n)} F=\operatorname{Inv}_{\mathbf{A}}^{\mathbb{T}}{ }^{(n)} \operatorname{Clo}(F)=\operatorname{Inv}_{\mathbf{A}}^{\mathbb{T}}{ }^{(n)} \operatorname{Loc}^{\mathbb{T}} \operatorname{Clo}(F)=\operatorname{Inv}_{\mathbf{A}}^{\mathbb{T}}{ }^{(n)}{ }-\operatorname{Loc}^{\mathbb{T}} \operatorname{Clo}(F) .
$$

Proof. Since the following arguments are analogue to those of the proof of (iii) from Lemma 5.1.28, we only sketch the proof: It is easy to see that the classes form a decreasing chain from left to right, so it remains to show that we have $\sigma \in \operatorname{Inv}_{\mathbf{A}}^{\mathbb{T}}{ }^{(n)}{ }_{\text {s-Loc }}{ }^{\mathbb{T}} \operatorname{Clo}(F)$ for $\sigma \in \operatorname{Inv}_{\mathbf{A}}^{\mathbb{T}^{(n)}} F$. We can deduce $\sigma \in \operatorname{Inv}_{\mathbf{A}}^{\mathbb{T}}{ }^{(n)} \mathrm{Clo}(F)$ by using Lemma 5.1.27 (page 85).

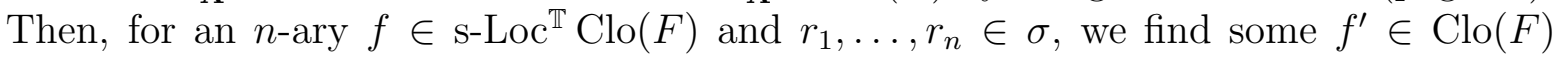
such that we have $f \circ\left\langle r_{1}, \ldots, r_{n}\right\rangle=f^{\prime} \circ\left\langle r_{1}, \ldots, r_{n}\right\rangle$. Therefore, $f \circ\left\langle r_{1}, \ldots, r_{n}\right\rangle \in \sigma$ and $\sigma \in \operatorname{Inv}_{\mathbf{A}}^{\mathbb{T}}{ }^{(n)}{ }_{\mathrm{S}-\operatorname{Loc}^{\mathbb{T}}} \mathrm{Clo}(F)$.

We can write the following proposition, which is analogue to statement (ii) from Theorem 5.1.33 (page 88):

5.1.48 Proposition (Galois closed sets of operations over $\mathbf{A}$ ). Let $F \subseteq O_{\mathbf{A}}$. Then, $\mathrm{s}-\operatorname{Loc}^{\mathbb{T}} \mathrm{Clo}(F)=\mathrm{Pol}_{\mathbf{A}} \operatorname{Inv}_{\mathbf{A}}^{\mathbb{T}}{ }^{(s)} F$ for every cardinal number $s \geq 1$.

Proof. Again, this proof is essentially analogue to a proof we have already seen (namely the one of (ii) from Theorem 5.1.33). Hence, we only present a sketch. By using that $\mathrm{Pol}_{\mathbf{A}}-\mathrm{Inv}_{\mathbf{A}}^{\mathbb{T}}$ is a Galois connection and with Lemma 5.1.47, we can show

$$
\mathrm{s}^{-\operatorname{Loc}^{\mathbb{T}}} \mathrm{Clo}(F) \subseteq \operatorname{Pol}_{\mathbf{A}} \operatorname{Inv}_{\mathbf{A}}^{\mathbb{T}}{ }^{(s)} F .
$$

For the other direction, let $f \in \operatorname{Pol}_{\mathbf{A}} \operatorname{Inv}_{\mathbf{A}}^{\mathbb{T}^{(s)}} F$ be an $n$-ary operation. In order to show $f \in \mathrm{s}-\operatorname{Loc}^{\mathbb{T}} \mathrm{Clo}(F)$, let $r_{1}, \ldots, r_{n} \in \mathrm{R}_{\mathbf{A}}^{(\mathbf{B})}$ where $|\mathbf{B}| \leq s$. Setting $\sigma:=\left\{r_{1}, \ldots, r_{n}\right\}$, we have $f \circ\left\langle r_{1}, \ldots, r_{n}\right\rangle \in \Gamma_{F}(\sigma)$, and by Proposition 5.1.30 (page 87) we find some $f^{\prime} \in \operatorname{Clo}(F)$ such that $f \circ\left\langle r_{1}, \ldots, r_{n}\right\rangle=f^{\prime} \circ\left\langle r_{1}, \ldots, r_{n}\right\rangle$. 


\subsection{A General Galois Theory for Dual Operations and Dual Relations}

For this section, let $X$ be a category that contains an object $\mathbf{X}$ and all finite nonempty copowers of $\mathbf{X}$. Recall that an $n$-ary dual operation over $\mathbf{X}$ is an $n$-ary operation over $\mathbf{X}$ in $X^{o p}$. Furthermore, $\mathbb{T}$ is a typeclass of $X$ if and only if it is a typeclass of $x^{o p}$. Therefore, we can dualize all the definitions from the last section to obtain a Galois connection $\overline{\operatorname{Pol}}_{\mathbf{X}}-\overline{\operatorname{Inv}}_{\mathbf{X}}^{\mathbb{T}}$ between classes of dual operations and classes of dualized relations such that the Galois closed classes are precisely the dualized local closures of clones of dual operations and the dualized local closures of the dualized clones of relations. We will describe this in the upcoming subsections.

\subsubsection{Dual Relations}

For the whole section, let $\mathbb{T} \subseteq X$ be a typeclass.

5.2.1 Definition. Let $\mathbf{Y} \in X$. A dual relation of type $\mathbf{Y}$ on $\mathbf{X}$ is a subset of $X(\mathbf{X}, \mathbf{Y})$. Denote the class of all dual relations of type $\mathbf{Y}$ on $\mathbf{X}$ by $\overline{\mathrm{R}}_{\mathbf{X}}^{(\mathbf{Y})}$. Moreover,

$$
\overline{\mathrm{R}}_{\mathbf{X}}^{\mathbb{T}}:=\bigcup_{\mathbf{Y} \in \mathbb{T}} \overline{\mathrm{R}}_{\mathbf{X}}^{(\mathbf{Y})}
$$

is called the class of dual relations of the typeclass $\mathbb{T}$. For a class of dual relations $R \subseteq \overline{\mathrm{R}}_{\mathbf{X}}^{\mathbb{T}}$, let $R^{(\mathbf{Y})}:=R \cap \overline{\mathrm{R}}_{\mathbf{X}}^{(\mathbf{Y})}$

It is easy to see that a dual relation of type $\mathbf{Y} \in X$ is a relation of type $\mathbf{Y}$ in $X^{o p}$. In other words, the notion of a dual relation is the dualized notion of a relation.

5.2.2 Example. Every dual relation of type $\{1, \ldots, k\}$ on a set $\mathbf{X} \in$ Set is a $k$-ary corelation on $X$ as introduced in [PR00].

We will now dualize the remaining notions of Subsection 5.1.1:

5.2.3 Definition. Let $\sigma$ be a dual relation of type $\mathbf{Y}$ on $\mathbf{X}$, and let $g$ be an $n$-ary dual operation over $\mathbf{X}$. We say that $\sigma$ is invariant for $g$ or that $g$ preserves $\sigma$, written $g \bar{\nabla} \sigma$, if $\left[r_{1}, \ldots, r_{n}\right] \circ g \in \sigma$ whenever $r_{1}, \ldots, r_{n} \in \sigma$. Furthermore, we say that a set of dual operations $G \subseteq \bar{O}_{\mathbf{x}}$ preserves $\sigma$, written $G \bar{\triangleright} \sigma$, if every $g \in G$ preserves $\sigma$.

It is easy to check that the injection morphisms preserve any dual relation on $\mathbf{X}$. This also follows by the Duality Principle from the fact that every projection morphism preserves each relation on $\mathbf{X} \in X^{o p}$.

5.2.4 Definition. For $G \subseteq \bar{O}_{\mathbf{X}}$ and $\sigma \in \overline{\mathrm{R}}_{\mathbf{X}}^{(\mathbf{Y})}$, define

$$
\bar{\Gamma}_{G}(\sigma):=\bigcap\left\{\sigma^{\prime} \in \overline{\mathrm{R}}_{\mathbf{X}}^{(\mathbf{Y})} \mid \sigma \subseteq \sigma^{\prime}, G \bar{\triangleright} \sigma^{\prime}\right\}
$$


Since $\bar{\Gamma}_{G}(\sigma)=\left(\Gamma_{G^{o p}}\left(\sigma^{o p}\right)\right)^{o p}$, it follows immediately from the last section that $\bar{\Gamma}_{G}(\sigma)$ is the least dual relation that is preserved by $G$ and contains $\sigma$.

5.2.5 Definition. A class $R \subseteq \overline{\mathrm{R}}_{\mathrm{X}}^{\mathbb{T}}$ is called a clone of dual relations of the typeclass $\mathbb{T}$ on $\mathbf{X}$, written $R \leq \overline{\mathrm{R}}_{\mathbf{X}}^{\mathbb{T}}$, if

(i) $\emptyset \in R$,

(ii) $R$ is closed under general superposition, that is, the following holds: Let $I$ be an index class, let $\sigma_{i} \in R^{\left(\mathbf{Y}_{i}\right)}(i \in I)$ and let $\varphi: \mathbf{Z} \rightarrow \mathbf{Y}$ and $\varphi_{i}: \mathbf{Z} \rightarrow \mathbf{Y}_{i}$ be morphisms where $\mathbf{Z} \in X$ and $\mathbf{Y} \in \mathbb{T}$. Then, the dual relation $\bar{\bigwedge}_{\left(\varphi_{i}\right)_{i \in I}}^{\varphi}\left(\sigma_{i}\right)_{i \in I} \in \overline{\mathrm{R}}_{\mathbf{X}}^{(\mathbf{Y})}$ defined by

$$
\bar{\bigwedge}_{\left(\varphi_{i}\right)_{i \in I}}^{\varphi}\left(\sigma_{i}\right)_{i \in I}:=\left\{\varphi \circ r \mid \forall i \in I: \varphi_{i} \circ r \in \sigma_{i}, r \in \mathbf{Z}^{\mathbf{X}}\right\}
$$

belongs to $R$.

Again, it is easy to see that $\overline{\mathrm{R}}_{\mathbf{X}}^{\mathbb{T}}$ is a clone of dual relations and that the intersection of clones of dual relations is a clone of dual relations. Hence, the following notion is well-defined:

5.2.6 Definition. For each $R \subseteq \overline{\mathrm{R}}_{\mathbf{X}}^{\mathbb{T}}$, denote by $\overline{\mathrm{Clo}}^{\mathrm{T}}(R)$ the least clone of dual relations that contains $R$. It is called the clone of dual relations generated by $R$.

Analogue to the arguments for clones of relations, clones of dual relations also form a complete lattice with respect to inclusion.

5.2.7 Definition. Denote by $\bar{L}_{\mathrm{X}}^{* \mathbb{T}}$ the class of clones of dual relations of the typeclass $\mathbb{T}$ on $\mathbf{X}$. Then, $\overline{\mathcal{L}}_{\mathbf{X}}^{* \mathbb{T}}:=\left\langle\bar{L}_{\mathbf{X}}^{* \mathbb{T}}, \subseteq\right\rangle$ is called the lattice of clones of dual relations of the typeclass $\mathbb{T}$ on $\mathbf{X}$.

5.2.8 Example. If $x$ is the category of sets and we choose $\mathbb{T}:=\left\{\{1, \ldots, k\} \mid k \in \mathbb{N}_{+}\right\}$, then the notion of clones of dual relations and that of clones of corelations as introduced in [PR00] coincide in $x$.

\subsubsection{The Galois Connection $\overline{\mathrm{Pol}}_{\mathrm{X}}-\overline{\operatorname{Inv}}_{\mathrm{X}}^{\mathrm{T}}$}

5.2.9 Definition. We define the two operators $\overline{\operatorname{Inv}}_{\mathbf{X}}^{\mathbb{T}}: \mathfrak{P}\left(\bar{O}_{\mathbf{X}}\right) \rightarrow \mathfrak{P}\left(\overline{\mathrm{R}}_{\mathbf{X}}^{\mathbb{T}}\right)$ and $\overline{\mathrm{Pol}}_{\mathbf{X}}: \mathfrak{P}\left(\overline{\mathrm{R}}_{\mathbf{X}}^{\mathbb{T}}\right) \rightarrow \mathfrak{P}\left(\bar{O}_{\mathbf{X}}\right)$ as follows: For $G \subseteq \bar{O}_{\mathbf{X}}$ and $R \subseteq \overline{\mathrm{R}}_{\mathbf{X}}^{\mathbb{T}}$, set

$$
\begin{aligned}
& \overline{\operatorname{Inv}}_{\mathbf{X}}^{\mathbb{T}} G:=\left\{\sigma \in \overline{\mathrm{R}}_{\mathbf{X}}^{\mathbb{T}} \mid \forall g \in G: g \bar{\triangleright} \sigma\right\}, \\
& {\overline{\mathrm{Pol}_{\mathbf{X}}}}_{\mathbf{X}} R:=\left\{g \in \bar{O}_{\mathbf{X}} \mid \forall \sigma \in R: g \bar{\triangleright} \sigma\right\} .
\end{aligned}
$$

5.2.10 Definition. For $G \subseteq \bar{O}_{\mathbf{X}}, R \subseteq \overline{\mathrm{R}}_{\mathbf{X}}^{\mathbb{T}}, \mathbf{Y} \in X$ and $n \in \mathbb{N}_{+}$, we use the following notation:

$$
\begin{aligned}
& \overline{\operatorname{Inv}}_{\mathbf{X}}^{(\mathbf{Y})} G:=\left\{\sigma \in \overline{\mathrm{R}}_{\mathbf{X}}^{(\mathbf{Y})} \mid \forall g \in G: g \bar{\triangleright} \sigma\right\}, \\
& \overline{\mathrm{Pol}}_{\mathbf{X}}^{(n)} R:=\overline{\operatorname{Pol}}_{\mathbf{X}} R \cap \bar{O}_{\mathbf{X}}^{(n)} .
\end{aligned}
$$


It is easy to see that these notions are the duals of the corresponding notions from the last section.

\subsubsection{Examples.}

(i) Let $\sigma:=\left\{i d_{\mathbf{X}}\right\}$. Then, $\overline{\operatorname{Pol}}_{\mathbf{X}}\{\sigma\}$ is the set of all dual idempotent operations over $\mathbf{X}$.

(ii) Let $C \leq \bar{O}_{\mathbf{X}}$. Note that $C^{(n)}$ is a dual relation of type $n \cdot \mathbf{X}$. Now, $\overline{\operatorname{Pol}}_{\mathbf{X}} C^{(n)}$ is the largest clone $C^{\prime}$ that agrees with $C$ on its $n$-ary part, i.e., $\overline{\operatorname{Pol}}_{\mathbf{X}}^{(n)} C^{(n)}=C^{(n)}$.

(iii) If two dual operations $g, g^{\prime}$ are essentially the same, then $\overline{\operatorname{Inv}}_{\mathbf{X}}^{\mathbb{T}}\{g\}=\overline{\operatorname{Inv}}_{\mathbf{X}}^{\mathbb{T}}\left\{g^{\prime}\right\}$.

(iv) Let $X$ be the category of finite bounded partially ordered sets and let $\mathbf{Y} \in X$. Moreover, let $\sigma \in \overline{\mathrm{R}}_{\mathbf{X}}^{(\mathbf{Y})}$ contain all morphisms $r: n \cdot \mathbf{X} \rightarrow \mathbf{Y}$ with $r(x) \notin\left\{0^{\mathbf{Y}}, 1^{\mathbf{Y}}\right\}$ for all $x \notin\left\{0^{n \cdot \mathbf{X}}, 1^{n \cdot \mathbf{X}}\right\}$. Then, $\overline{\operatorname{Pol}}_{\mathbf{X}}\{\sigma\}$ is the set of all $g \in \bar{O}_{\mathbf{X}}$ such that $g(x) \notin\left\{0^{n \cdot \mathbf{X}}, 1^{n \cdot \mathbf{X}}\right\}$ for all $x \in \mathbf{X} \backslash\left\{0^{\mathbf{X}}, 1^{\mathbf{X}}\right\}$.

(v) Again, let $X$ be the category of finite bounded partially ordered sets. Let $\mathbf{Y}$ be the (up to isomorphism) unique three-element bounded poset $\langle\{0, y, 1\}, 0,1, \leq\rangle$. Let $\sigma$ be the relation that contains each morphism $r: \mathbf{X} \rightarrow \mathbf{Y}$ with $r(x)=y$ for some $x \in \mathbf{X}$. Now, we have $g \in \overline{\mathrm{Pol}}_{\mathbf{X}}\{\sigma\}$ if and only if $g(\mathbf{X})=\iota_{i}^{n}(\mathbf{X})$ for some $i \in\{1, \ldots, n\}$.

The only thing left to dualize are the local closure operators.

5.2.12 Definition. Let $G \subseteq \bar{O}_{\mathbf{X}}, R \subseteq \overline{\mathrm{R}}_{\mathbf{X}}^{\mathbb{T}}, s \geq 1$ and let $\mathbf{Z} \in X$. We define the following local closure operators:

$$
\begin{gathered}
\overline{\mathbf{Z}-\operatorname{Loc}} G:=\left\{g \in \bar{O}_{\mathbf{X}}^{(n)} \mid n \geq 1, \forall r_{1}, \ldots, r_{n} \in \mathbf{Z}^{\mathbf{X}}:\right. \\
\left.\qquad g^{\prime} \in G:\left[r_{1}, \ldots, r_{n}\right] \circ g=\left[r_{1}, \ldots, r_{n}\right] \circ g^{\prime}\right\}, \\
\overline{\mathrm{s}-\mathrm{LOC}}^{\mathbb{T}} R:=\left\{\sigma \in \overline{\mathrm{R}}_{\mathbf{X}}^{\mathbb{T}}|\forall B \subseteq \sigma,| B \mid \leq s: \exists \sigma^{\prime} \in R: B \subseteq \sigma^{\prime} \subseteq \sigma\right\} .
\end{gathered}
$$

Furthermore, let

$$
\overline{\operatorname{Loc}}^{\mathbb{T}} G:=\bigcap_{\mathbf{Z} \in \mathbb{T}} \overline{\mathbf{Z}-\operatorname{Loc}} G
$$

and

$$
\overline{\mathrm{LOC}}^{\mathbb{T}} R:=\bigcap_{s \in \mathbb{N}_{+}}{\overline{\mathrm{s}-\mathrm{LOC}^{\mathrm{T}}}}^{\mathbb{T}} R
$$

In other words, $\overline{\mathbf{Z}-\operatorname{Loc}} G$ is the set of all dual operations $g \in \bar{O}_{\mathbf{X}}$ that cannot be distinguished from a dual operation $g^{\prime} \in G$ if a cotupling of $n$ morphism from $\mathbf{X}$ to some $\mathbf{Z}$ is applied after $g$ and $g^{\prime}$. Moreover, $\overline{\mathrm{s}-\mathrm{LOC}}^{\mathbb{T}} R$ is the class of all dual relations $\sigma \in \overline{\mathrm{R}}_{\mathbf{X}}^{\mathbb{T}}$ such that, for every $B \subseteq \sigma$ with at most $s$ elements, there exists a member $\sigma^{\prime}$ of $R$ that agrees with $\sigma$ on $B$ and is contained in $\sigma$.

We have dualized every definition of the last section. Thus, each proposition, lemma and theorem from Section 5.1 holds in its dualized version. For instance, we have the following statements (recall that we write $\mathbf{Z}_{1}<\mathbf{Z}_{2}$ if there exists a monomorphism from $\mathbf{Z}_{1}$ to $\mathbf{Z}_{2}$ ): 
5.2.13 Proposition. If $\mathbf{Z}_{1}<\mathbf{Z}_{2}$, then $\overline{\mathbf{Z}_{2}-\operatorname{Loc}} G \subseteq \overline{\mathbf{Z}_{1} \text {-Loc }} G$ for all $G \subseteq \bar{O}_{\mathbf{X}}$.

5.2.14 Proposition. Let $n \in \mathbb{N}_{+}$and let $G \subseteq \bar{O}_{\mathbf{X}}$. If we have $n \cdot \mathbf{X} \gtrless \mathbf{Y}$ for some $\mathbf{Y} \in \mathbb{T}$, then $\left(\overline{\mathrm{Loc}}^{\mathbb{T}} G\right)^{(n)}=G^{(n)}$ (that is, $\overline{\mathrm{Loc}}^{\mathbb{T}} G$ and $G$ agree on their $n$-ary part).

5.2.15 Corollary. If one of the following two conditions hold, then we have $\overline{\operatorname{Loc}}^{\mathbb{T}} C=C$ for all $C \leq \bar{O}_{\mathbf{X}}$ :

(i) For each $k \in \mathbb{N}_{+}$, there exists $n \geq k$ such that $n \cdot \mathbf{X}<\mathbf{Y}$ for some $\mathbf{Y} \in \mathbb{T}$.

(ii) Each $g \in \bar{O}_{\mathbf{X}}$ is essentially at most $n$-ary and $n \cdot \mathbf{X} \gtrless \mathbf{Y}$ for some $\mathbf{Y} \in \mathbb{T}$.

5.2.16 Proposition. We have $\overline{\mathrm{LOC}}^{\mathbb{T}} R=R$ for all $R \subseteq \overline{\mathrm{R}}_{\mathbf{X}}^{\mathbb{T}}$ if and only if $X(\mathbf{X}, \mathbf{Y})$ is finite for all $\mathbf{Y} \in \mathbb{T}$.

5.2.17 Examples. Except (i), the following examples are duals of the examples presented in Example 5.1.26 (page 85). Without using duality, they also follow from Proposition 5.2 .14 and 5.2.16.

(i) If $x=$ Set and $\mathbb{T}:=\left\{\{1, \ldots, k\} \mid k \in \mathbb{N}_{+}\right\}$, then both local closure operators can be dismissed if and only if $\mathbf{X}$ is a finite set.

(ii) If $\mathbb{T}$ is a skeleton of $x$, then $\overline{\operatorname{Loc}}^{\mathbb{T}} G=G$ for all $\mathbf{X} \in X$ and $G \subseteq \bar{O}_{\mathbf{X}}$.

(iii) If $x$ is the category of finite bounded posets and we define the typeclass by setting

$$
\mathbb{T}:=\left\{\left\langle\left\{0, a_{1}, \ldots, a_{k}, 1\right\}, 0,1, \leq\right\rangle \mid k \in \mathbb{N}_{+}, a_{1}, \ldots, a_{k} \text { antichain w.r.t. } \leq\right\},
$$

then $\overline{\mathrm{LOC}}^{\mathbb{T}} R=R$ for all $\mathbf{X} \in X$ and $R \subseteq \overline{\mathrm{R}}_{\mathbf{X}}^{\mathbb{T}}$. Moreover, we have $\overline{\mathrm{Loc}}^{\mathbb{T}} G=G$ for all $G \subseteq \bar{O}_{\mathbf{X}}$ if $\mathbf{X}$ is isomorphic to one of the posets among $\mathbb{T}$. Let us show that $\mathbf{X}$ being isomorphic to some $\mathbf{Y} \in \mathbb{T}$ is also a necessary condition. Assuming that $\mathbf{X}$ is not isomorphic to some object among $\mathbb{T}$ implies that there exist $x_{1}, x_{2} \in \mathbf{X}$ such that $0^{\mathbf{X}} \neq x_{1}<x_{2} \neq 1^{\mathbf{X}}$. Define $g \in \bar{O}_{\mathbf{X}}^{(1)}$ by setting

$$
g(x):= \begin{cases}0^{\mathbf{X}} & \text { if } x \leq x_{1} \\ 1^{\mathbf{X}} & \text { if } x \geq x_{2} \\ x & \text { otherwise }\end{cases}
$$

It is easy to see that $g$ is well-defined. We will show $g \in \overline{\operatorname{Loc}}^{\mathbb{T}}\left(\bar{O}_{\mathbf{X}}^{(1)} \backslash\{g\}\right)$. Take any $\mathbf{Y} \in \mathbb{T}$ and let $r \in \mathbf{Y}^{\mathbf{X}}$. If $r$ maps $x_{1}, x_{2}$ to the same element $y \in \mathbf{Y}$, then we have $r \circ g=r \circ g^{\prime}$ for $g^{\prime} \in \bar{O}_{\mathbf{X}} \backslash\{g\}$ defined as follows:

$$
g^{\prime}(x):= \begin{cases}x_{1} & \text { if } x_{1} \leq x \leq x_{2} \\ x & \text { otherwise }\end{cases}
$$

If $r\left(x_{1}\right) \neq r\left(x_{2}\right)$, then we have $r\left(x_{1}\right)=0^{\mathbf{Y}}$ or $r\left(x_{2}\right)=1^{\mathbf{Y}}$. In the first case, we obtain $r \circ g=r \circ g^{\prime}$ for $g^{\prime} \in \bar{O}_{\mathbf{X}} \backslash\{g\}$ defined as follows:

$$
g^{\prime}(x):= \begin{cases}1^{\mathbf{X}} & \text { if } x \geq x_{2} \\ x & \text { otherwise }\end{cases}
$$


An analogue trick works for the case $r\left(x_{2}\right)=1^{\mathbf{Y}}$. Thus, $g \in \overline{\operatorname{Loc}}^{\mathbb{T}}\left(\bar{O}_{\mathbf{X}}^{(1)} \backslash\{g\}\right)$. Therefore, $\overline{\mathrm{LOC}}^{\mathbb{T}} G=G$ for all $G \subseteq \bar{O}_{\mathbf{X}}$ if and only if $\mathbf{X} \cong \mathbf{Y}$ for some $\mathbf{Y} \in \mathbb{T}$. By the Duality Principle, this also proves (iii) from Example 5.1.26 since the elements of $\mathbb{T}$ are (up to isomorphism) the duals of Boolean lattices.

By the Duality Principle, we also immediately obtain our main theorems:

5.2.18 Theorem (Galois closed sets of dual operations). Let $G \subseteq \bar{O}_{\mathbf{X}}$. Then,

(i) $\overline{\operatorname{Loc}}^{\mathbb{T}} \overline{\mathrm{Clo}}(G)=\overline{\operatorname{Pol}}_{\mathbf{X}} \overline{\operatorname{Inv}}_{\mathbf{X}}^{\mathbb{T}} G$,

(ii) $\overline{\mathbf{Z}-\text { Loc }} \overline{\operatorname{Clo}}(G)=\overline{\operatorname{Pol}}_{\mathbf{X}} \overline{\operatorname{Inv}}_{\mathbf{X}}^{(\mathbf{Z})} G$ for every $\mathbf{Z} \in \mathbb{T}$.

5.2.19 Theorem (Galois closed classes of dual relations). Let $R \subseteq \overline{\mathrm{R}}_{\mathbf{X}}^{\mathbb{T}}$. Then,

(i) $\overline{\mathrm{LOC}}^{\mathbb{T}} \overline{\mathrm{Clo}}^{\mathbb{T}}(R)=\overline{\operatorname{Inv}}_{\mathbf{X}}^{\mathbb{T}} \overline{\mathrm{Pol}}_{\mathbf{X}} R$,

(ii) $\overline{\mathrm{s}-\mathrm{LOC}}^{\mathbb{T}} \overline{\mathrm{ClO}}^{\mathbb{T}}(R)=\overline{\mathrm{Inv}}_{\mathbf{X}}^{\mathbb{T}} \overline{\mathrm{Pol}}_{\mathbf{X}}^{(s)} R$ for every $s \geq 1$.

Thus, we are able to characterize those subsets $G \subseteq \bar{O}_{\mathbf{X}}$ and those subclasses $R \subseteq \overline{\mathrm{R}}_{\mathbf{X}}^{\mathbb{T}}$ which can be represented as $\overline{\operatorname{Pol}}_{\mathbf{X}} R^{\prime}$ and $\overline{\operatorname{Inv}}_{\mathbf{X}}^{\mathbb{T}} G^{\prime}$ for some $R^{\prime} \subseteq \overline{\mathrm{R}}_{\mathbf{X}}^{\mathbb{T}}$ and $G^{\prime} \subseteq \bar{O}_{\mathbf{X}}^{\mathbf{X}}$, respectively.

5.2.20 Corollary. For $G \subseteq \bar{O}_{\mathbf{X}}$, the following are equivalent:

(1) $G \leq \bar{O}_{\mathbf{X}}$ (i.e., $G=\overline{\mathrm{Clo}}(G)$ ) and $\overline{\mathrm{LOc}}^{\mathbb{T}} G=G$.

(2) $G=\overline{\operatorname{Pol}}_{\mathbf{X}} \overline{\operatorname{Inv}}_{\mathbf{X}}^{T} G$.

(3) $\exists R \subseteq \overline{\mathrm{R}}_{\mathbf{X}}^{\mathbb{T}}: G=\overline{\mathrm{Pol}}_{\mathbf{X}} R$.

5.2.21 Corollary. For $R \subseteq \overline{\mathrm{R}}_{\mathbf{X}}^{\mathbb{T}}$, the following are equivalent:

(1) $R \leq \overline{\mathrm{R}}_{\mathbf{X}}^{\mathbb{T}}$ (i.e., $R=\overline{\mathrm{Clo}}^{\mathbb{T}}(R)$ ) and $\overline{\mathrm{LOC}}^{\mathbb{T}} R=R$.

(2) $R=\overline{\operatorname{Inv}}_{\mathbf{X}}^{T} \overline{\operatorname{Pol}}_{\mathbf{X}} R$.

(3) $\exists G \subseteq \bar{O}_{\mathbf{X}}: R=\overline{\operatorname{Inv}}_{\mathbf{X}}^{\mathbb{T}} G$.

For the sake of completeness, let us also list the following obvious corollaries:

5.2.22 Corollary. Assume that the set of morphisms from $\mathbf{X}$ to any $\mathbf{Y} \in \mathbb{T}$ is finite and that, for each $k \in \mathbb{N}_{+}$, there exists $n \geq k$ such that $n \cdot \mathbf{X} \gtrless \mathbf{Y}$ for some $\mathbf{Y} \in \mathbb{T}$. Then, the Galois closed subclasses of $\overline{\mathrm{Pol}}_{\mathbf{X}}-\overline{\mathrm{Inv}}_{\mathbf{X}}^{\mathbb{T}}$ are precisely the clones of dual operations and the clones of dual relations, respectively. Consequently, $\overline{\mathcal{L}}_{\mathbf{X}}$ and $\overline{\mathcal{L}}_{\mathbf{X}}^{* \mathbb{T}}$ are dually isomorphic via $\overline{\operatorname{Inv}}_{\mathbf{X}}^{\mathbb{T}}$.

\subsubsection{Examples.}

(i) If $\mathbf{X}$ is a finite set in the category of sets and $\mathbb{T}$ the set of all finite cardinal numbers, then $\overline{\mathcal{L}}_{\mathbf{X}}$ and $\overline{\mathcal{L}}_{\mathbf{X}}^{* \mathbb{T}}$ are dually isomorphic via $\overline{\operatorname{Inv}}_{\mathbf{X}}^{\mathbb{T}}$. 
(ii) If $x$ is the category of bounded posets and $\mathbb{T}:=\mathbb{S P}(\langle\{0,1\}, \leq\rangle)$, then we obtain the same result as in (i).

5.2.24 Definition. Denote by $\overline{\mathrm{LOc}}^{\mathbb{T}} \overline{\mathcal{L}}_{\mathbf{X}}$ and $\overline{\mathrm{LOC}}^{\mathbb{T}} \overline{\mathcal{L}}_{\mathrm{X}}^{* \mathbb{T}}$ the lattice of locally closed clones of dual operations over $\mathbf{X}$ and the lattice of locally closed clones of dual relations on $\mathbf{X}$, respectively.

5.2.25 Corollary. $\overline{\operatorname{Loc}}^{\mathbb{T}} \overline{\mathcal{L}}_{\mathbf{X}}$ and $\overline{\mathrm{LOC}}^{\mathbb{T}} \overline{\mathcal{L}}_{\mathbf{X}}^{* \mathbb{T}}$ are dually isomorphic via $\overline{\operatorname{Inv}}_{\mathbf{X}}^{\mathbb{T}}$.

Concerning Subsection 5.1.3, we have analogue results. Of course, the objects in $\mathbb{T}$ do not have to form a chain with respect to $\leqslant$ but with respect to $₹$.

5.2.26 Proposition. Let $G \subseteq \bar{O}_{\mathbf{X}}$ and let $\mathbb{T}=\left(\mathbf{Z}_{i}\right)_{i \in \mathbb{N}_{+}} \subseteq x$ with $\mathbf{Z}_{i}<\mathbf{Z}_{j}$ if and only if $i \leq j$. Then

$$
\overline{\mathbf{Z}_{1}-\mathrm{Loc}} G \supseteq \overline{\mathbf{Z}_{2}-\mathrm{Loc}} G \supseteq \overline{\mathbf{Z}_{3}-\mathrm{Loc}} G \supseteq \ldots
$$

and

$$
\overline{\operatorname{Loc}}^{T} G=\bigcap_{i \in \mathbb{N}_{+}} \overline{\mathrm{Z}_{\mathrm{i}}-\operatorname{Loc}} G .
$$

\subsection{3. $\overline{\operatorname{Pol}}_{X}-\overline{\operatorname{Inv}}_{X}^{T}$ in Concrete Categories}

For this subsection, let $(X, U)$ be concrete. As discussed in Subsection 5.1.4, we can assign a cardinality to each object in $\mathcal{A}$ by looking at its image under the forgetful functor. Since $(X, U)$ is concrete if and only if $\left(X^{o p}, U \circ(-)^{o p}\right)$ is concrete and the cardinality of the objects in the two categories coincide, we can dualize all the results from Subsection 5.1 .4 without proofs.

5.2.27 Corollary. Assume that $\mathbf{X}$ is finite. If $\mathbb{T}$ consists only of finite objects, then $\overline{\mathrm{LOC}}^{\mathbb{T}} R=R$ for all $R \subseteq \bar{O}_{\mathbf{X}}$.

5.2.28 Definition. For a cardinal number $k \geq 1$, the class

$$
\overline{\mathrm{R}}_{\mathbf{X}}^{(k)}:=\bigcup_{\mathbf{Y} \in X,|\mathbf{Y}| \leq k} \overline{\mathrm{R}}_{\mathbf{X}}^{(\mathbf{Y})}
$$

is called the class of $k$-ary dual relations. The relations $\sigma$ for which we have $\sigma \notin \overline{\mathrm{R}}_{\mathbf{X}}^{(k)}$ for all $k \in \mathbb{N}_{+}$are called dual relations of infinite arity or infinitary dual relations. Moreover, for $G \subseteq \bar{O}_{\mathbf{X}}$, set $\overline{\operatorname{Inv}}_{\mathbf{X}}^{\mathbb{T}}{ }^{(s)} G:=\overline{\operatorname{Inv}}_{\mathbf{X}}^{\mathbb{T}} G \cap \overline{\mathrm{R}}_{\mathbf{X}}^{(k)}$.

5.2.29 Definition. Let $G \subseteq \bar{O}_{\mathbf{X}}$ and let $s \geq 1$ be a cardinal number. We define the following local closure operator:

$$
\begin{aligned}
\overline{\mathrm{s}-\mathrm{Loc}}^{\mathbb{T}} G:=\left\{g \in \bar{O}_{\mathbf{X}}^{(n)} \mid\right. & n \geq 1, \forall r_{1}, \ldots, r_{n} \in \mathbf{Y}^{\mathbf{X}}, \mathbf{Y} \in \mathbb{T},|\mathbf{Y}| \leq s: \\
& \left.\exists g^{\prime} \in G:\left[r_{1}, \ldots, r_{n}\right] \circ g=\left[r_{1}, \ldots, r_{n}\right] \circ g^{\prime}\right\} .
\end{aligned}
$$


We have

$$
\overline{\operatorname{Loc}}^{\mathbb{T}} G=\bigcap_{s \geq 1} \overline{\mathrm{s}-\mathrm{Loc}}^{\mathbb{T}} G
$$

and $\overline{\mathrm{r}-\mathrm{LOc}}^{\mathbb{T}} G \supseteq \overline{\mathrm{s}-\mathrm{Loc}}^{\mathbb{T}} G$ for all $r \leq s$.

5.2.30 Proposition. If $\mathbb{T}$ consists only of finite objects, then

$$
\overline{\mathrm{Loc}}^{\mathbb{T}} G=\bigcap_{s \in \mathbb{N}_{+}} \overline{\mathrm{s}-\mathrm{LOc}}^{\mathbb{T}} G .
$$

5.2.31 Proposition (Galois closed sets of dual operations over $\mathbf{X}$ ). Let $G \subseteq \bar{O}_{\mathbf{X}}$. Then, $\overline{\mathrm{S}-\mathrm{Loc}}^{\mathrm{T}} \overline{\mathrm{Clo}}(G)=\overline{\operatorname{Pol}}_{\mathbf{X}} \overline{\operatorname{Inv}}_{\mathbf{X}}^{\mathbb{T}}(s)$ for every cardinal number $s \geq 1$.

\subsection{Completing the Duality for Clones}

In Chapter 4, we have seen how to dualize clones of operations over an object $\mathbf{A}$ in a category $\mathcal{A}$ to clones of dual operations over the dual of $\mathbf{A}$ in a dually equivalent category $x$. Let $\langle D, E, e, \epsilon\rangle$ be the dual equivalence, and denote the dual of $\mathbf{A}$ by $\mathbf{X}$.

For any choice of typeclasses $\mathbb{T} \subseteq \mathcal{A}$ and $\mathbb{T}^{\prime} \subseteq \mathcal{X}$, we now have a Galois connection $\mathrm{Pol}_{\mathbf{A}}-\mathrm{Inv}_{\mathbf{A}}^{\mathbb{T}}$ between the subsets of $O_{\mathbf{A}}$ and the subclasses of $\mathrm{R}_{\mathbf{A}}^{\mathbb{T}}$, and a Galois connection $\overline{\mathrm{Pol}}_{\mathbf{X}}-\overline{\operatorname{Inv}}_{\mathbf{X}}^{\mathbb{T}^{\prime}}$ between the subsets of $\bar{O}_{\mathbf{X}}$ and the subclasses of $\overline{\mathrm{R}}_{\mathbf{X}}^{\mathbb{T}^{\prime}}$. Recall that $\mathcal{L}_{\mathbf{A}}^{* \mathbb{T}}$ and $\overline{\mathcal{L}}_{\mathbf{X}}^{* \mathbb{T}^{\prime}}$ denote the lattice of clones of relations on $\mathbf{A}$ and the lattice of clones of dual relations on $\mathbf{X}$, respectively. Diagram 5.1 summarizes the situation. Note that the mappings in the diagram commute only for the locally closed clones of the four lattices.

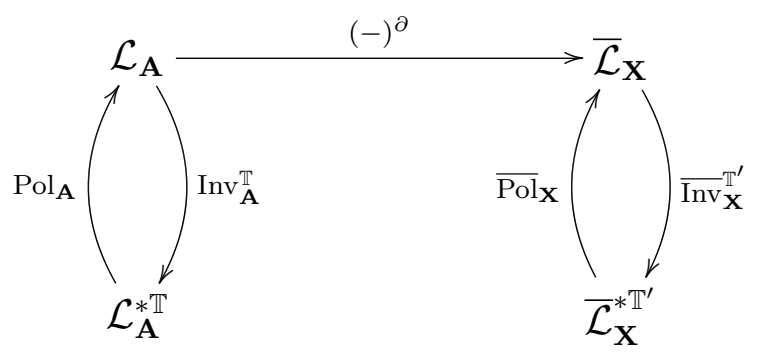

Figure 5.1.: How can the diagram be completed?

To complete this diagram, we need to find a mapping from $R_{\mathbf{A}}^{\mathbb{T}}$ to $\overline{\mathrm{R}}_{\mathbf{X}}^{\mathbb{T}^{\prime}}$ that induces a clone-isomorphism from $\mathcal{L}_{\mathbf{A}}^{* \mathbb{T}}$ to $\overline{\mathcal{L}}_{\mathbf{X}}^{* \mathbb{T}^{\prime}}$ and commutes with the other mappings in the diagram for the locally closed clones. To achieve this, it is obvious that the typeclasses $\mathbb{T}$ and $\mathbb{T}^{\prime}$ must correspond in a certain way. In fact, we require $\mathbb{T}^{\prime}$ to be the image of $\mathbb{T}$ under the functor $D$ up to isomorphism (and equivalently, $\mathbb{T}$ to be the image of $\mathbb{T}$ under the functor $E$ up to isomorphism). 
5.3.1 Definition. Let $\mathcal{A}_{1}$ and $\mathcal{A}_{2}$ be categories and let $F: \mathcal{A}_{1} \rightarrow \mathcal{A}_{2}$ be a full and faithful functor. Say that two typeclasses $\mathbb{T} \subseteq \mathcal{A}_{1}$ and $\mathbb{T}^{\prime} \subseteq \mathcal{A}_{2}$ are equivalent under $F$ if there exists a family of isomorphisms $\left(\psi_{\mathbf{B}}\right)_{\mathbf{B} \in \mathbb{T}}$ in $\mathcal{A}_{2}$ such that we have

$$
\mathbb{T}^{\prime}=\left\{\left(\psi_{\mathbf{B}} \circ F\right)(\mathbf{B}) \mid \mathbf{B} \in \mathbb{T}\right\} .
$$

In other words, $\mathbb{T}$ and $\mathbb{T}^{\prime}$ are equivalent under $F$ if and only if each $\mathbf{Y} \in \mathbb{T}^{\prime}$ is isomorphic to $F(\mathbf{B})$ for some $\mathbf{B} \in \mathbb{T}$ and, for each $\mathbf{B} \in \mathbb{T}$, we have some $\mathbf{Y} \in \mathbb{T}^{\prime}$ such that $\mathbf{Y} \cong F(\mathbf{B})$. Note that, for a dual equivalence $\langle D, E, e, \epsilon\rangle, \mathbb{T}$ and $\mathbb{T}^{\prime}$ are equivalent under $D$ if and only if they are equivalent under $E$. Of course, the easiest way to ensure the equivalence of $\mathbb{T}$ and $\mathbb{T}^{\prime}$ is to set $\mathbb{T}^{\prime}:=D(\mathbb{T})$ for a given typeclass $\mathbb{T} \subseteq \mathcal{A}$.

\subsubsection{Examples.}

(i) Two skeletons $\mathbb{T} \subseteq \mathcal{A}$ and $\mathbb{T}^{\prime} \subseteq X$ are always equivalent under $D$. In fact, if $\mathbb{T}$ is a skeleton of $\mathcal{A}$, then $\mathbb{T}$ and $\mathbb{T}^{\prime}$ are equivalent if and only if $\mathbb{T}^{\prime}$ is a skeleton of $x$.

(ii) Let $\mathcal{A}$ be the category of finite Boolean algebras, and let $x$ be the category of finite sets. Let $\langle D, E, e, \epsilon\rangle$ be the dual equivalence described in Subsection 4.2.2, and let $\mathbb{T}:=\{\mathbf{B} \in \mathcal{A}|| \mathbf{B} \mid \leq 1000\}$ and $\mathbb{T}^{\prime}:=\{\{1, \ldots, k\} \mid 1 \leq k \leq 9\}$. Then, $\mathbb{T}$ and $\mathbb{T}^{\prime}$ are equivalent under $D$.

(iii) Let $\mathcal{A}$ be the category of finite distributive lattices, let $x$ be the category of finite bounded partially ordered sets, and let $\langle D, E, e, \epsilon\rangle$ be the dual equivalence from Subsection 4.2.1. The typeclasses $\mathbb{T}:=\left\{\langle\{1, \ldots, k\}\right.$, max, $\left.\min \rangle \mid k \in \mathbb{N}_{+}\right\}$and $\mathbb{T}^{\prime}:=\left\{\langle\{1, \ldots, k\}, \leq, 1, k\rangle \mid k \in \mathbb{N}_{+}\right\}$are equivalent under $D$.

5.3.3 Lemma. Assume that $\mathbb{T}$ and $\mathbb{T}^{\prime}$ are equivalent under $D$. Then, there exists a bijective mapping $(-)^{\partial^{*}}: \mathrm{R}_{\mathbf{A}}^{\mathbb{T}} \rightarrow \overline{\mathrm{R}}_{\mathbf{X}}^{\mathbb{T}^{\prime}}$ such that $R^{\partial^{*}}$ is a clone of dual relations on $\mathbf{X}$ if and only if $R$ is a clone of relations on $\mathbf{A}$.

Proof. By assumption, there exists a family of morphisms $\left(\psi_{\mathbf{B}}\right)_{\mathbf{B} \in \mathbb{T}}$ such that we have $\mathbb{T}^{\prime}=\left\{\psi_{\mathbf{B}} \circ D(\mathbf{B}) \mid \mathbf{B} \in \mathbb{T}\right\}$. For $\sigma \in \mathbf{R}_{\mathbf{A}}^{(\mathbf{B})}$, we define

$$
\sigma^{\partial^{*}}:=\left\{\psi_{\mathbf{B}} \circ D(r) \mid r \in \sigma\right\}
$$

For each $r \in \sigma \subseteq \mathbf{R}_{\mathbf{A}}^{(\mathbf{B})}, D(r)$ is a morphism from $\mathbf{X}$ to $D(\mathbf{B})$. Hence, $\psi_{\mathbf{B}} \circ D(r)$ is a morphism from $\mathbf{X}$ to some $\mathbf{Y} \in \mathbb{T}^{\prime}$, and it follows $\sigma^{\partial^{*}} \in \overline{\mathrm{R}}_{\mathbf{X}}^{\mathbb{T}^{\prime}}$. Thus, $(-)^{\partial^{*}}$ is well-defined. Since $D$ is full and faithful and $\psi_{\mathbf{B}}$ is an isomorphism, it follows that the mapping $\psi_{\mathbf{B}} \circ D(-): \mathbb{T} \rightarrow \mathbb{T}^{\prime}$ is a bijection. Hence, $(-)^{\partial^{*}}: \mathbf{R}_{\mathbf{A}}^{\mathbb{T}} \rightarrow \overline{\mathbf{R}}_{\mathbf{X}}^{\mathbb{T}}$ is bijective.

Let us show that $R^{\partial^{*}}$ is a clone of dual relations if and only if $R$ is a clone of relations. Obviously, we have $\emptyset \in R^{\partial^{*}}$ if and only if $\emptyset \in R$. It remains to show that $R^{\partial^{*}}$ is closed under general superposition of dual relations on $\mathbf{X}$ if and only if $R$ is closed under general superposition of relations on $\mathbf{A}$. Let $I$ be an index class, let $\sigma_{i} \in R^{\left(\mathbf{B}_{i}\right)}(i \in I)$ and let $\varphi: \mathbf{B} \rightarrow \mathbf{C}$ and $\varphi_{i}: \mathbf{B}_{i} \rightarrow \mathbf{C}$ be morphisms where $\mathbf{C} \in \mathcal{A}$ and $\mathbf{B} \in \mathbb{T}$. We will show

$$
\left(\bigwedge_{\left(\varphi_{i}\right)_{i \in I}}^{\varphi}\left(\sigma_{i}\right)_{i \in I}\right)^{\partial^{*}}=\bar{\bigwedge}_{\left(\varphi_{i}^{\prime}\right)_{i \in I}}^{\varphi^{\prime}}\left(\sigma_{i}^{\partial^{*}}\right)_{i \in I}
$$


where

$$
\varphi^{\prime}:=\psi_{\mathbf{B}} \circ D(\varphi), \quad \varphi_{i}^{\prime}:=\psi_{\mathbf{B}_{i}} \circ D\left(\varphi_{i}\right)(i \in I) .
$$

We have

$$
\begin{aligned}
& \left(\bigwedge_{\left(\varphi_{i}\right)_{i \in I}}^{\varphi}\left(\sigma_{i}\right)_{i \in I}\right)^{\partial^{*}} \\
= & \left\{\psi_{\mathbf{B}} \circ D(r \circ \varphi) \mid \forall i \in I: r \circ \varphi_{i} \in \sigma_{i}, r \in \mathbf{A}^{\mathbf{C}}\right\} \\
= & \left\{\psi_{\mathbf{B}} \circ D(r \circ \varphi) \mid \forall i \in I: \psi_{\mathbf{B}_{i}} \circ D\left(r \circ \varphi_{i}\right) \in \sigma_{i}^{\partial^{*}}, r \in \mathbf{A}^{\mathbf{C}}\right\} \\
= & \left\{\psi_{\mathbf{B}} \circ D(\varphi) \circ D(r) \mid \forall i \in I: \psi_{\mathbf{B}_{i}} \circ D\left(\varphi_{i}\right) \circ D(r) \in \sigma_{i}^{\partial^{*}}, r \in \mathbf{A}^{\mathbf{C}}\right\} \\
= & \left\{\varphi^{\prime} \circ D(r) \mid \forall i \in I: \varphi_{i}^{\prime} \circ D(r) \in \sigma_{i}^{\partial^{*}}, r \in \mathbf{A}^{\mathbf{C}}\right\} \\
= & \left\{\varphi^{\prime} \circ s \mid \forall i \in I: \varphi_{i}^{\prime} \circ s \in \sigma_{i}^{\partial^{*}}, s \in D(\mathbf{C})^{\mathbf{X}}\right\} \\
= & \bigwedge_{\left(\varphi_{i}^{\prime}\right) i \in I}^{\varphi^{\prime}}\left(\sigma_{i}^{\partial^{*}}\right)_{i \in I} .
\end{aligned}
$$

It remains to show that each general superposition of relations in $R^{\partial^{*}}$ can be written as $\bar{\bigwedge}_{\left(\varphi_{i}^{\prime}\right) i \in I}^{\varphi^{\prime}}\left(\sigma_{i}^{\partial^{*}}\right)_{i \in I}$ for some $\mathbf{B}, \mathbf{B}_{i} \in \mathbb{T}, \mathbf{C} \in \mathcal{A}, \varphi: \mathbf{B} \rightarrow \mathbf{C}, \varphi_{i}: \mathbf{B}_{i} \rightarrow \mathbf{C}$ and $\sigma_{i} \in R$ $(i \in I)$. For this, let $I$ be an index class, let $\varrho_{i} \in R^{\partial^{*}\left(\mathbf{Y}_{i}\right)}(i \in I)$ and let $v: \mathbf{Z} \rightarrow \mathbf{Y}$ and $v_{i}: \mathbf{Z} \rightarrow \mathbf{Y}_{i}$ be morphisms in $X$ where $\mathbf{Y} \in \mathbb{T}^{\prime}$ and $\mathbf{Z} \in \mathcal{X}$. Without loss of generality, we can assume $\mathbf{Z}=D(\mathbf{C})$ for some $\mathbf{C} \in \mathcal{A}$. But now, there exist $\mathbf{B},\left(\mathbf{B}_{i}\right)_{i \in I} \in \mathbb{T}$ and $\varphi \in \mathcal{A}(\mathbf{B}, \mathbf{C})$ and $\varphi_{i} \in \mathcal{A}\left(\mathbf{B}_{i}, \mathbf{C}\right)(i \in I)$ such that $\varphi^{\prime}=v$ and $\varphi_{i}^{\prime}=v_{i}$ for each $i \in I$. By the bijectivity of $(-)^{\partial^{*}}$, there exist $\sigma \in R^{(\mathbf{B})}$ and $\sigma_{i} \in R^{\left(\mathbf{B}_{i}\right)}$ such that $\sigma^{\partial^{*}}=\varrho$ and $\sigma_{i}^{\partial^{*}}=\varrho_{i}$. We obtain

$$
\bar{\bigwedge}_{\left(v_{i}\right)_{i \in I}}^{v}\left(\varrho_{i}\right)_{i \in I}=\bar{\bigwedge}_{\left(\varphi_{i}^{\prime}\right) i \in I}^{\varphi^{\prime}}\left(\varrho_{i}^{\partial^{*}}\right)_{i \in I}
$$

Thus, we have found a bijective mapping between $R_{\mathbf{A}}^{\mathbb{T}}$ and $\bar{R}_{\mathbf{X}}^{\mathbb{T}^{\prime}}$ that maps clones of relations to clones of dual relations. It remains to show that Figure 5.1 (page 100) commutes for the locally closed clones if we draw $(-)^{\partial^{*}}$ into it.

5.3.4 Lemma. Assume that $\mathbb{T}$ and $\mathbb{T}^{\prime}$ are equivalent under $D$ and let $f \in O_{\mathbf{A}}, \sigma \in \mathrm{R}_{\mathbf{A}}^{\mathbb{T}}$. Then, $f \triangleright \sigma$ if and only if $f^{\partial} \bar{\triangleright} \sigma^{\partial^{*}}$.

Proof. Let $f \in O_{\mathbf{A}}^{(n)}$ and $r_{1}, \ldots, r_{n} \in \sigma \in \mathrm{R}_{\mathbf{A}}^{(\mathbf{B})}$. Recall that $(-)^{\partial}: O_{\mathbf{A}} \rightarrow \bar{O}_{\mathbf{X}}$ is defined by $f \mapsto \eta_{\operatorname{ar}(f)} \circ D(f)$ (see Section 4.1) and that, by Lemma 4.1.3 (page 41), we have

$$
D\left(\left\langle r_{1}, \ldots, r_{n}\right\rangle\right)=\left[D\left(r_{1}\right), \ldots, D\left(r_{n}\right)\right] \circ \eta_{n}
$$

Hence,

$$
\begin{aligned}
& f \circ\left\langle r_{1}, \ldots, r_{n}\right\rangle \in \sigma \\
\Longleftrightarrow & \left(f \circ\left\langle r_{1}, \ldots, r_{n}\right\rangle\right)^{\partial^{*}} \in \sigma^{\partial^{*}} \\
\Longleftrightarrow & \psi_{\mathbf{B}} \circ D\left(f \circ\left\langle r_{1}, \ldots, r_{n}\right\rangle\right) \in \sigma^{\partial^{*}}
\end{aligned}
$$




$$
\begin{aligned}
& \Longleftrightarrow \psi_{\mathbf{B}} \circ D\left(\left\langle r_{1}, \ldots, r_{n}\right\rangle\right) \circ D(f) \in \sigma^{\partial^{*}} \\
& \Longleftrightarrow \psi_{\mathbf{B}} \circ\left[D\left(r_{1}\right), \ldots, D\left(r_{n}\right)\right] \circ \eta_{n} \circ D(f) \in \sigma^{\partial^{*}} \\
& \Longleftrightarrow \psi_{\mathbf{B}} \circ\left[D\left(r_{1}\right), \ldots, D\left(r_{n}\right)\right] \circ f^{\partial} \in \sigma^{\partial^{*}} \\
& \Longleftrightarrow\left[\psi_{\mathbf{B}} \circ D\left(r_{1}\right), \ldots, \psi_{\mathbf{B}} \circ D\left(r_{n}\right)\right] \circ f^{\partial} \in \sigma^{\partial^{*}} .
\end{aligned}
$$

The claim follows since $r \mapsto \psi_{\mathbf{B}} \circ D(r)$ is bijective.

5.3.5 Lemma. Assume that $\mathbb{T}$ and $\mathbb{T}^{\prime}$ are equivalent under $D$, and let $\mathbf{C} \in \mathbb{T}, \mathbf{Z} \in \mathbb{T}^{\prime}$ such that $D(\mathbf{C}) \cong \mathbf{Z}$. Then, for all $F \subseteq O_{\mathbf{A}}$ and $R \subseteq \mathrm{R}_{\mathbf{A}}^{\mathbb{T}}$, we have

(i) $\overline{\mathbf{Z}-\operatorname{Loc}} F^{\partial}=(\mathbf{C}-\operatorname{Loc} F)^{\partial}$,

(ii) $\overline{\mathrm{Loc}^{\prime}} F^{\prime}=\left(\operatorname{Loc}^{\mathbb{T}} F\right)^{\partial}$,

(iii) ${\overline{\mathrm{s}-\mathrm{LOC}^{\mathbb{T}}}}^{\partial^{*}}=\left(\mathrm{s}-\mathrm{LOC}^{\mathbb{T}} R\right)^{\partial^{*}}$,

(iv) $\overline{\mathrm{LOC}}^{\mathbb{T}^{\prime}} R^{\partial^{*}}=\left(\mathrm{LOC}^{\mathbb{T}} R\right)^{\partial^{*}}$.

Proof. (i) Let $f \in O_{\mathbf{A}}^{(n)}$ and let $\psi_{\mathbf{C}}$ be the isomorphism from Definition 5.3.1. Set $g:=f^{\partial}$. We need to show that $g \in \overline{\mathbf{Z} \text {-Loc }} F^{\partial}$ is equivalent to $f \in \mathbf{C}$-Loc $F$. Before we do so, let us note the following equivalences for each $f^{\prime} \in F$ :

$$
\begin{aligned}
& {\left[\psi_{\mathbf{C}} \circ D\left(r_{1}\right), \ldots, \psi_{\mathbf{C}} \circ D\left(r_{n}\right)\right] \circ f^{\partial}=\left[\psi_{\mathbf{C}} \circ D\left(r_{1}\right), \ldots, \psi_{\mathbf{C}} \circ D\left(r_{n}\right)\right] \circ f^{\prime \partial}} \\
& \Longleftrightarrow \quad \psi_{\mathbf{C}} \circ\left[D\left(r_{1}\right), \ldots, D\left(r_{n}\right)\right] \circ f^{\partial}=\psi_{\mathbf{C}} \circ\left[D\left(r_{1}\right), \ldots, D\left(r_{n}\right)\right] \circ f^{\prime \partial} \\
& \Longleftrightarrow \quad\left[D\left(r_{1}\right), \ldots, D\left(r_{n}\right)\right] \circ f^{\partial}=\left[D\left(r_{1}\right), \ldots, D\left(r_{n}\right)\right] \circ f^{\prime \partial} \\
& \Longleftrightarrow \quad\left[D\left(r_{1}\right), \ldots, D\left(r_{n}\right)\right] \circ \eta_{n} \circ D(f)=\left[D\left(r_{1}\right), \ldots, D\left(r_{n}\right)\right] \circ \eta_{n} \circ D\left(f^{\prime}\right) \\
& \Longleftrightarrow \quad D\left(\left\langle r_{1}, \ldots, r_{n}\right\rangle\right) \circ D(f)=D\left(\left\langle r_{1}, \ldots, r_{n}\right\rangle\right) \circ D\left(f^{\prime}\right) \\
& \Longleftrightarrow \quad D\left(f \circ\left\langle r_{1}, \ldots, r_{n}\right\rangle\right)=D\left(f^{\prime} \circ\left\langle r_{1}, \ldots, r_{n}\right\rangle\right) \\
& \Longleftrightarrow \quad f \circ\left\langle r_{1}, \ldots, r_{n}\right\rangle=f^{\prime} \circ\left\langle r_{1}, \ldots, r_{n}\right\rangle \text {. }
\end{aligned}
$$

Moreover, $g \in \overline{\mathbf{Z}-\operatorname{Loc}} F^{\partial}$ is equivalent to the following statement: For all $s_{1}, \ldots, s_{n} \in \mathbf{Z}^{\mathbf{X}}$, there exists $g^{\prime} \in F^{\partial}$ such that:

$$
\left[s_{1}, \ldots, s_{n}\right] \circ g=\left[s_{1}, \ldots, s_{n}\right] \circ g^{\prime} .
$$

This, in turn, is equivalent to the following statement: For all $r_{1}, \ldots, r_{n} \in \mathbf{A}^{\mathbf{C}}$, there exists $f^{\prime} \in F$ such that

$$
\left[\psi_{\mathbf{C}} \circ D\left(r_{1}\right), \ldots, \psi_{\mathbf{C}} \circ D\left(r_{n}\right)\right] \circ f^{\partial}=\left[\psi_{\mathbf{C}} \circ D\left(r_{1}\right), \ldots, \psi_{\mathbf{C}} \circ D\left(r_{n}\right)\right] \circ f^{\prime \partial} .
$$

But now, we have seen above that this equation holds if and only if

$$
f \circ\left\langle r_{1}, \ldots, r_{n}\right\rangle=f^{\prime} \circ\left\langle r_{1}, \ldots, r_{n}\right\rangle,
$$

so $g \in \overline{\mathbf{Z}-\operatorname{Loc}} F^{\partial}$ is equivalent to $f \in\left(\operatorname{Loc}^{\mathbb{T}} F\right)^{\partial}$.

(ii) Since $\mathbb{T}$ and $\mathbb{T}^{\prime}$ are equivalent under $D$, (i) yields that, for each $\mathbf{C} \in \mathbb{T}$, there exists $\mathbf{Z} \in \mathbb{T}^{\prime}$ such that $\overline{\mathbf{Z}-\operatorname{Loc}} F^{\partial}=(\mathbf{C}-\operatorname{Loc} F)^{\partial}$. Thus,

$$
\operatorname{Loc}^{\mathbb{T}^{\prime}} F^{\partial}=\bigcap_{\mathbf{Z} \in \mathbb{T}^{\prime}} \overline{\mathbf{Z}-\operatorname{Loc}} F^{\partial}=\bigcap_{\mathbf{C} \in \mathbb{T}^{\prime}}(\mathbf{C}-\operatorname{Loc} F)^{\partial} \stackrel{(\star)}{=}\left(\bigcap_{\mathbf{C} \in \mathbb{T}^{\prime}} \mathbf{C}-\operatorname{Loc} F\right)^{\partial}=\left(\operatorname{Loc}^{\mathbb{T}} F\right)^{\partial}
$$


where $(\star)$ follows from the bijectivity of $(-)^{2}$.

(iii) Let $\varrho \in \mathrm{R}_{\mathbf{A}}^{\mathbb{T}}$ and let $\sigma \in R$ such that $\sigma^{\partial^{*}}=\varrho$. We have

$$
\begin{aligned}
\varrho \in \overline{\mathrm{s}-\mathrm{LOC}}^{\mathbb{T}} R^{\partial^{*}} & \Longleftrightarrow \forall B \subseteq \varrho,|B| \leq s: \exists \varrho^{\prime} \in R^{\partial^{*}}: B \subseteq \varrho^{\prime} \subseteq \varrho \\
& \Longleftrightarrow \forall B \subseteq \sigma^{\partial^{*}},|B| \leq s: \exists \sigma^{\prime} \in R: B \subseteq \sigma^{\prime \partial^{*}} \subseteq \sigma^{\partial^{*}} \\
& \Longleftrightarrow \forall B \subseteq \sigma,|B| \leq s: \exists \sigma^{\prime} \in R: B \subseteq \sigma^{\prime} \subseteq \sigma \\
& \Longleftrightarrow \sigma \in \overline{\mathrm{LOC}}^{\mathbb{T}} F .
\end{aligned}
$$

(iv) follows from (iii) in the same way that (ii) follows from (i).

We are ready to prove the following theorem:

5.3.6 Theorem. Assume that $\mathbb{T}$ and $\mathbb{T}^{\prime}$ are equivalent under $D$. There exists a bijective mapping $(-)^{\partial^{*}}: \mathrm{R}_{\mathbf{A}}^{\mathbb{T}} \rightarrow \overline{\mathrm{R}}_{\mathbf{X}}^{\mathbb{T}^{\prime}}$ that induces a clone-isomorphism from $\mathcal{L}_{\mathbf{A}}^{* \mathbb{T}}$ to $\overline{\mathcal{L}}_{\mathbf{X}}^{* \mathbb{T}^{\prime}}$ with the following properties:

(i) $\overline{\mathrm{Pol}}_{\mathbf{X}} R^{\partial^{*}}=\left(\mathrm{Pol}_{\mathbf{A}} R\right)^{\partial}$ for all $R \subseteq \mathrm{R}_{\mathbf{A}}^{\mathbb{T}}$,

(ii) $\overline{\operatorname{Inv}}_{\mathbf{X}}^{\mathbb{T}^{\prime}} F^{\partial}=\left(\operatorname{Inv}_{\mathbf{A}}^{\mathbb{T}} F\right)^{\partial^{*}}$ for all $F \subseteq O_{\mathbf{A}}$,

(iii) $\overline{\operatorname{Inv}}_{\mathbf{X}}^{\mathbb{T}^{\prime}}\left(\mathrm{Pol}_{\mathbf{A}} R\right)^{\partial}=R^{\partial^{*}}$ for locally closed $R \leq \mathrm{R}_{\mathbf{A}}^{\mathbb{T}}$,

(iv) $\overline{\mathrm{Pol}}_{\mathbf{X}}\left(\operatorname{Inv}_{\mathbf{A}}^{\mathbb{T}} F\right)^{\partial^{*}}=F^{\partial}$ for locally closed $F \leq O_{\mathbf{A}}$,

(v) The following diagram commutes:

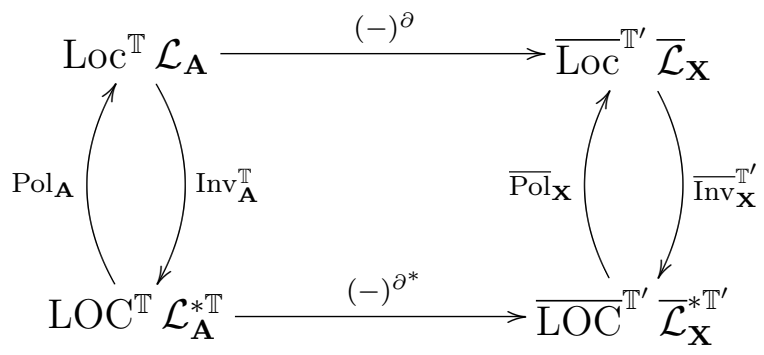

Proof. (i) Let $g \in \overline{\mathrm{Pol}}_{\mathbf{X}} R^{\partial^{*}}$, i.e., $g \bar{\triangleright} R^{\partial^{*}}$. Since $(-)^{\partial}: O_{\mathbf{A}} \rightarrow \bar{O}_{\mathbf{X}}$ is bijective, we can write $g$ as $f^{\partial}$ for some $f \in O_{\mathbf{A}}$. By Lemma 5.3.4, $f^{\partial} \bar{\triangleright} R^{\partial^{*}}$ is equivalent to $f \triangleright R$. Thus, $g \in \overline{\mathrm{Pol}}_{\mathbf{X}} R^{\partial^{*}}$ is equivalent to $g \in\left(\mathrm{Pol}_{\mathbf{A}} R\right)^{\partial}$.

(ii) Analogue to (i).

(iii) By (i), we have $\overline{\operatorname{Inv}}_{\mathbf{X}}^{\mathbb{T}^{\prime}}\left(\mathrm{Pol}_{\mathbf{A}} R\right)^{\partial}=\overline{\operatorname{Inv}}_{\mathbf{X}}^{\mathbb{T}^{\prime}} \overline{\operatorname{Pol}}_{\mathbf{X}} R^{\partial^{*}}$. Since $R$ is a locally closed clone of relations on $\mathbf{A}$, Lemma 5.3 .5 establishes that $R^{\partial^{*}}$ is a locally closed clone of dual relations on $\mathbf{X}$. Hence, $\overline{\operatorname{Inv}}_{\mathbf{X}}^{\mathbb{T}^{\prime}} \overline{\mathrm{Pol}}_{\mathbf{X}} R^{\partial^{*}}=R^{\partial^{*}}$.

(iv) Analogue to (iii).

(v) Follows directly from (i)-(iv).

The following corollary is an easy consequence: 
5.3.7 Corollary. Let $n \in \mathbb{N}_{+}$, let $\mathbf{B} \in \mathbb{T}$ and let $\mathbf{Y} \in \mathbb{T}$ such that $D(\mathbf{B}) \cong \mathbf{Y}$. Then,

(i) $\overline{\operatorname{Inv}}_{\mathbf{X}}^{(\mathbf{Y})} F^{\partial}=\left(\operatorname{Inv}_{\mathbf{A}}^{(\mathbf{B})} F\right)^{\partial^{*}}$ for all $F \subseteq O_{\mathbf{A}}$,

(ii) $\overline{\mathrm{Pol}}_{\mathbf{X}}^{(n)} R^{\partial^{*}}=\left(\mathrm{Pol}_{\mathbf{A}}^{(n)} R\right)^{\partial}$ for all $R \subseteq \mathrm{R}_{\mathbf{A}}^{\mathbb{T}}$.

Proof. (i) Since $(-)^{\partial^{*}}$ is bijective and $D(\mathbf{B}) \cong \mathbf{Y} \in \mathbb{T}$, we have $\mathrm{R}_{\mathbf{A}}^{(\mathbf{B})^{\partial^{*}}}=\overline{\mathrm{R}}_{\mathbf{X}}^{(\mathbf{Y})}$. Together with Theorem 5.3.6, we obtain

$\overline{\operatorname{Inv}}_{\mathbf{X}}^{(\mathbf{Y})} F^{\partial}=\overline{\mathrm{R}}_{\mathbf{X}}^{(\mathbf{Y})} \cap \overline{\operatorname{Inv}}_{\mathbf{X}}^{\mathbb{T}^{\prime}} F^{\partial}=\mathrm{R}_{\mathbf{A}}^{(\mathbf{B})^{\partial^{*}}} \cap\left(\operatorname{Inv}_{\mathbf{A}}^{\mathbb{T}} F\right)^{\partial^{*}}=\left(\mathrm{R}_{\mathbf{A}}^{(\mathbf{B})} \cap \operatorname{Inv}_{\mathbf{A}}^{\mathbb{T}} F\right)^{\partial^{*}}=\left(\operatorname{Inv}_{\mathbf{A}}^{(\mathbf{B})} F\right)^{\partial^{*}}$.

(ii) Follows in the same way (note that $(-)^{\partial}$ preserves the arity).

Among other things, these results offer us a possibility to dualize sets of operations without using $(-)^{\partial}$. As the following example shows, this comes in handy whenever applying $(-)^{\partial}$ would be rather difficult.

5.3.8 Example. Let $\mathcal{A}$ be the category of finite distributive lattices and let $\mathbf{A} \in \mathcal{A}$. By Subsection 4.2.1, $\mathcal{A}$ is dually equivalent to the category of finite bounded posets. Let $\langle D, E, e, \epsilon\rangle$ be the dual equivalence from Lemma 4.2.8 (page 49), let $\mathbf{X}$ be the dual of $\mathbf{A}$ and let $(-)^{\partial}: O_{\mathbf{A}} \rightarrow \bar{O}_{\mathbf{X}}$ be the corresponding clone duality. Recall that $\mathbf{X}=\langle\operatorname{Spec}(\mathbf{A}) \cup\{\emptyset, A\}, \emptyset, A, \subseteq\rangle$. Define $F \subseteq O_{\mathbf{A}}$ to be the set of operations $f \in O_{\mathbf{A}}$ such that $f\left(a_{1}, \ldots, a_{n}\right)>f\left(b_{1}, \ldots, b_{n}\right)$ whenever $a_{i}>b_{i}$ for all $i \in\{1, \ldots, n\}$. It seems as if dualizing $F$ via $(-)^{\partial}$ requires some nontrivial calculation. So, lets use the above results to dualize $F$ in another way. We have seen in Example 5.1 .18 (page 82 ) that we have $F=\operatorname{Pol}_{\mathbf{A}}\{\sigma\}$ for

$$
\sigma:=\left\{r \in \mathbf{A}^{\mathbf{B}} \mid r(0) \neq r(1)\right\}
$$

where $\mathbf{B}:=\langle\{0,1\}, \vee, \wedge\rangle$ may be in the typeclass $\mathbb{T}$. Let $\mathbb{T}^{\prime}:=D(\mathbb{T})$. Then, $\mathbb{T}$ and $\mathbb{T}^{\prime}$ are equivalent under $D$, and dualizing $\sigma$ via $(-)^{\partial^{*}}: \mathbf{R}_{\mathbf{A}}^{\mathbb{T}} \rightarrow \overline{\mathrm{R}}_{\mathbf{X}}^{\mathbb{T}}$ is easy. We have $D(\mathbf{B})=\langle\{\emptyset,\{1\}, B\}, \emptyset, B, \subseteq\rangle$ and

$$
\sigma^{\partial^{*}}=\left\{r \in D(\mathbf{B})^{\mathbf{X}} \mid \exists x \in X \backslash\{\emptyset, A\}: r(x)=\{1\}\right\} .
$$

But now, Example 5.2.11 (iv) (page 96) yields

$$
\overline{\mathrm{Pol}}_{\mathbf{X}}\left\{\sigma^{\partial^{*}}\right\}=\left\{g \in \bar{O}_{\mathbf{X}} \mid \exists i \in\{1, \ldots, n\}: g(\mathbf{X})=\iota_{i}^{n}(\mathbf{X})\right\} .
$$

By Theorem 5.3.6, we have $F^{\partial}=\overline{\mathrm{Pol}}_{\mathbf{X}}\left\{\sigma^{\partial^{*}}\right\}$, so we have successfully dualized $F$.

In the same way, one can dualize relations without using $(-)^{\partial^{*}}$.

If $\left(\mathcal{A}, U_{\mathfrak{A}}\right)$ and $\left(\mathcal{X}, U_{\mathcal{X}}\right)$ are concrete categories, we can also assign a cardinality to each object in the two categories, and we can assign arities to relations (see Subsection 5.1.4). However, $D$ does not preserve the cardinality of objects, and thus, we do not have $\left(\mathrm{R}_{\mathbf{A}}^{\mathbb{T}}{ }^{(k)}\right)^{\partial^{*}}=\overline{\mathrm{R}}_{\mathbf{X}}^{{\mathbb{T}^{\prime}}^{(k)}}$. If we want to write an equation strictly analogue to (ii) of Corollary 5.3.7, we need some restrictions. 
5.3.9 Lemma. Let $k, l$ be cardinal numbers such that, for all $\mathbf{B} \in \mathcal{A},|\mathbf{B}| \leq k$ if and only if $|D(\mathbf{B})| \leq l$. Then, $\overline{\operatorname{Inv}}_{\mathbf{X}}^{\mathbb{T}^{(l)}} F^{\partial}=\left(\operatorname{Inv}_{\mathbf{A}}^{\mathbb{T}}{ }^{(k)} F\right)^{\partial^{*}}$ for all $F \subseteq O_{\mathbf{A}}$.

Proof. By $|\mathbf{B}| \leq k \Longleftrightarrow|D(\mathbf{B})| \leq l$, we have $D\left(\mathrm{R}_{\mathbf{A}}^{\mathbb{T}^{(k)}}\right)=\overline{\mathrm{R}}_{\mathbf{X}}^{\mathbb{T}^{\prime}(l)}$. Thus, the claim follows analogously to the proof of Corollary 5.3.7.

Looking at the examples in Section 4.2 , it is easy to see that for $\mathcal{A}$ being the category of finite distributive lattices, finite median algebras or finite semilattices, there is no series of numbers $\left(l_{k}\right)_{k \in \mathbb{N}_{+}}$such that $|\mathbf{B}| \leq k \Longleftrightarrow|D(\mathbf{B})| \leq l_{k}$ for all $k \in \mathbb{N}_{+}$. For the other three examples, we obtain the following equations:

5.3.10 Examples. For all $F \subseteq O_{\mathbf{A}}$ and $k \in \mathbb{N}_{+}$, we have

(i) $\overline{\operatorname{Inv}}_{\mathbf{X}}^{\mathbb{T}^{\prime}} F^{\partial}=\left(\operatorname{Inv}_{\mathbf{A}}^{\mathbb{T}}{ }^{\left(2^{k}\right)} F\right)^{\partial^{*}}$ if $\mathcal{A}$ is the category of Boolean algebras.

(ii) $\overline{\operatorname{Inv}}_{\mathbf{X}}{ }_{\mathbb{T}^{\prime}}^{(k)} F^{\partial}=\left(\operatorname{Inv}_{\mathbf{A}}^{\mathbb{T}}{ }^{(k)} F\right)^{\partial^{*}}$ if $\mathcal{A}$ is the category of Boolean groups.

(iii) $\overline{\operatorname{Inv}}_{\mathbf{X}}^{\mathbb{T}^{\prime}} F^{\partial}=\left(\operatorname{Inv}_{\mathbf{A}}^{\mathbb{T}}{ }^{\left(2^{(k-2)}\right)} F\right)^{\partial^{*}}$ if $k \geq 2$ and $\mathcal{A}$ is the category of Boolean rings.

(iv) $\overline{\operatorname{Inv}}_{\mathbf{X}}^{\mathbb{T}^{\prime}} F^{\partial}=\left(\operatorname{Inv}_{\mathbf{A}}^{\mathbb{T}}{ }^{\left(2^{(k-1)}\right)} F\right)^{\partial^{*}}$ if $\mathcal{A}$ is the category of Boolean lattices.

\subsection{Example}

In the previous sections, we have included some small examples to illustrate the definitions and results. In particular, we have observed that, in the category of sets, $\operatorname{Pol}_{\mathbf{A}}-\operatorname{Inv}_{\mathbf{A}}^{\mathbb{T}}$ coincides with Pol-Inv if we choose $\mathbb{T}$ to be the class of finite cardinal numbers. Let us also refer to the upcoming proofs of Proposition 7.5.5, Theorem 8.3.20 and Lemma 8.7.3 for an illustration of how to use our new-found Galois theory as an efficient technique to obtain new results.

In this section, we will illustrate $\operatorname{Pol}_{\mathbf{A}}-\operatorname{Inv}_{\mathbf{A}}^{\mathbb{T}}$ by choosing $\mathcal{A}$ to be the category of finite distributive lattices. As we have seen in Subsection 4.2.1, $\mathcal{A}$ is dually equivalent to the category of finite bounded partially ordered sets. Let $X$ be this category. Since all objects in $\mathcal{A}$ and $x$ are finite, we can omit the local closure operators LOC $\mathbb{T}^{\mathbb{T}}$ and $\overline{\mathrm{LOC}^{T^{\prime}}}$. Whether we can also omit $\operatorname{Loc}^{\mathbb{T}}$ and $\overline{\operatorname{Loc}}^{\mathbb{T}^{\prime}}$ depends on the choice of the typeclasses $\mathbb{T}$ and $\mathbb{T}^{\prime}$.

Let $\mathbf{A}=\langle\{0, a, b, 1\}, \vee, \wedge\rangle$ be the distributive lattice given by the following diagram:

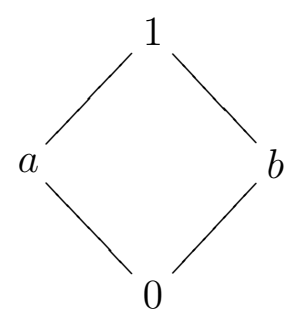

Let $\mathbf{X}:=D(\mathbf{A})$ so that we have $\mathbf{X}=\langle\{\emptyset, \uparrow a, \uparrow b, A\}, \emptyset, A, \subseteq\rangle$. We choose $\mathbb{T}$ and $\mathbb{T}^{\prime}$ to be skeletons of $\mathcal{A}$ and $X$, respectively. Thus, we have $\operatorname{Loc}^{\mathbb{T}} F=F$ for all $F \subseteq O_{\mathbf{A}}$ 
and $\overline{\mathrm{LOc}}^{\mathbb{T}} G=G$ for all $G \subseteq \bar{O}_{\mathbf{X}}$. Moreover, since $\mathbb{T}$ and $\mathbb{T}^{\prime}$ are both skeletons, they are equivalent under $D$. We have the following commuting diagram:

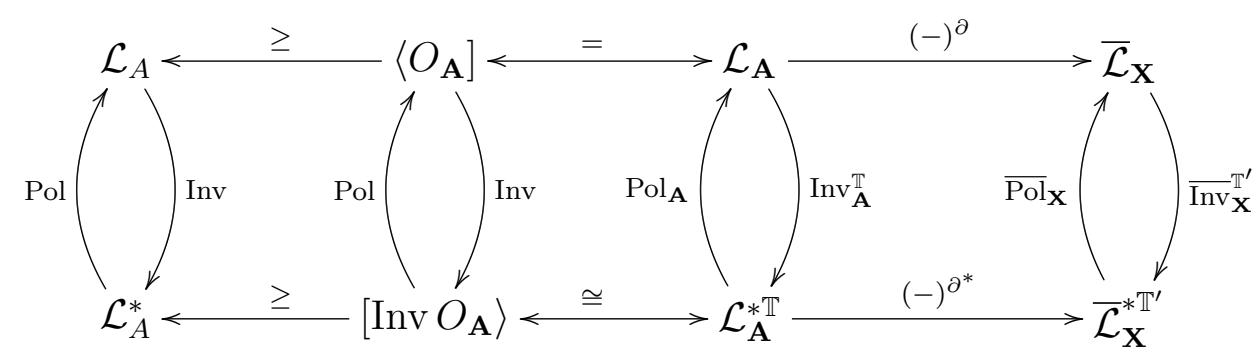

Figure 5.2.: The duality for clones over finite structures if $\mathbb{T}$ and $\mathbb{T}^{\prime}$ are equivalent

In Section 7.5 we will see that the minimal clones in the lattice of clones over a finite distributive lattice can be fully characterized: They are exactly the clones generated by nontrivial binary idempotent operations, nontrivial (unary) retractions and automorphisms of prime order. In our case, there are ten nontrivial retractions on $\mathbf{A}$, one automorphism of order 2 and (up to permutation of arguments) one nontrivial binary idempotent operation. Thus, there are twelve minimal clones over A (see Figure 5.3).

We will now describe these twelve minimal clones in terms of relations and dual relations. That is, for each minimal clone $C \leq O_{\mathbf{A}}$, we will determine the clone of relations $R \leq \mathrm{R}_{\mathbf{A}}^{\mathbb{T}}$ and the clone of dual relations $S \leq \overline{\mathrm{R}}_{\mathbf{X}}^{\mathbb{T}^{\prime}}$ such that $C=\operatorname{Pol}_{\mathbf{A}} R$ and $C=\left(\overline{\mathrm{Pol}}_{\mathbf{X}} S\right)^{\partial^{-1}}$. Since we can dismiss the local closure operators, this is equivalent to $R=\operatorname{Inv}_{\mathbf{A}}^{\mathbb{T}} C$ and $S=\overline{\operatorname{Inv}}_{\mathbf{X}}^{\mathbb{T}^{\prime}} C^{\partial}$ by Theorem 5.1.35 (page 89) and 5.2.19 (page 98). Note that this will give us all maximal clones in the lattice of clones of relations on $\mathbf{A}$ and in the lattice of clones of dual relations on $\mathbf{X}$.

In some cases, it will be easier to determine $R$ first and to obtain $S$ by dualizing $R$. For other clones, the opposite approach seems more promising.

For the four minimal clones generated by the morphisms $c_{0}, c_{a}, c_{b}, c_{1}$ we will take the first route and start by calculating $\operatorname{Inv}_{\mathbf{A}}^{\mathbb{T}} C$. Let $\mathbf{B} \in \mathbb{T}$. A relation $\sigma \in \mathrm{R}_{\mathbf{A}}^{(\mathbf{B})}$ is preserved by $c_{0}$ if and only if $r \in \sigma$ implies $c_{0} \circ r \in \sigma$. However, we have $c_{0} \circ r(x)=0$ for all $x \in \mathbf{B}$. Thus, for $\sigma \in \mathrm{R}_{\mathbf{A}}^{(\mathbf{B})}$, we have $c_{0} \triangleright \sigma$ if and only if $\sigma=\emptyset$ or $r_{0}^{\mathbf{B}} \in \sigma$ with $r_{0}^{\mathbf{B}}(x):=0$ for all $x \in \mathbf{B}$. Thus, we have

$$
R_{c_{0}}:=\operatorname{Inv}_{\mathbf{A}}^{\mathbb{T}} \operatorname{Clo}\left(c_{0}\right)=\operatorname{Inv}_{\mathbf{A}}^{\mathbb{T}}\left\{c_{0}\right\}=\{\emptyset\} \cup\left\{\sigma \in \mathrm{R}_{\mathbf{A}}^{(\mathbf{B})} \mid \mathbf{B} \in \mathbb{T}, r_{0}^{\mathbf{B}} \in \sigma\right\} .
$$

Furthermore, we can now simply apply $(-)^{\partial^{*}}$ to obtain

$$
S_{c_{0}}:=\left(R_{c_{0}}\right)^{\partial^{*}}=\{\emptyset\} \cup\left\{\sigma \in \overline{\mathrm{R}}_{\mathbf{X}}^{(\mathbf{Y})} \mid \mathbf{Y} \in \mathbb{T}^{\prime}, s_{0}^{\mathbf{Y}} \in \sigma\right\}
$$

where

$$
s_{0}^{\mathbf{Y}}(x):= \begin{cases}1^{\mathbf{Y}} & \text { if } x=A \\ 0^{\mathbf{Y}} & \text { otherwise }\end{cases}
$$




\begin{tabular}{|c||c|c|c|c|}
\hline$x$ & 0 & $a$ & $b$ & 1 \\
\hline \hline$c_{0}(x)$ & 0 & 0 & 0 & 0 \\
$c_{a}(x)$ & $a$ & $a$ & $a$ & $a$ \\
$c_{b}(x)$ & $b$ & $b$ & $b$ & $b$ \\
$c_{1}(x)$ & 1 & 1 & 1 & 1 \\
\hline$f_{1}(x)$ & 0 & 0 & $b$ & $b$ \\
$f_{2}(x)$ & 0 & $a$ & 0 & $a$ \\
$f_{3}(x)$ & 0 & 0 & 1 & 1 \\
$f_{4}(x)$ & 0 & 1 & 0 & 1 \\
$f_{5}(x)$ & $b$ & 1 & $b$ & 1 \\
$f_{6}(x)$ & $a$ & $a$ & 1 & 1 \\
\hline$p(x)$ & 0 & $b$ & $a$ & 1 \\
\hline
\end{tabular}

\begin{tabular}{|c|c||c|}
\hline$x_{1}$ & $x_{2}$ & $i\left(x_{1}, x_{2}\right)$ \\
\hline \hline 0 & 0 & 0 \\
0 & $a$ & 0 \\
0 & $b$ & $b$ \\
0 & 1 & $b$ \\
$a$ & 0 & $a$ \\
$a$ & $a$ & $a$ \\
$a$ & $b$ & 1 \\
$a$ & 1 & 1 \\
$b$ & 0 & 0 \\
$b$ & $a$ & 0 \\
$b$ & $b$ & $b$ \\
$b$ & 1 & $b$ \\
1 & 0 & $a$ \\
1 & $a$ & $a$ \\
1 & $b$ & 1 \\
1 & 1 & 1 \\
\hline
\end{tabular}

\begin{tabular}{|c||c|c|c|c|}
\hline$x$ & $\emptyset$ & $\uparrow a$ & $\uparrow b$ & $A$ \\
\hline \hline$c_{0}^{\partial}(x)$ & $\emptyset$ & $\emptyset$ & $\emptyset$ & $A$ \\
$c_{a}^{\partial}(x)$ & $\emptyset$ & $A$ & $\emptyset$ & $A$ \\
$c_{b}^{\partial}(x)$ & $\emptyset$ & $\emptyset$ & $A$ & $A$ \\
$c_{1}^{\partial}(x)$ & $\emptyset$ & $A$ & $A$ & $A$ \\
\hline$f_{1}^{\partial}(x)$ & $\emptyset$ & $\emptyset$ & $\uparrow b$ & $A$ \\
$f_{2}^{\partial}(x)$ & $\emptyset$ & $\uparrow a$ & $\emptyset$ & $A$ \\
$f_{3}^{\partial}(x)$ & $\emptyset$ & $\uparrow b$ & $\uparrow b$ & $A$ \\
$f_{4}^{\partial}(x)$ & $\emptyset$ & $\uparrow a$ & $\uparrow a$ & $A$ \\
$f_{5}^{\partial}(x)$ & $\emptyset$ & $\uparrow a$ & $A$ & $A$ \\
$f_{6}^{\partial}(x)$ & $\emptyset$ & $A$ & $\uparrow b$ & $A$ \\
\hline$p^{\partial}(x)$ & $\emptyset$ & $\uparrow b$ & $\uparrow a$ & $A$ \\
\hline$i^{\partial}(x)$ & $0^{2 \cdot \mathbf{X}}$ & $\langle 1, \uparrow a\rangle$ & $\langle 2, \uparrow b\rangle$ & $1^{2 \cdot \mathbf{X}}$ \\
\hline
\end{tabular}

Figure 5.3.: The minimal operations over $\mathbf{A}$ (up to generation of the respective minimal clone) and their duals

The cases for $c_{a}, c_{b}, c_{1}$ can be treated similarly. For $t \in\{a, b, 1\}$, we obtain

$$
R_{c_{t}}:=\operatorname{Inv}_{\mathbf{A}}^{\mathbb{T}} \operatorname{Clo}\left(c_{t}\right)=\{\emptyset\} \cup\left\{\sigma \in \mathrm{R}_{\mathbf{A}}^{(\mathbf{B})} \mid \mathbf{B} \in \mathbb{T}, r_{t}^{\mathbf{B}} \in \sigma\right\}
$$

and

$$
S_{c_{t}}:=\left(\operatorname{Inv}_{\mathbf{A}}^{\mathbb{T}} \operatorname{Clo}\left(c_{t}\right)\right)^{\partial^{*}}=\{\emptyset\} \cup\left\{\sigma \in \overline{\mathrm{R}}_{\mathbf{X}}^{(\mathbf{Y})} \mid \mathbf{Y} \in \mathbb{T}^{\prime}, s_{t}^{\mathbf{Y}} \in \sigma\right\},
$$


where $r_{t}^{\mathbf{B}}$ is the morphism that maps everything to $t$ and

$$
\begin{aligned}
& s_{a}^{\mathbf{Y}}(x):= \begin{cases}1^{\mathbf{Y}} & \text { if } x \in\{A, \uparrow a\}, \\
0^{\mathbf{Y}} & \text { otherwise },\end{cases} \\
& s_{b}^{\mathbf{Y}}(x):= \begin{cases}1^{\mathbf{Y}} & \text { if } x \in\{A, \uparrow b\}, \\
0^{\mathbf{Y}} & \text { otherwise },\end{cases} \\
& s_{1}^{\mathbf{Y}}(x):= \begin{cases}1^{\mathbf{Y}} & \text { if } x=\{A, \uparrow a, \uparrow b\}, \\
0^{\mathbf{Y}} & \text { otherwise. }\end{cases}
\end{aligned}
$$

For the six retractions $f_{1}, \ldots, f_{6}$, we will calculate $\overline{\operatorname{Inv}}_{\mathbf{X}}^{\mathbb{T}^{\prime}} \operatorname{Clo}\left(f_{i}\right)^{\partial}=\overline{\operatorname{Inv}}_{\mathbf{X}}^{\mathbb{T}^{\prime}}\left\{f_{i}^{\partial}\right\}$ and then obtain the clone of relations $\operatorname{Inv}_{\mathbf{A}}^{\mathbb{T}} \operatorname{Clo}\left(f_{i}\right)$ by applying $(-)^{\partial^{*-1}}$. We will only do the calculation for $f_{1}$ since the other cases are similar. Let $\mathbf{Y} \in \mathbb{T}^{\prime}$ and let $\sigma \in \overline{\mathrm{R}}_{\mathbf{X}}^{(\mathbf{Y})}$. For $r \in \sigma$, we have

$$
r \circ f_{1}^{\partial}(x)= \begin{cases}0^{\mathbf{Y}} & \text { if } x \in\{\emptyset, \uparrow a\}, \\ r(\uparrow b) & \text { if } x=\uparrow b, \\ 1^{\mathbf{Y}} & \text { if } x=A .\end{cases}
$$

Thus, $f_{1}^{\partial} \bar{\triangleright} \sigma$ if and only if, for all $r \in \sigma$, there exists $s \in \sigma$ with $s(\uparrow b)=r(\uparrow b)$ and $s(\uparrow a)=0^{\mathbf{Y}}$. In other words,

$$
\begin{aligned}
S_{f_{1}} & :=\overline{\operatorname{Inv}}_{\mathbf{X}}^{\mathbb{T}^{\prime}} \overline{\operatorname{Clo}}\left(f_{1}^{\partial}\right) \\
& =\overline{\operatorname{Inv}}_{\mathbf{X}}^{\mathbb{T}^{\prime}}\left\{f_{1}^{\partial}\right\} \\
& =\left\{\sigma \in \overline{\mathrm{R}}_{\mathbf{X}}^{(\mathbf{Y})} \mid \mathbf{Y} \in \mathbb{T}, \forall r \in \sigma \exists s \in \sigma: s(\uparrow b)=r(\uparrow b) \text { and } s(\uparrow a)=0^{\mathbf{Y}}\right\} .
\end{aligned}
$$

We obtain the desired $R_{f_{1}} \leq \mathrm{R}_{\mathrm{A}}^{\mathbb{T}}$ by setting

$$
\begin{aligned}
R_{f_{1}} & :=\left(S_{f_{1}}\right)^{\partial^{*-1}} \\
& =\left\{\sigma \in \mathrm{R}_{\mathbf{A}}^{(\mathbf{B})} \mid \mathbf{B} \in \mathbb{T}, \forall r \in \sigma \exists s \in \sigma: s^{-1}(\uparrow b)=r^{-1}(\uparrow b) \text { and } s^{-1}(\uparrow a)=\emptyset\right\} \\
& =\left\{\sigma \in \mathrm{R}_{\mathbf{A}}^{(\mathbf{B})} \mid \mathbf{B} \in \mathbb{T}, \forall r \in \sigma \exists s \in \sigma: s^{-1}(b)=r^{-1}(\uparrow b) \text { and } s^{-1}(\uparrow a)=\emptyset\right\} .
\end{aligned}
$$

The results for $f_{2}, \ldots, f_{6}$ can be seen in Figure 5.4.

For $p$, we will also determine $\overline{\operatorname{Inv}}_{\mathbf{X}}^{\mathbb{T}^{\prime}}\left\{p^{\partial}\right\}$. Let $\mathbf{Y} \in \mathbb{T}^{\prime}$ and let $\sigma \in \overline{\mathrm{R}}_{\mathbf{X}}^{(\mathbf{Y})}$. For $r \in \sigma$, we have

$$
r \circ p^{\partial}(x)= \begin{cases}0^{\mathbf{Y}} & \text { if } x=\emptyset, \\ r(\uparrow a) & \text { if } x=\uparrow b, \\ r(\uparrow b) & \text { if } x=\uparrow a, \\ 1^{\mathbf{Y}} & \text { if } x=A .\end{cases}
$$

Thus,

$$
\begin{aligned}
S_{p} & :=\overline{\operatorname{Inv}}_{\mathbf{X}}^{\mathbb{T}^{\prime}} \overline{\operatorname{Clo}}\left(p^{\partial}\right) \\
& =\overline{\operatorname{Inv}}_{\mathbf{X}}\left\{p^{\prime}\right\} \\
& =\left\{\sigma \in \overline{\mathrm{R}}_{\mathbf{X}}^{(\mathbf{Y})} \mid \mathbf{Y} \in \mathbb{T}, \forall r \in \sigma \exists s \in \sigma: s(\uparrow a)=r(\uparrow b) \text { and } s(\uparrow b)=r(\uparrow a)\right\} .
\end{aligned}
$$


We obtain the desired $R \leq \mathrm{R}_{\mathbf{A}}^{\mathbb{T}}$ by setting

$$
\begin{aligned}
R_{p} & :=\left(S_{p}\right)^{\partial^{*-1}} \\
& =\left\{\sigma \in \mathrm{R}_{\mathbf{A}}^{(\mathbf{B})} \mid \mathbf{B} \in \mathbb{T}, \forall r \in \sigma \exists s \in \sigma: s^{-1}(\uparrow a)=r^{-1}(\uparrow b) \text { and } s^{-1}(\uparrow b)=r^{-1}(\uparrow a)\right\} \\
& =\left\{\sigma \in \mathrm{R}_{\mathbf{A}}^{(\mathbf{B})} \mid \mathbf{B} \in \mathbb{T}, \forall r \in \sigma \exists s \in \sigma: s^{-1}(a)=r^{-1}(b) \text { and } s^{-1}(b)=r^{-1}(a)\right\} .
\end{aligned}
$$

Finally, we will also determine $\overline{\operatorname{Inv}}_{\mathbf{X}}^{\mathbb{T}^{\prime}}\left\{i^{\partial}\right\}$. Again, let $\mathbf{Y} \in \mathbb{T}^{\prime}$ and let $\sigma \in \overline{\mathrm{R}}_{\mathbf{X}}^{(\mathbf{Y})}$. For $r_{1}, r_{2} \in \sigma$, we have

$$
\left[r_{1}, r_{2}\right] \circ i^{\partial}(x)= \begin{cases}0^{\mathbf{Y}} & \text { if } x=\emptyset \\ r_{1}(\uparrow a) & \text { if } x=\uparrow a, \\ r_{2}(\uparrow b) & \text { if } x=\uparrow b, \\ 1^{\mathbf{Y}} & \text { if } x=A .\end{cases}
$$

Thus,

$$
\begin{aligned}
S_{i} & :=\overline{\operatorname{Inv}}_{\mathbf{X}}^{\mathbb{T}^{\prime}} \overline{\operatorname{Clo}}\left(i^{\partial}\right) \\
& =\left\{\sigma \in \overline{\mathrm{R}}_{\mathbf{X}}^{(\mathbf{Y})} \mid \mathbf{Y} \in \mathbb{T}, \forall r_{1}, r_{2} \in \sigma \exists s \in \sigma: s(\uparrow a)=r_{1}(\uparrow a) \text { and } s(\uparrow b)=r_{2}(\uparrow b)\right\} .
\end{aligned}
$$

We obtain the desired $R \leq \mathrm{R}_{\mathbf{A}}^{\mathbb{T}}$ by setting

$$
\begin{aligned}
R_{i} & :=\left(S_{i}\right)^{\partial^{*-1}} \\
& =\left\{\sigma \in \mathrm{R}_{\mathbf{A}}^{(\mathbf{B})} \mid \mathbf{B} \in \mathbb{T}, \forall r \in \sigma \exists s \in \sigma: s^{-1}(\uparrow a)=r_{1}^{-1}(\uparrow a) \text { and } s^{-1}(\uparrow b)=r_{2}^{-1}(\uparrow b)\right\} .
\end{aligned}
$$

We have summarized the results in the table on the next page. 


$$
\begin{aligned}
& R_{c_{0}}=\{\emptyset\} \cup\left\{\sigma \in \mathrm{R}_{\mathbf{A}}^{(\mathbf{B})} \mid \mathbf{B} \in \mathbb{T}, r_{0}^{\mathbf{B}} \in \sigma\right\} \\
& R_{c_{a}}=\{\emptyset\} \cup\left\{\sigma \in \mathrm{R}_{\mathbf{A}}^{(\mathbf{B})} \mid \mathbf{B} \in \mathbb{T}, r_{a}^{\mathbf{B}} \in \sigma\right\} \\
& R_{c_{b}}=\{\emptyset\} \cup\left\{\sigma \in \mathrm{R}_{\mathbf{A}}^{(\mathbf{B})} \mid \mathbf{B} \in \mathbb{T}, r_{b}^{\mathbf{B}} \in \sigma\right\} \\
& R_{c_{1}}=\{\emptyset\} \cup\left\{\sigma \in \mathrm{R}_{\mathbf{A}}^{(\mathbf{B})} \mid \mathbf{B} \in \mathbb{T}, r_{1}^{\mathbf{B}} \in \sigma\right\} \\
& R_{f_{1}}=\left\{\sigma \in \mathrm{R}_{\mathbf{A}}^{(\mathbf{B})} \mid \mathbf{B} \in \mathbb{T}, \forall r \in \sigma \exists s \in \sigma: s^{-1}(\uparrow a)=\emptyset \text { and } s^{-1}(b)=r^{-1}(\uparrow b)\right\} \\
& R_{f_{2}}=\left\{\sigma \in \mathrm{R}_{\mathbf{A}}^{(\mathbf{B})} \mid \mathbf{B} \in \mathbb{T}, \forall r \in \sigma \exists s \in \sigma: s^{-1}(a)=r^{-1}(\uparrow a) \text { and } s^{-1}(\uparrow b)=\emptyset\right\} \\
& R_{f_{3}}=\left\{\sigma \in \mathrm{R}_{\mathbf{A}}^{(\mathbf{B})} \mid \mathbf{B} \in \mathbb{T}, \forall r \in \sigma \exists s \in \sigma: s^{-1}(\{a, b\})=\emptyset \text { and } s^{-1}(1)=r^{-1}(\uparrow b)\right\} \\
& R_{f_{4}}=\left\{\sigma \in \mathrm{R}_{\mathbf{A}}^{(\mathbf{B})} \mid \mathbf{B} \in \mathbb{T}, \forall r \in \sigma \exists s \in \sigma: s^{-1}(\{a, b\})=\emptyset \text { and } s^{-1}(1)=r^{-1}(\uparrow a)\right\} \\
& R_{f_{5}}=\left\{\sigma \in \mathrm{R}_{\mathbf{A}}^{(\mathbf{B})} \mid \mathbf{B} \in \mathbb{T}, \forall r \in \sigma \exists s \in \sigma: s^{-1}(\{0, a\})=\emptyset \text { and } s^{-1}(1)=r^{-1}(\uparrow a)\right\} \\
& R_{f_{6}}=\left\{\sigma \in \mathrm{R}_{\mathbf{A}}^{(\mathbf{B})} \mid \mathbf{B} \in \mathbb{T}, \forall r \in \sigma \exists s \in \sigma: s^{-1}(\{0, b\})=\emptyset \text { and } s^{-1}(1)=r^{-1}(\uparrow b)\right\} \\
& R_{p}=\left\{\sigma \in \mathrm{R}_{\mathbf{A}}^{(\mathbf{B})} \mid \mathbf{B} \in \mathbb{T}, \forall r \in \sigma \exists s \in \sigma: s^{-1}(a)=r^{-1}(b) \text { and } s^{-1}(b)=r^{-1}(a)\right\} \\
& R_{i}=\left\{\sigma \in \mathrm{R}_{\mathbf{A}}^{(\mathbf{B})} \mid \mathbf{B} \in \mathbb{T}, \forall r_{1}, r_{2} \in \sigma \exists s \in \sigma: s^{-1}(\uparrow a)=r_{1}^{-1}(\uparrow a) \text { and } s^{-1}(\uparrow b)=r_{2}^{-1}(\uparrow b)\right\} \\
& S_{c_{0}}=\{\emptyset\} \cup\left\{\sigma \in \overline{\mathrm{R}}_{\mathbf{X}}^{(\mathbf{Y})} \mid \mathbf{Y} \in \mathbb{T}^{\prime}, s_{0}^{\mathbf{Y}} \in \sigma\right\} \\
& S_{c_{a}}=\{\emptyset\} \cup\left\{\sigma \in \overline{\mathrm{R}}_{\mathbf{X}}^{(\mathbf{Y})} \mid \mathbf{Y} \in \mathbb{T}^{\prime}, s_{a}^{\mathbf{Y}} \in \sigma\right\} \\
& S_{c_{b}}=\{\emptyset\} \cup\left\{\sigma \in \overline{\mathrm{R}}_{\mathbf{X}}^{(\mathbf{Y})} \mid \mathbf{Y} \in \mathbb{T}^{\prime}, s_{b}^{\mathbf{Y}} \in \sigma\right\} \\
& S_{c_{1}}=\{\emptyset\} \cup\left\{\sigma \in \overline{\mathrm{R}}_{\mathbf{X}}^{(\mathbf{Y})} \mid \mathbf{Y} \in \mathbb{T}^{\prime}, s_{1}^{\mathbf{Y}} \in \sigma\right\} \\
& S_{f_{1}}=\left\{\sigma \in \overline{\mathrm{R}}_{\mathbf{X}}^{(\mathbf{Y})} \mid \mathbf{Y} \in \mathbb{T}^{\prime}, \forall r \in \sigma \exists s \in \sigma: s(\uparrow a)=0^{\mathbf{Y}} \text { and } s(\uparrow b)=r(\uparrow b)\right\} \\
& S_{f_{2}}=\left\{\sigma \in \overline{\mathrm{R}}_{\mathbf{X}}^{(\mathbf{Y})} \mid \mathbf{Y} \in \mathbb{T}^{\prime}, \forall r \in \sigma \exists s \in \sigma: s(\uparrow a)=r(\uparrow a) \text { and } s(\uparrow b)=0^{\mathbf{Y}}\right\} \\
& S_{f_{3}}=\left\{\sigma \in \overline{\mathrm{R}}_{\mathbf{X}}^{(\mathbf{Y})} \mid \mathbf{Y} \in \mathbb{T}^{\prime}, \forall r \in \sigma \exists s \in \sigma: s(\uparrow a)=s(\uparrow b)=r(\uparrow b)\right\} \\
& S_{f_{4}}=\left\{\sigma \in \overline{\mathrm{R}}_{\mathbf{X}}^{(\mathbf{Y})} \mid \mathbf{Y} \in \mathbb{T}^{\prime}, \forall r \in \sigma \exists s \in \sigma: s(\uparrow a)=s(\uparrow b)=r(\uparrow a)\right\} \\
& S_{f_{5}}=\left\{\sigma \in \overline{\mathrm{R}}_{\mathbf{X}}^{(\mathbf{Y})} \mid \mathbf{Y} \in \mathbb{T}^{\prime}, \forall r \in \sigma \exists s \in \sigma: s(\uparrow b)=1^{\mathbf{Y}} \text { and } s(\uparrow a)=r(\uparrow a)\right\} \\
& S_{f_{6}}=\left\{\sigma \in \overline{\mathrm{R}}_{\mathbf{X}}^{(\mathbf{Y})} \mid \mathbf{Y} \in \mathbb{T}^{\prime}, \forall r \in \sigma \exists s \in \sigma: s(\uparrow a)=1^{\mathbf{Y}} \text { and } s(\uparrow b)=r(\uparrow b)\right\} \\
& S_{p}=\left\{\sigma \in \overline{\mathrm{R}}_{\mathbf{X}}^{(\mathbf{Y})} \mid \mathbf{Y} \in \mathbb{T}^{\prime}, \forall r \in \sigma \exists s \in \sigma: r(\uparrow a)=s(\uparrow b) \text { and } r(\uparrow b)=s(\uparrow a)\right\} \\
& S_{i}=\left\{\sigma \in \overline{\mathrm{R}}_{\mathbf{X}}^{(\mathbf{Y})} \mid \mathbf{Y} \in \mathbb{T}, \forall r_{1}, r_{2} \in \sigma \exists s \in \sigma: s(\uparrow a)=r_{1}(\uparrow a) \text { and } s(\uparrow b)=r_{2}(\uparrow b)\right\}
\end{aligned}
$$

Figure 5.4.: The twelve maximal clones of relations in $\mathcal{L}_{\mathbf{A}}^{* \mathbb{T}}$ and $\overline{\mathcal{L}}_{\mathbf{X}}^{* \mathbb{T}^{\prime}}$ 


\section{Clones of Dual Operations in Concrete Categories}

In this chapter, we will assume $x$ to be a concrete category. Recall that, formally, a concrete category is a category equipped with some faithful functor to the category of sets. This faithful functor allows one to think of the objects in $X$ as sets with some additional structure and of the morphisms as structure-preserving functions. Thus, without loss of generality, we can omit the faithful functor and directly assume that all objects in $X$ are sets with some additional structure and that the morphisms are structure-preserving functions between them.

For the whole chapter, let $\mathbf{X}$ be a finite object in $X$ and assume that all finite nonempty copowers of $\mathbf{X}$ also belong to $X$.

Our goal in this chapter is to examine the clones of dual operations over $\mathbf{X}$ and the structure of $\overline{\mathcal{L}}_{\mathbf{X}}$. Since each $g \in \bar{O}_{\mathbf{X}}^{(n)}$ is a morphism from $\mathbf{X}$ to $n \cdot \mathbf{X}$, it seems promising to find some information about the clones by looking at the form of the copowers of $\mathbf{X}$. Motivated by this, we will introduce certain properties that the copowers of $\mathbf{X}$ might or might not have, and we will discuss the effects that these properties have on the clones in $\overline{\mathcal{L}}_{\mathbf{X}}$. This will turn out to be a rather fruitful task. For example, we will see that $\bar{O}_{\mathbf{X}}$ having bounded essential arity is equivalent to a condition on the copowers of $\mathbf{X}$. Moreover, some conditions on the copowers of $\mathbf{X}$ will allow us to give a full characterization of the minimal clones in $\overline{\mathcal{L}}_{\mathbf{X}}$.

In the next chapter, we will then use the outcomes of this chapter to obtain new results for clones over sets.

Before we start our task, we need to deal with a technicality. It is possible that $\overline{\mathcal{L}}_{\mathbf{X}}$ contains only one clone (e.g., if $\mathbf{X}$ is an initial object in $X$ ). In the following, we will assume that this is not the case 1

\subsection{Respecting the Images of the Injection Morphisms}

We introduce the following notation: For $n, k \in \mathbb{N}_{+}, k \geq n$, let

$$
I_{n}^{k}:=\left\{\left[\iota_{i_{1}}^{k}, \ldots, \iota_{i_{n}}^{k}\right] \mid 1 \leq i_{1}<\ldots<i_{n} \leq k\right\} .
$$

Note that we have $I_{n}^{k} \subseteq X(n \cdot \mathbf{X}, k \cdot \mathbf{X})$ and that the cardinality of $I_{n}^{k}$ is $\left(\begin{array}{l}k \\ n\end{array}\right)$. We will now define a property for sets of dual operations $G \subseteq \bar{O}_{\mathbf{X}}$ that will be the key definition

${ }^{1}$ The reason for this is that we do not want to deal with some of the properties that can only occur in this very special case. For instance, if (and only if) $\left|\overline{\mathcal{L}}_{\mathbf{X}}\right|=1$, then it is possible that the injection morphisms $\iota_{1}^{3}, \iota_{2}^{3}, \iota_{3}^{3}$ coincide and that they are dual minority and dual majority operations. 
for this chapter:

6.1.1 Definition. A $k$-ary dual operation $g$ is said to respect the images of the injection morphisms to the degree $n$ provided that each $y \in g(\mathbf{X})$ can be written as $\widehat{\iota}(x)$ for some $\widehat{\iota} \in I_{n}^{k}$ and $x \in \mathbf{X}$. A set of dual operations $G$ is said to respect the images of the injection morphisms to the degree $n$ whenever each $g \in G$ respects the images of the injection morphisms to the degree $n$.

Equivalently formulated, a set of dual operations $G \subseteq \bar{O}_{\mathbf{X}}$ respects the images of the injection morphisms to the degree $n$ if we have

$$
\bigcup_{g \in G^{(k)}} g(\mathbf{X}) \subseteq \bigcup_{\widehat{\iota} \in I_{n}^{k}} \widehat{\iota}(n \cdot \mathbf{X})
$$

for all $k \geq n$. Figure 6.1 visualizes this property.

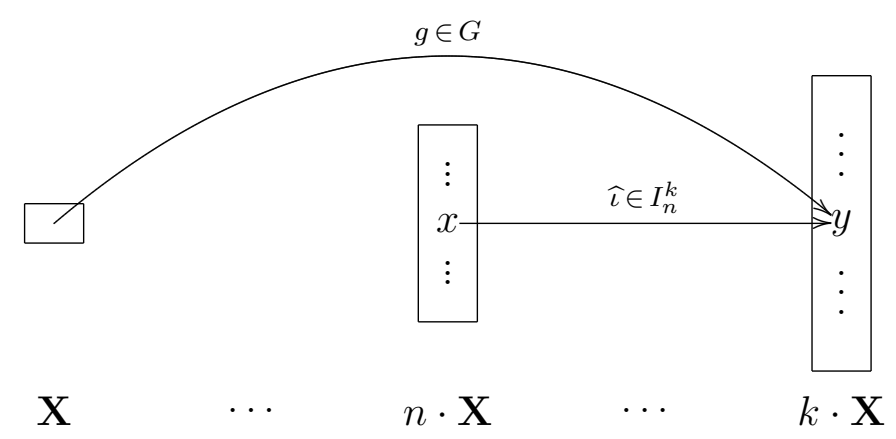

Figure 6.1.: $G \subseteq \bar{O}_{\mathbf{X}}$ respects the images of the injection morphisms to the degree $n$ if, for each $k$-ary dual operations $g \in G$ with $k \geq n$, every $y \in g(\mathbf{X})$ is in the image of some $\widehat{\iota} \in I_{n}^{k}$

Whenever a set of dual operations $G$ respects the images of the injection morphisms to the degree $n$, then it also respects the images of the injection morphisms to the degree $m$ for all $m \geq n$. This will be shown in the next proposition:

6.1.2 Proposition. Let $m \geq n$. If a set of dual operations $G \subseteq \bar{O}_{\mathbf{X}}$ respects the images of the injection morphisms to the degree $n$, then $G$ respects the images of the injection morphisms to the degree $m$.

Proof. Let $k \geq m \geq n$ and let $y=\bigcup_{g \in G^{(k)}} g(\mathbf{X})$. By assumption, there exist integers $1 \leq i_{1}<\ldots<i_{n} \leq k$ and $x \in n \cdot \mathbf{X}$ such that $y=\left[\iota_{i_{1}}^{k}, \ldots, \iota_{i_{n}}^{k}\right](x)$. Now take any $\widehat{\iota} \in I_{m}^{k}$ and integers $j_{1}, \ldots, j_{n} \in\{1, \ldots, m\}$ such that

$$
\widehat{\iota} \circ\left[\iota_{j_{1}}^{m}, \ldots, \iota_{j_{n}}^{m}\right]=\left[\iota_{i_{1}}^{k}, \ldots, \iota_{i_{n}}^{k}\right] .
$$

It is easy to see that this is always possible. We obtain

$$
y=\left[\iota_{i_{1}}^{k}, \ldots, \iota_{i_{n}}^{k}\right](x)=\widehat{\iota}(\underbrace{\left[\iota_{i_{1}}^{m}, \ldots, \iota_{i_{n}}^{m}\right](x)}_{\in m \cdot \mathbf{X}}) .
$$


It is important to note that $G$ respecting the images of the injection morphisms to the degree $n$ does not imply that $\overline{\mathrm{Clo}}(G)$ respects the images of the injection morphisms to the degree $n$.

To assure that any set of dual operations $G \subseteq \bar{O}_{\mathbf{x}}$ respects the images of the injection morphisms to the degree $n$, we can require that $\mathbf{X}$ has the following property:

6.1.3 Definition. For $n \in \mathbb{N}_{+}, \mathbf{X}$ is said to have non-deformed copowers of degree $n$ or the copowers of $\mathbf{X}$ are said to be non-deformed to the degree $n$ provided that each $y \in k \cdot \mathbf{X}$ can be written as $\widehat{\iota}(x)$ for some $\widehat{\iota} \in I_{n}^{k}$ and $x \in \mathbf{X}$.

In other words, $\mathbf{X}$ has non-deformed copowers of degree $n$ if we have

$$
k \cdot \mathbf{X}=\bigcup_{\widehat{\iota} \in I_{n}^{k}} \widehat{\iota}(n \cdot \mathbf{X})
$$

for all $k \geq n$.

If $\mathbf{X}$ has non-deformed copowers of degree $n$, then it is evident that each set of dual operations $G \subseteq \bar{O}_{\mathbf{x}}$, and in particular each clone $C \leq \bar{O}_{\mathbf{X}}$, respects the images of the injection morphisms to the degree $n$. In general, the converse is not true.

6.1.4 Proposition. Let $m \geq n$. If $\mathbf{X}$ has non-deformed copowers of degree $n$, then $\mathbf{X}$ has non-deformed copowers of degree $m$.

Proof. The proof is analogue to the one of Proposition 6.1.2.

In many well-known categories, all objects have non-deformed copowers of degree 1. Among them are the category of sets, the category of (bounded) posets, the category of pointed spaces, the category of graphs, the category of topological spaces and any quasivariety of relational structures. We also know categories in which almost no objects have non-deformed copowers of any degree from Section 4.2: In the category of Boolean groups or the category of bounded semilattices, only the trivial objects (Boolean groups with one element and bounded semilattices with at most two elements) have non-deformed copowers. It can also happen that an object $\mathbf{X}$ has non-deformed copowers to the degree $k$ but not to the degree $l$ for some $l<k$. We will see such a case in Example 7.1.4 (page 128. Hence, the converse of Proposition 6.1.4 is not true, and neither is the converse of Proposition 6.1.2.

\subsection{The Essential Arity of Clones of Dual Operations}

In this section, we will show that the essential arity of the dual operations in a clone $C \leq \bar{O}_{\mathbf{x}}$ is bounded if and only if there exists an integer $n$ such that $C$ respects the images of the injection morphisms to the degree $n$.

We start by showing the following lemma:

6.2.1 Lemma. Let $k, m \in \mathbb{N}_{+}$and let $M \subseteq\{1, \ldots, k\}$ be an m-element set. For all $g \in \bar{O}_{\mathbf{X}}^{(k)}$, the following two statements are equivalent: 
(1) There exist pairwise distinct integers $i_{1}, \ldots, i_{m} \in M$ and $g^{\prime} \in \bar{O}_{\mathbf{X}}^{(m)}$ such that $g=\left[\iota_{i_{1}}^{k}, \ldots, \iota_{i_{m}}^{k}\right] \circ g^{\prime}$.

(2) For all $t \in\{1, \ldots, k\} \backslash M, g$ does not depend on its $t$-th argument.

Proof. (1) $\Longrightarrow(2)$. We will show a stronger claim by proving that (2) holds whenever we have $g(\mathbf{X}) \subseteq \bigcup_{i_{1}, \ldots, i_{n} \in M}\left[\iota_{i_{1}}^{k}, \ldots, \iota_{i_{n}}^{k}\right](n \cdot \mathbf{X})$ for some integer $n \in\{1, \ldots, k\}$. Let $t \in\{1, \ldots, k\} \backslash M$. We need to show that there exists $h \in \bar{O}_{\mathbf{X}}^{(k-1)}$ such that $g=\widetilde{\iota}_{t}^{k} \circ h$. Let $x \in \mathbf{X}$. By assumption, there exists $y \in n \cdot \mathbf{X}$ and integers $i_{1}, \ldots, i_{n} \neq t$ such that

$$
\left[\iota_{i_{1}}^{k}, \ldots, \iota_{i_{n}}^{k}\right](y)=g(x)
$$

Setting $h:=\left[\iota_{1}^{k-1}, \ldots, \iota_{t-1}^{k-1}, \iota_{t-1}^{k-1}, \iota_{t}^{k-1}, \ldots, \iota_{k-1}^{k-1}\right] \circ g$, we obtain

$$
\begin{aligned}
h(x) & =\left(\left[\iota_{1}^{k-1}, \ldots, \iota_{t-1}^{k-1}, \iota_{t-1}^{k-1}, \iota_{t}^{k-1}, \ldots, \iota_{k-1}^{k-1}\right] \circ g\right)(x) \\
& \stackrel{6.1]}{=}\left(\left[\iota_{1}^{k-1}, \ldots, \iota_{t-1}^{k-1}, \iota_{t-1}^{k-1}, \iota_{t}^{k-1}, \ldots, \iota_{k-1}^{k-1}\right] \circ\left[\iota_{i_{1}}^{k}, \ldots, \iota_{i_{n}}^{k}\right]\right)(y) \\
& =\left[\iota_{i_{1}^{\prime}}^{k-1}, \ldots, \iota_{i_{n}^{\prime}}^{k-1}\right](y),
\end{aligned}
$$

where $i_{j}^{\prime}=i_{j}$ if $i_{j} \leq t-1$ and $i_{j}^{\prime}=i_{j}-1$ if $i_{j}>t-1$. In any case, it follows

$$
\begin{aligned}
\left(\widetilde{\iota}_{t}^{k} \circ h\right)(x) & =\left(\widetilde{\iota}_{t}^{k} \circ\left[\iota_{i_{1}^{\prime}}^{k}, \ldots, \iota_{i_{n}^{\prime}}^{k}\right]\right)(y) \\
& =\left(\left[\iota_{1}^{k}, \ldots, \iota_{t-1}^{k}, \iota_{t+1}^{k}, \ldots, \iota_{k}^{k}\right] \circ\left[\iota_{i_{1}^{\prime}}^{k-1}, \ldots, \iota_{i_{n}^{\prime}}^{k-1}\right]\right)(y) \\
& =\left[\iota_{i_{1}}^{k} \ldots, \iota_{i_{n}}^{k}\right](y) \\
& \stackrel{6.1}{=} g(x) .
\end{aligned}
$$

$(2) \Longrightarrow(1)$. Without loss of generality, we can assume $M=\{1, \ldots, k-l\}$ for some $1 \leq l<k$. Since $g$ does not depend on its $k$-th argument, there exists $g_{1} \in \bar{O}_{\mathbf{X}}^{(k-1)}$ such that

$$
g=\left[\iota_{1}^{k}, \ldots, \iota_{k-1}^{k}\right] \circ g_{1} .
$$

If $l=1$, we are done. For the case $l \geq 2$, we will now show that there exists $g_{2} \in \bar{O}_{\mathbf{X}}^{(k-2)}$ such that $g_{1}=\left[\iota_{1}^{k-1}, \ldots, \iota_{k-2}^{k-1}\right] \circ g_{2}$. We know that $g$ does not depend on its $(k-1)$-th argument. Hence, there exists $h \in \bar{O}_{\mathbf{X}}^{(k-1)}$ such that $g=\left[\iota_{1}^{k}, \ldots, \iota_{k-2}^{k}, \iota_{k}^{k}\right] \circ h$. But now,

$$
\begin{aligned}
g_{1} & =\left[\iota_{1}^{k-1}, \ldots, \iota_{k-1}^{k-1}\right] \circ g_{1} \\
& =\left[\iota_{1}^{k-1}, \ldots, \iota_{k-1}^{k-1}, \iota_{k-2}^{k-1}\right] \circ\left[\iota_{1}^{k}, \ldots, \iota_{k-1}^{k}\right] \circ g_{1} \\
& \stackrel{6.2}{=}\left[\iota_{1}^{k-1}, \ldots, \iota_{k-1}^{k-1}, \iota_{k-2}^{k-1}\right] \circ g \\
& =\left[\iota_{1}^{k-1}, \ldots, \iota_{k-1}^{k-1}, \iota_{k-2}^{k-1}\right] \circ\left[\iota_{1}^{k}, \ldots, \iota_{k-2}^{k}, \iota_{k}^{k}\right] \circ h \\
& =\left[\iota_{1}^{k-1}, \ldots, \iota_{k-2}^{k-1}, \iota_{k-2}^{k-1}\right] \circ h \\
& =\left[\iota_{1}^{k-1}, \ldots, \iota_{k-2}^{k-1}\right] \circ\left[\iota_{1}^{k-2}, \ldots, \iota_{k-2}^{k-2}, \iota_{k-2}^{k-2}\right] \circ h .
\end{aligned}
$$




\section{Clones of Dual Operations in Concrete Categories}

Thus, for $g_{2}:=\left[\iota_{1}^{k-2}, \ldots, \iota_{k-2}^{k-2}, \iota_{k-2}^{k-2}\right] \circ h$, we have $g_{1}=\left[\iota_{1}^{k-1}, \ldots, \iota_{k-2}^{k-1}\right] \circ g_{2}$, and hence,

$$
g \stackrel{[6.2]}{=}\left[\iota_{1}^{k}, \ldots, \iota_{k-1}^{k}\right] \circ g_{1}=\left[\iota_{1}^{k}, \ldots, \iota_{k-1}^{k}\right] \circ\left[\iota_{1}^{k-1}, \ldots, \iota_{k-2}^{k-1}\right] \circ g_{2} .
$$

We can repeat this argument until we finally obtain $g_{l} \in \bar{O}_{\mathbf{X}}^{(k-l)}$ with

$$
g=\left[\iota_{1}^{k}, \ldots, \iota_{k-1}^{k}\right] \circ\left[\iota_{1}^{k-1}, \ldots, \iota_{k-2}^{k-1}\right] \circ \ldots \circ\left[\iota_{1}^{k-l+1}, \ldots, \iota_{k-l}^{k-l+1}\right] \circ g_{l}=\left[\iota_{1}^{k}, \ldots, \iota_{k-l}^{k}\right] \circ g_{l} .
$$

The claim follows for $g^{\prime}:=g_{l}$.

If we additionally assume that $g$ respects the images of the injection morphisms to the degree $n$, then we can state a stronger version of this lemma:

6.2.2 Lemma. Let $k, n \in \mathbb{N}_{+}$with $k \geq n$. Let $g$ be a $k$-ary dual operation that respects the images of the injection morphisms to the degree $n$ and let $M \subseteq\{1, \ldots, k\}$. The following two statements are equivalent:

(1) $g(\mathbf{X}) \subseteq \bigcup_{i_{1}, \ldots, i_{n} \in M}\left[\iota_{i_{1}}^{k}, \ldots, \iota_{i_{n}}^{k}\right](n \cdot \mathbf{X})$.

(2) For all $t \in\{1, \ldots, k\} \backslash M, g$ does not depend on its $t$-th argument.

Proof. (1) $\Longrightarrow(2)$. We have already shown the claim in the proof of Lemma 6.2.1.

$(2) \Longrightarrow(1)$. Let $z \in \mathbf{X}$. We need to show that there exist $j_{1}, \ldots, j_{n} \in M$ and $x \in n \cdot \mathbf{X}$ such that $g(z)=\left[\iota_{j_{1}}^{k}, \ldots, \iota_{j_{n}}^{k}\right](x)$. Let $m:=|M|$. Without loss of generality, we can assume $M=\{1, \ldots, m\}$. Since $g$ can only depend on the arguments among $M$, we can apply Lemma 6.2.1 to obtain $g^{\prime} \in \bar{O}_{\mathbf{X}}^{(m)}$ such that $g=\left[\iota_{1}^{k}, \ldots, \iota_{m}^{k}\right] \circ g^{\prime}$. Thus,

$$
\begin{aligned}
g & =\left[\iota_{1}^{k}, \ldots, \iota_{m}^{k}\right] \circ g^{\prime} \\
& =\left[\iota_{1}^{k}, \ldots, \iota_{m}^{k}, \iota_{1}^{k}, \ldots, \iota_{1}^{k}\right] \circ\left[\iota_{1}^{k}, \ldots, \iota_{m}^{k}\right] \circ g^{\prime} \\
& =\left[\iota_{1}^{k}, \ldots, \iota_{m}^{k}, \iota_{1}^{k}, \ldots, \iota_{1}^{k}\right] \circ g .
\end{aligned}
$$

Since $g$ respects the images of the injection morphisms to the degree $n$, there exist integers $i_{1}, \ldots, i_{n} \in\{1, \ldots, k\}$ and some $x \in n \cdot \mathbf{X}$ such that $g(z)=\left[\iota_{i_{1}}^{k}, \ldots, \iota_{i_{n}}^{k}\right](x)$. We obtain

$$
\begin{aligned}
g(z) & \stackrel{[6.3]}{=}\left(\left[\iota_{1}^{k}, \ldots, \iota_{m}^{k}, \iota_{1}^{k}, \ldots, \iota_{1}^{k}\right] \circ g\right)(z) \\
& =\left(\left[\iota_{1}^{k}, \ldots, \iota_{m}^{k}, \iota_{1}^{k}, \ldots, \iota_{1}^{k}\right] \circ\left[\iota_{i_{1}}^{k}, \ldots, \iota_{i_{n}}^{k}\right]\right)(x) \\
& =\left[\iota_{i_{1}^{\prime}}^{k}, \ldots, \iota_{i_{n}^{\prime}}^{k}\right](x),
\end{aligned}
$$

where $i_{j}^{\prime}=i_{j}$ if $i_{j} \leq m$ and $i_{j}^{\prime}=1$ if $i_{j}>m$. Hence, $\left\{i_{1}^{\prime}, \ldots, i_{n}^{\prime}\right\} \subseteq\{1, \ldots, m\}=M$.

We are ready to prove the main result of this section.

6.2.3 Theorem. Let $C \leq \bar{O}_{\mathbf{x}}$. The following two statements are equivalent:

(1) There exists $k \in \mathbb{N}_{+}$such that every $g \in C$ is essentially at most $k$-ary. 
(2) There exists $n \in \mathbb{N}_{+}$such that $C$ respects the images of the injection morphisms to the degree $n$.

Proof. (1) $\Longrightarrow(2)$. We assume that, for all $n \in \mathbb{N}_{+}, C$ does not respect the images of the injection morphisms to the degree $n$. Then, there exist $g \in C^{(n+1)}$ and $x \in \mathbf{X}$ such that $g(x) \notin \widehat{\iota}(n \cdot \mathbf{X})$ for all $\widehat{\iota} \in I_{n}^{n+1}$. If $g$ has a nonessential argument, then there exists $g^{\prime} \in \bar{O}_{\mathbf{X}}^{(n)}$ such that $g(x)=\widetilde{\iota}_{i}^{n} \circ g^{\prime}(x)$ for some $i \in\{1, \ldots, n\}$. Since $\widetilde{\iota}_{i}^{n} \in I_{n}^{n+1}$, this is a contradiction to $g(x) \notin \widehat{\iota}(n \cdot \mathbf{X})$ for all $\widehat{\iota} \in I_{n}^{n+1}$. Thus, for each $n \in \mathbb{N}_{+}$, there exists an $n$-ary dual operation $g \in C$ with only essential arguments. It follows that the essential arity of all dual operations in $C$ is not bounded.

$(2) \Longrightarrow(1)$. Set $l:=|\mathbf{X}|$ and let $x_{1}, \ldots, x_{l}$ be the elements in $X$. Furthermore, set $k:=n l$. We will show that every $g \in C$ is essentially at most $k$-ary. Let $r>k$ and let $g \in C^{(r)}$. Since $r>n$, it follows by $C$ respecting the images of the injection morphisms to the degree $n$ that, for all $x_{j} \in \mathbf{X}$, there exist integers $1 \leq i_{j, 1}<\ldots<i_{j, n} \leq r$ and $y_{j} \in n \cdot \mathbf{X}$ such that $\left[\iota_{i_{j, 1}}^{r}, \ldots, \iota_{i_{j, n}}^{r}\right]\left(y_{j}\right)=g\left(x_{j}\right)$. Set

$$
M:=\left\{i_{1,1}, \ldots, i_{1, n}, \ldots, i_{l, 1}, \ldots, i_{l, n}\right\} .
$$

Each $y \in g(\mathbf{X})$ can be written as $\left[\iota_{i_{1}}^{r}, \ldots, \iota_{i_{n}}^{r}\right](x)$ for some $x \in \mathbf{X}$ and $i_{1}, \ldots, i_{n} \in M$. Thus, by Lemma 6.2.2, $g$ can only depend on its $t$-th argument if $t \in M$. This implies that $g$ is essentially at most $|M|$-ary, and the claim follows since we have $|M| \leq k$.

Note that the second part of the proof establishes the following upper bound for the essential arity of clones that respect the images of the injection morphisms to a certain degree:

6.2.4 Corollary. Let $n \in \mathbb{N}_{+}$and let $C$ be a clone of dual operations over $\mathbf{X}$ that respects the images of the injection morphisms to the degree $n$. Then, the essential arity of the dual operations among $C$ is bounded by $n *|\mathbf{X}|$.

The following corollary is another consequence:

6.2.5 Corollary. Assume that there exists $n \in \mathbb{N}_{+}$such that $\bar{O}_{\mathbf{X}}$ respects the images of the injection morphisms to the degree $n$. Then,

(i) $\overline{\mathcal{L}}_{\mathbf{X}}$ is finite,

(ii) $\bar{O}_{\mathbf{X}}$ is finitely generated.

Proof. By Theorem 6.2.3, the essential arity of the dual operations among $\bar{O}_{\mathbf{X}}$ is bounded by some $k \in \mathbb{N}_{+}$. Thus, every clone in $\overline{\mathcal{L}}_{\mathbf{X}}$ is generated by its $k$-ary part. Both claims follow immediately.

\footnotetext{
${ }^{2}$ The symbol $*$ denotes the usual multiplication of numbers. It is chosen to avoid confusion with the notation of copowers.
} 


\subsection{Identities of Clones of Dual Operations}

From now on until the end of this chapter, most of the results will be applicable for clones that respect the images of the injection morphisms to the degree 1. As a motivation, let us recall that, in many well-known categories, all dual operations in the category respect the images of the injection morphisms to the degree 1.

In this section, we will examine the identities that hold for the dual operations in a given clone $C \leq \bar{O}_{\mathbf{X}}$. We will see that if $C$ respects the images of the injection morphisms to the degree 1 , surprisingly many identities can be ruled out.

6.3.1 Theorem. Let $g \in \bar{O}_{\mathbf{X}}^{(n)}$ and let $h \in \bar{O}_{\mathbf{X}}^{(r)}$. Assume that $g$ respects the images of the injection morphisms to the degree 1 and that the following identity holds:

$$
\left[\iota_{i_{1}}^{n}, \iota_{i_{2}}^{n}, \ldots, \iota_{i_{n}}^{n}\right] \circ g=\left[\iota_{j_{1}}^{n}, \ldots, \iota_{j_{r}}^{n}\right] \circ h .
$$

Then, $g$ does not depend on its $t$-th argument for all $t \in\{1, \ldots, n\}$ with $i_{t} \notin\left\{j_{1}, \ldots, j_{r}\right\}$.

Proof. Let $t \in\{1, \ldots, n\}$ such that $i_{t} \notin\left\{j_{1}, \ldots, j_{r}\right\}$. Without loss of generality, we can assume $t=1$ and $i_{1}=1$. We need to show that the identity

$$
\left[\iota_{1}^{n}, \iota_{i_{2}}^{n}, \ldots, \iota_{i_{n}}^{n}\right] \circ g=\left[\iota_{j_{1}}^{n}, \ldots, \iota_{j_{r}}^{n}\right] \circ h
$$

implies that $g$ does not depend on its first argument. For contradiction, assume that $g$ depends on its first argument. Lemma 6.2.2 yields $g(\mathbf{X}) \nsubseteq \bigcup_{i=2}^{n} \iota_{i}^{n}(\mathbf{X})$, so there exists $x \in \mathbf{X}$ such that $g(x) \notin \iota_{i}^{n}(\mathbf{X})$ for all $i \in\{2, \ldots, n\}$. Since $g(\mathbf{X}) \subseteq \bigcup_{i=1}^{n} \iota_{i}^{n}(\mathbf{X})$, this implies that there exists $y \in \mathbf{X}$ such that $g(x)=\iota_{1}^{n}(y)$. Hence,

$$
\left(\left[\iota_{1}^{n}, \iota_{i_{2}}^{n}, \ldots, \iota_{i_{n}}^{n}\right] \circ g\right)(x)=\left(\left[\iota_{1}^{n}, \iota_{i_{2}}^{n}, \ldots, \iota_{i_{n}}^{n}\right] \circ \iota_{1}^{n}\right)(y)=\iota_{1}^{n}(y)=g(x) \notin \iota_{i}^{n}(\mathbf{X})
$$

for all $i \in\{2, \ldots, n\}$. On the other hand, we can write $h(x)$ as $\iota_{s}^{r}(z)$ for some integer $s \in\{1, \ldots, r\}$ and some $z \in \mathbf{X}$. Thus,

$$
\left(\left[\iota_{j_{1}}^{n}, \ldots, \iota_{j_{r}}^{n}\right] \circ h\right)(x)=\left(\left[\iota_{j_{1}}^{n}, \ldots, \iota_{j_{r}}^{n}\right] \circ \iota_{s}^{r}\right)(z)=\iota_{j_{s}}^{n}(z) \in \iota_{j_{s}}^{n}(\mathbf{X}) .
$$

Since $j_{s} \in\left\{j_{1}, \ldots, j_{r}\right\} \subseteq\{2, \ldots, n\}$, we have successfully contradicted

$$
\left[\iota_{1}^{n}, \iota_{i_{2}}^{n}, \ldots, \iota_{i_{n}}^{n}\right] \circ g=\left[\iota_{j_{1}}^{n}, \ldots, \iota_{j_{r}}^{n}\right] \circ h
$$

as the two sides of the equation do not coincide for $x$.

In other words, if $g$ respects the images of the injection morphisms to the degree 1 and satisfies an identity that is irregular in its $t$-th argument (see Definition 3.1.12, page 37), then $g$ does not depend on its $t$-th argument.

This is a rather strong condition on the dual operations in $C$. For example, it implies that $C$ cannot contain dual operations that depend on all their arguments but satisfy an irregular identity (such as dual majority operations or dual minority operations). Furthermore, many other types of dual operations cannot occur. As an example, we show that a clone that respects the images of the injection morphisms to the degree 1 cannot contain a dual near-unanimity operation or a proper semiinjection of arity at least 3: 
6.3.2 Corollary. Let $C \leq \bar{O}_{\mathbf{x}}$. Assume that $C$ respects the images of the injection morphisms to the degree 1 . Then, $C$ cannot contain dual near-unanimity operations and proper semiinjection of arity greater than 2 .

Proof. Let $m \in C$ be an $n$-ary near-unanimity operation $(n \geq 3)$. The following identities hold:

$$
\begin{gathered}
{\left[\iota_{2}^{n}, \iota_{1}^{n}, \iota_{1}^{n}, \ldots, \iota_{1}^{n}\right] \circ m=\iota_{1}^{n},} \\
{\left[\iota_{1}^{n}, \iota_{2}^{n}, \iota_{1}^{n}, \ldots, \iota_{1}^{n}\right] \circ m=\iota_{1}^{n},} \\
\vdots \\
{\left[\iota_{1}^{n}, \iota_{1}^{n}, \ldots, \iota_{1}^{n}, \iota_{2}^{n}\right] \circ m=\iota_{1}^{n} .}
\end{gathered}
$$

Recall that we assume $\left|\overline{\mathcal{L}}_{\mathbf{X}}\right|>1$. It is easy to see that this implies that $m$ must have some essential arguments. But now, by Theorem 6.3.1, the first identity implies that $m$ does not depend on its first argument. The second identity implies that $m$ does not depend on its second argument, and so on. Thus, $m$ does not depend on any of its arguments, a contradiction.

Now let $s \in C$ be an $n$-ary semiinjection for $n \geq 3$. Without loss of generality, we may assume that we have $\left[\iota_{i_{1}}^{n}, \iota_{i_{2}}^{n}, \ldots, \iota_{i_{n}}^{n}\right] \circ s=\iota_{1}^{n}$ whenever $i_{1}, \ldots, i_{n} \in\{1, \ldots, n\}$ are not pairwise distinct. The following two identities hold:

$$
\begin{aligned}
& {\left[\iota_{1}^{n}, \iota_{1}^{n}, \iota_{2}^{n}, \iota_{3}^{n}, \ldots, \iota_{n-1}^{n}\right] \circ s=\iota_{1}^{n},} \\
& {\left[\iota_{1}^{n}, \iota_{2}^{n}, \iota_{1}^{n}, \iota_{3}^{n}, \ldots, \iota_{n-1}^{n}\right] \circ s=\iota_{1}^{n} .}
\end{aligned}
$$

Using Theorem 6.3.1 again, the first identity implies that $s$ can only depend on its first two arguments. Furthermore, the second identity implies that $m$ does not depend on its second argument. Thus, $s$ depends only on its first argument. But now, for $x \in \mathbf{X}$, Lemma 6.2.2 yields that $s(x)$ can be written as $\iota_{1}^{n}(y)$ for some $y \in \mathbf{X}$. We obtain

$$
s(x)=\iota_{1}^{n}(y)=\left(\left[\iota_{1}^{n}, \iota_{1}^{n}, \iota_{2}^{n}, \iota_{3}^{n}, \ldots, \iota_{n-1}^{n}\right] \circ \iota_{1}^{n}\right)(y)=\left(\left[\iota_{1}^{n}, \iota_{1}^{n}, \iota_{2}^{n}, \iota_{3}^{n}, \ldots, \iota_{n-1}^{n}\right] \circ s\right)(x)=\iota_{1}^{n}(x) .
$$

Thus, $s=\iota_{1}^{n}$.

\subsection{Dual Idempotent Operations}

In this section, we will show that dual idempotent operations have rather surprising properties if they respect the images of the injection morphisms to the degree 1.

Recall that an $n$-ary dual operation $g$ is called idempotent if $\left[\iota_{1}^{n}, \ldots, \iota_{1}^{n}\right] \circ g=\iota_{1}^{n}$. Of course, the index 1 in this identity can be replaced by any $i \in\{1, \ldots, n\}$. In other words, $g$ is idempotent if and only if $\left[\iota_{i}^{n}, \ldots, \iota_{i}^{n}\right] \circ g=\iota_{i}^{n}$ for all $i \in\{1, \ldots, n\}$.

We denote by $\overline{\mathcal{I}}_{\mathbf{X}}$ the clone of all dual idempotent operations over $\mathbf{X}$.

6.4.1 Definition. A clone $C \leq \bar{O}_{\mathbf{X}}$ is called idempotent if each dual operation in $C$ is idempotent. 
Obviously, the ideal $\left\langle\overline{\mathcal{I}}_{\mathbf{X}}\right]$ is the lattice of all idempotent clones of dual operations over $\mathbf{X}$.

As our first result of this section, we will show that dual idempotent operations can be characterized nicely whenever they preserve the injection morphisms to the degree 1 .

6.4.2 Theorem. Assume that $g \in \bar{O}_{\mathbf{X}}^{(n)}$ respects the images of the injection morphisms to the degree 1 . The following two statements are equivalent:

(1) $g$ is idempotent.

(2) For each $x \in \mathbf{X}, g(x)$ can be written as $\iota_{i}^{n}(x)$ for some $i \in\{1, \ldots, n\}$.

Proof. (1) $\Longrightarrow(2)$. Let $x \in \mathbf{X}$. Since $g$ respects the images of the injection morphisms to the degree 1 , we can write $g(x)$ as $\iota_{i}^{n}(y)$ for some $i \in\{1, \ldots, n\}$ and $y \in \mathbf{X}$. Moreover, we have $\iota_{i}^{n}=\left[\iota_{i}^{n}, \ldots, \iota_{i}^{n}\right] \circ g$ as $g$ is idempotent. Hence,

$$
\iota_{i}^{n}(x)=\left(\left[\iota_{i}^{n}, \ldots, \iota_{i}^{n}\right] \circ g\right)(x)=\left(\left[\iota_{i}^{n}, \ldots, \iota_{i}^{n}\right] \circ \iota_{i}^{n}\right)(y)=\iota_{i}^{n}(y)=g(x) .
$$

$(2) \Longrightarrow(1)$. We have to show $\iota_{1}^{n}=\left[\iota_{1}^{n}, \ldots, \iota_{1}^{n}\right] \circ g$. For each $x \in \mathbf{X}$, there exists $j_{x} \in\{1, \ldots, n\}$ such that $g(x)=\iota_{j_{x}}^{n}(x)$. Hence,

$$
\iota_{1}^{n}(x)=\left(\left[\iota_{1}^{n}, \ldots, \iota_{1}^{n}\right] \circ \iota_{j_{x}}^{n}\right)(x)=\left(\left[\iota_{1}^{n}, \ldots, \iota_{1}^{n}\right] \circ g\right)(x)
$$

for all $x \in \mathbf{X}$.

With this theorem, we can establish a close connection between dual idempotent operations and partitions.

6.4.3 Definition. Let $X^{\sharp}$ be the set of all $x \in \mathbf{X}$ such that $\iota_{i}^{n}(x) \neq \iota_{j}^{n}(x)$ for all $n \in \mathbb{N}_{+}$ and $i \neq j$.

It is an easy exercise to show that $x \notin X^{\sharp}$ is equivalent to $\iota_{1}^{n}(x)=\iota_{2}^{n}(x)=\ldots=\iota_{n}^{n}(x)$ for all $n \in \mathbb{N}_{+}$.

\subsubsection{Examples.}

(i) If $\mathbf{X}$ is a set in the category of sets, then $X^{\sharp}=\mathbf{X}$.

(ii) If $\mathbf{X}=\langle X, 0,1, \leq\rangle$ is a bounded poset in the category of bounded posets, then $X^{\sharp}=X \backslash\{0,1\}$.

6.4.5 Definition. For a dual idempotent operation $g \in \bar{O}_{\mathbf{X}}^{(n)}$ that respects the images of the injection morphisms to the degree 1 , we denote by $\Pi(g)$ the partition of $X^{\sharp}$ defined as follows:

$$
\Pi(g):=\left\{X_{1}, \ldots, X_{n}\right\} \backslash\{\emptyset\} \text {, where } x \in X_{i}: \Longleftrightarrow g(x)=\iota_{i}^{n}(x) .
$$

Note that $\Pi(g)$ is well-defined since, for each $x \in X^{\sharp}, g(x)$ is contained in exactly one of the sets among $\iota_{1}^{n}(\mathbf{X}), \ldots, \iota_{n}^{n}(\mathbf{X})$. 
6.4.6 Lemma. Let $g_{1}, g_{2}$ be two dual idempotent operations that respect the images of the injection morphisms to the degree 1 . Then, $g_{1}$ and $g_{2}$ are essentially the same dual operation if and only if $\Pi\left(g_{1}\right)=\Pi\left(g_{2}\right)$.

Proof. Let $g_{1}$ be $k$-ary and let $g_{2}$ be $n$-ary. Without loss of generality, assume $k \leq n$.

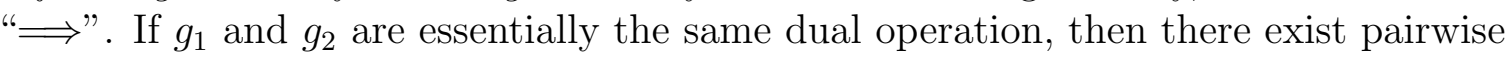
distinct integers $i_{1}, \ldots, i_{k} \in\{1, \ldots, n\}$ such that $g_{2}=\left[\iota_{i_{1}}^{n}, \ldots, \iota_{i_{k}}^{n}\right] \circ g_{1}$. This clearly implies $\Pi\left(g_{1}\right)=\Pi\left(g_{2}\right)$.

"£". The equation $\Pi\left(g_{1}\right)=\Pi\left(g_{2}\right)$ implies that two elements $x, y \in X^{\sharp}$ belong to the same set among $\Pi\left(g_{1}\right)$ if and only if they belong to the same set among $\Pi\left(g_{2}\right)$. Thus, we can choose pairwise distinct $j_{1}, \ldots, j_{k} \in\{1, \ldots, n\}$ such that, for $i \in\{1, \ldots, k\}$, we have $g_{1}(x), g_{1}(y) \in \iota_{i}^{k}(\mathbf{X})$ if and only if $g_{2}(x), g_{2}(y) \in \iota_{j_{i}}^{n}(\mathbf{X})$. We obtain $g_{2}=\left[\iota_{j_{1}}^{n}, \ldots, \iota_{j_{k}}^{n}\right] \circ g_{1}$, and we are done.

This lemma yields an upper bound on the number of dual idempotent operations among $\bar{O}_{\mathbf{X}}$.

6.4.7 Definition. For two integers $k, n$ with $k \geq n$, denote by $S_{k}^{n}$ the Stirling number of the second kind. That is, $S_{k}^{n}$ is the number of ways to partition a set of $k$ elements into $n$ non-empty subsets.

6.4.8 Corollary. Assume that all dual idempotent operations over $\mathbf{X}$ respect the images of the injection morphisms to the degree 1 . Then, the number of essentially different, essentially n-ary dual idempotent operations over $\mathbf{X}$ is bounded by $S_{\left|X^{\sharp}\right|}^{n}$.

Proof. By Lemma 6.4.6, the number of essentially different, essentially $n$-ary dual idempotent operations is bounded by the number of partitions of $X^{\sharp}$ into $n$ parts. The claim follows.

Note that we can also give an upper bound without calculating $X^{\sharp}$. Since $\left|X^{\sharp}\right| \leq|\mathbf{X}|$, $S_{|\mathbf{X}|}^{n}$ is also an upper bound on the number of essentially different, essentially $n$-ary dual operations among $\overline{\mathcal{I}}_{\mathbf{X}}$.

To obtain more results for dual idempotent operations, we will now introduce an order relation on partitions.

6.4.9 Definition. Let $\Pi_{1}=\left\{Y_{1}, \ldots, Y_{p}\right\}$ and $\Pi_{2}=\left\{Z_{1}, \ldots, Z_{q}\right\}$ be two partitions of the same set. We say that $\Pi_{1}$ is smaller than $\Pi_{2}$, in symbols $\Pi_{1} \preccurlyeq \Pi_{2}$, if $\Pi_{2}$ is finer. That is, $\Pi_{1} \preccurlyeq \Pi_{2}$ if an only if, for every $j \in\{1, \ldots, q\}$, there is an $i \in\{1, \ldots, p\}$ such that $Z_{j} \subseteq Y_{i}$.

With this definition, we can now show a more precise connection between idempotent clones of dual operations and partitions of $X^{\sharp}$.

6.4.10 Lemma. Let $g, g^{\prime} \in \bar{O}_{\mathbf{X}}$ be dual idempotent operations that respect the images of the injection morphisms to the degree 1 . We have $g \in \overline{\mathrm{Clo}}\left(g^{\prime}\right)$ if and only if $\Pi(g) \preccurlyeq \Pi\left(g^{\prime}\right)$. Consequently, $\overline{\mathrm{Clo}}(g)=\overline{\mathrm{Clo}}\left(g^{\prime}\right)$ if and only if $\Pi(g)=\Pi\left(g^{\prime}\right)$. 
Proof. "£". Let $n$ be the arity of $g^{\prime}$ and denote by $r(g)$ the minimal number of appearances of the operational symbol $g^{\prime}$ needed to write $g$ as a superposition of $g^{\prime}$ and the injection morphisms. We will show the claim by induction over $r(g)$. For $r(g)=0, g$ is an injection morphism, and we obtain $\Pi(g)=\left\{X^{\sharp}\right\}$, which clearly implies $\Pi(g) \preccurlyeq \Pi\left(g^{\prime}\right)$. Now suppose $r(g)>0$. Then, $g$ can be written as $\left[g_{1}, \ldots, g_{n}\right] \circ g^{\prime}$ where $r\left(g_{1}\right), \ldots, r\left(g_{n}\right)<r(g)$. By the induction hypothesis, we have $\Pi\left(g_{1}\right), \ldots, \Pi\left(g_{n}\right) \preccurlyeq \Pi\left(g^{\prime}\right)$. But now, Theorem 6.4 .2 establishes $\Pi(g) \preccurlyeq \Pi\left(g^{\prime}\right)$.

“”". Let $\Pi(g)=\left\{Y_{1}, \ldots, Y_{p}\right\}$ and $\Pi\left(g^{\prime}\right)=\left\{Z_{1}, \ldots, Z_{q}\right\}$ such that $\Pi(g) \preccurlyeq \Pi\left(g^{\prime}\right)$. Without loss of generality, we can assume that all arguments of $g$ and $g^{\prime}$ are essential (that is, $g$ is $p$-ary and $g^{\prime}$ is $q$-ary) and that there exist $i_{1}, \ldots, i_{p} \in \mathbb{N}_{+}$such that $i_{1}+\ldots+i_{p}=q$ and

$$
\begin{aligned}
Z_{1} \cup \ldots \cup Z_{i_{1}} & =Y_{1}, \\
Z_{i_{1}+1} \cup \ldots \cup Z_{i_{1}+i_{2}} & =Y_{2}, \\
\vdots & \vdots \\
Z_{\left(\sum_{j=1}^{p-1} i_{j}\right)+1} \cup \ldots \cup Z_{\sum_{j=1}^{p} i_{j}} & =Y_{p} .
\end{aligned}
$$

Moreover, for $x \in X^{\sharp}$ and $i \in\{1, \ldots, p\}$, we can assume $g(x)=\iota_{i}^{p}(x)$ if and only if $x \in Y_{i}$ and, for $j \in\{1, \ldots, q\}$, we can assume $g^{\prime}(x)=\iota_{j}^{q}(x)$ if and only if $x \in Z_{j}$. But now, by Theorem 6.4.2, we obtain

$$
g=[\underbrace{\iota_{1}^{p}, \ldots, \iota_{1}^{p}}_{i_{1} \text { times }}, \underbrace{\iota_{2}^{p}, \ldots, \iota_{2}^{p}}_{i_{2} \text { times }}, \ldots, \underbrace{\iota_{p}^{p}, \ldots, \iota_{p}^{p}}_{i_{p} \text { times }}] \circ g^{\prime} .
$$

Hence, $g \in \overline{\mathrm{Clo}}\left(g^{\prime}\right)$.

We can also show the following lemma:

6.4.11 Lemma. Let $C$ be an idempotent clone that respects the images of the injection morphisms to the degree 1 . Then, $C$ is generated by a single dual operation.

Proof. Let $k:=|\mathbf{X}|$. By Corollary 6.2.4 (page 117), $C$ is generated by $C^{(k)}$. Let $C^{(k)}=\left\{g_{1}, \ldots, g_{s}\right\}$ and set

$$
g:=\llbracket \underbrace{g_{s}, \ldots, g_{s}}_{k^{s-1} \text { times }} \rrbracket \circ \ldots \circ \llbracket \underbrace{g_{3}, \ldots, g_{3}}_{k^{2} \text { times }} \rrbracket \circ \llbracket \underbrace{g_{2}, \ldots, g_{2}}_{k \text { times }} \rrbracket \circ g_{1} .
$$

Since clones of dual operations are closed under expanded superposition (see Proposition 3.1.10, page 36), we have $g \in \overline{\mathrm{Clo}}\left(g_{1}, \ldots, g_{s}\right)$. Furthermore, with the help of Theorem 6.4.2 it is easy to see that we have $\Pi\left(g_{1}\right), \ldots, \Pi\left(g_{s}\right) \preccurlyeq \Pi(g)$. By Lemma 6.4.10, this yields $g_{1}, \ldots, g_{s} \in \overline{\mathrm{Clo}}(g)$. Thus, $\overline{\mathrm{Clo}}\left(g_{1}, \ldots, g_{s}\right)=\overline{\mathrm{Clo}}(g)$.

With the last two lemmas, we can show the following proposition: 
6.4.12 Proposition. Assume that all dual idempotent operations over $\mathbf{X}$ respect the images of the injection morphisms to the degree 1. Then, the number of essentially different dual idempotent operations over $\mathbf{X}$ coincides with the number of idempotent clones over $\mathbf{X}$.

Proof. By Lemma 6.4.11, each idempotent clone is generated by a single dual idempotent operation. It remains to show that two essentially different dual idempotent operations generate a different clone. To this end, let $g, g^{\prime} \in \bar{O}_{\mathbf{X}}$ be essentially different and idempotent. By Lemma 6.4.6, we have $\Pi(g) \neq \Pi\left(g^{\prime}\right)$. But now, by Lemma 6.4.10, $g$ and $g^{\prime}$ generate a different clone.

We have now collected all the facts to show that the ideal $\left\langle\overline{\mathcal{I}}_{\mathbf{X}}\right]$ can be found in a certain lattice of partitions provided that $\overline{\mathcal{I}}_{\mathbf{X}}$ respects the images of the injection morphisms to the degree 1.

6.4.13 Definition. For a finite set $A$, denote by $\operatorname{Part}(A)$ the set of partitions of $A$. The poset $\langle\operatorname{Part}(A), \preccurlyeq\rangle$ is called the lattice of partitions of $A$.

It is straightforward to check that $\langle\operatorname{Part}(A), \preccurlyeq\rangle$ is indeed a complete lattice.

6.4.14 Theorem. Assume that all dual idempotent operations over $\mathbf{X}$ respect the images of the injection morphisms to the degree 1. Then, the ideal $\left\langle\overline{\mathcal{I}}_{\mathbf{X}}\right]$ can be order-embedded into the lattice $\left\langle\operatorname{Part}\left(X^{\sharp}\right), \preccurlyeq\right\rangle$.

Proof. We define the mapping $\varphi:\left\langle\overline{\mathcal{I}}_{\mathbf{X}}\right] \rightarrow \operatorname{Part}\left(X^{\sharp}\right)$ by setting $\varphi(C):=\Pi(g)$ where $g$ is one of the single dual idempotent operations that generate $C$. The existence of $g$ is ensured by Lemma 6.4.11, and Lemma 6.4.10 yields $\Pi(g)=\Pi\left(g^{\prime}\right)$ whenever $g$ and $g^{\prime}$ both generate $C$. Thus, $\varphi$ is a well-defined mapping. By Lemma 6.4.10, it is also an order embedding.

Since $\left\langle\operatorname{Part}\left(X^{\sharp}\right), \preccurlyeq\right\rangle$ is a sublattice of $\langle\operatorname{Part}(X), \preccurlyeq\rangle$, this theorem also implies that $\left\langle\overline{\mathcal{I}}_{\mathbf{X}}\right]$ can be order-embedded into $\langle\operatorname{Part}(X), \preccurlyeq\rangle$. Moreover, the theorem implies that the number of essentially different dual idempotent operations over $\mathbf{X}$ and the number of clones of dual operations over $\mathbf{X}$ are both bounded by $\sum_{i=1}^{\left|X^{\sharp}\right|} S_{\left|X^{\sharp}\right|}^{i}$, that is, the Bell number $B_{\left|X^{\sharp}\right|}$.

\subsection{Minimal Clones}

In the final section of this chapter, we will show that we can fully characterize the minimal clones below a clone $C \leq \bar{O}_{\mathbf{X}}$ (that is, the minimal clones in the ideal $\langle C] \leq \overline{\mathcal{L}}_{\mathbf{X}}$ ) if we assume that $C$ respects the images of the injection morphisms to the degree 1 .

6.5.1 Theorem. Assume that $C$ respects the images of the injection morphisms to the degree 1. A nontrivial dual operation $g \in C$ is minimal if and only if it is of one of the following three types:

1. a unary retraction, 
2. an automorphism of prime order,

3. a binary dual idempotent operation.

Proof. By the Dualized RCT (Theorem 3.2.2, page 38), we know that every minimal dual operation is either of one of the three types from above or it is a dual majority operation, a dual minority operations or a proper semiinjection. However, by the remark after Theorem 6.3.1 (page 118) and Corollary 6.3.2, we know that the latter three cases are impossible. Thus, every minimal clone must be generated by a dual operation of one of the three types from above. It remains to show that each clone generated by these dual operations is in fact minimal. In the first two cases, there is nothing to show since unary retractions and automorphisms of prime order are necessarily minimal (see the remark after Theorem 3.2.2). For the third case, let $g \in C^{(2)}$ be a nontrivial dual idempotent operation. Suppose that $\overline{\mathrm{Clo}}(g)$ contains a minimal clone $C^{\prime}$. We need to show $C^{\prime}=\overline{\mathrm{Clo}}(g)$. Since $C^{\prime}$ is minimal, it must be generated by a nontrivial unary operation or by a binary dual idempotent operation. However, it is obvious that there are no nontrivial unary operations among $\overline{\mathrm{Clo}}(g)$. Hence, $C^{\prime}$ must be generated be a dual idempotent operation $g^{\prime}$. But now, by Lemma 6.4.10, $g^{\prime} \in \overline{\operatorname{Clo}}(g)$ implies $\Pi\left(g^{\prime}\right) \preccurlyeq \Pi(g)$. Since $\Pi\left(g^{\prime}\right)$ and $\Pi(g)$ are both two-element sets, this means $\Pi\left(g^{\prime}\right)=\Pi(g)$. Applying Lemma 6.4.10 again, we can infer $\overline{\mathrm{Clo}}(g)=\overline{\mathrm{Clo}}\left(g^{\prime}\right)$, and we have successfully shown that $\overline{\mathrm{Clo}}(g)$ is minimal.

As a very easy example, we can apply this theorem (and all the other results from this chapter) on the lattice of coclones over sets. However, we can also apply it in numerous other situations, some of which we will find in the next chapter. 


\section{Applying the Duality for Clones over Sets}

So far, we have generalized the notion of a clone, introduced a duality for clones, generalized Pol-Inv and examined clones of dual operations. In the remaining two chapters of this thesis we will come back to something we have promised earlier: We will show that all these results are also useful if we want to obtain results for clones over sets.

Let us recall the results from Section 4.2. Any given clone $C$ on a finite set $A$ can be written as $O_{\mathbf{A}}$ where $\mathbf{A}$ is a structure with carrier set $A$ in the finite part of a quasivariety $\mathcal{A}$ of structures. In this setting, $\mathcal{L}_{\mathbf{A}}$ is the order ideal $\langle C]$ in the lattice of clones over the set $A$. Moreover, the category $\mathcal{A}$ is dually equivalent to some category $\mathcal{X}$ of finite structures, so we have a clone duality $(-)^{2}: O_{\mathbf{A}} \rightarrow \bar{O}_{\mathbf{X}}$ that dualizes each clone of operations over $\mathbf{A}$ to a clone of dual operations over $\mathbf{X}$. Therefore, we can examine $\langle C]$ by studying $\overline{\mathcal{L}}_{\mathbf{X}}$. This is precisely what we will do in this chapter.

To this end, for the whole chapter, let $\mathbf{A}=\langle A, F, H, R\rangle$ be a finite structure with at least two elements (recall that, for $|A|=1, \mathcal{L}_{\mathbf{A}}$ would only contain $O_{\mathbf{A}}$ ), let $\mathcal{A}$ be the finite part of a quasivariety that contains $\mathbf{A}$, and let $\mathcal{X}$ be a category of finite structures that is dually equivalent to $\mathcal{A}$. Denote the dual of $\mathbf{A}$ by $\mathbf{X}$ and let $(-)^{\partial}: O_{\mathbf{A}} \rightarrow \bar{O}_{\mathbf{X}}$ be the clone duality. Choose $\mathbb{T}$ and $\mathbb{T}^{\prime}$ to be skeletons of $\mathcal{A}$ and $\mathcal{X}$, respectively. By Section 5.3. we also have a duality $(-)^{\partial^{*}}: \mathbf{R}_{\mathbf{A}}^{\mathbb{T}} \rightarrow \overline{\mathbf{R}}_{\mathbf{X}}^{\mathbb{T}^{\prime}}$ between the clones of relations on $\mathbf{A}$ and the clones of dual relations on $\mathbf{X}$. The following diagram summarizes the scenario:

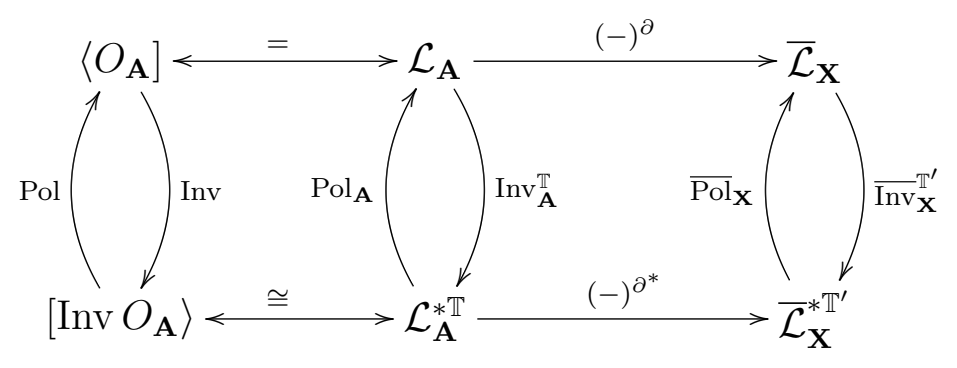

To study the clones of dual operations over $\mathbf{X}$, we can use $\overline{\mathrm{Pol}}_{\mathbf{X}}-\overline{\operatorname{Inv}}_{\mathbf{X}}^{\mathbb{T}}$ and exploit the results obtained in the last chapter. We will collect some general results and see what we can obtain for the seven examples introduced in Section 4.2 . 


\subsection{Respecting the Images of the Injection Morphisms and Clones over Structures}

In the last chapter we have seen that many properties of the clones in $\overline{\mathcal{L}}_{\mathbf{X}}$ depend on the form of the copowers of $\mathbf{X}$. Consequently, looking at the properties of the copowers of $\mathbf{X}$ might give us a new way to obtain results for the clone lattice $\mathcal{L}_{\mathbf{A}}$. One of these properties was respecting the images of the injection morphisms to a certain degree. Recall that, for a clone of dual operations $C \leq \bar{O}_{\mathbf{X}}$, we say that $C$ respects the images of the injection morphisms to the degree $n$ whenever we have

$$
\bigcup_{g \in C^{(k)}} g(\mathbf{X}) \subseteq \bigcup_{\widehat{\iota} \in I_{n}^{k}} \widehat{\iota}(n \cdot \mathbf{X})
$$

for all $k \geq n$.

In [Ker10], the author of this thesis showed that we do not have to check this equation for all $k \geq n$ if the object $\mathbf{X}$ is isomorphic to the dual of $\mathbf{A}$ under a natural dual equivalence.

7.1.1 Lemma ([Ker10]). Assume that $\mathbf{X}$ is isomorphic to the dual of $\mathbf{A}$ under a natural dual equivalence. For $n \in \mathbb{N}_{+}$and $C \leq \bar{O}_{\mathbf{X}}$, the following two statements are equivalent:

(1) $C$ respects the images of the injection morphisms to the degree $n$.

(2) $\bigcup_{g \in C^{(k)}} g(\mathbf{X}) \subseteq \bigcup_{\widehat{\iota} \in I_{n}^{k}} \widehat{\iota}(n \cdot \mathbf{X})$ for $k:=\max (|A|, n+2)$.

Since basically all well-studied dualities between structures are natural dualities or modifications of natural dualities in the sense of Lemma 4.2.3 (page 46), the above lemma is very useful to check whether $\bar{O}_{\mathbf{X}}$ preserves the images of the injection morphisms to a certain degree. In particular, we can apply the lemma if we use one of the clone dualities constructed in Section 4.2. Recall that, in each of these cases, $\mathbf{X}$ is isomorphic to $D^{\prime}(\mathbf{A})$, where $D^{\prime}: \mathcal{A} \rightarrow X$ is the functor from the natural dual equivalence.

Figure 7.1 visualizes the result of this lemma.

Recall that we say that $\mathbf{X}$ has non-deformed copowers of degree $n$ if

$$
k \cdot \mathbf{X}=\bigcup_{\widehat{\iota} \in I_{n}^{k}} \widehat{\iota}(n \cdot \mathbf{X})
$$

for all $k \geq n$. We already know that $\bar{O}_{\mathbf{X}}$ respects the images of the injection morphisms to the degree $n$ if $\mathbf{X}$ has non-deformed copowers of degree $n$. However, the two conditions are not equivalent. Hence, in general, we can only decide whether $\bar{O}_{\mathbf{X}}$ respects the images of the injection morphisms to a certain degree if we know the operations among $\bar{O}_{\mathbf{X}}$. Luckily, if $\mathbf{X}$ is isomorphic to the dual of $\mathbf{A}$ under a natural dual equivalence, then it is a different story: In this case, $\bar{O}_{\mathbf{X}}$ respects the images of the injection morphisms to the degree $n$ if and only if $\mathbf{X}$ has non-deformed copowers of degree $n$. Moreover, it is enough to check Equation (7.2) for $k:=\max (n+2,|A|)$. This result was shown in Ker10] and it is stated in the next lemma. It should be noted that a small restriction is necessary: We 


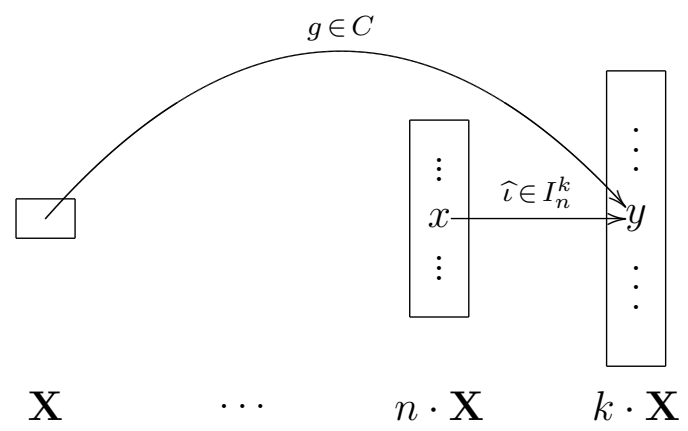

Figure 7.1.: $C \leq \bar{O}_{\mathbf{X}}$ respects the images of the injection morphisms to the degree $n$ if and only if, for $k:=\max (|A|, n+2)$, every $k$-ary dual operations $g \in C$, each $y \in g(\mathbf{X})$ is in the image of some $\widehat{\iota} \in I_{n}^{k}$

have to assume that $\mathcal{A}$ is generated by a retract of $\mathbf{A}$. That is, $\mathcal{A}=\mathbb{I S P}(\mathbf{M})_{\text {fin }}$ for some retract $\mathbf{M}$ (see Definition 1.4.6, page 17). However, the restriction is only a technical one since such a structure $\mathbf{M}$ can always be chosen (we can take $\mathbf{M}:=\mathbf{A}$ if no other structure is at hand).

7.1.2 Lemma ([Ker10]). Assume that $\mathcal{A}$ is generated by a retract $\mathbf{M}$ of $\mathbf{A}$. Moreover, assume that $\mathbf{X}$ is isomorphic to the dual of $\mathbf{A}$ under a natural duality yielded by an alter ego of $\mathbf{M}$. Then, for $n \in \mathbb{N}_{+}$, the following statements are equivalent:

(1) $\bar{O}_{\mathbf{X}}$ respects the images of the injection morphisms to the degree $n$.

(2) $\mathbf{X}$ has non-deformed copowers of degree $n$.

(3) $k \cdot \mathbf{X}=\bigcup_{\widehat{\iota} \in I_{n}^{k}} \widehat{\iota}(n \cdot \mathbf{X})$ for $k:=\max (|A|, n+2)$.

We will now take a look at the examples and the dualities introduced in Section 4.2. In each of the seven cases, we have $\mathcal{A}=\mathbb{I} \mathbb{S P}(\mathbf{M})_{\text {fin }}$, where $\mathbf{M}$ is a retract of all nontrivial structures in $\mathcal{A}$. Thus, by Lemma $7.1 .2, \bar{O}_{\mathbf{X}}$ respects the images of the injection morphisms to the degree $n$ if and only if the copowers of $\mathbf{X}$ are non-deformed to the degree $n$. If $\mathbf{A}$ is a distributive lattice, a Boolean algebra, a Boolean ring, a Boolean lattice or a median algebra, then it is fairly obvious that $\mathbf{X}$ has non-deformed copowers of degree 1 . We will now show that, in the other two cases (that is, $\mathbf{A}$ is a Boolean group or a semilattice), $\mathbf{X}$ does not have non-deformed copowers of any degree:

7.1.3 Lemma. If $\mathbf{A}$ is a Boolean group or a semilattice, then there exists no $n \in \mathbb{N}_{+}$ such that the copowers of $\mathbf{X}$ are non-deformed to the degree $n$.

Proof. Let $\mathbf{X}=\langle X,+, 0\rangle$ be a Boolean group with $|X|=2^{l}$ for some $l \in \mathbb{N}_{+}$, and let $B_{\mathbf{X}}$ be a basis of $\mathbf{X}$. For each $r \geq 2$, let $B_{r \cdot \mathbf{X}}:=\bigcup_{i=1}^{r} \iota_{i}^{r}\left(B_{\mathbf{X}}\right)$. Clearly, $B_{r \cdot \mathbf{X}}$ is a basis of $r \cdot \mathbf{X}$. Note that $B_{r \cdot \mathbf{X}}$ has exactly $l r$ elements. Let $n \in \mathbb{N}_{+}$and $\widehat{\iota} \in I_{n}^{n+1}$. We need to show that there exists $y \in(n+1) \cdot \mathbf{X}$ such that $y \neq \widehat{\imath}(x)$ for all $x \in n \cdot \mathbf{X}$. To this end, let $c_{1}, \ldots, c_{l n+1} \in B_{(n+1) \cdot \mathbf{x}}$ be pairwise distinct base elements. Set

$$
y:=c_{1}+^{(n+1) \cdot \mathbf{X}} \ldots+{ }^{(n+1) \cdot \mathbf{X}} c_{l n+1} .
$$


Since $\widehat{\iota}\left(0^{n \cdot \mathbf{X}}\right)=0^{(n+1) \cdot \mathbf{X}} \neq y$, we have $x \neq 0^{n \cdot \mathbf{X}}$. Hence, we can assume that there exists some integer $1 \leq k \leq \ln$ and base elements $b_{1}, \ldots, b_{k} \in B_{n \cdot \mathbf{x}}$ such that

$$
x=b_{1}+{ }^{n \cdot \mathbf{X}} \ldots+{ }^{n \cdot \mathbf{X}} b_{k} .
$$

But now, there exist $i_{1}, \ldots, i_{n} \in\{1, \ldots, n+1\}$ such that

$$
\widehat{\iota}(x)=\left[\iota_{i_{1}}^{n+1}, \ldots, \iota_{i_{n}}^{n+1}\right](x)=\left[\iota_{i_{1}}^{n+1}, \ldots, \iota_{i_{n}}^{n+1}\right]\left(b_{1}\right)+{ }^{(n+1) \cdot \mathbf{X}} \ldots+{ }^{(n+1) \cdot \mathbf{X}}\left[\iota_{i_{1}}^{n+1}, \ldots, \iota_{i_{n}}^{n+1}\right]\left(b_{k}\right) .
$$

For all $j \in\{1, \ldots, k\}$, we have $\left[\iota_{i_{1}}^{n+1}, \ldots, \iota_{i_{n}}^{n+1}\right]\left(b_{j}\right) \in B_{(n+1)} \mathbf{x}$. Hence, $\widehat{\iota}(x)$ is the sum of at most $k$ base elements of $(n+1) \cdot \mathbf{X}$. This implies $\widehat{\iota}(x) \neq y$ since $y$ is the sum of $l n+1$ base elements and $k \leq \ln <\ln +1$.

Now let $\mathbf{X}=\left\langle X, 0^{\overline{\mathbf{x}}}, 1^{\mathbf{X}}, \vee^{\mathbf{X}}\right\rangle$ be a bounded semilattice with $|X| \geq 3$. Let $n \in \mathbb{N}_{+}$and $\widehat{\iota} \in I_{n}^{n+1}$. Again, we need to show that there exists $y \in(n+1) \cdot \mathbf{X}$ such that $y \neq \widehat{\imath}(x)$ for all $x \in n \cdot \mathbf{X}$. For this, let $z \in X \backslash\left\{0^{\mathbf{X}}, 1^{\mathbf{X}}\right\}$ and set

$$
y:=\langle 1, z\rangle \vee^{(n+1) \cdot \mathbf{X}} \ldots \vee^{(n+1) \cdot \mathbf{X}}\langle n+1, z\rangle \text {. }
$$

Since $\widehat{\iota}\left(0^{n \cdot \mathbf{X}}\right)=0^{(n+1) \cdot \mathbf{X}} \neq y$ and $\widehat{\iota}\left(1^{n \cdot \mathbf{X}}\right)=1^{(n+1) \cdot \mathbf{X}} \neq y$, we obtain $x \notin\left\{0^{n \cdot \mathbf{X}}, 1^{n \cdot \mathbf{X}}\right\}$. There exist $j_{1}, \ldots, j_{n} \in\{1, \ldots, n\}$ and $x_{1}, \ldots, x_{n} \in X$ such that

$$
x=\left\langle j_{1}, x_{1}\right\rangle \vee^{n \cdot \mathbf{X}} \ldots \vee^{n \cdot \mathbf{X}}\left\langle j_{n}, x_{n}\right\rangle
$$

But now, there exist $s_{1}, \ldots, s_{n} \in\{1, \ldots, n+1\}$ such that $\widehat{\iota}\left(\left\langle j_{i}, x_{i}\right\rangle\right)=\left\langle s_{i}, x_{i}\right\rangle$ for all $i \in\{1, \ldots, n\}$. Therefore,

$$
\widehat{\iota}(x)=\left\langle s_{1}, x_{1}\right\rangle \vee^{(n+1) \cdot \mathbf{X}} \ldots \vee^{(n+1) \cdot \mathbf{X}}\left\langle s_{n}, x_{n}\right\rangle,
$$

which cannot coincide with $y$ since $n<n+1$.

Table 7.1 summarizes the situation for our examples.

Note that we can conclude that $\mathcal{L}_{\mathbf{A}}$ is finite and that $O_{\mathbf{A}}$ is finitely generated if $\mathbf{A}$ is a distributive lattice, a Boolean algebra, a Boolean ring, a Boolean lattice or a median algebra.

When we look at Table 7.1, it stands out that each $\bar{O}_{\mathbf{x}}$ either respects the images of the injection morphisms to the degree 1 or not at all. Although this happens for most well-known categories, we will see in the next example that it does not hold in general, even if the dual equivalence is natural.

7.1.4 Example. Let $\mathbf{M}=\mathbf{A}=\left\langle\left\{0,1, a_{1}, a_{2}\right\}, \vee, \wedge, 0,1, a_{1}, a_{2}\right\rangle$ be a distributive lattice where the two atoms $a_{1}, a_{2}$ are also constants. Set $\mathcal{A}:=\mathbb{I S P}(\mathbf{M})_{\text {fin }}$ and let $x$ be the category of finite structures that is dually equivalent to $\mathcal{A}$ via the natural duality. Then, $\bar{O}_{\mathbf{X}}$ respects the images of the injection morphisms to the degree 2 , but not to the degree 1. In fact, since there are no proper substructures of $\mathbf{M}$, it is easy to see that there is no natural dual equivalence for a quasivariety that contains $\mathbf{A}$ such that $D(\mathbf{A})$ respects the images of the injection morphisms to degree 1. 


\begin{tabular}{|l|c|}
\hline \multicolumn{1}{|c|}{$\mathbf{A}$} & $\begin{array}{c}\text { Least } n \in \mathbb{N}_{+} \text {such that } \bar{O}_{\mathbf{X}} \text { respects the images of } \\
\text { the injection morphisms to the degree } n\end{array}$ \\
\hline \hline Distributive lattice & 1 \\
\hline Boolean algebra & 1 \\
\hline Boolean ring & 1 \\
\hline Boolean lattice & 1 \\
\hline Median algebra & 1 \\
\hline Boolean group & - \\
\hline Semilattice & - \\
\hline
\end{tabular}

Table 7.1.: The degree to which $\bar{O}_{\mathbf{X}}$ respects the images of the injection morphisms for our examples

\subsection{The Essential Arity of Clones over Structures}

In this section, we will characterize the structures $\mathbf{A}$ for which the essential arity of operations among $O_{\mathbf{A}}$ is bounded.

7.2.1 Theorem. Let $C$ be a clone over A. The following two statements are equivalent:

(1) There exists $k \in \mathbb{N}_{+}$such that every $f \in C$ is essentially at most $k$-ary.

(2) There exists $n \in \mathbb{N}_{+}$such that $C^{\partial}$ respects the images of the injection morphisms to the degree $n$.

Proof. This is a direct consequence of Theorem 6.2.3 (page 116) since we know that the clone duality $(-)^{\partial}$ preserves the essentiality of arguments.

If we only consider the case $C=O_{\mathbf{A}}$, then we can use Lemma 7.1.2 to obtain the following corollary:

7.2.2 Corollary. Assume that $\mathcal{A}$ is generated by a retract $\mathbf{M}$ of $\mathbf{A}$. Moreover, assume that $\mathbf{X}$ is isomorphic to the dual of $\mathbf{A}$ under the natural duality yielded by an alter ego of $\mathbf{M}$. Then, the following two statements are equivalent:

(1) There exists $k \in \mathbb{N}_{+}$such that every $f \in O_{\mathbf{A}}$ is essentially at most $k$-ary.

(2) There exists $n \in \mathbb{N}_{+}$such that $\mathbf{X}$ has non-deformed copowers of degree $n$.

Thus, if the assumptions of the corollary are met (which is the case for basically all of the known dualities for structures), then we only need to look at the copowers of $\mathbf{X}$ to decide whether the essential arity of the operations among $O_{\mathbf{A}}$ is bounded.

Let us apply this result to the examples presented in Section 4.2. By looking back at Table 7.1, we can infer that the essential arity in $O_{\mathbf{A}}$ is not bounded if $\mathbf{A}$ is a nontrivial Boolean group or a nontrivial semilattice. However, if $\mathbf{A}$ is a distributive lattice, a Boolean algebra, a Boolean ring, a Boolean lattice or a median algebra, then 
the essential arity of operations among $O_{\mathbf{A}}$ must be bounded. A first bound, namely $|\mathbf{X}|$, is provided by Corollary 6.2.4 (page 117). In the following, we will examine how tight this bound is for these five examples. The case of clones over distributive lattices will be postponed to Chapter 8 in which we will deal with this example in detail (this also includes the case of clones over Boolean lattices).

\subsubsection{The Essential Arity of Clones over Boolean Algebras}

If $\mathbf{A}$ is a Boolean algebra, then the bound $|\mathbf{X}|=|\operatorname{At}(\mathbf{A})|$ is tight. This can be seen as follows: For $X=\left\{x_{1}, \ldots, x_{k}\right\}$ define $g \in \bar{O}_{\mathbf{X}}$ by setting $g\left(x_{i}\right):=\left\langle i, x_{i}\right\rangle$. Evidently, $g$ depends on all of its arguments.

\subsubsection{The Essential Arity of Clones over Boolean Rings}

In this subsection, we will determine a tight bound for the essential arity of operations over finite Boolean rings.

7.2.3 Theorem. Let $\mathbf{A}$ be a Boolean ring. Then, $\log _{2}|\mathbf{A}|$ is a sharp bound on the essential arity of operations among $O_{\mathbf{A}}$.

Proof. Let $k:=\log _{2}|\mathbf{A}|$ and let $(-)^{\partial}: O_{\mathbf{A}} \rightarrow \bar{O}_{\mathbf{X}}$ be the clone duality from Subsection 4.2.4. Recall that we have $\mathbf{X}=\langle\operatorname{Spec}(\mathbf{A}) \cup\{A\}, A\rangle$. Hence, $|\mathbf{X}|=k+1$. Since the duality preserves essential arguments, it suffices to show that each $g \in \bar{O}_{\mathbf{X}}$ can have at most $k$ essential arguments and that there exists $g^{*} \in \bar{O}_{\mathbf{X}}$ with exactly $k$ essential arguments. Evidently, the $i$-th argument of $g$ is nonessential if and only if $g(\mathbf{X}) \cap \iota_{i}^{n}(\mathbf{X})=\left\{1^{n \cdot \mathbf{X}}\right\}$. However, since $g(A)=1^{n \cdot \mathbf{X}}$ and $|\mathbf{X}|=k+1, g(x) \neq 1^{n \cdot \mathbf{X}}$ can only happen for at most $k$ elements. Since $\iota_{1}^{n}(\mathbf{X}), \ldots, \iota_{n}^{n}(\mathbf{X})$ share no common elements except $1^{n \cdot \mathbf{X}}$, this establishes that $g$ has at most $k$ essential arguments.

For the second part, let $X=\left\{x_{1}, \ldots, x_{k}\right\}$ and define $g^{*} \in \bar{O}_{\mathbf{X}}^{(k)}$ as follows:

$$
g^{*}(x):= \begin{cases}1^{k \cdot \mathbf{X}} & \text { if } x=A \\ \left\langle i, x_{i}\right\rangle & \text { if } x=x_{i}\end{cases}
$$

It is obvious that $g^{*}$ is well-defined and essentially $k$-ary.

Note that we have $\log _{2}|\mathbf{A}|=|\mathbf{X}|-1$, so the bound from Corollary 6.2.4 was actually very close.

\subsubsection{The Essential Arity of Clones over Median Algebras}

In this subsection, we will turn our attention to operations over finite median algebras. This time, the maximum essential arity will be notably lower than the bound provided by Corollary 6.2.4 
7.2.4 Theorem. Let $\mathbf{A}$ be a finite median algebra and let $k$ be the greatest number of pairwise distinct ideals $I_{1}, \ldots, I_{k} \subseteq A$ such that $I_{1}, \ldots, I_{k}, A \backslash I_{1}, \ldots, A \backslash I_{k}$ is an antichain with respect to inclusion. Then, $k$ is a sharp bound on the essential arity of operations among $O_{\mathbf{A}}$.

Proof. Let $(-)^{\partial}: O_{\mathbf{A}} \rightarrow \bar{O}_{\mathbf{X}}$ be the clone duality from Subsection 4.2 .3 . Recall that we have $\mathbf{X}=\left\langle\operatorname{Spec}(\mathbf{A}), \emptyset, A,{ }^{-}, \subseteq\right\rangle$, where

$$
-: \operatorname{Spec}(\mathbf{A}) \rightarrow \operatorname{Spec}(\mathbf{A}): x \mapsto A \backslash x .
$$

Again, it suffices to show that each $g \in \bar{O}_{\mathbf{X}}$ can have at most $k$ essential arguments and that there exists $g^{*} \in \bar{O}_{\mathbf{X}}$ with exactly $k$ essential arguments. By assumption, $k$ is the greatest number of pairwise distinct $x_{1}, \ldots, x_{k} \in \mathbf{X}$ such that $x_{1}, \ldots, x_{k}, \overline{x_{1}}, \ldots, \overline{x_{k}}$ is an antichain with respect to $\subseteq$. Assume that there exists $g \in \bar{O}_{\mathbf{X}}^{(n)}$ with at least $k+1$ essential arguments. Evidently, the $i$-th argument of $g$ is nonessential if and only if $g(\mathbf{X}) \cap \iota_{i}^{n}(\mathbf{X})=\left\{0^{n \cdot \mathbf{X}}, 1^{n \cdot \mathbf{X}}\right\}$. Hence, $g$ being essentially at least $(k+1)$-ary implies that there exist pairwise distinct integers $i_{1}, \ldots, i_{k+1} \in\{1, \ldots, n\}$ and $x_{1}, \ldots, x_{k+1} \in \mathbf{X}$ such that $g\left(x_{j}\right) \in g(\mathbf{X}) \cap \iota_{i_{j}}^{n}(\mathbf{X}) \backslash\left\{0^{n \cdot \mathbf{X}}, 1^{n \cdot \mathbf{X}}\right\}$ for all $j \in\{1, \ldots, k+1\}$. Since this implies that $g\left(x_{1}\right), \ldots, g\left(x_{k+1}\right)$ is an antichain in $n \cdot \mathbf{X}$, we can infer that $x_{1}, \ldots, x_{k+1}$ is an antichain in $\mathbf{X}$. Let us show that we cannot have $x_{r} \leq \overline{x_{s}}$ for any $r, s \in\{1, \ldots, k+1\}$ : For $r=s$, this is obvious. For $r \neq s$, assume $x_{r} \leq \overline{x_{s}}$. Then, $g\left(x_{r}\right) \leq g\left(\overline{x_{s}}\right)=\overline{g\left(x_{s}\right)}$, which is impossible since $g\left(x_{s}\right) \notin \iota_{i_{r}}^{n}(\mathbf{X})$ implies $\overline{g\left(x_{s}\right)} \notin \iota_{i_{r}}^{n}(\mathbf{X}) \ni g\left(x_{r}\right)$. We have reached a contradiction since we can conclude that $x_{1}, \ldots, x_{k+1}, \overline{x_{1}}, \ldots, \overline{x_{k+1}}$ is a $2(k+1)$-element antichain.

For the second part, define $g^{*} \in \bar{O}_{\mathbf{X}}^{(k)}$ as follows (we write $x<y$ to indicate $x \leq y$ and $x \neq y)$ :

$$
g^{*}(x):= \begin{cases}0^{k \cdot \mathbf{X}} & \text { if } x<x_{i} \text { or } x<\overline{x_{i}} \text { for some } i \in\{1, \ldots, k\} \\ 1^{k \cdot \mathbf{X}} & \text { if } x>x_{i} \text { or } x>\overline{x_{i}} \text { for some } i \in\{1, \ldots, k\} \\ \langle i, x\rangle & \text { if } x \in\left\{x_{i}, \overline{x_{i}}\right\} .\end{cases}
$$

We have to show that $g^{*}$ is a well-defined morphism. We start by showing that our definition assigns a unique $g^{*}(x) \in n \cdot \mathbf{X}$ to each $x \in \mathbf{X}$. For $x \in \mathbf{X}$, we must have $x \leq x_{i}$ or $x \leq \overline{x_{i}}$ or $x \geq x_{i}$ or $x \geq \overline{x_{i}}$ for at least one $i \in\{1, \ldots, k\}$ since otherwise $x_{1}, \ldots, x_{k}, x, \overline{x_{1}}, \ldots, \overline{x_{k}}, \bar{x}$ would be an antichain of length greater than $2 k$. We will now show that two of the three cases given in the definition of $g^{*}$ cannot hold at the same time: Assuming they do, we get $x_{i} \leq x \leq x_{j}$ or $x_{i} \leq x \leq \overline{x_{j}}$ or $\overline{x_{i}} \leq x \leq x_{j}$ or $\overline{x_{i}} \leq x \leq \overline{x_{j}}$ for some $i, j \in\{1, \ldots, k\}$, which contradicts that $x_{1}, \ldots, x_{k}, \overline{x_{1}}, \ldots, \overline{x_{k}}$ is an antichain. Thus, $g^{*}$ is a well-defined mapping between the carrier sets of $\mathbf{X}$ and $k \cdot \mathbf{X}$. Since it is easy to see that $g^{*}$ has no nonessential arguments, we can finish the proof by showing that $g^{*}$ is structure-preserving. It is obvious that we have $g^{*}(\emptyset)=0^{k \cdot \mathbf{X}}, g^{*}(A)=1^{k \cdot \mathbf{X}}$ and that $x \leq y$ implies $g^{*}(x) \leq g^{*}(y)$. It remains to show that we have $g^{*}(\bar{x})=\overline{g^{*}(x)}$. Assume $x<x_{i}$ for some $i \in\{1, \ldots, k\}$. Then $\bar{x}>\overline{x_{i}}$, and it follows $g^{*}(\bar{x})=1^{k \cdot \mathbf{X}}=\overline{0^{k \cdot \mathbf{X}}}=\overline{g^{*}(x)}$. A similar argument works for all the other possibilities such that $g^{*}(x) \in\left\{0^{k \cdot \mathbf{X}}, 1^{k \cdot \mathbf{x}}\right\}$. If $x=x_{i}$ for some $i \in\{1, \ldots, k\}$, then $g^{*}(\bar{x})=\left\langle i, \overline{x_{i}}\right\rangle=\overline{\left\langle i, x_{i}\right\rangle}=\overline{g^{*}(x)}$. The same argument works for the case $x=\overline{x_{i}}$. Thus, $g^{*}$ is a dual operation with exactly $k$ essential arguments. 
Note that $k$ from above is usually much smaller than $|\mathbf{X}|$, which was the bound provided by Corollary 6.2.4. In fact, for arbitrarily large $n \in \mathbb{N}_{+}$, it is possible to construct a median algebra $\mathbf{A}$ such that $|\mathbf{X}| \geq n$ and $k=2$.

Table 7.2 summarizes the results we obtained for the essential arity of the clones $O_{\mathbf{A}}$ for our seven examples. Recall that each $\mathbf{A}$ is assumed to have more than one element (otherwise, the essential arity is clearly bounded by 1).

\begin{tabular}{|c|c|c|}
\hline A & $\begin{array}{l}\text { Essential } \\
\text { arity } \\
\text { bounded? }\end{array}$ & Maximum essential arity \\
\hline Distributive lattice $^{1}$ & yes & $\begin{array}{l}\text { Greatest number of pairwise distinct } \\
\text { join-irreducible elements } a_{1}, \ldots, a_{k} \\
\text { such that } a_{1}, \ldots, a_{k} \text { is an anti-chain }\end{array}$ \\
\hline Distributive lattice with $0^{2}$ & yes & Number of atoms of $\mathbf{A}$ \\
\hline Distributive lattice with $1^{2}$ & yes & Number of coatoms of $\mathbf{A}$ \\
\hline Bounded distributive lattice $^{2}$ & yes & $|\operatorname{Con}(\mathbf{X})|^{3}$ \\
\hline Boolean algebra & yes & $\log _{2}|\mathbf{A}|($ number of atoms of $\mathbf{A})$ \\
\hline Boolean ring & yes & $\begin{array}{c}\log _{2}|\mathbf{A}| \text { (number of atoms of the } \\
\text { lattice induced by } \vee \text { ) }\end{array}$ \\
\hline Boolean lattice $^{1}$ & yes & $\log _{2}|\mathbf{A}|($ number of atoms of $\mathbf{A})$ \\
\hline Median algebra & yes & $\begin{array}{c}\text { Greatest number of pairwise distinct } \\
\text { ideals } I_{1}, \ldots, I_{k} \subseteq A \text { such that } \\
I_{1}, \ldots, I_{k}, A \backslash I_{1}, \ldots, A \backslash I_{k} \text { is an } \\
\text { antichain with respect to } \subseteq\end{array}$ \\
\hline Boolean group & no & - \\
\hline Semilattice & no & - \\
\hline $\begin{array}{l}{ }^{1} \text { see Section } 8.2 \\
2 \text { see Section } 8.4 \\
{ }^{3} \text { for } \operatorname{Con}(\mathbf{X}), \text { see Definition }\end{array}$ & pag & \\
\hline
\end{tabular}

Table 7.2.: The essential arity in $O_{\mathbf{A}}$ for our examples

\subsection{Identities of Clones over Structures}

In this section, we will rely on the results of Section 6.3 to obtain some information about the identities that hold in a given clone $C \leq O_{\mathbf{A}}$.

The following corollary is a direct consequence of Theorem 6.3.1 (page 118):

7.3.1 Corollary. Let $f \in O_{\mathbf{A}}^{(n)}$ and $g \in O_{\mathbf{A}}^{(r)}$. Assume that $f^{\partial}$ respects the images of the 
injection morphisms to the degree 1 and that the following identity holds:

$$
f\left(x_{i_{1}}, \ldots, x_{i_{n}}\right) \approx g\left(x_{j_{1}}, \ldots, x_{j_{r}}\right) .
$$

Then, $f$ does not depend on its $t$-th argument for all $t \in\{1, \ldots, n\}$ with $i_{t} \notin\left\{j_{1}, \ldots, j_{r}\right\}$.

We can conclude that, whenever $C^{\partial}$ respects the images of the injection morphisms to the degree 1, many types of operations cannot occur in $C$. For instance, $C$ cannot contain operations that depend on all their arguments but satisfy an irregular identity. Moreover, the dualized version of Corollary 6.3.2 (page 119) holds:

7.3.2 Corollary. Let $C \leq O_{\mathbf{A}}$. Assume that $C^{\partial}$ respects the images of the injection morphisms to the degree 1 . Then, $C$ cannot contain near-unanimity operations and proper semiprojections of arity greater than 2 .

Looking at the examples presented in Section 4.2, we can conclude that for clones over distributive lattices, Boolean algebras, Boolean lattices, Boolean rings or median algebras, all these types of operations cannot occur.

Note that we have already established that $\langle C]$ is finite if $C^{\partial}$ respects the images of the injection morphisms to the degree 1 . Thus, all such clones have only finitely many subclones but do not contain near-unanimity operations. This is a somewhat surprising observation since a near-unanimity operation in a clone $C$ also implies that $C$ has only finitely many subclones. Therefore, we have found a class of finite clone ideals that is disjoint to the class of ideals generated by a clone with a near-unanimity term.

\subsection{Idempotent Operations}

In this section, we will examine the idempotent operations among $O_{\mathbf{A}}$. Recall that an operation $f$ is called idempotent if $f(x, \ldots, x) \approx x$. Denote by $\mathcal{I}_{\mathbf{A}}$ the set of all idempotent operations over A. A clone $C \leq O_{\mathbf{A}}$ is called idempotent if each operation in $C$ is idempotent.

Combining the statements of Corollary 6.4.8, Proposition 6.4.12 and Theorem 6.4.14 (pages 121, 123), we obtain the following corollary (recall the definitions of $X^{\sharp}, \Pi(-)$ and the Stirling numbers, page 120 and 121):

7.4.1 Corollary. Assume that $\left(\mathcal{I}_{\mathbf{A}}\right)^{\partial}$ respects the images of the injection morphisms to the degree 1. Then,

(i) the number of essentially different, essentially n-ary idempotent operations over $\mathbf{A}$ is bounded by $S_{\left|X^{\sharp}\right|}^{n}$,

(ii) the ideal $\left\langle\mathcal{I}_{\mathbf{A}}\right]$ can be order-embedded into $\left\langle\operatorname{Part}\left(X^{\sharp}\right), \preccurlyeq\right\rangle$,

(iii) the number of idempotent clones over $\mathbf{A}$ and the number of essentially different idempotent operations over $\mathbf{A}$ coincide, and they are both bounded by the Bell number $B_{|X|}$. 
Recall that we have $X^{\sharp} \subseteq X$, so all the statements in the corollary also hold if we replace $X^{\sharp}$ by $X$.

Looking at our examples, recall that $\bar{O}_{\mathbf{X}}$ respects the images of the injection morphisms to the degree 1 if $\mathbf{A}$ is a distributive lattice, a Boolean algebra, a median algebra, a Boolean ring or a Boolean lattice. Thus, we can apply the corollary to all these cases.

Statement (i) raises the questions of how we have to interpret the bound $S_{\left|X^{\sharp}\right|}^{n}$ for our examples and how tight it is. For A being a Boolean algebra, a Boolean ring or a Boolean lattice, we can give a straight-forward answer to both questions.

7.4.2 Proposition. If $\mathbf{A}$ is a Boolean algebra, a Boolean ring or a Boolean lattice, then the number of essentially different, essentially n-ary idempotent operations over $\mathbf{A}$ is $S_{l}^{n}$ for $l:=\log _{2}|\mathbf{A}|$ (the number of atoms of $\mathbf{A}$ ).

Proof. Let A be a Boolean algebra. If we take the clone duality described in Subsection 4.2.2, we have $|\mathbf{X}|=l$. Evidently, we have $X^{\sharp}=X$. Furthermore, it is easy to see that every partition of $X^{\sharp}$ can be written as $\Pi\left(f^{\partial}\right)$ for some $f \in O_{\mathbf{A}}$. Thus, we have $S_{\left|X^{\sharp}\right|}^{n}=S_{l}^{n}$ essentially different, essentially $n$-ary idempotent operations over A.

Now assume that $\mathbf{A}$ is a Boolean ring. If we take the clone duality described in Subsection 4.2.4 then $|\mathbf{X}|=l+1$. This time, we have $X^{\sharp}=X \backslash\{A\}$, and each partition of $X^{\sharp}$ can again be written as $f^{\partial}$ for some $f \in O_{\mathbf{A}}$. Thus, the number of essentially different, essentially $n$-ary idempotent operations is $S_{\left|X^{\sharp}\right|}^{n}=S_{l}^{n}$.

If $\mathbf{A}$ is a Boolean lattice, the proof is analogue. However, this time, we have $|\mathbf{X}|=l+2$, $X^{\sharp}=X \backslash\{A, \emptyset\}$ and, consequently, $S_{\left|X^{\sharp}\right|}^{n}=S_{l}^{n}$.

Thus, $S_{\left|X^{\sharp}\right|}^{n}$ is a sharp bound in all three cases.

If $\mathbf{A}$ is a distributive lattice or a median algebra, then $\left|X^{\sharp}\right|$ and the tightness of the bound do not only depend on the cardinality of $\mathbf{A}$, but also on the inner properties of A. In fact, for some choices of $\mathbf{A}$, the bound $S_{\left|X^{\sharp}\right|}^{n}$ can be tight, for others it can be very loose. We will discuss this for distributive lattices in Section 8.3, and since median algebras will turn out to be very similar in this aspect, we will treat them in the same section.

Continuing with applying Corollary 7.4.1 to our examples, we will now specify the order embedding in (ii) and the bound in (iii) for Boolean algebras, Boolean lattices and Boolean rings:

7.4.3 Proposition. If $\mathrm{A}$ is a Boolean algebra, a Boolean ring or a Boolean lattice, then $\left\langle\mathcal{I}_{\mathbf{A}}\right] \cong\left\langle\operatorname{Part}\left(\left\{1, \ldots, \log _{2}|\mathbf{A}|\right\}\right), \preccurlyeq\right\rangle$. Consequently, the number of idempotent clones is

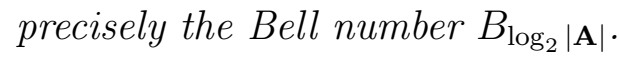

Proof. As presented in the previous proof, we have $\left|X^{\sharp}\right|=\log _{2}|\mathbf{A}|$ in all three cases. Moreover, each partition of $X^{\sharp}$ can be written as $\Pi\left(f^{\partial}\right)$ for some $f \in O_{\mathbf{A}}$. Thus, the order embedding $\varphi:\left\langle\overline{\mathcal{I}}_{\mathbf{X}}\right] \rightarrow\left\langle\operatorname{Part}\left(X^{\sharp}\right), \preccurlyeq\right\rangle$ constructed in the proof of Theorem 6.4.14 (page 123) is an isomorphism. Therefore,

$$
\left\langle\mathcal{I}_{\mathbf{A}}\right] \cong\left\langle\overline{\mathcal{I}}_{\mathbf{X}}\right] \cong\left\langle\operatorname{Part}\left(X^{\sharp}\right), \preccurlyeq\right\rangle \cong\left\langle\operatorname{Part}\left(\left\{1, \ldots, \log _{2}|\mathbf{A}|\right\}\right), \preccurlyeq\right\rangle .
$$




\begin{tabular}{|l|c|c|c|}
\hline \multicolumn{1}{|c|}{ A } & $\left\langle\mathcal{I}_{\mathbf{A}}\right]$ & $\begin{array}{c}\text { Number of } \\
\text { idempotent } \\
\text { clones }\end{array}$ & $\begin{array}{c}\text { Number of } \\
\text { essentially different, } \\
\text { essentially } n \text {-ary } \\
\text { idempotent } \\
\text { operations }\end{array}$ \\
\hline \hline Distr. lattice $^{1}$ & $\cong\langle\operatorname{Part}(\operatorname{Con}(\mathbf{X})), \preccurlyeq\rangle^{2}$ & $B_{|\operatorname{Con}(\mathbf{X})|}$ & $S_{|\operatorname{Con}(\mathbf{X})|}^{n}$ \\
\hline Median algebra $^{1}$ & $\cong\langle\operatorname{Part}(\operatorname{Con}(\mathbf{X})), \preccurlyeq\rangle^{2}$ & $B_{\left|\operatorname{Con}_{(}(\mathbf{X})\right|}$ & $S_{|\operatorname{Con}(\mathbf{X})|}^{n}$ \\
\hline Boolean algebra $^{3}$ & $\cong\left\langle\operatorname{Part}\left(\left\{1, \ldots, \log _{2}|\mathbf{A}|\right\}\right), \preccurlyeq\right\rangle$ & $B_{\log _{2}|\mathbf{A}|}$ & $S_{\log _{2}|\mathbf{A}|}^{n}$ \\
\hline Boolean ring & $\cong\left\langle\operatorname{Part}\left(\left\{1, \ldots, \log _{2}|\mathbf{A}|\right\}\right), \preccurlyeq\right\rangle$ & $B_{\log _{2}|\mathbf{A}|}$ & $S_{\log _{2}|\mathbf{A}|}$ \\
\hline Boolean lattice & $\cong\left\langle\operatorname{Part}\left(\left\{1, \ldots, \log _{2}|\mathbf{A}|\right\}\right), \preccurlyeq\right\rangle$ & $B_{\log _{2}|\mathbf{A}|}$ & $S_{\log _{2}|\mathbf{A}|}$ \\
\hline
\end{tabular}

${ }^{1}$ see Section 8.3

2 for $\operatorname{Con}(\mathbf{X})$, see Definition 8.0 .3 , page 148

${ }^{3}$ cf. Maš06]

Table 7.3.: Idempotent clones in $O_{\mathbf{A}}$ for our examples

A similarly nice result can be obtained for the case in which $\mathbf{A}$ is a distributive lattice or a median algebra (see Section 8.3). Table 7.3 summarizes the results for our examples.

Corollary 7.4.1 is based on the nice characterization of dual idempotent operations over $\mathbf{X}$ that is provided by Theorem 6.4.2 (page 120). However, this theorem only works if the dual idempotent operation in question respects the images of the injection morphisms to the degree 1 . Thus, the duals of idempotent operations over Boolean groups and over semilattices can not be characterized as in Theorem 6.4.2. In the next two subsections, we will find alternative characterizations for these cases.

\subsubsection{The Duals of Idempotent Operations over Boolean Groups}

In this subsection, let $\mathbf{A}=\langle A,+, 0\rangle$ be a finite Boolean group and let $(-)^{\partial}: O_{\mathbf{A}} \rightarrow \bar{O}_{\mathbf{X}}$ be the clone duality from Subsection 4.2.6. Recall the notation from Subsection 4.2.6. We denote by $B_{\mathbf{A}}$ a basis of $\mathbf{A}$ and by

$$
B_{\mathbf{A}^{n}}:=\left\{(0, \ldots, \underset{\substack { i \\
\begin{subarray}{c}{i{ i \\
\begin{subarray} { c } { i } }\end{subarray}}{a}, 0, \ldots, 0) \mid i \in\{1, \ldots, n\}, a \in B_{\mathbf{A}}\right\}
$$

the according basis of $\mathbf{A}^{n}$. Furthermore, we denote by $\chi_{\mathbf{A}^{n}}(a)$ the (unique) subset $B^{\prime} \subseteq B_{\mathbf{A}^{n}}$ such that $a=\sum B^{\prime}$, and for a subset $B^{\prime} \subseteq B_{\mathbf{A}^{n}}$, we denote by $\left[B^{\prime}\right]_{\text {odd }}$ the set of elements $a \in A^{n}$ for which $\left|B^{\prime} \cap \chi_{\mathbf{A}^{n}}(a)\right|$ is odd. The set $\left[B^{\prime}\right]_{\text {even }}$ is defined accordingly.

7.4.4 Proposition. An operation $f \in O_{\mathbf{A}}^{(n)}$ is idempotent if and only if the following two conditions hold for each $a \in B_{\mathbf{A}}$ :

(i) $f^{\partial}(a) \in[\{(a, 0, \ldots, 0),(0, a, 0, \ldots, 0), \ldots,(0, \ldots, 0, a)\}]_{o d d}$, 
(ii) $f^{\partial}(a) \in[\{(b, 0, \ldots, 0),(0, b, 0, \ldots, 0), \ldots,(0, \ldots, 0, b)\}]_{\text {even }}$ for all $b \in B_{\mathbf{A}} \backslash\{a\}$.

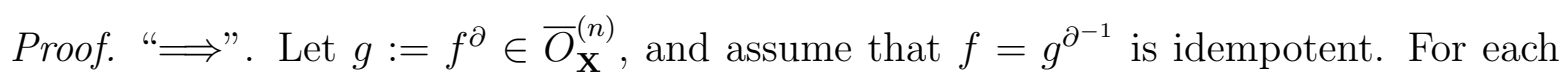
$a \in B_{\mathbf{A}}$, we have

$$
\begin{aligned}
a=f(a, \ldots, a) & =g^{\partial^{-1}}(a, \ldots, a) \\
& =\sum\left(g^{-1}\left(\left[\chi_{\mathbf{A}^{n}}(a, \ldots, a)\right]_{o d d}\right) \cap B_{\mathbf{A}}\right) \\
& =\sum\left(g^{-1}\left([\{(a, 0, \ldots, 0), \ldots,(0, \ldots, 0, a)\}]_{\text {odd }}\right) \cap B_{\mathbf{A}}\right),
\end{aligned}
$$

which implies

$$
\{a\}=g^{-1}\left([\{(a, 0, \ldots, 0), \ldots,(0, \ldots, 0, a)\}]_{o d d}\right) \cap B_{\mathbf{A}} .
$$

Thus,

$$
f^{\partial}(a)=g(a) \in[\{(a, 0, \ldots, 0), \ldots,(0, \ldots, 0, a)\}]_{o d d} .
$$

Moreover, for $b \in B_{\mathbf{A}} \backslash\{a\}$, we obtain

$$
\{b\}=g^{-1}\left([\{(b, 0, \ldots, 0), \ldots,(0, \ldots, 0, b)\}]_{o d d}\right) \cap B_{\mathbf{A}},
$$

which implies

$$
f^{\partial}(a)=g(a) \notin[\{(b, 0, \ldots, 0), \ldots,(0, \ldots, 0, b)\}]_{o d d} .
$$

That is,

$$
f^{\partial}(a) \in[\{(b, 0, \ldots, 0), \ldots,(0, \ldots, 0, b)\}]_{\text {even }} .
$$

"£". Again, let $g:=f^{\partial}$. It is sufficient to establish $f(a, \ldots, a)=a$ for all $a \in B_{\mathbf{A}}$. Thus, we need to show

$$
\sum\left(g^{-1}\left([\{(a, 0, \ldots, 0), \ldots,(0, \ldots, 0, a)\}]_{\text {odd }}\right) \cap B_{\mathbf{A}}\right)=a .
$$

By (i), we have $a \in g^{-1}\left([\{(a, 0, \ldots, 0), \ldots,(0, \ldots, 0, a)\}]_{\text {odd }}\right)$. Now let $a^{\prime} \in B_{\mathbf{A}} \backslash\{a\}$. By (ii), we have $a^{\prime} \notin g^{-1}\left([\{(b, 0, \ldots, 0), \ldots,(0, \ldots, 0, b)\}]_{\text {odd }}\right)$ for all $b \in B_{\mathbf{A}} \backslash\left\{a^{\prime}\right\}$. In particular, we can choose $b=a$, and it follows

$$
\left\{a^{\prime}\right\} \notin g^{-1}\left([\{(a, 0, \ldots, 0), \ldots,(0, \ldots, 0, a)\}]_{\text {odd }}\right) .
$$

Thus,

$$
\{a\}=g^{-1}\left([\{(a, 0, \ldots, 0), \ldots,(0, \ldots, 0, a)\}]_{o d d}\right) \cap B_{\mathbf{A}} .
$$

We obtain

$$
\sum\left(g^{-1}\left([\{(a, 0, \ldots, 0), \ldots,(0, \ldots, 0, a)\}]_{o d d}\right) \cap B_{\mathbf{A}}\right)=\sum\{a\}=a .
$$

For binary operations, we can simplify this criterion. 
7.4.5 Corollary. An operation $f \in O_{\mathbf{A}}^{(2)}$ is idempotent if and only if we have

$$
f^{\partial}(a) \in\{(a, 0)+(x, x) \mid x \in A\}
$$

for all $a \in B_{\mathbf{A}}$.

Proof. By Proposition 7.4.4 $f$ is idempotent if and only if $f^{\partial}(a) \in[\{(a, 0),(0, a)\}]_{\text {odd }}$ and $f^{\partial}(a) \in[\{(b, 0),(0, b)\}]_{\text {even }}$ for all $b \in B_{\mathbf{A}} \backslash\{a\}$. The first condition means that $\chi_{\mathbf{A}^{2}}\left(f^{\partial}(a)\right)$ contains exactly one of the two elements $(a, 0)$ and $(0, a)$. Thus, it contains $(a, 0)$ or $(a, 0)+(a, a)$. The latter condition means that, for each $b \in B_{\mathbf{A}} \backslash\{a\}, \chi_{\mathbf{A}^{2}}\left(f^{\partial}(a)\right)$ either contains $(b, 0)$ and $(0, b)$ or none of the two elements. Thus, $f^{\partial}(a)$ is idempotent if and only if there exist base elements $b_{1}, \ldots, b_{n}$ such that

$$
f^{\partial}(a)=(a, 0)+\left(b_{1}, b_{1}\right)+\ldots+\left(b_{n}, b_{n}\right) .
$$

The claim follows directly.

7.4.6 Example. Let $\mathbf{A}:=\langle\{0, a, \bar{a}, 1\},+, 0\rangle$ be the (up to isomorphism) unique Boolean group with 4 elements. Let $B_{\mathbf{A}}:=\{a, \bar{a}\}$. We will list all dual binary idempotent operations over $\mathbf{X}$. By Corollary 7.4.5, a dual operation $g \in \bar{O}_{\mathbf{X}}^{(2)}$ is idempotent if and only if $g(a) \in\{(a, 0),(0, a),(1, \bar{a}),(\bar{a}, 1)\}$ and $g(\bar{a}) \in\{(\bar{a}, 0),(0, \bar{a}),(1, a),(a, 1)\}$. Thus, we obtain 16 binary dual idempotent operations over $\mathbf{X}$, eight of which are essentially different. They can be seen in the table below. The other eight arise by interchanging the two arguments of $g_{1}, \ldots, g_{8}$.

\begin{tabular}{|c||c|c|c|c|}
\hline$x$ & 0 & $a$ & $\bar{a}$ & 1 \\
\hline \hline$g_{1}(x)$ & $(0,0)$ & $(a, 0)$ & $(\bar{a}, 0)$ & $(1,0)$ \\
$g_{2}(x)$ & $(0,0)$ & $(a, 0)$ & $(0, \bar{a})$ & $(a, \bar{a})$ \\
$g_{3}(x)$ & $(0,0)$ & $(a, 0)$ & $(1, a)$ & $(\bar{a}, a)$ \\
$g_{4}(x)$ & $(0,0)$ & $(a, 0)$ & $(a, 1)$ & $(0,1)$ \\
$g_{5}(x)$ & $(0,0)$ & $(1, \bar{a})$ & $(\bar{a}, 0)$ & $(a, \bar{a})$ \\
$g_{6}(x)$ & $(0,0)$ & $(1, \bar{a})$ & $(0, \bar{a})$ & $(1,0)$ \\
$g_{7}(x)$ & $(0,0)$ & $(1, \bar{a})$ & $(1, a)$ & $(0,1)$ \\
$g_{8}(x)$ & $(0,0)$ & $(1, \bar{a})$ & $(a, 1)$ & $(\bar{a}, a)$ \\
\hline
\end{tabular}

Note that $g_{1}$ is $\iota_{1}^{2}$, so we have seven essentially different nontrivial binary idempotent operations over A.

\subsubsection{The Duals of Idempotent Operations over Semilattices}

For this subsection, let $\mathbf{A}=\langle A, \vee\rangle$ be a finite semilattice, and let $(-)^{\partial}: O_{\mathbf{A}} \rightarrow \bar{O}_{\mathbf{X}}$ be the clone duality from Subsection 4.2.7. Recall that $\mathbf{X}=\langle\operatorname{Spec}(\mathbf{A}) \cup\{\emptyset, A\}, \emptyset, A, \cup\rangle$.

7.4.7 Proposition. An operation $f \in O_{\mathbf{A}}^{(n)}$ is idempotent if and only if one of the following two conditions holds for each $x \in X \backslash\{\emptyset, A\}$ : 
(i) $f^{\partial}(x)=\langle j, x\rangle$ for some $j \in\{1, \ldots, n\}$,

(ii) $f^{\partial}(x)=\left\{\left\langle j_{1}, x_{1}\right\rangle, \ldots,\left\langle j_{k}, x_{k}\right\rangle\right\}$ for some $j_{1}, \ldots, j_{k} \in\{1, \ldots, n\}$ and $x_{1}, \ldots, x_{k} \in \mathbf{X}$ with $x=x_{1} \vee^{\mathbf{X}} \ldots \vee^{\mathbf{X}} x_{k}$.

Proof. For this proof, recall the description of the cotuplings of morphisms over $\mathbf{X}$ given in Subsection 4.2.7 (page 74).

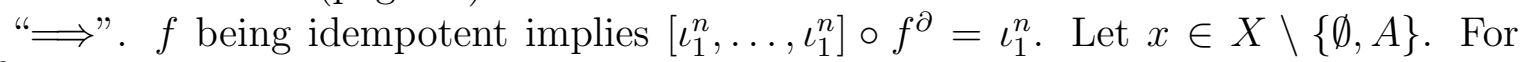
$f^{\partial}(x)=0^{n \cdot \mathbf{X}}$, we obtain

$$
\left(\left[\iota_{1}^{n}, \ldots, \iota_{1}^{n}\right] \circ f^{\partial}\right)(x)=\left[\iota_{1}^{n}, \ldots, \iota_{1}^{n}\right]\left(0^{n \cdot \mathbf{X}}\right)=0^{n \cdot \mathbf{X}} \neq\langle 1, x\rangle=\iota_{1}^{n}(x),
$$

a contradiction. Hence, $f^{\partial}(x) \neq 0^{n \cdot \mathbf{X}}$. A similar argument implies $f^{\partial}(x) \neq 1^{n \cdot \mathbf{X}}$. Now, let $f^{\partial}(x)=\left\langle j, x^{\prime}\right\rangle$. Then

$$
\left\langle 1, x^{\prime}\right\rangle=\left[\iota_{1}^{n}, \ldots, \iota_{1}^{n}\right]\left(\left\langle j, x^{\prime}\right\rangle\right)=\left(\left[\iota_{1}^{n}, \ldots, \iota_{1}^{n}\right] \circ f^{\partial}\right)(x)=\iota_{1}^{n}(x)=\langle 1, x\rangle,
$$

and thus, $x=x^{\prime}$. Finally, let $f^{\partial}(x)=\left\{\left\langle j_{1}, x_{1}\right\rangle, \ldots,\left\langle j_{k}, x_{k}\right\rangle\right\}$. But now,

$$
\left\langle 1, x_{1} \vee^{\mathbf{X}} \ldots \vee^{\mathbf{X}} x_{k}\right\rangle=\left(\left[\iota_{1}^{n}, \ldots, \iota_{1}^{n}\right] \circ f^{\partial}\right)(x)=\iota_{1}^{n}(x)=\langle 1, x\rangle
$$

implies $x=x_{1} \vee^{\mathbf{X}} \ldots \vee^{\mathbf{X}} x_{k}$.

"£". Let $f$ have the property described in the claim and let $x \in \mathbf{X}$. We have to show $\left(\left[\iota_{1}^{n}, \ldots, \iota_{1}^{n}\right] \circ f^{\partial}\right)(x)=\iota_{1}^{n}(x)$. For $x \in\{\emptyset, A\}$, this is obvious. For $x \notin\{\emptyset, A\}$, we have (i) or (ii) as above. In the first case, we obtain

$$
\left(\left[\iota_{1}^{n}, \ldots, \iota_{1}^{n}\right] \circ f^{\partial}\right)(x)=\left[\iota_{1}^{n}, \ldots, \iota_{1}^{n}\right](\langle j, x\rangle)=\langle 1, x\rangle=\iota_{1}^{n}(x) .
$$

In the second case, we have

$$
\begin{aligned}
{\left[\iota_{1}^{n}, \ldots, \iota_{1}^{n}\right] \circ f^{\partial}(x) } & =\left[\iota_{1}^{n}, \ldots, \iota_{1}^{n}\right]\left(\left\{\left\langle j_{1}, x_{1}\right\rangle, \ldots,\left\langle j_{k}, x_{k}\right\rangle\right\}\right) \\
& =\left\langle 1, x_{1} \vee^{\mathbf{X}} \ldots \vee^{\mathbf{x}} x_{k}\right\rangle \\
& =\langle 1, x\rangle \\
& =\iota_{1}^{n}(x) .
\end{aligned}
$$

In the following example, we will see that this characterization of dual idempotent operations can be used to simplify the problem of determining all idempotent operations over a given finite semilattice $\mathbf{A}$.

7.4.8 Example. Let $\mathbf{A}=\langle\{0,1,2\}, \vee\rangle$ be a semilattice where $x \vee y:=\max (x, y)$. As we have seen in Example 4.2.46 (page 74), the dual of $\mathbf{A}$ is given by

$$
\mathbf{X}=\langle\{\emptyset,\{2\},\{1,2\}, A\}, \emptyset, A, \cup\rangle .
$$

We will now count the dual binary idempotent operations over $\mathbf{X}$. Recall that $2 \cdot \mathbf{X}$ can be represented as in Example 4.2.46 (page 74). Denote by $\leq_{2 \cdot \mathbf{X}}$ the order relation that is induced by this semilattice and let $g \in \bar{O}_{\mathbf{X}}^{(2)}$ be idempotent. By Proposition 7.4.7, there 
are three possible choices for $g(\{2\})$, namely $\langle 1,\{2\}\rangle,\langle 2,\{2\}\rangle$ and $\{\langle 1,\{2\}\rangle,\langle 2,\{2\}\rangle\}$. For $g(\{2\})=\langle 1,\{2\}\rangle$, we can use Proposition 7.4.7 and the fact that we must have $g(\{2\}) \leq_{2} \cdot \mathbf{X} g(\{1,2\})$ to conclude that there are exactly four possible values for $g(\{1,2\})$, and we obtain the four dual idempotent operations $g_{1}, \ldots, g_{4}$ that are listed in Table 7.4. The case $g(\{2\})=\langle 2,\{2\}\rangle$ is analogue. In fact, we obtain exactly those dual operations that arise from $g_{1}, \ldots, g_{4}$ by interchanging the two arguments. Call these operations $g_{1}^{\prime}, \ldots, g_{4}^{\prime}$. Finally, for $g(\{2\})=\{\langle 1,\{2\}\rangle,\langle 2,\{2\}\rangle\}$, we can use the same arguments and conclude that there are three possible values for $g(\{1,2\})$, and thus, three dual binary operations $g_{5}, g_{5}^{\prime}, g_{6}$. However, as the name suggests, $g_{5}$ and $g_{5}^{\prime}$ are essentially the same operation as they arise from each other by interchanging the two arguments (note that $g_{6}$ is invariant under permutation of arguments).

\begin{tabular}{|c||c|c|c|c|}
\hline$x$ & $\emptyset$ & $\{2\}$ & $\{1,2\}$ & $A$ \\
\hline \hline$g_{1}(x)$ & $0^{2 \cdot \mathbf{X}}$ & $\langle 1,\{2\}\rangle$ & $\langle 1,\{1,2\}\rangle$ & $1^{2 \cdot \mathbf{X}}$ \\
$g_{1}^{\prime}(x)$ & $0^{2 \cdot \mathbf{X}}$ & $\langle 2,\{2\}\rangle$ & $\langle 2,\{1,2\}\rangle$ & $1^{2 \cdot \mathbf{X}}$ \\
\hline$g_{2}(x)$ & $0^{2 \cdot \mathbf{X}}$ & $\langle 1,\{2\}\rangle$ & $\{\langle 1,\{1,2\}\rangle,\langle 2,\{2\}\rangle\}$ & $1^{2 \cdot \mathbf{X}}$ \\
$g_{2}^{\prime}(x)$ & $0^{2 \cdot \mathbf{X}}$ & $\langle 2,\{2\}\rangle$ & $\{\langle 1,\{2\}\rangle,\langle 2,\{1,2\}\rangle\}$ & $1^{2 \cdot \mathbf{X}}$ \\
\hline$g_{3}(x)$ & $0^{2 \cdot \mathbf{X}}$ & $\langle 1,\{2\}\rangle$ & $\{\langle 1,\{2\}\rangle,\langle 2,\{1,2\}\rangle\}$ & $1^{2 \cdot \mathbf{X}}$ \\
$g_{3}^{\prime}(x)$ & $0^{2 \cdot \mathbf{X}}$ & $\langle 2,\{2\}\rangle$ & $\{\langle 1,\{1,2\}\rangle,\langle 2,\{2\}\rangle\}$ & $1^{2 \cdot \mathbf{X}}$ \\
\hline$g_{4}(x)$ & $0^{2 \cdot \mathbf{X}}$ & $\langle 1,\{2\}\rangle$ & $\{\langle 1,\{1,2\}\rangle,\langle 2,\{1,2\}\rangle\}$ & $1^{2 \cdot \mathbf{X}}$ \\
$g_{4}^{\prime}(x)$ & $0^{2 \cdot \mathbf{X}}$ & $\langle 2,\{2\}\rangle$ & $\{\langle 1,\{1,2\}\rangle,\langle 2,\{1,2\}\rangle\}$ & $1^{2 \cdot \mathbf{X}}$ \\
\hline$g_{5}(x)$ & $0^{2 \cdot \mathbf{X}}$ & $\{\langle 1,\{2\}\rangle,\langle 2,\{2\}\rangle\}$ & $\{\langle 1,\{2\}\rangle,\langle 2,\{1,2\}\rangle\}$ & $1^{2 \cdot \mathbf{X}}$ \\
$g_{5}^{\prime}(x)$ & $0^{2 \cdot \mathbf{X}}$ & $\{\langle 1,\{2\}\rangle,\langle 2,\{2\}\rangle\}$ & $\{\langle 1,\{1,2\}\rangle,\langle 2,\{2\}\rangle\}$ & $1^{2 \cdot \mathbf{X}}$ \\
\hline$g_{6}(x)$ & $0^{2 \cdot \mathbf{X}}$ & $\{\langle 1,\{2\}\rangle,\langle 2,\{2\}\rangle\}$ & $\{\langle 1,\{1,2\}\rangle,\langle 2,\{1,2\}\rangle\}$ & $1^{2 \cdot \mathbf{X}}$ \\
\hline
\end{tabular}

Table 7.4.: The nontrivial binary idempotent operations over $\mathbf{A}$

Thus, we have nine nontrivial binary idempotent operations over $\mathbf{A}$, five of which are essentially different.

\subsection{Minimal Clones}

In this section, we will apply the results of Section 6.5. For a clone $C \leq O_{\mathbf{A}}$ with $C^{\partial}$ respecting the images of the injection morphisms to the degree 1, we can use Theorem 6.5.1 (page 123), and we obtain the following full description of all minimal clones in the ideal $\langle C]$ :

7.5.1 Theorem. Assume that $C^{\partial}$ respects the images of the injection morphisms to the degree 1. A nontrivial operation $f \in C$ is minimal if and only if it is of one of the following three types:

1. a unary retraction, 
2. an automorphism of prime order,

3. a binary idempotent operation.

Looking at the examples presented in Section 4.2, we now have a full description of the minimal clones in $\mathcal{L}_{\mathbf{A}}$ for $\mathbf{A}$ being a distributive lattice, a Boolean algebra, a Boolean lattice, a Boolean ring or a median algebra. For three of theses cases, we can directly determine the number of minimal clones in $\mathcal{L}_{\mathbf{A}}$.

7.5.2 Proposition. Let $k$ be the number of minimal clones in $\mathcal{L}_{\mathbf{A}}$, and let $P_{n}^{p}$ denote the number of permutations of order $p$ on an n-element set. Then,

(i) $k=S_{n}^{2}+\sum_{p \leq n, p \text { prime }} P_{n}^{p}+\sum_{i=1}^{n-1}\left(\begin{array}{c}n \\ i\end{array}\right) i^{(n-i)}$ if $\mathbf{A}$ is a $2^{n}$-element Boolean algebra.

(ii) $k=S_{n}^{2}+\sum_{p \leq n, p \text { prime }} P_{n}^{p}+\sum_{i=0}^{n-1}\left(\begin{array}{c}n \\ i\end{array}\right)(i+1)^{(n-i)}$ if $\mathbf{A}$ is an $2^{n}$-element Boolean ring.

(iii) $k=S_{n}^{2}+\sum_{p \leq n, p \text { prime }} P_{n}^{p}+\sum_{i=0}^{n-1}\left(\begin{array}{c}n \\ i\end{array}\right)(i+2)^{(n-i)}$ if $\mathbf{A}$ is an $2^{n}$-element Boolean lattice.

Proof. By Theorem 7.5.1, we have to count the number of essentially different binary idempotent operations over $\mathbf{A}$, the number of automorphisms on $\mathbf{A}$ that are of prime order and the number of retraction over $\mathbf{A}$.

We have seen in Proposition 7.4.2 (page 134) that the number of essentially different binary idempotent operations is $S_{n}^{2}$ in all three cases. Now, let us count the number of automorphisms that are of prime order. For this, let $\mathbf{X}$ be the dual of $\mathbf{A}$ under the respective clone duality from Section 4.2. If $\mathbf{A}$ is a Boolean algebra, then $\mathbf{X}$ has $n$ elements and each bijective mapping on $\mathbf{X}$ is an isomorphism. If $\mathbf{A}$ is a Boolean ring, then $\mathbf{X}$ has $n+1$ elements and a bijective mapping $g: X \rightarrow X$ is an automorphism on $\mathbf{X}$ if and only if $g\left(1^{\mathbf{X}}\right)=1^{\mathbf{X}}$. If $\mathbf{A}$ is a Boolean lattice, then $\mathbf{X}$ has $n+2$ elements and a bijective mapping $g: X \rightarrow X$ is an automorphism on $\mathbf{X}$ if and only if $g\left(1^{\mathbf{X}}\right)=1^{\mathbf{X}}$ and $g\left(0^{\mathbf{X}}\right)=0^{\mathbf{X}}$. Hence, in each case, each automorphism of order $p$ on $\mathbf{X}$ can be uniquely associated to a permutation of order $p$ on $n$ elements. Therefore, the number of isomorphisms over $\mathbf{X}$ of order $p$ is $P_{n}^{p}$. It remains to count the number of retractions over $\mathbf{X}$. Let $G_{i}$ be the set of retractions $g: \mathbf{X} \rightarrow \mathbf{X}$ with $|g(\mathbf{X})|=i$. If $\mathbf{A}$ is a Boolean algebra, we have $\left|G_{i}\right|=\left(\begin{array}{c}n \\ i\end{array}\right) i^{(n-i)}$ since we can choose $i$ fixpoints, and each of the $n-i$ remaining elements can be mapped to each of the $i$ fixpoints. By $|\mathbf{X}|=n$, we end up with $\sum_{i=1}^{n-1}\left(\begin{array}{c}n \\ i\end{array}\right) i^{(n-i)}$ retractions over $\mathbf{X}$. If $\mathbf{A}$ is a Boolean ring, the situation is similar except that $1^{\mathbf{X}} \in \mathbf{X}$ is always a fixpoint. Thus, we have $\left|G_{1}\right|=1$, and for $2 \leq i \leq n$, we have $\left|G_{i}\right|=\left(\begin{array}{c}n \\ i-1\end{array}\right) i^{(n-i)}$. Therefore, the number of retractions over $\mathbf{X}$ is

$$
1+\sum_{i=2}^{n}\left(\begin{array}{c}
n \\
i-1
\end{array}\right) i^{(n-i)}=\sum_{i=0}^{n-1}\left(\begin{array}{c}
n \\
i
\end{array}\right)(i+1) i^{(n-i)} .
$$

For $\mathbf{A}$ being a Boolean lattice, $0^{\mathbf{X}}$ and $1^{\mathbf{X}}$ are always fixpoints. Thus, $\left|G_{1}\right|=0,\left|G_{2}\right|=2^{n}$ and $\left|G_{i}\right|=\left(\begin{array}{c}n \\ i-2\end{array}\right) i^{(n-i)}$ for $3 \leq i \leq n$. It follows in the same way as above that we have $\sum_{i=0}^{n-1}\left(\begin{array}{c}n \\ i\end{array}\right)(i+2)^{(n-i)}$ retractions over $\mathbf{X}$. 
Table 7.5 gives the number of minimal clones in $\mathcal{L}_{\mathbf{A}}$ for the three cases discussed in the proposition above.

\begin{tabular}{|l|c|c|c|}
\hline$n$ & $\begin{array}{c}\text { \# minimal clones if } \\
\text { A Boolean algebra }\end{array}$ & $\begin{array}{c}\text { \# minimal clones } \\
\text { if A Boolean ring }\end{array}$ & $\begin{array}{c}\text { \# minimal clones if } \\
\text { A Boolean lattice }\end{array}$ \\
\hline \hline 1 & $0^{1}$ & 1 & 2 \\
\hline 2 & 4 & 7 & 12 \\
\hline 3 & 17 & 30 & 55 \\
\hline 4 & 64 & 127 & 264 \\
\hline 5 & 279 & 620 & 1441 \\
\hline 6 & 1386 & 3429 & 8774 \\
\hline 7 & 8189 & 21560 & 59123 \\
\hline 8 & 50618 & 145289 & 428458 \\
\hline 9 & 331193 & 1050930 & 3327787 \\
\hline 10 & 2443990 & 8282713 & 27713418 \\
\hline
\end{tabular}

${ }^{1} \mathcal{L}_{\mathrm{A}}$ consists of only one clone.

Table 7.5.: The number of minimal clones for $|\mathbf{A}|=2^{n}$ in the case of A being a Boolean algebra, a Boolean ring or a Boolean lattice

If $\mathbf{A}$ is a Boolean group or a semilattice, the copowers of $\mathbf{X}$ are not non-deformed to any degree (let alone to the degree 1). Hence, we cannot apply Theorem 7.5.1. However, we can still obtain some partial results for the minimal clones which will be presented in the next two subsections.

\subsubsection{The Minimal Clones over Boolean Groups}

In this subsection, let $\mathbf{A}=\langle A,+, 0\rangle$ be a finite Boolean group.

7.5.3 Proposition. Every minimal clone in $\mathcal{L}_{\mathbf{A}}$ is generated by a nontrivial operation $f$ that is of one of the following four types:

1. a unary retraction

2. an automorphism of prime order,

3. a binary idempotent operation,

4. a minority operation.

Proof. We have to show that majority operations and proper semiprojections cannot occur. First, assume that $f: \mathbf{A}^{3} \rightarrow \mathbf{A}$ is a majority operation. Then, for any $a \neq 0$, we have

$$
f(a, a, a)=f(a, 0,0)+f(0, a, 0)+f(0,0, a)=0+0+0=0
$$


a contradiction. Now let us assume that $f: \mathbf{A}^{n} \rightarrow \mathbf{A}$ is a semiprojection. Without loss of generality, we can assume $f\left(a_{1}, \ldots, a_{n}\right)=a_{1}$ whenever $a_{1}, \ldots, a_{n}$ are not pairwise distinct. But now,

$$
\begin{aligned}
f\left(a_{1}, \ldots, a_{n}\right) & =f\left(a_{1}, 0, \ldots, 0\right)+f\left(0, a_{2}, 0, \ldots, 0\right)+\ldots+f\left(0, \ldots, 0, a_{n}\right) \\
& =a_{1}+0+\ldots+0 \\
& =a_{1}
\end{aligned}
$$

for all $a_{1}, \ldots, a_{n} \in A$, implying that $f$ is a projection.

Concerning the fourth case, it was shown in Ros83 that every minimal minority operation over a finite set $A$ is necessarily $x \oplus y \oplus z$ in a Boolean group $\mathbf{A}^{\prime}=\langle A, \oplus, 0\rangle$. From this, we can conclude the following proposition:

7.5.4 Proposition. Over $\mathbf{A}$, there is only one minority operation, and this operation is minimal. It is also (up to conjugation) the only minimal minority operation over the set $A$.

Proof. Let $f \in O_{A}$ be a minimal minority operation. By [Ros83], it can be written as $f(x, y, z)=x \oplus y \oplus z$ where $\mathbf{A}^{\prime}=\langle A, \oplus, 0\rangle$ is a Boolean group. Since $\mathbf{A}^{\prime}$ and $\mathbf{A}$ are Boolean groups on the same set, there exists an isomorphism $\varphi: \mathbf{A}^{\prime} \rightarrow \mathbf{A}$. Let $h(x, y, z):=x+y+z$. Clearly, $h$ is a minority operation over $\mathbf{A}$, and we have

$$
f(x, y, z)=x \oplus y \oplus z=\varphi^{-1}(\varphi(x)+\varphi(y)+\varphi(z))=\varphi^{-1}(h(\varphi(x), \varphi(y), \varphi(z))),
$$

which means that $f$ is a conjugate of $h$ (and vice versa). This also implies that $h$ and all its other conjugates must be minimal as well. It remains to show that $h$ is the only minority operation in $O_{\mathbf{A}}$. For this, let $h^{\prime} \in O_{\mathbf{A}}$ be a minority operations. Then,

$$
h^{\prime}(x, y, z)=h^{\prime}(x, 0,0)+h^{\prime}(0, y, 0)+h^{\prime}(0,0, z)=x+y+z=h(x, y, z) .
$$

In contrast, not every nontrivial binary idempotent operation is minimal even in the four-element case. However, we will see in the next proposition that, in this case, almost all nontrivial binary idempotent operations are minimal.

7.5.5 Proposition. Let $\mathbf{A}=\langle\{0, a, \bar{a}, 1\}, 0,+\rangle$ be the (up to isomorphism) unique fourelement Boolean group. A clone in $\mathcal{L}_{\mathbf{A}}$ is minimal if and only if it is generated by a nontrivial operation $f$ that is of one of the following four types:

1. a unary retraction,

2. a permutation of prime order,

3. a binary dual idempotent operation $f$ for which the following two conditions hold:

- $f(a, 0) \neq 1$ or $f(\bar{a}, a) \neq a$,

- $f(a, 0) \neq \bar{a}$ or $f(\bar{a}, 0) \neq 1$,

4. the minority operation $m(x, y, z):=x+y+z$. 
Proof. By our previous work, everything is clear expect the binary case. Note that we have already looked at the binary idempotent operations over $\mathbf{A}$ in Example 7.4.6 (page 137). Let $g_{1}, \ldots, g_{8}$ be defined as in this example. Evidently, $g_{1}$ is trivial, and it is an easy calculation that each $g \in\left\{g_{2}, \ldots, g_{8}\right\}$ generates only four binary operations: Itself, $\left[\iota_{2}^{1}, \iota_{1}^{1}\right] \circ g$ and the two projections. This implies that $g \in\left\{g_{2}, \ldots, g_{8}\right\}$ is minimal if and only if it does not generate $m^{\partial}$, which can easily be calculated to be the following ternary dual operation:

\begin{tabular}{|c||c|c|c|c|}
\hline$x$ & 0 & $a$ & $\bar{a}$ & 1 \\
\hline$m^{\partial}(x)$ & $(0,0,0)$ & $(a, a, a)$ & $(\bar{a}, \bar{a}, \bar{a})$ & $(1,1,1)$ \\
\hline
\end{tabular}

We have

$$
m^{\partial}=\left[\left[\iota_{1}^{3}, \iota_{2}^{3}\right] \circ g_{8},\left[\iota_{3}^{3}, \iota_{1}^{3}\right] \circ g_{8}\right] \circ g_{8},
$$

so $g_{8}$ is not minimal. We will now show that $g_{2}, \ldots, g_{7}$ are minimal. For this, let us show that there exist dual relations $\sigma_{2}, \ldots, \sigma_{7}$ such that $g_{i}$ preserves $\sigma_{i}$, whereas $m^{\partial}$ does not preserve $\sigma_{i}$ for all $i \in\{2, \ldots, 7\}$. To this end, we define the following six morphisms from $\mathbf{B}$ to $\mathbf{A}$, where $\mathbf{B}=\left\langle\left\{0^{\mathbf{B}}, b, \bar{b}, 1_{\mathbf{B}}\right\},+, 0^{\mathbf{B}}\right\rangle$ is the unique element of $\mathbb{T}$ with $\mathbf{B} \cong \mathbf{A}$ :

\begin{tabular}{|c||c|c|c|c|}
\hline$x$ & 0 & $a$ & $\bar{a}$ & 1 \\
\hline \hline$r_{1}(x)$ & $0^{\mathbf{B}}$ & $\bar{b}$ & $1_{\mathbf{B}}$ & $b$ \\
\hline$r_{2}(x)$ & $0^{\mathbf{B}}$ & $b$ & $1_{\mathbf{B}}$ & $\bar{b}$ \\
\hline$r_{3}(x)$ & $0^{\mathbf{B}}$ & $1_{\mathbf{B}}$ & $1_{\mathbf{B}}$ & $0^{\mathbf{B}}$ \\
\hline$r_{4}(x)$ & $0^{\mathbf{B}}$ & $1_{\mathbf{B}}$ & $\bar{b}$ & $b$ \\
\hline$r_{5}(x)$ & $0^{\mathbf{B}}$ & $0^{\mathbf{B}}$ & $b$ & $b$ \\
\hline$r_{6}(x)$ & $0^{\mathbf{B}}$ & $1_{\mathbf{B}}$ & $b$ & $\bar{b}$ \\
\hline
\end{tabular}

Now, let $\sigma_{2}, \ldots, \sigma_{7}$ be the following dual relations of type $\mathbf{B}$ :

$$
\begin{aligned}
\sigma_{2}, \sigma_{5}, \sigma_{6} & :=\left\{r_{1}, r_{2}, r_{3}\right\}, \\
\sigma_{3}, \sigma_{4} & :=\left\{r_{3}, r_{4}, r_{6}\right\}, \\
\sigma_{7} & :=\left\{r_{1}, r_{4}, r_{5}\right\} .
\end{aligned}
$$

We have

$$
\begin{aligned}
& {\left[r_{1}, r_{2}, r_{3}\right] \circ m^{\partial}(a)=\left[r_{1}, r_{2}, r_{3}\right](a, a, a)=\bar{b}+b+1_{\mathbf{B}}=0^{\mathbf{B}} \notin\left\{r_{1}(a), r_{2}(a), r_{3}(a)\right\},} \\
& {\left[r_{1}, r_{4}, r_{5}\right] \circ m^{\partial}(\bar{a})=\left[r_{1}, r_{4}, r_{5}\right](\bar{a}, \bar{a}, \bar{a})=1_{\mathbf{B}}+\bar{b}+b=0^{\mathbf{B}} \notin\left\{r_{1}(\bar{a}), r_{4}(\bar{a}), r_{5}(\bar{a})\right\},} \\
& {\left[r_{3}, r_{4}, r_{6}\right] \circ m^{\partial}(\bar{a})=\left[r_{3}, r_{4}, r_{6}\right](\bar{a}, \bar{a}, \bar{a})=1^{\mathbf{B}}+\bar{b}+b=0^{\mathbf{B}} \notin\left\{r_{3}(\bar{a}), r_{4}(\bar{a}), r_{6}(\bar{a})\right\},}
\end{aligned}
$$

which implies that $m^{\partial}$ does not preserve $\sigma_{i}$ for all $i \in\{2, \ldots, 7\}$. In contrast, it can be checked that, for $r, r^{\prime} \in \sigma_{i}$, we have $\left[r, r^{\prime}\right] \circ g_{i} \in\left\{r, r^{\prime}\right\}$, and thus, $g_{i} \bar{\triangleright} \sigma_{i}$ for all $i \in\{2, \ldots, 7\}$. This implies that $g_{2}, \ldots, g_{7}$ cannot generate $m^{\partial}$ and are therefore minimal. It remains to show that a nontrivial idempotent operation $f \in O_{\mathbf{A}}^{(2)}$ falls under case (2) if and only if $f^{\partial}$ is essentially $g_{8}$. Assume that $f$ does not fall under case (2). 
It follows that $f$ is one of the following two operations $f_{1}, f_{2}$ (recall that it is enough to give $f(x)$ for all $\left.x \in B_{\mathbf{A}}\right)$ :

\begin{tabular}{|c||c|c|c|c|}
\hline$x$ & $(a, 0)$ & $(\bar{a}, 0)$ & $(0, a)$ & $(0, \bar{a})$ \\
\hline \hline$f_{1}(x)$ & 1 & $a$ & $\bar{a}$ & 1 \\
\hline$f_{2}(x)$ & $\bar{a}$ & 1 & 1 & $a$ \\
\hline
\end{tabular}

Since $f_{1}$ and $f_{2}$ arise from each other by interchanging the arguments and $f_{1}^{\partial}=g_{8}$, we have finished the proof.

Thus, over a four-element Boolean group, there is essentially only one nontrivial binary idempotent operation that is not minimal. For the next bigger Boolean group (that is, an eight-element Boolean group), the ratio of non-minimal binary idempotent operations is already notably higher.

\subsubsection{The Minimal Clones over Semilattices}

For this subsection, let $\mathbf{A}=\langle A, \vee\rangle$ be a finite semilattice and let $(-)^{\partial}: O_{\mathbf{A}} \rightarrow \bar{O}_{\mathbf{X}}$ be the clone duality from Subsection 4.2.7.

7.5.6 Proposition. Every minimal clone in $\mathcal{L}_{\mathbf{A}}$ is generated by an operation $f$ that is of one of the following three types:

1. a unary retraction,

2. an automorphism of prime order,

3. a binary idempotent operation.

Proof. Denote by $1_{\mathbf{A}}$ the join of all elements in $A$. We have to show that semiprojections, majority operations and minority operations cannot occur. First, assume that $f: \mathbf{A}^{3} \rightarrow \mathbf{A}$ is a majority operation. Then, for any $a \neq 1_{\mathbf{A}}$, we have

$$
f\left(1_{\mathbf{A}}, 1_{\mathbf{A}}, 1_{\mathbf{A}}\right)=f\left(1_{\mathbf{A}}, a, a\right) \vee f\left(a, 1_{\mathbf{A}}, a\right) \vee f\left(a, a, 1_{\mathbf{A}}\right)=a \vee a \vee a=a,
$$

a contradiction. If $f$ is a minority operation, we obtain

$$
f\left(1_{\mathbf{A}}, 1_{\mathbf{A}}, 1_{\mathbf{A}}\right)=f\left(a, 1_{\mathbf{A}}, 1_{\mathbf{A}}\right) \vee f\left(1_{\mathbf{A}}, a, 1_{\mathbf{A}}\right) \vee f\left(1_{\mathbf{A}}, 1_{\mathbf{A}}, a\right)=a \vee a \vee a=a,
$$

and we have reached a contradiction again. Now assume that $f: \mathbf{A}^{n} \rightarrow \mathbf{A}$ is a semiprojection. Without loss of generality, assume that $f$ is a projection on the first component whenever two arguments coincide. We have to show $f\left(a_{1}, \ldots, a_{n}\right)=a_{1}$ for all $a_{1}, \ldots, a_{n} \in A$. Let $f\left(a_{1}, \ldots, a_{n}\right)=b$. But now, we have

$$
\begin{aligned}
a_{1} & =f\left(a_{1}, 1_{\mathbf{A}}, \ldots, 1_{\mathbf{A}}\right) \\
& =f\left(a_{1} \vee a_{1}, 1_{\mathbf{A}} \vee a_{2}, \ldots, 1_{\mathbf{A}} \vee a_{n}\right) \\
& =f\left(a_{1}, 1_{\mathbf{A}}, \ldots, 1_{\mathbf{A}}\right) \vee f\left(a_{1}, \ldots, a_{n}\right) \\
& =f\left(a_{1}, 1_{\mathbf{A}}, \ldots, 1_{\mathbf{A}}\right) \vee b
\end{aligned}
$$




$$
\begin{aligned}
& =f\left(a_{1}, 1_{\mathbf{A}}, \ldots, 1_{\mathbf{A}}\right) \vee f\left(b, 1_{\mathbf{A}}, \ldots, 1_{\mathbf{A}}\right) \\
& =f\left(a_{1} \vee b, 1_{\mathbf{A}}, \ldots, 1_{\mathbf{A}}\right) \\
& =f\left(a_{1} \vee b, a_{2} \vee 1_{\mathbf{A}}, \ldots, a_{n} \vee 1_{\mathbf{A}}\right) \\
& =f\left(a_{1}, \ldots, a_{n}\right) \vee f\left(b, 1_{\mathbf{A}}, \ldots, 1_{\mathbf{A}}\right) \\
& =b \vee b \\
& =b
\end{aligned}
$$

Although this leaves us with the same three cases that also remain in the case in which $\bar{O}_{\mathbf{X}}$ respects the images of the injection morphisms to the degree 1 , we do not have that every binary idempotent operation is minimal as the following example shows:

7.5.7 Example. Let $\mathbf{A}=\langle\{0,1,2\}, \vee\rangle$ be a semilattice where $x \vee y:=\max (x, y)$. We have already seen all the dual idempotent operations over $\mathbf{X}=D(\mathbf{A})$ in Table 7.4 (page 139). Using the notation from this example, it is straightforward to check that we have $g_{4}=\left[g_{3}, g_{3}^{\prime}\right] \circ g_{3} \in \operatorname{Clo}\left(g_{3}\right)$, but $g_{3} \notin \overline{\operatorname{Clo}}\left(g_{4}\right)$.

As a side-note, for the setting as in the example above, $g_{3}$ and $g_{3}^{\prime}$ are the only two non-minimal, nontrivial, binary dual idempotent operations over $\mathbf{X}$, and their duals are therefore the only two non-minimal, nontrivial binary idempotent operations over $\mathbf{A}$. Furthermore, except these two operations (and their isomorphic copies), every nontrivial binary idempotent operation over any semilattice with at most three elements is minimal (besides $\mathbf{A}$, there is, up to isomorphism only one more three-element semilattice, and each nontrivial binary operation over this semilattice is minimal). 


\section{The Lattice of Clones over Distributive Lattices}

In Chapter 7, we have used the clone duality $(-)^{\partial}$ to obtain some general results for clones over sets and some specific results for the clones over the structures discussed in Section 4.2. This chapter will serve as an example of how to use our clone duality to obtain some in-depth results about parts of the lattice of clones over sets.

Throughout this chapter, we denote by $\mathcal{A}$ the category of finite distributive lattices and by $\mathbf{A}=\langle A, \vee, \wedge\rangle$ a finite distributive lattice of which 0 is the smallest and 1 the largest element. Our goal is to investigate the lattice $\mathcal{L}_{\mathbf{A}}$. Recall that $O_{\mathbf{A}}$ can be thought of as the centralizer clone of $\mathbf{A}$, so our study of $O_{\mathbf{A}}$ and $\mathcal{L}_{\mathbf{A}}$ is an investigation of the centralizer clones of distributive lattices and their subclones.

Recall what we have seen in Subsection 4.2.1: The category $\mathcal{A}$ of finite distributive lattices is dually equivalent to the category of finite bounded partially ordered sets. Denote the latter category by $\mathcal{X}$, and let $\langle D, E, e, \epsilon\rangle$ be the dual equivalence between $\mathcal{A}$ and $x$ that we have constructed in Lemma 4.2 .8 (page 49). Furthermore, set $\mathbf{X}:=D(\mathbf{A})$. We have

$$
\mathbf{X}=\left\langle\operatorname{Spec}(\mathbf{A}) \cup\left\{0^{\mathbf{X}}, 1^{\mathbf{X}}\right\}, 0^{\mathbf{X}}, 1^{\mathbf{X}}, \leq^{\mathbf{X}}\right\rangle
$$

where $0^{\mathbf{X}}=\emptyset, 1^{\mathbf{X}}=A$ and $\leq^{\mathbf{X}}=\subseteq$. Whenever the structure will be clear from the context, we will simply write $\leq$ instead of $\leq \mathbf{X}$. Moreover, we write $x<y$ to indicate $x \leq y$ and $x \neq y$. Recall that the clone duality $(-)^{\partial}: O_{\mathbf{A}} \rightarrow \bar{O}_{\mathbf{X}}$ is defined by setting

$$
f^{\partial}(x):= \begin{cases}0^{n \cdot \mathbf{X}} & \text { if } f^{-1}(x)=\emptyset \\ 1^{n \cdot \mathbf{X}} & \text { if } f^{-1}(x)=A^{n} \\ \langle i, y\rangle & \text { if } f^{-1}(x)=A^{i-1} \times y \times A^{n-i} \text { for some } y \in \operatorname{Spec}(\mathbf{A})\end{cases}
$$

for each $f \in O_{\mathbf{A}}^{(n)}$ (see page 53 . . Before we use this clone duality to investigate $\mathcal{L}_{\mathbf{A}}$, let us look at a motivating example to illustrate that this is indeed a promising task. In Figure 8.1. we can see a distributive lattice A with 30 elements. Investigating the clones over this lattice seems to be an exhaustive task. However, $\mathbf{A}$ dualizes to the bounded poset $\mathbf{X}$ given in the same figure. Since $\mathbf{X}$ has only 8 elements and easy copowers, it seems to be a much more convenient undertaking to investigate the clones of dual operations over $\mathbf{X}$ instead of the clones of operations over $\mathbf{A}$. This distributive lattice (and its dual) will serve as a running example throughout this chapter.

To work with clones of dual operations over $\mathbf{X}$, it is important to understand the cotupling of dual operations over $\mathbf{X}$. For $g_{1}, \ldots, g_{k} \in \bar{O}_{\mathbf{X}}^{(n)}$, the morphism

$$
\left[g_{1}, \ldots, g_{k}\right]: k \cdot \mathbf{X} \rightarrow n \cdot \mathbf{X}
$$




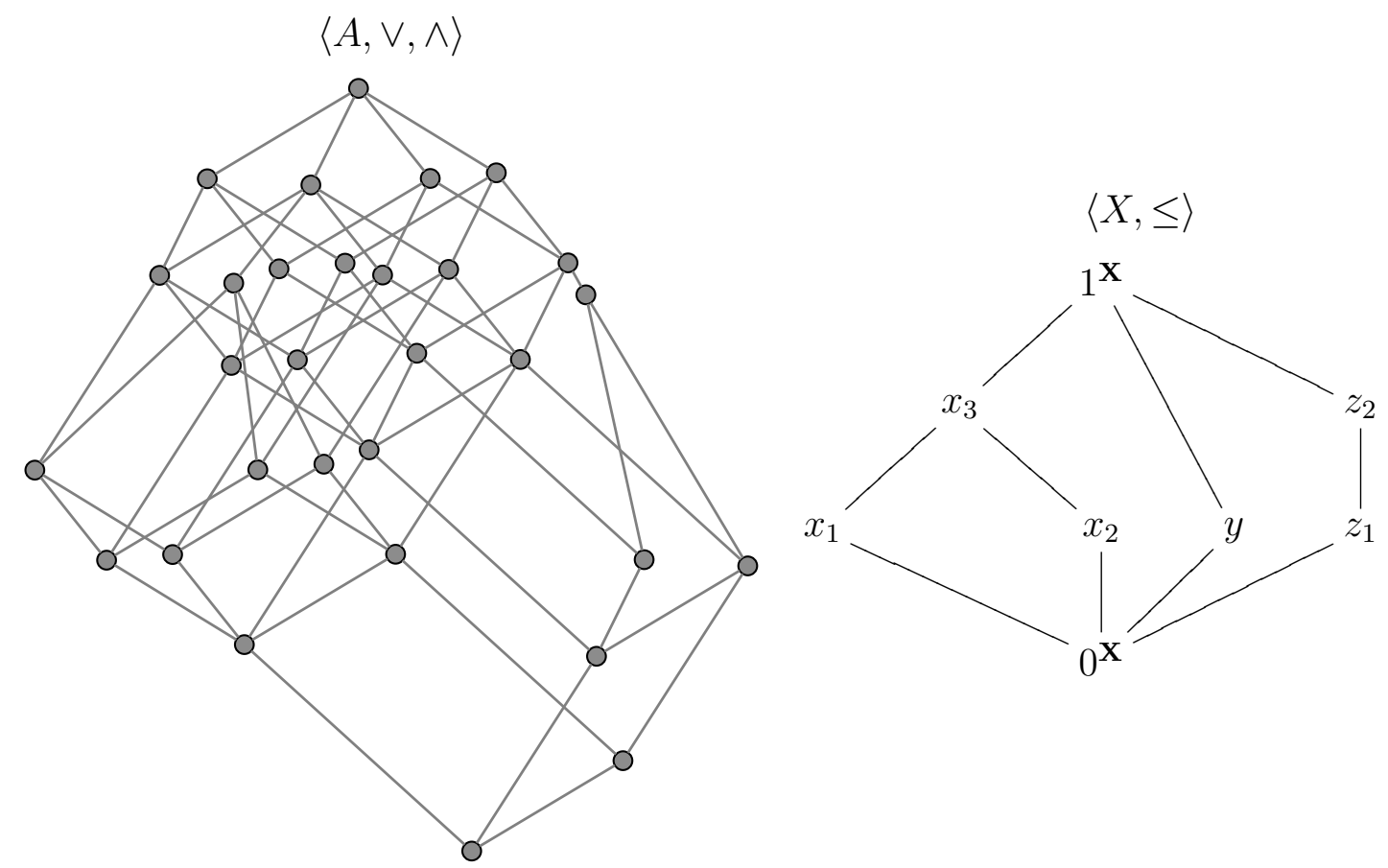

Figure 8.1.: A distributive lattice $\mathbf{A}$ and its dual $\mathbf{X}$

is given by

$$
\left[g_{1}, \ldots, g_{k}\right](x)= \begin{cases}0^{n \cdot \mathbf{X}} & \text { if } x=0^{k \cdot \mathbf{X}} \\ 1^{n \cdot \mathbf{X}} & \text { if } x=1^{k \cdot \mathbf{X}} \\ g_{i}(y) & \text { if } x=\langle i, y\rangle\end{cases}
$$

In particular,

$$
\left[\iota_{i_{1}}^{n}, \ldots, \iota_{i_{k}}^{n}\right](x)= \begin{cases}0^{n \cdot \mathbf{X}} & \text { if } x=0^{k \cdot \mathbf{X}}, \\ 1^{n \cdot \mathbf{X}} & \text { if } x=1^{k \cdot \mathbf{X}}, \\ \left\langle i_{j}, y\right\rangle & \text { if } x=\langle j, y\rangle .\end{cases}
$$

Figure 8.2 visualizes $\left[\iota_{3}^{5}, \iota_{4}^{5}, \iota_{1}^{5}\right]$ for a five-element $\mathbf{X}$.

We will now start our examination by showing a connection between $\mathbf{A}$ and $\mathbf{X}$ that will be helpful for our upcoming work.

8.0.1 Lemma. The number of atoms of $\mathbf{A}$ coincides with the number of coatoms of $\mathbf{X}$, and the number of coatoms of $\mathbf{A}$ coincides with the number of atoms of $\mathbf{X}$.

Proof. Since $\mathbf{A} \cong E D(\mathbf{A})=E(\mathbf{X})$, we can prove the first statement by showing that the number of atoms of $E(\mathbf{X})$ coincides with the number of coatoms of $\mathbf{X}$. Recall that the carrier set of $E(\mathbf{X})$ is $\operatorname{Inc}(\mathbf{X})$. An element $a \in \operatorname{Inc}(\mathbf{X})$ is an atom of $E(\mathbf{X})=\langle\operatorname{Inc}(\mathbf{X}), \cup, \cap\rangle$ if and only if $a=\left\{x, 1^{\mathbf{X}}\right\}$ for some coatom $x \in \mathbf{X}$. The claim follows.

For the second statement, let $x_{1}, \ldots, x_{k}$ be the atoms of $\mathbf{X}$. An element $a \in \operatorname{Inc}(\mathbf{X})$ is a coatom of $E(\mathbf{X})=\langle\operatorname{Inc}(\mathbf{X}), \cup, \cap\rangle$ if and only if we have

$$
a=\uparrow x_{1} \cup \ldots \cup \uparrow x_{i-1} \cup \uparrow x_{i+1} \cup \ldots \cup \uparrow x_{s}
$$




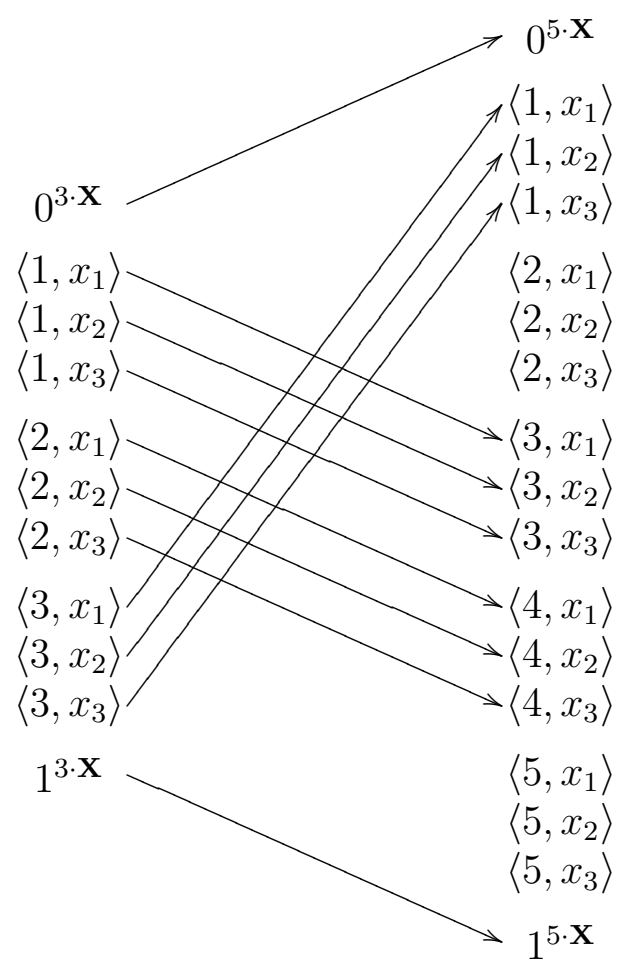

$3 \cdot \mathrm{X} \quad 5 \cdot \mathrm{X}$

Figure 8.2.: $\left[\iota_{3}^{5}, \iota_{4}^{5}, \iota_{1}^{5}\right]$ for $X=\left\{0^{\mathbf{X}}, x_{1}, x_{2}, x_{3}, 1^{\mathbf{X}}\right\}$

for some $i \in\{1, \ldots, k\}$. Thus, there exist exactly $k$ coatoms of $E(\mathbf{X}) \cong \mathbf{A}$.

The following lemma recalls what we have observed in Example 4.2 .9 (page 50):

8.0.2 Lemma. Let $f$ be an n-ary operation over $\mathbf{A}$. Then,

(i) $f$ is a 0-homomorphism if an only if $f^{\partial}(x)=1^{n \cdot \mathbf{X}}$ implies $x=1^{\mathbf{X}}$,

(ii) $f$ is a 1-homomorphism if an only if $f^{\partial}(x)=0^{n \cdot \mathbf{X}}$ implies $x=0^{\mathbf{x}}$.

For certain problems we are about to attack, the following definition will be useful:

8.0.3 Definition. Let $\mathbf{Y}=\left\langle Y, 0^{\mathbf{Y}}, 1^{\mathbf{Y}}, \leq^{\mathbf{Y}}\right\rangle \in X$. Define $G_{\mathbf{Y}}$ to be the undirected graph whose set of vertices is $Y \backslash\left\{0^{\mathbf{Y}}, 1^{\mathbf{Y}}\right\}$ and in which two vertices $y_{1}$ and $y_{2}$ are connected by an edge if and only if $y_{1} \leq^{\mathbf{Y}} y_{2}$ or $y_{2} \leq^{\mathbf{Y}} y_{1}$. A subset $Z \subseteq Y \backslash\left\{0^{\mathbf{Y}}, 1^{\mathbf{Y}}\right\}$ is said to be connected if there exists a path in $Z$ between each two elements of the set. For $y \in Y \backslash\left\{0^{\mathbf{Y}}, 1^{\mathbf{Y}}\right\}$, denote by $\langle y\rangle_{G_{\mathbf{Y}}}$ the largest connected subset of $Y \backslash\left\{0^{\mathbf{Y}}, 1^{\mathbf{Y}}\right\}$ that contains $y$. Then,

$$
\operatorname{Con}(\mathbf{Y}):=\left\{\langle y\rangle_{G_{\mathbf{Y}}} \mid y \in G_{\mathbf{Y}}\right\}
$$

is called the set of connected components of $\mathbf{Y}$. 
8.0.4 Example. Consider the poset $\mathbf{X}$ given in Figure 8.1. The set $\left\{x_{1}, x_{2}, x_{3}\right\}$ is connected, whereas $\left\{x_{1}, x_{2}\right\}$ is not. Moreover, the connected components of $\mathbf{X}$ are the three sets $\left\{x_{1}, x_{2}, x_{3}\right\},\{y\}$ and $\left\{z_{1}, z_{2}\right\}$.

Note that, for $n \in \mathbb{N}_{+}$, the connected components of $n \cdot \mathbf{X}$ are precisely the images of the connected components of $\mathbf{X}$ under the injection morphisms. That is,

$$
\operatorname{Con}(n \cdot \mathbf{X})=\left\{\iota_{i}^{n}(Y) \mid Y \in \operatorname{Con}(\mathbf{X}), i \in\{1, \ldots, n\}\right\} .
$$

In particular, two elements $x_{1}, x_{2} \in n \cdot \mathbf{X} \backslash\left\{0^{n \cdot \mathbf{X}}, 1^{n \cdot \mathbf{X}}\right\}$ belong to two different connected components of $n \cdot \mathbf{X}$ whenever they belong to different sets among $\iota_{1}^{n}(\mathbf{X}), \ldots, \iota_{n}^{n}(\mathbf{X})$.

Before we start examining the lattice of clones over a finite distributive lattice, we will present some examples of distributive lattices that have special properties. In fact, these properties will heavily influence the difficulty of our upcoming tasks.

\subsection{Three Cases of Distributive Lattices}

When we deal with dual operations over $\mathbf{X}$, it is obvious that the order relation $\leq$ can be a source of trouble, since every dual operation has to respect this relation. Therefore, it is a significant simplification to assume that the order relation is of the smallest possible size. That is, $\leq=\left(\left\{0^{\mathbf{X}}\right\} \times X\right) \cup\left(X \times\left\{1^{\mathbf{X}}\right\}\right)$. Then, clearly, every set in $\operatorname{Con}(\mathbf{X})$ consists of exactly one element, and $\mathbf{X}$ has the form of the poset in Figure 8.3 .

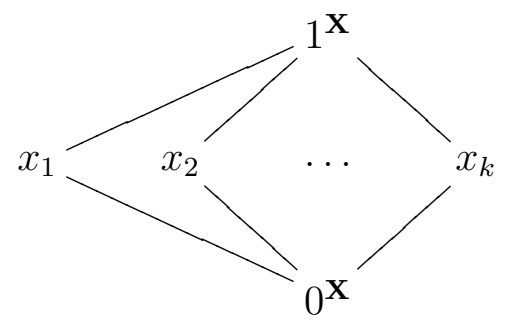

Figure 8.3.: $\mathbf{X}$ if $\mathbf{A}$ is a Boolean lattice

As noted in Subsection 4.2.5, this occurs if and only if $\mathbf{A}$ is a Boolean lattice. In this case, we can ignore the order relation $\leq\left(\right.$ since $0^{\mathbf{X}}$ and $1^{\mathbf{X}}$ are constants anyway) and many problems become much easier to solve.

Now we turn to a slightly more general case by assuming that the connected components of $\mathbf{X}$ might contain more than one element, but are still totally ordered with respect to $\leq$. We can see the prototype of such a partially ordered set in Figure 8.4. In this setting, each $n$-ary dual operation $g \in \bar{O}_{\mathbf{X}}$ must map a connected component $Y \in \operatorname{Con}(\mathbf{X})$ into the same connected component of $n \cdot \mathbf{X}$. This also simplifies the structure of $\mathcal{L}_{\mathrm{A}}$.

As the last example for $\mathbf{X}$, Figure 8.5 shows a lattice that can be seen as a prototype for the most difficult cases. Here, $x_{1}$ and $x_{2}$ belong to the same connected component 


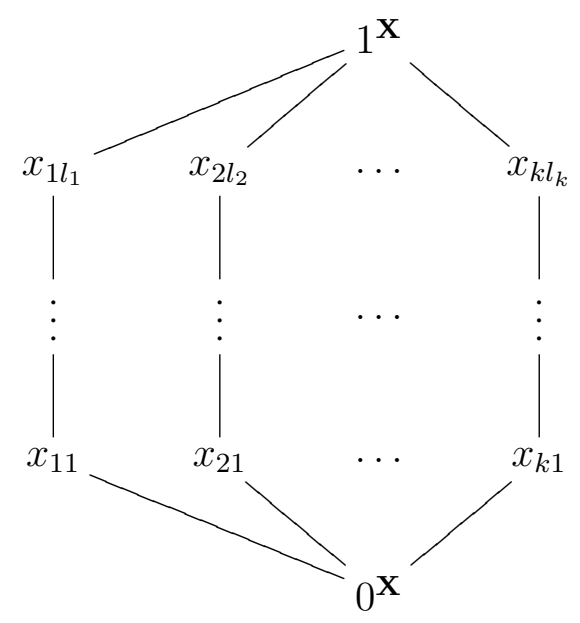

Figure 8.4.: $\mathbf{X}$ if each $Y \in \operatorname{Con}(\mathbf{X})$ is a chain

of $\mathbf{X}$ but are not directly connected. Thus, if we map $x_{0}$ to $0^{n \cdot \mathbf{X}}$, then $x_{1}$ and $x_{2}$ can go to different connected components of $n \cdot \mathbf{X}$. In particular, they can be mapped into different sets among $\iota_{1}^{1}(\mathbf{X}), \ldots, \iota_{n}^{n}(\mathbf{X})$. As we will see later, this can be a major source of trouble.

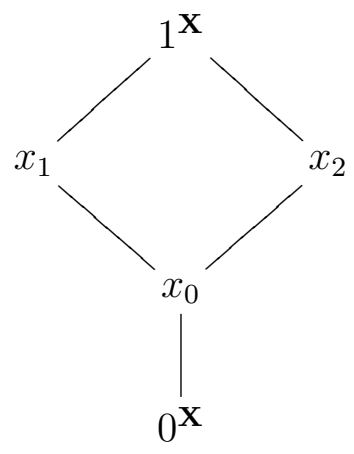

Figure 8.5.: A prototype for $\mathbf{X}$ not consisting of chains

\subsection{The Essential Arity of Operations in $O_{\mathrm{A}}$}

By Corollary 7.2.2 (page 129), the essential arity of operations among $O_{\mathbf{A}}$ must be bounded. In this section, we will determine the exact size of this bound.

8.2.1 Theorem. Let $k$ be the length of the longest antichain of $\vee$-irreducible elements in $\mathbf{A}$. Then, $k$ is a sharp bound on the essential arity of operations among $O_{\mathbf{A}}$.

Proof. Note that $a_{1}, \ldots, a_{k}$ is an antichain of join-irreducible elements in $\mathbf{A}$ if and only if $\uparrow a_{1}, \ldots, \uparrow a_{k}$ is an antichain in $\mathbf{X}$. Therefore, $k$ is also the length of the longest 
antichain in $\mathbf{X}$. We will show the claim by proving that each $g \in \bar{O}_{\mathbf{X}}$ is at most $k$-ary and that there exists $g^{*} \in \bar{O}_{\mathbf{X}}$ with exactly $k$ essential arguments. For the first part, let $g \in \bar{O}_{\mathbf{X}}$ and assume that $g$ has at least $k+1$ essential arguments. The $i$-th argument of $g$ is nonessential if and only if $g(\mathbf{X}) \cap \iota_{i}^{n}(\mathbf{X})=\left\{0^{n \cdot \mathbf{X}}, 1^{n \cdot \mathbf{X}}\right\}$. Hence, there exist $x_{1}, \ldots, x_{k+1} \in g(\mathbf{X}) \backslash\left\{0^{n \cdot \mathbf{X}}, 1^{n \cdot \mathbf{X}}\right\}$ such that $g\left(x_{1}\right), \ldots, g\left(x_{k+1}\right)$ pairwise belong to different connected components of $n \cdot \mathbf{X}$. Since this implies that $g\left(x_{1}\right), \ldots, g\left(x_{k+1}\right)$ is an antichain in $n \cdot \mathbf{X}$, it follows that $x_{1}, \ldots, x_{k+1}$ is an antichain in $\mathbf{X}$. This contradicts our assumption that an antichain in $\mathbf{X}$ can contain at most $k$ elements.

For the second part, let $x_{1}, \ldots, x_{k}$ be an antichain in $\mathbf{X}$ and define $g^{*} \in \bar{O}_{\mathbf{X}}^{(k)}$ as follows:

$$
g^{*}(x):= \begin{cases}0^{n \cdot \mathbf{X}} & \text { if } x<x_{i} \text { for some } i \in\{1, \ldots, k\} \\ 1^{n \cdot \mathbf{X}} & \text { if } x>x_{i} \text { for some } i \in\{1, \ldots, k\} \\ \langle i, x\rangle & \text { if } x=x_{i}\end{cases}
$$

We have to show that $g^{*}$ is well-defined. For $x \in \mathbf{X}$, we must have $x \leq x_{i}$ or $x \geq x_{i}$ for at least one $i \in\{1, \ldots, k\}$ since otherwise we would get an antichain of length $k+1$. Hence, at least one of the three cases in the definition of $g^{*}$ is satisfied. Assume that two of the three cases hold simultaneously. If we have $x<x_{i}$ and $x>x_{j}$ for some $i, j \in\{1, \ldots, k\}$, then we obtain $x_{j}<x<x_{i}$, which is impossible since $x_{1}, \ldots, x_{k}$ is an antichain. For the same reason we cannot have $x=x_{i}$ for some $i \in\{1, \ldots, k\}$ at the same time as $x<x_{j}$ or $x_{j}<x$ for some $j \in\{1, \ldots, k\}$. Thus, $g^{*}$ is a well-defined mapping from $\mathbf{X}$ to $k \cdot \mathbf{X}$. Evidently, we have $g^{*}\left(0^{\mathbf{X}}\right)=0^{k \cdot \mathbf{X}}$ and $g^{*}\left(1^{\mathbf{X}}\right)=1^{k \cdot \mathbf{X}}$. Moreover, $x \leq y$ implies $g^{*}(x) \leq g^{*}(y)$. Thus, $g^{*} \in \bar{O}_{\mathbf{X}}^{(k)}$. This finishes the proof since it is obvious that $g^{*}$ has no nonessential arguments.

8.2.2 Example. Let A be our running example from Figure 8.1. Since the longest antichain of join-irreducible elements in $\mathbf{A}$ consists of 4 elements, we can conclude that 4 is a sharp bound on the essential arity of operations over $\mathbf{A}$.

For Boolean lattices, we obtain the following corollary:

8.2.3 Corollary. Let $\mathbf{A}$ be a Boolean lattice and let $k$ be the number of atoms of $\mathbf{A}$. Then, $k$ is a sharp bound on the essential arity of operations among $O_{\mathbf{A}}$.

Proof. The claim follows directly from the fact that, in a Boolean lattice, only the atoms and the bottom element are join-irreducible.

\subsection{Idempotent Operations in $O_{\mathrm{A}}$}

Recall that we denote by $\mathcal{I}_{\mathbf{A}}$ the clone of all idempotent operations over $\mathbf{A}$ and by $\overline{\mathcal{I}}_{\mathbf{X}}$ the clone of all dual idempotent operations over $\mathbf{X}$. Moreover, recall that $\left(\mathcal{I}_{\mathbf{A}}\right)^{\partial}=\overline{\mathcal{I}}_{\mathbf{X}}$.

While the ideal $\left\langle\mathcal{I}_{\mathbf{A}}\right]$ is the lattice of idempotent clones over $\mathbf{A}$, the filter $\left[\mathcal{I}_{\mathbf{A}}\right\rangle$ is the lattice of clones over $\mathbf{A}$ that contain all idempotent operations over $\mathbf{A}$. In this section, we will collect some facts about the idempotent operations over $\mathbf{A}$, and we will explore $\left\langle\mathcal{I}_{\mathbf{A}}\right]$ as well as $\left[\mathcal{I}_{\mathbf{A}}\right\rangle$. 
Since the copowers of $\mathbf{X}$ are non-deformed to the degree 1, we can use Theorem 6.4.2 (page 120) to obtain the following characterization of the dual idempotent operations over X:

8.3.1 Lemma. Let $X_{1}, \ldots, X_{k}$ be the connected components of $\mathbf{X}$. An n-ary dual operation $g \in \bar{O}_{\mathbf{X}}$ is idempotent if and only if there exists a (unique) function

$$
j_{g}:\{1, \ldots, k\} \rightarrow\{1, \ldots, n\}
$$

such that

$$
g(x)= \begin{cases}0^{n \cdot \mathbf{X}} & \text { if } x=0^{\mathbf{X}}, \\ 1^{n \cdot \mathbf{X}} & \text { if } x=1^{\mathbf{x}} \\ \left\langle j_{g}(i), x\right\rangle & \text { if } x \in X_{i} .\end{cases}
$$

Proof. " $\Longleftarrow$ ". The idempotency of $g$ follows immediately from Theorem 6.4.2.

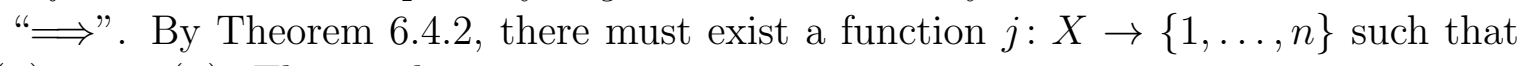
$g(x)=\iota_{j(x)}^{n}(x)$. This implies

$$
g(x)= \begin{cases}0^{n \cdot \mathbf{X}} & \text { if } x=0^{\mathbf{X}} \\ 1^{n \cdot \mathbf{X}} & \text { if } x=1^{\mathbf{x}} \\ \langle j(x), x\rangle & \text { otherwise }\end{cases}
$$

It remains to show that we have $j(x)=j(y)$ whenever $x$ and $y$ belong to the same connected component of $\mathbf{X}$. Without loss of generality, let $x, y \in X_{1}$. By the definition of connected components, there must exist $z \in X_{1}$ such that we either have $x, y \geq z$ or $z \geq x, y$. Without loss of generality, we assume the latter case. It follows $g(z) \geq g(x)$ and $g(z) \geq g(y)$. As $g(z) \neq 1^{n \cdot \mathbf{X}}$, this implies that $g(x)$ and $g(y)$ belong to the same connected component. Thus, $j(x)=j(y)$.

With this lemma, we can identify each idempotent operation $f \in O_{\mathbf{A}}$ with a partition of $\operatorname{Con}(\mathbf{X})$ :

8.3.2 Definition. Let $f \in O_{\mathbf{A}}^{(n)}$ be idempotent. For $i \in\{1, \ldots, n\}$, set

$$
P_{i}:=\left\{Y \in \operatorname{Con}(\mathbf{X}) \mid f^{\partial}(Y) \subseteq \iota_{i}^{n}(\mathbf{X})\right\}
$$

Denote by $\Pi(f)$ the set $\left\{P_{1}, \ldots, P_{n}\right\} \backslash\{\emptyset\}$.

Note that $\Pi(f)$ is well-defined since, by Lemma 8.3.1, the image of each $Y \in \operatorname{Con}(\mathbf{X})$ under $f^{\partial}$ can only be contained in one of the sets $\iota_{1}^{n}(\mathbf{X}), \ldots, \iota_{n}^{n}(\mathbf{X})$. Moreover, it is evident that $|\Pi(f)|$ is the essential arity of $f$.

8.3.3 Example. Let $\mathbf{A}$ be the distributive lattice from Figure 8.1 (page 147). Recall that it dualizes to the bounded poset $\mathbf{X}$ that is given in the same figure and of which the connected components are the three sets $\left\{x_{1}, x_{2}, x_{3}\right\},\{y\}$ and $\left\{z_{1}, z_{2}\right\}$. Let $f \in O_{\mathbf{A}}^{(2)}$ be the idempotent operation that dualizes to $f^{\partial} \in \bar{O}_{\mathbf{X}}^{(2)}$ as given in the following table:

\begin{tabular}{|c||cccccc|}
\hline$x$ & $x_{1}$ & $x_{2}$ & $x_{3}$ & $y$ & $z_{1}$ & $z_{2}$ \\
\hline$f^{\partial}(x)$ & $\left\langle 1, x_{1}\right\rangle$ & $\left\langle 1, x_{2}\right\rangle$ & $\left\langle 1, x_{3}\right\rangle$ & $\langle 2, y\rangle$ & $\left\langle 1, z_{1}\right\rangle$ & $\left\langle 1, z_{2}\right\rangle$ \\
\hline
\end{tabular}


Then,

$$
\Pi(f)=\left\{\left\{\left\{x_{1}, x_{2}, x_{3}\right\},\left\{z_{1}, z_{2}\right\}\right\},\{\{y\}\}\right\} .
$$

8.3.4 Lemma. For each partition $\left\{P_{1}, \ldots, P_{n}\right\}$ of $\operatorname{Con}(\mathbf{X})$, there exists an idempotent operation $f \in O_{\mathbf{A}}^{(n)}$ such that $\Pi(f)=\left\{P_{1}, \ldots, P_{n}\right\}$.

Proof. Let $k:=|\operatorname{Con}(\mathbf{X})|$ and let $X_{1}, \ldots, X_{k}$ be the connected components of $\mathbf{X}$. Define $j_{g}:\{1, \ldots, k\} \rightarrow\{1, \ldots, n\}$ by setting $j_{g}(i):=r$ whenever $X_{i} \in P_{r}$. Since $\left\{P_{1}, \ldots, P_{n}\right\}$ is a partition of $\operatorname{Con}(\mathbf{X}), j_{g}$ is well-defined. Let

$$
g(x)= \begin{cases}0^{n \cdot \mathbf{X}} & \text { if } x=0^{\mathbf{X}}, \\ 1^{n \cdot \mathbf{X}} & \text { if } x=1^{\mathbf{x}}, \\ \left\langle j_{g}(i), x\right\rangle & \text { if } x \in X_{i},\end{cases}
$$

and set $f:=g^{\partial^{-1}}$. By Lemma 8.3.1, $f$ is idempotent, and it is obvious that we have $\Pi(f)=\left\{P_{1}, \ldots, P_{n}\right\}$.

We can now show a more precise connection between clones of idempotent operations and partitions of $\operatorname{Con}(\mathbf{X})$ (recall the definition of $\preccurlyeq$, page 121 ):

8.3.5 Theorem. The ideal $\left\langle\mathcal{I}_{\mathbf{A}}\right]$ is order-isomorphic to the lattice $\langle\operatorname{Part}(\operatorname{Con}(\mathbf{X})), \preccurlyeq\rangle$ of partitions of $\operatorname{Con}(\mathbf{X})$.

Proof. Let $\Phi$ be a partition of $\operatorname{Con}(\mathbf{X})$. By Lemma 8.3.4 there exists an operation $f_{\Phi}$ such that $\Pi\left(f_{\Phi}\right)=\Phi$. This operation is not unique, but, in the light of Lemma 6.4.10 (page 121), it becomes obvious that all operations with this property generate the same clone. Thus, the mapping

$$
\varphi: \operatorname{Part}(\operatorname{Con}(\mathbf{X})) \rightarrow \mathcal{L}_{\mathbf{A}}: \Phi \mapsto \operatorname{Clo}\left(f_{\Phi}\right)
$$

is well-defined. Furthermore, as another consequence of Lemma 6.4.10, it is also an order embedding from $\langle\operatorname{Part}(\operatorname{Con}(\mathbf{X})), \preccurlyeq\rangle$ to $\left\langle\mathcal{I}_{\mathbf{A}}\right]$. It remains to show that $\varphi$ is bijective. Define the mapping $\varphi^{\prime}: \mathcal{L}_{\mathbf{A}} \rightarrow \operatorname{Part}(\operatorname{Con}(\mathbf{X}))$ by setting $\varphi^{\prime}(C)=\Pi(f)$ where $f$ is one of the idempotent operations that generate $C$ (the existence of such an operation is given by Lemma 6.4.11, page 122. Evidently, $\varphi^{\prime}$ is the inverse of $\varphi$, and the claim follows.

By Corollary 7.4.1 (page 133), this theorem also implies that the number of essentially different idempotent operations over $\mathbf{A}$ and the number of idempotent clones over $\mathbf{A}$ is precisely the Bell number $B_{|\operatorname{Con}(\mathbf{X})|}$.

8.3.6 Example. Once again, let us turn to our running example from page 147. We have $|\operatorname{Con}(\mathbf{X})|=3$, so $\left\langle\mathcal{I}_{\mathbf{A}}\right]$ is isomorphic to $\langle\operatorname{Part}(\{1,2,3\}), \preccurlyeq\rangle$. This implies that there are exactly 5 clones of idempotent operations in $\mathcal{L}_{\mathbf{A}}$.

For a given $n \in \mathbb{N}_{+}$, we can also easily infer the number of essentially different, essentially $n$-ary idempotent operations over A. For this, recall that $S_{k}^{n}$ denote the Stirling numbers of the second kind (see Definition 6.4.7, page 121). 
8.3.7 Corollary. Let $l:=|\operatorname{Con}(\mathbf{X})|$. The number of essentially different, essentially $n$-ary idempotent operations over $\mathbf{A}$ is $S_{l}^{n}$.

Proof. As presented above, $f$ and $g$ generate a distinct clone if and only if $\Pi(f) \neq \Pi(g)$. Thus, the number of essentially different, essentially $n$-ary operations is the number of possible partitions of $\operatorname{Con}(\mathbf{X})$ into $n$ parts, i.e., the number is $S_{l}^{n}$.

Note that this number is exactly the bound given in Corollary 7.4.1 (page 133) if and only if $\operatorname{Con}(\mathbf{X})$ consists of only one-element sets, that is, $\mathbf{A}$ is a Boolean lattice (cf. Proposition 7.4.2, page 134). The more elements the sets in $\operatorname{Con}(\mathbf{X})$ contain, the looser the upper bound from Corollary 7.4.1 becomes. In fact, as the following corollary states, it is possible that, for arbitrarily large $\mathbf{A}$, there is not a single nontrivial idempotent operation over A:

8.3.8 Corollary. There are no nontrivial idempotent operations over $\mathbf{A}$ if and only if $\operatorname{Con}(\mathbf{X})=1$.

Before we turn to the filter $\left[\mathcal{I}_{\mathbf{A}}\right\rangle$, let us take a look at median algebras. Recall that clones of operations over median algebras dualize to clones of dual operations over strongly complemented bounded posets (see Subsection 4.2.3). For them, we can define connected components analogue to the definition of connected components in bounded posets.

8.3.9 Definition. Let $\mathbf{Y}=\left\langle Y, 0^{\mathbf{Y}}, 1^{\mathbf{Y}},{ }^{-}, \leq\right\rangle$be a strongly complemented bounded poset. Define $G_{\mathbf{Y}}$ to be the undirected graph whose set of vertices is $\mathbf{Y} \backslash\left\{0^{\mathbf{Y}}, 1^{\mathbf{Y}}\right\}$ and in which two vertices $y_{1}$ and $y_{2}$ are connected by an edge if and only if $y_{1} \leq \mathbf{Y} y_{2}$ or $y_{2} \leq \mathbf{Y} y_{1}$ or $y_{1}=\overline{y_{2}}$. A subset of $Z \subseteq Y \backslash\left\{0^{\mathbf{Y}}, 1^{\mathbf{Y}}\right\}$ is said to be connected if there exists a path in $Z$ between each two elements of the set. For $y \in Y \backslash\left\{0^{\mathbf{Y}}, 1^{\mathbf{Y}}\right\}$, denote by $\langle y\rangle_{G_{\mathbf{Y}}}$ the largest connected subset of $Y \backslash\left\{0^{\mathbf{Y}}, 1^{\mathbf{Y}}\right\}$ that contains $y$. Then,

$$
\operatorname{Con}(\mathbf{Y}):=\left\{\langle y\rangle_{G_{\mathbf{Y}}} \mid y \in G_{\mathbf{Y}}\right\}
$$

is called the set of connected components of $\mathbf{Y}$.

It is very easy to see that we can use arguments completely analogue to the ones used for distributive lattices to infer the following result:

8.3.10 Proposition. Let $\mathbf{B}$ be a finite median algebra that dualizes to the strongly complemented bounded poset $\mathbf{Y}$. Let $l:=|\operatorname{Con}(\mathbf{Y})|$. Then,

(i) the number of essentially different, essentially n-ary idempotent operations over $\mathbf{B}$ is exactly $S_{l}^{n}$,

(ii) $\left\langle\mathcal{I}_{\mathbf{B}}\right] \cong\langle\operatorname{Part}(\operatorname{Con}(\mathbf{Y})), \preccurlyeq\rangle \cong\langle\operatorname{Part}(\{1, \ldots, l\}), \preccurlyeq\rangle$,

(iii) the number of idempotent clones over $\mathbf{B}$ and the number of essentially different idempotent operations over $\mathbf{B}$ are both the Bell number $B_{l}$.

We will now explore the structure of the filter $\left[\mathcal{I}_{\mathbf{A}}\right\rangle$. 
8.3.11 Definition. For $\operatorname{dom}(p) \subseteq X$, a partial homomorphism $p$ : $\operatorname{dom}(p) \rightarrow n \cdot \mathbf{X}$ is called an $n$-ary partial dual operation over $\mathbf{X}$. Denote by $\bar{P}_{\mathbf{X}}^{(n)}$ the set of all $n$-ary partial dual operations over $\mathbf{X}$ whose domain is a connected component of $\mathbf{X}$ and set $\bar{P}_{\mathbf{X}}:=\bigcup \bar{P}_{\mathbf{X}}^{(n)}$.

We extend the definition of essential and nonessential arguments to partial operations in the obvious way.

8.3.12 Lemma. Let $(-)^{\lambda}: \mathfrak{P}\left(\bar{O}_{\mathbf{X}}\right) \rightarrow \mathfrak{P}\left(\bar{P}_{\mathbf{X}}\right)$ be defined by setting

$$
G^{\lambda}:=\left\{\left.g\right|_{Y} \mid g \in G, Y \in \operatorname{Con}(\mathbf{X})\right\} .
$$

Then, each $C \in\left[\overline{\mathcal{I}}_{\mathbf{X}}\right\rangle$ is uniquely determined by $C^{\lambda}$. Hence, $(-)^{\lambda}$ is an order embedding of $\left[\overline{\mathcal{I}}_{\mathbf{X}}\right\rangle$ into $\left\langle\mathfrak{P}\left(\bar{P}_{\mathbf{X}}\right), \subseteq\right\rangle$.

Proof. We will show that, for the mapping $(-)^{\varkappa}: \mathfrak{P}\left(\bar{P}_{\mathbf{X}}\right) \rightarrow \mathfrak{P}\left(\bar{O}_{\mathbf{X}}\right)$ defined by

$$
H^{\varkappa}:=\left\{g \in \bar{O}_{\mathbf{X}}|\forall Y \in \operatorname{Con}(\mathbf{X}): g|_{Y} \in H\right\},
$$

we have $C=\left(C^{\lambda}\right)^{\varkappa}$ for all $C \in\left[\overline{\mathcal{I}}_{\mathbf{X}}\right\rangle$. The first direction, $C \subseteq\left(C^{\lambda}\right)^{\varkappa}$, is obvious. For the other direction, let $g \in\left(C^{\lambda}\right)^{\varkappa}$ be $n$-ary, and let $X_{1}, \ldots, X_{k}$ be the connected components of $\mathbf{X}$. Hence, there exist $p_{1}, \ldots, p_{k} \in C^{\lambda}$ such that

$$
g(x)=\left\{\begin{array}{cc}
0^{n \cdot \mathbf{X}} & \text { if } x=0^{\mathbf{X}}, \\
p_{1}(x) & \text { if } x \in X_{1}, \\
\vdots & \vdots \\
p_{k}(x) & \text { if } x \in X_{k}, \\
1^{n \cdot \mathbf{X}} & \text { if } x=1^{\mathbf{X}}
\end{array}\right.
$$

This, in turn, implies that there exist $g_{1}, \ldots, g_{k} \in C^{(n)}$ such that $\left.g_{i}\right|_{X_{i}}=p_{i}$ for all $i \in\{1, \ldots, k\}$. Hence,

$$
g(x)=\left\{\begin{array}{cc}
0^{n \cdot \mathbf{X}} & \text { if } x=0^{\mathbf{X}}, \\
g_{1}(x) & \text { if } x \in X_{1} \\
\vdots & \vdots \\
g_{k}(x) & \text { if } x \in X_{k} \\
1^{n \cdot \mathbf{X}} & \text { if } x=1^{\mathbf{X}}
\end{array}\right.
$$

Furthermore, let $s \in \bar{O}_{\mathbf{X}}^{(k)}$ be defined as follows:

$$
s(x):=\left\{\begin{array}{cc}
0^{k \cdot \mathbf{X}} & \text { if } x=0^{\mathbf{X}} \\
\langle 1, x\rangle & \text { if } x \in X_{1} \\
\vdots & \vdots \\
\langle k, x\rangle & \text { if } x \in X_{k} \\
1^{k \cdot \mathbf{X}} & \text { if } x=1^{\mathbf{X}}
\end{array}\right.
$$


By Lemma 8.3.1, $s$ is a well-defined dual idempotent operation over $\mathbf{X}$. Hence, $s \in C$. But now, we have

$$
g=\left[g_{1}, \ldots, g_{k}\right] \circ s .
$$

Since $s, g_{1}, \ldots, g_{k} \in C$, this yields $g \in C$. We have established that $(-)^{\lambda}$ is injective. Since $(-)^{\lambda}$ and $(-)^{\varkappa}$ are obviously monotone with respect to inclusion, it follows that $(-)^{\lambda}$ is an order embedding.

Note that $\left|\bar{P}_{\mathbf{X}}\right|$ is generally much smaller than $\left|O_{\mathbf{A}}\right|$. For instance, if we take the distributive lattice from Figure 8.1 (page 147), then we can calculate that there are exactly 826, 336 operations over $\mathbf{A}$ that have only essential arguments (13 of which are idempotent). However, there are only 266 partial operations that have a connected component as their domain and have exclusively essential arguments.

The next lemma shows that each $C \in\left[\overline{\mathcal{I}}_{\mathbf{X}}\right\rangle$ is already uniquely determined by $\left(C^{(l)}\right)^{\lambda}$, where $l$ is the length of the longest antichain that is contained in one connected component. Note that we have $l=1$ if and only if each connected component of $\mathbf{X}$ is totally ordered.

8.3.13 Lemma. Let $C \in\left[\mathcal{I}_{\mathbf{A}}\right\rangle$ and let $l$ be the length of the longest antichain in $\mathbf{X}$ that is contained in one connected component. Then, $C$ is uniquely determined by $\left(C^{(l)}\right)^{\lambda}$.

Proof. By Lemma 8.3.12, $C$ is uniquely determined by $C^{\lambda} \subseteq \bar{P}_{\mathbf{X}}$. An argument similar to that from the proof of Theorem 8.2.1 (page 150 establishes that each $p \in \bar{P}_{\mathbf{X}}$ is essentially at most $l$-ary. Hence, for each $p \in \bar{P}_{\mathbf{X}}$, there exists $p^{\prime} \in \bar{P}_{\mathbf{X}}^{(l)}$ such that $p$ and $p^{\prime}$ are essentially the same partial dual operation. But now, it is easy to see that we have $p \in C^{\lambda}$ if and only if $p^{\prime} \in C^{\lambda}$. Thus, each $C$ is already uniquely determined by $\left(C^{(l)}\right)^{\lambda}$.

Note that this lemma implies that each $C \in\left[\mathcal{I}_{\mathbf{A}}\right\rangle$ is uniquely determined by its $l$-ary part (which also gives us an upper bound on the size of $\left[\mathcal{I}_{\mathbf{A}}\right\rangle$ ).

8.3.14 Example. For our running example from Figure 8.1, the longest antichain that is contained in one connected component is $\left\{x_{1}, x_{2}\right\}$, so each $C \in\left[\mathcal{I}_{\mathbf{A}}\right\rangle$ is uniquely determined by its binary part.

As another consequence of Lemma 8.3.13, $C \mapsto\left(C^{(l)}\right)^{\lambda}$ is an order embedding from $\left[\overline{\mathcal{I}}_{\mathbf{X}}\right\rangle$ into $\left\langle\mathfrak{P}\left(\bar{P}_{\mathbf{X}}^{(l)}\right), \subseteq\right\rangle$, so $\left[\mathcal{I}_{\mathbf{A}}\right\rangle$ is isomorphic to a certain sublattice of $\left\langle\mathfrak{P}\left(\bar{P}_{\mathbf{X}}^{(l)}\right), \subseteq\right\rangle$. In the general case, a characterization of this lattice is (as far as the author can provide one) rather technical. We will therefore not elaborate it here. However, we will show that a comparably simple description is available if the connected components of $\mathbf{X}$ are totally ordered.

8.3.15 Corollary. If each connected component of $\mathbf{X}$ is a chain, then $C \in\left[\mathcal{I}_{\mathbf{A}}\right\rangle$ is isomorphic to the lattice of subalgebras of

$$
\mathbf{P}:=\left\langle\bar{P}_{\mathbf{X}}^{(1)},\left(\left.i d\right|_{Y}\right)_{Y \in \operatorname{Con}(\mathbf{X})}, \diamond\right\rangle,
$$


where $\diamond: \bar{P}_{\mathbf{X}}^{(1)} \times \bar{P}_{\mathbf{X}}^{(1)} \rightarrow \bar{P}_{\mathbf{X}}^{(1)}$ is defined as follows: For $h(\operatorname{dom}(h)) \subseteq \operatorname{dom}(p) \cup\left\{0^{\mathbf{X}}, 1^{\mathbf{X}}\right\}$, set

$$
p \diamond h(x):= \begin{cases}p \circ h(x) & \text { if } h(x) \in \operatorname{dom}(p), \\ 0^{\mathbf{X}} & \text { if } h(x)=0^{\mathbf{X}}, \\ 1^{\mathbf{X}} & \text { if } h(x)=1^{\mathbf{x}}\end{cases}
$$

and $p \diamond h:=h$ otherwise.

Proof. In the light of Lemma 8.3.13, we only need to show that a subset $H \subseteq \bar{P}_{\mathbf{X}}^{(1)}$ is the universe of a subalgebra of $\mathbf{P}$ if and only if we have $\left(C^{(1)}\right)^{\lambda}=H$ for some $C \in\left[\overline{\mathcal{I}}_{\mathbf{X}}\right\rangle$. " $\Longrightarrow$ ". Let $H$ be the universe of a subalgebra of $\mathbf{P}$. Let $(-)^{x}$ be the mapping from the proof of Lemma 8.3.12, and set $C:=\overline{\operatorname{Clo}}\left(H^{\varkappa} \cup \overline{\mathcal{I}}_{\mathbf{X}}\right)$. Clearly, $C \in\left[\overline{\mathcal{I}}_{\mathbf{X}}\right\rangle$. It remains to show that we have $\left(C^{(1)}\right)^{\lambda}=H$. It is obvious that $H \subseteq\left(C^{(1)}\right)^{\lambda}$ holds. For the other direction, let $g \in C^{(1)}$. We need to show $\left.g\right|_{Y} \in H$ for all $Y \in \operatorname{Con}(\mathbf{X})$. For $g \in H^{\varkappa}$, this is obvious. For $g \in \overline{\mathcal{I}}_{\mathbf{X}}$, we have $\left.g\right|_{Y}=\left.i d\right|_{Y} \in H$. Thus, we can finish the proof by showing that we have $\left.g\right|_{Y} \in H$ whenever $g$ can be written as a superposition $\left[g_{1}, \ldots, g_{n}\right] \circ g^{\prime}$, where $g^{\prime} \in H^{\varkappa} \cup \overline{\mathcal{I}}_{\mathbf{X}}$ and $\left.g_{i}\right|_{Z} \in H$ for all $i \in\{1, \ldots, n\}$ and $Z \in \operatorname{Con}(\mathbf{X})$. If $g^{\prime}$ is idempotent, it follows by Lemma 8.3 .12 that we have $\left.g\right|_{Y}=\left.g_{i}\right|_{Y} \in H$ for some integer $i \in\{1, \ldots, n\}$. If $g^{\prime} \in H^{\varkappa}$, then $g^{\prime}$ is unary, so we have $n=1$ and $g=g_{1} \circ g^{\prime}$. But now, since $Y$ is a chain, there exists $Z \in \operatorname{Con}(\mathbf{X})$ such that

$$
g^{\prime}(Y) \subseteq Z \cup\left\{0^{\mathbf{X}}, 1^{\mathbf{X}}\right\}=\operatorname{dom}\left(\left.g_{1}\right|_{Z}\right) \cup\left\{0^{\mathbf{X}}, 1^{\mathbf{X}}\right\} .
$$

By $\left.g_{1}\right|_{Z} \in H$, this implies $\left.g\right|_{Y}=\left.\left.g_{1}\right|_{Z} \diamond g^{\prime}\right|_{Y} \in H$.

"£". Let $C \in\left[\overline{\mathcal{I}}_{\mathbf{X}}\right\rangle$ and assume $H=\left(C^{(1)}\right)^{\lambda}$. The first direction, $H \subseteq \bar{P}_{\mathbf{X}}^{(1)}$, is obvious. Since $i d \in C^{(1)}$, it follows that we have $\left.i d\right|_{Y} \in H$ for all $Y \in \operatorname{Con}(\mathbf{X})$. For $p, h \in H$ and $h(\operatorname{dom}(h)) \nsubseteq \operatorname{dom}(p) \cup\left\{0^{\mathbf{X}}, 1^{\mathbf{X}}\right\}$, we have $p \diamond h=h \in H$. Assume $h(\operatorname{dom}(h)) \subseteq \operatorname{dom}(p) \cup\left\{0^{\mathbf{x}}, 1^{\mathbf{x}}\right\}$. By the definition of $H$, there exist $g, g^{\prime} \in C^{(1)}$ such that $\left.g\right|_{\operatorname{dom}(p)}=p$ and $\left.g^{\prime}\right|_{\operatorname{dom}(h)}=h$. But now, $p \diamond h=\left.\left(g \circ g^{\prime}\right)\right|_{\operatorname{dom}(h)} \in H$. Thus, $H$ is a subalgebra of $\mathbf{P}$.

We can use this corollary to give a very nice description of $\left[\mathcal{I}_{\mathbf{A}}\right\rangle$ if $\mathbf{A}$ is a Boolean lattice:

8.3.16 Corollary. If $\mathbf{A}$ is a Boolean lattice, then $\left[\mathcal{I}_{\mathbf{A}}\right\rangle$ is isomorphic to the lattice of binary, reflexive, transitive relations $\theta$ on $X$ where $\left(0^{\mathbf{X}}, x\right) \in \theta$ implies $x=0^{\mathbf{X}}$ and $\left(1^{\mathbf{X}}, x\right) \in \theta$ implies $x=1^{\mathbf{x}}$. That is,

$$
\left[\mathcal{I}_{\mathbf{A}}\right\rangle \cong\left\langle\operatorname{Ref} \operatorname{Tra}(X) \backslash R_{\theta_{01}}, \subseteq\right\rangle
$$

where $R_{\theta_{01}}$ is the set of all binary relations on $X$ that contain a tuple from the set $\theta_{01}:=\left\{(x, y) \mid 1^{\mathbf{X}}=x \neq y\right.$ or $\left.0^{\mathbf{X}}=y \neq x\right\}$.

Proof. Since $\mathbf{A}$ is a Boolean lattice, we have

$$
\operatorname{Con}(\mathbf{X})=\left\{\{x\} \mid x \in \mathbf{X}, x \notin\left\{0^{\mathbf{X}}, 1^{\mathbf{X}}\right\}\right\} .
$$


By Corollary 8.3.15, $\left[\mathcal{I}_{\mathbf{A}}\right\rangle$ is isomorphic to the lattice formed by the subalgebras of $\mathbf{P}=\left\langle\bar{P}_{\mathbf{X}}^{(1)},\left(\left.i d\right|_{Y}\right)_{Y \in \operatorname{Con}(\mathbf{X})}, \diamond\right\rangle$. Hence, we can show the claim by proving that the latter lattice is isomorphic to $\left\langle\operatorname{Ref} \operatorname{Tra}(X) \backslash R_{\theta_{01}}, \subseteq\right\rangle$. Each $p \in \bar{P}_{\mathbf{X}}^{(1)}$ has a domain that consists of only one element. Moreover, $p$ is uniquely determined by the binary tuple $(x, p(x))$ where $\operatorname{dom}(p)=\{x\}$. Vice versa, each binary tuple $r \in X^{2} \backslash \theta_{01}$ uniquely determines a nontrivial unary partial dual operation in $\bar{P}_{\mathbf{X}}^{(1)}$. Thus, there is a one-to-one correspondence between the subsets of $\bar{P}_{\mathbf{X}}^{(1)}$ and the subsets of $X^{2} \backslash \theta_{01}$. Let $H \subseteq \bar{P}_{\mathbf{X}}^{(1)}$ and let $\theta_{H}$ be the corresponding relation. Clearly, we have $\left.i d\right|_{Y} \in H$ for all $Y \in \operatorname{Con}(\mathbf{X})$ if and only if $\theta_{H}$ is reflexive. Furthermore, $H$ is closed under $\diamond$ if and only if $\theta_{H}$ is also transitive. Thus, $H$ is the universe of a subalgebra of $\mathbf{P}$ if and only if $\theta_{H} \in \operatorname{Ref} \operatorname{Tra}(X) \backslash R_{\theta_{01}}$. Hence, the lattice of substructures of $\left\langle\bar{P}_{\mathbf{X}}^{(1)},\left(\left.i d\right|_{Y}\right)_{Y \in \operatorname{Con}(\mathbf{X})}, \diamond\right\rangle$ is isomorphic to the lattice $\left\langle\operatorname{Ref} \operatorname{Tra}(X) \backslash R_{\theta_{01}}, \subseteq\right\rangle$.

As a curiosity, we will now look at the clone generated by the union of $\mathcal{I}_{\mathbf{A}}$ and End $\mathbf{A}$. That is, we look at the least clone that contains all unary and all idempotent operations over A. In the lattice of clones over sets, this clone is the full clone. One way to see this is to apply the Słupecki criterion [Słu39]. The same is true for clones over Boolean algebras (see [Maš06]). However, in the lattice of clones over distributive lattices, this is only true in certain cases. To characterize these cases, we have to introduce some more notation.

8.3.17 Definition. For $Y \in \operatorname{Con}(\mathbf{X})$, denote by $\operatorname{Spl}(Y)$ the set of pairs

$$
\left(Y_{1}, Y_{2}\right) \in(\mathfrak{P}(Y) \backslash\{\emptyset\}) \times(\mathfrak{P}(Y) \backslash\{\emptyset\}),
$$

such that each of the two sets $Y_{1}$ and $Y_{2}$ is connected (see Definition 8.0.3, page 148) and we have $y_{1} \not \leq y_{2}$ and $y_{2} \not \leq y_{1}$ for all $y_{1} \in Y_{1}, y_{2} \in Y_{2}$.

8.3.18 Example. Let $\mathrm{X}$ be the bounded poset illustrated by the following diagram:

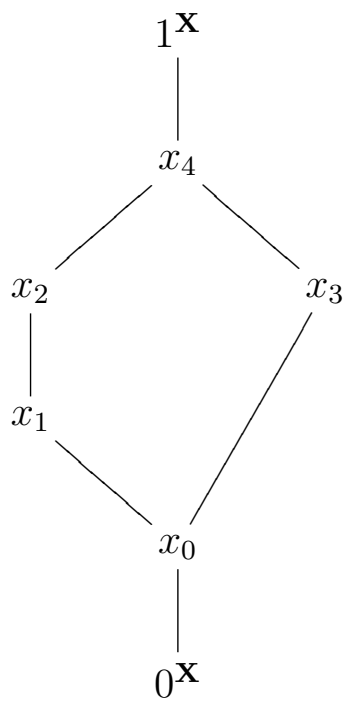

$$
\begin{aligned}
\operatorname{Spl}\left(\left\{x_{0}, \ldots, x_{4}\right\}\right)=\{ & \left(\left\{x_{1}\right\},\left\{x_{3}\right\}\right), \\
& \left(\left\{x_{3}\right\},\left\{x_{1}\right\}\right), \\
& \left(\left\{x_{2}\right\},\left\{x_{3}\right\}\right), \\
& \left(\left\{x_{3}\right\},\left\{x_{2}\right\}\right), \\
& \left(\left\{x_{1}, x_{2}\right\},\left\{x_{3}\right\}\right), \\
& \left.\left(\left\{x_{3}\right\},\left\{x_{1}, x_{2}\right\}\right)\right\}
\end{aligned}
$$


Clearly, $\operatorname{Spl}(Y)$ is a symmetric relation. The notation $\operatorname{Spl}(Y)$ is due to the fact that $\left(Y_{1}, Y_{2}\right) \in \operatorname{Spl}(Y)$ indicates that $Y_{1}$ and $Y_{2}$ can be split into different connected components via some dual operation $g \in \bar{O}_{\mathbf{X}}$. This is shown in the next lemma:

8.3.19 Lemma. Let $Y \in \operatorname{Con}(\mathbf{X})$. For two connected subsets $Y_{1}, Y_{2} \subseteq Y$, we have $\left(Y_{1}, Y_{2}\right) \in \operatorname{Spl}(Y)$ if and only if there exists $g \in \bar{O}_{\mathbf{X}}^{(n)}$ such that, for each $x_{1} \in g\left(Y_{1}\right)$ and $x_{2} \in g\left(Y_{2}\right)$, the two elements $x_{1}$ and $x_{2}$ belong to different connected components of $n \cdot \mathbf{X}$.

Proof. Let $\left(Y_{1}, Y_{2}\right) \in \operatorname{Spl}(Y)$. We define the following mapping:

$$
g(x):= \begin{cases}0^{2 \cdot \mathbf{X}} & \text { if } x<y \text { for some } y \in Y_{1} \cup Y_{2}, \\ 1^{2 \cdot \mathbf{X}} & \text { if } x>y \text { for some } y \in Y_{1} \cup Y_{2}, \\ \langle 1, x\rangle & \text { if } x \in Y_{1} \\ \langle 2, x\rangle & \text { if } x \in Y_{2} \\ \langle 1, x\rangle & \text { otherwise. }\end{cases}
$$

Since $y_{1} \not \leq y_{2}$ and $y_{2} \not \leq y_{1}$ for all $y_{1} \in Y_{1}$ and $y_{2} \in Y_{2}, g$ is a well-defined binary dual operation over $\mathbf{X}$. Conversely, let $g \in \bar{O}_{\mathbf{X}}^{(n)}$ be an operation that maps $Y_{1}$ and $Y_{2}$ into two different connected components of $n \cdot \mathbf{X}$. Then, clearly, for $y_{1} \in Y_{1}$ and $y_{2} \in Y_{2}$, we have $g\left(y_{1}\right) \not \leq g\left(y_{2}\right)$ and $g\left(y_{2}\right) \not \leq g\left(y_{1}\right)$. Since $g \in \bar{O}_{\mathbf{X}}$, this implies $y_{1} \not \leq y_{2}$ and $y_{2} \not \leq y_{1}$. Thus, $\left(Y_{1}, Y_{2}\right) \in \operatorname{Spl}(Y)$.

With this notation, we can now formulate the desired characterization.

8.3.20 Theorem. The following two statements are equivalent:

(1) $\operatorname{Clo}\left(\mathcal{I}_{\mathbf{A}} \cup\right.$ End $\left.\mathbf{A}\right)=O_{\mathbf{A}}$.

(2) For each $Y \in \operatorname{Con}(\mathbf{X})$ and $\left(Y_{1}, Y_{2}\right) \in \operatorname{Spl}(Y)$ there exists $Y^{\prime} \in \operatorname{Con}(\mathbf{X}) \backslash\{Y\}$ such that $Y_{1}$ or $Y_{2}$ can be order-embedded into $Y^{\prime}$.

Proof. (1) $\Longrightarrow(2)$. Assume that there exist $Y \in \operatorname{Con}(\mathbf{X})$ and $\left(Y_{1}, Y_{2}\right) \in \operatorname{Spl}(Y)$ such that $Y_{1}$ and $Y_{2}$ cannot be order-embedded into any $Y^{\prime} \in \operatorname{Con}(\mathbf{X}) \backslash\{Y\}$. We will show $\overline{\mathrm{Clo}}\left(\overline{\mathcal{I}}_{\mathbf{X}} \cup\right.$ End $\left.\mathbf{X}\right) \neq \bar{O}_{\mathbf{X}}$. For $\left(Z_{1}, Z_{2}\right) \in \operatorname{Spl}(Y)$, we write $\left(Z_{1}, Z_{2}\right) \sim\left(Y_{1}, Y_{2}\right)$ to indicate that $Z_{i}$ and $Y_{i}$ are order-isomorphic for $i \in\{1,2\}$. Define $g \in \bar{O}_{\mathbf{X}}^{(2)}$ as follows:

$$
g(x):= \begin{cases}0^{2 \cdot \mathbf{X}} & \text { if } x<y \text { for some } y \in Y_{1} \cup Y_{2}, \\ 1^{2 \cdot \mathbf{X}} & \text { if } x>y \text { for some } y \in Y_{1} \cup Y_{2}, \\ \langle 1, x\rangle & \text { if } x \in Y_{1} \\ \langle 2, x\rangle & \text { if } x \in Y_{2} \\ \langle 1, x\rangle & \text { otherwise. }\end{cases}
$$

Since $\left(Y_{1}, Y_{2}\right) \in \operatorname{Spl}(Y), g$ is well-defined (see Lemma 8.3.19). We will show

$$
g \notin \overline{\operatorname{Clo}}\left(\overline{\mathcal{I}}_{\mathbf{X}} \cup \text { End } \mathbf{X}\right)
$$

by defining a dual relation $\sigma$ of type $2 \cdot \mathbf{X}$ such that $\overline{\mathcal{I}}_{\mathbf{X}} \cup$ End $\mathbf{X} \bar{\nabla} \sigma$, whereas $g \not \nabla$. Let $\sigma$ contain all morphisms $r: \mathbf{X} \rightarrow 2 \cdot \mathbf{X}$ such that, for all $\left(Z_{1}, Z_{2}\right) \in \operatorname{Spl}(Y)$ with $\left(Z_{1}, Z_{2}\right) \sim\left(Y_{1}, Y_{2}\right), r$ satisfies one of the following two cases: 
(a) $r$ maps $Z_{1}$ and $Z_{2}$ into the same connected component,

(b) $r$ is not order-reflecting on $Z_{1}$ or $Z_{2}$.

Let $r_{1}, \ldots, r_{n} \in \sigma$ and let $h \in \overline{\mathcal{I}}_{\mathbf{X}} \cup$ End $\mathbf{X}$. If $h$ is idempotent, then $\left[r_{1}, \ldots, r_{n}\right] \circ h \in \sigma$ is immediate since Lemma 8.3.1 (page 152) implies that there exists $i \in\{1, \ldots, n\}$ such that $h(Y)=r_{i}(Y)$. Assume that $h$ is unary. Consequently, we have $n=1$ and it remains to show that $r_{1} \circ h \in \sigma$. Let $\left(Z_{1}, Z_{2}\right) \in \operatorname{Spl}(Y)$ with $\left(Z_{1}, Z_{2}\right) \sim\left(Y_{1}, Y_{2}\right)$. Since, by assumption, $Z_{1}$ and $Z_{2}$ cannot be order-embedded into different connected components of $\mathbf{X}$, it follows that $h$ satisfies (a) or (b). If $h$ satisfies case (b), then so does $r_{1} \circ h$. Hence, we assume that $h$ is order-reflecting on $Z_{1}$ and $Z_{2}$. Since, by assumption, $Z_{1}$ and $Z_{2}$ cannot be order embedded into a connected component different from $Y$, this implies $h\left(Z_{1}\right), h\left(Z_{2}\right) \subseteq Y$. If $\left(h\left(Z_{1}\right), h\left(Z_{2}\right)\right) \notin \operatorname{Spl}(Y)$, then it follows by Lemma 8.3.19 that $h\left(Z_{1}\right)$ and $h\left(Z_{2}\right)$ cannot be mapped into different connected components, and so $r_{1} \circ h \in \sigma$. If $\left(h\left(Z_{1}\right), h\left(Z_{2}\right)\right) \in \operatorname{Spl}(Y)$, then $\left(h\left(Z_{1}\right), h\left(Z_{2}\right)\right) \sim\left(Z_{1}, Z_{2}\right) \sim\left(Y_{1}, Y_{2}\right)$, and the claim follows since $r_{1}$ must satisfy (a) or (b) for $\left(h\left(Z_{1}\right), h\left(Z_{2}\right)\right)$. Thus, $\overline{\mathcal{I}}_{\mathbf{X}} \cup$ End $\mathbf{X} \bar{\triangleright} \sigma$. However, since $g$ does not satisfy (a) or (b) for $\left(Y_{1}, Y_{2}\right)$, we obtain

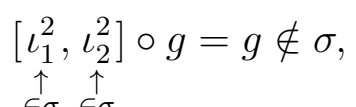

and this means that $g$ does not preserve $\sigma$.

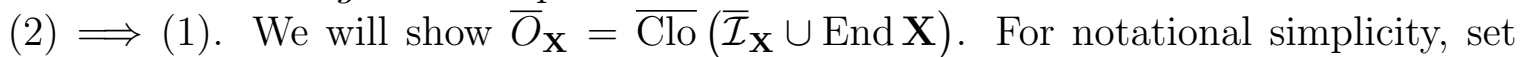
$C:=\overline{\operatorname{Clo}}\left(\overline{\mathcal{I}}_{\mathbf{X}} \cup\right.$ End $\left.\mathbf{X}\right)$. Since $C, \bar{O}_{\mathbf{X}} \in\left[\overline{\mathcal{I}}_{\mathbf{X}}\right\rangle$, we can use Lemma 8.3 .12 which enables us to finish the proof by showing $\left(\bar{O}_{\mathbf{X}}\right)^{\lambda} \subseteq C^{\lambda}$. Let $p \in\left(\bar{O}_{\mathbf{X}}\right)^{\lambda}$. Then, we have $Y:=\operatorname{dom}(p) \in \operatorname{Con}(\mathbf{X})$, and it suffices to show that there exists $g \in C$ with $\left.g\right|_{Y}=p$. Without loss of generality, we can assume that there exists $k \in \mathbb{N}_{+}$and a partition $\left\{Y_{0}, \ldots, Y_{k+1}\right\}$ of $Y$ such that

$$
\begin{aligned}
p\left(Y_{0}\right) & =0^{k \cdot \mathbf{X}}, \\
p\left(Y_{1}\right) & \subseteq \iota_{1}^{k}(\mathbf{X}) \backslash\left\{0^{k \cdot \mathbf{X}}, 1^{k \cdot \mathbf{X}}\right\}, \\
\vdots & \vdots \\
p\left(Y_{k}\right) & \subseteq \iota_{k}^{k}(\mathbf{X}) \backslash\left\{0^{k \cdot \mathbf{X}}, 1^{k \cdot \mathbf{X}}\right\}, \\
p\left(Y_{k+1}\right) & =1^{k \cdot \mathbf{X}} .
\end{aligned}
$$

We will only show the claim for the case in which each of the sets $Y_{1}, \ldots, Y_{k}$ is connected since the general case is similar. Since, for each $i \in\{1, \ldots, k\}, p$ maps $Y_{i}$ and $Y_{i+1} \cup \ldots Y_{k}$ into different connected components, Lemma 8.3.19 establishes

$$
\left(Y_{1}, Y_{2} \cup \ldots \cup Y_{k}\right),\left(Y_{2}, Y_{3} \cup \ldots \cup Y_{k}\right), \ldots,\left(Y_{k-1}, Y_{k}\right) \in \operatorname{Spl}(Y) .
$$

By (2), this implies that all except at most one of the subsets $Y_{1}, \ldots, Y_{k}$ can be orderembedded into a connected component different from $Y$. Hence, without loss of generality, we can assume that there exist $Z_{1}, \ldots, Z_{k-1} \in \operatorname{Con}(\mathbf{X}) \backslash\{Y\}$ such that $Y_{i}$ can be order-embedded into $Z_{i}$ for each $i \in\{1, \ldots, k-1\}$. For $i \in\{1, \ldots, k-1\}$, let $h_{i}: Y_{i} \rightarrow Z_{i}$ 
be the corresponding order embedding. For $i \in\{1, \ldots, k\}$, let the mapping $p_{i}: Y_{i} \rightarrow X$ be defined as follows:

$$
p_{i}(x)=y: \Longleftrightarrow p(x)=\langle i, y\rangle \text {. }
$$

Moreover, set

$$
u_{i}(x):= \begin{cases}0^{\mathbf{X}} & \text { if } y \in Y_{0} \text { or } x<y \text { for some } y \in Y_{i}, \\ 1^{\mathbf{X}} & \text { if } y \in Y_{k+1} \text { or } x>y \text { for some } y \in Y_{i}, \\ h_{i}(x) & \text { if } x \in Y_{i}, \\ x & \text { otherwise }\end{cases}
$$

and

$$
u_{i}^{\prime}(x):= \begin{cases}0^{\mathbf{X}} & \text { if } x<y \text { for some } y \in Z_{i}, \\ 1^{\mathbf{X}} & \text { if } x>y \text { for some } y \in Z_{i}, \\ p_{i} \circ h_{i}^{-1}(x) & \text { if } x \in Z_{i}, \\ x & \text { otherwise. }\end{cases}
$$

It is easy to verify that $u_{i}$ and $u_{i}^{\prime}$ are well-defined unary operations over $\mathbf{X}$. Furthermore, define $s_{i}$ by setting

$$
s_{i}(x):= \begin{cases}0^{k \cdot \mathbf{X}} & \text { if } x=0^{\mathbf{X}} \\ 1^{k \cdot \mathbf{X}} & \text { if } x=1^{\mathbf{X}} \\ \langle i, x\rangle & \text { if } x \in Z_{i} \\ \langle k, x\rangle & \text { otherwise. }\end{cases}
$$

Clearly, $s_{i}$ is a well-defined $k$-ary dual idempotent operation over $\mathbf{X}$. Set

$$
r_{1}:=\left[\iota_{1}^{k} \circ u_{1}^{\prime}, \iota_{2}^{k}, \ldots, \iota_{k}^{k}\right] \circ s_{1} \circ u_{1} .
$$

Now, $r_{1}$ is a $k$-ary dual operation that maps each $y \in Y_{0} \cup Y_{1} \cup Y_{k+1}$ to $p(y)$ and each $z \in Y_{2} \cup \ldots \cup Y_{k}$ to $\langle k, z\rangle$. Now, we set

$$
r_{2}:=\left[\iota_{1}^{k}, \iota_{2}^{k} \circ u_{2}^{\prime}, \iota_{3}^{k}, \ldots, \iota_{k}^{k}\right] \circ\left[\iota_{1}^{k}, \ldots, \iota_{k-1}^{k}, s_{2} \circ u_{2}\right] \circ r_{1} .
$$

Then, $r_{2}$ is a $k$-ary dual operation that maps each $y \in Y_{0} \cup Y_{1} \cup Y_{2} \cup Y_{k+1}$ to $p(y)$ and each $z \in Y_{2} \cup \ldots \cup Y_{k}$ to $\langle z, y\rangle$. We can repeat this argument until we obtain a $k$-ary dual operation $r_{k-1}$ that maps $y \in Y_{i}$ to $p(y)$ for each $i \in\{0, \ldots, k-1, k+1\}$ and each $z \in Y_{k}$ to $\langle k, z\rangle$. Finally, we set

$$
g:=\left[\iota_{1}^{k}, \ldots, \iota_{k-1}^{k}, \iota_{k}^{k} \circ p_{k}\right] \circ r_{k-1},
$$

and we obtain $\left.g\right|_{Y}=p$.

\subsubsection{Examples.}

(i) Let $\mathbf{A}$ be our running example from Figure 8.1. It is easy to see that condition (2) from the theorem is satisfied. Thus, $\operatorname{Clo}\left(\mathcal{I}_{\mathbf{A}} \cup\right.$ End $\left.\mathbf{A}\right)=O_{\mathbf{A}}$.

(ii) Let $\mathbf{A}$ be a distributive lattices that dualizes to the poset $\mathbf{X}$ from Example 8.3.18. For $\left(\left\{x_{1}, x_{2}\right\},\left\{x_{3}\right\}\right) \in \operatorname{Spl}\left(\left\{x_{0}, \ldots, x_{4}\right\}\right)$, there exists no $Y^{\prime} \in \operatorname{Con}(\mathbf{X}) \backslash\{Y\}$ such that $\left\{x_{1}, x_{2}\right\}$ or $\left\{x_{2}\right\}$ can be order-embedded into $Y^{\prime}$. Thus, $\operatorname{Clo}\left(\mathcal{I}_{\mathbf{A}} \cup\right.$ End $\left.\mathbf{A}\right)$ is not the full clone. 
(iii) Let $\mathbf{A}$ be a distributive lattice with exactly one atom or one coatom. Since this implies that $\mathbf{X}$ has only one connected component, condition (2) of the theorem is satisfied if and only if $\mathbf{X}$ is totally ordered, which, in turn, is equivalent to $\mathbf{A}$ being totally ordered. Thus, $\operatorname{Clo}\left(\mathcal{I}_{\mathbf{A}} \cup\right.$ End $\left.\mathbf{A}\right)=O_{\mathbf{A}}$ if and only if $\mathbf{A}$ is a chain.

The following corollary is an obvious consequence:

8.3.22 Corollary. If $\mathbf{A}$ is a Boolean lattice, then $O_{\mathbf{A}}=\operatorname{Clo}\left(\mathcal{I}_{\mathbf{A}} \cup\right.$ End $\left.\mathbf{A}\right)$.

Note that $\operatorname{Clo}\left(\mathcal{I}_{\mathbf{A}} \cup\right.$ End $\left.\mathbf{A}\right)$ contains the clone of all 01-homomorphisms over $\mathbf{A}$ (recall that an $n$-ary operation over $\mathbf{A}$ is a 01-homomorphism provided that it maps the maximum and minimum element of $\mathbf{A}^{n}$ to the maximum and minimum element of $\mathbf{A}$, respectively). This follows from Lemma 8.3 .12 (page 155) and the fact that the restriction of each 01-homomorphism to a connected component $Y \in \operatorname{Con}(\mathbf{X})$ is essentially unary. In the next section, we will take a closer look at the clone of all 01-homomorphisms.

\subsection{0-Homomorphisms and 1-Homomorphisms over A}

For this section, set $\mathbf{A}_{0}:=\langle A, \vee, \wedge, 0\rangle, \mathbf{A}_{1}:=\langle A, \vee, \wedge, 1\rangle$ and $\mathbf{A}_{01}:=\langle A, \vee, \wedge, 0,1\rangle$. Evidently, $\mathcal{L}_{\mathbf{A}_{0}}, \mathcal{L}_{\mathbf{A}_{1}}$ and $\mathcal{L}_{\mathbf{A}_{01}}$ are ideals of $\mathcal{L}_{\mathbf{A}}$.

By exploring these three lattices, we obtain general results about the centralizer clones of distributive lattices with 0 , distributive lattices with 1 and so-called bounded distributive lattices (that is, a distributive lattice with 0 and 1 ).

Note that, by Lemma 8.0.2 (page 148), we have

$$
\begin{aligned}
\left(O_{\mathbf{A}_{0}}\right)^{\partial} & =\left\{g \in \bar{O}_{\mathbf{X}}^{(n)} \mid n \in \mathbb{N}_{+}, g^{-1}\left(1^{n \cdot \mathbf{X}}\right)=\left\{1^{\mathbf{X}}\right\}\right\} \\
\left(O_{\mathbf{A}_{1}}\right)^{\partial} & =\left\{g \in \bar{O}_{\mathbf{X}}^{(n)} \mid n \in \mathbb{N}_{+}, g^{-1}\left(0^{n \cdot \mathbf{X}}\right)=\left\{0^{\mathbf{X}}\right\}\right\} \\
\left(O_{\mathbf{A}_{01}}\right)^{\partial} & =\left\{g \in \bar{O}_{\mathbf{X}}^{(n)} \mid n \in \mathbb{N}_{+}, g^{-1}\left(\left\{1^{n \cdot \mathbf{X}}, 0^{n \cdot \mathbf{X}}\right\}\right)=\left\{0^{\mathbf{X}}, 1^{\mathbf{X}}\right\}\right\} .
\end{aligned}
$$

We will start this section by determining the maximum essential arity in each of these clones.

8.4.1 Theorem. Let $k_{0}$ be the number of the atoms of $\mathbf{A}$, and let $k_{1}$ be the number of the coatoms of $\mathbf{A}$. Then,

(i) $k_{0}$ is a sharp bound on the essential arity of operations among $O_{\mathbf{A}_{0}}$,

(ii) $k_{1}$ is a sharp bound on the essential arity of operations among $O_{\mathbf{A}_{1}}$,

(iii) $|\operatorname{Con}(\mathbf{X})|$ is a sharp bound on the essential arity of operations among $O_{\mathbf{A}_{01}}$.

Proof. (i) Let $f \in O_{\mathbf{A}_{0}}$ be $n$-ary. Since we have $f^{\partial}(x) \neq 1^{n \cdot \mathbf{X}}$ for all $x \neq 1^{\mathbf{X}}, f^{\partial}$ must map any element below a coatom $y \in \mathbf{X}$ into the same set among $\iota_{i}^{1}(\mathbf{X}), \ldots, \iota_{i}^{n}(\mathbf{X})$. Since we have seen in Lemma 8.0.1 (page 147) that the number of the coatoms of $\mathbf{X}$ is the number of atoms of $\mathbf{A}$, this implies that $f^{\partial}$ is essentially at most $k_{0}$-ary. To construct an 
operation $f^{*} \in O_{\mathbf{A}}$ with exactly $k_{0}$ essential arguments, let $x_{1}, \ldots, x_{k_{0}}$ be the coatoms of $\mathbf{X}$, and define a $k_{0}$-ary dual operation $g \in \bar{O}_{\mathbf{X}}$ as follows:

$$
g(x):= \begin{cases}1^{k_{0}} \cdot \mathbf{X} & \text { if } x=1^{\mathbf{X}} \\ \langle i, x\rangle & \text { if } x \in\left\{x_{1}, \ldots, x_{k_{0}}\right\} \\ 0^{k_{0}} \cdot \mathbf{X} & \text { otherwise }\end{cases}
$$

It is straight-forward to check that $g$ is well-defined and has $k_{0}$ essential arguments. Setting $f^{*}=g^{\partial^{-1}}$ finishes the proof.

(ii) Analogue to (i).

(iii) Let $f \in O_{\mathbf{A}_{01}}$ be $n$-ary and let $k:=|\operatorname{Con}(\mathbf{X})|$. Since we have $f^{\partial}(x) \notin\left\{0^{n \cdot \mathbf{X}}, 1^{n \cdot \mathbf{X}}\right\}$ for all $x \notin\left\{1^{\mathbf{X}}, 0^{\mathbf{X}}\right\}, f^{\partial}$ must map each connected component into the same set among $\iota_{1}^{n}(\mathbf{X}), \ldots, \iota_{n}^{n}(\mathbf{X})$. Hence, $f^{\partial}$ is essentially at most $k$-ary which implies the same for $f$. To show that the bound is sharp, let $X_{1}, \ldots, X_{k}$ be the connected components of $\mathbf{X}$, and define $g \in \bar{O}_{\mathbf{X}}^{(k)}$ by setting

$$
g(x):= \begin{cases}0^{k \cdot \mathbf{X}} & \text { if } x=0^{\mathbf{X}} \\ 1^{k \cdot \mathbf{X}} & \text { if } x=1^{\mathbf{X}} \\ \langle i, x\rangle & \text { if } x \in X_{i}\end{cases}
$$

Evidently, $g$ is well-defined and has exactly $k$ essential arguments.

8.4.2 Example. As usual, we will take a look at our running example from Figure 8.1. Since $\mathbf{A}$ has three atoms and four coatoms and $\mathbf{X}$ has three connected components, we can infer that the maximum essential arities in $O_{\mathbf{A}_{0}}, O_{\mathbf{A}_{1}}$ and $O_{\mathbf{A}_{01}}$ are 3,4 and 3, respectively.

We will now show that $\mathcal{L}_{\mathbf{A}_{01}}$ is essentially an ideal in the lattice of clones of dual operations over a finite set.

8.4.3 Theorem. Let $l$ be the number of join-irreducible elements among $A \backslash\{0\}$ (i.e., $l=|\operatorname{Spec}(\mathbf{A})|)$. Then, $\mathcal{L}_{\mathbf{A}_{01}}$ is order-isomorphic to an ideal in the lattice of clones of dual operations over the set $\{1, \ldots, l\}$ in Set.

Proof. It is a well-known result from the field of lattice theory that there exists a (up to isomorphism) unique minimal Boolean algebra $\mathbf{B}$ such that $\mathbf{A}$ can be order-embedded into B. Moreover, this Boolean algebra is known to have exactly as many atoms as $\mathbf{A}$ has join-irreducible elements without taking the bottom element into account. Clearly, for each $n \in \mathbb{N}_{+}$, the lattice $\mathbf{A}^{n}$ is now order-embeddable into $\mathbf{B}^{n}$. Let $\varphi_{n}: \mathbf{A}^{n} \rightarrow \mathbf{B}^{n}$ be the corresponding order embedding. For each $f \in O_{\mathbf{A}_{01}}^{(n)}$, there exists a unique operation $f_{\varphi}: \mathbf{B}^{n} \rightarrow \mathbf{B}$ such that $f_{\varphi}(x)=\left(\varphi_{1} \circ f \circ \varphi_{n}^{-1}\right)(x)$ for each $x \in \varphi_{n}(A)$. Furthermore, it is easy to see that the set $\left\{f_{\varphi} \mid f \in O_{\mathbf{A}_{01}}\right\}$ is a clone over $\mathbf{B}$ in the category of finite Boolean algebras. Thus $f \mapsto f_{\varphi}$ is a clone-isomorphism, whence it follows $\mathcal{L}_{\mathbf{A}_{01}} \cong\left\langle\left\{f_{\varphi} \mid f \in O_{\mathbf{A}_{01}}\right\}\right] \leq \mathcal{L}_{\mathbf{B}}$. But now, the clone duality introduced in Subsection 4.2.2 yields $\mathcal{L}_{\mathbf{B}} \cong \overline{\mathcal{L}}_{\mathrm{At}(\mathbf{B})} \cong \overline{\mathcal{L}}_{\{1, \ldots, l\}}$, and the claim follows.

Note that the number of join-irreducible elements among $A \backslash\{0\}$ is precisely $|\mathbf{X}|-2$. 
8.4.4 Example. Again, let A be the distributive lattice from Figure 8.1. Since $\mathbf{A}$ has six join-irreducible elements besides the bottom element, we can conclude that $\mathcal{L}_{\mathbf{A}_{01}}$ is orderisomorphic to an ideal in the lattice of clones of dual operations over $\{1, \ldots, 6\} \in$ Set.

The last theorem becomes stronger if $\mathbf{A}$ is a Boolean lattice:

8.4.5 Corollary. If $\mathbf{A}$ is a Boolean lattice, then $\mathcal{L}_{\mathbf{A}_{01}}$ is isomorphic to the lattice of clones of dual operations over the set $\mathrm{At}(\mathbf{A})$ in the category of sets.

Proof. If $\mathbf{A}$ is a Boolean lattice, the order embeddings $\varphi_{n}: \mathbf{A}^{n} \rightarrow \mathbf{B}^{n}$ from the proof of Theorem 8.4 .3 become bijective. This establishes $\left\{f_{\varphi} \mid f \in O_{\mathbf{A}_{01}}\right\}=O_{\mathbf{B}}$, and we obtain $\mathcal{L}_{\mathbf{A}_{01}} \cong\left\langle O_{\mathbf{B}}\right]=\mathcal{L}_{\mathbf{B}} \cong \overline{\mathcal{L}}_{\mathrm{At}(\mathbf{B})} \cong \overline{\mathcal{L}}_{\mathrm{At}(\mathbf{A})}$.

Now, we will turn our attention to the filter $\left[O_{\mathbf{A}_{01}}\right\rangle$. If the connected components of $\mathbf{X}$ are totally ordered, then we can give a very nice description. The result is stated in the next theorem, but since the proof is long and technical, we have moved it to the appendix.

8.4.6 Theorem. Assume that each connected component of $\mathbf{X}$ is a chain, and let $l$ be the cardinality of the greatest connected component (that is, $l+2$ is the length of the longest chain in $\mathbf{X})$. Define

$$
M:=\{1,2, \ldots, 2 l\}^{2} \backslash\{(v, w) \mid v \neq w, \max (v, w) \equiv 1(\bmod 2)\} .
$$

Then,

$$
\begin{aligned}
{\left[O_{\mathbf{A}_{01}}\right\rangle } & \cong\langle M,(\leq, \leq)\rangle, \\
{\left[O_{\mathbf{A}_{0}}\right\rangle } & \cong\langle\{1, \ldots, 2 l\}, \leq\rangle, \\
{\left[O_{\mathbf{A}_{1}}\right\rangle } & \cong\langle\{1, \ldots, 2 l\}, \leq\rangle
\end{aligned}
$$

Note that, alternatively stated, $M$ is the set of tuples $(v, w) \in\{1, \ldots, 2 l\}^{2}$ where $\max \{v, w\}$ is even or $v=w$.

8.4.7 Example. Let $\mathbf{A}$ be a lattice such that each connected component of $\mathbf{X}$ is a chain, and assume that the cardinality of the greatest connected component is 2 . Then, $\left[O_{\mathbf{A}_{01}}\right\rangle$ is isomorphic to $\langle M,(\leq, \leq)\rangle$ as given in Figure 8.6.

If $\mathbf{A}$ is a Boolean lattice, then $\operatorname{Con}(\mathbf{X})$ consists of one-element sets, so the cardinality of the maximal chain in each connected component is 1 . The following corollary is now obvious:

8.4.8 Corollary. If $\mathbf{A}$ is a Boolean lattice, then the filter $\left[O_{\mathbf{A}_{01}}\right\rangle$ is the following fourelement lattice:

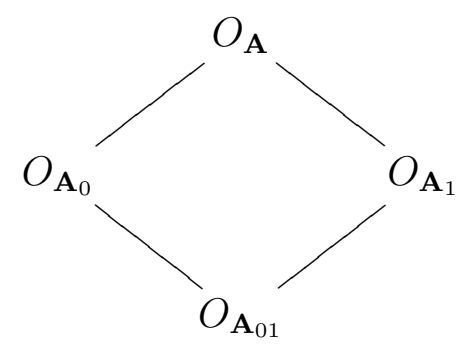




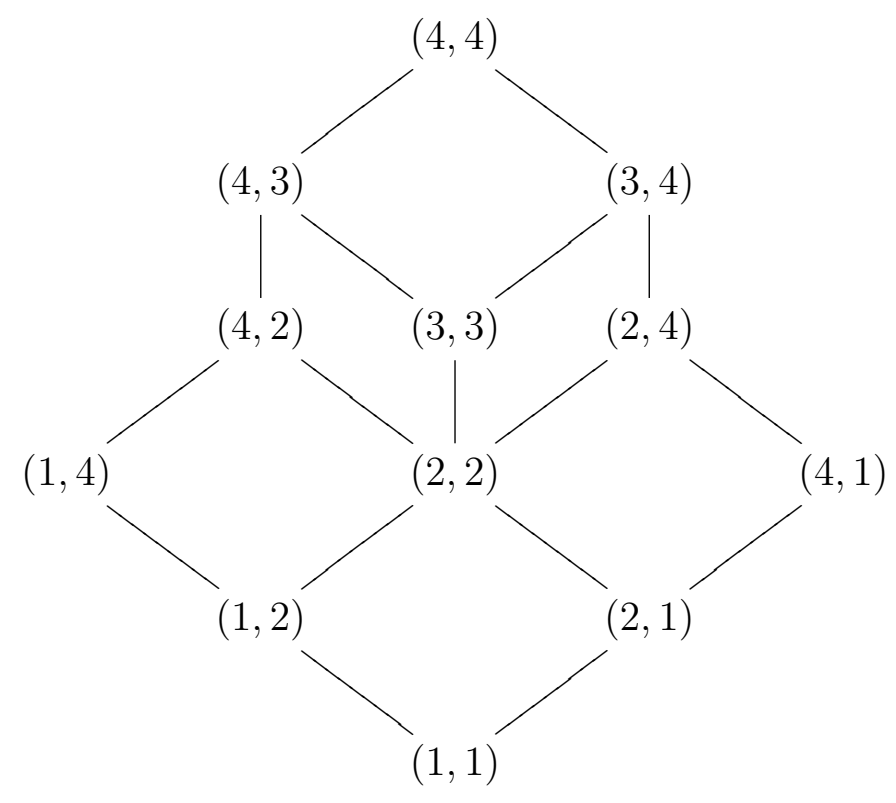

Figure 8.6.: $\langle M,(\leq, \leq)\rangle$ for $l=2$

For the general case, a similarly nice description seems out of reach. However, we have $\mathcal{I}_{\mathbf{A}} \subseteq O_{\mathbf{A}_{01}}$, so we can use the results from Section 8.3 about $\left[\mathcal{I}_{\mathbf{A}}\right\rangle$ to collect a few facts about $\left[O_{\mathbf{A}_{01}}\right\rangle$. For instance, we can apply Lemma 8.3.13 (page 156) to conclude that each $C \in\left[O_{\mathbf{A}_{01}}\right\rangle$ is uniquely determined by its $l$-ary part where $l$ is the cardinality of the longest antichain in $\mathbf{X}$ that is contained in one connected component.

\subsection{Unary Clones and Clones Containing End A}

In this section, we will turn our attention to the structure of $\mathcal{L}_{\mathbf{A}}$ above and below the clone $\mathrm{Clo}$ (End $\mathbf{A}$ ) of all essentially at most unary operations over $\mathbf{A}$.

Evidently, the ideal of clones below $\mathrm{Clo}($ End $\mathbf{A})$ in $\mathcal{L}_{\mathbf{A}}$ is order-isomorphic to the lattice of endomorphism monoids on $\mathbf{X}$ via $C \mapsto\left(C^{(1)}\right)^{\partial}$. This establishes the following proposition:

8.5.1 Proposition. Let $B:=\operatorname{Spec}(\mathbf{A}) \cup\{\emptyset, A\}$. Then, the following two statements hold:

(i) The ideal $\langle\mathrm{Clo}($ End $\mathbf{A})] \leq \mathcal{L}_{\mathbf{A}}$ is order-isomorphic to the lattice of endomorphism monoids on the bounded poset $\langle B, \emptyset, A, \subseteq\rangle$.

(ii) If $\mathbf{A}$ is a Boolean lattice, then $\langle\mathrm{Clo}($ End $\mathbf{A})]$ is order-isomorphic to the lattice of endomorphism monoids on the doubly pointed set $\langle B, \emptyset, A\rangle$.

Note that the set $B$ is generally much smaller than the carrier set of $\mathbf{A}$. For instance, in our running example from Figure 8.1, we have $|\mathbf{A}|=30$, but $|B|=8$. In particular, if $\mathbf{A}$ is a Boolean lattice with $2^{n}$ elements, then $B$ contains only $n+2$ elements. Only if $\mathbf{A}$ 
consists of just one chain, then we have $|B|>|A|$ (in fact, this yields $|B|=|A|+1$ ). But then, $\langle\mathrm{Clo}$ (End $\mathbf{A})]$ is simply order-isomorphic to the lattice of monotone endomorphism monoids on the set $A$.

We will now look at the filter $[\operatorname{Clo}($ End $\mathbf{A})\rangle$, i.e., the clones above the clone of all essentially at most unary operations. In the lattice of clones of operations over a finite set $A$, this filter has a rather surprising structure, first described in [Bur67]: It is a $(|A|+1)$-element chain

$$
\operatorname{Clo}\left(A^{A}\right)=C_{1}<C^{*}<C_{2}<\ldots<C_{|A|-1}<C_{|A|}=O_{A}
$$

where $C^{*}$ is the clone known as the Burle clone. For a finite Boolean algebra $\mathbf{B}$, $[\mathrm{Clo}($ End $\mathbf{B})\rangle$ is also a chain, but without the Burle "deviation" [Maš06]. We will now show that the same is true if $\mathbf{A}$ is a finite Boolean lattice.

For an operation $g \in \bar{O}_{\mathbf{X}}$, denote the rank of $g$, written $r(g)$, as follows:

$$
r(g):= \begin{cases}1 & \text { if } g \text { is essentially unary, } \\ |g(\mathbf{X})|-2 & \text { otherwise. }\end{cases}
$$

Note that $g$ cannot be essentially nullary and that $|g(\mathbf{X})| \geq 4$ whenever $g$ is essentially at least binary. Thus, $r(g)=1$ if and only if $g$ is essentially unary.

Let us assume that $\mathbf{A}$ is a Boolean lattice with $k$ atoms. We have $r(g) \in\{1, \ldots, k\}$ for each $g \in \bar{O}_{\mathbf{X}}$. For $i \in\{1, \ldots, k\}$, we set

$$
U_{i}:=\overline{\mathrm{Clo}}\left(\text { End } \mathbf{X} \cup\left\{g \in \bar{O}_{\mathbf{X}} \mid r(g) \leq i\right\}\right) .
$$

It is easy to see that we have

$$
\overline{\mathrm{Clo}}(\text { End } \mathbf{X})=U_{1}<U_{2}<\ldots<U_{k}=\bar{O}_{\mathbf{X}} .
$$

The following lemma is a technicality, but it is needed to prove our claim:

8.5.2 Lemma. Let $\mathbf{A}$ be a Boolean lattice. For each $g \in \bar{O}_{\mathbf{X}}$ with essential arity $j$, the set of dual operations End $\mathbf{X} \cup\{g\}$ generates some $g^{\prime} \in \bar{O}_{\mathbf{X}}$ with $r\left(g^{\prime}\right)=r(g)$ and essential arity $\min \{j+1, r(g)\}$.

Proof. Assume $X=\left\{0^{\mathbf{X}}, x_{1}, \ldots, x_{k}, 1^{\mathbf{X}}\right\}$. If $g$ is essentially unary or essentially $r(g)$-ary, the claim is trivial. Hence, we can assume that there exists $i \in\{1, \ldots, r(g)\}$ such that

$$
\left|\iota_{i}^{n}(\mathbf{X}) \backslash\left\{0^{n \cdot \mathbf{X}}, 1^{n \cdot \mathbf{X}}\right\}\right|>1
$$

Without loss of generality, we assume $i=1$. Then, there exists an integer $l \geq 2$ such that $\left\langle 1, y_{1}\right\rangle, \ldots,\left\langle 1, y_{l}\right\rangle$ are all the (pairwise distinct) elements in $g(\mathbf{X}) \cap \iota_{1}^{n}(\mathbf{X}) \backslash\left\{0^{n \cdot \mathbf{X}}, 1^{n \cdot \mathbf{X}}\right\}$. Hence, there exist $x_{1}, \ldots, x_{l} \in \mathbf{X}$ such that $g\left(x_{i}\right)=\left\langle 1, y_{i}\right\rangle$ for all $i \in\{1, \ldots, l\}$. Moreover, since $g$ is essentially at least binary, there exists $z \in \mathbf{X}$ such that $g(z) \notin \iota_{1}^{n}(\mathbf{X})$. Define the unary operation $u$ as follows:

$$
u(x):= \begin{cases}0^{\mathbf{X}} & \text { if } x=0^{\mathbf{X}} \\ z & \text { if } x=y_{1} \\ x_{i} & \text { if } x=y_{i} \text { and } i \in\{2, \ldots, l\} \\ 1^{\mathbf{X}} & \text { otherwise. }\end{cases}
$$


Now set $g^{\prime}:=\left[g \circ u, \iota_{n+1}^{2 n}, \ldots, \iota_{2 n}^{2 n}\right] \circ g$. Evidently, $g^{\prime} \in \overline{\mathrm{Clo}}($ End $\mathbf{X} \cup\{g\})$. Moreover,

$$
g^{\prime}(x)= \begin{cases}g(z) & \text { if } x=x_{1} \\ \left\langle 1, y_{i}\right\rangle & \text { if } x=x_{i} \text { and } i \in\{2, \ldots, l\} \\ \iota_{n+j}^{2 n}(y) & \text { if } x \notin\left\{x_{1}, \ldots, x_{l}\right\} \text { and } g(x)=\iota_{j}^{n}(y) .\end{cases}
$$

We have

$$
g(z) \notin \iota_{1}^{2 n}(\mathbf{X}) \cup \iota_{n+1}^{2 n}(\mathbf{X}) \cup \ldots \cup \iota_{2 n}^{2 n}(\mathbf{X}),
$$

so it follows that the rank of $g$ and $g^{\prime}$ coincide and that $g^{\prime}$ has one more essential argument than $g$.

8.5.3 Lemma. Let $\mathbf{A}$ be a Boolean lattice, and let $g \in \bar{O}_{\mathbf{X}}$ such that $r(g)=i$. Then $U_{i} \leq \overline{\mathrm{Clo}}($ End $\mathbf{X} \cup\{g\})$.

Proof. It is easy to see that $U_{i}$ is generated by End $\mathbf{X} \cup\left\{g_{i}\right\}$ for any $g_{i} \in \bar{O}_{\mathbf{X}}$ with exactly $i$ essential arguments and $r\left(g_{i}\right)=i$. In the light of this observation, it suffices to show that End $\mathbf{X} \cup\{g\}$ generates such an operation. Let $j$ be the essential arity of $g$. By Lemma 8.5.2, End $\mathbf{X} \cup\{g\}$ generates an essentially $(\min \{j+1, i\})$-ary operation $g^{\prime}$ of rank $i$. Applying the lemma again, End $\mathbf{X} \cup\left\{g^{\prime}\right\}$ generates a dual operation $g^{\prime \prime}$ of the same rank with essential arity $\min \{j+2, i\}$. Repeating this argument finally leaves us with an essentially $i$-ary dual operation of rank $i$. Thus, $U_{i} \leq \overline{\operatorname{Clo}}($ End $\mathbf{X} \cup\{g\})$.

8.5.4 Theorem. If $\mathbf{A}$ is a Boolean lattice with $k$ atoms, then $[\mathrm{Clo}($ End $\mathbf{A})\rangle$ is a chain of length $k$.

Proof. We will show that $[\overline{\mathrm{Clo}}($ End $\mathbf{X})\rangle$ is a $k$-element chain. We have already established

$$
\overline{\mathrm{Clo}}(\text { End } \mathbf{X})=U_{1}<U_{2}<\ldots<U_{k}=\bar{O}_{\mathbf{X}},
$$

so it suffices to show that $U_{1}, \ldots, U_{k}$ are the only clones above $\overline{\mathrm{Clo}}($ End $\mathbf{X})$. For this, let $C \in[\operatorname{End} \mathbf{X}\rangle$. Let $i=\max \{r(g) \mid g \in C\}$. Clearly, $C \leq U_{i}$. Let $g \in C$ such that $r(g)=i$. But now, Lemma 8.5.3 yields $U_{i} \leq \overline{\mathrm{Clo}}($ End $\mathbf{X} \cup\{g\}) \leq C$. Thus, $C=U_{i}$.

However, if $\mathbf{A}$ is not a Boolean lattice, then we do not obtain a chain, not even in the simplified case of assuming that each connected component of $\mathbf{X}$ is totally ordered.

8.5.5 Example. Let $\mathbf{X}$ be the bounded partially ordered set given by the diagram below, and let $g_{1}, g_{2}$ be the two binary dual operations as defined in the table next to it.

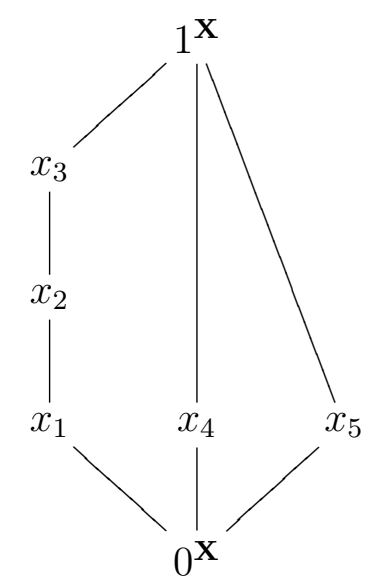

\begin{tabular}{|c||ccccc|}
\hline$x$ & $x_{1}$ & $x_{2}$ & $x_{3}$ & $x_{4}$ & $x_{5}$ \\
\hline \hline$g_{1}(x)$ & $\left\langle 1, x_{1}\right\rangle$ & $\left\langle 1, x_{1}\right\rangle$ & $\left\langle 1, x_{1}\right\rangle$ & $\left\langle 2, x_{4}\right\rangle$ & $\left\langle 2, x_{5}\right\rangle$ \\
$g_{2}(x)$ & $\left\langle 1, x_{1}\right\rangle$ & $\left\langle 1, x_{2}\right\rangle$ & $\left\langle 1, x_{3}\right\rangle$ & $\left\langle 2, x_{4}\right\rangle$ & $\left\langle 2, x_{4}\right\rangle$ \\
\hline
\end{tabular}


Set $C_{1}:=\overline{\mathrm{Clo}}\left(\right.$ End $\left.\mathbf{X} \cup\left\{g_{1}\right\}\right)$ and $C_{2}:=\overline{\mathrm{Clo}}\left(\right.$ End $\left.\mathbf{X} \cup\left\{g_{2}\right\}\right)$. The image of $g_{2}$ consists of two maximal chains. Thus, the image of every essentially at least binary operation $g \in C_{2}$ consists of at most two maximal chains. Since the image of $g_{1}$ consists of three maximal chains, this implies $C_{1} \nsubseteq C_{2}$. Similarly, the fact that the image of $g_{1}$ contains only three elements implies that each essentially at least binary operation $g \in C_{1}$ contains at most three elements. Since the image of $g_{2}$ contains four elements, this yields $C_{2} \not \subset C_{1}$.

A similar argument can be used to show that, for our running example from Figure 8.1. we also have that $[\mathrm{Clo}($ End $\mathbf{A})\rangle$ is not a chain.

\subsection{Minimal Clones}

Since the copowers of $\mathbf{X}$ are non-deformed to the degree 1, we can apply Theorem 7.5.1 (page 139), and we obtain that the minimal clones in $\mathcal{L}_{\mathbf{A}}$ are exactly the clones that are generated by nontrivial unary retractions, automorphisms of prime order and nontrivial binary idempotent operations, respectively.

8.6.1 Example. Once again, let A be the distributive lattice from Figure 8.1 (page 147). In Example 8.3.6 (page 153), we have already mentioned that there are five idempotent clones over $\mathbf{A}$. Three of them are generated by nontrivial binary operations and are therefore minimal (the other two idempotent clones are $J_{\mathbf{A}}$ and $\mathcal{I}_{\mathbf{A}}$ ). Concerning the automorphisms of prime order, we can easily see that there is only one automorphism of prime order on $\mathbf{X}$, namely the one that interchanges $x_{1}$ and $x_{2}$. Thus, with four exceptions, the minimal operations over $\mathbf{A}$ are precisely the retractions, of which we have exactly 2085 .

In the lattice of clones over finite sets, the join of all minimal clones is the full clone (in fact, it was shown in [CHK ${ }^{+}$01] that the join of two minimal clones is enough). For clones over distributive lattices, the story is quite different:

8.6.2 Proposition. The join of all minimal clones over $\mathbf{A}$ is the full clone if and only if the following two conditions are met:

(i) For each $Y \in \operatorname{Con}(\mathbf{X})$ and $\left(Y_{1}, Y_{2}\right) \in \operatorname{Spl}(Y)$ there exists $Y^{\prime} \in \operatorname{Con}(\mathbf{X}) \backslash\{Y\}$ such that $Y_{1}$ or $Y_{2}$ can be order-embedded into $Y^{\prime}$.

(ii) The join of all minimal clones contains End $\mathbf{A}$.

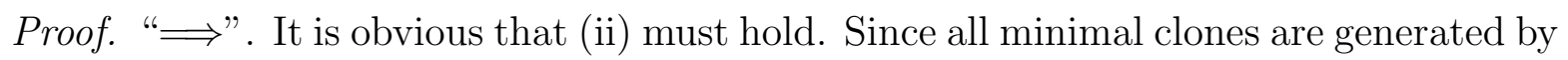
unary operations or binary idempotent operations, we must have $\operatorname{Clo}\left(\mathcal{I}_{\mathbf{A}} \cup\right.$ End $\left.\mathbf{A}\right)=O_{\mathbf{A}}$. By Theorem 8.3.20 (page 159), this is equivalent to (i).

"£". By (ii), the join of minimal clones contains End A. Moreover, it follows easily from Lemma 8.3 .1 (page 152 that $\mathcal{I}_{\mathbf{A}}^{(2)}$ generates $\mathcal{I}_{\mathbf{A}}$. Since each operation in $\mathcal{I}_{\mathbf{A}}^{(2)}$ is either minimal or trivial, we can infer that the join of all minimal clones contains $\mathcal{I}_{\mathbf{A}}$. Thus, the join of all minimal clones contains $\operatorname{Clo}\left(\mathcal{I}_{\mathbf{A}} \cup\right.$ End $\left.\mathbf{A}\right)$. But now, by (i) and Theorem 8.3.20, the latter is the full clone. 


\subsubsection{Examples.}

(i) Let A be our running example from Figure 8.1. As noted in Example 8.3.21 (page 161), condition (i) is satisfied. Moreover, it is easy to see that each unary operation over $\mathbf{X}$ is generated by the automorphisms over $\mathbf{X}$ (each of them is either trivial or has prime order), the retractions over $\mathbf{X}$ and the binary idempotent operations over $\mathbf{X}$. Hence, End $\mathbf{X}$ is contained in the join of all minimal clones. This implies (ii), and we obtain that the join of all minimal clones over $\mathbf{A}$ is $O_{\mathbf{A}}$.

(ii) Let $\mathbf{A}$ be a distributive lattices that dualizes to the poset $\mathbf{X}$ from Example 8.3.18 (page 158). As learned in Example 8.3.21, condition (i) is not satisfied. Thus, the join of all minimal clones over $\mathbf{A}$ is not $O_{\mathbf{A}}$.

(iii) Let $\mathbf{A}$ be a distributive lattice with exactly one atom or one coatom. As presented in Example 8.3.21, we have (i) if and only if $\mathbf{X}$ is totally ordered. In this case, it follows easily that each unary operation over $\mathbf{X}$ is generated by a composition of retractions and the identity on $\mathbf{X}$. Thus, the join of all minimal clones is $O_{\mathbf{A}}$ if and only if $\mathbf{A}$ is a chain.

(iv) Let $\mathbf{Y}$ be any bounded poset over which we have some nontrivial automorphisms that cannot be written as a composition of automorphisms of prime order (for instance, take the bounded poset whose automorphism group is isomorphic to $\left\langle\mathbb{Z}_{4},+, 0\right\rangle$; its existence is provided by the well-known result from G. D. BIRKHOFF [Bir46] that any finite group is isomorphic to the automorphism group of a finite bounded poset). Now, let $\mathbf{X}$ be the following bounded poset:

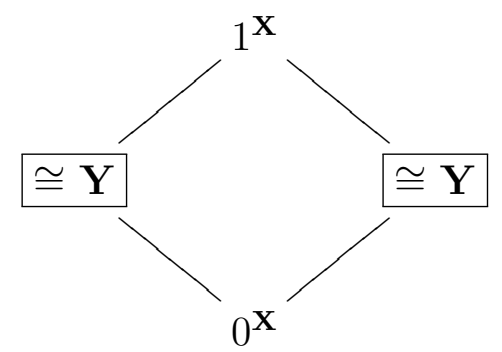

Since there exists an automorphisms over $\mathbf{Y}$ that is not generated by the automorphisms of prime order over $\mathbf{Y}$, it follows that there exists an automorphism over $\mathbf{X}$ that is not generated by the set of retractions, idempotent dual operations and automorphisms of prime order over $\mathbf{X}$. Thus, condition (ii) is not met. However, it should be noted that $\mathbf{X}$ fulfils (i) since there are only two connected components and these components are isomorphic. In fact, the sole purpose of this example was to show that there are distributive lattices for which (i) holds, but (ii) does not. That is, (ii) cannot be eliminated from the proposition.

Moreover, we can state the following corollary for Boolean lattices:

8.6.4 Corollary. If $\mathbf{A}$ is a Boolean lattice, then the join of all minimal clones is $O_{\mathbf{A}}$.

Proof. By Corollary 8.3.22 (page 162), condition (i) of Proposition 8.6.2 is met. It remains to show that (ii) is satisfied. Let $g$ be a unary operation over $\mathbf{X}$. We need to 
show that $g$ is in the join of all minimal clones over $\mathbf{X}$. Let $X=\left\{0^{\mathbf{X}}, x_{1}, \ldots, x_{k}, 1^{\mathbf{X}}\right\}$. We define $s \in \bar{O}_{\mathbf{X}}^{(k)}$ by setting

$$
s(x):=\left\{\begin{array}{cc}
0^{k \cdot \mathbf{X}} & \text { if } x=0^{\mathbf{X}} \\
\langle 1, x\rangle & \text { if } x=x_{1} \\
\vdots & \vdots \\
\langle k, x\rangle & \text { if } x=x_{k} \\
1^{k \cdot \mathbf{X}} & \text { if } x=1^{\mathbf{X}}
\end{array}\right.
$$

It is obvious that $s$ is generated by the (minimal) dual binary idempotent operations over $\mathbf{X}$. Now, for $i \in\{1, \ldots, k\}$, let $r_{i}$ be the retraction defined as follows:

$$
r_{i}(x):= \begin{cases}g(x) & \text { if } x=x_{i} \\ x & \text { otherwise. }\end{cases}
$$

We obtain $g=\left[r_{1}, \ldots, r_{k}\right] \circ s$. Hence, $g$ is generated by the minimal dual operations over $\mathbf{X}$. Thus, (ii) is satisfied and the claim follows.

\subsection{On the Size of $\mathcal{L}_{\mathrm{A}}$}

In the last section of this chapter, we will give very rough upper and lower bounds on the size of $\mathcal{L}_{\mathbf{A}}$.

We will start with an upper bound that can be derived from our observation that the essential arity of operations among $O_{\mathbf{A}}$ is sharply bounded by the length $l$ of the longest antichain of join irreducible elements in A. For such $l$, every clone of dual operations is uniquely determined by $\bar{O}_{\mathbf{X}}^{(l)} \backslash \overline{\mathcal{J}}_{\mathbf{X}}$. We have $\left|\bar{O}_{\mathbf{X}}^{(l)} \backslash \overline{\mathcal{J}}_{\mathbf{X}}\right| \leq k^{2+l k}-l$, where $k:=|\operatorname{Spec}(\mathbf{A})|=|\mathbf{X}|-2$. In general, this is a rather rough upper bound, but it is tight for the case in which $\mathbf{A}$ is a Boolean lattice. The following proposition is now immediate:

8.7.1 Proposition. $\left|\mathcal{L}_{\mathbf{A}}\right| \leq 2^{k^{2+l k}-l}$.

Note that this bound is very rough since it merely counts the number of subsets of $\left|\bar{O}_{\mathbf{X}}^{(l)} \backslash \overline{\mathcal{J}}_{\mathbf{X}}\right|$. For instance, for our running example from Figure 8.1 , we have $k=6$ and $l=4$, so the proposition yields $2^{6^{26}-4}\left(\approx 2^{10^{20}}\right)$ as an upper bound.

To obtain a lower bound on the size of $\mathcal{L}_{\mathrm{A}}$, we use the concept of independence:

8.7.2 Definition. A non-empty set of operations $F \subseteq O_{\mathbf{A}}$ is said to be independent if $f \notin \operatorname{Clo}(F \backslash\{f\})$ for all $f \in F$.

For an independent set $F$ of operations, we have $\left|\mathcal{L}_{\mathbf{A}}\right| \geq 2^{|F|}$ since $\langle\mathfrak{P}(F), \subseteq\rangle$ is orderembeddable in $\mathcal{L}_{\mathbf{A}}$. Hence, we can obtain a lower bound by finding an independent set of operations $F \subseteq O_{\mathbf{A}}$.

Let $y_{1}, \ldots, y_{l}$ be the elements of a longest antichain of join-irreducible elements in $\mathbf{A}$ and let $Y:=\left\{y_{1}, \ldots, y_{l}\right\}$. In the following we will assume $l \geq 2$ (note that $l=1$ implies that the elements of $\mathbf{A}$ are totally ordered and that every operation over $\mathbf{A}$ is essentially at most unary). We will determine a lower bound that depends on $l$ and the number of join-irreducible elements in $\mathbf{A}$. To do so, we will consider four cases: 
(a) $X=\left\{0^{\mathbf{X}}, y_{1}, \ldots, y_{l}, 1^{\mathbf{X}}\right\}$.

(b) $X \neq\left\{0^{\mathbf{X}}, y_{1}, \ldots, y_{l}, 1^{\mathbf{x}}\right\}, \forall x \in X \backslash\left\{1^{\mathbf{x}}\right\}: \exists y \in Y: x \leq y$.

(c) $X \neq\left\{0^{\mathbf{X}}, y_{1}, \ldots, y_{l}, 1^{\mathbf{X}}\right\}, \forall x \in X \backslash\left\{0^{\mathbf{X}}\right\}: \exists y \in Y: x \geq y$.

(d) None of the above three cases hold.

Note that case (a) holds if any only if $\mathbf{A}$ is a Boolean lattice.

Now, let $\prec$ be a total order on $Y$ defined by setting $y_{1} \prec y_{2} \prec \ldots \prec y_{l}$. Then, on every partition $\Phi$ of $Y$, the order $\prec$ induces a total ordering of blocks in a natural way: Let $A_{1} \in \Phi$ be the block of $\Phi$ that contains $y_{1}$, let $A_{2} \in \Phi$ be the block of $\Phi$ that contains $\min \left(Y \backslash A_{1}\right)$, let $A_{3} \in \Phi$ be the block of $\Phi$ that contains $\min \left(Y \backslash\left(A_{1} \cup A_{2}\right)\right)$, and so on. Therefore, we can always assume that for a partition $\Phi=\left\{A_{1}, \ldots, A_{m}\right\}$ of $Y$ we have $\min A_{1} \prec \min A_{2} \prec \ldots \prec \min A_{m}$.

Depending on which of the cases (a)-(d) from above applies to our situation, we define a dual operation $g_{\Phi}: \mathbf{X} \rightarrow m \cdot \mathbf{X}$ for each partition $\Phi=\left\{A_{1}, \ldots, A_{m}\right\}$ of $Y$ for which we assume $\min A_{1} \prec \ldots \prec \min A_{m}$ :

(a) $g_{\Phi}(x):= \begin{cases}0^{m \cdot \mathbf{X}} & \text { if } x=0^{\mathbf{X}} \\ 1^{m \cdot \mathbf{X}} & \text { if } x=1^{\mathbf{X}} \\ \left\langle j, y_{1}\right\rangle & \text { if } x \in A_{j}, j \in\{1, \ldots, m\} .\end{cases}$

(b) $g_{\Phi}(x):= \begin{cases}0^{m \cdot \mathbf{X}} & \text { if } x<y \text { for some } y \in Y, \\ 1^{m \cdot \mathbf{X}} & \text { if } x \in A_{m} \text { or } x=1^{\mathbf{X}}, \\ \left\langle j, y_{1}\right\rangle & \text { if } x \in A_{j}, j \in\{1, \ldots, m-1\} .\end{cases}$

(c) $g_{\Phi}(x):= \begin{cases}0^{m \cdot \mathbf{X}} & \text { if } x \in A_{m} \text { or } x=0^{\mathbf{X}} \\ 1^{m \cdot \mathbf{X}} & \text { if } x>y \text { for some } y \in Y, \\ \left\langle j, y_{1}\right\rangle & \text { if } x \in A_{j}, j \in\{1, \ldots, m-1\} .\end{cases}$

(d) $g_{\Phi}(x):= \begin{cases}0^{m \cdot \mathbf{X}} & \text { if } x<y \text { for some } y \in Y, \\ 1^{m \cdot \mathbf{X}} & \text { if } x>y \text { for some } y \in Y, \\ \left\langle j, y_{1}\right\rangle & \text { if } x \in A_{j}, j \in\{1, \ldots, m\} .\end{cases}$

It is easy to see that $g_{\Phi}$ is a well-defined mapping in each of the four cases. Moreover, by arguments similar to those from the proof of Theorem 8.2.1 (page 150), we can also infer $g_{\Phi} \in \bar{O}_{\mathbf{X}}^{(m)}$.

Furthermore, we define a unary operation $c_{\omega}: \mathbf{X} \rightarrow \mathbf{X}$ for each $\omega \in X \backslash\left\{y_{1}\right\}$ : For $\omega \in\left\{0^{\mathbf{X}}, 1^{\mathbf{X}}\right\}$, let $c_{\omega}$ be the unique morphism that maps each $x \in X \backslash\left\{0^{\mathbf{X}}, 1^{\mathbf{X}}\right\}$ to $\omega$. For $\omega \notin\left\{0^{\mathbf{X}}, 1^{\mathbf{X}}\right\}$, we define $c_{\omega}$ depending on which of the cases (a)-(d) applies to $\mathbf{X}$ :

(a) $c_{\omega}(x):= \begin{cases}0^{\mathbf{X}} & x=0^{\mathbf{X}}, \\ 1^{\mathbf{X}} & x=1^{\mathbf{X}}, \\ \omega & \text { otherwise. }\end{cases}$
(b) $c_{\omega}(x):= \begin{cases}0^{\mathbf{X}} & \text { if } x<y \text { for some } y \in Y, \\ 1^{\mathbf{X}} & \text { if } x \in\left\{y_{1}, 1^{\mathbf{X}}\right\}, \\ \omega & \text { otherwise. }\end{cases}$
(c) $c_{\omega}(x):= \begin{cases}0^{\mathbf{X}} & \text { if } x \in\left\{y_{1}, 0^{\mathbf{X}}\right\}, \\ 1^{\mathbf{X}} & \text { if } x>y \text { for some } y \in Y, \\ \omega & \text { otherwise. }\end{cases}$ 
(d) $c_{\omega}(x):= \begin{cases}0^{\mathbf{X}} & x<y \text { for some } y \in Y, \\ 1^{\mathbf{X}} & x>y \text { for some } y \in Y, \\ \omega & \text { otherwise. }\end{cases}$

Now, for $2 \leq m \leq l$, we put

$$
G_{m}:=\left\{g_{\Phi} \mid \Phi \text { is a partition of } Y \text { into } m \text { blocks }\right\} \cup\left\{c_{\omega} \mid \omega \in X \backslash\left\{y_{1}\right\}\right\} .
$$

8.7.3 Lemma. $G_{m}$ is an independent set of operations for every $2 \leq m \leq l$.

Proof. Let $\mathbf{Z}$ be the bounded poset visualized by the following diagram:

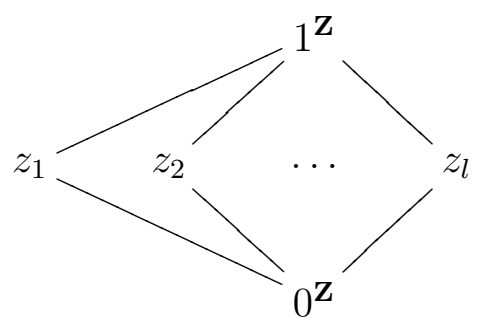

Let $\Phi=\left\{A_{1}, \ldots, A_{m}\right\}$ be a partition of $Y$ into $m$ blocks. We will define a dual relation $\sigma_{\Phi}$ of type $\mathbf{Z}$, and we will show $g_{\Phi} \notin \mathrm{Clo}\left(G_{m} \backslash\left\{g_{\Phi}\right\}\right)$ by proving that each $g \in G_{m} \backslash\left\{g_{\Phi}\right\}$ preserves $\sigma_{\Phi}$, whereas $g_{\Phi}$ does not. Define $\sigma_{\Phi} \in \overline{\mathrm{R}}_{\mathbf{X}}^{(\mathbf{Z})}$ by setting $r \in \sigma_{\Phi}$ if and only if one of the following two conditions is satisfied:

(i) $\left\{r^{-1}\left(0^{\mathbf{Z}}\right) \cap Y, r^{-1}\left(z_{1}\right), \ldots, r^{-1}\left(z_{l}\right), r^{-1}\left(1^{\mathbf{Z}}\right) \cap Y\right\} \backslash\{\emptyset\} \neq \Phi$,

(ii) $r\left(y_{1}\right) \in\left\{0^{\mathbf{Z}}, 1^{\mathbf{Z}}\right\}$.

Let us show that $G_{m} \backslash\left\{g_{\Phi}\right\} \bar{\triangleright} \sigma_{\Phi}$. For this, we need to show that $\left[r_{1}, \ldots, r_{m}\right] \circ g$ belongs to $\sigma_{\Phi}$ for all $r_{1}, \ldots, r_{m} \in \sigma_{\Phi}$ and $g \in G_{m} \backslash\left\{g_{\Phi}\right\}$. For $g=c_{\omega}$, we need to distinguish between the four cases (a)-(d) from above. In the cases (a) and (d), we have $g\left(y_{i}\right)=\omega$ for all $i \in\{1, \ldots, l\}$. Thus,

$$
\left|\left\{r^{-1}\left(0^{\mathbf{z}}\right) \cap Y, r^{-1}\left(z_{1}\right), \ldots, r^{-1}\left(z_{l}\right), r^{-1}\left(1^{\mathbf{z}}\right) \cap Y\right\} \backslash\{\emptyset\}\right| \leq 1 .
$$

Since $|\Phi|=m \geq 2$, this establishes that $\left[r_{1}, \ldots, r_{m}\right] \circ g$ satisfies condition (i). In cases (b) and (c), we have $g\left(y_{1}\right) \in\left\{0^{\mathbf{X}}, 1^{\mathbf{X}}\right\}$. Hence, $\left(\left[r_{1}, \ldots, r_{m}\right] \circ g\right)\left(y_{1}\right) \in\left\{0^{\mathbf{Z}}, 1^{\mathbf{Z}}\right\}$, that is, condition (ii) is met. Now, let us assume that $g$ can be written as $g_{\Phi^{\prime}}$ for some partition $\Phi^{\prime} \neq \Phi$ of $Y$ into $m$ parts. Since $\Phi^{\prime}$ and $\Phi$ are different partitions of $Y$ into $m$ parts, there must exist two distinct elements $x_{1}, x_{2} \in Y$ that belong to different sets in $\Phi$, but to the same set in $\Phi^{\prime}$. Hence, $g_{\Phi^{\prime}}\left(x_{1}\right)=g_{\Phi^{\prime}}\left(x_{2}\right)$, and consequently

$$
\left(\left[r_{1}, \ldots, r_{m}\right] \circ g_{\Phi^{\prime}}\right)\left(x_{1}\right)=\left(\left[r_{1}, \ldots, r_{m}\right] \circ g_{\Phi^{\prime}}\right)\left(x_{2}\right) .
$$

This establishes (i), so we have $\left[r_{1}, \ldots, r_{m}\right] \circ g_{\Phi^{\prime}} \in \sigma_{\Phi}$.

To see that $g_{\Phi}$ does not preserve $\sigma_{\Phi}$, let $r_{1}, \ldots, r_{m}: \mathbf{X} \rightarrow \mathbf{Z}$ be defined as follows:

$$
r_{i}(x)= \begin{cases}0^{\mathbf{Z}} & \text { if } x<y \text { for some } y \in Y, \\ 1^{\mathbf{Z}} & \text { if } x>y \text { for some } y \in Y \\ z_{i} & \text { if } x \in Y\end{cases}
$$


Evidently, $r_{1}, \ldots, r_{m}$ satisfy (i), so they belong to $\sigma_{\Phi}$. However, $r:=\left[r_{1}, \ldots, r_{m}\right] \circ g_{\Phi} \notin \sigma_{\Phi}$ since

$$
\left\{r^{-1}\left(0^{\mathbf{Z}}\right) \cap Y, r^{-1}\left(z_{1}\right), \ldots, r^{-1}\left(z_{l}\right), r^{-1}\left(1^{\mathbf{Z}}\right) \cap Y\right\} \backslash\{\emptyset\}=\left\{A_{1}, \ldots, A_{m}\right\}=\Phi
$$

and

$$
\left(\left[r_{1}, \ldots, r_{m}\right] \circ g_{\Phi}\right)\left(\underset{\substack{\uparrow \\ \in A_{1}}}{y_{1}}\right)=\left[r_{1}, \ldots, r_{m}\right]\left(\left\langle 1, y_{1}\right\rangle\right)=r_{1}\left(y_{1}\right)=z_{1} \notin\left\{0^{\mathbf{Z}}, 1^{\mathbf{Z}}\right\} .
$$

Thus, $g_{\Phi} \notin \overline{\operatorname{Clo}}\left(G_{m} \backslash\left\{g_{\Phi}\right\}\right)$ for all partitions $\Phi$ of $Y$ into $m$ parts.

It remains to show that $c_{\omega} \notin \overline{\mathrm{Clo}}\left(G_{m} \backslash\left\{c_{\omega}\right\}\right)$ for all $\omega \in X \backslash\left\{y_{1}\right\}$. For $\omega \notin\left\{0^{\mathbf{X}}, 1^{\mathbf{X}}\right\}$, we have $\omega \in c_{\omega}(X)$ but $\langle j, \omega\rangle \notin g(X)$ for all $k$-ary dual operations $g \in G_{m} \backslash\left\{c_{\omega}\right\}$ and $j \in\{1, \ldots, k\}$. Thus, $c_{\omega} \notin \overline{\mathrm{Clo}}\left(G_{m} \backslash\left\{c_{\omega}\right\}\right)$. For the case $\omega \in\left\{0^{\mathbf{X}}, 1^{\mathbf{X}}\right\}$, we need to distinguish between the cases (a)-(d) from above:

(a) For $\omega=0^{\mathbf{x}}$, the claim follows since $c_{\omega}$ is the only dual operation among $G_{m}$ that maps an element $x \in X \backslash\left\{0^{\mathbf{X}}\right\}$ to $0^{\mathbf{X}}$ or $0^{m \cdot \mathbf{X}}$. An analogue argument yields the claim for $\omega=1^{\mathbf{x}}$.

(b) For $\omega=0^{\mathbf{X}}$, the claim follows since $c_{\omega}$ is the only dual operation among $G_{m}$ that maps only the element $1^{\mathbf{X}}$ to $1^{\mathbf{X}}$ or $1^{m \cdot \mathbf{X}}$. For $\omega=1^{\mathbf{X}}$, the claim follows since $c_{\omega}$ is the only dual operation among $G_{m}$ that maps the set $X \backslash\left\{0^{\mathbf{x}}, y_{1}, \ldots, y_{l}, 1^{\mathbf{x}}\right\} \neq \emptyset$ not to $0^{\mathbf{X}}$ or $0^{m \cdot \mathbf{X}}$.

(c) For $\omega=0^{\mathbf{X}}$, the claim follows since $c_{\omega}$ is the only dual operation among $G_{m}$ that maps the set $X \backslash\left\{0^{\mathbf{X}}, y_{1}, \ldots, y_{l}, 1^{\mathbf{X}}\right\} \neq \emptyset$ not to $1^{\mathbf{X}}$ or $1^{m \cdot \mathbf{X}}$. For $\omega=1^{\mathbf{X}}$, the claim follows since $c_{\omega}$ is the only dual operation among $G_{m}$ that maps only the element $0^{\mathbf{X}}$ to $0^{\mathbf{X}}$ or $0^{m \cdot \mathbf{X}}$.

(d) For $\omega=0^{\mathbf{X}}$, the claim follows since $c_{\omega}$ is the only dual operation among $G_{m}$ that maps only the element $1^{\mathbf{X}}$ to $1^{\mathbf{X}}$ or $1^{m \cdot \mathbf{X}}$. An analogue argument yields the claim for $\omega=1^{\mathbf{X}}$.

This finishes the proof.

Recall that $S_{m}^{l}$ denotes the Stirling number of the second kind (see Definition 6.4.7, page 121. Set

$$
S(l):=\max \left\{S_{l}^{m} \mid 1 \leq m \leq l\right\} .
$$

Since $\left|G_{m}\right|=S_{l}^{m}+|\mathbf{X}|-1$ for every $m \in\{2, \ldots, l\}$, we have the following theorem:

8.7.4 Theorem. $\left|\mathcal{L}_{\mathbf{A}}\right| \geq 2^{S(l)+|\mathbf{X}|-1}=2^{S(l)+|\operatorname{Spec}(\mathbf{A})|+1}$.

Table 8.1 contains the first values of $2^{S(l)+|\operatorname{Spec}(\mathbf{A})|+1}$. As we can see, increasing the number of join-irreducible elements of $\mathbf{A}$ by one only doubles the lower bound, whereas increasing the length of the longest antichain makes the size of $\mathcal{L}_{\mathbf{A}}$ grow very rapidly. Let us also note that the estimate given in the theorem is pretty rough. For instance, for $\mathbf{A}$ with $|\operatorname{Spec}(\mathbf{A})|=l=3$ (that is, $\mathbf{A}$ is a nine-element Boolean lattice), we get $\left|\mathcal{L}_{\mathbf{A}}\right| \geq 128$, while the ideal of 01-homomorphisms in $\mathcal{L}_{\mathbf{A}}$ contains already 3551 clones (by Corollary 8.4.5, page 164, the ideal of 01-homomorphisms is isomorphic to the lattice of coclones over a three-element set and the size of this lattice was determined in [Maš99]). 
8. The Lattice of Clones over Distributive Lattices

\begin{tabular}{|c|c|c|c|}
\hline$l$ & $|\operatorname{Spec}(\mathbf{A})|$ & $S(l)$ & $2^{S(l)+|\operatorname{Spec}(\mathbf{A})|+1}$ \\
\hline 2 & 2 & 1 & 16 \\
2 & 3 & 1 & 32 \\
$\vdots$ & $\vdots$ & $\vdots$ & $\vdots$ \\
\hline 3 & 3 & 3 & 128 \\
3 & 4 & 3 & 256 \\
$\vdots$ & $\vdots$ & $\vdots$ & $\vdots$ \\
\hline 4 & 4 & 7 & 4096 \\
4 & 5 & 7 & 8192 \\
$\vdots$ & $\vdots$ & $\vdots$ & $\vdots$ \\
\hline 5 & 5 & 25 & $\approx 2.14 * 10^{9}$ \\
$\vdots$ & $\vdots$ & $\vdots$ & $\vdots$ \\
\hline 6 & 6 & 90 & $\approx 1.58 * 10^{29}$ \\
$\vdots$ & $\vdots$ & $\vdots$ & $\vdots$ \\
\hline 7 & 7 & 350 & {$\left[\sim 10^{107}\right]$} \\
$\vdots$ & $\vdots$ & $\vdots$ & $\vdots$ \\
\hline 8 & 8 & 1701 & {$\left[\sim 10^{514}\right]$} \\
$\vdots$ & $\vdots$ & $\vdots$ & $\vdots$ \\
\hline 9 & 9 & 7770 & {$\left[\sim 10^{2342}\right]$} \\
$\vdots$ & $\vdots$ & $\vdots$ & $\vdots$ \\
\hline & & & \\
\hline
\end{tabular}

Table 8.1.: First values of $2^{S(l)+|\operatorname{Spec}(\mathbf{A})|+1}$

8.7.5 Example. Let us take a final look at our running example from Figure 8.1 (page 147). As we have $|\operatorname{Spec}(\mathbf{A})|=6$ and $l=4$, we obtain

$$
16,384 \leq \mathcal{L}_{\mathbf{A}} \leq 2^{6^{26}-4}
$$




\section{Remarks and Conclusion}

In this thesis, we have introduced a notion of clones that generalizes Lawvere Theories and clones over sets into one concept. Based on this concept, we have introduced a general duality theory for clones. In this chapter, we will give some concluding remarks and a prospect of possible further research on the topic.

\subsection{Clones in Quasi-Categories}

One might ask if our terminology and all the results can be generalized to quasicategories.

9.1.1 Definition. Let $\mathcal{A}$ be a conglomerate consisting of:

- a conglomerate of objects,

- a class $\mathcal{A}(\mathbf{A}, \mathbf{B})$ for each pair of objects $\mathbf{A}, \mathbf{B}$, called the morphisms from $\mathbf{A}$ to $\mathbf{B}$,

- a binary operation $\circ$, called composition, on the conglomerate of all morphisms of $\mathcal{A}$ such that $u \in \mathcal{A}(\mathbf{A}, \mathbf{B})$ and $v \in \mathcal{A}(\mathbf{B}, \mathbf{C})$ implies $v \circ u \in \mathcal{A}(\mathbf{A}, \mathbf{C})$.

$\mathcal{A}$ is a quasi-category if it satisfies the axioms (i) and (ii) from the definition of a category (see Definition 1.4.1, page 16.

Note that the only difference between categories and quasi-categories is that the collection of objects is allowed to be a proper conglomerate and that $\mathcal{A}(\mathbf{A}, \mathbf{B})$ can be a proper class. A natural example for a quasi-category that is not a category is the quasi-category that contains all categories as objects and all functors between them as morphisms. A proper quasi-category can never be concretizable.

Coming back to the above question, the answer is yes. In fact, every definition and (almost) every result of this thesis can easily be generalized to clones over objects in quasi-categories. However, if we allow $O_{\mathbf{A}}$ to be a proper class, then we lose the connection between clones over $\mathbf{A}$, abstract clones and Lawvere Theories. Indeed, $O_{\mathbf{A}}$ can not be seen as an abstract clone, and it is clearly not the image of a Lawvere Theory under a product-preserving functor.

\subsection{Clones with Constants}

Clones as introduced in this thesis do not have constants (that is, nullary operations). Although this is a usual convention followed by almost every paper about clones, it 
should at least be remarked that, with some adjustments, clone theory with nullary operations can also be pursued, see for example [TS09].

The theory as presented here can also be a modified accordingly by including $\mathcal{A}\left(\mathbf{A}^{0}, \mathbf{A}\right)$ into the definition of $O_{\mathbf{A}}$. This requires $\mathbf{A}^{0} \in \mathcal{A}$, which is equivalent to $\mathcal{A}$ containing a terminal object (see Definition 1.4.9, page 18). Note that, in the light of the footnote on page 24, this would make our notion of a clone more similar to the standard definition of a Lawvere Theory. Similarly, to keep the duality theory, we had to include $x(\mathbf{X}, 0 \cdot \mathbf{X})$ into $\bar{O}_{\mathbf{X}}$. This requires $0 \cdot \mathbf{X} \in \mathcal{X}$, which is equivalent to $x$ containing an initial object (see Definition 1.4.15, page 20.

We will not elaborate the consequences of this change, but it should be noted that the theory outlined in this thesis would stay essentially the same. However, some adjustments would become necessary. For instance, it would cause some changes in the Galois theory presented in Chapter 5 since nullary operations do not preserve the empty relation.

\subsection{Further Applications for Clones over Sets}

As we have explained in Section 4.2, each clone over a finite set $A$ can be interpreted as $O_{\mathbf{A}}$ for some finite structure $\mathbf{A}=\langle A, F, H, R\rangle$ in a quasivariety $\mathcal{A}$ of structures. Then, with the help of natural dualities, we can dualize $O_{\mathbf{A}}$ to the full clone of dual operations over some finite structure $\mathbf{X}$. In Chapter 7 and 8 , we have used this technique to obtain several new results for clones over sets. However, to maximize the algebraic content, the theory of natural dualities was mainly developed for the case in which $\mathcal{A}$ is a quasivariety of algebras. As a consequence, almost all of the well-studied natural dualities are dualities for algebras. For our purposes, this is somewhat unfortunate as only finitely many clones over $A$ can be written as $O_{\mathbf{A}}$ where $\mathbf{A}$ is an algebra. For all the other clones over $A$, we would like to have well-understood natural dualities for structures different from algebras.

Only within the last few years, some authors started to generalize the theory of natural dualities to non-algebraic structures (see for example [Dav06]). Having said that, the theory is not yet as rich and as well-understood as natural dualities for algebras. Furthermore, most of the natural dualities that are known for non-algebraic structures arise from a natural duality for algebras by swapping the topology from one side to the other (see [Dav06] and [Joh10]). Since the swapped dualities were originally aimed to simplify problems on the algebraic side, they make most problems on the relational side harder when they are dualized into the algebraic sides. Thus, most of these dualities are not very useful to simplify clone-theoretic problems by dualizing the environment.

In the future, however, with the framework built in this thesis and a richer theory of natural dualities for structures, many more results about clones over sets might be obtained by treating them as clones over structures and dualizing them. 


\section{A. Appendix}

We show Theorem 8.4.6 (page 164):

Theorem 8.4.6. Assume that each connected component of $\mathbf{X}$ is a chain, and let $l$ be the cardinality of the greatest connected component (that is, $l+2$ is the length of the longest chain in $\mathbf{X})$. Define

$$
M:=\{1, \ldots, 2 l\}^{2} \backslash\{(v, w) \mid v \neq w, \max (v, w) \equiv 1(\bmod 2)\} .
$$

Then, $\left[O_{\mathbf{A}_{01}}\right\rangle \cong\langle M,(\leq, \leq)\rangle,\left[O_{\mathbf{A}_{0}}\right\rangle \cong\langle\{1, \ldots, 2 l\}, \leq\rangle$ and $\left[O_{\mathbf{A}_{1}}\right\rangle \cong\langle\{1, \ldots, 2 l\}, \leq\rangle$.

Henceforth, let $\mathbf{X}$ and $l$ be as in the theorem. Recall the definition of the algebra $\mathbf{P}=\left\langle\bar{P}_{\mathbf{X}}^{(1)},\left(\left.i d\right|_{Y}\right)_{Y \in \operatorname{Con}(\mathbf{X})}, \diamond\right\rangle$ from Corollary 8.3.15 (page 156). We start the proof with the following lemma:

A.1 Lemma. $\left[O_{\mathbf{A}_{01}}\right\rangle \cong\left\langle\left[\mathbf{P}_{\mathbf{0 1}}, \mathbf{P}\right], \leq\right\rangle$, where $\mathbf{P}_{\mathbf{0 1}}$ is the subalgebra of $\mathbf{P}$ with universe

$$
P_{01}:=\left\{p \in \bar{P}_{\mathbf{X}}^{(1)} \mid \forall x \in \operatorname{dom}(p): p(x) \notin\left\{0^{\mathbf{X}}, 1^{\mathbf{X}}\right\}\right\}
$$

and $\left[\mathbf{P}_{\mathbf{0 1}}, \mathbf{P}\right]$ denotes the set of algebras $\mathbf{H}$ with $\mathbf{P}_{\mathbf{0 1}} \leq \mathbf{H} \leq \mathbf{P}$.

Proof. By Corollary 8.3.15, $\left[O_{\mathbf{A}_{01}}\right\rangle$ is isomorphic to the lattice formed by those subalgebras of $\mathbf{P}$ of which $\left(O_{\mathbf{A}_{01}}^{(1)}\right)^{\lambda}$ is a subuniverse. Since $\left(O_{\mathbf{A}_{01}}^{(1)}\right)^{\lambda}=P_{01}$, the claim follows.

First, we will show $\left[O_{\mathbf{A}_{01}}\right\rangle \cong\langle M,(\leq, \leq)\rangle$. In the light of Lemma A.1, it suffices to show $\left\langle\left[\mathbf{P}_{\mathbf{0 1}}, \mathbf{P}\right], \leq\right\rangle \cong\langle M,(\leq, \leq)\rangle$. For $p \in \bar{P}_{\mathbf{X}}^{(1)}$, define

$$
\xi_{0}(p)= \begin{cases}1 & \text { if } 0^{\mathbf{X}} \notin p(\operatorname{dom}(p)), \\ 2\left|g(\operatorname{dom}(p)) \backslash\left\{1^{\mathbf{X}}\right\}\right|+\left|g(\operatorname{dom}(p)) \cap\left\{1^{\mathbf{X}}\right\}\right| & \text { otherwise }\end{cases}
$$

and analogously,

$$
\xi_{1}(p)= \begin{cases}1 & \text { if } 1^{\mathbf{X}} \notin p(\operatorname{dom}(p)), \\ 2\left|g(\operatorname{dom}(p)) \backslash\left\{0^{\mathbf{X}}\right\}\right|+\left|g(\operatorname{dom}(p)) \cap\left\{0^{\mathbf{x}}\right\}\right| & \text { otherwise }\end{cases}
$$

Note that $\xi_{0}(p), \xi_{1}(p) \in\{1, \ldots, 2 l\}$. Furthermore, we have $\xi_{0}(p)=1$ if and only if $0^{\mathbf{X}} \notin p(\operatorname{dom}(p))$, and similarly, $\xi_{1}(p)=1$ if and only if $1^{\mathbf{X}} \notin p(\operatorname{dom}(p))$. For $\mathbf{H} \leq \mathbf{P}$, set

$$
\begin{aligned}
& r_{0}(\mathbf{H}):=\max \left\{\xi_{0}(p) \mid p \in H\right\}, \\
& r_{1}(\mathbf{H}):=\max \left\{\xi_{1}(p) \mid p \in H\right\} .
\end{aligned}
$$


A.2 Lemma. Let $\mathbf{H} \in\left[\mathbf{P}_{\mathbf{0 1}}, \mathbf{P}\right]$. Then, $\left(r_{0}(\mathbf{H}), r_{1}(\mathbf{H})\right) \in M$.

Proof. Clearly, we have $\left(r_{0}(\mathbf{H}), r_{1}(\mathbf{H})\right) \in\{1,2, \ldots, 2 l\}^{2}$. Let $p \in H$. If $\xi_{0}(p) \neq 1$ is odd, then we have $1^{\mathbf{X}} \in p(\operatorname{dom}(p))$, and thus, $\xi_{1}(p)=\xi_{0}(p)$. Thus, whenever $r_{0}(\mathbf{H})$ is odd, we have $r_{1}(\mathbf{H}) \geq r_{0}(\mathbf{H})$. Analogously, $\xi_{1}(p) \neq 1$ being odd implies $\xi_{0}(p)=\xi_{1}(p)$. Hence, $r_{1}(\mathbf{H})$ being odd implies $r_{0}(\mathbf{H}) \geq r_{1}(\mathbf{H})$. Thus, $\max \left\{r_{0}(\mathbf{H}), r_{1}(\mathbf{H})\right\}$ is even or $r_{0}(\mathbf{H})=r_{1}(\mathbf{H})$. That is, $\left(r_{0}(\mathbf{H}), r_{1}(\mathbf{H})\right) \in M$.

A.3 Lemma. For all $(v, w) \in M$, there exists $\mathbf{H} \in\left[\mathbf{P}_{\mathbf{0 1}}, \mathbf{P}\right]$ such that

$$
\left(r_{0}(\mathbf{H}), r_{1}(\mathbf{H})\right)=(v, w) .
$$

Proof. Choose $Z \in \operatorname{Con}(\mathbf{X})$ such that $|Z|=l$. Let $Z=\left\{z_{1}, \ldots, z_{l}\right\}$, and assume $z_{1} \leq \ldots \leq z_{l}$. We define the unary partial operation $h_{v} \in \bar{P}_{\mathbf{X}}$ with $\operatorname{dom}\left(h_{v}\right)=Z$ as follows:

$$
\text { If } v=1: \quad h_{v}(x):=x .
$$

If $v$ is even:

$$
h_{v}(x):= \begin{cases}0^{\mathbf{X}} & \text { if } x \in\left\{z_{1}, \ldots, z_{l-\frac{v}{2}+1}\right\}, \\ x & \text { if } x \in\left\{z_{l-\frac{v}{2}+2}, \ldots, z_{l}\right\}\end{cases}
$$

If $v \neq 1$ and $v$ is odd: $h_{v}(x):= \begin{cases}0^{\mathbf{X}} & \text { if } x \in\left\{z_{1}, \ldots, z_{l-\frac{v-3}{2}-1}\right\}, \\ x & \text { if } x \in\left\{z_{l-\frac{v-3}{2}}, \ldots, z_{l-1}\right\}, \\ 1^{\mathbf{X}} & \text { if } x=z_{l} .\end{cases}$

Analogously, we define the unary partial operations $h_{w}$ with $\operatorname{dom}\left(h_{w}\right)=Z$ :

$$
\text { If } w=1: \quad h_{w}(x):=x .
$$

If $w$ is even:

$$
h_{w}(x):= \begin{cases}x & \text { if } x \in\left\{z_{1}, \ldots, z_{\frac{w}{2}}-1\right\} \\ 1^{\mathbf{X}} & \text { if } x \in\left\{z_{\frac{w}{2}}, \ldots, z_{l}\right\}\end{cases}
$$

If $w \neq 1$ and $w$ is odd: $h_{w}(x):= \begin{cases}0^{\mathbf{X}} & \text { if } x=x_{1}, \\ x & \text { if } x \in\left\{z_{2}, \ldots, z_{\frac{w-1}{2}}\right\}, \\ 1^{\mathbf{X}} & \text { if } x \in\left\{z_{\frac{w-1}{2}+1}, \ldots, z_{l}\right\} .\end{cases}$

We have $\xi_{0}\left(h_{v}\right)=v$ and $\xi_{1}\left(h_{w}\right)=w$. Furthermore, we have $\xi_{1}\left(h_{v}\right)=1$ if $v$ is even, and $\xi_{1}\left(h_{v}\right)=v$ if $v$ is odd. In the latter case, it follows $w \geq v$ since $(v, w) \in M$. Thus, $\xi_{1}\left(h_{v}\right) \leq w$. Similarly, we obtain $\xi_{1}\left(h_{w}\right)=w$ and $\xi_{0}\left(h_{w}\right) \leq v$. Now, let $\mathbf{H}$ be the subalgebra of $\mathbf{P}$ generated by $\left\{h_{v}, h_{w}\right\} \cup P_{01}$. Clearly, $\left(r_{0}(\mathbf{H}), r_{1}(\mathbf{H})\right) \geq(v, w)$. Furthermore, it is easy to check that $\xi_{0}\left(\left.i d\right|_{Y}\right)=\xi_{1}\left(\left.i d\right|_{Y}\right)=1$ for all $Y \in \operatorname{Con}(\mathbf{X})$ and that $\xi_{0}\left(p_{1}\right), \xi_{0}\left(p_{2}\right) \leq v$ and $\xi_{0}\left(p_{1}\right), \xi_{0}\left(p_{2}\right) \leq w$ imply $\xi_{0}\left(p_{1} \diamond p_{2}\right) \leq v$ and $\xi_{1}\left(p_{1} \diamond p_{2}\right) \leq w$ for all $p_{1}, p_{2} \in \bar{P}_{\mathbf{X}}^{(1)}$. Thus, we also have $\left(r_{0}(\mathbf{H}), r_{1}(\mathbf{H})\right) \leq(v, w)$, and the claim follows. 
A.4 Lemma. Let $\mathbf{H}_{1}, \mathbf{H}_{2} \in\left[\mathbf{P}_{\mathbf{0 1}}, \mathbf{P}\right]$. Then $\mathbf{H}_{1} \leq \mathbf{H}_{2}$ if and only if $r_{0}\left(\mathbf{H}_{1}\right) \leq r_{0}\left(\mathbf{H}_{2}\right)$ and $r_{1}\left(\mathbf{H}_{1}\right) \leq r_{1}\left(\mathbf{H}_{2}\right)$.

Proof. We can prove the claim by showing that, for $\mathbf{H} \in\left[\mathbf{P}_{\mathbf{0 1}}, \mathbf{P}\right]$ and $p_{1}, p_{2} \in H$, we have $p \in H$ for all $p \in \bar{P}_{\mathbf{X}}^{(1)}$ with $\xi_{0}(p) \leq \max \left\{\xi_{0}\left(p_{1}\right), \xi_{0}\left(p_{2}\right)\right\}$ and $\xi_{1}(p) \leq \max \left\{\xi_{1}\left(p_{1}\right), \xi_{1}\left(p_{2}\right)\right\}$. For notational simplicity, let $(v, w):=\left(\max \left\{\xi_{0}\left(p_{1}\right), \xi_{0}\left(p_{2}\right)\right\}, \max \left\{\xi_{1}\left(p_{1}\right), \xi_{1}\left(p_{2}\right)\right\}\right)$. Without loss of generality, we can assume $v \geq w$ (note that this implies that $v$ is even or $v=w)$. Let $p \in \bar{P}_{\mathbf{X}}^{(1)}$ such that $\xi_{0}(p) \leq v$ and $\xi_{1}(p) \leq w$. We will finish the proof by showing that $p$ is in the smallest subalgebra $\mathbf{H} \leq \mathbf{P}$ that contains $\left\{p_{1}, p_{2}\right\} \cup P_{01}$. If $v=1$, we have $w=1$, and it follows that $p \in P_{01}$. Hence, we can assume $v>1$. Note that $\operatorname{dom}(p)$ is a chain. Let this chain be $x_{1}<\ldots<x_{k}$. The image of $p$ must be contained in $Z \cup\left\{0^{\mathbf{X}}, 1^{\mathbf{X}}\right\}$ for some $Z \in \operatorname{Con}(\mathbf{X})$. Thus, there exists an integer $s \leq k$ and $z_{1}, \ldots, z_{s} \in Z$ such that $z_{1}<\ldots<z_{s}$ and $\left\{z_{1}, \ldots, z_{s}\right\}=p(\operatorname{dom}(p)) \backslash\left\{0^{\mathbf{X}}, 1^{\mathbf{X}}\right\}$. Now, there exist $i_{0}, \ldots, i_{s} \geq 1$ and $i_{s+1} \geq 0$ with $i_{0}+\ldots+i_{s+1}=k$ such that we can write $p$ as follows:

$$
p(x)=\left\{\begin{array}{cc}
0^{\mathbf{X}} & \text { if } x \in\left\{x_{1}, \ldots, x_{i_{0}}\right\} \\
z_{1} & \text { if } x \in\left\{x_{i_{0}+1}, \ldots, x_{i_{0}+i_{1}}\right\} \\
\vdots & \vdots \\
z_{s} & \text { if } x \in\left\{x_{\sum_{n=0}^{s-1} i_{n}+1}, \ldots, x_{\sum_{n=0}^{s} i_{n}}\right\}, \\
1^{\mathbf{X}} & \text { if } x \in\left\{x_{\sum_{n=0}^{s} i_{n}+1}, \ldots, x_{\sum_{n=0}^{s+1} i_{n}}\right\} .
\end{array}\right.
$$

Note that $i_{s+1} \geq 1$ if and only if $\xi_{0}(p)$ is odd. Now let $h \in\left\{p_{1}, p_{2}\right\}$ such that $\xi_{0}(h)=v$. Again, $\operatorname{dom}(h)$ is a chain. Let this chain be $y_{1}<\ldots<y_{k^{\prime}}$. The image of $h$ must be contained in $Z^{\prime} \cup\left\{0^{\mathbf{X}}, 1^{\mathbf{X}}\right\}$ for some $Z^{\prime} \in \operatorname{Con}(\mathbf{X})$. Thus, there exists an integer $t \leq k^{\prime}$ and $z_{1}^{\prime}, \ldots, z_{t}^{\prime} \in Z$ such that $z_{1}^{\prime}<\ldots<z_{t}^{\prime}$ and $\left\{z_{1}^{\prime}, \ldots, z_{t}^{\prime}\right\}=h(\operatorname{dom}(h)) \backslash\left\{0^{\mathbf{X}}, 1^{\mathbf{X}}\right\}$. Since $\xi_{0}(h)=v \geq \xi_{0}(p)$ and $\xi_{1}(h) \leq w \leq v$, we have $t \geq s$. Again, there exist $j_{0}, \ldots, j_{t+1} \geq 1$ and $j_{t} \geq 0$ with $j_{0}+\ldots+j_{t+1}=k^{\prime}$ such that we can write $h$ as follows:

$$
h(y)=\left\{\begin{array}{cc}
0^{\mathbf{X}} & \text { if } y \in\left\{y_{1}, \ldots, y_{j_{0}}\right\}, \\
z_{1}^{\prime} & \text { if } y \in\left\{y_{j_{0}+1}, \ldots, y_{j_{0}+j_{1}}\right\}, \\
\vdots & \vdots \\
z_{t}^{\prime} & \text { if } y \in\left\{y_{\sum_{n=0}^{t-1} j_{n}+1}, \ldots, y_{\sum_{n=0}^{t} j_{n}}\right\}, \\
1^{\mathbf{X}} & \text { if } y \in\left\{y_{\sum_{n=0}^{t} j_{n}+1}, \ldots, y_{\sum_{n=0}^{t+1} j_{n}}\right\} .
\end{array}\right.
$$

This time, we have $j_{t+1}=0$ if and only if $v$ is odd. We define a partial operation $h^{\prime}: \operatorname{dom}(p) \rightarrow \operatorname{dom}(h)$ by setting

$$
h^{\prime}(x):= \begin{cases}y_{j_{0}} & \text { if } x \in\left\{x_{1}, \ldots, x_{i_{0}}\right\}, \\ y_{j_{0}+j_{1}} & \text { if } x \in\left\{x_{i_{0}+1}, \ldots, x_{i_{0}+i_{1}}\right\}, \\ \vdots & \quad \vdots \\ y_{\sum_{n=0}^{s} j_{n}} & \text { if } x \in\left\{x_{\sum_{n=0}^{s-1} i_{n}+1}, \ldots, x_{\sum_{n=0}^{s} i_{n}}\right\}, \\ y_{\sum_{n=0}^{t+1} j_{n}} & \text { if } x \in\left\{x_{\sum_{n=0}^{s} i_{n}+1}, \ldots, x_{\sum_{n=0}^{s+1} i_{n}}\right\} .\end{cases}
$$

We will show that $h^{\prime}$ is well-defined. By $t \geq s$, we have

$$
y_{j_{0}}, \ldots, y_{\sum_{n=0}^{s} j_{n}}, y_{\sum_{n=0}^{t+1} j_{n}} \in\left\{y_{1}, \ldots, y_{k^{\prime}}\right\}=\operatorname{dom}(h) .
$$


Therefore, $h^{\prime}$ is a well-defined mapping on $\operatorname{dom}(p)$, and it is obvious that it also preserves the order relation. Thus, $h^{\prime}$ is a partial operation. Set $p^{\prime}:=h \diamond h^{\prime}$. Since $h \in\left\{p_{1}, p_{2}\right\}$ and $h^{\prime} \in P_{01}$, we have $p^{\prime} \in H$. Since $j_{t+1}=0$ if and only if $v$ is odd, $p^{\prime}$ is given as follows:

$$
\begin{aligned}
& \text { If } v \text { is odd: } p^{\prime}(x)=\left\{\begin{array}{cc}
0^{\mathbf{X}} & \text { if } x \in\left\{x_{1}, \ldots, x_{i_{0}}\right\}, \\
z_{1}^{\prime} & \text { if } x \in\left\{x_{i_{0}+1}, \ldots, x_{i_{0}+i_{1}}\right\}, \\
\vdots & \vdots \\
z_{s}^{\prime} & \text { if } x \in\left\{x_{\sum_{n=0}^{s-1} i_{n}+1}, \ldots, x_{\sum_{n=0}^{s} i_{n}}\right\}, \\
1^{\mathbf{X}} & \text { if } x \in\left\{x_{\sum_{n=0}^{s} i_{n}+1}, \ldots, x_{\sum_{n=0}^{s+1} i_{n}}\right\} .
\end{array}\right. \\
& \text { If } v \text { is even: } p^{\prime}(x)=\left\{\begin{array}{cc}
0^{\mathbf{X}} & \text { if } x \in\left\{x_{1}, \ldots, x_{i_{0}}\right\}, \\
z_{1}^{\prime} & \text { if } x \in\left\{x_{i_{0}+1}, \ldots, x_{i_{0}+i_{1}}\right\}, \\
\vdots & \vdots \\
z_{s}^{\prime} & \text { if } x \in\left\{x_{\sum_{n=0}^{s-1} i_{n}+1}, \ldots, x_{\sum_{n=0}^{s} i_{n}}\right\}, \\
z_{t}^{\prime} & \text { if } x \in\left\{x_{\sum_{n=0}^{s} i_{n}+1}, \ldots, x_{\sum_{n=0}^{s+1} i_{n}}\right\} .
\end{array}\right.
\end{aligned}
$$

If $v$ is odd, it is obvious that there exists an operation $p^{\prime \prime} \in P_{01}$ such that $p^{\prime \prime} \diamond p^{\prime}=p$, and we have shown the claim for this case. Thus, from now on, we can assume that $v$ is even.

If $\xi_{0}(p)$ is even, then $i_{s+1}=0$, and consequently, $p$ does not map any element to $\mathbf{A}$, and $p^{\prime}$ does not map any element to $z_{t}^{\prime}$. But now, there exists again an operation $p^{\prime \prime} \in P_{01}$ such that $p^{\prime \prime} \diamond p^{\prime}=p$. It remains the case in which $\xi_{0}(p)$ is odd. If $\xi_{1}(p)=1$, it follows $i_{s+1}=0$, and once again, there exists $p^{\prime \prime} \in P_{01}$ such that $p^{\prime \prime} \diamond p^{\prime}=p$. Finally, $\xi_{1}(p)>1$ implies $\xi_{1}(p) \geq \xi_{0}(p)$ and $w \geq \xi_{1}(p)>1$. Now, let $h_{2} \in\left\{p_{1}, p_{2}\right\}$ such that $\xi_{1}\left(h_{2}\right)=w$. Let $y_{1}<\ldots<y_{k_{2}}$ be the chain that is $\operatorname{dom}\left(h_{2}\right)$. The image of $h_{2}$ must be contained in $Z^{\prime \prime} \cup\left\{0^{\mathbf{X}}, 1^{\mathbf{X}}\right\}$ for some $Z^{\prime \prime} \in \operatorname{Con}(\mathbf{X})$. Thus, there exist $z_{1}^{\prime \prime}, \ldots, z_{r}^{\prime \prime} \in Z$ such that $z_{1}^{\prime \prime}<\ldots<z_{r}^{\prime \prime}$ and $\left\{z_{1}^{\prime \prime}, \ldots, z_{r}^{\prime \prime}\right\}=h_{2}\left(\operatorname{dom}\left(h_{2}\right)\right) \backslash\left\{0^{\mathbf{X}}, 1^{\mathbf{X}}\right\}$. Once again, there exist $j_{0}, \ldots, j_{r+1} \in\left\{0, \ldots, k_{2}\right\}$ with $j_{0}+\ldots+j_{r+1}=k_{2}$ such that we can write $h_{2}$ as follows:

$$
h_{2}(y)=\left\{\begin{array}{cc}
0^{\mathbf{X}} & \text { if } y \in\left\{y_{1}, \ldots, y_{j_{0}}\right\}, \\
z_{1}^{\prime \prime} & \text { if } y \in\left\{y_{j_{0}+1}, \ldots, y_{j_{0}+j_{1}}\right\}, \\
\vdots & \vdots \\
z_{r}^{\prime \prime} & \text { if } y \in\left\{y_{\sum_{n=0}^{r-1} j_{n}+1}, \ldots, y_{\sum_{n=0}^{r} j_{n}}\right\}, \\
1^{\mathbf{X}} & \text { if } y \in\left\{y_{\sum_{n=0}^{r} j_{n}+1}, \ldots, y_{\sum_{n=0}^{r+1} j_{n}}\right\} .
\end{array}\right.
$$

Since $\xi_{1}\left(h_{2}\right)=w \geq \xi_{1}(p) \geq \xi_{0}(p)$ implies $r \geq s$, we can define the following partial operation $h_{2}^{\prime}: Z^{\prime} \rightarrow \operatorname{dom}\left(h_{2}\right)$ (recall that $Z^{\prime}$ denotes the connected component of $\mathbf{X}$ that contains $\left.\left\{z_{1}^{\prime}, \ldots, z_{s}^{\prime}\right\}\right)$ :

$$
h_{2}^{\prime}(x):=\left\{\begin{array}{lc}
y_{j_{0}+j_{1}} & \text { if } x=z_{1}^{\prime} \\
y_{j_{0}+j_{1}+j_{2}} & \text { if } x=z_{2}^{\prime} \\
\vdots & \vdots \\
y_{\sum_{n=1}^{s} j_{n}} & \text { if } x=z_{s}^{\prime} \\
y_{\sum_{n=1}^{r+1} j_{n}} & \text { if } x>z_{s}^{\prime} .
\end{array}\right.
$$


Set $p^{\prime \prime}:=h_{2}^{\prime} \diamond\left(h^{\prime} \diamond p^{\prime}\right)$. Since $h_{2} \in\left\{p_{1}, p_{2}\right\}$ and $h_{2}^{\prime} \in P_{01}$, we have $p^{\prime \prime} \in H$. Note that $p^{\prime \prime}$ is given as follows:

$$
p^{\prime \prime}(x)=\left\{\begin{array}{cc}
0^{\mathbf{X}} & \text { if } x \in\left\{x_{1}, \ldots, x_{i_{0}}\right\}, \\
z_{1}^{\prime \prime} & \text { if } x \in\left\{x_{i_{0}+1}, \ldots, x_{i_{0}+i_{1}}\right\}, \\
\vdots & \vdots \\
z_{s}^{\prime \prime} & \text { if } x \in\left\{x_{\sum_{n=0}^{s-1} i_{n}+1}, \ldots, x_{\sum_{n=0}^{s} i_{n}}\right\}, \\
1^{\mathbf{X}} & \text { if } x \in\left\{x_{\sum_{n=0}^{s} i_{n}+1}, \ldots, x_{\sum_{n=0}^{s+1} i_{n}}\right\} .
\end{array}\right.
$$

Now, it is obvious that there exists $p^{\prime \prime \prime} \in P_{01}$ such that $p^{\prime \prime \prime} \diamond p^{\prime \prime}=p$. We have finally reached $p \in H$ for all possible cases.

Combining the last three lemmas yields $\left[O_{\mathbf{A}_{01}}\right\rangle \cong\left\langle\left[\mathbf{P}_{\mathbf{0 1}}, \mathbf{P}\right], \leq\right\rangle \cong\langle M,(\leq, \leq)\rangle$. Together with the next lemma, the proof of Theorem 8.4.6 is complete.

A.5 Lemma. Let

$$
\begin{aligned}
& \mathbf{P}_{\mathbf{0}}:=\left\langle\left(O_{\mathbf{A}_{0}}^{(1)}\right)^{\lambda},\left(\left.i d\right|_{Y}\right)_{Y \in \operatorname{Con}(\mathbf{X})}, \diamond\right\rangle, \\
& \mathbf{P}_{\mathbf{1}}:=\left\langle\left(O_{\mathbf{A}_{1}}^{(1)}\right)^{\lambda},\left(\left.i d\right|_{Y}\right)_{Y \in \operatorname{Con}(\mathbf{X})}, \diamond\right\rangle .
\end{aligned}
$$

Then, $\mathbf{P}_{\mathbf{0}}, \mathbf{P}_{\mathbf{1}} \in\left[\mathbf{P}_{\mathbf{0 1}}, \mathbf{P}\right]$ and

$$
\begin{aligned}
& \left(r_{0}\left(\mathbf{P}_{\mathbf{0}}\right), r_{1}\left(\mathbf{P}_{\mathbf{0}}\right)\right)=(2 l, 1), \\
& \left(r_{0}\left(\mathbf{P}_{\mathbf{1}}\right), r_{1}\left(\mathbf{P}_{\mathbf{1}}\right)\right)=(1,2 l) .
\end{aligned}
$$

Proof. $\mathbf{P}_{\mathbf{0}}, \mathbf{P}_{\mathbf{1}} \in\left[\mathbf{P}_{\mathbf{0 1}}, \mathbf{P}\right]$ follows from $O_{\mathbf{A}_{01}} \leq O_{\mathbf{A}_{0}}$ and $O_{\mathbf{A}_{01}} \leq O_{\mathbf{A}_{1}}$. Furthermore, $p \in\left(O_{\mathbf{A}_{0}}^{(1)}\right)^{\lambda}$ yields $1^{\mathbf{X}} \notin p(\operatorname{dom}(p))$, whence it follows $r_{1}\left(\mathbf{P}_{\mathbf{0}}\right)=1$. Similarly, we obtain $r_{0}\left(\mathbf{P}_{\mathbf{1}}\right)=1$. It remains to show that there exist $p_{0} \in\left(O_{\mathbf{A}_{0}}^{(1)}\right)^{\lambda}$ and $p_{1} \in\left(O_{\mathbf{A}_{1}}^{(1)}\right)^{\lambda}$ such that $\xi_{0}\left(p_{0}\right)=2 l$ and $\xi_{1}\left(p_{1}\right)=2 l$. For this, choose $Z \in \operatorname{Con}(\mathbf{X})$ such that $|Z|=l$. Let $Z=\left\{x_{1}, \ldots, x_{l}\right\}$ with $x_{1} \leq \ldots \leq x_{l}$. Define $g_{0}, g_{1} \in \bar{O}_{\mathbf{X}}^{(1)}$ as follows:

$$
g_{0}(x):=\left\{\begin{array}{ll}
0^{\mathbf{X}} & \text { if } x \in\left\{0^{\mathbf{X}}, x_{1}\right\}, \\
x & \text { otherwise. }
\end{array} \quad g_{1}(x):= \begin{cases}1^{\mathbf{X}} & \text { if } x \in\left\{1^{\mathbf{X}}, x_{l}\right\} \\
x & \text { otherwise }\end{cases}\right.
$$

It is easy to see that we have $g_{0} \in O_{\mathbf{A}_{0}}^{(1)}$ and $g_{1} \in O_{\mathbf{A}_{1}}^{(1)}$. The claim follows for $p_{0}:=\left.g_{0}\right|_{Z}$ and $p_{1}:=\left.g_{1}\right|_{z}$. 


\section{Nomenclature}

$(-)^{\partial} \quad$ The clone duality $(-)^{\partial}: O_{\mathbf{A}} \rightarrow \bar{O}_{\mathbf{X}}$, page 39

$(-)^{c} \quad$ The complement-operation, page 54

$(-)^{\bar{\partial}} \quad$ The clone duality $(-)^{\bar{\partial}}: \bar{O}_{\mathbf{X}} \rightarrow O_{\mathbf{A}}$, page 41

$(-)^{\partial^{*}} \quad$ The clone duality $(-)^{\partial^{*}}: R_{\mathbf{A}}^{\mathbb{T}} \rightarrow \bar{R}_{\mathbf{X}}^{\mathbb{T}}$ for relations, page 99

$(-)^{o p} \quad$ The morphism-reversing functor from a category to its opposite, page 21

$[B]_{\text {even }} \quad$ The set of elements in the Boolean group for which an even number of elements among $B$ is needed to write them as a sum of base elements, page 63

$[B]_{\text {odd }} \quad$ The set of elements in the Boolean group for which an odd number of elements among $B$ is needed to write them as a sum of base elements, page 63

$[C\rangle \quad$ The order-filter generated by $C$, page 149

$\left[g_{1}, \ldots, g_{n}\right] \quad$ The cotupling of $g_{1}, \ldots, g_{n}$, page 17

$\mathbf{A} \cong \mathbf{B} \quad$ Indicates that there exists an isomorphism from $\mathbf{A}$ to $\mathbf{B}$, page 15

$\mathbf{A} \leqslant \mathbf{B} \quad$ Indicates that there exists an epimorphism from $\mathbf{B}$ to $\mathbf{A}$, page 15

$\mathbf{A}<\mathbf{B} \quad$ Indicates that there exists an monomorphism from $\mathbf{A}$ to $\mathbf{B}$, page 15

$\mathbf{A}^{n} \quad$ The $n$-th power of the object $\mathbf{A}$, page 16

$\operatorname{At}(\mathbf{A}) \quad$ The set of atoms of the lattice or poset $\mathbf{A}$, page 51

$\operatorname{At}(a) \quad$ The set of atoms below $a$, page 51

$\mathbf{B}^{\mathbf{A}} \quad$ The set of morphisms from $\mathbf{A}$ to $\mathbf{B}$ in the underlying category, page 14

$\bigwedge_{\left(\varphi_{i}\right)_{i \in I}}^{\varphi}\left(\sigma_{i}\right)_{i \in I}$ The general superposition of relations, page 78

$\mathcal{A}(\mathbf{A}, \mathbf{B}) \quad$ The set of morphisms from $\mathbf{A}$ to $\mathbf{B}$ in $\mathcal{A}$, page 14

$\mathcal{A}^{o p} \quad$ The opposite category of $\mathcal{A}$, page 21

$\mathcal{A}_{\text {fin }} \quad$ The full subcategory of finite objects among $\mathcal{A}$, page 15 
$\chi_{\mathbf{A}}(a)$

o

$\operatorname{Clo}(F)$

C-Loc

$\mathrm{Clo}^{\mathbb{T}}(R)$

$\operatorname{Con}(\mathbf{Y})$

$\overline{\mathrm{Clo}}(G)$

$\diamond$

$\operatorname{dInc}(\mathbf{X})$

$\overline{\operatorname{Inv}}_{\mathbf{X}}^{\mathbb{T}} G$

$\overline{\operatorname{InV}}_{\mathbf{X}}^{(\mathbf{Y})} G$

$\overline{\mathrm{LOC}}^{\mathbb{T}} \overline{\mathcal{L}}_{\mathrm{X}}^{* \mathbb{T}}$

$\overline{\operatorname{Loc}}^{\mathbb{T}} \overline{\mathcal{L}}_{\mathbf{X}}$

$\overline{\operatorname{Loc}}^{\mathbb{T}} G$

$\overline{\mathrm{LOC}}^{\mathbb{T}} R$

$\operatorname{dom}(p)$

$\bullet$

$\overline{\mathrm{Pol}}_{\mathbf{x}} R$

$\overline{\mathrm{s}-\mathrm{LOC}}^{\mathbb{T}}$

$\overline{\mathrm{s}-\mathrm{Loc}}^{\mathrm{T}}$

$\overline{\mathbf{Z}-\mathrm{LOC}}$

End A

$\eta_{n}$
The (unique) subset $B^{\prime} \subseteq B_{\mathbf{A}}$ such that $a=\sum B^{\prime}$, page 63

The composition of morphisms ( $f \circ g$ means $f$ after $g$ ), page 14

The least clone of operations that contains $F$, page 28

A local closure operator for sets of operations, page 81

The least clone of relations of the typeclass $\mathbb{T}$ that contains $R$, page 79

The set of connected components of the bounded poset $\mathbf{Y}$, page 146

The least clone of dual operations that contains $G$, page 34

A binary operation on partial operations, page 155

The set of increasing subsets of $X$ that contain exactly one of the two elements $x, \bar{x}$ for each $x \in X$, page 54

The class of dual relations of the typeclass $\mathbb{T}$ invariant for each $g \in G$, page 93

The class of all dual relations of type $\mathbf{Y}$ preserved by each $g \in G$, page 94

The lattice of locally closed clones of dual relations of the typeclass $\mathbb{T}$ on $\mathbf{X}$, page 97

The lattice of locally closed clones of dual operations over $\mathbf{X}$, page 97

$\bigcap_{\mathbf{Z} \in \mathbb{T}} \overline{\mathbf{Z} \text {-Loc }} G$, page 94

$\bigcap_{s \in \mathbb{N}_{+}} \overline{\mathrm{s}-\mathrm{LOC}}^{\mathbb{T}} R$, page 94

The domain of the (partial) operation $p$, page 9

The disjoint union, page 17

The set of dual operations preserving each $\sigma \in R$, page 93

A local closure operator for classes of dual relations, page 94

A local closure operator for sets of dual operations (in concrete categories), page 97

A local closure operator for sets of dual operations, page 94

The set of endomorphisms on the structure A, page 156

A special isomorphism from $D\left(\mathbf{A}^{n}\right)$ to $n \cdot \mathbf{X}$, page 38 
$\left\langle\left\langle f_{1}, \ldots, f_{n}\right\rangle\right\rangle \quad$ The expanded tupling of $f_{1}, \ldots, f_{n}$, page 17

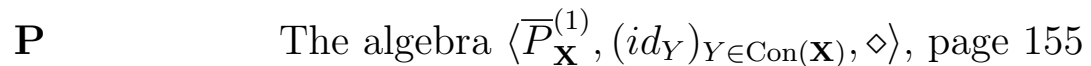

$\Gamma_{F}(\sigma) \quad$ The least relation that is preserved by $F$ and contains $\sigma$, page 77

$\operatorname{Inc}(\mathbf{X}) \quad$ The set of non-empty, proper, increasing subsets of $X$, page 46

Inv $F \quad$ The set of relations invariant for each $f \in F$ (in the classical sense), page 12

$\operatorname{Inv}^{(n)} F \quad$ The set of all $n$-ary relations among $\operatorname{Inv} F$, page 12

$\operatorname{Inv}_{\mathbf{A}}^{\mathbb{T}} F \quad$ The class of relations of the typeclass $\mathbb{T}$ invariant for each $f \in F$, page 79

$\operatorname{Inv}_{\mathbf{A}}^{(\mathbf{B})} F \quad$ The class of all relations of type $\mathbf{B}$ preserved by each $f \in F$, page 79

$\iota_{i}^{n} \quad$ The $i$-th injection morphism associated with the $n$-th copower of an object, page 17

$\mathcal{L}_{\mathbf{A}} \quad$ The lattice of clones of operations over the object $\mathbf{A}$, page 28

$\langle C] \quad$ The order-ideal generated by $C$, page 28

$\left\langle f_{1}, \ldots, f_{n}\right\rangle \quad$ The tupling of $f_{1}, \ldots, f_{n}$, page 16

$\llbracket g_{1}, \ldots, g_{n} \rrbracket \quad$ The expanded cotupling of $g_{1}, \ldots, g_{n}$, page 18

$\operatorname{Loc}^{\mathbb{T}} \mathcal{L}_{\mathbf{A}} \quad$ The lattice of locally closed clones of operations over $\mathbf{A}$, page 89

$\mathrm{LOC}^{\mathbb{T}} \mathcal{L}_{\mathbf{A}}^{* \mathbb{T}} \quad$ The lattice of locally closed clones of relations of the typeclass $\mathbb{T}$ on $\mathbf{A}$, page 89

$\operatorname{Loc}^{\mathbb{T}} F \quad \bigcap_{\mathbf{C} \in \mathbb{T}} \mathbf{C}$-Loc $F$, page 81

$\mathrm{LOC}^{\mathbb{T}} R \quad \bigcap_{s \in \mathbb{N}_{+}}{ }^{\text {s-LOC }}{ }^{\mathbb{T}} R$, page 81

$\mathcal{L}_{\mathbf{A}}^{* \mathbb{T}} \quad$ The lattice of clones of relations of the typeclass $\mathbb{T}$ on $\mathbf{A}$, page 79

$\overline{\mathcal{L}}_{\mathbf{X}}^{* \mathbb{T}} \quad$ The lattice of clones of dual relations of the typeclass $\mathbb{T}$ on $\mathbf{X}$, page 93

$\overline{\mathcal{L}}_{\mathbf{X}} \quad$ The lattice of clones of dual operations over the object $\mathbf{X}$, page 34

M A finite structure, page 22

$\mathbb{H}(\mathcal{K}) \quad$ The class of homomorphic images of structures from $\mathcal{K}$, page 10

$\operatorname{ISP}(\mathcal{K}) \quad$ The quasivariety generated by $\mathcal{K}$, page 10

$\mathbb{I}(\mathcal{K}) \quad$ The class of isomorphic images of structures from $\mathcal{K}$, page 10 
$\mathbb{P}(\mathcal{K}) \quad$ The class of products of structures from $\mathcal{K}$, page 10

$\mathbb{P}^{+}(\mathcal{K}) \quad$ The class of products of structures from $\mathcal{K}$ over non-empty index sets, page 10

$\mathbb{S}(\mathcal{K}) \quad$ The class of non-empty substructures of structures from $\mathcal{K}$, page 10

$\mathbb{S}^{0}(\mathcal{K}) \quad$ The class of (possibly empty) substructures of structures from $\mathcal{K}$, page 10

$\mathcal{I}_{\mathbf{A}} \quad$ The clone of all idempotent operations over $\mathbf{A}$, page 131

$\mathbb{N}_{+} \quad$ The set of natural numbers not including 0 , page 9

$O_{\mathbf{A}} \quad$ The set of finitary (non-nullary) operations over the object A, page 26

$O_{\mathbf{A}}^{(n)} \quad$ The set of $n$-ary operations over the object $\mathbf{A}$, page 26

$\bar{\bigwedge}_{\left(\varphi_{i}\right)_{i \in I}}^{\varphi}\left(\sigma_{i}\right)_{i \in I}$ The general superposition of dual relations, page 93

$\bar{\eta}_{n} \quad$ A special isomorphism from $\mathbf{A}^{n}$ to $E(n \cdot \mathbf{X})$, page 41

$\bar{\Gamma}_{G}(\sigma) \quad$ The least dual relation that is preserved by $G$ and contains $\sigma$, page 93

$\overline{\mathcal{I}}_{\mathbf{X}} \quad$ The clone of all dual idempotent operations over $\mathbf{X}$, page 117

$\overline{\mathrm{R}}_{\mathbf{X}}^{(k)} \quad$ The class of $k$-ary dual relations on $\mathbf{X}$ (in concrete categories), page 97

$\bar{J}_{\mathbf{X}} \quad$ The clone of all injection morphisms over $\mathbf{X}$, page 34

$\overline{\mathrm{Pol}}_{\mathbf{X}}^{(n)} R \quad$ The set of all $n$-ary dual operations among $\mathrm{Pol}_{\mathbf{A}} R$, page 94

$\bar{O}_{\mathbf{X}} \quad$ The set of finitary (non-nullary) dual operations over the object $\mathbf{X}$, page 33

$\bar{O}_{\mathbf{X}}^{(n)} \quad$ The set of $n$-ary dual operations over the object $\mathbf{X}$, page 33

$\operatorname{Part}(X) \quad$ The set of partitions of the set $X$, page 121

$\pi_{i}^{n} \quad$ The $i$-th projection morphism of the $n$-th power of an object, page 16

Pol $R$ The set of operations preserving each $\sigma \in R$ (in the classical sense), page 12

$\mathrm{Pol}^{(n)} R \quad$ The set of all $n$-ary operations among Pol $R$, page 12

$\mathrm{Pol}_{\mathbf{A}}^{(n)} R \quad$ The set of all $n$-ary operations among $\mathrm{Pol}_{\mathbf{A}} R$, page 79

$\operatorname{Pol}_{\mathbf{A}} R \quad$ The set of operations preserving each $\sigma \in R$, page 79

$\mathfrak{P}(A) \quad$ The power set/class of the set/class $A$, page 48 
$\preccurlyeq \quad$ An order relation on the set of partitions, page 119

$\bar{P}_{\mathbf{X}} \quad$ The set of partial dual operations over $\mathbf{X}$ whose domain is a connected component of $\mathbf{X}$, page 153

$\mathrm{R}_{\mathbf{A}}^{(k)}$

The class of $k$-ary relations on $\mathbf{A}$ (in concrete categories), page 91

$\mathrm{R}_{\mathbf{A}}^{\mathbb{T}} \quad \bigcup_{\mathbf{B} \in \mathbb{T}} R_{\mathbf{A}}^{(\mathbf{B})}$, the class of relations of the typeclass $\mathbb{T}$ on $\mathbf{A}$, page 78

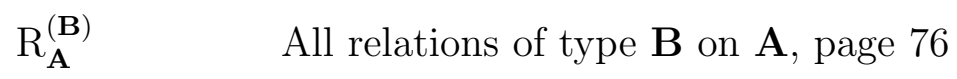

$\operatorname{Ref} \operatorname{Tra}(X) \quad$ The set of reflexive, transitive relations on the set $X$, page 155

$\overline{\mathrm{R}}_{\mathbf{X}}^{\mathbb{T}}$

$\bigcup_{\mathbf{Y} \in \mathbb{T}} \overline{\mathrm{R}}_{\mathbf{X}}^{(\mathbf{Y})}$, the class of dual relations of the typeclass $\mathbb{T}$ on $\mathbf{X}$, page 92

$\overline{\mathrm{R}}_{\mathbf{X}}^{(\mathbf{Y})}$

The class of dual relations of type $\mathbf{Y}$, page 92

Set

The category of sets, page 14

$\sigma^{\partial^{*}}$

The dual of the relation $\sigma$ under the clone duality for relations, page 99

s-LOC ${ }^{\mathbb{T}} \quad$ A local closure operator for classes of relations, page 81

$\mathrm{s}-\mathrm{Loc}^{\mathbb{T}}$

A local closure operator for sets of operations (in concrete categories), page 91

$\operatorname{Spec}(\mathbf{A}) \quad$ The spectrum of $\mathbf{A}$ (usually the set of prime filters or ideals), page 46

$\operatorname{Spec}^{*}(\mathbf{A}) \quad$ The set of $\cup$-irreducible prime filters among $\operatorname{Spec}(\mathbf{A})$, page 70

$\operatorname{Spl}(Y) \quad$ Pairs $\left(Y_{1}, Y_{2}\right)$ of pointwise incomparable non-empty connected subsets of $Y$, page 156

$\mathbb{T}$

$\uplus$

$\underline{\mathrm{M}}$

$\widetilde{\iota}_{i}^{n}$

$\widetilde{\pi}_{i}^{n}$

$\operatorname{ar}(f)$

$B_{\mathbf{A}}$

$f \triangleright \sigma$

$F^{\partial}$
A typeclass, page 78

The exclusive union, $X_{1} \uplus X_{2}:=\left(X_{1} \cup X_{2}\right) \backslash\left(X_{1} \cap X_{2}\right)$, page 59

The alter-ego of $\mathbf{M}$ (with our without discrete topology), page 22

The morphism $\left[\iota_{1}^{n}, \ldots, \iota_{i-1}^{n}, \iota_{i+1}^{n}, \ldots, \iota_{n}^{n}\right]$, page 33

The morphism $\left\langle\pi_{1}^{n}, \ldots, \pi_{i-1}^{n}, \pi_{i+1}^{n}, \ldots, \pi_{n}^{n}\right\rangle$, page 26

The arity of $f$, page 11

The basis of the Boolean group A, page 62

Indicates that the operation $f$ preserves the relation $\sigma$, page 76

The set $\left\{f^{\partial} \mid f \in F\right\}$, page 39 
$f^{\partial}$

$F^{(n)}$

$g \bar{\triangleright} \sigma$

$g^{\bar{\partial}}$

$I_{n}^{k}$

$J_{\mathbf{A}}$

$n \cdot \mathbf{X}$

$P_{n}^{p}$

$R^{(\mathbf{B})}$

$S_{k}^{n}$

$X^{\sharp}$

$\uparrow a$
The dual of $f$ under the clone duality, page 39

The set of $n$-ary operations among $F$, page 26

Indicates that the dual operation $g$ preserves the dual relation $\sigma$, page 92

The dual of $g$ under the clone duality $(-)^{\bar{\partial}}$, page 41

The set $\left\{\left[\iota_{i_{1}}^{k}, \ldots, \iota_{i_{n}}^{k}\right] \mid 1 \leq i_{1}<\ldots<i_{n} \leq k\right\}$, page 110

The clone of all projection morphisms over $\mathbf{A}$, page 28

The $n$-th copower of the object $\mathbf{X}$, page 18

The number of permutations of order $p$ on an $n$-element set, page 138

The class of relations of type $\mathbf{B}$ among $R$, page 78

The number of ways to partition a set of $k$ elements into $n$ non-empty subsets (Stirling number of the second kind), page 119

The set of all $x \in \mathbf{X}$ such that $\iota_{i}^{n}(x) \neq \iota_{j}^{n}(x)$ for all $i \neq j$, page 118

$\{b \geq a \mid b \in A\}$, the upset generated by $a$, page 46 


\section{Bibliography}

[AHS90] J. Adamek, H. Herrlich, and G. Strecker, Abstract and concrete categories, WileyInterscience, New York, NY, 1990.

[BH83] H.-J. Bandelt and J. Hedlíková, Median algebras, Discrete Math. 45 (1983), no. 1, $1-30$.

[Bir37] G. Birkhoff, Rings of sets., Duke Math. J. 3 (1937), 443-454.

[Bir46] — On groups of automorphisms, Revista Unión Mat. Argentina 11 (1946), 155-157 (Spanish).

[Bir67]_ Lattice theory, Third edition. American Mathematical Society Colloquium Publications, Vol. XXV, American Mathematical Society, Providence, R.I., 1967.

[Bor94] F. Borceux, Handbook of categorical algebra. 1, Encyclopedia of Mathematics and its Applications, vol. 50, Cambridge University Press, Cambridge, 1994, Basic category theory.

[BP75] K. A. Baker and F. Pixley, Polynomial interpolation and the chinese remainder theorem for algebraic systems, Math. Zeitschrift 143 (1975), 165-174.

[BS81] S. Burris and H.P. Sankappanavar, A course in universal algebra, Graduate Texts in Mathematics, vol. 78, Springer Verlag, New York, 1981.

[Bur67] G. A. Burle, The classes of k-valued logics containing all one-variable functions, Diskret. Analiz 10 (1967), 3-7 (Russian).

[BW87] S. Burris and R. Willard, Finitely many primitive positive clones, Proc. Amer. Math. Soc. 101(3) (1987), 427-430.

[CD73] P. Crawley and R. P. Dilworth, Algebraic theory of lattices, Prentice-Hall Englewood Cliffs, N.J., 1973.

[CD98] D. M. Clark and B. A. Davey, Natural dualities for the working algebraist, Cambridge studies in advanced mathematics, vol. 57, Cambridge Unviersity Press, Cambridge, 1998.

[CDFJ04] D. M. Clark, B. A. Davey, R. S. Freese, and M. G. Jackson, Standard topological algebras: syntactic and principal congruences and profiniteness, Algebra Universalis 52 (2004), no. 2-3, 343-376.

$\left[\mathrm{CDH}^{+} 03\right]$ D. M. Clark, B. A. Davey, M. Haviar, J. G. Pitkethly, and M. R. Talukder, Standard topological quasi-varieties, Houston J. Math. 29 (2003), no. 4, 859-887 (electronic). 
[CDJP08] D. M. Clark, B. A. Davey, M. G. Jackson, and J.G. Pitkethly, The axiomatizability of topological prevarieties, Adv. Math. 218 (2008), no. 5, 1604-1653.

$\left[\mathrm{CHK}^{+} 01\right]$ G. Czédli, R. Halaš, K.A. Kearnes, P.P. Pálfy, and Á. Szendrei, The join of two minimal clones and the meet of two maximal clones, Algebra Universalis 45 (2001), no. 2-3, 161-178, Conference on Lattices and Universal Algebra (Szeged, 1998).

[Coh65] P. M. Cohn, Universal algebra, Harper and Row, New York, NY, 1965.

[Csá83] B. Csákány, All minimal clones on the three-element set, Acta Cybernet. 6 (1983), $227-238$.

[Csá85] _ Completeness in coalgebras, Acta Sci. Math. 48 (1985), 75-84.

[Csá05] _ Minimal clones - a minicourse, Algebra Universalis 54 (2005), 73-89.

[Dav06] B. A. Davey, Natural dualities for structures, Acta Univ. M. Belii Ser. Math. (2006), no. $13,3-28$.

[DP02] B. A. Davey and H. A. Priestley, Introduction to lattices and order, second ed., Cambridge University Press, New York, 2002.

[DPW] B. A. Davey, J. G. Pitkethly, and R. Willard, The lattice of alter egos, preprint.

[Drb71] K. Drbohlav, On quasicovarieties, Acta Fac. Rerum Natur. Univ. Comenian. (1971), 17-20.

[DT05] B. A. Davey and M. R. Talukder, Dual categories for endodualisable Heyting algebras: optimization and axiomatization, Algebra Universalis 53 (2005), no. 2-3, $331-355$.

[GBGL08] T. Gowers, J. Barrow-Green, and I. Leader (eds.), The Princeton companion to mathematics, Princeton University Press, Princeton, NJ, 2008.

[Grä79] G. Grätzer, Universal algebra, second ed., Springer-Verlag, New York, 1979.

[Haz95] M. Hazewinkel (ed.), Encyclopaedia of mathematics. Vol. 1-6. A-Zyg, index, Kluwer Academic Publishers, Dordrecht, 1995, Translated from the Russian, Reprint of the 1988-1994 English translation.

[Isb80] J. Isbell, Median algebras, Trans. Amer. Math. Soc. 260 (1980), 319-362.

[Joh10] S. Johansen, Natural dualities for three classes of relational structures, Algebra Universalis 63 (2010), 149-170.

[Ker10] S. Kerkhoff, The essential arity of clones over algebras, Proceedings of the Olomouc Conference 2010, Contributions to general algebra, vol. 19, Heyn, Klagenfurt, 2010.

[Law63] W. F. Lawvere, Functorial semantics of algebraic theories and some algebraic problems in the context of functorial semantics of algebraic theories, Ph.D. thesis, Columbia University, 1963. 
[LP96] L. Lévai and P.P. Pálfy, On binary minimal clones., Acta Cybern. 12 (1996), no. 3, 279-294.

[Maš99] D. Mašulović, The lattice of clones of co-operations, Ph.D. thesis, University of Novi Sad, Yugoslovia, 1999.

[Maš06] _ On dualizing clones as lawvere theories, International Journal of Algebra and Computation 16 (2006), 675-687.

[Maš08]_, On clones of operations over finite boolean algebras, Algebra Universalis 59 (2008), 447-461.

[MMT87] R. N. McKenzie, G. F. McNulty, and W. Taylor, Algebras, lattices, varieties. Vol. I, The Wadsworth \& Brooks/Cole Mathematics Series, Wadsworth \& Brooks/Cole Advanced Books \& Software, Monterey, CA, 1987.

[Pál86] P.P. Pálfy, The arity of minimal clones., Acta Sci. Math. 50 (1986), 331-333.

[PK79] R. Pöschel and L. A. Kalužnin, Funktionen- und relationenalgebren, Deutscher Verl. der Wiss., Berlin, 1979.

[Pos41] E. L. Post, The Two-Valued Iterative Systems of Mathematical Logic, Annals of Mathematics Studies, no. 5, Princeton University Press, Princeton, N. J., 1941.

[Pös79] R. Pöschel, Concrete representation of algebraic structures and a general galois theory, Contributions to General Algebras, Proc. Klagenfurt Conf., May 1978 (1979), 249-272.

[Pös80] _ A general Galois theory for operations and relations and concrete characterization of related algebraic structures, Report 1980, vol. 1, Akademie der Wissenschaften der DDR Institut für Mathematik, Berlin, 1980.

[PR00] R. Pöschel and M. Rössiger, A general galois theory for cofunctions and corelations, Algebra universalis 43 (2000), 331-345.

[Pri72] H. A. Priestley, Representation of distributive lattices by means of ordered stone spaces, Bull Lonson Math. Soc. 324 (1972), 186-190.

[Qua95] R. W. Quackenbush, A survey of minimal clones, Aequationes Math. 50 (1995), $3-16$.

[Ros83] I. G. Rosenberg, Minimal clones I: the five types, Lectures in universal algebra (Szeged, 1983), Colloq. Math. Soc. Janos Bolyai, vol. 43, North-Holland, Amsterdam, 1983, pp. 405-427.

[Ros72] I. Rosenberg, A classification of universal algebras by infinitary relations, Algebra Universalis 1 (1971/72), 350-354.

[Słu39] J. Słupecki, Kriterium petnosci wielowartosciewych systemow logiki zdań, C. R. Seane Soc. Sci. Varsovie 32 (1939), 102-109.

[Sto36] M. H. Stone, The theory of representations of boolean algebras, Trans. Amer. Math. Soc. 40 (1936), 37-111. 
[Świ61] S. Świerczkowski, Algebras which are independently generated by every $n$ elements, Fun. Math. 49 (1960/61), 93-104.

[Sze86] Á. Szendrei, Clones in universal algebra, Séminaire de Mathématiques Supérieures [Seminar on Higher Mathematics], vol. 99, Presses de l'Université de Montréal, Montreal, QC, 1986.

[Szé89] Z. Székely, On maximal clones of co-operations, Acta. Sci. Math. 53 (1989), 43-50.

[Tay73] W. Taylor, Characterizing mal'cev conditions, Algebra Universalis 3 (1973), 351397.

[Tay86] W. Taylor, The clone of a topological space, Research and Exposition in Mathematics, vol. 13, Heldermann Verlag, Berlin, 1986.

[Tay93] W. Taylor, Abstract clone theory, Algebras and orders (Montreal, PQ, 1991), NATO Adv. Sci. Inst. Ser. C Math. Phys. Sci., vol. 389, Kluwer Acad. Publ., Dordrecht, 1993, pp. 507-530.

[TS09] V. Trnková and J. Sichler, All clones are centralizer clones, Algebra Universalis 61 (2009), 77-95.

[Wal07] T. Waldhauser, Minimal clones with few majority operations., Acta Sci. Math. 73 (2007), no. 3-4, 471-486. 



\section{Affirmation}

(a) Hereby I affirm that I wrote the present thesis without any inadmissible help by a third party and without using any other means than indicated. Thoughts that were taken directly or indirectly from other sources are indicated as such. This thesis has not been presented to any other examination board in this or a similar form, neither in this nor in any other country.

(b) The present thesis has been produced since October 2009 at the Institut für Algebra, Department of Mathematics, Faculty of Science, TU Dresden under the supervision of Prof. Dr. Reinhard Pöschel.

(c) There have been no prior attempts to obtain a $\mathrm{PhD}$ at any university.

(d) I accept the requirements for obtaining a PhD (Promotionsordnung) of the Faculty of Science of the TU Dresden, issued March 20, 2000 with the changes in effect since April 16, 2003 and October 1, 2008.

\section{Versicherung}

(a) Hiermit versichere ich, dass ich die vorliegende Arbeit ohne unzulässige Hilfe Dritter und ohne Benutzung anderer als der angegebenen Hilfsmittel angefertigt habe; die aus fremden Quellen direkt oder indirekt übernommenen Gedanken sind als solche kenntlich gemacht. Die Arbeit wurde bisher weder im Inland noch im Ausland in gleicher oder ähnlicher Form einer anderen Prüfungsbehörde vorgelegt.

(b) Die vorliegende Dissertation wurde seit Oktober 2009 am Institut für Algebra, Fachrichtung Mathematik, Fakultät Mathematik und Naturwissenschaften, Technische Universität Dresden unter der Betreuung von Prof. Dr. Reinhard Pöschel angefertigt.

(c) Es wurden zuvor keine Promotionsvorhaben unternommen.

(d) Ich erkenne die Promotionsordnung der Fakultät Mathematik und Naturwissenschaften der TU Dresden vom 20. März 2000, in der geänderten Fassung mit Gültigkeit vom 16. April 2003 und vom 1. Oktober 2008 an.

Dresden, den 07. April 2011 